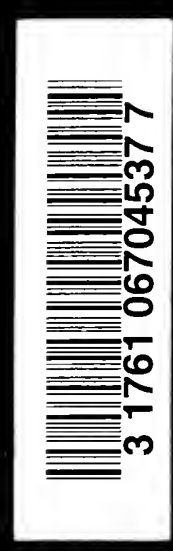

DISEASES OF THE STOMACH AND

'JUIIIR SURGICAI, TRENTMENT

MAYO ROBSON \& MOYNIHAN

SECOND KDITION 
WORKS BY MR. MAYO ROBSON.

DISEASES OF THE GALL-BLADDER AND BILEDUCTS. Third Edition.

Baillière, Tindall, \& Cox. IgO4.

A GUIDE TO THE INSTRUMENTS AND APPLIANCES REQUIRED IN VARIOUS OPERATIONS. Third Edition.

Casseli \& Co.

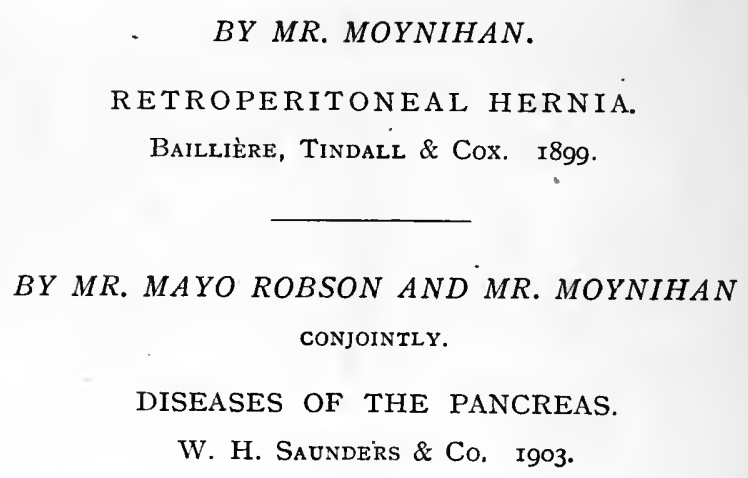


DISEASES OF THE STOMACH

AND THEIR

SURGICAL TREATMENT 

MTi

R

\title{
DISEASES OF THE STOMACH
}

\author{
AND THEIR
}

\section{SURGICAL TREATMENT}

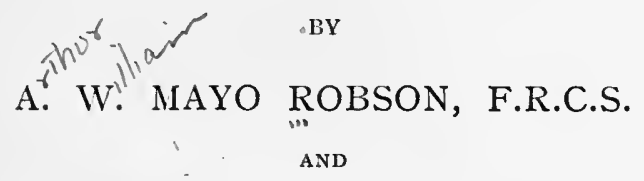

B. G. A. MOYNIHAN, M.S. Lond., F.R.C.S.

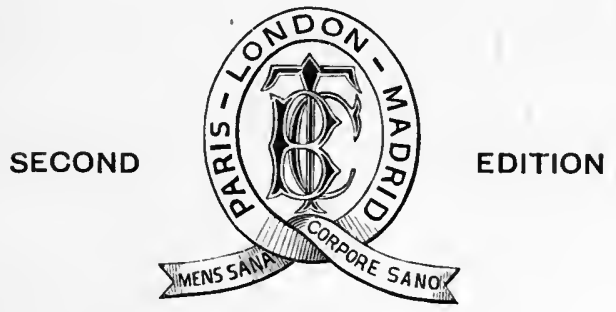

\footnotetext{
LONDON

BAILLIÉRE, TINDALL AND COX 8, HENRIETTA STREET, COVENT GARDEN 1904 


\section{Digitized by the Internet Archive}

in 2007 with funding from Microsoft Corporation 


\section{PREFACE TO SECOND EDITION}

IN the preface to the first edition of this work we ventured to forecast that in the near future there would be a great activity in the surgical treatment of the diseases of the stomach. This forecast has been abundantly justified. During the two years that have passed since then the number of papers, discussions, and addresses dealing with one aspect or another of the various surgical diseases of the stomach has been very remarkable. Indeed, it has been no light task to keep abreast of the literature of the subject.

Our own experience has been considerable. Our combined records show a total of over 600 operations. During the last two years the mortality has been greatly reduced. In cases of simple gastro-enterostomy, for example, the deathrate is well under 5 per cent., and many of these patients were operated upon when almost in a desperate plight as the result of hæmorrhage or of chronic indigestion with frequent vomiting. The malignant cases still show, and must necessarily always show, a greater mortality than this. This is due, of course, to the nature and extent of the disease, and to the extremely enfeebled condition or to the age of the patient. We may briefly sum up our experience of cancer of the stomach by saying that our tendency seems to be to the more frequent performance of gastrectomy as compared with gastro-enterostomy. Though the latter operation is sometimes remarkably successful, it cannot be doubted that 
even as a palliative operation there is much to be said in favour of partial gastrectomy.

The whole work has undergone a most careful revision. Indeed, the greater part of it has been rewritten, for where our knowledge has so greatly increased, the form and method of the first edition seemed inappropriate, and it was easier and more satisfactory to rewrite than to modify.

We desire to express our thanks to Dr. Campbell Thomson for the loan of three blocks used to illustrate the chapter on 'Acute Dilatation,' and to Dr. Porter Parkinson for the loan of one block, showing the perforation of a gastric ulcer in a child aged two. This illustration is reproduced from vol. i. of the Transactions of the Society for the Study of Disease in Children.

A. W. M. R.

8, Park Crescent, London, W.

B. G. A. M.

33, Park Square, Leeds.

June, 1904. 


\section{PREFACE TO FIRST EDITION}

THERE can, we think, be no need for an apologetic preface to a work dealing with the 'Surgery of the Stomach.' This branch of our art has made so great advances within the last few years that a review of its progress seems both fitting and desirable.

The present work took its origin in the Hunterian Lectures delivered by one of us at the Royal College of Surgeons of England in Igoo. The attention called to the subject by those lectures resulted in a large increase in our experience of the various gastric conditions amenable to surgical treatment. To deal fully with subjects lightly touched upon in the lectures, to expand, in fact, those lectures into a volume, was a work which the lecturer unaided would have been hard pressed to accomplish in the space of time within which it was felt desirable to publish the volume. A collaboration seemed therefore desirable, and as we had been associated in hospital work more or less closely since 1887 , when our relative positions were those of Honorary Officer and House Surgeon, the present collaboration seemed both natural and appropriate.

For every statement.made in the book we jointly hold ourselves responsible. The whole work has undergone a careful revision by both of us, and much of it has been frequently discussed while in preparation and after completion.

That the aid of surgery must be called in to deal with 
both simple and malignant disease of the stomach, in the future far more often than in the past, seems inevitable. We consider that we are justified in saying that our joint record shows that the risks of such surgery are far less than has been generally believed.

In support of this statement, one of us, in a series of over 200 operations on the stomach, can refer to the last seventyfive consecutive operations in his private clinic, including malignant and simple cases, with only one death (and that from accidental perforation on the twelfth day), and the other can point to a consecutive series of forty-seven hospital and private cases, with three deaths.

We take this opportunity to thank Dr. W. MacGregor Young, M.A., for his valuable assistance in illustrating the work by original drawings and diagrams, which will be found of great service in elucidating the text, and one of us also accepts the occasion of the preface to acknowledge the great help he received during the preparation of the Hunterian Lectures from Dr. Farquhar Macrae, now on the staff of the Western Hospital, Glasgow, but at that time assisting us in his private clinical work.
A. W. M. R.
B. G. A. M. 


\section{CONTENTS}

CHAPTER

I. ANATOMICAL CONSIDERATIONS - - - - $\quad$ - I

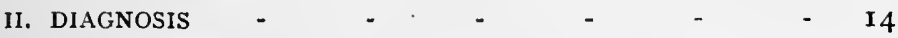

III. GASTROTOMY - $\quad$ - $\quad$ - $\quad$ -

IV. CONGENITAL HYPERTROPHIC STENOSIS OF THE PYLORUS 44

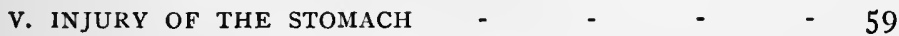

VI. SIMPLE TUMOURS OF THE STOMACH - $\quad$ - $\quad$ - 75

VII. CANCER OF THE STOMACH -

VIII. THE OPERATION OF GASTRECTOMY, PARTIAL AND COM-

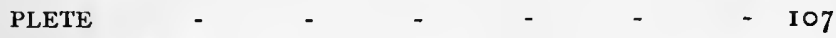

IX. GASTROSTOMY - $\quad$ - $\quad$ - $\quad$ -

X. UlCUS CARCINOMATOSUM - - - - - $\quad$ - 146

XI. SARCOMA OF THE STOMACH - - - - $\quad-$ I55

XII. GASTRIC ULCER - - $\quad$ - $\quad$ - $\quad$ - $\quad$ - I60

XIII. GASTRIC ULCER AND ITS TREATMENT - - - I

XIV. HYPERCHLORHYDRIA - $\quad$ - $\quad$ - $\quad$ - $\quad-202$

XV. THE OPERATION OF GASTRO-ENTEROSTOMY, WITH INDI-

CATIONS FOR ITS PERFORMANCE - $\quad-\quad-207$

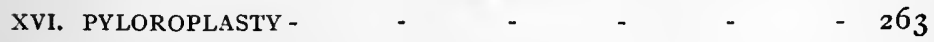

XVII. GASTRORRHAGIA $\quad-\quad$ - $\quad$ - $\quad-\quad-\quad 275$

XVIII. PERFORATION OF GASTRIC OR DUODENAL ULCER - 3OI

XIX. HOUR-GLASS STOMACH - $\quad$ - $\quad$ - $\quad-\quad-338$

XX. Dilatation of THE StOMaCh - - $\quad-\quad 378$

XXI. ACUTE Dilatation OF THE STOMACH- - $\quad$ - 389

XXI. ATONIC DILATATION OF THE STOMACH - 403

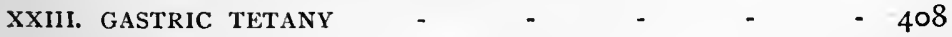

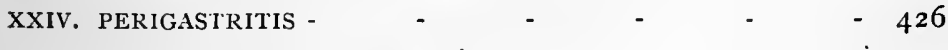




\section{CONTENTS}

CHAPTER

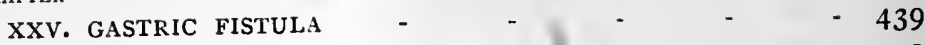

XXVI. GASTROPTOSIS -

- 448

XXVII. TUBERCLE-SYPHILIS

XXVIII. PHLEGMONOUS GASTRITIS

- 455

LIST OF AUTHORITIES -

$-463$

INDEX -

- 497 


\section{LIST OF ILLUSTRATIONS}

FIG.

I. Foreign Bodies successfully removed from the Stomach of a Girl

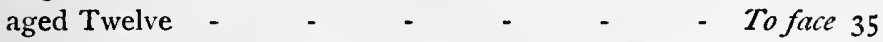

2. A Roll of Black Human Hair, 12 Inches Long, weighing $5 \mathrm{lb}$. 3 oz., removed successfully from a Girl of Twenty - To face 35

3. Photograph of Stomach from a Married Woman, aged Fortyone, who had suffered from Hæmatemesis at Seventeen and

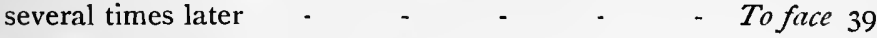

4. Bent Pins removed from Specimen 2,379 - $\quad$ - $\quad$ " 39

5. Pins removed from Specimen 2,379 - - - $\quad$ - $\quad$ " 39

6. Relations of Anterior Surface of Stomach. (Testut.) - - 63

7. Relations of Posterior Surface of Stomach. (Testut.) - - 64

8. Preceding Figures superimposed. (Forgue and Jeanbrau.) - 65

9. Villous Growth near Lesser Curvature, found Post-mortem in an Aged Wonian - $\quad$ - $\quad$ - $\quad$ - $\quad$ - $\quad$ - To face 75

ı. Polypus near Pylorus, which caused Death by Vomiting " 75

I1. Adenoma removed from Stomach - - - " 76

12. Polypi growing from the Mucous Membrane of the Stomach of a Gentleman, Seventy-six Years of Age, who suffered from

Constant Dyspepsia - $\quad$ - $\quad$ - $\quad$ - $\quad$ - $\quad 76$

I3, Polypus near Pylorus which caused Fatal Intussusception of

Duodenum in a Man aged Twenty-one - $\quad$ - To face 77

I4. Lymphadenoma of Stomach - - - $\quad$ - $\quad$ - $\quad$ " 77

15. Malignant Plaque in Stomach Wall-Polypoid Growth at the

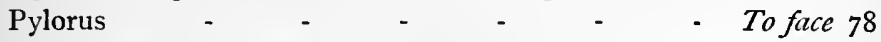

16. Villous Growth near Lesser Curvature, found Post-mortem " 78

17. The Lymphatics of the Stomach. (After Cunéo.) - - 81

18. Showing the Lymphatic Areas of the Stomach - - $\quad$ - 83

19. Cancer of Cardiac End of Stomach with Dilated CEsophagus

20. Cancer of Cardiac End of Stomach, associated with Cancer of the Lower End of the CEsophagus - - - - To face 106 
FIG.

2I. Cancer of Cardiac Orifice of the Stomach - To face 106

22. Showing Lines of Section of the Stomach in Partial Gastrectomy

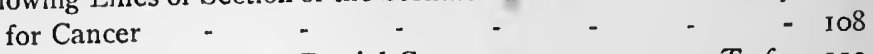
23. Gastro-enterostomy after Partial Gastrectomy - - To fuce I1 2 24. Cancer of Pylorus, producing Stenosis, in a Woman aged Thirty-six - - - - - - $\quad$ - To fuce II4

25. Perforation of the Stomach due to Sloughing Cancer - " 116 26. Extremely Small Stomach dependent on Neoplasm-'Leather-

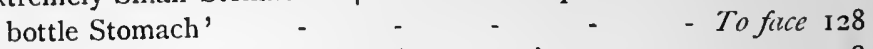

27. Colloid Cancer of Pylorus producing Stenosis - - " $\quad$ - 128

28. Jejunostomy-Adaptation of Witzel's Method - - $\quad$ - 130

29. Jejunostomy-Maydl's Method - - - - - $\quad$ - I3I

30. Gastrostomy. (Frank's Method modified by Mayo Robson.) - I37

31. Gastrostomy. (Frank's Method modified by Mayo Robson.) - $13^{8}$

32. Gastrostomy. (Frank's Method modified by Mayo Robson.) - I39

33 and 34. Gastrostomy. (Senn's Method.) - _ - - . $\quad$ I40

35 and 36. Gastrostomy. (Witzel's Method.) - - - I4I

37. Gastrostomy. (Kader's Method.) - - - - - $\quad$ - 142

38. Gastrostomy. (Kader's Method.) - - - - $\quad$ - I43

39 and 40. Depage's Gastrostomy - _ - _ n - $\quad$ - 144

4I. Multiple Round Ulcers - $\quad$ - $\quad$ - $\quad$ - $\quad$ - To face I6I

42. Acute Round Ulcers in the Stomach of a Woman aged Twenty To face I6I

43. 'Simple Chronic Ulcer' of Cruveilhier, or the 'Perforating Ulcer' of Rokitansky - $\quad$ - $\quad$ - $\quad$ - $\quad$ - To fuce I6I

44. A Small, Universally Ulcerated Stomach, 5 Inches Long, 6 Inches in Widest Circumference, with the Coats Three or Four Lines

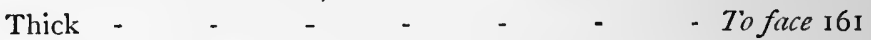

45. Chronic Ulcer of Posterior Wall of Stomach eroding Pancreas

To face $\mathbf{1 6 8}$

46. Large Chronic Ulcer, $3 \frac{1}{2}$ by $1 \frac{1}{4}$ Inches, on the Posterior Wall, adherent to Pancreas and Liver - - - To face I68

47. Chronic Ulcer of Stomach showing Characteristic Puckering and Contraction the Result of Healing - - To face 169

48. Pyloric Stenosis from Ulcer with Hypertıophy of Stomach " I69

49. Showing the Posterior Wall of the Stomach projecting through the Rent in the Transverse Mesocolon - - To face 227

50. Showing the Oblique Application of the Clamp to the Stomach

51. Clamps Side by Side ; the First Line of Suture - - - 228

52. The Stomach and Jejunum opened; the Inner, Hæmostatic

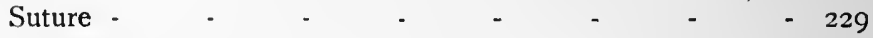

53. Inner Suture Complete - $\quad$ - $\quad$ - $\quad$ - $\quad$ - $\quad$ - 230

54. Clamps removed ; Completion of Outer Suture _ - $\quad 230$

55. Mayo Robson's Decalcified Bone Bobbins - $\quad$ - 232 
FIG.

56. Anterior Gastro-enterostomy, showing Proper and Improper

Locations of Opening. (After Mayo.) - - To face 234

57. Showing the Various Operations performed - - - 237

58. Showing the Varieties of Misdirected Current after Gastro-

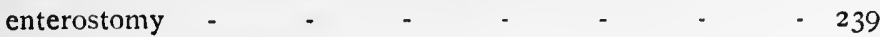

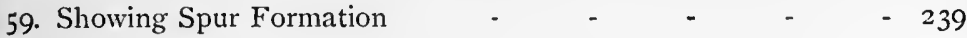

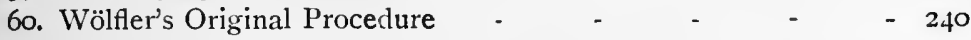

6r. Von Hacker's Operation - $\quad$ - $\quad$ - $\quad$ - $\quad$ - $\quad$ - $24 \mathrm{I}$

62. Von Hacker's Operation seen from Behind. (After Petersen.) - 24I

63. Gastro-duodenostomy. (Jaboulay.) - - - - - 24I

64. Gastro-duodenostomy. (Kümmell.) - - - - - 24I

65. Gastro-duodenostomy. (Villard.) - - - - - $\quad-242$

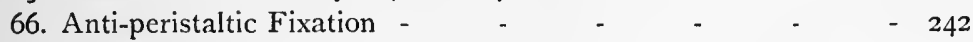

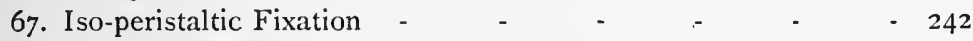

68. Gastro-enterostomy in Y. (Roux's Method.) - - - - 243

69. Entero-anastomosis as suggested by Lauenstein - - 243

70. Entero-anastomosis between Afferent and Efferent Loops.

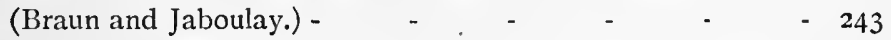

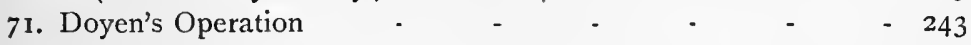

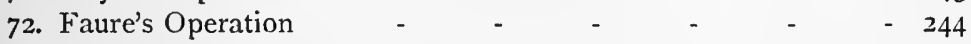

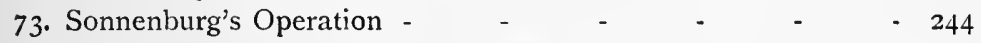

74. Gastro-enterostomy and Gastrostomy - $\quad-\quad e_{2} \quad-\quad-245$

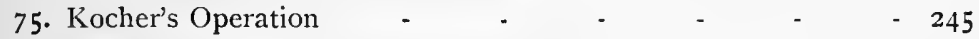

76. Suspension of Loop. (Hadra's Method.) - - - - 245

77. Suspension of Loop. (Lauenstein's Method.) - - - 245

78. I, Diagram of Condition found in Peptic Jejunal Ulcer, May, 1903, showing Perforation. 2, Diagram of Portion of Ulcer Adherent to, and Detached from, Anterior Abdominal Wall. 3, Diagram of Portion of Intestine Excised. 4, Diagram of Anastomosis Performed after the Enterectomy - - $\quad 252$

79. Pyloroplasty as Modified by the Use of a Decalcified Bone

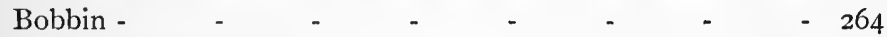

80. Finney's Operation - $\quad$ - $\quad$ - $\quad$ - $\quad$ - To face $27 \mathrm{I}$

81. Stomach showing Large Varicose Veins - - " $\quad 286$

82. Ulcer Midway between Cardiac and Pyloric Orifices, near Lesser Curvature, eroding Splenic Artery, on which is a Small

Aneurysmal Dilatation

83. Method of Exposure of Ulcer

84. Ligature of Bleeding-point

85. Perforation of an Ulcer in the Centre of an Hour-glass Stomach

To face 303

86. Perforating UIcer of the Stomach in a Child aged Two Years. (Porter Parkinson.) - - - - - - To face 303

87. Pyloric Ulcer showing Small Perforation, and Another causing Contraction of Pylorus 
FIG.

88. A Perforating Round Ulcer causing Death in a Lady Twentytwo Years of Age - $\quad$ - $\quad$ - $\quad$ - $\quad$ - $\quad$ - To face 304

89. Stomach showing Two Perforating Ulcers : one on the Anterior, one on the Posterior, Surface _. _ . . To face 312

9o. Pyopneumothorax - $\quad$ - $\quad$ - $\quad$ - $\quad$ - $\quad$ - 3I5

9r. 'Pyopneumothorax Subphrenicus': Subphrenic Abscess - 317

92. Posterior Perigastric Abscess - $\quad$ - $\quad$ - $\quad$ - $\quad-318$

93. Anterior Perigastric Abscess - $\quad-\quad$ - $\quad-\quad$ - 318

93a. Illustrating Site of Abscess in Case 6, and Position of Incision

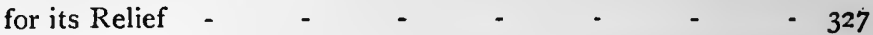

94. Cancer of the Cardiac End of the Stomach causing the Fundus to be partially shut off, so as to form a Large Pouch. (Modification of Hour-glass Stomach.) - - To face 342

95. Hour-glass Stomach; possibly Congenital, with Growth round Cardiac Orifice - To face 343

96. Cancer of Anterior Wall of the Stomach producing Hour-glass

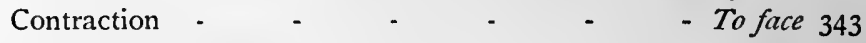

97. Types of Hour-glass Stomach - $\quad$ - $\quad-\quad-\quad 345$

98. Gastroplasty with the Aid of the Decalcified Bone Bobbin - 350

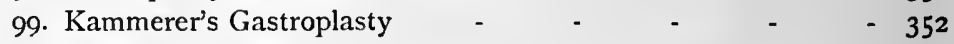

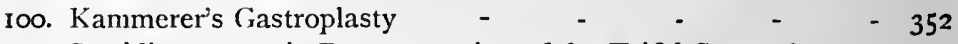

IOI. Semidiagrammatic Representation of the Trifid Stomach

To face 373

102. Acute Dilatation of the Stomach complicating Pneumonia and

Pleurisy. (Campbell Thomson.) - - - $\quad$ - To face 389

I03. Acute Dilatation of Stomach and Upper Part of Intestine.

(Campbell Thomson.)

- To face 394

104. Side View of the same Case - - - - $\quad$ - $\quad$ - 394

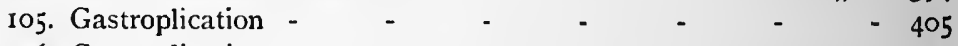

I06. Gastroplication - $\quad$ - $\quad$ - $\quad$ - $\quad$ - $\quad$ - $\quad$ - 406

107. Gastroptosis, the Pancreas showing above the Lesser Curvature $45 \mathrm{I}$

108. Cardiac Orifice of Stomach obstructed by a Gumma, which also involves the Adjoining Portion of the Liver - To face 459 


\section{SURGERY OF THE STOMACH}

\section{CHAPTER I}

\section{ANATOMICAL CONSIDERATIONS}

THE stomach, when normal, is a somewhat pear-shaped hollow organ situated in the epigastric and left hypochondriac regions, the larger part (fully two-thirds) being under cover of the liver and diaphragm, but the anterior surface in its lower part lying against the anterior abdominal wall. Its general axis is not quite vertical, but inclined obliquely, the cardiac orifice being on the left of the tenth dorsal vertebra, while the pyloric outlet is situated on the right of the eleventh or twelfth dorsal vertebra, about an inch to the right of the middle line, and nearer the anterior abdominal wall. The channel of entrance into the stomach is vertical, but that of exit is directed downwards, backwards, and to the right.

Between the cardiac and pyloric orifices the stomach is curved along both its upper and lower borders. The upper border, known as the lesser curvature, between 3 and 4 inches in length, is slightly concave, looks upward and towards the right, and is nearly all situated to the left of the middle line. The greater curvature is about three times the length of the "lesser, and, except for a small part near the pylorus, is convex throughout. Tracing it from the cardiac orifice, it is found to arch upwards to the left for about 2 inches; it then sweeps downwards and to the right until it reaches the middle line, where it again changes its direction and passes upwards, to the right and 
slightly forwards, till it reaches the pylorus. When the stomach is filled with air under medium tension, the lower border reaches to within $1 \frac{1}{2}$ to $2 \frac{1}{2}$ inches of the umbilicus, being rather higher in women than in men (Ewald, 'Diseases of the Stomach,' p. II5).

The only fixed part of the stomach is the cardiac orifice, which lies at a point on the posterior abdominal wall corresponding in front with the junction of the seventh left costal cartilage with the sternum.

When the stomach is distended, its position is somewhat altered. The greater curvature comes forward, and the anterior surface is thus caused to look upward as well as to the front, while the posterior wall faces downwards and backwards. The pylorus, which is freely movable, is under these circumstances usually displaced 2 or 3 inches to the right of the middle line.

In its greatest length the normal stomach measures rather over 10 inches, and its diameter at its widest part (toward the cardiac end) is between 4 and 5 inches. The normal capacity of the stomach varies greatly in different individuals, but is probably never more than $2 \frac{1}{2}$ pints (Ewald, 'Diseases of the Stomach,' p. I20).

The wall of the stomach, which is thinner than that of the œsophagus, though thicker than that of the small intestine, is composed of four layers-the serous, muscular, submucous, and mucous.

The serous coat is composed of peritoneum, which closely invests the whole viscus, except at the greater and lesser curvature, where the attachment is looser, allowing space for the larger bloodvessels.

The muscular coat consists of unstriped muscular fibres arranged in three more or less distinct layers-longitudinal, circular, and oblique. Of these three, that which is of special interest is the middle layer. This, toward the pylorus, becomes thicker and stronger, and when it reaches the exit from the stomach the circular fibres are heaped up so as to project inward into the lumen of the passage and form a distinct sphincter. Under normal circumstances the calibre of the pyloric orifice has a diameter of rather 
less than half an inch. This diminution in the calibre is caused entirely by the increase of the muscular fibres, the longitudinal fibres taking no part in the process, but passing on into the first part of the duodenum.

The submucous coat is composed of areolar tissue, and is the tunic in which the larger arterioles break up. The submucous tissue does not bind the mucous membrane very closely to the muscular layer, but permits considerable sliding one upon the other.

The mucous membrane, which is thickest in the pyloric region and thinnest in the great sac, is richly supplied with glands. The whole interior of the stomach is covered by a single layer of columnar epithelial cells. Scattered throughout the mucous membrane, but most abundant toward the pylorus, are small masses of lymphoid tissue, which are of importance as occasionally ulcerating in Hodgkin's disease.

The stomach receives its blood-supply from all three of the branches of the coliac axis. The coronary artery of the stomach reaches the viscus at the cardiac end, and, after giving off branches to the lower part of the œsophagus, it runs along the lesser curvature from left to right, and anastomoses with the pyloric branch of the hepatic artery. From the hepatic artery two branches in part aid the supply of the stomach. The smaller of these, the pyloric branch, reaches the stomach at the upper margin of the pylorus, and passes toward the left along the lesser curvature to inosculate with the terminal branches of the coronary artery. The gastro-duodenal artery passes behind the first part of the duodenum close to the pylorus, and, after giving off the superior pancreatico-duodenal branch, continues from right to left along the greater curvature of the stomach as the right gastro-epiploic artery. The splenic artery runs along the upper margin of the pancreas from right to left, and supplies several small branches to the stomach before it gives rise to the left gastro-epiploic artery, which lies between the layers of the gastro-splenic omentum, and is continued along the great curvature of the stomach to anastomose with the terminal branches of the right gastro-epiploic artery. 
From the two arches thus formed at the upper and lower margins of the stomach, vessels pass at right angles to supply the body of the viscus. The ultimate branches of these form an intricate network in the interglandular tissue, and from the capillaries round the mouths of the glands the veins take origin. These in the mucous membrane are fewer but larger than the arteries. They form a plexus in the submucous tissue, and then pass along with the arteries to form larger veins corresponding to the large arteries already describedviz., coronary, left gastro-epiploic, right gastro-epiploic, and pyloric veins. These all empty into the portal vein, either directly, as in the case of the pyloric and coronary veins, or by joining the superior mesenteric or splenic veins.

The lymphatics of the stomach, which are very numerous, arise in intimate relation with the gland tubules. They form a plexus of dilated lymph sinuses in the submucous tissue, and then pass toward the upper and lower margins, where they traverse a number of lymphatic glands which lie along the gastric borders of the small and great omenta respectively. Thence they pass to the coliac glands, which lie beside the aorta above the origin of the superior mesenteric artery, those of the lesser curvature following the course of the coronary vessels until the cardiac orifice is reached, when they turn down behind the pancreas to reach the cœliac glands. Those on the greater curvature run with the right gastro-epiploic vessels, and in part with the splenic vessels, and reach the same lymphatic glands. Thence they pass, together with the vessels which drain the mesenteric glands, to open into the lower end of the thoracic duct.

The nerves of the stomach, derived from the terminal branches of both pneumogastrics and from sympathetic branches from the solar plexus, are very abundant, and not only account for the very severe pain caused by ulceration, but also for the severe collapse produced by injury, though it is a mistake to suppose that manipulation of the pylorus is always attended by the severe shock suggested by the experiments made by Dr. Crile; for in many cases we have freely handled the stomach and pylorus, and operated on them without our patient experiencing more shock than 
would be expected. after any abdominal operation. When the pylorus is adherent and the parts have to be much dragged on, severe shock is not infrequently seen, but this is due to interference with the large sympathetic nerves and ganglia behind the pylorus.

The relation of the sympathetic nerves with the seventh, eighth and ninth spinal roots accounts for the superficial tenderness of the epigastrium in ulceration, and for the reflected left shoulder-blade pain. This is well shown in pyloric adhesions complicating cholelithiasis, where, though the pain is originally on the right, passing to the right infrascapular region, as soon as the pylorus becomes involved in the inflammation or tied down by adhesions, the pain passes also to the left subscapular region.

The anterior surface of the stomach is in relation above with the under surface of the left lobe of the liver and the diaphragm, and below with the abdominal parietes opposite the epigastric region. The posterior surface rests upon the transverse meso-colon, behind which are the pancreas and great vessels.

Above, the stomach is connected to the liver by the gastrohepatic omentum, in the free (right) border of which run the common bile-duct, the portal vein, and the hepatic artery. To the left of the cardiac orifice, between the œsophagus and diaphragm, is a small fold of peritoneum, the gastro-phrenic omentum.

The gastro-splenic omentum lies at the extreme left of the stomach, but is of more importance in the surgery of the spleen than of the stomach.

The great omentum is attached to the whole lower surface of the stomach, from which it passes down over the transverse portion of the colon.

The above description applies to the normal stomach, but there is probably no organ in the body which varies so much in size, position, and relations under pathological circumstances.

It may be so contracted, as in some cases of cancerous or simple stricture of the œsophagus, as to lie quite away from the surface and be tucked under the liver and ribs, forming 
little more than a thick-walled tube; on the other hand, in some cases of stenosis of the pylorus, it may be so dilated as to occupy every region of the abdomen, reaching even behind the pubes into the true pelvis.

The pylorus, instead of readily permitting passage of the forefinger, may be so contracted as barely to admit a No. 6 catheter, and cicatricial contraction in the body of the stomach may so reduce its calibre at a particular point as to render it less than that of the normal pylorus.

The free border of the lesser omentum may be so shortened as to anchor the pylorus higher up than normal, and as a consequence of ulceration the pylorus or other part of the stomach may be fixed to the parietes or to neighbouring organs in such a way as seriously to interfere with the gastric functions; or the lesser omentum may be so wide as to allow the stomach to descend well below the umbilicus, as in Glénard's disease, and under such circumstances the pancreas can be easily felt, and when the abdomen is opened even seen above the lesser curvature of the stomach.

Since the introduction of formalin as a hardening agent, several alterations have been made in our conception of the normal shape and position of several of the viscera. The following account of the anatomy of the stomach, based upon the observations made upon bodies hardened by formalin, was given by Professor Birmingham in the Journal of Anatomy and Physiology, vol. xxxv. We are indebted to him for permission to reproduce his description in full.

\section{The Stomach}

The Natural Form of the Stomach.-As seen in male bodies the viscera of which have been hardened by the intravascular injection of formalin, the empty stomach is of an attenuated or slender pear shape, generally flattened from above downwards, but preserving in its whole length more or less of an irregularly rounded or cylindrical form. In this, the empty condition, it is much bent on itself, particularly at the junction of the cardiac and pyloric portions, where the lesser curvature is generally reduced to a sharp angle. The long axis of the organ (tracing it from the 
summit of the fundus) is in the cardiac portion, directed from behind forwards and to the right, with a slight inclination downwards; then it bends almost at a right angle, and in the pyloric portion runs to the right towards the pylorus. Even in the empty condition the cardiac portion retains, as a rule, an appearance of rotundity, and rarely or never assumes a completely collapsed and flattened form; whilst the pyloric portion, contracting, assumes a narrow, cylindrical shape, and resembles in size and general appearance a bit of thick-walled small intestine.

It would appear that the collapsed, flat-walled, and flaccid bag, usually pictured as the empty stomach, does not represent its true condition, but is rather the result of postmortem softening, relaxation, and pressure. The stomach, like the bladder, and like other hollow viscera with muscular walls, is an extensile organ, capable of considerable expansion, and also of contraction, in virtue of the properties of its muscular coat. When food enters its cavity it expands, the expansion being proportionate to the amount of food that enters; when the food passes away or is absorbed, it contracts until its cavity is reduced to little more than a stellate lumen, containing, perhaps, some mucous gastric juice or gas. In other words, the stomach is not an inert bag, hanging down when empty like a flapping sail, with its walls in contact, but an active, living organ, capable of expansion and contraction, which adapts the size of its cavity to the amount of its contents.

Whilst all the other coats of the stomach are highly extensile, the mucous coat is so only to a slight degree; this coat must therefore be sufficient in amount to surround the cavity when largest ; hence, when the stomach is empty and the other coats contracted round the diminished lumen, the inelastic mucous coat is thrown into folds or rugæ, which project into and practically fill the cavity of the organ, and give to it, even in the empty state, a rounded or cylindrical form.

The Distended Stomach.-In the gradual passage of the stomach from the empty to the distended condition, we may recognise three stages.

First Stage.-This commences with an enlargement of the 
fundus in a backward direction, and is followed by an expansion of the cardiac portion, which passes upwards towards the left cupola of the diaphragm, and also to the left, displacing the coils of the transverse colon which lie over the stomach and to its left when the organ is empty. The pyloric portion for 3 or 4 inches remains contracted and cylindrical, the whole organ bearing some resemblance to a Florence flask, sharply bent at the junction of the neck with the body, and in this condition the stomach is frequently found after death.

Second Stage.-As distension goes on, the lesser curvature opens out, the pyloric portion (with the exception of its last inch) becomes distended, but its junction with the cardiac portion is, as a rule, still marked by a faint constriction, most evident at the curvatures, which persists until distension is almost complete.

Third Stage.-A further general expansion of the whole stomach takes place; the diameters of both cardiac and pyloric portions, as well as the length of the organ, are increased; the great curvature presses forwards against the anterior abdominal wall in front, where the restraining influence of the ribs is absent. The pyloric end for about I inch $(2.5$ centimetres) from the pylorus remains narrow (constituting the pyloric canal of Jonnesco), but to the left of this it is dilated, forming the antrum pylori, which is most distinct at the great curvature. By the increase in length of the organ, the antrum is carried a considerable distance to the right beneath the liver-even further than the pylorus itself-so that the terminal part of the stomach is directed backwards in order to reach the pylorus, which latter rarely passes more than 2 inches to the right from its normal position-namely, in the empty condition, within half an inch (I2 millimetres) of the middle line. And, finally, as the stomach becomes distended, it gradually becomes more oblique, so that in the distended condition the long axis of the posterior two-thirds of the organ is directed forwards, downwards, and to the right, and it forms an angle of about 40 to 45 with both the horizontal and sagittal planes. This obliquity is due to the ascent of the fundus and adjacent parts of the upper surface into the 
cupola of the diaphragm during distension, and the descent of the under surface as it passes forwards towards the yielding anterior abdominal wall, where it is supported only by intestines.

There is, however, as pointed out by Jonnesco, no distinct rotation of the organ on its long axis, no turning of the great curvature more forwards, nor of the so-called anterior surface more upwards.

In the change from the distended to the empty state, these stages are reversed: the whole stomach is contracted or drawn in from all directions towards the lesser curvature ; this latter is bent upon itself to an acute angle, and, the long axis of the organ becoming less oblique, approaches the horizontal.

Although this description of the shape and direction of the stomach is at variance with the generally accepted accounts, it is based upon the examination of a considerable number of hardened bodies, and has been found to apply so generally in well-formed males that it is advanced here as the condition most frequently found during life, or immediately after death.

In connection with the usual description-viz, that the cardiac two-thirds or three-fourths of the stomach is placed vertically, it may be pointed out that the antero-posterior depth of the portion of the abdominal cavity in which this part of the stomach lies is often over 5-or even 6-inches, and this with a stomach the greatest diameter of which is 4 or $4 \frac{1}{2}$ inches. Again, with the arrangement of the pancreas and transverse mesocolon which is found to exist in hardened specimens, and which will be described in connection with the stomach bed, it is impossible for the cardiac portion of the stomach to lie vertically without displacement of the pancreas and other parts. It must, however, be admitted that in the female, as a result of tight-lacing, the stomach is often found to assume an abnormal and almost vertical position; but this condition is associated with displacement of other abdominal organs in the neighbourhood.

It should perhaps be mentioned in this connection that the posterior part of the under surface of the stomach, as far 
forwards, namely, as the anterior edge of the pancreas, is nearer to the horizontal than the portion in front of it. This is due to the firm support which the kidney and pancreas afford to the former part. Indeed, there is often a distinct bend in this surface corresponding to the anterior edge of the pancreas.

In brief, it may be said that the stomach when empty is contracted, not collapsed; that it assumes a narrow, attenuated shape, its cavity being practically obliterated, and its pyloric portion contracted to the size of small intestine; and, in addition, that its long axis lies in an almost horizontal plane. With distension there comes a general enlargement of the various diameters, an elongation of the whole stomach, with a consequent passage of its pyloric portion to the right beneath the liver, the development of the antrum pylori, and a general inclination of the organ from behind downwards and forwards without any rotation.

Surfaces.-There is little doubt, considering the position of the empty stomach, that its two surfaces should be called superior and inferior, rather than anterior and posterior, respectively. The superior is the more convex and the more extensive of the two.

If the attachment of the lesser omentum be traced towards the cardia, it will be found to wind gradually on to the upper surface, so that the œsophagus joins the stomach rather on its upper surface than at the lesser curvature, as described in a paper in the Journal of Anatomy and Physiology, October, I 896 .

The Stomach Chamber and Stomach Bed.-The portion of the abdominal cavity in which the stomach lies has such definite boundaries and such constant surroundings that it seems to merit the title of stomach chamber, particularly as the boundaries or walls of the space-which is completely filled by the distended stomach-do not collapse when the stomach is empty, but maintain the form of the cavity, which then becomes occupied by the transverse colon-as a rule-which doubles up over the stomach when this latter is empty.

The chamber presents an arched roof, an irregular sloping floor, and an anterior wall. The roof is formed on the right 
by the visceral surface of the left lobe of the liver, and in the rest of its extent by the left cupola of the diaphragm, which arches gradually downwards behind and on the left to meet the floor.

The floor, or stomach bed, is a distinct sloping shelf; not a mere indiscriminate collection of underlying viscera, but a definite bed, constant in its formation, on which the under surface of the stomach rests, and by which the organ is supported. The bed is formed behind by the top of the left kidney (with its suprarenal capsule) and the gastric surface of the spleen, both of which, taken together, form a fairly regular concave surface, sloping backwards and upwards to meet the roof, and thus completing the posterior part of the chamber, which receives the fundus of the stomach. In front of the spleen and kidney the bed is formed, first, by the wide upper surface of the pancreas, then by the transverse mesocolon sloping forwards and slightly downwards from the anterior edge of the pancreas to the colon, which latter completes the floor anteriorly. This portion of the transverse mesocolon on which the stomach rests is stretched over and buoyed up by a large mass of small intestine (jejunum), which invariably will be found packed in beneath the mesocolon on the front of the left kidney when the transverse colon is turned up.

Finally, the anterior wall of the stomach chamber is formed by the abdominal wall between the ribs on the left and the liver on the right side.

Behind, as already indicated, the chamber is completed by the meeting of the floor and roof, but the line along which the two meet is variable; for sometimes the spleen extends up for but a little way at the back of the chamber, whilst in other cases it constitutes the greater part of its posterior boundary, and forms a cuplike surface on which the inferior aspect of the fundus of the stomach rests.

This chamber, as already mentioned, is completely filled by the stomach when the organ is distended. When, on the other hand, the stomach is empty and contracted, it still rests on the floor or stomach bed, but occupies only the lower portion of the chamber, whilst the rest of this space is 
filled by the transverse colon, which turns gradually upwards as the stomach retracts, and finally comes to lie both above and in front of that organ and immediately beneath the diaphragm - a fact of some importance in clinical examination of this region. Possibly one of the reasons why the transverse colon is so tortuous and its mesentery so long is to permit of its doubling up in the manner described in order to fill the space vacated by the contracted stomach. Sometimes part of the small intestine, too, may be found in the chamber.

The Pyloric Portion of the Stomach and the Antrum Pylori.When the stomach is empty the pyloric portion for a distance of 3 or 4 inches is contracted and cylindrical; it resembles in appearance a bit of thick-walled small intestine, and runs transversely to the right, lying, as a rule, beneath the left lobe of the liver. When the stomach is distended, this part is carried to the right beneath the quadrate lobe, and its terminal part is directed backwards, or even backwards and to the left, to reach the duodenum; at the same time it becomes curved like an italic $S$ placed horizontally. This $S$-shaped curve (which is maintained by the ligamenta pylori). gives rise to two grooves, one at the upper, the other at the lower border and nearer to the pylorus. The first curve of the $S$-i.e., that nearest to the cardiac end-is convex downwards and forwards, and this, becoming more prominent with distension, forms the antrum pylori. The terminal curve of the $S$ extends up to the pylorus; it is nearly $x$ inch in length, and it appears never to become dilated to any noticeable extent. This is the part described by Jonnesco as the pyloric canal.

When examined post-mortem in the ordinary way, the pyloric aperture, viewed from the duodenal side, is somewhat oval in form, and closely resembles the external os uteri, as pointed out by Cunningham. From the opposite side it presents an irregular or stellate appearance, owing to the rugæ of the gastric mucous membrane being continued up to the orifice. In formalin-hardened bodies with empty and contracted stomach and duodenum the aperture is closed, and presents a stellate or purse-mouth appearance when 
viewed from either side, the appearance of firm closure being much more marked on the gastric than the duodenal aspect. In hardened bodies with distended stomach and duodenum, the aperture, which is somewhat oval, is practically closed, and from the duodenal side resembles the os uteri. But both in the empty and the distended state the pylorus seems to be a tubular narrowing, extending over half an inch to an inch of the canal, rather than an abrupt constriction. On section, it will also be remarked that the muscular thickening which produces the valve is gradual in its development when traced from the gastric side, but that it ends abruptly as the duodenum is reached.

When the stomach is empty the pylorus lies about 2 inches below, $I_{2} \frac{1}{2}$ inches to the right, and the same distance in front of the cardia; in the distended condition its position is about $2 \frac{1}{2}$ or 3 inches below, 2 to $2 \frac{1}{2}$ inches to the right, and 2 inches in front of the cardia.

We have never seen a really patent pylorus in a hardened body, with either an empty or a distended stomach, which leads us to think that in its ordinary condition the pylorus is naturally closed, and that the opening of the orifice (like that of the anus) is an active process.

The ' uncovered area'-that is to say, the small triangular surface of the stomach which is free from peritoneum-is situated on the inferior (or posterior) surface, below and to the left of the cardia. It is irregularly triangular in shape, and measures about 2 inches in width and $I \frac{1}{2}$ inches from above downwards. From its left angle the attachment of the great omentum (gastro-splenic part) starts; and at its right angle the coronary artery reaches the stomach. 


\section{CHAPTER II}

\section{DIAGNOSIS}

ALTHOUGH diseases of the stomach naturally first come under the notice of the physician, the time has passed when the surgeon can feel content to accept and act on the diagnosis already made for him by his medical colleague, leaving with the latter the responsibility of a possible error; rather, he must himself go over the whole of the medical evidence, and be prepared to supplement it by surgical methods, should such be required to elucidate the case or to render possible an accurate diagnosis.

The diagnosis of any case of gastric trouble necessitates. both a general and a special inquiry. The former, which involves the questions of age, sex, occupation, habits, mental, moral, and physical condition, and the history both of the patient and the disease, can be best discussed when considering the special disease; the latter includes a consideration of all the information which the surgeon can elicit by a physical examination.

Inspection.-Inspection will always constitute our first effort in diagnosis, and it is important that the patient should be in the dorsal decubitus, lying comfortably in a warm and light room with the abdomen exposed to view. Dilatation of the stomach can often be ascertained by inspection, and it may in extreme cases be seen to occupy every region of the abdomen. If the dilatation is atonic, the swelling persists without visible peristalsis; but if it is due to narrowing of the pylorus, visible peristalsis from left to right can frequently be plainly seen. Tumours of the pylorus and of the body of the stomach are frequently visible 
through the abdominal wall, and in an early stage they may be seen to move downwards on inspiration; this more especially applies to cases of cancer, since in tumours depending on ulcer of the pylorus adhesions form early, and fix the stomach under cover of the liver. Inspection will reveal the shallow, rapid, costal breathing induced by pain at the commencement of peritonitis, and by distension in the later stages, just as it will demonstrate the sighing respiration in internal hæmorrhage, and the irregular, catching breathing in diaphragmatic peritonitis or subphrenic abscess. It will show the fixed and bulging ribs in subphrenic abscess, and the swelling in the upper abdominal region when the pus tends to make its way forwards.

Palpation.-Palpation immediately follows inspection, and, of all methods which are adopted for diagnosis, it is the one which we can least afford to omit. With the patient supine in bed or on a couch, the head slightly elevated on a cushion, and the knees drawn up, the muscles of the abdomen are relaxed as much as possible. They can then easily be kept relaxed by talking to the patient, so as to divert his attention. The flat warm hand placed on the abdomen at once perceives any irregularity or abnormality. If the upper abdomen is rigid, it will raise the suspicion of local peritonitis, especially if, in addition, there is tenderness on pressure. Should there be a tumour, its nodular character, if malignant, will easily be felt; and if it be cancer of the pylorus in an early stage, it will in all probability be freely movable. If there be malignant disease of the body of the organ, the tumour will move downward on inspiration, but usually not freely from side to side, though we have seen a growth of the centre of the stomach forming an hour-glass contraction as freely movable as a pyloric tumour sometimes is.

It will be found better, as a rule, to slide the hand over the abdomen, and not to raise it from the surface, thus avoiding fresh defensive contraction of the muscles, which will occur if the hand be raised and replaced on the abdomen. It may sometimes be advisable to employ gentle massage in order to elicit the presence of a tumour of the body of the 
stomach, or a swelling of the pylorus due to muscular spasm, or visible peristalsis from left to right as in pyloric stenosis.

In some cases of ulcer a distinct tumour may be present at one time, owing to the contraction of the muscular coat, and absent at another, the tumour only being distinctly felt when the spasm is present. In diagnosing between atonic dilatation of the stomach and that due to obstructed pylorus, the vermicular contractions felt under the palpating hand are of the first importance. Where, as occasionally happens, palpation is difficult on account of rigidity of the muscles or of obesity, an examination under an anæsthetic may be advisable. Bimanual examination, with one hand in the loin and the other on the surface of the abdomen, will frequently give increased information as to the size of the tumour.

A tender and rigid epigastrium suggests ulcer of the stomach, and the situation of the tenderness is frequently a good guide to the site of the ulcer; for instance, if the tenderness be under the left costal margin and the left rectus be rigid, especially if the patient be relieved by assuming the dorsal decubitus, in all probability the ulcer will be on the anterior surface, whereas if the tenderness is on the right of the middle line, between the umbilicus and the right costal margin, the probability is that the pylorus is the affected part.

When there are symptoms of ulceration without marked epigastric tenderness, and with increase of pain on dorsal decubitus, and relief is found by lying on the face, the probability is that the ulcer is on the posterior wall of the stomach, in which case there will usually be found a tender spot opposite the tenth dorsal vertebra on the left of the spine. It is important to distinguish between surface tenderness due to reflected pain and pain on deep pressure due to the disease itself.

Palpation will usually bring out the character of the tumour as to hardness and softness, smoothness or irregularity. A nodular character of the tumour, especially if associated with fluid in the peritoneal cavity, usually indicates malignant disease. 
Pulsation in an epigastric tumour is often felt, but if the swelling be due to cancer or other tumour of the stomach, it will be non-expansible.

Fixation of a tumour is suggestive of antecedent perigastritis, especially if tenderness be present; but it may also be dependent on infiltration due to malignant disease. A change of posture may be useful; for instance, with the patient on his left side a pyloric tumour will frequently change its position considerably-especially is this so in the early stages of pyloric cancer. Or, by making the patient assume the knee-elbow position, it may be more easy to distinguish and locate a small tumour at the pylorus or on the anterior surface of the stomach, as in this position the mass may be more readily felt by the hand placed flat on the upper abdomen than with the patient lying on his back.

A modification of palpation is succussion, which is of considerable use in determining the presence of a splash in a dilated stomach. It may be safely asserted that if the stomach splash can be obtained habitually three hours after a meal the stomach is abnormally dilated. No succussion splash should ever be obtained two hours after a test breakfast or five hours after a full meal, otherwise there is motor insufficiency, which may be confirmed by washing out the stomach and finding remnants of the meal.

The motility of the stomach is seriously affected by organic disease, but not by neurosis.

Palpation of the stomach may be made more distinct by distending the cavity of the stomach with gas. In this way one can diagnose hour-glass contraction, as well as ordinary dilatation, and can estimate the size of the cavity or cavities.

In subphrenic abscess on the right side, palpation will show if the liver is depressed, and this will form a link in the chain of evidence going to show that the abscess is between the liver and diaphragm, and not in the liver itself.

Percussion.-Deviation from the normal size of the stomach can usually be ascertained by percussion, first with the patient recumbent, and afterwards by mapping out the region of dulness when the patient is erect after he has drunk freely of water. After distending the stomach with gas, either 
through a tube introduced through the mouth or by giving from 40 to 60 grains of carbonate of soda in solution, followed by 30 grains of tartaric acid, percussion readily demonstrates the outlines of the stomach, and shows the position of any tumour, both with regard to the orifices and to the greater or lesser curvature; and it also enables the diagnosis to be made between a pancreatic and a gastric tumour.

If distension occurs after the administration of the carbonate of soda, before the acid dose has been given, it shows the presence of free acid, and is, primâ facie, in favour of hyperchlorhydria and possibly ulcer, rendering the presence of cancer extremely improbable.

Percussion is also of use in demonstrating the presence of free fluid in the peritoneal cavity due to the rupture of a gastric ulcer, and for showing the absence of liver dulness under the like circumstance when sufficient free gas is present in the peritoneal cavity.

It must not, however, be forgotten that dulness in the flanks may be dependent on distension of the colon with fluid, and the similarity to free fluid in the peritoneum may be accentuated by change of posture, increasing the dulness in the dependent area and creating resonance in the elevated area. Again, resonance in the flanks may be created by distension of the colon with gas, and excessive distension of the colon may lead to error in diagnosis by pushing the liver well under cover of the diaphragm, thus leading to the belief that the liver dulness is abolished by free gas in the peritoneal cavity. We have known this to occur in a case of ruptured extra-uterine gestation which was thought to be one of rupture of a gastric ulcer.

Where a tumour is manifest to inspection or rendered evident by palpation, percussion is useful in eliciting the presence of a hollow viscus in front of it, as in diagnosing between a pancreatic and a gastric tumour; for in the former case light percussion will give resonance in front of the tumour, whereas in the latter light percussion will reveal dulness.

Auscultation.-Auscultation is of much more importance in 
the diagnosis of chest than of abdominal conditions, but it is sometimes useful in demonstrating the splashing sounds in gastric dilatation, and the gurgling or metallic sounds in subphrenic abscess containing gas. In the diagnosis of stricture of the œsophagus, where gastrostomy is in question, the gurgling sound on fluid reaching the stomach is usually delayed several seconds in the presence of a constriction. Before performing gastrostomy it is of the first importance to be certain of the diagnosis. Dr. Ogston has pointed out that in a healthy person food occupies about four seconds in passing from the mouth to the stomach, and that if the ear be placed 3 inches below the angle of the left scapula an amphoric rushing sound is heard when fluid enters the stomach. But when there is a stricture of the gullet, it will usually be found that fourteen or sixteen seconds elapse before fluid reaches the stomach. This is a valuable addition to the ordinary well-known means of diagnosis.

In this connection it may be pointed out that spasm of the pharynx or of the œsophagus at times closely resembles organic stricture; hence, it may sometimes be necessary to make an examination with the patient anæsthetized. In estimating the size of the stomach by percussion, greater precision may be obtained by the observer applying the mouth of a stethoscope over the stomach while an assistant performs the percussion, when the altered note is readily recognised on the borders of the stomach being reached.

Pain.-Though pain may be absent in some few examples, it is present at some stage of the disease in the greater number of stomach ailments calling for surgical treatment, and its character and course frequently afford the most valuable means at the disposal of the surgeon for interpreting and diagnosing ailments which present few physical signs.

The history of the onset of pain may show the local origin of more general trouble. For instance, in the diffused pain of general peritonitis due to the rupture of a gastric ulcer it is of the utmost importance to know whether it was preceded by pain after food and by epigastric tenderness, so that an exploration of the abdomen may be made over the site of the disease, and thus a great saving of time may be effected in 
the operation. In one case of ruptured gastric ulcer that we operated on, there was absolutely no previous symptoms of stomach disorder, and thinking, from the profound collapse, the sudden onset, the presence of fluid in the abdominal cavity, and the history of a missed period followed a fortnight later by irregular uterine bleeding, that the case was one of ruptured extra-uterine gestation, the first incision was made above the pubes.

As a rule, however, the pain from perforated gastric ulcer, though ultimately diffused, will have been first felt in the epigastrium, and the history will disclose the past existence of pain after the ingestion of food, and probably indigestion of long standing; there will also frequently be a history of reflected pain in the left scapular region and of tenderness to the left of the spine from the ninth to the twelfth dorsal vertebræ, though in some cases of pyloric ulceration, with adhesions to the gall-bladder and liver, we have found the pain reflected to the right scapular region.

Obscure recurrent abdominal pains may be dependent on a stricture or kink at the pylorus produced by adhesions, which, though not sufficient to cause obstruction, may produce considerable distress and disability. In this class of cases the local and reflected pain frequently affords much help.

Instrumental Aids to Diagnosis.-In subphrenic abscess the exploring syringe affords useful aid in diagnosing the presence of pus and enabling the surgeon to decide on the exact site of the incision for its evacuation.

Esophageal bougies are employed in cases of dysphagia, not only for treatment, but to ascertain the site of the obstruction and its nature, when the question of gastrostomy arises. We prefer instruments made after the pattern of bougies $\dot{a}$ boule employed in urethral cases; they are not generally known, but they present great advantage, both in the ease and safety with which they can be utilized, and the facility with which they can be employed to dilate a stricture.

In a case of starvation from stricture of the œsophagus, the question of gastrostomy has to be considered; but it must first be shown that the disease is incapable of being overcome 
by other means. Simple stricture, which is a rare condition, may frequently be efficiently treated by dilatation. Cancerous stricture, on the other hand, cannot be safely dilated, and will require other treatment. If there be a history of steady loss of flesh and gradually increasing dysphagia in a middleaged or elderly patient, malignant stricture will be suspected; and if on passing a bougie it be arrested just before entering the stomach, the diagnosis is rendered very probable; while if there be slight bleeding caused by the gentle use of the bougie, little doubt can be left.

Spasm is most frequent in the pharynx, though it may occur in any part of the gullet. It usually occurs in subjects younger than those who are affected by malignant stricture, and nearly always in women. It is, however, frequently associated with other nervous symptoms, it varies in intensity, and it usually yields to the passage of bougies without difficulty. If, however, a bougie cannot be passed in any case where spasm is suspected, an anæsthetic will at once settle the doubt, as under its influence a full-sized bougie usually reaches the stomach when the stricture depends on spasm alone. It is noteworthy that in cases of spasm the larger sizes of bougies are more likely to pass than the smaller, while in organic stricture the exact opposite holds good.

Electric illumination may sometimes be useful. It is accomplished by an apparatus consisting of a small electric lamp, fixed to the end of a bougie, which is passed into the stomach. If the stomach is full of water, an illuminated area is seen, which corresponds exactly to the limits of the organ. It is best demonstrated in the erect posture and in a dark room. In this way abnormal dilatation of the stomach can be detected, and a tumour appears as a dark spot in the light field.

With regard to the Röntgen rays, we have found both the screen and the skiagram of use in demonstrating the position of impacted coins, metallic anastomosis buttons, and other foreign bodies. The Röntgen rays have also been suggested for localizing the position and size of the pyloric orifice, after letting the patient swallow balls of different sizes made of 
bismuth nitrate or carbonate coated with keratin, which will be only temporarily arrested at the pylorus if it be patent, and the keratin covering of which will be dissolved on reaching the intestine, keratin not being soluble in the gastric juice. The gastro-endoscope has not yet proved to be of any material service in diagnosis.

Vomited Matters and the Contents of the Stomach.-With regard to vomit, the first thing to consider is the quantity vomited at one time. Nurses should be trained to estimate this carefully, and also to preserve specimens on all occasions. In dilated stomach vomiting usually does not occur more than once daily, sometimes only every second or third day, and the quantity at any time is correspondingly large.

In ulcer of the stomach a considerable portion of the last meal may be brought up within an hour or two of its ingestion, and the pain it has caused be thereby relieved.

The smell should also be considered, a yeasty smell being characteristic of dilatation of the stomach ; a habitually fœetid odour, of cancer of the stomach; and a feculent odour, of intestinal obstruction.

Vomit is usually acid in reaction; but it may be alkaline in some cases of chronic dyspepsia, or when there is much blood present.

The most important abnormal constituent of vomit is blood. In large quantities its nature is obvious, and the event is suggestive of simple ulcer; but in cirrhosis of the liver profuse hæmatemesis may occur owing to rupture of dilated veins. In smaller quantities the vomit has a characteristic dark appearance, resembling coffee-grounds, and this may be due to cancer or simple ulcer. When the existence of blood in vomited matter is doubtful, the most reliable guide is the hæmin test, which may be done in the following manner :

Evaporate a small quantity of the gastric contents to dryness, powder the residue and place some along with a crystal of common salt on a microscopic slide, add a drop of glacial acetic acid and boil over a spirit lamp, cover with a coverglass and examine under a high power for the small darkbrown crystals of hæmin. As a rule, it is not necessary to 
add sodium chloride, since fresh blood contains sufficient of it; but, since excess of the salt does not interfere with the reaction, it is well to use a crystal or two.

In cancer of the stomach, blood is frequently present in the vomit-often in small, sometimes in considerable, only rarely in large quantity.

Pus is sometimes, but not often, vomited. In considering both pus and blood in a fluid said to have been vomited, it must be remembered that when large quantities of fluid are expelled from the lungs-e.g., on the rupture of an empyema into the lung or a profuse hæmoptysis-the sensation to the patient is often as if vomiting had occurred. The presence of food and the general absence of frothiness will help to distinguish true vomit, while vomited blood is generally much darker than blood from the lungs. But the only reliable way to make the distinction is to inquire carefully into the facts of the occurrence. Pus in the vomit may arise from an empyema of the gall-bladder, or a pancreatic or other abscess bursting into the stomach or esophagus.

Examination by the microscope of vomited material is usually of secondary importance, but it sometimes affords great assistance, as in the case of a subdiaphragmatic abscess bursting into the lung referred to later, where the presence of half-digested muscular fibres and the absence of elastic tissue distinctly proved the source of the pus to be from the stomach, and not from an abscess of the lung or an empyema, and in some cases of cancer where portions of growths or groups of cells are occasionally obtained by means of lavage. In dilatation of the stomach the sarcina ventriculi is frequently to be seen, together with yeast cells. In cancer, where macroscopically there is no evidence of blood, red blood corpuscles may often be found on microscopic examination.

In reference to the diagnosis of malignant disease of the stomach, the relative abundance or absence of free hydrochloric acid has been pointed out as being of importance by Ewald. In order to determine its existence, the patient should take a 'test breakfast,' consisting of a cup of weak tea and a little dry toast. An hour later the stomach-tube 
should be passed, and the contents of the stomach drawn off. These are to be tested by Gunsberg's test for free hydrochloric acid. The reagent consists of 2 parts phloroglucin and I part of vanillin in 30 parts by weight of absolute alcohol. When a few drops of the filtered contents of the stomach are evaporated to dryness in a porcelain dish with an equal quantity of the reagent, if free hydrochloric acid be present red crystals will form; should there be much peptone present no crystals, but a red paste, will result. The absence or deficiency of free hydrochloric acid occurs in several morbid states, but its presence is a strong point against a diagnosis of malignant disease of the stomach. Hyperacidity, on the other hand, is as characteristic of ulcer as diminished acidity is of cancer.

For more elaborate methods of analysis we would refer our readers to papers in the Lancet, by Drs. C. E. Ham and J. J. MacLeod, August I, I903, p. 3I3; and by Dr. F. Shufflebotham, September I5, I900, p. 795; also to a leader in the same journal, July 5, I9o2.

The mere presence of an acid reaction should not be held as proving the presence of free hydrochloric acid, since this may be caused by acid salts or by free organic acids. Of these latter the most important is lactic acid, and it the practitioner should be able to recognise, since its presence in appreciable quantity in the later stages of digestion is of considerable diagnostic import, implying as it does that excessive fermentation is going on in the stomach. It can be readily recognised by the use of Uffelmann's reagent, which can be made by adding I drop of liq. ferri perchlor. to I ounce of a I per cent. solution of carbolic acid. This will give an amethyst blue solution, the colour of which is changed to yellow on the addition of the merest trace of lactic acid. Since inorganic acids decolourize Uffelmann's reagent, while sugar, alcohol, and phosphates give the same reaction with it as lactic acid, it is necessary to extract the lactic acid by shaking the filtrate, left after filtering a small quantity of gastric contents, with ether, to allow the ether to separate from the watery solution, and after decanting it to evaporate the ethereal solution until only a few drops 
remain. If any free lactic acid be present, on adding some of this to Uffelmann's reagent the alteration in colour noted above will take place. The fatty acids, especially butyric acid, give a somewhat similar reaction, but only when present in larger proportions than they are found to occur in the stomach. The presence of lactic acid and the absence of free hydrochloric acid are strongly suggestive of cancer.

Diagnosis of Stomach Tumours.-Having determined that the gastric mucous membrane can be divided into two physiologically distinct segments, the fundus, which has a large supply of glands, and the pylorus, which has but few glands, K. Glaessner (Berl. klin. Woch., July 21, I902, British Medical Journal supplement) describes how the localization of tumours can be made. Pepsin and rennet are both produced by the mucosa of the fundus, but only pepsin and no rennet is secreted by that of the pylorus. It therefore occurred to Glaessner that if one examined the contents of the stomach in cases of gastric tumours, which can be gained by a trial meal (Ewald), one might be able to learn more of the situation of the growth. In the cases in which he was able to test this, he estimated the total acidity by means of phenolphthalein, $\mathrm{HCl}$ by Toepfer's reagent, pepsin by Mett's method, and rennet by its direct action on milk, within a given time. He considers that normally the pepsin should be present at 5 millimetres-that is, that ro cubic centimetres of normal gastric juice should be able to completely digest a column of albumin in Mett's test-tube measuring 5 millimetres within twenty-four hours; and that rennet should have the value of $I$ in Ioo-that is, that $0^{\circ} \mathrm{I}$ cubic centimetre of normal neutralized juice should be able to coagulate ro cubic centimetres of milk at $30^{\circ}$ to $40^{\circ} \mathrm{C}$. within half an hour. In six cases of carcinoma of the pylorus, confirmed at the operation or at the necropsy, the pepsin was between $\mathbf{I}$ and 3 millimetres, while the rennet was normal. In seven cases of carcinoma of the fundus he found that not only was the pepsin much diminished, as in the pyloric cases, but the rennet also was diminished, or was entirely absent. He looks upon this method of diagnosis as highly valuable. 
The motor activity of the stomach has been estimated in various ways, of which two may be mentioned:

I. Leube's method consists in washing out the stomach at various times after the administration of a fairly large meal-a quarter of a pound of freshly minced meat and some bread. Within six or seven hours the stomach should be empty; but in cases of dilatation of the stomach, or other conditions in which the functional value of the walls of the stomach is diminished, some food may be found many hours after.

2. Ewald's method depends on the fact that salol does not split up in the stomach, but that when subjected to the action of alkaline pancreatic juice it is decomposed, absorbed into the circulation, and, in part, excreted as salicyluric acid in the urine, where it can be readily detected by the addition of neutral ferric chloride solution, a violet coloration occurring. Ewald ('Diseases of the Stomach,' p. 55) advises that ${ }_{5}$ grains of salol be given along with food, and the urine tested at intervals thereafter. Normally, he says, salicyluric acid will appear forty to sixty-at most within seventy-five-minutes after the administration of the drug; whereas in dilatation of the stomach its appearance will be delayed. A simple method of applying the test, devised by Ewald and Einhorn (Einhorn, 'Diseases of the Stomach,' p. 87), is to place a drop of urine on a filter-paper, and then let a drop of Io per cent. ferric chloride solution fall on the moistened spot. The edge of the drop will in the presence of salicyluric acid assume a violet colour.

Various ingenious mechanical contrivances have been suggested for estimating the power of the stomach, but these appear to us scarcely to be suitable for practical use, even if the results to be obtained from any yet devised were certainly reliable.

Examination of the Blood for Leucocytosis.-See p. 88.

Exploratory Incision.--In only a small percentage of cases is simple exploratory incision, as a means of diagnosis, necessary, and there can be no doubt but that in some cases it has served as a cloak for carelessness or incompetence. A careful physical examination, and time and care in study- 
ing the history of the case, will usually enable either an exact diagnosis or an approximate one to be made; and in the odd case the experienced surgeon can usually say it is one likely to be relieved only by surgical treatment, though possibly the exact pathological condition may be a matter of some doubt. Where there is doubt, and delay will not be injurious, a second or even a third examination should be made, as it is well known that at a second visit new facts may be brought to light, the patient's memory may be stirred up, and small data, really important, but thought by the patient or friends to be too trivial to mention at the first visit, may form the key to the situation; moreover, the mind of the surgeon may on the next visit focus his attention on different points, or group his data differently, and so perhaps arrive at a truer conclusion. In case of doubt a consultation, if it has not already been held, is advisable, in order that the case may be also considered from the point of view of the physician. It must not be forgotten, however, that the surgeon must bear the responsibility that awaits the decision, and he should be careful, if a positive diagnosis is decided on, to be able to answer two questions in the affirmative. First, Can I perform an exploratory operation without adding serious risk to the life of the patient, already threatened by the disease? Second, Is it possible that good will result from the operation? At times the surgeon may be called on to explore the abdomen for tumour of the stomach, which it is just possible may be amenable to surgical treatment, but where it is impossible to say beforehand whether the lymphatic glands are involved, or the disease has invaded the adjoining parts to such an extent that the removal of the growth would be useless. Under such circumstances, when the tumour is exposed, the great gift (usually a matter of common-sense combined with experience) of ' knowing when to stop' comes in; for although having made an exploratory incision may, and probably, with due precautions, will, have involved no risk to the patient, any interference with the tumour may inflict such injury on the growth that the surgeon is compelled to convert his harmless exploratory procedure into one of the most serious of operations, which, if it does not cause 
death on the table or shortly after, can lead to no ultimate good. This is sheer meddlesomeness, and for the credit of surgery, if from no higher motive, should be avoided.

Very frequently, however, when organic disease of the stomach is diagnosed, it is impossible to decide what operation is required until the stomach is exposed and handled. This applies very strongly to the early stages of cancer of the stomach, for which operation in an early stage, before adhesions have formed and glands have become affected, holds out the only hope of cure, and also to cases of chronic ulcer and to disabling adhesions of the pylorus or stomach.

An exploratory operation is justified wherever cancer of the stomach is suspected, for the smaller the tumour, the greater will be the hope of a radical cure.

In some cases of abdominal injury, either gunshot or stab wound, or even injury without external wound, the severe shock, the vomiting of blood, and the general condition of the patient, may lead to a suspicion that the stomach has been injured, and an exploratory incision may be justified, since, in case of the stomach having been ruptured, such an injury, if not repaired, would inevitably prove fatal. The following is a case in point :

A youth was admitted to the Leeds Infirmary suffering from shock, with vomiting of blood, following on a stab wound over the stomach. As there was free fluid in the peritoneal cavity, it was thought that bleeding was going on, and that possibly the stomach had been injured and its contents had become extravasated. An exploration showed a wound of the superior mesenteric vein, with bruising, but no wound of the stomach. The vein was ligatured and the abdomen cleared of blood, the patient making a good recovery.

At times all the symptoms of perforating ulcer may be present without a previous history of ulceration, and all that can be said is that a peritoneal catastrophe has occurred which threatens life. Under such circumstances an exploratory operation may be required. A case of this kind came under our care, where a lady was seized with violent abdominal pain at the railway-station. As she became profoundly collapsed, she was removed to the station hotel, and 
was seen by a medical man, who suggested a consultation. The only history obtainable was that there had been no stomach symptoms, but that the patient had missed her last menstrual period for nearly a fortnight. As there was free fluid in the peritoneal cavity, which was thought to be blood due to a ruptured extra-uterine gestation, and as the liver dulness was present, the abdomen was opened above the pubes, when free non-odorous gas escaped, showing that the case was one of ruptured gastric ulcer. The incision was therefore closed, and another opening made over the stomach, in which a perforated ulcer was found. The perforation was closed and the abdomen cleansed by sponging, the patient making a good recovery.

Incision for Exposure of the Stomach.-Although incision in the middle line between the xiphoid and the umbilicus gives the readiest access to the stomach, it is not the best site, for three reasons :

r. It is apt to be followed by yielding of the scar and hernia, unless long rest is enjoined after the operation.

2. The round ligament of the liver, with its irregular adipose envelope, is apt to be in the way and to prove confusing.

3. To prolong the incision for further investigation is inconvenient, because of the umbilicus.

These difficulties, of which the first only is of importance, are obviated by an incision through the inner margin of the rectus muscle just to the right of the middle line. An incision on either side gives ample room; but if the pylorus has to be attacked or gastro-enterostomy has to be done, it is better on the right; if gastrostomy has to be performed, it is better on the left. Afterwards, if the abdominal wall is sutured layer by layer, there is no fear of hernia or of a weak scar being left; moreover, there is no difficulty in extending the incision if more room is required. For routine work we find the incision through the right rectus most satisfactory, as it can be very quickly performed, and with careful suturing we have not found it give rise to subsequent hernia. The oblique incision along the costal margin is undesirable, because it divides muscle so freely, and exact repair by 
suture is difficult; hence permanent weakness of the abdominal wall and ventral hernia are more likely to occur. As a matter of fact, with due care in applying sutures, any one of these methods may be employed, and the operator should select that which will give him the best access to the parts to be manipulated. 


\section{HAPTER III}

\section{GASTROTOMY}

Gastrotomy is the term applied to the operation of opening the cavity of the stomach. It is one of the oldest operations on the stomach, having been practised by Matthis of Brandenburg so long ago as 1602 for the removal of a knife that had been accidentally swallowed.

There is a case, possibly this one, more probably a different one, referred to in Evelyn's diary under date August 28, I64I. The following is the account :

'But amongst all the rarities of this place (Leyden), I was much pleased with their anatomy school, theatre, and repository adjoining, which is well furnished with natural curiosities: skeletons from the whale and elephant to the fly and spider, which last is a very delicate piece of art to see how the bones (if I may so call them of so tender an insect) could be separated from the mucilaginous parts of that minute animal. Amongst a great variety of other things, I was shown the knife newly taken out of a drunken Dutchman's guts by an incision in his side after it had slipped from his fingers into his stomach. The pictures of the chirurgeon and of his patient, both living, were there.'

But as a diagnostic method with a view to further treatment gastrotomy is a modern procedure. Exploratory gastrotomy has only been performed within the last few years; by its means an extensive and minute examination of the interior of the stomach may be made with thoroughness and safety, so that in any case calling for operation, where by other means a positive diagnosis cannot be arrived at, the surgeon is justified in opening and exploring the stomach. 
It will be convenient in this place to consider the operation of gastrotomy as a whole, bearing in mind that one of its purposes may be simply exploratory.

It may be performed-

I. For the removal of foreign bodies from the stomach.

2. For the removal of foreign bodies from the lower end of the œsophagus.

3. For dilating a stricture of the œsophagus.

4. For dilating a stricture of the pylorus.

5. For the removal of a polypus or other tumour projecting into the stomach.

6. For exploration in case of intractable or bleeding ulcer.

7. For curetting a cancer of the pylorus, as in Bernay's operation.

The preliminary incision of the parietes, which has already been described, requires to be rather free for gastrotomy. After the abdomen has been opened, flat sponges or gauze pads should be packed round the stomach, so as to avoid soiling of the peritoneal cavity. The edges of the wound having been retracted, the stomach can now be palpated; and if palpation from the front does not give the information required, a tear may be made in the gastro-colic omentum, so as to admit the fingers to palpate the posterior surface. The stomach should now be drawn forward, surrounded by a sheet of sterilized gauze, and opened by a free incision in its longitudinal axis. Divided vessels must be seized and ligatured, and the liquid contents of the stomach mopped or siphoned out, if they have not been pushed on into the duodenum before the stomach was opened. On retracting the edges of the gastric incision, the interior of the stomach will be brought into view, and may be further explored by the electric cystoscope, if thought necessary, as, for instance, in hæmatemesis from a small bleeding ulcer. If the posterior wall be the part affected, it may be invaginated by the fingers, inserted through the omental slit, pushing it forward into the wound. A tumour may now be removed, an ulcer excised, a bleeding-point ligatured or cauterized, the pylorus exposed and divulsed, the œsophageal opening exposed and dilated, or other treatment may be carried out. 
In the case of foreign bodies in the stomach, there is, of course, no need to make the omental wound; but an anterior incision may at once be made, and the object or objects seized and removed. The incision in the stomach can be rapidly closed by a continuous catgut suture for the mucous membrane and a continuous silk suture for the serous edges, a few Lembert's sutures of silk sometimes being applied over all for greater security. The exposed parts may then be gently sponged, the pads or sponges removed, and the abdominal wound closed without drainage. Beyond teaspoonfuls of water for the first twenty-four to forty-eight hours, all feeding is best done by enemata; but after forty-eight hours the amount of liquid foods may be increased. Later pultaceous food may be given, but it is better not to give solid food for a week, after which the treatment should be as in any other abdominal case.

Dr. Keen (Philadelphia Medical Journai, May 7, 1898) insists on the necessity in this, as in all similar operations on the stomach, of washing out the organ before it is opened, of bringing it out of the peritoneal cavity, and of protecting the peritoneum by a wall of iodoform gauze. Lavage of the stomach is, however, undesirable before gastrotomy for foreign bodies and for hæmorrhage, and is unnecessary as a routine preliminary to operation; but it is desirable, if practicable, to have the stomach emptied before proceeding to open its cavity.

Gastrotomy for Foreign Bodies.-Foreign bodies found in the stomach are of infinite variety. Nails, hair-balls, knives open or shut, spoons, forks, braces, pieces of wood, razor, stones, needles, safety-pins, ordinary pins, false teeth, buttons, coins, broken glass, keys, are among the commonest forms. In many of the cases the bodies are swallowed by the insane, in other cases by accident, in others for the amusement of the victim or his friends, or as a means of livelihood. They may remain for months or even years. Kortman relates the case of a painter, aged fifty-five, who had two shellac stones in the stomach for over sixteen years. The man, while working in a factory, had often drank some alcoholic solution of shellac. A movable tumour in the epigastrium had been 
observed for sixteen years, and had caused a sense of weight and oppression and, occasionally, vomiting.

Friedenwald and Rosenthal (New York Medical Journal, July I8, I903) have collected from the literature a series of go cases of gastrotomy for the removal of foreign bodies from the stomach. In the 90 cases, one foreign body was found in 68 instances, more than one in the remainder. The following table gives the sex and age in 78 patients:

\begin{tabular}{llllrlr} 
& & \multicolumn{4}{c}{ Male. } & \multicolumn{2}{c}{ Female. } \\
Under I year $\ldots$ & $\ldots$ & $\ldots$ & $\ldots$ & o & $\ldots$ & I \\
I to Io years $\ldots$ & $\ldots$ & $\ldots$ & $\ldots$ & 0 & $\ldots$ & 3 \\
I0 to 20 years & $\ldots$ & $\ldots$ & $\ldots$ & 7 & $\ldots$ & 7 \\
20 to 30 years & $\ldots$ & $\ldots$ & $\ldots$ & I 5 & $\ldots$ & I 5 \\
30 to 40 years & $\ldots$ & $\ldots$ & $\ldots$ & I 4 & $\ldots$ & 6 \\
40 to 60 years & $\ldots$ & $\ldots$ & $\ldots$ & 5 & $\ldots$ & 3 \\
60 to 80 years & $\ldots$ & $\ldots$ & $\ldots$ & I & $\ldots$ & I
\end{tabular}

In the series of 90 , adhesions were found in $\mathbf{I}_{3}$ cases. In 6 an abscess had formed and had pointed on the anterior abdominal wail. In 3 perforation occurred and resulted fatally.

The symptoms caused by the presence of numerous or large foreign bodies are singularly few. There is nothing characteristic about them. In many cases a tumour has been felt the exact nature of which has remained unsuspected until an operation has been performed. The diagnosis, which was often a matter of doubt, is now made easier by skiagraphy, though even this may be misleading. Kränzle records one case in which the X-ray photograph seemed to show that the foreign body lay in the intestine about the level of the iliac crest ; it.was nevertheless found in the stomach. In several cases the patients complained of nothing more than chronic indigestion, pain after food radiating to the interscapular region, occasional vomiting, and rarely hæmatemesis.

The removal by gastrotomy of foreign bodies lodged in the stomach has been attended with considerable success.

A preliminary distension of the stomach with fluid or air is neither advantageous nor desirable. The indications for operation are the presence of a foreign body which can neither be safely dissolved nor allowed to pass through the gut, and 

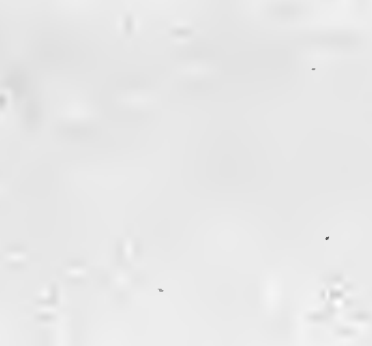

$\because$ 
PLATE I.

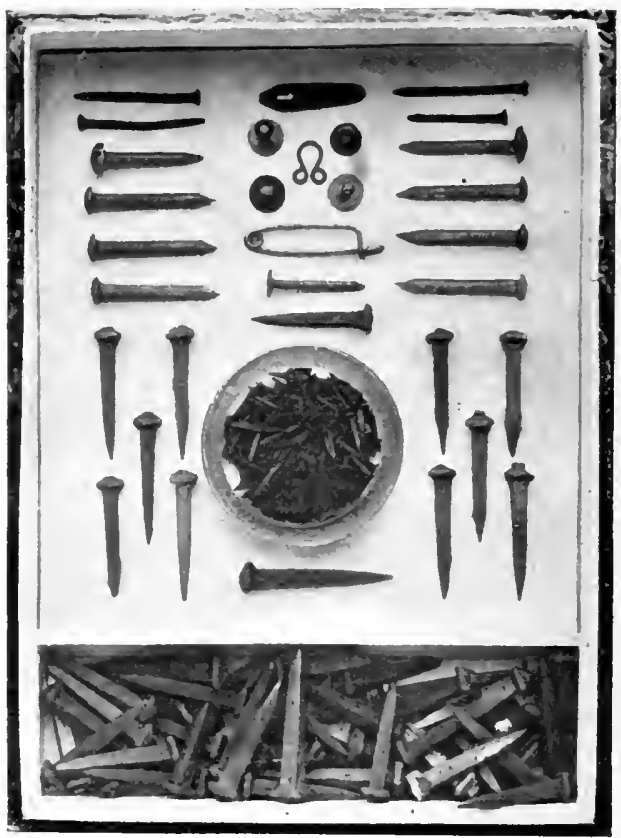

Fig. t.-Foreigi Bodies successfelly removed from the Stomach of a Girl agei) Twelle. (Royal College of Surgeons' Museum.)

For detailed report, see Lancet, November, i894.

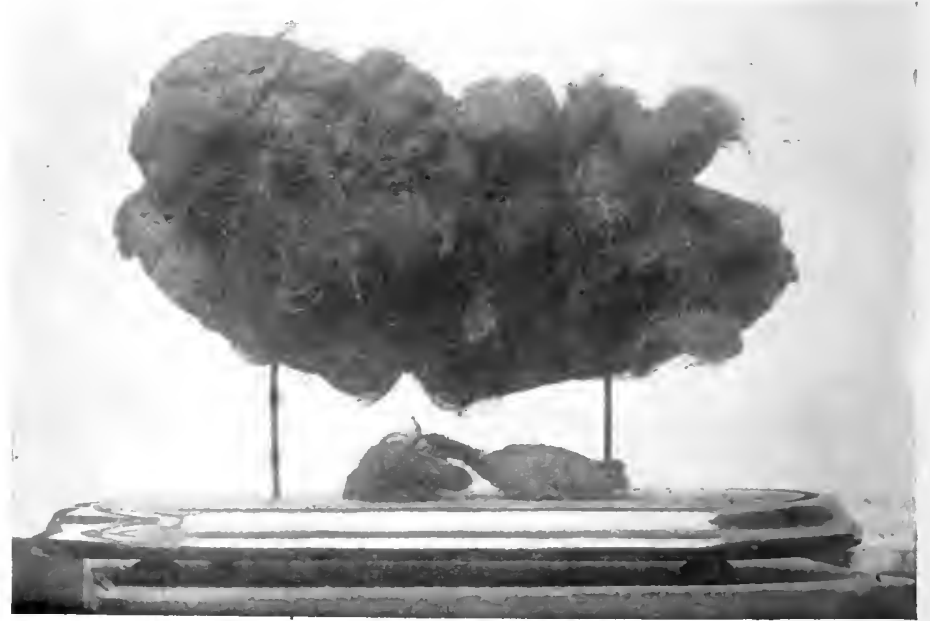

Fig? 2.-A Roll of Black Humal hair i2 inches long, weighing 5 LB. 3 OZ, Removed successfully from a Gre of Twenty. Lancet, I895, vol.i., p. I58r. (No. 2,38r, Royal College of Surgeons' Museum.) To face $f$. 35.] 
which is actually producing, or is likely to produce, serious symptoms. We have described a case (Lancet, November 3, I 894, p. I028) in which 'forty-two cast-iron garden nails $I_{8}^{5}$ inches long, ninety-three brass and tin tacks from $\frac{1}{2}$ inch to I inch long, twelve large nails (some brass-headed), three collarstuds, one safety-pin, and one sewing-needle,' were removed by gastrotomy, the patient, a girl, twelve years of age, making a complete recovery and being now in good health (Fig. J). The photograph here reproduced from the College of Surgeons' Museum shows the objects removed. The value of transfusion of saline fluid in the treatment of shock was well shown in this case. Although practically no blood was lost and the operation was not very prolonged, the patient, who had been much exhausted from excessive vomiting before the operation, was pulseless after its termination, yet transfusion restored the pulse and rendered recovery possible.

Dr. A. G. Bernays described a case in which, through an incision 5 inches long in the linea alba, he successfully removed a table knife from the stomach of a man who had swallowed it within an hour previously. The patient was fed by the rectum only for four days, but after that time food was given by the mouth.

Professor Loreta mentions the case of a woman who, having swallowed needles with suicidal intent, suffered severe pain. On gastrotomy being performed, several needles were removed from the stomach and others were drawn out of the left lobe of the liver. The patient recovered without a bad symptom, and was entirely freed from her suffering.

Among the foreign bodies removed by gastrotomy are masses of hair forming a dense ball. These tumours are described by French authors as 'egagropiles,' by the Germans as 'trichobezoars.' Very few symptoms are caused by them, but a tumour can often be felt. A mistaken diagnosis of omental tumour, renal tumour, floating kidney, or enlarged spleen has been made in some of the cases recorded.

The most remarkable specimen was successfully removed by Mr. Paul Swain (Fig. 2). It weighed 5 pounds 3 ounces. It can be seen in the Museum of the Royal College of Surgeons of England. A photograph here reproduced of the mass of hair 
is taken from the specimen in the museum. Schopf (Wiener Medicinische Wochenschrift, November 16, 1899) has collected I6 cases of hair-ball in the human stomach, of which 8 were removed by operation, all successfully. Cathelin (Bull. de la Soc. Anatomique, January, I902) has collected 2I cases, and to these may be added a case recorded by E. Percy Paton (Lancet, vol.i., p. I63, I902), making 22 in all up to I903. As a rule, the patients have been young girls of a highly neurotic tendency or insane. The youngest patient was a girl of nine, in whose stomach a hair-ball had lain for three years (Potter). Bandamant's patient was ten years old, Jacobsen's eleven, Schopf's sixteen, Bollinger's seventeen. The oldest patient was forty-five.* There is no record of a hair-ball having been found in the stomach of a male.

Statistics show the mortality in gastrotomy for foreign bodies to be 15 per cent. in a series of 79 cases; but the later cases show a smaller rate, and with the improved technique of to-day it will doubtless diminish considerably. Crede (Archiv für Klinische Chirurgie, 1886) and Ericker (Deutsche Med. Wochenschrift, 1897) collected 54 cases, with Io deaths; Heydenreich (La Semaine Médicale r89I) I3 cases, with 2 deaths; and Schopf (Wien. Med. Wochenschrift, November, I899) 8 cases, with no death. Friedenwald and Rosenthal divide the operations recorded up to July, I903-9o in number-into those done in the preantiseptic days and those done since. In the former period I9 cases were operated upon, with I5 recoveries and 4 deaths. During the later period there have been $7 \mathrm{I}$ cases with 7 deaths. The earlier the operation was done, the greater was the success attending it.

* Schreiber and Eiselsberg (Mitt. a. d. Grenzgeb. d. Mcd. u. Chir., 1896 , i., p. 729) record the following case of removal of a 'vegetable tumour' from the stomach: 'A woman, forty-five years of age, was admitted into hospital for an abdominal tumour accompanied by pain and vomiting. The tumour resembled a large spleen, but as it was ascertained that the patient had eaten a quantity of a plant which superstition endowed with marvellous powers of healing, a diagnosis of "phytobezoar" was made, and a large mass of fibrous roots was successfully removed from the stomach by operation.'

A similar, though fatal, case is related by Kooyker (Zeit.f. Klin. Med., 1888, xiv., p. 203). 
The following case, though not actually a gastrotomy, is of interest from a historical point of view, and it seems to be convenient to place the account here while discussing the operation for removal of foreign bodies from the stomach. The account is taken from the British Medical Journal, Igor.

'It may be remembered by those among us whose professional memory can carry them back a quarter of a century that in the mid seventies there was much talk in the newspapers of a Frenchman who had swallowed a fork. The fork was successfully removed by M. Labbé, surgeon to the Charité, now a member of the French Senate, and the case is quoted in the books as marking a step in the development of the surgery of the stomach. The man, whose name was Lausseur, lately died, twenty.five years after the operation. He was an assistant in the Magasins du Printemps, a large drapery establishment, and he was in the habit of amusing his fellow-employés by putting into his mouth down to the handle the big scissors-30 centimetres long-with which he cut samples. One day at luncheon in the early part of I875, by way of what our ancestors would have called a "merrie and conceited jest," he introduced a fork into his mouth with the handle downwards, holding the points between his teeth, and closing his lips so as to hide the fork. On opening his mouth to take it out again he drew in his breath, and the fork slipped down into the gullet. Attempts to seize it with forceps failed, and it finally found its way into the stomach. The affair for some weeks figured prominently in the newspapers. "L'homme à la fourchette "was the sensation of the day, and he must have been a good advertisement for the Magasins du Printemps, for people wrote there from Russia, from America, and nearly every part of the world, asking for particulars of the accident and as to the condition of the patient. Lausseur, who was a man of cheerful disposition, did not allow himself to be depressed by the knowledge of the formidable foreign body which he had in his stomach. $\mathrm{He}$ actually composed a waltz about it, which he called "La Fourchette," and he used to sing it, accompanying himself on the piano. He did not, as a matter of fact, suffer so much inconvenience as might have been expected, and it was only when the fork got into an awkward position in the stomach that acute pain was caused. After a time, however, an abscess formed, which pointed under the umbilicus, and symptoms of constitutional disturbance showed themselves. M. Labbé opened the abscess and removed the fork, which he found projecting into the abscess 
cavity, the stomach being adherent to the abdominal wall. This was the first instance in which a foreign body was removed from the stomach by a surgical procedure, though it was not, strictly speaking, a gastrotomy.'

The following case, recorded in the British Medical Journal, November I7, I900, p. I423, by Mr. Walter Spencer, is worth relating here :

'A married woman, aged forty-eight, was transferred to me by Dr. Murrell with the following history: She had been in very good health until ten months ago, when suddenly one day, whilst walking in the street, she was seized with a violent pain in the epigastrium, which made her feel as though she was going to burst. The abdomen swelled up, but she did not pass flatus; for seven weeks she was in bed on a fluid diet, with poultices on the abdomen. Then she got up and tried to take ordinary food, but it caused a choking sensation, with an aching pain in the epigastrium. On several occasions the attacks of pain seized her as at the first, with swelling of the abdomen, constipation, and scantiness of urine. A fixed lump appeared in the epigastrium, and the pain became worse whilst moving about, and caused her to suffer from dyspnœea.

'In the medical ward the patient could only take fluids, and did not improve. It was decided, therefore, to explore the painful lump in the epigastrium, and the case is an admirable instance of the value of surgical exploration in obscure and stationary cases. The pyloric third of the stomach was found adherent to the back of the abdominal wall, being pinned there by a fishbone $2 \frac{1}{2}$ inches in length and of the size of a stout needle. One end was fixed in the left rectus muscle, and the other end of the fishbone projected into the pyloric third of the cavity of the stomach, the middle of the bone being surrounded by a small thick-walled abscess cavity. The fishbone, after being swallowed, having arrived at the pylorus, instead of passing through end on, had becomed wedged athwart the orifice, and subsequently, by the peristaltic movements of the stomach, one end had been driven through the stomach into the abdominal wall. This accounts for the sudden attack, the immediate result of which was a reflex inhibition of intestinal peristalsis, causing the abdomen to become swollen by tympanites, and inducing constipation and scanty flow of urine. At the same time there was partial obstruction to the escape of food from the stomach, hence the subsequent difficulties in digestion. The patient could never recall having swallowed the bone. The fishbone was extracted, and the adhesions of the stomach to the 
$\because$ 
PLATE II.

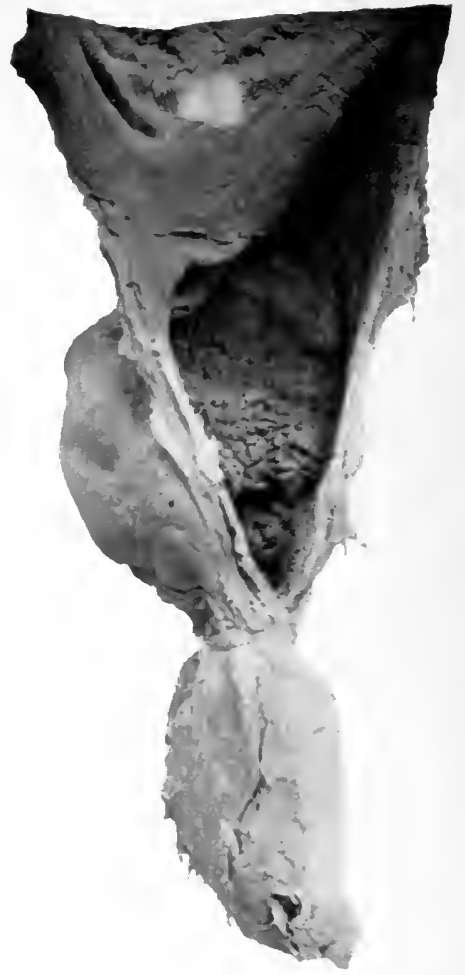

Fig, 3.-Photograph of Stomach from a Married Woman, aged Forty-one, who had suffered from Hanatenesis at Seventeen AND SEVERAL TIMES LATER.

Ultimately, three weeks' incessant vomiting led to death. A post-mortem examination showed the pylorus behind the pubes, the duodenum being distended by a mass of pins weighing nearly a pound and causing obstruction. There were no peritoneal adhesions. (No. 2,379, Royal College of Surgeons' Museum.)

To face p. 39 ] 


\section{PLATE III.}

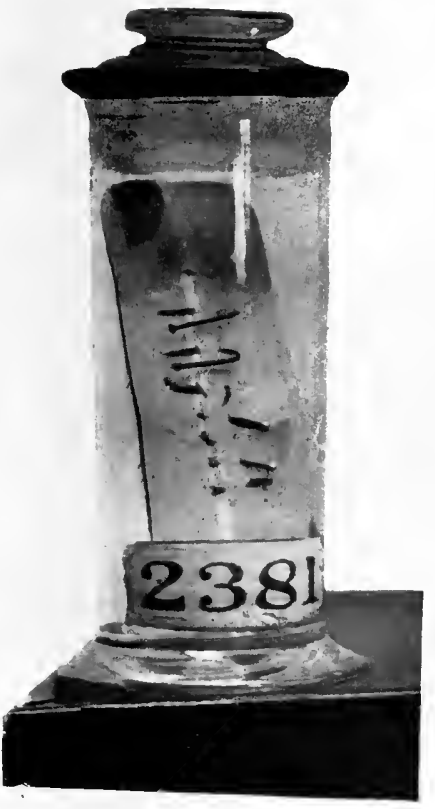

Fig. 4-Bent Pins Removed from Specimen 2,379 (FIG. 3).

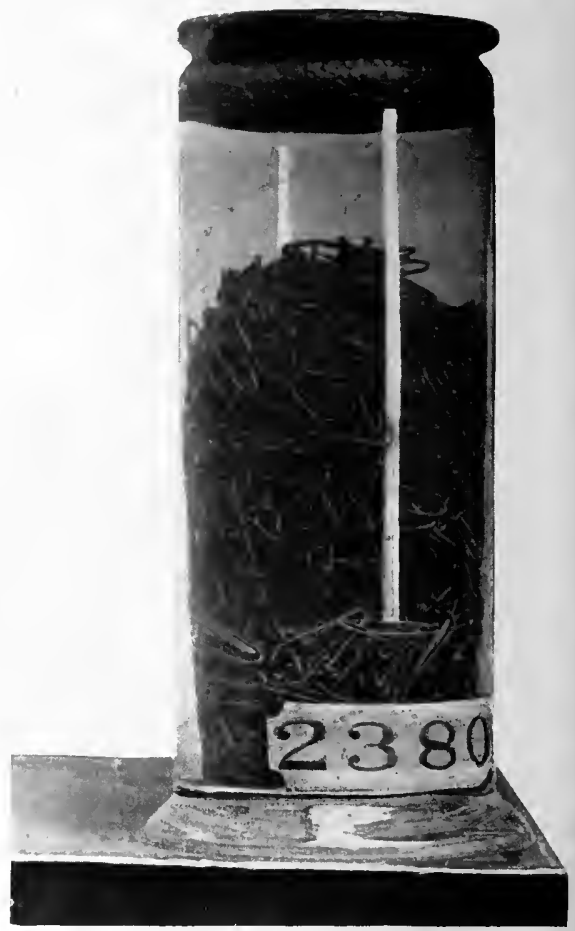

Fig. 5.-Pins Removed from Specimen 2,379 (FIG, 3).

To face p. 39.] 
abdominal wall divided. The portion of the anterior wall of the stomach, which had formed a thickened mass about $\mathrm{I} \frac{1}{2}$ inches in diameter around the bone, was excised; then the gap was closed by a double row of sero-muscular sutures. The abdominal wound healed at once, a sinus leading down to the abscess cavity closed after a short delay, and she was seen some time after her discharge from the hospital in good health.'

The effect produced on the stomach by various foreign bodies accidentally or intentionally swallowed can be studied in a series of specimens in the Hunterian Museum at the Royal College of Surgeons. Photographs of several of the specimens are here reproduced.

No. 2,377, Royal College of Surgeons, shows the remains of a knife swallowed two months before death. No. 2,378, Royal College of Surgeons, shows the blades and iron portions of three or more clasp-knives swallowed by a man and voided per anum; the man recovered. No. 2,379, Royal College of Surgeons (Fig. 3), shows the stomach from a woman, aged forty-one years, who had suffered from hæmatemesis at seventeen years of age and several times later; ultimately incessant vomiting led to death. A post-mortem examination. showed the pylorus behind the pubes, and the duodenum distended by a mass of pins weighing nearly a pound, which had caused the obstruction. The bent pins removed from the last specimen are shown in No. 2,380, Royal College of Surgeons (Figs. 4 and 5). No. 2,381a, Royal College of Surgeons, shows a roll of black human hair, 12 inches long and weighing 5 pounds 3 ounces, removed from a girl, twenty years of age, by Mr. Paul Swain; the patient recovered (Fig. 2, p. 35).

Gastrotomy may be performed for the removal of foreign bodies impacted in the lower end of the esophagus when all attempts to remove them by the mouth or to push them on by bougies have failed.

When a foreign body, except in the case of a coin, is impacted in the lower part of the osophagus, and cannot be removed by ordinary means, the surgeon may pursue one of three courses :

(a) Esophagotomy in the neck, which is not to be recommended. 
(b) Intra-mediastinal œsophagctomy, an extremely difficult and dangerous operation, which has, we believe, never been done successfully.

(c) Gastrotomy, the operation we would recommend as being safer and more efficient than any other.

In the case of impacted coins, our experience is that by the use of the instrument known as a coin-catcher, which we believe was invented by the late William Hey, of Leeds, they can always be safely removed.

We have had under our care four cases of this kind in children where, after the coins had been localized by skiagraphy, they were removed by means of the coin-catcher with the greatest facility, although they had been impacted for four months (British Medical Journal, vol. i., r898).

At the American International Medical Congress Dr. Maurice H. Richardson (Boston Medical Journal, December $I 6, I 886$ ) read a paper on the possibility of operation on the œesophagus through the stomach. He had verified his statements by experiments on sixty bodies, and had in one case operated successfully on a man, thirty-seven years of age, who had eleven months previously swallowed a denture of four teeth, which had become impacted at the lower end of the œsophagus. The patient had lost 50 pounds in weight, and it was found to be quite impossible to extricate the plate by means of forceps introduced through the mouth. After the stomach had been opened, the hand was inserted; on reaching the cardiac orifice, the finger discovered the plate about 2 inches above the diaphragm. By means of digital manipulation it was detached and withdrawn; the stomach was then carefully sutured, and the abdomen closed. The patient rapidly gained weight, and was soon able to resume his work. The conclusions Dr. Richardson drew from his experiments were: that the oblique incision below the margin of the ribs is the best; that the stomach, after being withdrawn from the abdomen a little, should be opened to the right of the convexity of the lesser curve; that the assistant should, by traction on the stomach, put the lesser curve on the stretch; and that the sulcus so formed is always a certain guide to the cardiac orifice. When a foreign body 
is impacted at the lowest part of the cesophagus, it is about 6 inches below the opening made in œsophagotomy; and if it be firmly impacted and require much force to detach it, the safer plan will probably be to perform gastrotomy, and draw it down a little way, than to perform œsophagotomy, and draw it forcibly a distance of 6 inches past very important structures.

Dr. Bull, of New York, has recorded (New York Medical Journal, October 29, I897) an operation in which he successfully detached an impacted peach-stone from the lower end of the œsophagus in a thin boy, aged sixteen years, and then, by attaching a piece of string to a sponge, and dragging the sponge upwards from the gastrotomy opening to the mouth, he was able to bring the stone with it.

In the Lancet for February 23, I9or, is an account of a case by Mr. Flavell Edmunds, where gastrotomy was successfully employed in a man, aged forty-five, for a toothplate impacted in the lower end of the osophagus.

Gastrotomy as a preliminary to forcible dilatation of the pyloric or cardiac orifice of the stomach was suggested by Richter, and carried out by Loreta in September, I882.

In stenosis of the pylorus, however, divulsion has, speaking generally, given way to pyloroplasty or gastro-enterostomy, for the double reason that pylorodiosis has a greater deathrate, and is more liable to be followed by relapses. It may nevertheless be advisable in those cases due to spasm and hypertrophy of the pyloric sphincter. In a certain number of these cases, however, the sphincter may be divulsed without gastrotomy by invaginating the stomach wall, and gradually pushing the finger through the pyloric opening, as suggested by Hahn. This subject will be considered more in detail under simple dilatation of the stomach.

In simple cicatricial stricture of the esophagus at or near the cardiac orifice, if dilatation by bougies is impossible or has failed to keep the stricture open, the operation of gastrotomy may be performed, and an attempt made to dilate the orifice from below. This operation has proved very successful. Gissler (Beiträge für Klin. Chirurgie, Tubingen, I 892, vol. viii., p. Iog) has collected ten cases, and Kendal Franks twenty- 
one cases, all the patients recovering. The operation may be performed in two stages, in which case a large gastrotomy opening must be made and the dilatation proceeded with later. The one-stage operation is to be preferred, as the finger can then palpate the cardiac orifice, and a mechanical dilator may be used if necessary. For subsequent operation a gastric fistula may be left, if it be thought that there will be difficulty in keeping the passage patent by bougies introduced through the mouth. The dilator that Loreta employed was after the pattern of Dupuytren's lithotomy dilator, only longer, and incapable of dilating beyond 5 centimetres. Kendal Franks used Otis's urethrotome, from which he had previously removed the knife. Weiss's rectal dilator may be conveniently employed. Abbé has recently devised a method of dividing a simple stricture of the lower end of the œesophagus, or of the cardiac orifice of the stomach, by passing a string through the stricture from below until it emerges at the mouth; then, by putting the string on the stretch, and running it rapidly upwards and downwards, the stricture is soon divided. The stomach and the abdominal opening are then closed, and the stricture is kept permeable by the regular passage of bougies. The diagnosis is of the first importance, as in malignant disease the operation will be useless and may be very dangerous. In simple stricture of the cardiac orifice there will probably be a history of corrosive fluid having been swallowed some time previously, and the symptoins will also be of longer duration than if the stricture be malignant.

Gastrotomy for the removal of a polypus or other growth projecting into the cavity of the stomach or occluding the pylorus is an operation seldom required, since simple tumours of the stomach are uncommon. The following is a good example: A middle-aged man, with a history of longcontinued dyspepsia and of loss of flesh, came under our care for dilatation of the stomach, which was producing the usual symptoms with great loss of flesh. The pylorus appeared to be thickened, and on opening the stomach the lumen of the pylorus was seen to be occupied by a polypoid projection with a wide base, so that the passage of the 
stomach contents onward was almost completely prevented. The removal of the growth by scissors, and the suture of the longitudinal wound transversely, led to such relief that the patient gained weight at first at the rate of $\frac{1}{2}$ pound a day, and in three months he weighed between 2 and 3 stones more than before the operation. The growth proved to be an adenoma.

Chaput (Bulletin et Mémoire de la Société de Chirurgie, I894) records a case in which he excised a pedunculated adenoma from the posterior wall of the stomach; and C. B. Lyman (Annals of Surgery) reports a case of removal of a pedunculated carcinoma of the stomach through a gastrotomy opening. Sir W. H. Bennett (British Medical Journal, February 3, I900) has reported an interesting case of the kind, where the removal of a simple papilloma that occluded the pylorus from time to time, and led to intermittent dilatation of the stomach, cured the patient, a man aged thirty-five years, who had been ill for years.

Gastrotomy for exploration in cases of intractable gastric ulcer not yielding to ordinary treatment, and for gastrorrhagia, will be considered more conveniently when we come to discuss ulcer of the stomach (see p. I86).

Gastrotomy for partial removal of cancerous growths in the stomach by the curette is an operation that has been performed by Dr. Bernays, of St. Louis. He has recorded (A nnals of Surgery, December, I887) some cases in which he had given relief by this method; but in view of the better results from gastrectomy or gastro-enterostomy, it is scarcely likely that this operation will continue to hold a place in surgery, both from its uncertainty and from the danger of hæmorrhage and perforation; moreover, the relief given by such an operation can only be of a very temporary nature. 


\section{CHAPTER IV}

\section{CONGENITAL HYPERTROPHIC STENOSIS OF THE PYLORUS}

THIs condition, to which attention has been especially directed during recent years, has been described in the older records as 'scirrhus of the pylorus.' Dr. Osler calls attention to a case which he had found recorded in 1788 in the Transactions of the Newhaven Medical Association. The case was reported by Dr. Hezekiah Beardsley as one of 'scirrhus of the pylorus in an infant.' The symptoms noted are constant vomiting, the leanness and the wizened old look of the child. The necropsy showed a dilated and hypertrophied stomach. "The pylorus was invested with a hard compact substance or scirrhosity, which so completely obstructed the passage into the duodenum as to admit with the greatest difficulty the finest fluid' (Osler, Boston Medical and Surgical Journal, March I2, 1903).

Dr. John Thomson, of Edinburgh, who has observed eleven cases since I894, has drawn attention to two early recordsthe one by Williamson (London and Edinburgh Monthly Journal of Medical Science, 184r, p. 23), the second by Dawosky (Caspar's Wochenschrift, I842, No. 7). The title of Williamson's paper was 'A Case of Scirrhus of the Stomach, probably Congenital'; of Dawosky's, 'A Case of Hypertrophy of the Submucous Cellular Tissue of the Pylorus in an Infant Six Weeks Old.' These early records, however, sank out of remembrance, and were only recalled after the description of two cases by Hirschsprung, of Copenhagen, in I888.

The condition is one to which many names have been 
given. Hirschsprung entitled his paper 'Congenital Stenosis of the Pylorus.' Newton Pitt used the term 'hypertrophy of the pylorus.' J. Thomson, in his second publication, called the condition 'congenital gastric spasm.' De Bruyn Kops related one case as an example of 'congenital tumour of the pylorus.'

During the last five years, and especially during the last two years, the condition has been recognised with rapidly increasing frequency. From being one of the freaks of medicine having little more than pathological interest, it has now become a well-established clinical entity. The disease, indeed, is probably not uncommon, for it is more than possible that many children who have been certified as dying from marasmus, vomiting, or even intestinal obstruction, have, in truth, been its victims.

\section{Symptoms.}

The symptoms of the disease are strikingly similar in the majority of the recorded cases. The child at birth is a wellnourished, healthy baby of normal weight. Soon after birth, sometimes within the first day or two, sometimes not until two, three, or even four weeks have elapsed, it is noticed that the child begins to vomit after its food. At the first it is only after the completion of a meal or towards the end that the food is returned. The quantity vomited is only small, but it increases day by day. It may then be observed that if only a small quantity of food is given it is retained, a larger quantity is at once rejected. An alteration of the food from breast milk to peptonized milk or to diluted cow's milk is of little or no avail in checking the vomiting. The change may seem to answer for the first meal, or even for all the meals of the first day, but soon the vomiting returns. As day succeeds day, the quantity of food that will excite vomiting gradually lessens, until finally even a teaspoonful of milk or water will return. The vomited matters are very slightly altered from their condition when swallowed; a little mucus alone is added. Bile is never present in the vomit. When the vomiting is thoroughly established as an enduring symptom, its violence is remarkable. As soon as the fluid is 
taken it is instantly ejected with considerable force. Thomson calls attention to the circumstance that when the stomach tube is periodically passed the fact is revealed that when the fluid is not vomited it is often retained for long periods in the stomach unabsorbed, thus seeming to indicate not only that the pylorus is impassable, but that there is something interfering with the absorption of fluids by the stomach. The condition of the child changes progressively for the worse; weight is steadily lost-a few ounces dailyuntil finally the child may weigh little more than three, or perhaps four, pounds; the little face is pinched and shrunken, the child looks wizened and old, and the whole body is parched; the temperature is constantly subnormal; constipation is almost invariable-the bowels do not empty because they are never filled; the mouth and tongue remain clean and moist, as a rule.

If the abdomen be examined at the first, little or nothing may be found; but when the symptoms have been present for a few days the outline of the stomach may be clearly seen. Faint waves of contraction, daily increasing in vigour, may pass across the stomach from left to right. At the pylorus a tumour may be felt. The tumour is hard, cylindrical, easily movable, and it lies transversely in the epigastrium. It may at times be difficult or impossible to discover, owing to the shelter it receives from the liver. An cxamination under an anæsthetic will then disclose it. The stomach is, as a rule, dilated. In one case, related by Cautley and Dent, it is said that 'the stomach was dilated, and peristaltic movements could be seen passing onwards to the pylorus, there pausing, and then continuing onward down the duodenum. Deep down under the liver at the point of temporary pause in the peristaltic wave could be felt an illdefined, rounded, movable tumour.' The intestines soon become collapsed, and the contrast then between the dilated and thickened stomach projecting forward in the epigastrium and the sunken, hollowed abdomen has excited frequent comment. From this account it may be gathered that there is, in the early stages, very considerable difficulty in the diagnosis. Vomiting in the young infant is so common a 
symptom that but little interest is aroused by it. Nevertheless, the persistence of vomiting for a few days should excite suspicion. If the vomiting be found to occur soon after a meal, and especially after a large meal or a meal quickly taken; if all fluids are alike rejected; if constipation be present, and if on examination of the abdomen it be found shrunken and hollow, and the stomach be seen to be dilated or hypertrophied, with visible waves of peristalsis hastening across it; and if, finally, a transverse epigastric tumour be found, the diagnosis of pyloric stenosis can no longer be in doubt.

\section{Morbid Anatomy.}

The appearances presented on post-mortem examination are almost precisely similar in all cases. The stomach is large, and its walls are thickened by a hypertrophy of the muscular fibres; the pyloric region is greatly altered, and shows a funnel-shaped, circular thickening. When handled, it feels like a solid cylinder, hard and incompressible. On cutting through it the walls are seen to be very thick and dense. The mucous membrane lining the pylorus is thrown into longitudinal folds. Cautley and Dent remark that 'a single longitudinal reduplication of the mucous membrane, much more marked than any other fold, forms a conspicuous feature in many of the specimens. This prominent fold in its appearance may be compared to the verumontanum of the male urethra. Indeed, these stomachs in appearance and feel curiously resemble the dissected-out bladder and prostate, the latter being comparable to the thickened pyloric portion.' Several writers have noticed that if the duodenum be opened and the pylorus inspected from its distal side, the appearances presented resemble very closely the cervix uteri seen from below. There is a central opening, and around it a thick, hard, circular band. On making a section of the pylorus, it is seen that the thickening is due to an enormous hypertrophy, or hyperplasia, rather, of the circular muscular fibres. This thickening is chiefly limited to the pylorus, but it may extend onwards a little way in the duodenum. The longitudinal muscular fibres are not, as a 
rule, much affected, though a slight increase may be observed. Finkelstein in one case attributed the whole thickening to an increase in this layer. The submucous layer was, in one instance, found by Thomson to be greatly increased in amount. If the stomach and duodenum be examined before removal from the body, it will be found that, though fluid may be present in fair quantity in the stomach, it is not possible to force any through the pylorus into the duodenum. To the passage of fluid the pylorus is completely blocked, though a fine probe may pass readily enough. The serous and mucous coats are generally unimpaired. The walls of the stomach at the cardiac end are rarely thickened; Thomson says that they are as thin as, or thinner than, usual. In some few cases, however, the coats of the whole of the stomach and of the lower part of the osophagus also have been thickened and the muscular fibres grossly hypertrophied.

\section{Etiology.}

No very satisfactory explanation of the causation of the condition has yet been given. The muscular hyperplasia, it is thought, may be primary, a local overgrowth, or secondary, due to disturbances elsewhere, which result in spasm. The arguments on either side are not convincing, and for the present, at least, our judgment in the matter must be suspended. Dr. Cautley, who supports the view that the hyperplasia is primary and is due to a simple redundance of growth, says: 'Nature, in her extreme anxiety to provide an efficient pyloric sphincter, has overexerted herself, and produced too great a quantity of muscular tissue.' One observation which would seem to give support to this theory has been made in some few cases. It is that when the child dies at an early age-between the third and fourth weeksthe amount of hypertrophy found may be far greater than could have been produced since birth. If the pyloric hypertrophy and stenosis existed at birth, they could only have been formed as a primary developmental exuberance, or have been caused by spasm, excited when amniotic fluid was swallowed by the foetus. And in favour of this latter possibility there is no tangible evidence. It is important to note 
that the condition of pyloric hypertrophy has never yet been observed in the fotus. Dr. J. W. Ballantyne, whose experience of fotal pathology is unrivalled, has never once met with this abnormality in all the number of malformed foetuses which he has examined.* Dr. John Thomson is the originator and the foremost exponent of the theory that the hypertrophy is secondary. He has described two cases of the disease under the title 'Congenital Gastric Spasm '; he considers that there is both a structural and a functional change in these cases. The essential structural change consists in true hypertrophy of the muscular coat of the pylorus, stomach, and sometimes also of the œesophagus. The other changes found-the narrowing of the lumen of the pylorus and the dilatation of the stomach and esophagus-are secondary. The main functional abnormality consists in a tendency to spasmodic closure of the thickened pylorus, which prevents the normal periodic opening to allow of the onward passage of food. Dr. Thomson says that there is no symptom characteristic of these cases which may not be regarded with great probability as the result of the excessive, ill-timed, spasmodic contraction of the hypertrophied muscular apparatus of the pylorus, stomach, and œesophagus. In support of this theory he refers to certain facts which seem to him to throw some light upon the matter. These are:

I. The comparatively isolated character of this malformation, which tells against its being really a teratological malformation. In all the recorded cases there were only six which showed other congenital defects or aberrations.

2. The prominence of the spasmodic element in the symptoms after birth, which suggests the probability of a similar spasm in utero. As great muscular hypertrophy is the main anatomical, so spasmodic contraction of the stomach seems to be the main clinical, feature in these cases.

3. The apparent continued growth in size of the pylorus after birth while the symptoms last, which suggests the probability of a similar growth in fotal life.

* Henschel has reported three cases occurring in the same family, a remarkable occurrence suggesting the probability of some congenital dystrophy. 
4. The gradual disappearance of the symptoms in some cases, while there is reason to believe that the anatomical condition remains unchanged (see the record of Batten's case later); and the immediate improvement after stretching of the pylorus in others, which are points in favour of a functional causation.

The whole question as to the etiology of this disease will require further investigation in the light of a wider experience. It is, perhaps, not irrelevant to mention that spasm at the pylorus and in the body of the stomach is a condition which can be seen from time to time on the operation-table. In some cases the muscle, as it contracts, may so stiffen and harden the pylorus in cramp that a tumour, palpable through the abdominal wall, is produced.

A degree of congenital stenosis is doubtless a frequent, though often an unrecognised, cause of dilatation of the stomach in young adults. Maier (Virch. Archiv, Bd. I02) cites a number of cases, mostly in adults, of chronic catarrh of the gastric mucous membrane, with dilatation, in which stenosis of the pylorus, varying from 3 millimetres to I centimetre (the normal being I to 3 centimetres), was found. $\mathrm{He}$ is careful to distinguish between the congenital form and that due to catarrh, ulcer, and cancer, the acquired forms of stenosis, and give his reasons for considering the stenosis primary and the catarrh secondary in the cases mentioned. He acknowledges, however, that clinically the distinction is extremely difficult. Basing his classification on the anatomical structure of the pylorus, he distinguishes between a simple form of stenosis, in which there is a round or slit-like narrowing of the pyloric orifice, and a complicated form, due to a hypertrophy of the longitudinal or of the circular fibres of the pyloric region. In the first case, where the longitudinal fibres are hypertrophied, the stenosis is usually conical in shape, with the apex of the cone at the pylorus, and projecting into the duodenum after the manner of the os uteri into the vagina; but sometimes it is ringlike, the constriction then measuring as low as from 4 millimetres to 5 millimetres. When the circular fibres, on the other hand, are increased, the form taken is usually that of a thick 
swelling, the lumen of the canal not being so much reduced as in the former case.

This form of stenosis may explain many of the cases of dilatation and catarrh of the stomach coming on in young adults without apparent cause, and it may also explain many of the cases of persistent vomiting set down to a vicious habit, or to hysteria. To judge from the literature of the subject, this condition is not of frequent occurrence; but it may be more common than is supposed, as necropsies are not frequently made in the case of children dying out of hospital, and persistent vomiting in infants is not a very uncornmon event.

The transition from the acute cases, speedily ending in death, occurring in infants, and the chronic cases, where symptoms are noticed for the first time in young adults, or where symptoms long troublesome in a slight degree become aggressive, is clearly shown by the following series of cases:

I. Batten's Case. - A male infant eleven weeks old, weighing $7 \frac{1}{4}$ pounds. Up to the age of five weeks the child was quite well ; he then began to throw up his food, and had continued to vomit afterwards. A diagnosis of pyloric hypertrophy was made from the following points: (I) A healthy baby at birth. (2) Vomiting. (3) Constipation. (4) Subnormal temperature in rectum. (5) Wasting. (6) Marked dilatation and peristalsis of stomach. (7) A tumour in the position of the pylorus. (8) Absence of the usual signs of gastritis. The infant was fed by a nasal tube. Treatment was begun in December, I 898. In May, I 899, the patient weighed 16 pounds. In August an attack of acute gastroenteritis with broncho-pneumonia proved fatal. At the postmortem a hypertrophied pylorus and stomach were found.

2. KenR's Case.-During the first few months of life the child had developed normally; then it began to vomit every time it took food, and to lose weight. Stenosis of the pylorus with hypertrophy was found at the time a gastro-enterostomy was performed.

3. Sonnenburg's Case. - In which gastro-enterostomy was performed in a child of five years of age for congenital stenosis.

4. Hunsy's Case. - A boy aged eleven, who had suffered from infancy from general alimentary disorder, whose principal symptoms were swelling of the abdomen, especially in the left lower region, vomiting of large quantities of half-digested food, false appetite, constipation, etc. The signs were splashing or 
gurgling in the swollen region, cyanosis, and undoubted emaciation. On distending the stomach with carbonic acid gas, it was found to be enormously enlarged, extending downwards to the symphysis pubis. On opening the abdomen a 'concentric thickened ring of hypertrophy round the pylorus' was found, about a finger's breadth in width, without any cicatrix or other adhesions that would raise any suspicions of preceding inflammation. Gastro-enterostomy was performed with good results. Hunsy adds that the case is " doubtless one of congenital muscular hypertrophy of the pylorus.'

5. Mayo Robson's Case.-Mr. A. J. H., aged twenty-four; had suffered from indigestion which had been present for five years, and which had had a gradual and painless onset. $\mathrm{He}$ had discomfort and fulness after meals with flatulency, evidently associated with fermentation of the stomach contents. He was 5 feet ro inches in height, but only weighed 8 stones ro pounds, and, as he had recently further lost weight and strength, his friends were naturally anxious about him. He had already had his stomach washed out twice a week for some time, but without benefit. On making a physical examination no tumour could be felt ; but the stomach splash was easily obtained, and on distending the stomach with $\mathrm{CO}_{2}$ it was found to reach three fingers' breadth below the umbilicus. On June 9 an operation was performed on him, when the pylorus was found to be contracted, so that the little finger could only with difficulty be passed through it.

Pylorodiosis was performed by Hahn's method, and the pyloric sphincter was stretched until it readily admitted two fingers by invagination. The operation was performed at Huddersfield with the assistance of Dr. Irving, under whose care he remained. He made a good recovery, and gained about $1 \frac{1}{3}$ stone in weight in the course of the next few weeks. The improvement lasted for three months, when he arrived at a standstill for a month, and during the next three months he rapidly lost what he had gained, and his weight diminished to 8 stones 7 pounds. There was no vomiting, and he had no pain, but the fulness and flatulency with acidity continued. We saw him again in January of the following year. On distending the stomach, we found that it had returned to its former volume. He was thin from illness and weak, and in spite of lavage of the stomach, careful feeding, and rest in bed, he made no improvement, and it became clear that unless something could be done he would probably die.

A posterior gastro-enterostomy was therefore performed on February 25, 1900. It was interesting to note that a distinct scar over the pylorus could now be seen, as if the pylorodiosis 
had led to ulceration, and so to cicatricial contraction. He made a satisfactory recovery from the gastro-enterostomy.

There can be little hesitation in affirming that a congenital abnormality in the pylorus of some, at present indeterminate, character may after the lapse of few or many years be so altered, or added to, as to cause symptoms of pyloric obstruction. The conditions and the frequency of such cases require further investigation.

\section{Treatment.}

In the earlier stages of the disease, before the vomiting has become incessant, much good may be done by careful washing of the stomach, and feeding in small quantities through an indiarubber catheter. A doubt may perhaps at first be felt as to the accuracy of the diagnosis in cases which recover under this treatment, but the doubt is not valid. A series of cases are now recorded-three by Finkelstein from the private practice of Professor Heubner, and isolated examples by F. E. Batten, Coates, Still, Senator, and others, which prove beyond dispute that the condition is not necessarily fatal. Batten's case has already been given in detail ; a clearer proof of the possibility of the subsidence of symptoms under treatment and of recovery afforded by this case could not be needed.

In feeding the little sufferers from this disease, it is better to pass an indiarubber catheter and gently to wash out the stomach with a little sterile salt solution before introducing the food. The best food is probably diluted and sweetened cow's milk. It should be given in small quantities of 2 ounces or slightly more, and be gradually increased as experience may sanction. The catheter may be passed through the nose, and the sucking action, which is the starting of a peristaltic wave, thereby avoided. If all goes well for eight or ten days, an attempt may be made to feed the child by the mouth, either by teat or with a spoon. If the food is quietly retained the nasal feeding may be gradually abandoned.

Despite the fact that recovery from this condition, under medical treatment on the plan just described, is possible, there will doubtless remain a large proportion of cases that 
can only be dealt with satisfactorily by surgical measures. Many cases have already been treated by operation, and varying opinions as to the most appropriate means to be adopted have been discussed. The operations which have been performed are Loreta's operation, dilatation of the pylorus, pyloroplasty, gastro-enterostomy, and pylorectomy. The latter is so obviously unsuited to the tender age and the prostrate condition of the infant that, as equally effective methods of overcoming the mechanical obstruction exist, it is not worthy of further thought. The operations of pylorodiosis, pyloroplasty, and gastro-enterostomy have each been performed on several occasions, and their relative merits have not, as yet, been definitely determined.

Loreta's Operation, Pylorodiosis, consists in opening the stomach by a small incision near the pylorus, and through this incision introducing a small pair of forceps, which is passed through the stenosed pyloric orifice. The blades of the forceps are then gently separated, and the tissues around them stretched as widely as is possible without rupturing the serous coat. In one case, related by Harold Stiles, a rupture of the duodenum was produced by the forcible dilatation, and the little patient died from peritonitis. In two other cases Stiles dilated by means of tracheal dilators with a satisfactory result. Burghard prefers this operation on account of its ease, the rapidity with which it can be performed, and the absence of shock. He employs Hegar's dilators. In one case a rupture of the peritoneal coat was produced. The disadvantage of the operation would appear to be the likelihood of a recurrence of symptoms after the paralysis of the pyloric sphincter has passed away. Experience has shown that when the sphincter ani, for example, is stretched to its utmost limit a fair degree of control is established by the third or fourth day. But it must be admitted that in all the patients who have recovered from the operation the after-results seem to have been most satisfactory.

Pyloroplasty has been practised and especially advocated by Clinton Dent. He believes that pyloroplasty is preferable to dilatation for the following reasons : 
I. It can be done at least as quickly.

2. It is a more definite proceeding, and allows more range, as the length of incision can be graduated according to the condition found.

3. The lumen of the tube can be examined, and, if thought desirable, the longitudinal fold of mucous membrane can be removed.

4. The exact amount of injury done to the parts is known.

Pyloroplasty in the adult has been found to be, on the whole, an unsatisfactory operation, partly because a very striking tendency to subsequent narrowing has been observed, and partly because the formation of adhesions of the sutured line to the abdominal wall, omentum, liver, etc., crippling the stomach in its action, has been frequently noticed.

Clinton Dent records two cases of pyloroplasty. The first operation was performed on June 10, 1902. The patient, a boy, was born on April I4. Vomiting began at the age of three weeks, and persisted. When seven weeks old, a dilated stomach, showing visible peristalsis, was seen. After the operation a teaspoonful of hot water was given by the mouth every quarter of an hour; then he received a like quantity of whey, the amount being doubled forty-eight hours later. The vomiting continued after the operation, and up to the time of discharge the patient 'was occasionally a little sick.'

Eight days after the operation the child weighed exactly a pound more than he did the day before operation, but 'some of the gain was due to œdema.' The child was discharged on July 6 , weighing an ounce less than on admission. The food passed readily through the pylorus, and was taken eagerly and without vomiting. On July 29 he was readmitted for an attack of diarrhoea due to unsuitable food. Under treatment he improved gradually until September 3, when his weight was 9 pounds to ounces, and he 'looked well and happy.' On September 9 the little patient died from zymotic enteritis. Dent adds: "The case may fairly be claimed as one of recovery from the original mischief.' No post-mortem report is given.

The influence of the operation, pyloroplasty, upon the 
recovery of this patient is perhaps open to question. The vomiting continued after the operation to such an extent that practically all that was given by the mouth was returned. It might well be urged that if the child had been submitted to the dietary discipline that followed the operation, without the pyloroplasty having been performed, an cqually satisfactory ' recovery from the original mischief' might have been attained.

The second case operated upon by Clinton Dent made a perfect recovery. The following are the notes: The patient, a boy, was born on July 6, I902, and weighed ro pounds. He was the third child, the two previous boys being strong and well. From the first the mother noticed that he did not take the breast readily like the previous children, and that he was soon satisfied. On July 27 he weighed ro pounds 6 ounces, but he then started severe vomiting, and lost 12 ounces in four days. At first the vomiting was very bad, everything being brought up in from ten minutes to an hour. The stools were variable, and not noticed to be markedly small. During the first ten days of life he was breast-fed, and then for a short period he was partially bottle-fed on account of pyrexia and a tender breast in the mother. On August I 3 he was seen in consultation with Dr. James Morrison, who had recognised the presence of some obstruction. The child looked ill, the eyes were sunken, the tongue was clean, and the weight was 9 pounds 6 ounces. The stomach was dilated, and peristaltic movements could be seen passing onwards to the pylorus, there pausing, and then continuing onward down the duodenum. Deep down under the liver at the point of temporary pause in the peristaltic wave could be felt an ill-defined, rounded, movable tumour. The last stool was greenish, and contained a little fæcal matter. Temporary measures in the way of diet and drugs were tried for a few days, but as the child steadily lost ground, and the vomiting was characteristic, pyloroplasty was performed by Mr. Dent on August Ig. A little altered blood was brought up during the next night. Fæcal matter was passed on the third day, and on August 28 the child was gaining weight. During the next four weeks 
he gained no less than 8 pounds. His recovery was steady and uneventful. The post-operative treatment and feeding were conducted on the same lines as in the other case.

Gastro-enterostomy was first performed by W. Abel. The patient was a male, eight weeks old, upon whom anterior gastro-enterostomy was successfully performed. Kehr relates two successful cases, and Monnier one. Fatal cases are recorded by Meltzer, Stern, Adler, and others.

The anastomosis between the stomach and the jejunum may be made upon the anterior or the posterior surface. Posterior gastro-enterostomy in adults is now the operation most frequently selected. Its disadvantages are said to be the greater length of time required for its performance and the greater exposure of viscera. Neither of these objections is sound. A considerable experience of the operation entitles us to say that no more than half an hour need ever be expended in the operation. In desperate cases twenty minutes will be adequate to allow of its careful completion. During the operation there need be absolutely no exposure of the viscera. Only those parts of the stomach and jejunum which are to be united lie outside the abdomen. The soiling of the peritoneum is certainly less in gastroenterostomy than in either pyloroplasty or in Loreta's operation, and there is no blood lost from the cut vessels of the stomach. It is certainly necessary in the early stages of the operation of gastro-enterostomy to handle the stomach and to prepare it for the application of the clamp, if clamps are employed, whereas in pyloroplasty the stomach need hardly be touched, and the intestines are not seen.

Which of the three operations-pyloroplasty, pylorodiosis, or gastro-enterostomy-will finally prove to be the generaliyaccepted procedure it is impossible to say. The statistical results, up to the end of 1902, were as follows: Pyloroplasty had been performed three times successfully. In a fourth case, recorded by Sonnenburg, a pyloroplasty was performed, and, as the relief was imperfect, a gastro-enterostomy was subsequently performed with a good result. This patient was six years old, and the case is therefore hardly in the same category as those occurring in infancy. Gastro- 
enterostomy, always anterior, had been performed nine times. Five patients recovered, four died. In one of these death was due to acute obstruction, caused by a Murphy button, which had been used to effect the anastomosis. Loreta's operation had been performed nine times, with seven recoveries.

\section{Congenital Atresia of the Pylorus.}

Hammer (quoted by Nicoll, loc. sup. cit.) distinguished between congenital stenosis, characterized by great thickening, and congenital atresia, in which no thickening is found. $\mathrm{He}$ describes a case of congenital atresia in which the pyloric end of the stomach and the upper end of the duodenum formed culs-de-sac abutting on one another, and united by a fibrous band in which there was ultimately found an exceedingly fine channel, which, however, had proved impervious to fluid under pressure, used as a test.

The disease has in all recorded examples run a rapidly fatal course. If diagnosed early, it should, nevertheless, prove amenable to surgical treatment. 


\section{CHAPTER V \\ INJURY OF THE STOMACH}

Wounds of the stomach may be incised, lacerated, or gunshot. Incised (punctured) wounds are caused by stabs with sharp-pointed instruments of metal or wood. Lacerated wounds are the result of severe contusions, kicks, blows with the fist, falls, or buffer accidents. The stomach is less frequently injured than the intestine, owing to its protection by the chest-wall and liver. The stomach may also rupture spontaneously or by injury from within.

A wound of the stomach may involve the mucous coat alone, the mucous and muscular, the muscular and serous, the serous alone, or all the coats; it may or may not be associated with a wound of the abdominal wall, and may be found on either the anterior or the posterior surface. The symptoms will vary according to the nature and extent of the wound. If the mucous surface alone is wounded, gastrorrhagia of greater or less severity will be noticed. In some cases there has been copious vomiting of bright arterial blood, showing that a vessel of some size has been wounded. If the serous coat alone is torn, there will be a localized peritonitis, which may possibly lead to a perigastric abscess. If a complete solution of all the coats occurs, the symptoms and signs will depend in some degree upon the amount and character of the food present in the viscus. If the stomach be empty, the extravasated fluid will consist of gastric juice, with remnants of food and mucus, and it will be, comparatively speaking, innocuous. If the stomach be laden with recently-acquired food, the material escaping into the peritoneal cavity will be abundant in quantity, and 
will contain various forms of micro-organisms. Septic peritonitis will result in either case, but the measure of its virulence will depend upon the quantity and character of the extravasation. The symptoms following immediately upon the receipt of the injury are pain, collapse, and hæmatemesis.

Pain is of widely varying intensity, but is generally severe; the whole of the abdomen, but especially the umbilical and epigastric regions, are sensitive and tender. Any attempt at movement excites or increases the suffering. The pain may pass upwards to the sternal region, or laterally. Shortness of breath or difficulty of breathing are often mentioned as appearing early.

Collapse is sometimes immediate and profound, and is then due to hæmorrhage from a large vessel. The pulse is rapid, thin, compressible, the skin cold and pallid; there is a bursting forth of sweat over all the body. Gradually the abdomen, at first held rigid and tense, becomes swollen, and the signs of a general peritoneal infection swiftly assert themselves.

Hæmatemesis is the most important symptom. It may be noticed immediately after the accident, or only after the lapse of an hour or more. Clayton records a case of persistent hæmatemesis, after a crush, from which the patient died; a laceration of the mucous membrane of the stomach was found.

The following case is worthy of record :

Hematemesis following on Stab Wound of Abdomen: Abdominal Section.

On January 27, I897, a young mechanic, aged twenty, was admitted to the Leeds General Infirmary after vomiting of bright blood following on a stab wound of the abdomen, the wound having been inflicted by a long, fine, triangular file that had caught in the lathe at which the youth had been turning.

On admission he was pale and collapsed, with a pulse of 190 ; and as there were signs of free fluid in the peritoneal cavity, it was thought that the stomach had been perforated, and that the bleeding was from a ruptured gastric artery.

On opening the abdomen a large quantity of blood and clot was found free in the abdominal cavity, and in the kidney pouches 
and pelvis, but on carefully examining the stomach no perforation could be found. After some searching the superior mesenteric vein was found bleeding freely, the file having bruised and pushed aside the stomach, pierced the great onentum, and then wounded the vein.

The abdomen was washed out with hot water after the vein had been ligatured, and this also arrested the gastrorrhagia, as the patient made a good recovery and had no further hæmatemesis. The shock was relieved by the infusion of 3 pints of normal saline solution into the median basilic vein, and by injections of liq. strychniæ.

The bleeding was evidently from bruising, and possibly laceration of the mucous coat of the stomach, and not from a large vessel, as it was apparently arrested by the hot lavage, without any actual operation on the stomach itself.

If the wound, or the chief of several wounds, is on the posterior surface of the stomach, the symptoms and signs are equivocal, and a localized effusion in the lesser bag will result. The first case of this kind was recorded by $\mathrm{E}$. Rose of Berlin.

Rehn of Frankfort related, at the German Surgical Congress of 1896 , the case of a girl, aged nineteen, who fell some distance heavily on to the abdomen. The symptoms suggested injury to the stomach. A laparotomy was performed five hours after the accident. A turbid fluid escaped through the incision. On the anterior wall of the stomach were two wounds, which involved the serous and muscular coats. A tear was seen in the great omentum close to the stomach, from which fluid issued. This opening was cautiously enlarged, and the posterior wall of the stomach exposed. A wound 4 inches in length was then laid bare, involving all the coats of the posterior wall of the stomach. This was stitched, a wound in the spleen sutured, and the abdomen closed.

\section{Traumatic Ulcer of the Stomach.}

Traumatic ulcer of the stomach is more often found in men. As a rule, the patient, in hurrying, strikes the epigastrium against some heavy or sharp object-the corner of a table, or of a box or staircase, etc.; in other cases a blow from a fist or a kick is received in the course of a struggle. In one case recorded by Rendu the patient was squeezed between the opposed seats of a railway carriage 
during an accident. The injury may arise from within, as when, for example, a piece of sharply-spiculed bone has been swallowed, or it may arise from a perforating wound, as from a stab with a knife or from a bullet wound. The lesion which results in an ulcer may be a tearing of the mucosa or a hæmatoma beneath the unbroken surface of the mucous membrane. Ulceration is more apt to follow a wound of the stomach if the viscus has been filled with food and the process of digestion active. The actual cause of the ulceration is the digestion, by an excessive secretion of gastric juice aroused by the lesion of the damaged tissue, of the mucous membrane. Very rarely the submucous or the muscular layers are invaded by the wound. Hæmatemesis is noticed at one or more of these stages, when the rent is made in the mucosa, when the slough which results from the damage separates, or when an ulcer has formed, and in the course of its deepening a vessel is opened. Perforation may occur either when the slough separates or when the process of ulceration is spreading. When the ulcer has become chronic, there may be thickening or stenosis of the pylorus, as recorded by Micaëlis, or an hour-glass stomach may be caused, as related by Potain.

The occurrence of traumatic ulcer of the stomach was first recorded by Pinel in the early part of the nineteenth century. A full record of cases will be found in a Paris thesis, 'L'Ulcère traumatique de l'Estomac,' by Dumeny, I903.

\section{Gunshot Wounds of the Stomach.}

Gunshot wounds of the stomach vary greatly in character and in treatment, according to the nature of the weapon which produces them. In the South African War the rifles used by both the combatants delivered a bullet of small size travelling with an extremely high velocity. The result was that there was a cleavage rather than an actual destruction of the tissues, and in those cases where a bullet traversed the abdominal cavity it was found that when the stomach or intestines were wounded there was no leakage of the visceral contents, and that healing generally occurred without complication. 
The lessons of this war, however, have no application in civil practice. The rude weapons of the suicide or the would-be murderer are often clumsy and uncertain. With them a larger bullet is used, and the rate of velocity is very much less. There is, as a result, a large destruction of tissue and a crushing or bruising over a wide area. In addition to the perforation in a gunshot wound made by

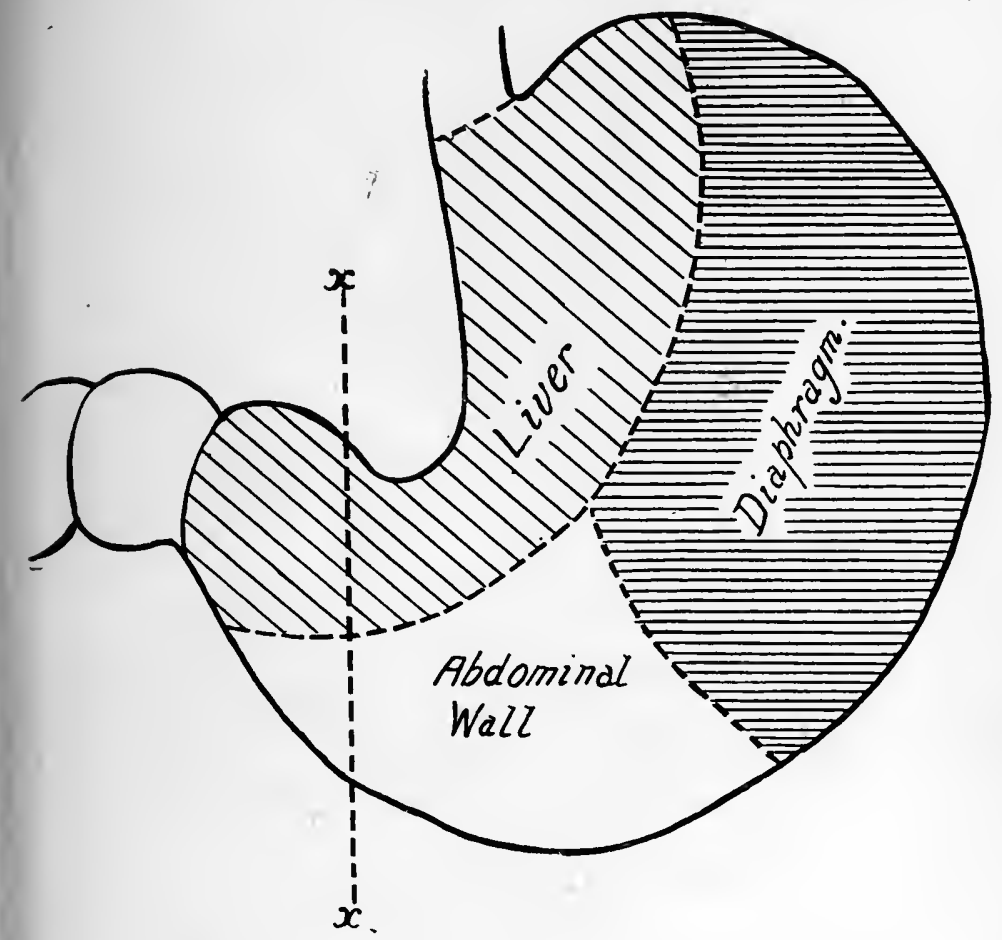

Fig. 6.-Relations of Anterior Surface of Stomach. (Testut.)

such a weapon, there is a considerable amount of contusion and of rough damage to the immediately adjacent parts.

Forgue and Jeanbrau (Rev. de Chir., September and October, I903) have collected the notes of II 2 cases of gunshot wound of the stomach in which the lesion was verified either at the post-mortem examination or at an operation. In 64 of these cases the ball passed completely through the stomach, wounding both surfaces. In some cases only one 
wall was injured, the ball remaining in the stomach, or passing, in one case, into the intestine. In other cases the bullet had struck a curvature and clipped a piece away.

Seven of the cases died of hæmorrhage, which was due generally to the wounding of a large arterial trunk, such as the coronary. In one case, related by Guinard as having occurred at the Lariboisière Hospital, an abdominal explora-

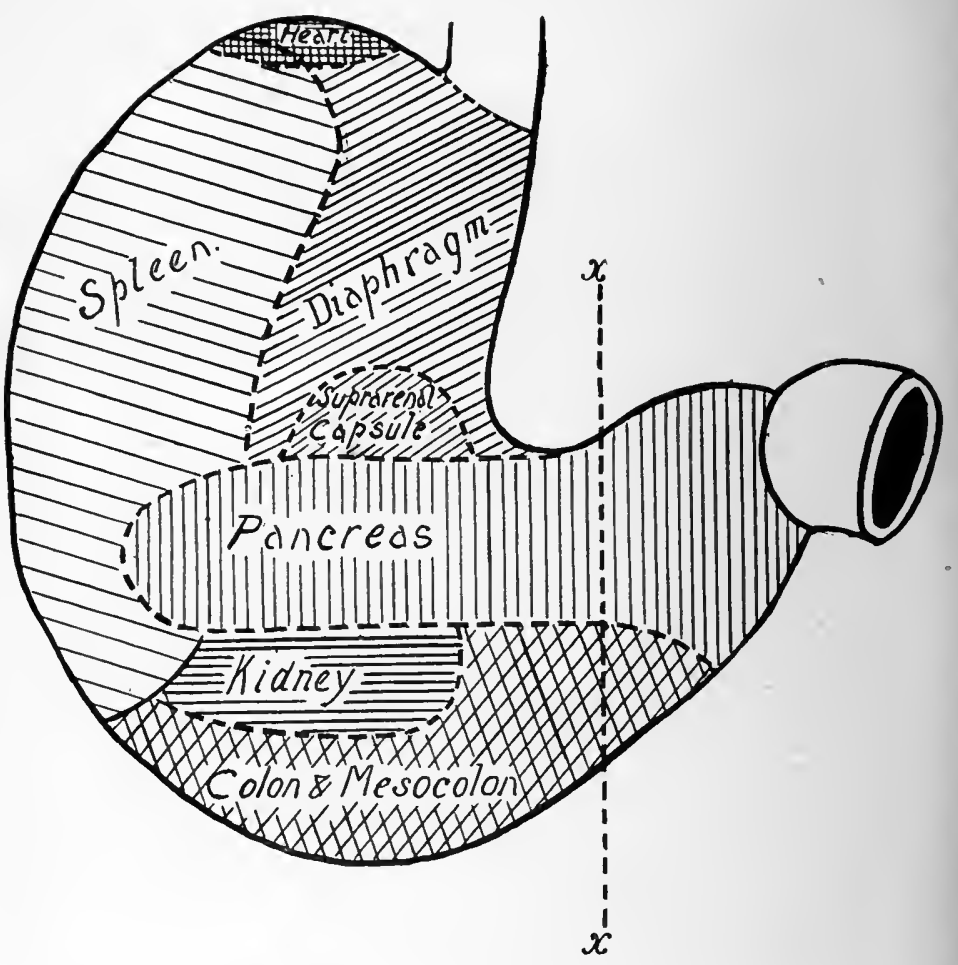

Fig. 7--Relations of Posterior Surface of Stomach. (Testut.)

tion was undertaken in a patient who presented no signs of injury except an abundant hæmatemesis. A very minute examination of the stomach was made, but nothing abnormal was found. The patient died, and at the autopsy the bullet was found free in the peritoneal cavity. It had not perforated any viscus. But on examining the interior of the stomach a wound of the mucosa close to the pylorus was 
found, of the size of a franc piece. This was the source of the hæmorrhage.

Peritoneal infection depends upon the size of the wound, and upon the state of repletion of the organ. If the stomach is full, and if vomiting occurs, the contents escape freely into the general cavity of the peritoneum.

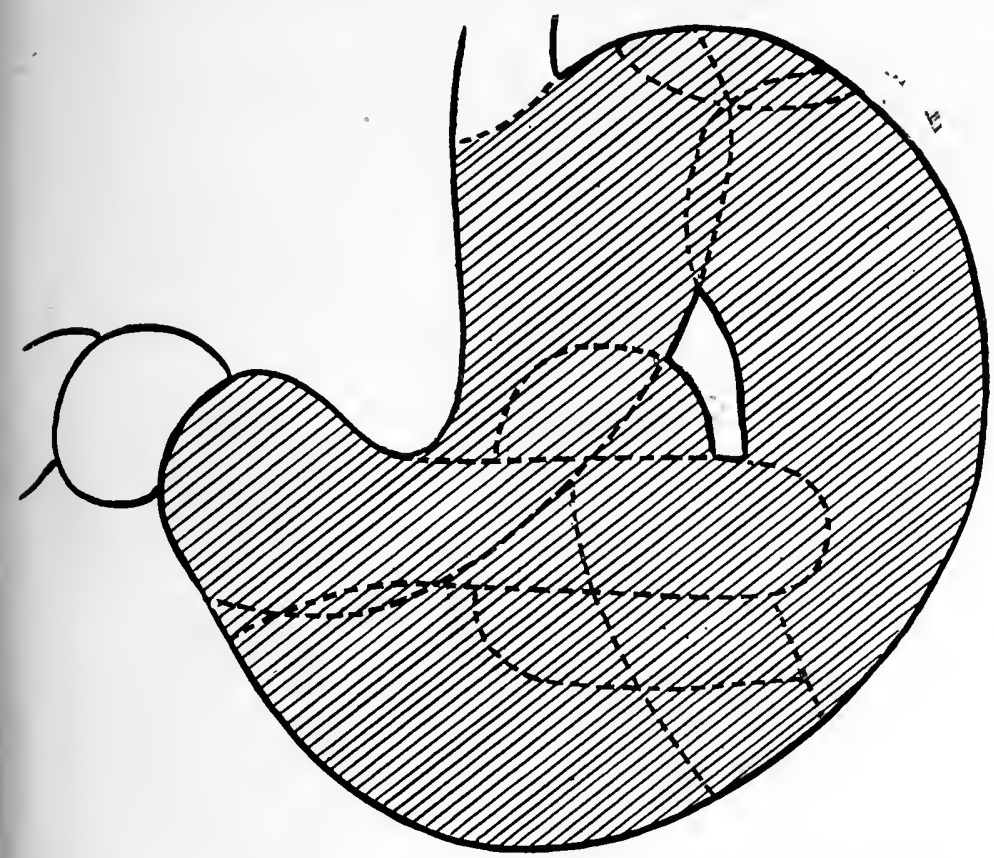

Fig. 8.-Preceding Figdres superimposed (Forgue and Jeanbrau.)

The light area shows the only part of the stomach which can be penetrated without the wounding of other structures.

Spontaneous recovery is possible. Socin records the case of a man who was shot in the abdomen. It was thought that the stomach was wounded, but the patient recovered without operation. Five months later he died of a 'medical illness.' At the autopsy two wounds of the stomach soundly cicatrized were seen. Spontaneous healing such as this depends upon the emptiness of the stomach, the small size of the wound, and the plugging of the wound by omentum; or upon the formation of a gastric fistula, which permits 
the instant escape of contents to the exterior, as in the oftquoted case of Alexis St. Martin, and in others related by Baudens, Cannizaro, and T. Smith.

In all cases of revolver or pistol wound-indeed, it may be said in all forms of gunshot wound-of the stomach in civil practice the abdomen should be opened with the utmost expedition. The records of the cases collected by Forgue and Jeanbrau show that the mortality increases in direct proportion to the delay. The result of their inquiries is shown by the following table:

I. Wound of the Stomach alone, without other Viscera.

Recovery. Death.

(a) Intervention within 6 hours $\quad \ldots \quad 96 \quad \cdots \quad 4$ cases.

(b) " without mention of time 2 .. 4 ,

2. Wound of the Stomach, with other Viscera.

(a) Intervention within 6 hours

Recovery. Death.

(b)

(c),

(d) ,

" 6 to 12 hours

... I 3 ... I6 cases.

In examining the stomach the utmost care should be exercised. The figures given by Forgue and Jeanbrau show that it is not unlikely that some other damage will be inflicted by a bullet which traverses the stomach. Search for such an injury must be made, and any wounds so found must be appropriately dealt with. So far as the wound in the stomach is concerned, the edges must be trimmed or turned in by suture,

The following case is recorded by Dr. Roswell Park, of Buffalo, a surgeon entitled to speak with high authority on this subject (Annals of Surgery, vol. xxxviii., p. 228). It exemplifies as well as any case could the attitude the surgeon should adopt in such an emergency.

'About 6 p.m., February 3, I902, a woman of twenty-six years turned the point of a 22-calibre pistol towards the centre of her body, and fired it in a suicidal attempt. She was soon after removed to the Buffalo General Hospital, where I saw her about 
7.30 p.m. But one shot had been fired. This evidently took effect in the middle line about an inch above the tip of the sternum. Between the time of the injury and that when I saw her she had vomited more or less fluid and bloody material. With the exception of complaint of considerable pain and the vomiting as above, her general condition was good. I at once prepared her for operation, and had the back of the body as well as the anterior surface scrubbed and sterilized.

' Gas and ether were given as the anæsthetic. The area of the gunshot wound was excised by a wide elliptical incision, which was then extended downward as a straight line incision to the region of the umbilicus. The xiphoid appendix was not only perforated by the injury, but broken loose from its attachment, although not so loosely that its removal was called for. Upon opening into the upper abdomen, a large quantity of fluid and clotted blood presented, and was removed with the hand used as a scoop. Upon withdrawing the stomach, it was evident that the bullet, which had passed through the left lobe of the liver, had cut across the upper curvature a little to the pyloric side of the middle of the curved line. This opening was like a notch in the upper border, which probably had been a double perforation at first, with a very slight intervening bridge, which latter was torn in the handling of the viscus. Through it the little finger could be easily passed into the stomach. The stomach seeming reasonably empty, I made no particular effort to clean it out, but at once carefully closed the opening with three rows of fine silk sutures, the first of which closed the mucosa, and the last of which took in some of the peritoneal fat, making a sort of omental graft. After replacing the stomach, it was evident that there had been considerably more hæmorrhage from the bullet track in the liver. I again removed a large amount of blood from the lesser peritoneal cavity, at first with the hand and later by sponging. Altogether, at least two quarts of fluid and clotted blood were thus removed.

' Of the bullet I found no further trace. Exploring backward through the gastrohepatic omentum, I could not make out any wound or injury of the pancreas; nevertheless, I made a posterior opening on the left side at the costospinal angle, and here cut down upon the point with a long pair of forceps introduced through the front and held in the left hand. Through this opening a good-sized drainage-tube was drawn with the forceps, being drawn from without inward to a depth of about 6 inches from the skin, so that its inner end lay in the cavity of the lesser omentum in front of the pancreas.

' This still left a somewhat gaping punctured wound of the liver, the hæmorrhage from which was easily checked by tam- 
poning with a strip of gauze. This strip was left hanging out of the upper end of the abdominal wound. Before closing this wound, and before making the final toilet of the peritoneum, I inserted a large gauze drain wrapped in perforated oil-silk, which was passed through the gastrohepatic omentum to such a depth that its lower end was close to the inner end of the posterior drainage-tube. The abdominal wound was then closed with silkworm sutures, save for the point where the drain emerged, where secondary sutures were used.

' The patient developed no unpleasant symptoms after the operation, save that on the following day her temperature was $1025^{\circ} \mathrm{F}$. She was given two enemata, each of which contained 2 grammes of antipyrin. She vomited no blood and raised scarcely any fluid at all. After the second day her temperature never went above $100^{\circ} \mathrm{F}$. The anterior drain was removed on the third day, and the gauze tampon removed from the liver on the fifth. The posterior drain was shortened on the third day and removed on the fifth. Absolutely no fluid was allowed in her stomach for four days, and nothing except water until the eighth day, she being nourished meantime by the rectum. Abdominal sutures were removed the fifteenth day. She left the hospital, March II, I902, in apparently perfect health. I have not subjected her to X-ray examination for the purpose of detecting the present location of the bullet, and consequently have no idea just where it may be located.'

\section{Injury of the Stomach from within.}

There are on record a number of cases of spontaneous rupture of the stomach-of rupture, that is, independent of ulcer or carcinoma, or other visible disease. Such a bursting results from overdistension. Arton reports a case in a negro aged fifty years. Fulton mentions a case of rupture of the cesophageal end of the stomach in a child owing to distension. Collins describes a. spontaneous rupture in a woman seventy-four years of age; this was followed by collapse and death, and a necropsy showed a rupture 2 inches long, about 2 inches from the pyloric extremity. The records of St. Bartholomew's Hospital contain the account of a man, thirty-four years of age, who for two years had been the subject of paroxysmal pain in the stomach. At the hospital he had an attack of vomiting after a debauch, and after a 
sudden attack of pain at the pit of the stomach he died. A ragged opening on the surface of the stomach near the cardiac extremity was found post-mortem, there being no signs of gastric ulcer or cancer. Clarke (Indian Medical Gazette, I885) reports two cases-one in a Hindoo, twentytwo years of age, and another in a woman who was supposed to have died from cholera. Recovery probably never occurs naturally in these spontaneous ruptures.

Perforation may occur from within, as in the case of sword and knife swallowers. A remarkable case of this kind occurred at Guy's Hospital in I807. After death there were thirty or forty fragments of knives found in the stomach, one of which had transfixed the colon and the rectum. Needless to say that in all these cases the sooner after the injury the patient is treated the greater will be the chance of recovery.

\section{Treatment.}

In every case of wound of the stomach, however produced, in which all the coats are involved, operation should be resorted to at the earliest moment.

If any doubt be felt as to the existence of a perforating wound of the stomach, the osophageal tube may be passed, and the stomach inflated with air. If the organ is intact it will distend, and its outline will be plainly visible; if burst, the gas will escape into the peritoneal cavity, and probably cause an immediate loss in the liver dulness.

The abdomen will be opened through a central incision, and the stomach at once exposed. Careful search, both on the anterior and posterior surfaces, must be made, and the entire organ examined. If the wound be small, the bubbling of fluid or gas through the aperture will at once indicate its locality; if large, there will, of course, be little or no difficulty in discovering it. The wound should be stitched up with a continuous suture of catgut, including the mucous membrane alone or all the coats, and a suture of silk picking up the serous coat only. If there has been, as in gunshot wound, a destruction of a portion of the wall of the stomach, an omental flap or graft may be usefully employed to close the 
resulting gap. Enderlen has recently shown (Deut. Zeit. f. Chir., I90o) experimentally in the cat that, when a portion of the stomach wall is excised, there is a free prolapse of mucous membrane. To the cut edges of mucous membrane he stitched a graft of omentum, and the gap in the serous coat was then closed by a large omental flap. The experiment proved, not only that the transplantation succeeded, but that the omental tissue gradually assumed the character of gastric mucous membrane, and well-formed glands developed.

Care must in all cases be taken to insure that a wound of the posterior surface is not overlooked. It would, we think, be a safe and wise precaution in all operations to turn the great omentum upwards, tear through the transverse mesocolon, and expose the posterior surface of the stomach, as in Von Hacker's operation. The existence of multiple wounds must be always contemplated as a possibility. In one reported case there were no less than three wounds in the stomach, two of which were sutured; the third, being undiscovered, led to death from peritonitis.

Dr. G. Woolsey reports a case of wound from a pistol bullet in which he had successfully sutured two wounds of the stomach, two of the transverse, and two of the ascending, colon.

' Kopfstein has related the case of a schoolboy who shot himself in the stomach playing with a revolver. It was about one o'clock in the day, shortly after a meal, when the accident occurred, and he was straightway taken to hospital. The abdomen was not found to be tender; the pulse was accelerated, vomiting present, and the patient was very pale. The wound was observed in the parasternal line, and deviating towards the left, directly under the margin of the ribs.

' One hour later it was resolved to perform laparotomy. The abdomen was freely opened in the median line, and the whole of the stomach to the left laid bare. On the upper, or convex, side of the liver a star-shaped mass of blood was found on the peritoneal covering, which was continued into the parenchyma of the organ as far as the sound would go without injury. A sharp-cut perforation was present in the stomach, admitting the free passage of a pencil with no prolapse of mucous membrane, which is often 
assumed to close these wounds rapidly. Close to the wound in the gastric wall a large vessel was seen pulsating; none of the stomach contents were observed in the liver or the track of the bullet.

"The hepatic hæmorrhage was checked by "Paquelin," and the walls of the stomach were closed with Lembert's sutures.

- The stomach was then lifted up and its posterior aspect examined, but nothing abnormal could be found. The abdomen was then washed out, and the wound closed in three stages.

' After the operation he vomited once. Temperature, $37^{\circ} 7^{\circ}$ Cent., equals $99^{\circ} 86^{\circ}$ Fahr.; pulse, I 20. Next day he took fluids with comfort. The first dressing was removed twelve days after the operation, and the patient dismissed from hospital twenty days after admission.'-Medical Press, Igoo.

\section{Ulceration of the Stomach due to the Swallowing of Caustic Fluids.}

Caustic fluids, such as soap 'leys,' hydrochloric acid, carbolic acid, and other strongly acid or alkaline fluids, may be swallowed accidentally or with suicidal intent. The parts most frequently and most seriously injured in such cases are the mouth, pharynx, the upper end of the osophagus, the lower end of the oesophagus just above the cardiac orifice, and the pyloric antrum of the stomach. The mid portion of the œesophagus and the greater part of the stomach may escape, and in any event are less deeply destroyed than the spots that have been mentioned.

The symptoms noticed at once are burning of the mucous membrane of the mouth and pharynx, which, on examination, are seen to be more or less deeply destroyed; inability to swallow fluids, or to swallow them only in very small quantity and with excessive pain; thirst, which is intolerable and unquenchable; and occasionally vomiting, the ejected fluids containing blood in small or large quantity. In severe cases the shock is profound, and the patient may die after a few days of the greatest agony. Every attempt to drink causes unbearable distress; thirst and restlessness never cease.

In some cases pneumonia may set in, and may speedily prove fatal. In the less severe cases the ability to take thin 
fluids in small quantity may return after a few days, but even then there may be unceasing epigastric pain. Bronchitis of greater or less intensity may occur, and blood or blackish fluid may be expectorated. The patient may, in some cases, recover entirely the power to take fluid or semi-solid foods in fair quantity, but in many cases it is found that after a brief respite there is a serious defect in the stomach. The pylorus and the pyloric antrum are those parts of the stomach chiefly affected. When the ulceration which the caustic has caused begins to heal a dense cicatricial mass is found, which forms a gross mechanical impediment to the onward passage of food. The result is that the stomach becomes enlarged, often to an enormous degree, and its muscular walls show a great hypertrophy. In some of the recorded cases it is said that the stomach filled apparently the whole abdomen. C. B. Keetley remarks of one of his cases :

' Hers was the most striking case of dilatation of the stomach I have ever seen, and it is much to be regretted that no photograph was taken. When she stood up, whether she was viewed from the front or from the side, the whole anterior abdominal wall, except in the left iliac region, could be seen pushed forward by the stomach. The patient was very emaciated, and the shape and movements of the organ could be seen with ease.'

In such a case there may also be a cicatricial stenosis at the lower part of the œsophagus, similarly caused. As a result there will be great difficulty in getting food into the stomach, and a greater difficulty for the food to pass out. In a large proportion of cases there have been pulmonary complications, pneumonia, or acute or chronic bronchitis. These are probably due to the inhalation of septic material from the foul and freely discharging ulcers in the pharynx. In a few of the recorded instances there have been found at the operation or at the post-mortem examination a number of widespread, dense adhesions between the stomach and its neighbours. The ulceration of the mucosa has extended to a sufficient depth to cause a local intense peritonitis. In these circumstances no dilatation results, but often, rather, an actual shrinking in the size of the organ, owing to its continual compression by contracting adhesions. A patient 
so affected will be unable to take fluid in any but the smallest quantities, and vomiting will always be a conspicuous and distressing feature.

In the treatment of all cases there should be a complete abstention from food from the first. It seems to be always the desire of the medical attendant to feed the patient at the earliest chance, and as often as may be, with fluid foods. The result of this is that the process of ulceration is quickened, that ulceration extends more widely and more deeply, and that owing to septic material lodging upon the ulcers their discharge becomes offensive and abundant. The first and chief attempt should be to aid the process of healing by keeping the damaged surfaces sweet and clean. For this purpose mouth washes, gargles, throat sprays, and so forth are useful. But the chief aid to healing will be avoidance of food. In the severer cases the question of operation will have to be considered. If we consider the case of a patient whose mouth, cesophagus, and stomach are deeply corroded with an acid fluid, it will clearly be desirable to avoid infection and irritation by withholding food, and yet to feed the patient so as to carry him through a grave crisis.

Rectal feeding is insufficient and operative treatment is urgently required. The operations suggested are gastrostomy, duodenostomy, jejunostomy, and the combination of the operations of gastrostomy and gastro-enterostomy as suggested by Witzel and Rutkowski. Gastrostomy does not afford rest to the injured pylorus, and is therefore not applicable. Jejunostomy and duodenostomy both fulfil the theoretical requirements satisfactorily, but in a later stage, when cicatricial stenosis has developed in the pyloric region, life cannot be long maintained by their aid alone. It is therefore better to perform the double operation of gastrostomy and gastro-enterostomy. The gastro-enterostomy is first performed, and then through a second opening into the stomach through the anterior abdominal wall a catheter is passed through the stomach into the distal loop of the jejunum. The patient is then fed through the catheter directly into the jejunum, and, when healing in the corroded areas is complete, the catheter may be removed, the gas- 
trostomy opening allowed to close, and feeding begun by the mouth.

The treatment may be summed up, therefore, in the following way :

I. It is necessary to avoid feeding, even with fluids in small quantity.

2. The mouth, pharynx, and the accessible damaged areas should be cleansed and kept clean by frequent wiping, spraying, or gargling.

3. Gastro-enterostomy, combined with gastrostomy, after the method of Witzel and Rutkowski, should be performed, for in this way both the immediate pressing needs of the patient are relieved, and his future distress, otherwise inevitable, anticipated and prevented.

4. If the case is not seen until a late stage, when cicatricial stenosis of the pylorus and consecutive dilatation of the stomach have developed, gastro-enterostomy should be performed.

C. B. Keetley, who has recorded three cases of ulceration of the œsophagus and stomach, due to swallowing strong hydrochloric acid, writes (Trans. Roy. Med.Chir. Soc., Igor):

'The main conclusion that I draw from a study of these three cases is that in cases of poisoning by the more corrosive acids surgical intervention should be almost immediate, and that it is a mistake to postpone resort to surgery until there is no other alternative, except that of letting the patient die of inanition.'

An opinion with which we are in complete accord. 
$\therefore$

- 


\section{PLATE IV.}

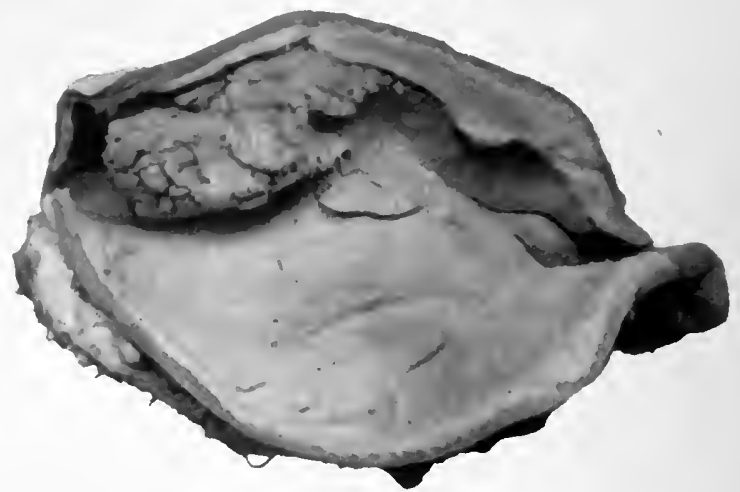

Fig. 9.-Villous Growth near Lesser Curvature, found Post-mortem IN AN AGEd WoMan.

(No. 2,407a, Royal College of Surgeons' Museum.)

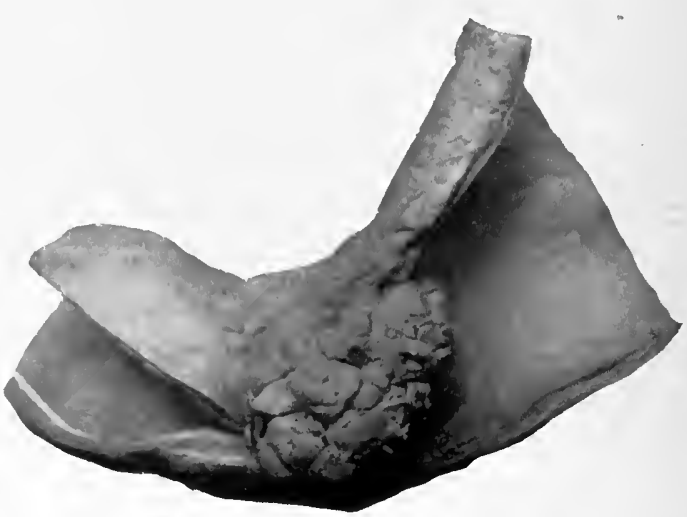

Fig. io.-Polyyus near Pylorus, which caused Death by Vomiting.

The patient was a woman aged ninety-two. (No. 2,405d, Royal College of Surgeons' Museum.)

To face $\rho .75$. 


\section{C.HAPTER VI}

\section{SIMPLE TUMOURS OF THE STOMACH}

THESE tumours are rare. Their clinical importance is small, and their symptoms, though frequently trifling and quite inconspicuous, may suggest a diagnosis of pyloric stenosis or cancer. The following forms are known :

I. Adenoma.

2. Lymphadenoma.

3. Lipoma. Lipo-myoma.

4. Myoma. Fibro-myoma.

5. Cysts.

I. Adenoma may be found at any portion of the stomach, though it is most commonly found near the pylorus (Figs. 9 and 10). At first a rounded, smooth projection is formed on the surface of the organ; gradually, owing to a narrowing at the periphery, the little tumour becomes pedunculated, and then hangs pendulous from a narrow stalk. One of us, in operating upon such a case, was able to see the cherryshaped nodule carried on into the pylorus, which it blocked with the completeness of a ball-valve. In another case a sessile polypus which was obstructing the pylorus was removed. The patient gained weight at the rate of half a pound a day, so that in three months he weighed nearly 3 stones more than at the time of operation. The growth proved to be a simple adenoma. When the tumour is of large size it may form a readily-palpable mass of extreme mobility, generally situated above the umbilicus, but occasionally descending below it. According to Sutton, these tumours are sometimes 'so mobile that they may be shifted into all the regions of the abdomen' (Fig. Io). 
Adenoma of Stomach - Fig. II (under the care of Dr. Hinds, Worthing, who has kindly supplied us with the accompanying notes) :

'A. C., female, sixty-eight years of age. Complained of painful "gripings" in the stomach and frequent action of the bowels at irregular times; she had wasted and was unable to work. On examination of the abdomen a hard tumour was felt in the epigastrium, freely movable and capable of being pushed to the left of the spine as low as the umbilicus, and to the back under the right ribs, where it was lost.

'Operation.-Median incision; the tumour was found to be within the stomach. The anterior wall of the stomach was opened, and a tumour the size of a golf-ball found attached to the posterior wall by a base about I inch in diameter, which involved the pyloric opening. The tumour was removed by cutting through the mucous membrane around the base, but at one point the posterior wall at the junction of the stomach and duodenum was opened; bleeding was slight. The posterior wound was closed from within the stomach. The patient recovered from the operation, and was well for twelve months, putting on flesh, and returning to her work. Then sickness, flatulence, and a feeling as if the food "could not get past somewhere," with wasting and inability to work. Gastro-enterostomy by Von Hacker's method was performed eighteen months after the first operation, but the patient died of exhaustion in fortyeight hours. At the autopsy an ulcer $\frac{3}{4}$ inch in diameter, involving the pylorus, was found. 'There was no sign of growth.'

Hayem describes two cases of adenomatous growth, the structure of which resembled Brunner's glands, chiefly affecting the submucosa. The growth appears to be derived from the glandular cul-de-sac of the mucosa, but it rapidly passes through the muscularis mucosæ, and develops in the submucous tissue. In Hayem's cases the adenoma was associated, in the first with the perforation of an ulcer, and in the second with malignant disease (Fig. I2). Hayem regards them as benign tumours which may become malignant.

A case of adenoma simulating cancer was shown in 1894 to the Société de Chirurgie at Paris by Chaput. The patient was a man aged sixty-four. The symptoms were chiefly emaciation, vomiting of coffee-ground material, and an epigastric tumour was observed. At the operation an adenoma 


\section{PLATE V.}

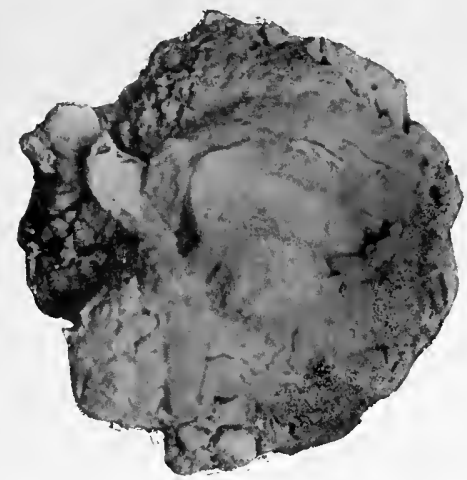

Fig. II.-Adenoma removed from Stomach.

(Dr. Hind's Case.)

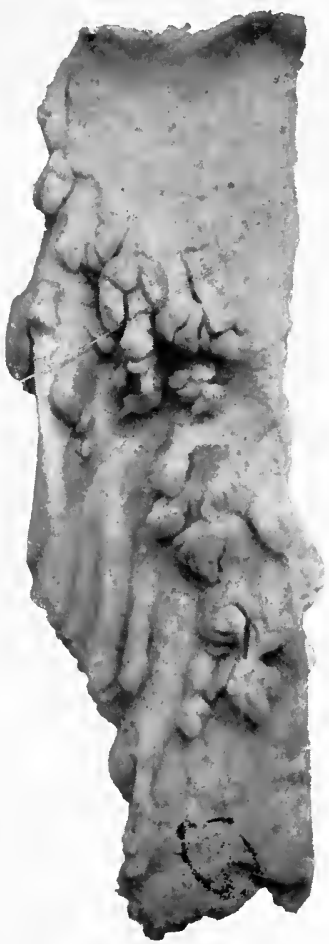

Fig. I2,-Polypi growing from the Mucous Membrane of the Stomach of a Gentleman, Seventy-six Years of Age, who suffered from Constant Dyspepsia.

(No. 2,405, Royal College of Surgeons' Museum.)

To face p. 76.$]$ 


\section{PLATE VI.}

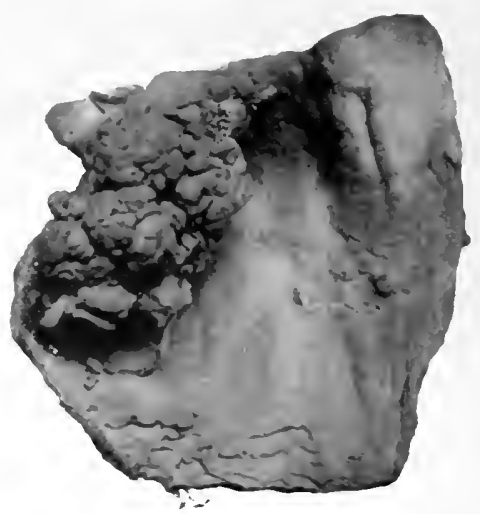

Fig, 13. - Polipus Neak l'ylorus which caused Fatal INTussusceptiox of Duodenum in a Max aged Twenty-one.

(No. 2,405c, Royal College of Surgeons' Museum.)

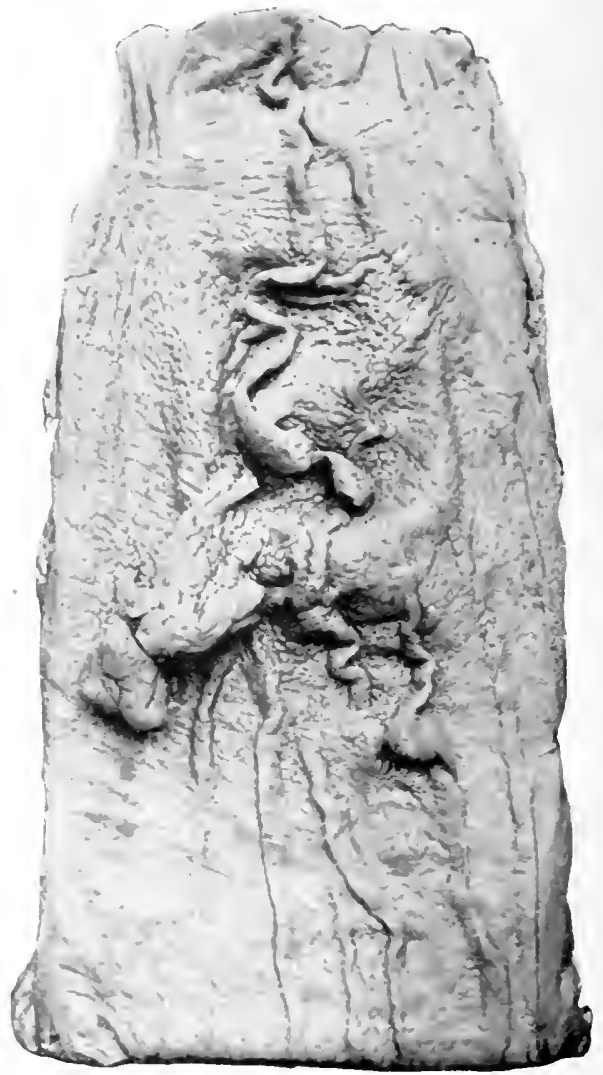

Fig. I4.-Lymphadexoma of Stomach. 
covered with normal mucous membrane was found attached by a small pedicle to the posterior wall of the stomach.

Adenomata are occasionally multiple. Adenoma and malignant disease may be present in the same stomach (Leeds Museum).

2. Lymphadenoma, $\mathrm{A}$ rare form of tumour, characterized by exuberant polyposis of the mucous membrane (Figs. I3, I4). A very good example is described and figured by Pitt (Trans. Path. Soc., I889). Several masses of growth projected into the cavity of the stomach, one measuring 2 inches across and I inch thick. Norman (Dublin Journal of Medical Science, vol. xcv.) reports a case in which symptoms were absent. The mucous membrane was everywhere thickened, and presented all varieties of polyposis, from large dendriform projections to small wartlike growths.

3. Lipoma.-Lipomata are occasionally found in the submucous layer of the stomach near the pylorus. Such a specimen is figured in Virchow's work ('Die Krankhaft. Geschwülste').

A case of lipo-myoma is reported by Kunze (Ann. of Univ. Med. Sci., I8gr). The patient was a man aged fifty-two, who complained of a tumour in the epigastrium. The chief points remarked were that the swelling was hard, nodular, and extremely mobile. There were no stomach symptoms. A diagnosis of mesenteric tumour was made and an abdominal section performed. The growth was found in the anterior wall of the stomach at the cardiac end. The patient succumbed in fifteen days.

4. Myoma and Fibro-myoma.-These forms are generally found as prominent, rounded, hard swellings in the coats of the stomach. They may in their enlargement become pedunculated and project either towards the mucous or the serous coat of the stomach, forming the varieties known as 'internal' and 'external.' Wilks and Moxon state that 'cases are recorded where a polypus of this kind has been vomited up.' These tumours grow slowly, are smooth or nodular upon the surface, and, if projecting into the stomach, are attended by hæmorrhage.

Myoma of the stomach was first observed in 1762 by 
Morgagni. Vogel in 1845 , Förster in 1858 , and Sangalli in I860, recorded examples.

Herhold (Deutsche Med. Wochensch., r898, No. 4) records an example of myoma in a woman thirty-seven years of age. There was a three years' history of intractable vomiting occurring soon after food. The stomach was moderately dilated and the vomit contained free $\mathrm{HCl}$. As there was a history pointing to old peritonitis, it was supposed that there were adhesions pulling on the pylorus. On opening the abdomen, a tumour was found in the pyloric region of the stomach of the size of a hazel-nut, obstructing the duodenum to a moderate degree. The tumour, which was removed, proved to be a myoma.

The following cases are also recorded:

Ruprecht of Dresden (Archiv f. Klin. Chir., Bd. 40) removed a myoma, Io centimetres by 7 centimetres, from the anterior wall of the stomach near the cardia. The patient died of pneumonia.

Von Erlach (Wien. Klin. Woch., 1895, No. I5) removed an enormous myoma weighing $5 \frac{1}{2}$ kilogrammes from the anterior wall of the stomach with success.

The successful removal of a very large fibro-myoma has been accomplished by Professor von Eiselsberg. The growth was 'the size of a man's head,' and hung downwards from the greater curvature. The portion of the stomach bearing it was also removed (Archiv f. Klin. Chir., Bd. 54).

Nicoladoni has also removed a myoma of the stomach (Steiner, Beit. z. Klin. Chir., 22). The patient was in extremis, and died from purulent peritonitis. An area of the stomach wall, 26 centimetres by I4 centimetres, was removed from near the greater curvature (Fig. r6).

Altogether, nineteen cases are recorded in surgical literature. Of these, eleven are external, six internal, and in two details are not given. Of the eleven external, seven grew from the greater curvature, one from the lesser, one on the anterior wall, and two from near the pylorus. The external growths are generally not recognised as arising from the stomach until an operation is performed for their removal.

Myosarcoma is described by Virchow and Brodowski. 


\section{PLATE VII.}

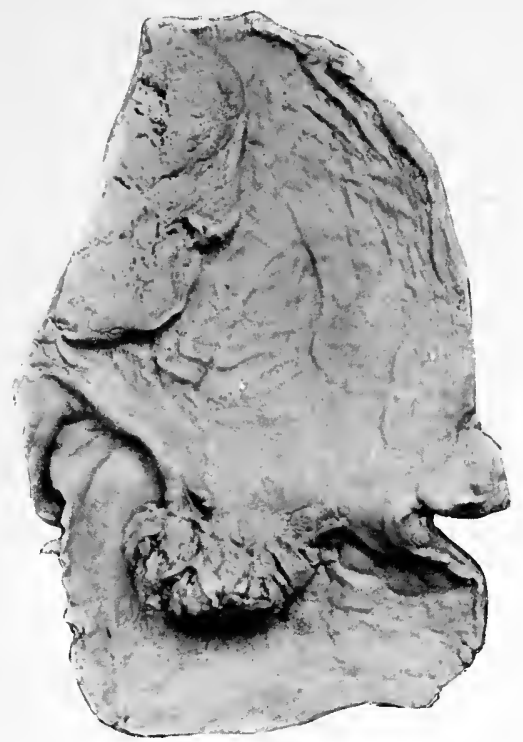

Fig. 15-Malignant Plague in Stomach Wall-Polypoid Growth at THE Pylorus.

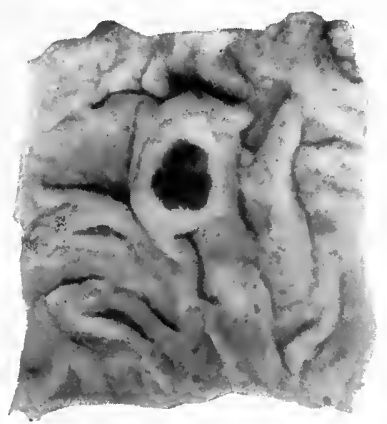

Fig. i6. - Villous Growth near Lesser Curvature, found PostMORTEM.

(No. 2,407a, Royal College of Surgeons' Museum.)

To face p. 78.$]$ 

5. Cysts.-Wilks and Moxon describe a case of cyst in the walls of the stomach of about the size of a walnut. Cysts of small size from dilatation of the glands are not very infrequent.

$Z$ iegler has recorded a case occurring in the Munich Clinic in which, as the result of an injury, a tumour formed in the walls of the stomach. Laparotomy was performed, and a cyst found lying between the muscular and mucous coats of the stomach. The cyst was emptied, and did not refill.

In the Trans. Path. Soc., I857, Mr. J. Hutchinson describes a cystic tumour of the stomach the size and shape of a walnut, situated near the pylorus, between the peritoneal and muscular coats.

H. Read (Medical Record, I882) records the case of a man aged sixty-two who died after an illness of five weeks. At the autopsy a large cyst was found completely encircling the stomach. It contained clear fluid and lumps of fatty substance, with black streaks of extravasated blood.

Anderson (British Medical Journal, I898) gives an account of a case of multiple cysts of the stomach and intestines, which he believed 'originated, after the fashion of dermoids, from inclusion or embryonal rests left over in the process of development of the alimentary canal.' 


\section{CHAPTER VII}

\section{CANCER OF THE STOMACH}

\section{Pathological Anatomy.}

CARCINoma of the stomach is either primary or secondary. The primary form alone is of interest to the surgeon.

The classification of carcinoma which is at once the simplest and most accurate is that adopted by Perry and Shaw in their admirable paper in the Guy's Hospital Reports (I89I). They describe cylindrical carcinomata, including the forms variously termed adeno-carcinoma, cylinder-epithelioma, or destructive adenoma, and spheroidal carcinomata. If the fibrous stroma is abundant, the term 'scirrhus' is applied; if scanty, the term 'medullary.' In both varieties the cells are liable to colloid degeneration. The cylindrical carcinomata are more amenable to surgical treatment. They are slower in rate of growth, and they do not develop adhesions at an early period.

Situation.-Cancer may be found at any part of the organ. Gussenbauer states that in 6o per cent. of cases the growth is in the pyloric region, in 30 per cent. in the body, and in Io per cent. at the cardia. Lebert gives the following percentages :

$\begin{array}{lllll}\text { Pylorus } \ldots & \ldots & \ldots & 54 \text { per cent. } \\ \text { Lesser curvature } & \ldots & \ldots & \text { I6 } & " \\ \text { Cardia } \ldots & \ldots & \ldots & 9 & " \\ \text { Anterior wall } & \ldots & \ldots & 3 & " \\ \text { Posterior wall } & \ldots & \ldots & 4 & , \\ \text { Both walls } \ldots & \ldots & \ldots & 4 & " \\ \text { Greater curvature } & \ldots & \ldots & 4 & " \\ \text { Diffuse } \quad \ldots & \ldots & \ldots & 6 & \\ & & 80 & & \end{array}$


Furnivall in 1,795 cases, compiled from various authors, found the pylorus affected in I,IIO, the lesser curvature in I97, the cardiac orifice in $\mathrm{r}_{5} 8$, and the rest of the stomach in 33I. The cardiac area is rarely affected primarily : in many cases the growth extends downwards from the œesophagus; in others the growth spreads from the body.

Extension.-Adhesions found in association with malignant

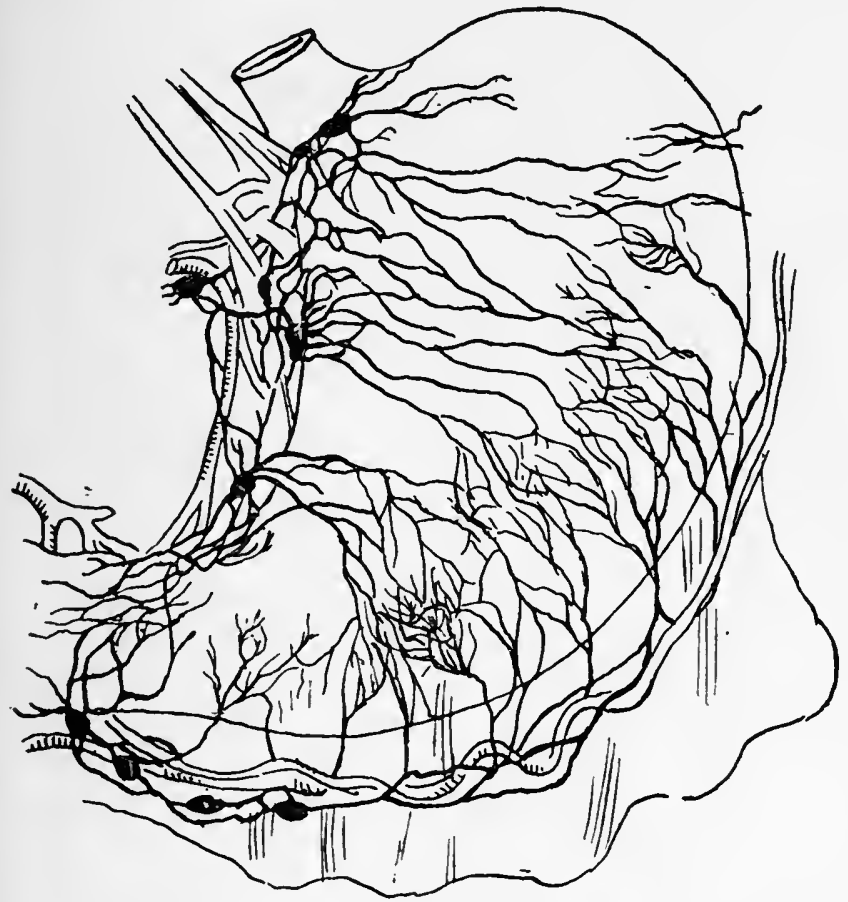

Fig. I7.-The Lymphatics of the Stomach. (After Cunéo.)

disease of the stomach are either simple in character-the result of local septic inflammation-or malignant. Spread of the growth away from the stomach may occur along the line of adhesions, by direct implantation upon a neighbouring surface, through the bloodvessels, or through the lymphatic vessels, which drain into glands situated (a) along the whole of the lesser curvature in large numbers; $(b)$ along the greater curvature, chiefly near the pylorus; $(c)$ a few in the great omentum; $(d)$ near the head of the pancreas. 
Cunéo has recently made an exhaustive examination of specimens of malignant disease of the stomach, and the lymphatic and glandular implication resulting therefrcm. The points of especial importance from the surgical standpoint are (See Figs. I7 and I8):

I. The early and wide extension in the submucosa.

2. The tendency of cancer to drift towards the lesser curvature.

3. The habitual integrity of the duodenum.

I. The early and wide extension in the submucosa is well seen on making sections of the margin of the growth.

The infiltration of the mucous membrane marks the limit of induration. Beyond this there is a continuous layer of growth in the submucosa for some distance, which gradually thins off and becomes broken up, as it were, so that the growth for several centimetres is represented by scattered and isolated groups of cells. The area of infiltration of the muscular and serous layer is always less than that of the mucosa. In order to remove the whole disease, the line of section must be at a minimum distance of 3 centimetres from the margin of the induration.

2. The tendency of cancer to drift towards the lesser curvature. In eleven specimens an extension to the lesser curvature was found in nine. The stomach along the curve is hardened, and a thick, white, cordlike band is formed. Glandular enlargement is found always, for the chief lymph current is directed towards the lesser curvature. The thickening ceases generally at the point where the coronary artery reaches the stomach-that is, where the lymphatics also pass away.

When the glands along the lesser curvature are all infiltrated, the only method of extirpating them is by removing the stomach wall, to which they are attached.

3. The habitual integrity of the duodenum was first pointed out by Rokitansky and Brinton. Though growth rarely extends far into the duodenum, Carle and Fantino have shown that islets of growth may exceptionally be found between 2 and 3 centimetres from the pylorus. The section of the gut, in pylorectomy, should be inade at least 2 centimetres from the margin of the induration. 
The frequency of adhesions, glandular enlargements and metastases has been variously estimated. Gussenbauer and Winiwarter state that adhesions are present in 63 per cent. of cases of pyloric cancer; Sutton that glandular enlargements are found in 50 per cent. of cancer affecting any part of the stomach; and Gussenbauer and Winiwarter that 59 per cent. of cases show metastatic deposits. McArdle collected records of 1,342 cases of cancer of the stomach, of which 802 were limited to the pyloric region; and out of this number 496 were not associated with any important lymphatic involvement. Lindner records 28 recurrences after local removal; of these 15 were local, I 2 metastatic,

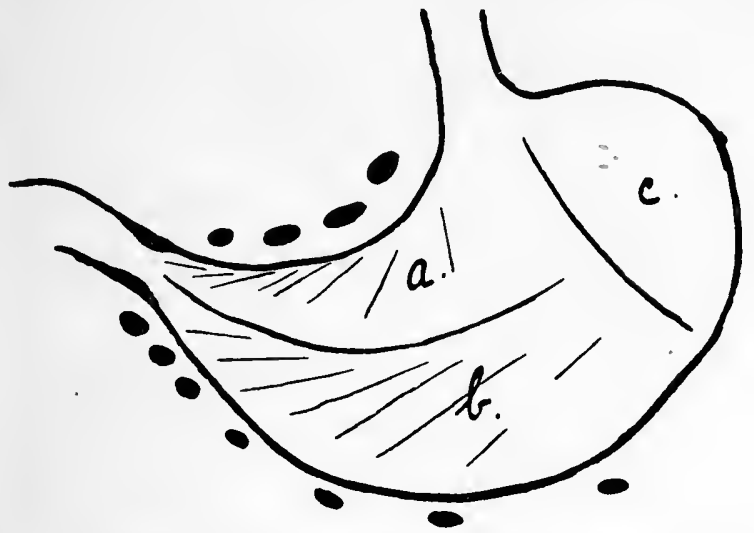

Fig. I8.-Showing the Lymphatic Areas of the Stomach.

and I glandular. These figures, which are, of course, drawn from post-mortem records, are evidence, sufficiently striking, of the local character of malignant disease of the stomachevidence the worth of which is borne out by clinical experience, which indubitably goes to show that it is the local growth which entails the suffering and determines the death of the patient.

The surgery of carcinoma of the stomach must in the future concern itself with a much wider local removal. It seems not unlikely that, in order to secure freedom from local recurrence, the lesser curvature up to the point where the coronary artery reaches the stomach will in all cases 
have to be removed. By so doing, the chief lymphatic vessels and glands will be, of necessity, extirpated also.

\section{Clinical History and Symptoms.}

Cancer of the stomach affects males more commonly than females. Welch gives the proportion as five to four. At the Leeds Infirmary, during ten years, there were thirty-six males to twenty-three females. Osler in I 50 cases observed 126 males and 24 females. The age of patients affected is generally between forty and sixty years of age, though no period of life is exempt. Widerhofen has recorded a case of congenital carcinoma of the stomach; Cullingworth has observed the disease in a child of five weeks; and we have operated on a girl of twenty. The first symptoms of this disease are a gradual loss of appetite and wasting, generally, though not necessarily, progressive, coming on brusquely in an individual previously in good health. Concomitant with the loss of flesh is a sapping of the strength of the patient. He becomes anæmic, listless, disinclined for, and unequal to, any exertion, and readily tired. Pain, or a sense of heaviness, fulness or discomfort, at a varying interval after food, is complained of early, and may lead to a feeling of dislike or disgust for food. In not a few cases, however, despite the loss of health, the appetite is unimpaired, but a wary and wise caution is observed in the taking of food, because of the known penalties which follow indulgence. Vomiting of food, little or much altered, with or without blood, or of blood alone, is noticed in most of the cases. The early symptoms, then, are very similar to those of chronic gastritis. It is, however, rare to elicit a history of very old-standing stomach disorder; the first evidences of local disease appear suddenly in persons of perfectly sound health and robust digestion. After some weeks or months a palpable tumour develops in the majority of instances, and the symptoms then become pronounced. The difficulties of taking and digesting food are increased, wasting is rapid, and cachexia develops. In some cases a remarkable desiccation of the tissues, the result of the copious outpouring of fluid from the stomach wall, is observed. Thrombosis of 
veins, œdema of the legs, enlargement of the supraclavicular and other glands, are evidences of the approaching end.

Cases of cancer of the stomach may be acute, running all their course in three months; or latent, giving rise to no characteristic signs or symptoms, the cause of illness and death being accidentally discovered at the autopsy. The former constitute, according to Osler, ro per cent., and the latter 5 per cent., of all cases. Lindner and Kuttner record two cases presenting symptoms of pulmonary tubercle, in which carcinoma of the stomach was accidentally discovered at the autopsy, the lungs being healthy.

The most important of these symptoms are pain, vomiting, and the presence of a tumour.

Pain is the earliest and most constant symptom. At the outset of the disease complaint is made of heaviness, of undue fulness, of a feeling of oppression and distension in the epigastrium after food. Pain, limited to the gastric area or radiating to one or the other side, and occasionally penetrating to the back, soon follows. The suffering is of widely varying intensity, and comes at a shorter or longer interval after food. We have noticed in not a few cases that, as with ulcer, the earlier the onset of pain the nearer to the cardiac orifice is the growth situated. In a small minority of cases the patients assure us that, at least in the earlier stages, the taking of food has given ease, as in hyperchlorhydria. The severity of the pain is liable to considerable fluctuation in the same patient, and individual complaints vary much in their intensity. It is not often that the pain is acute and disabling.

Vomiting is almost as constant a symptom as pain. According to Osler, it is present in $85^{\circ} 3$ per cent. of patients. The character of the vomiting depends largely upon the area of the viscus affected. In pyloric growth with obstruction and consecutive dilatation, the vomiting is copious in quantity, and the intervals between seizures are long. Food taken a few days before may be recognised by its appearance or by flavour. When the body of the stomach is affected, and the capacity of the organ is lessened, the vomiting is noticed soon after-sometimes immediately after-food has been taken, and is therefore small in quantity, becoming progres- 
sively smaller. In cases of mural cancer the vomiting is not usually an early, and from the statistics of Osler would not appear to be so pronounced a symptom.

The appearance of the vomited matter will depend upon the length of time the food has been in the stomach, and upon the presence or absence of adventitious products, such as bile or blood. When food is ejected from a contracted stomach during or immediately after a meal, the appearance is but little altered. When the food has remained a day or two in the stomach, the vomited matters will consist of profoundly altered, ill-digested food, gastric outpourings, and probably blood. The appearance of such vomit is described by the epithet 'coffee-ground.' The odour is faint and sour ; when sloughing of the growth takes place, the smell may be extremely offensive and penetrating. Blood, though generally intimately mixed with the stomach contents, may be vomited alone. In a few instances it has been the first recorded symptom of gastric disease. In amount it varies from a mere tinge to a quantity sufficient to cause fainting and anæmia. Blood poured out rapidly into the stomach induces immediate vomiting; when slowly escaping, it has time to undergo partial digestion, and its appearance becomes, therefore, altered. Death rarely, if ever, results from hæmorrhage alone.

Tumour.-In the great majority of cases of cancer of the stomach a tumour can be felt at some period of the disease. This is due in part to the frequency with which the pyloric region of the stomach is attacked, and in part to the fact that the presence of a tumour entails a certain descent of the viscus, which brings the mass into the field of palpation.

It is of the first importance to remember that the presence of a tumour is evidence of large and late involvement of the stomach. It has been said by Czerny and Rindfleisch and by Kraske that when a malignant tumour of the stomach can be felt through the abdominal wall, the growth is no longer a local one, and is therefore unsuited to a radical operation. That this statement is not rigidly accurate has been proved by the experience of Kocher and other surgeons, but there can be no question but that by the time a tumour 
has developed a radical operation is more difficult, attended with a greater danger, and is the more likely to be followed by a speedy recurrence. The endeavours of clinicians now and in the future must be towards the perfecting of our methods of diagnosis in the days before a palpable tumour has developed.

Character of the Tumour.-For examination of the abdomen the patient must be upon the back, with the knees slightly flexed. The abdomen must be freely uncovered and exposed to a good light. A tumour, if of large size or if projecting, can occasionally be seen on inspection. Deep respiration will cause the ascent and descent of the tumour to the extent, perhaps, of some inches. Lateral movement is chiefly noted in cases of pyloric tumour, but may be equally present when the body of the stomach is implicated. The position of the tumour under the varying degrees of artificial distension of the stomach should be studied, and will giye useful information. Complete emptying of the stomach will sometimes reveal an otherwise impalpable tumour. As the growth enlarges and adhesions form, its mobility becomes impeded, but the greater or lesser range of the movement affords no precise indication as to the character and extent of the adhesions. The tumour may be hard and smooth, or irregularly nodular, and may seem, when situated in or near the pylorus, to vary in size. The apparent sudden increase is probably due to the hardening by tonic contraction of the hypertrophied muscle fibres at the pyloric antrum.

Dilatation of the stomach is the inevitable result of narrowing of the outlet. According to Osler, the commonest cause of dilatation of the stomach is malignant disease of the pylorus. The extent of the dilatation is determined by the narrowing of the exit, and upon the duration of the obstruction. All degrees of distension are met with; the stomach may even, as in one of our cases, almost fill the abdomen, and descend behind the pubes into the true pelvis. Waves of contraction will generally be seen readily; they may be elicited, or, if sluggish, increased by artificial distension of the organ with carbonic acid gas. Percussion and auscultation give little information of value. 
In about half the cases observed, a rise of temperature of greater or less range will be noticed. As a rule, the fever is trivial and inconspicuous. Deep ulceration of the growth with pus formation, loculi of pus lying between an ulcer on the posterior wall and the body of the pancreas, for example, may cause chills and high fever. The importance of enlargement of the supraclavicular glands, as positive evidence of carcinoma of the stomach, is pointed out by Riegel. The gland which first enlarges is situated on the left side of the neck at the posterior border of the sterno-mastoid, just above the clavicle. The absence of glandular enlargement here is, however, void of significance. Ascites is occasionally present, and may be of such grossness as to mask the original disease. Hampeln and Strauss (Deut. Med. Woch., Igor) have noticed the frequency with which pleural effusion on the left side is associated with carcinoma of the stomach, and consider that the association may at times be of diagnostic import. Jaundice, œdema of the legs, thrombosis of one or many veins, perforation of an ulcerating growth followed by peritonitis, and metastases in the umbilicus, abdominal wall, ribs, or elsewhere, are signs observed in the last days of the disease.

\section{The Blood in Carcinoma of the Stomach.}

The blood, as a rule, shows the changes found in secondary anemia. Beyond this, the information given by an examination is of doubtful value. Krokiewic $z$ states that there is no change in the red blood corpuscles. In thirteen cases digestion leucocytosis was absent. Krokiewicz agrees with Löwitt that this sign is 'of equal value with absence of $\mathrm{HCl}$ and presence of lactic acid.' In nearly all cases the alkalinity of the blood was lessened. Osler and McCrae come to the following conclusions :

x. Neither an increase in the leucocytes nor special variations in the forms appears to be of any moment in the diagnosis of cancer of the stomach.

2. The presence or absence of digestion leucocytosis is too uncertain to be of much assistance in diagnosis (in twentytwo cases was present in ten, absent in twelve). 
According to Lindner and Kuttner, absence of digestion leucocytosis is noticed rather more frequently in malignant than in simple disease. Hartmann and Silhol (Rev. de Chir., IgoI, No. 2) have recently communicated to the Société de Chirurgie the results of some researches made on the blood of surgical patients. In the course of these researches they have become convinced that in cancer of the stomach an examination of the blood is more likely to prove useful than a chemical investigation of the gastric contents. The authors made particular.investigations on two questions: (a) The degree of anæmia characterized by diminution of the quantity of hæmoglobin, which may depend on the reduction of the number of globules or on the reduced proportion of hæmoglobin in their contents; and $(b)$ the existence of leucocytosis. The presence of cancer of the stomach, it is held, is indicated by a well-marked association of decided anæmia with decided leucocytosis. Anæmia is marked less by the diminished number of globules than by (I) a diminished proportion of the hæmoglobin in the globules; (2) by irregularity in the form of the globules, indicating a profound modification of the elasticity and texture of the red globules; and (3) by inequality in the size of those globules that are not misshapen. The leucocytosis, to have any value as a symptomatic sign, should be very marked, and should affect especially the mono-nucleated cells.

\section{Examination of the Stomach Contents.}

In all cases of suspected cancer of the stomach, the examination of the contents is of the greatest importance. From such examination, information is afforded upon the following points :

r. The motility of the stomach.

2. The defective secretion of the gastric juice, as manifested by the diminution of free $\mathrm{HCl}$.

3. The presence of adventitious products, the result of stagnation, such as lactic acid and the OpplerBoas bacillus.

4. The presence of fragments of growth. 
I. The Motility of the Stomach is best tested by the examination of the stomach after the administration of Ewald's breakfast. At the end of three, or at the most four, hours all traces of this meal should be absent from the stomach. If any be found, the quantity affords a rough estimate of the impairment of the motor function of the viscus. Such impairment may be due to stenosis of the pylorus, or invasion of the wall of the stomach by growth. The employment of any mechanical appliance, such as the 'deglutable, indiarubber stomach-shaped bag' of Hemmeter, is unnecessary.

The motility, according to Hemmeter, who has devoted considerable attention to the subject, is chiefly affected in disease of the orifices of the stomach. In chronic gastritis the motility, if impaired, is less so than in cancer ; in neuroses the motility is increased.

2. Defective Secretion of the Gastric Juice was first observed by Von der Velder in I879. The contents of the stomach after the administration of Ewald's test-breakfast are examined. It is important to emphasize the fact that a single examination of this kind is not enough. Repeated examinations are necessary in order to arrive at the truth. The reliance to be placed upon chemical examination is very differently estimated by authors. We are accustomed to consider that, if free hydrochloric acid is permanently absent and lactic acid is present in a patient whose symptoms are those described above, there is strong presumption in favour of a diagnosis of malignant disease. In a series of 343 cases of cancer recorded by Boas, Hammerschlag, Schneider, Rosenheim, and Osler, $89^{\circ} 7$ per cent. showed an absence of free $\mathrm{HCl}$. It will be seen from this, however, that there are $10^{*} 3$ per cent. of cases in which a diagnosis based upon this point alone would be misleading. In some of these it is probable that there has been a malignant implantation upon a simple ulcer. It is recognised as a well-established fact, to which Rosenheim first called attention, that in such cases (ulcus carcinomatosum) free $\mathrm{HCl}$ is frequently present, sometimes in excess. Hemmeter says: 'In all carcinomas that have arisen from ulcers, free $\mathrm{HCl}$ in normal, or even in increased, amounts may continue until death.' In others the carcinoma 
may be limited to a small area at the pyloric end, where there are no oxyntic or border cells, which alone, according to Heidenhain and Mall, are concerned in the production of hydrochloric acid. (In all the cases recorded by Osler, in which $\mathrm{HCl}$ was present, a tumour was observed.) The changes occurring in the mucous membrane of the stomach in cases of cancer, and determining the alteration in the secretion, are described as $(a)$ simple catarrhal inflammation, (b) interstitial gastritis, and (c) atrophy of the glands. Hammerschlag quotes certain cases in which the histological condition could be compared with the chemical findings: 'It was found that in cases of carcinoma in which hydrochloric acid was present there were very slight, or no, changes in the mucous membrane. But in cases where the $\mathrm{HCl}$ and the ferments were absent and lactic acid present there was found atrophy of the specific gland elements, and substitution by cylindrical epithelium or fibrous connective tissue.' This atrophy will account also for the diminished and delayed absorption pointed out by Eichhorst, $Z$ weifel, and others. In a condition of health, the iodine reaction should be obtained from the saliva a quarter of an hour after the administration of a cachet of 5 grains of potassium iodide. In carcinoma the reaction may be delayed an hour or more.

3. The Presence of Adventitious Substances-Lactic Acid.-The discussion as to the precise significance to be attached to the presence of lactic acid in the stomach contents has been given a considerable prominence in medical journalism in recent years. Lactic acid is either introduced into the stomach as such, in small quantities, or is formed by abnormal fermentative processes. It is evidence of gastric stagnation and impairment of the motor capacity, and is associated with a reduction in the amount of the digestive ferments. In order to test for it, a special meal, Boas's oatmeal and water breakfast, must be given, after carefully washing out the stomach. Uffelmann's test is employed (see p. 24).

In 268 cases of cancer quoted by Schiff, lactic acid was found in 197 cases $=73.5$ per cent. Osler, in 73 cases, found lactic acid in $55=75^{\circ} 3$ per cent. The presence of lactic acid 
is, therefore, evidence of some value. Its absence, however, cannot be held to be of any significance. Unfortunately, though so frequently present in cancer, it is not found at an early stage, and its clinical importance is therefore diminished.

The value of the chemical findings may be expressed in this manner :

If $\mathrm{HCl}$ is permanently absent, and lactic acid present, the evidence in favour of cancer is strong.

If $\mathrm{HCl}$ is permanently absent, and lactic acid is absent, the evidence is in favour of cancer.

If $\mathrm{HCl}$ is present, and lactic acid is present, the evidence is against malignant disease, though the possibility of ulcus carcinomatosum should be borne in mind.

The 0ppler-Boas bacillus-a long, non-motile bacillus ' of the shape of a base-ball bat.' In twenty cases of malignant disease of the stomach recorded by Kaufmann, the bacilli were present in large numbers in nineteen. In sixteen recorded by Hemmeter, they were present in fourteen. According to Schlesinger and Kaufmann (Wien.Klin. Runds., I 895, No. I5), ' the presence of a large number of these bacilli in the stomach contents is an indication of carcinoma.' This observation is supported by Riegel. Further investigation is required before an opinion of worth can be expressed, but it would seem as though the presence of these bacilli is of the same value, as an evidence of cancer, as the presence of lactic acid. Their absence does not prejudice the diagnosis in any way. Kaufmann states that the bacilli are capable of forming lactic acid from sugar.

4. The Presence of Fragments of Growth.--Various means are described, and by some surgeons employed, for the purpose of obtaining fragments of growth from the stomach for microscopical investigation. Efforts in this direction are to be deprecated. Purposeful attempts to scrape or brush away portions of growth are not devoid of danger. Hemmeter uses an indiarubber stomach tube, with a sharpened edge to the eye. This is moved slowly backwards and forwards in the stomach. A fragment of growth may in that way be caught in the opening. After ordinary lavage of the stomach, the tube should always be compressed 
during withdrawal, and any fragments in the tube carefully examined. If a particle of growth is thereby discovered, the diagnosis is complete. It is not likely that such a fragment will be spontaneously or by artifice detached from the growth in the earlier stages, when its discovery would be most helpful.

\section{A New Sign of Gastric Cancer.}

The success of surgical operations for cancer of the stomach depends to such a large extent upon the early diagnosis of the disease that any sign which offers a fair prospect of proving trustworthy should be carefully scrutinized. For this reason we would draw attention to a communication by Dr. H. Ehret of Strasburg, in La Semaine Médicale (March 6, I9or), upon the value of the presence of the Bacillus filiformis in the stomach contents. This organism was first described by Boas; it is a very long, slender, threadlike bacillus, forming masses large enough to fill the greater part of the field of the microscope. It grows well in agar-agar containing I per cent. of glucose, and forms colonies visible on the second day which do not exceed a pin's head in size; with a lens they look like little tufts of cotton. It forms, after three days, a thick woolly precipitate in acidulated bouillon, but does not grow upon gelatine. These cultural characters are not, however, of clinical importance. The bacillus can be early recognised by the microscope, and it is only when present abundantly that it has, according to Dr. Ehret, any special significance, and this is more particularly the case when there is no great stagnation of the stomach contents. In his opinion, if masses of this bacillus are found in the contents of the fasting stomach where those contents are only moderate in quantity - that is to say, not more than Ioo cubic centimetres-the diagnosis of cancer may be affirmed.

\section{Diagnosis.}

It cannot be too often repeated nor too strenuously emphasized that cases of cancer of the stomach should 
be dealt with surgically at the earliest possible moment. The evidence to which we have alluded certainly goes to show that for some length of time the disease is a purely local one. In the surgery of the breast, the tongue, the uterus, and other organs, a vast improvement has been accomplished since early diagnoses (of 'precancerous' conditions) were followed by early and extensive operations. In cases of cancer of the stomach a like, if not an equal, improvement should be forthcoming. It is therefore necessary that the very earliest beginnings of malignant disease of this organ should be exhaustively and persistently investigated. Whenever a patient over forty years of age complains, somewhat suddenly, of indefinite symptoms of gastric uneasiness, pain, and vomiting, followed by progressive loss of weight, secondary ancmia, and so forth, the possibility of cancer should at once be recognised. As Osler has said, 'If we hear that a woman of a certain age complains of a bloody discharge from the uterus, the possibility of malignant disease is at once thought of ; but how many of us consider a like grave possibility when a patient of the same age complains of stomach symptoms, perhaps with a moderately sudden onset ?' As our knowledge stands at present, we cannot but recognise that our only sure method of making the diagnosis in an early stage is by an exploratory operation. Although the chemical examination of the stomach contents and the general examination of the patient may give us a very strong impression that cancer is present, our diagnosis can only be rendered certain by (I) the discovery of fragments of growth in the stomach or in the evacuated contents (probably a late sign), or (2) the digital exploration of the organ through an abdominal incision (the 'lesser abdominal section' of Mikulicz). Such a digital exploration by a competent surgeon is devoid of risk, and may, if necessary, be performed (as we have frequently performed gastrostomy) under cocaine anæsthesia. We feel, therefore, compelled very strongly to advocate, in all cases where there is any doubt, an early exploratory operation. In thus advising, we would still impress upon both physicians and surgeons the urgent need of the most painstaking and 
the fullest examination, in the hope that some result from their labours may, by establishing a criterion, thereby do away with the need of an 'exploratory' incision. Though such careful investigation is absolutely essential, it should not be unduly prolonged. To await the development of a 'tumour' before consenting to a diagnosis of malignant disease and advising operation is to prove one's self in ignorance of the real issues at stake. Cancer of the stomach should be dealt with surgically before a tumour is clinically recognisable. In this alone lies our hope for successful treatment, and it may be also our means of establishing an early diagnosis.

In the recent admirable work on 'Cancer of the Stomach,' by Osler and McCrae, the following statements are made: 'The important aid of an exploratory operation should be more frequently advised.' 'The risk is comparatively slight, and is much less than that of an undiagnosed neoplasm.' 'In a suspected case, when under treatment there is no improvement in a few weeks, an exploratory operation is justifiable.'

The exploratory examination, however, does not give information which is infallible. Several observers, Czerny, ourselves, and others, have recorded cases where malignant disease was diagnosed, in which the event proved the tumour to have been simple inflammatory thickening round a chronic ulcer. Such cases, however, are exceptional, and only afford additional support to our advocacy of the earliest possible exploration.

\section{The Choice of Operation in Cancer of the Stomach.}

i. In Pyloric Cancer.-Surgeons of some experience in operations upon the stomach are divided in their opinions as to the better operation in cases of malignant disease of the pylorus. On the one hand are those who, believing that a diagnosis of malignant disease cannot be made while yet the disease is local, advocate a palliative operation, gastroenterostomy, with the idea of giving rest to the diseased area, and thereby retarding growth, as in colotomy for 
inalignant disease of the rectum. On the other hand are those who, having been tempted to employ a radical operation in some favourable case or series of cases, are so impressed with its advantages that they become apostles of a broader creed, and advocate local extirpation. Our opinion emphatically is that, in all cases, whenever possible, a radical operation should be attempted. Under present conditions of diagnosis, the probability is that, when a patient is submitted to operation, gastro-enterostomy is, in general, a safer operation than pylorectomy. But although the comfort and sense of well-being of the patient may improve very decidedly for a time after the former operation, the tumour is still slowly enlarging in size, and will eventually cause death. How much of the general ill-health, cachexia, and so forth, are induced by absorption from the growth, by necrotic changes in its mass, by ulceration and hæmorrhages upon the surface, is quite unknown, but one may presume that such influences are not trivial. Krokiewicz and Pilliet believe, indeed, that cancer cachexia is the result of intoxication with the by-products of metabolism of the cancer cells. A local extirpation, then, even if followed by a recurrence, will probably prolong life for a greater period and in greater comfort than a gastro-enterostomy. It was doubtless an opinion similar to this which led Terrier to remark that 'the best form of gastro-enterostomy was done after removal of the pylorus.' But increasing experience in the most competent hands all tends to show that in properly-selected cases pylorectomy is not an operation of very grave risk, and is an operation of generous promise.

Partial gastrectomy in the early days of its employment was an exceedingly serious operation, with an appalling death-rate. Latterly the mortality is seen to be a gradually, but persistently, diminishing one.

In order to form some estimate as to the chances of life, of the condition of health, and of the relative values of different operations in patients afflicted with malignant disease of the stomach, it is desirable to inquire closely into the records of a number of cases, preferably in the practice of one surgeon, observed over a series of years. The fullest 
account of the surgical side of the question has been given by Krönlein of $Z$ urich and by Von Mikulic $z$ of Breslau. The questions that are in urgent need of settlement are the following :-

I. Will a palliative operation upon the stomach prolong life?

2. Will it make the remnant of life more tolerable? Will it make the patient feel that the ordeal of operation is justified in the greater comfort of his later days?

3. Will a resection give a reasonable prospect of cure ?

4. Will a resection, if followed by recurrence, give increased length of days and better health?

It is necessary, in fact, to know whether, in the stage in which we now meet with the cases, an operation is worth doing, and, if it is, whether it should be palliative, or whether an attempt should be made to eradicate the disease.

In order to have some means of comparing the surgical cases with those not operated upon, it is necessary that exact details should be kept of all cases coming to the surgeon, whatever their destiny, to operation or to internal medication or to absence of treatment, may chance to be.

Krönlein tabulates the cases which came under his observation from April I, I88I, to February, I902. All the cases were recorded, without exception. There were 264, and the following table gives a brief sketch of their classification :

A. Not operated upon.

I. Inoperable

2. Refusing operation

$$
\begin{aligned}
& \begin{array}{llll}
\cdots & \ldots & \ldots & 53
\end{array} \\
& \begin{array}{llll}
\ldots & \ldots & \ldots & \text { I } 4
\end{array} \\
& \begin{array}{llll}
\text { Total } & \ldots & \ldots & \overline{67}
\end{array}
\end{aligned}
$$

B. Operated upon.

I. Exploratory laparotomies

Deaths under Operation.

2. Gastro-enterostomies _.. 74

3. Gastrectomies $\quad \ldots \quad \ldots \quad 50$

Total $7=9.5$ per cent.

$$
\begin{aligned}
& \text { 1 } 8=24.3 \\
& \text { 1 } 4=28 \\
& \hline 39=19.8
\end{aligned}
$$

Of the 264 patients, all but $I_{3}$ were traced. Of these $I_{3}$ the majority had not been treated by operation, and not one of them had been submitted to gastrectomy. 
The fate of these patients is shown in the following table:

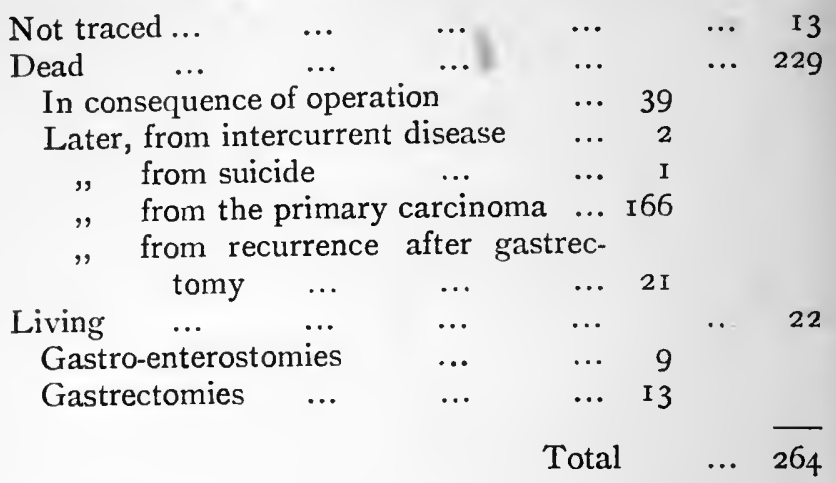

Of the 264 it will be seen that 67 were not operated upon $\left(25^{\circ} 3\right.$ per cent.). In 53 of these an operation was contraindicated, in $\mathrm{I}_{4}$ it was refused by the patients.

Of the 264,73 had exploratory laparotomy performed (27.6 per cent.). After the surgeon had opened the abdomen and had become convinced of the impossibility of radical extirpation, and of the unsuitability of a palliative operation, the abdominal wound was closed.

Of the 73 patients, 7 died within the first week after operation (4 from exhaustion, 2 from pneumonia, and I from pulmonary embolism).

In 74 patients gastro-enterostomy was performed. At first sight this number appears small, but Krönlein has laid it down as a rule of his practice that in cases of carcinoma of the stomach, where extirpation is impossible, the palliative operation of gastro-enterostomy shall not be performed unless there is evidence of stenosis. If this evidence fails, the operation becomes merely 'exploratory.' The only exception that has been made is in those cases in which there has been marked stagnation of food without evidence of stenosis. Of the 74 gastro-enterostomies, I 8 (or $24^{\circ} 3$ per cent.) died under the operation.

Fifty patients were submitted to the operation of gastrectomy. Of these 14 died in direct consequence of the operation. Of the total 264 cases, therefore, only 18.9 per cent. underwent the radical operation. One of the 50 was 
the well-known case of total extirpation of the stomach performed by Schlatter.

The subjoined table shows the percentage in which each form of operation was attempted :

$\begin{array}{lccccc}\text { No operation in } & \ldots & \ldots & \ldots & 25 \cdot 3 & \text { per cent. } \\ \text { Operation in } & \ldots & \ldots & \ldots & 74 \cdot 7 & , \\ \text { Exploratory } & \ldots & \ldots & 27 \cdot 6 & & \\ \text { Gastro-enterostomy } & \ldots & 28 \cdot 0 & \\ \text { Gastrectomy } & \ldots & \ldots & 18 \cdot 9 & \end{array}$

$$
264 \text { Cases. }
$$

Operation Mortality.

Exploratory laparotomy

Gastro-enterostomy ...

Gastrectomy

$\begin{array}{llll}\ldots & \ldots & 9 \cdot 5 & \text { per cent. } \\ \ldots & \ldots & 24 \cdot 3 & \\ \ldots & \ldots & 28 & \end{array}$

In order to obtain a comparison between the life-chances of those patients who survive operation and those who are not operated upon, it is necessary to cancel the following:

Patients who withdrew after examination

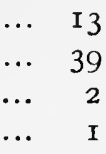

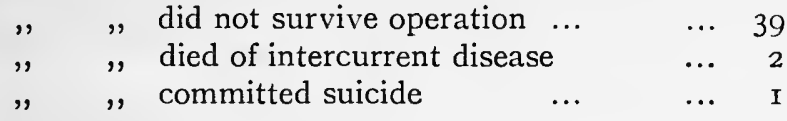

Total ... 55

Deducting these 55 from the total of 264 , we have 209 patients, who may be classified thus :

A. Not operated upon.

I. Inoperable

$$
\cdots \quad \cdots \quad 5 \mathrm{I}
$$

2. Declining operation $\quad \ldots \quad \ldots .12-63$

B. Operated on.

I. Exploratory laparotomy $\quad \ldots \quad \ldots \quad \ldots$

2. Gastro-enterostomy $\quad \ldots \quad \ldots \quad 54$

3. Gastrectomy $\quad \ldots \quad \ldots \quad \ldots \quad 34-146$

$$
\text { Total ... } \quad \text {... } 209
$$

The report as to these 209 cases at the beginning of March, I902, when these inquiries were concluded, was as follows :

$\begin{array}{llll}\text { Died from carcinoma of the stomach } & \ldots & \ldots & \text { I } 87\end{array}$

$\begin{array}{llllllll}\text { Living } & \ldots & \ldots & \ldots & \ldots & \ldots & 22\end{array}$ 
There died :

A. All those not operated on.

I. Inoperable cases...

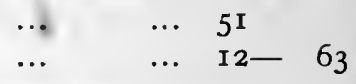

2. Declining operation

$B$. Of those operated on.

I. All the exploratory laparotomies $\ldots \quad 5^{8}$

2. Of the gastro-enterostomies $\quad \ldots .45$

3. Of the gastrectomies $\ldots$
Of those operated on and not operated on

There are still living :

I. Of gastro-enterostomies $\quad \ldots \quad$ ‥

2. Of gastrectomies... $\quad \ldots \quad \ldots \quad$ I $3-\quad 22$

$$
\text { Total ... } \quad \ldots \overline{209}
$$

The duration of disease in patients suffering from carcinoma of the stomach was also reckoned. It was found that on the average the time from the first onset of symptoms up to the day of the patient's admission to the hospital, or up to the day of operation, was from eight to nine months. Means were also taken to discover the number of days from the patient's admission or from the date of operation to the patient's death.

The duration of time from entrance into hospital (time of operation) to date of death :

A. In the non-operated cases

B. In the operated cases:

I. Exploratory laparotomies ...

2. Gastro-enterostomies

3. Gastrectomies $\ldots \quad \ldots \quad \ldots \quad 5^{20} \quad$ "

$\ldots \quad \ldots \quad$ IO2 days

If in these groups we sum up the values found for both periods of duration of the disease, we gather the following to be the average time for the whole duration of the carcinoma from the onset of the first symptoms till time of death :

A. In the non-operated, 9 months and 102 days-about I $2 \frac{1}{2}$ months.

B. In the operated cases :

I. Exploratory laparotomies, 9 months and II4 days are I 3 months.

2. Gastro-enterostomies, 9 months and 193 days are $15 \frac{1}{2}$ months.

3. Gastrectomies, 9 months and 520 days are $26 \frac{1}{2}$ months. 
Krönlein, from this experience, draws the following conclusions :

I. That carcinoma of the stomach, without operation, has a fatal termination in about a year.

2. That gastro-enterostomy prolongs the life of the patients suffering from this disease for about three months, on the average.

3. That gastrectomy, so far as it is followed by recurrence, prolongs life on an average about fourteen months.

At the time this report was published there were 22 patients still living after operation. Of these, 9 were cases of gastro-enterostomy and $\mathrm{I} 3$ of gastrectomy. The former will, of course, prove fatal within a few months. So far as the latter are concerned, their length of life since operation is shown in the following table:

I Case is in the 8th year since gastrectomy

\begin{tabular}{|c|c|c|c|c|c|}
\hline $\begin{array}{l}\text { I Cases } \\
2 \text { Ch }\end{array}$ & are & " & $\begin{array}{l}4^{\text {th }} \\
3^{\text {rd }}\end{array}$ & ", & ", \\
\hline ", & ", & " & 2nd & " & " \\
\hline " & $"$ & ", & Ist & "n & $"$ \\
\hline
\end{tabular}

Von Mikulicz, in rgor, published the results of his experience from April, I89r, to March, Igor. During this period $45^{8}$ cases of cancer of the stomach had been under observation in the clinic at Breslau. This number includes 46 cases of cancer of the cardia. The diagnosis was confirmed either at the operation, or by observation of the progress of the disease, or by examination after death. In several cases of cancer of the cardia the diagnosis was confirmed by osophagoscopy or by the removal and examination of a small portion of the growth. In 128 cases no operation was performed, chiefly because radical removal was no longer possible, and because there was no indication for any palliative procedure, such as gastro-enterostomy. In exceptional cases the operative interference was declined by the patient. The total duration of life from the commencement of the disease (as inferred from the symptoms in 67 cases in which it was possible to ascertain the facts) varied from a few months to 38 months, an average of $11 \frac{1}{5}$ months. 
In 320 cases recourse was had to operation as follows :

I. Simple exploratory incision

2. Gastrostomy (in cancer of cardia)

3. Jejunostomy

4. Gastro-enterostomy

5. Resection of stomach

6. Extirpation of stomach

$\begin{array}{rrr}\ldots & \ldots & 44 \\ \ldots & \ldots & 27 \\ \ldots & \ldots & \text { 1 } 2 \\ \ldots & \ldots & \text { I43 } \\ \ldots & \ldots & \text { 100 } \\ \ldots & \ldots & 3\end{array}$

Exploratory Incision.-Four deaths followed this operation, giving a mortality of 9 per cent. The average duration of life after operation was $4 \frac{8}{10}$ months, and from the beginning of the disease $I 4 \frac{3}{10}$ months. If the cases submitted to exploratory laparotomy be added to those in which no operation was performed, we get an average duration of life of a little over 13 months from the beginning of the disease, a figure which is adopted by the author as a basis for judging of the results of the operative treatment of gastric cancer.

Gastro-enterostomy.-There were 48 deaths in 143 cases, a mortality of $33 \frac{7}{10}$ per cent. Although during the last three years the mortality has been reduced to $26 \frac{1}{2}$ per cent., it is still very high in proportion to the same operation in noncancerous conditions. The average duration of life after operation was $6 \frac{4}{10}$ months, and from the beginning of the disease I4 months; but if the operation deaths are included this is reduced to $12: 3$ months, slightly shorter than the duration of life when no operation is performed.

Resection of the Stomach.-There were 37 deaths in Ioo cases. In the last three years the death-rate has been reduced to 25 per cent. ; that is to say, a mortality slightly lower than that of gastro-enterostomy. The results, as observed in $5^{8}$ patients who survived the operation, and whose subsequent history is known, are as follows-20 are still alive between 6 months and $8 \frac{1}{4}$ years :

Longer than I year I7 are alive
$", \quad, 2$ years I0,$\quad$,
$, \quad, 3 \frac{1}{2}$ years $4 ", \quad$,

These last four may be regarded as radically cured. The author regards these results as quite as good as those after operations for cancer of the tongue or rectum. 
It will thus be seen that there is no great divergence in the results taken from the two clinics, allowing for the fact that the number of cases in the one is almost double that in the other, while the period covered by the observations is only one-half. Krönlein records 264 cases extending over 21 years, Von Mikulicz $45^{8}$ in ro years. The results may be compared in this way:

\section{KRöNLEIN. MiKULICZ.}

\section{Months. Months.}

The non-operated cases lived, from the beginning of the disease $\quad \ldots \quad \ldots \quad$ I $2 \frac{1}{2} \quad$ I I $\frac{1}{5}$

The cases treated by exploratory lapar-

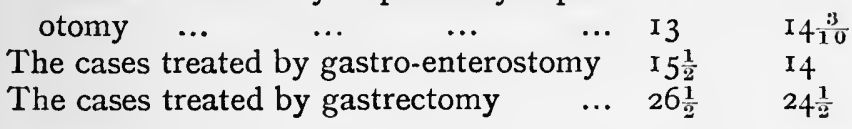

A careful study of the wealth of experience laid bare in these records will enable us more clearly to formulate our ideas as to the principles that should guide us in dealing with this most serious disease. In the first place, it must be admitted that our means of obtaining cases sufficiently early are almost as meagre as it is possible for them to be. Until more accurate methods of diagnosis are established it is absolutely imperative that recourse should be had earlier and more often to the exploratory laparotomy. At present that operation is limited to the examination of patients when the diagnosis has been made, and when the only question to be settled is whether the growth is removable or not. But in order to better our results we must explore, not to confirm, but to make, a diagnosis.

When the diagnosis has been made, and the patient is submitted to operation, it is difficult to decide upon the exact surgical procedure which it is wisest to adopt. Von Mikulicz, Krönlein, and not a few other surgeons have spoken strongly upon the question of gastro-enterostomy, saying that this method should be adopted only in cases where stenosis, either at or near the pylorus, is caused, or in cases where stasis of food is marked. There can be no doubt that in such cases gastro-enterostomy is productive of the most remarkable benefit to the health and well-being of the patient. The.weight increases, the appetite and the 
power of gratifying it return, and vomiting, often the most distressing and unceasing symptom, stops at once. But there can also be no doubt that in some instances, when the growth does not actually obstruct by its bulk the onward passage of food, a decided benefit results from the operation. The stomach is better and more quickly drained, and, as a rule, food can be taken more frequently and with greater comfort. In the majority of these cases, however, little or no benefit results from gastro-enterostomy. The position, therefore, may be thus briefly stated: If, after exploration, a growth is found to be obstructing the pylorus, or to be narrowing the stomach and causing an hour-glass condition, or if stasis of food has been a marked symptom, then gastro-enterostomy will give very decided relief. If, on the other hand, the growth be confined to one or other of the curvatures, and if neither stenosis nor stasis be present, gastro-enterostomy will give little or no relief; it will not prolong life nor give a greater degree of comfort.

The operation of gastrectomy in the hands of Von Mikulicz and of Carle and Fantino has given a less mortality than gastro-enterostomy. Its advantages as compared with gastro-enterostomy are that it gives a greater prolongation of life, ten or eleven months longer, and that it affords a greater degree of comfort to the patient. Though recurrence may follow, yet in the majority of cases the patient is relieved greatly by the removal of a foul, ulcerating growth, from the surface of which an offensive and septic discharge is constantly occurring, and from which hæmorrhages, more or less copious, are often likely to take place. If, after removal of a malignant growth of the stomach, the surgeon will open the viscus and inspect the surface of the tumour he will realize, when he sees the foul, ulcerous mass, that its removal cannot but be of vast benefit to the patient. It is true that in the great majority of cases the growth will recur either locally or generally; but the question may arise as to whether gastrectomy should not be performed deliberately as a palliative operation in cases where an early secondary deposit can be seen in the liver, or inaccessible or irremovable glands be found in the pancreas or along the aorta and vena 
cava. If we take into account the following advantages of gastrectomy as compared with gastro-enterostomy-that in the most competent hands its mortality is not greater, but is even less, than the mortality of gastro-enterostomy; that a prolongation of life for ten months longer than the period given by gastro-enterostomy is the rule; that the comfort, the general health, appetite, and well-being of the patient are all emphatically better; and, finally, that the patient has always a chance, even though it is of the slenderest, of a complete recovery from his disease-if we take all these into our consideration, there can be no doubt that the operation of choice will always be gastrectomy. Gastrectomy will be done always when a radical operation is attempted; it will probably be done often when nothing more than a palliative operation is intended.

If, after the abdomen has been opened, it is found impossible or imprudent to attempt either gastro-enterostomy or gastrectomy, some relief may be obtained by performing duodenostomy or jejunostomy. The scope of these procedures is very strictly limited; but in rare instances, when the prolongation of life for even two or three weeks is of the greatest importance, then either of these operations can be done with propriety. The operations are simple, speedily done, and cause little or no shock, and they can, if need be, be readily performed with cocaine anæsthesia.

In Mural Cancer.-In these cases obstruction may be absent. Diagnosis is, therefore, not so early, so that when the abdomen is opened a large area of stomach may already be affected. Even if no narrowing is produced, a gastroenterostomy, by determining rest, will assuage pain and lessen the rate of growth. A complete local removal, however, is the ideal for whose attainment we should strive. The extent of such removal will vary from the minimum of an hour-glass stomach to the maximum of a general infiltration of both walls. The surgeon will be guided in a decision by the extent of such growth and by his personal capacity and preference. It is, we think, possibly open to question whether a complete gastrectomy is a scientific operation or a brilliant exploit in surgical gymnastics. The records of 
the cases so far performed are certainly far better than could have been anticipated.

In all cases of local excision, whether in the body of the stomach or at the pylorus, a wide healthy area surrounding the growth should be removed. Experience goes forcibly to show that it is from local recurrence that patients die, even when the incisions have been made, as it would seem, wide of the disease. The direction of the spread of the growth should be carefully noticed. If the growth is spreading circularly, in the line of the vessels, it shows little tendency to recur after removal ; if it is spreading transversely along the curvatures, there is said to be a strong tendency to recurrence (Мayo). The importance of Cunéo's observations, already referred to (p. 82), may be again emphasized.

In Growth at the Cardiac End.-Only palliative operations are possible when the growth involves the cardiac orifice and the adjacent portion of the stomach (Figs. I9, 20 and 2I). Levy has, indeed, planned an operation, and practised it upon the cadaver, for the purpose of removing such a growth (Langenbeck Archiv, I898), but, so far as we know, a procedure of this kind has only once been attempted during life.

This was by Mikulicz, who removed a primary carcinoma of the cardia and a portion of the cesophagus between 3 and 4 centimetres in length. The operation was exceedingly difficult on account of spreading of the growth towards the pancreas and implication of the retroperitoneal lymphatic glands. The patient died of peritonitis. Mikulicz expresses the hope that not only carcinoma of the cardia, but even of the lower end of the cesophagus, may prove within the safe reach of a capable surgeon.

Krehl has shown that in dogs the two pneumogastrics may be completely destroyed at the lower end of the œsophagus without interfering in any degree with the processes of digestion.

In all cases gastrostomy should be performed at the earliest moment after the diagnosis is assured. 


\section{PLATE VIII.}

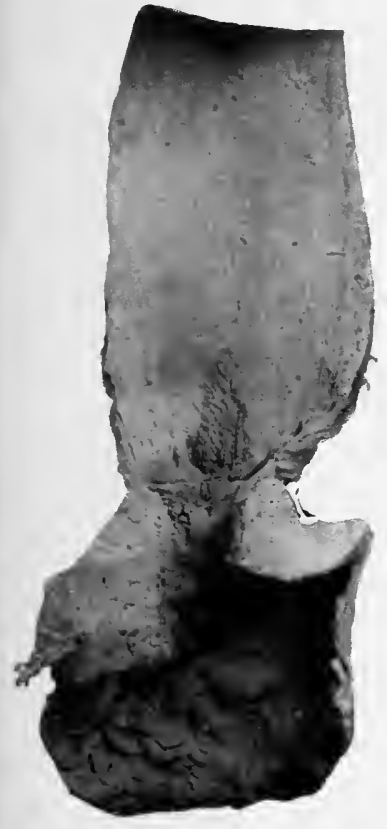

Fig. 19.-Cancer of Cardiac END of Stomach with DiLAtEd Esophagus.

(No. 2,417, Royal College of Surgeons' Museum.)

To face p. 106.]

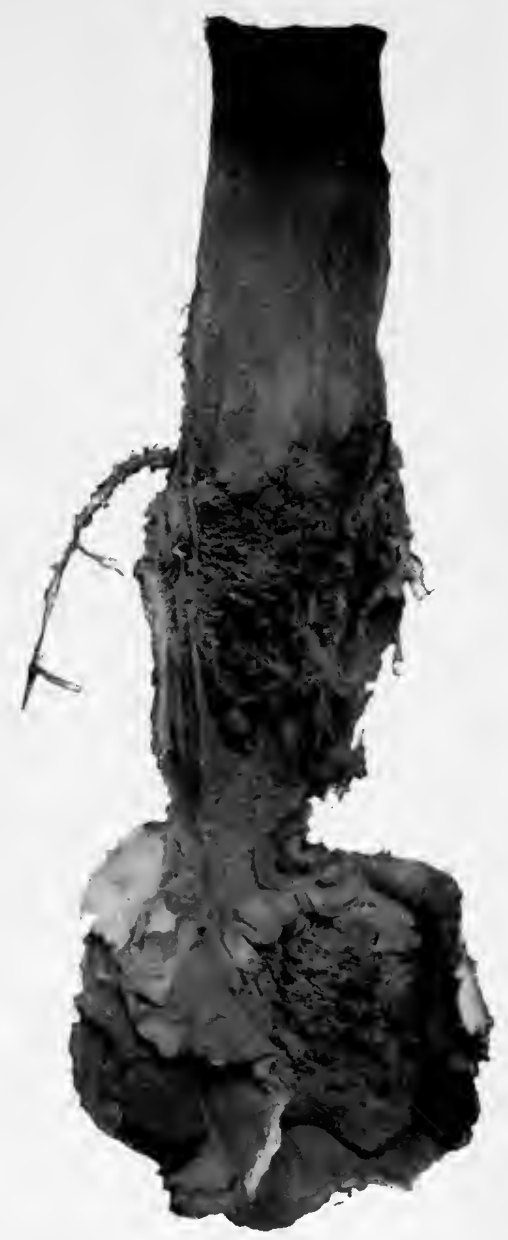

Fig. 20. - Cancer of Cardiac End of Stomach, associated with Cancer of THE Lower END of THE EsoPHAGUS.

(No. 2,421, Royal College of Surgeons' Museum.) 

PLATE IX.

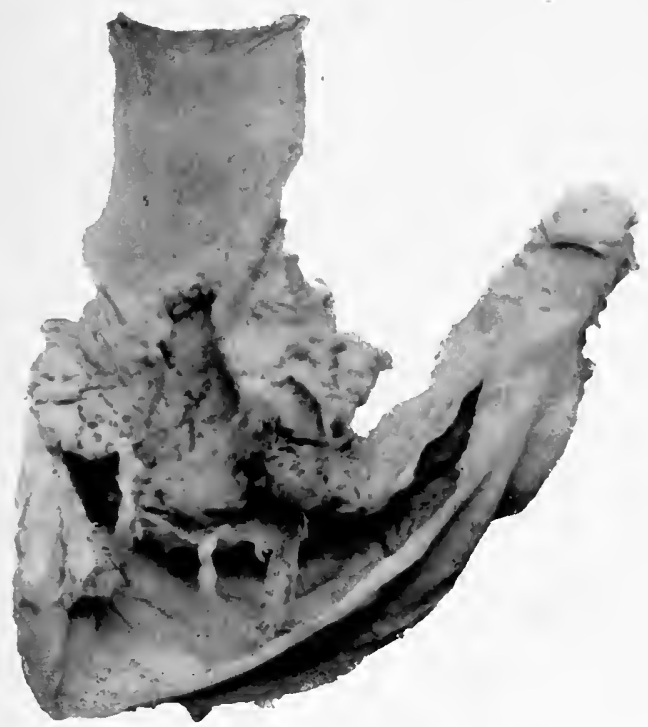

Fig. 2I.-. Cancer of Cardiac Orifice of the Stomach (No. 2,422, Royal College of Surgeons' Museum.)

To face p. I06.] 



\section{THE OPERATION OF GASTRECTOMY, PARTIAL AND COMPLETE}

\section{Pylorectomy, or Partial Gastrectomy}

MerRem in I81o showed by experiment upon a dog the possibility of successful removal of the pylorus. In 1876 Gussenbauer and Winiwarter, and later Czerny and Kaiser, performed many successful operations upon dogs, and suggested the feasibility of the procedure in the human subject. On April 9, I879, Péan performed the first pylorectomy in man ; in 1880 Rydygier operated-both unsuccessfully. On February 28, I88I, Billroth performed the first operation, followed by recovery.

The abdomen is opened in the manner previously described. The area to be operated upon is surrounded with compresses or sponges. The vessel in the small omentum is ligatured in two places and divided. The fingers are then passed from above behind the pylorus, and made to define the vessels of the great omentum, which are similarly ligatured. Any adhesions are gently separated. Those between the pylorus and the pancreas are the most serious and the most difficult to deal with. The majority of surgeons consider that dense adhesions here forbid any attempt at extirpation, but it is interesting to note that in two cases recorded by Obalinski and $\mathrm{M}$. Richardson, in which the adhesions were so tough as to prevent their stripping, a portion of the head of the pancreas was successfully removed. A similar removal in a case of cancer is recorded by Christy Wilson. If the pancreas be denuded, it should be covered by peritoneum borrowed from the lesser sac. Lymphatic glands are now 
sought for and removed. As shown by Mikulicz, Kader and Cunéo, the most numerous and the most important lie along the lesser curvature. All these, as they lie along the coronary artery, must be removed. In excising glands from the great omentum there is great danger of wounding the middle colic artery, and thereby causing gangrene of the transverse colon. The glands along the greater curvature are most numerous near the pylorus. The stomach and duodenum are then clamped and divided. The most serviceable clamps are Doyen's hysterectomy clamps, a modification made for us by Messrs. Down, Bros., shielded with indiarubber tubing, and specially devised for operations on the stomach.

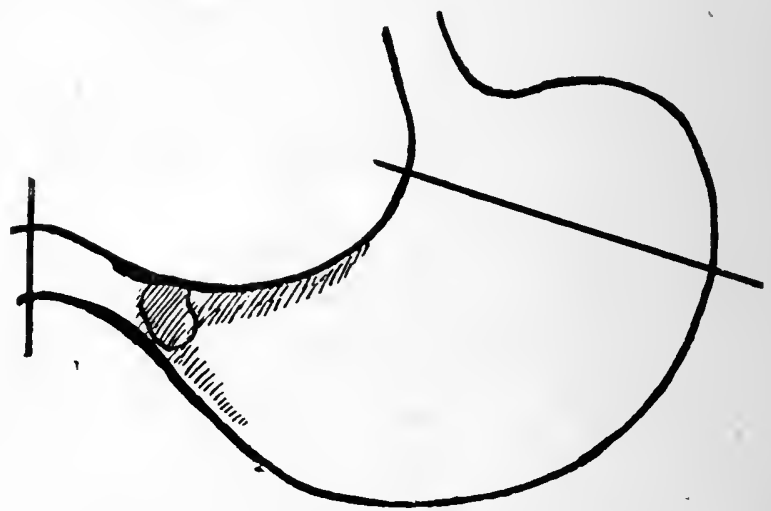

Fig. 22.-Showing Lines of Section of the Stomach in Partial Gastrectomy for Cancer.

Examination of a large number of specimens shows clearly that malignant disease of the stomach rarely infiltrates far into the duodenum, but does extend readily and rapidly towards the cardiac end of the stomach. In dividing the duodenum, then, it will suffice to remove 2 centimetres of apparently healthy tissue beyond the growth. In cutting across the stomach the incisions should be $I_{\frac{1}{2}}$ to 2 inches wide of the disease at least. Pathological observations show that implication of the stomach along the lesser curvature and of the glands there is early and rapid, and the record of cases operated upon in which the disease recurs clearly proves the marked frequency of local return, rather than metastasis, as the cause of death. It is therefore probable 
that permanently successful operations will depend upon a very wide local removal, especially of the upper border of the stomach. We are strongly inclined to believe that, even in the earliest examples of pyloric growth, a removal of at least half,- possibly more, of the stomach is necessary.

Union of the stomach and duodenum may be carried out in one of three ways :

I. Immediate suture of the cut ends (Billroth's first method).

2. Suture of the stomach opening; implantation of the duodenal cut end to the posterior surface of the stomach (Kocher).

3. Closure of both openings, and performance of a gastrojejunostomy (Billroth's 'second method ').

I. Immediate union of cut ends, advocated by Mikuiicz and Krönlein, is best carried out by means of the simple suture or the decalcified bone bobbin. The inequality in the size of the two openings is overcome by partial suture of the stomach incision, or by the making of a horizontal slit in the duodenal end. It is of the first importance that the new opening should be large, as several cases are recorded in which subsequent contraction of the orifice demanded a further operation. The method of suture is similar to that already described in gastro-enterostomy. The bobbin is of the greatest possible advantage here. A very large proportion of deaths in cases of simple suture have been due to leakage at what has been termed 'the fatal suture angle of Billroth.' This danger is wholly avoided by the use of the bobbin.

If simple suture is the method chosen, it may be carried out by the plan advocated by Rutherford Morison.

After removal of the pylorus, the stomach opening is larger than the duodenal. An incision over $\mathrm{I}$ inch in length is made down the centre of the anterior wall of the duodenum. By spreading this longitudinal cut transversely the duodenal opening is so widened as to correspond in size with the stomach opening. The two viscera are now united. The posterior wall of the opening which is to serve as the artificial pylorus is first made. A long catgut suture threaded in 
an ordinary needle is introduced through all the coats in the position of one of the outer three temporary sutures used to show the exact relation of parts. This suture is securely tied, has its short end clamped in hæmostatic forceps, and takes the place of the temporary suture. The longer threaded end is used as a continuous stitch through all the coats of the stomach and duodenum, and brings their apposed surfaces into firm apposition. The middle temporary suture being reached, it is withdrawn, and the continuous stitch travels on to the further temporary one, which it replaces.

The posterior wall is now formed by a continuous catgut suture passed from within outwards through all the coats, and bringing the peritoneal surfaces of stomach and duodenum into firm apposition. The anterior wall is next made in a similar way by a continuous catgut stitch through all the coats, but here the suture cannot be passed from within, and of course does not bring the peritoneal coats into apposition as it does posteriorly. The object of this continuous catgut stitch is to secure a temporary watertight junction between the two viscera. To secure broad permanent adhesion between stomach and duodenum, a ring of fine interrupted silk sutures through peritoneal and muscular coats only is placed all round, and completely buries the temporary junction.

2. Kocher's Method.-Suture of the stomach incision; implantation of the cut end of the duodenum into the posterior wall of the stomach. The union may be effected by a simple suture, bobbin or button. The suture adopted by Kocher is most satisfactory. Kocher has recorded fifty-seven cases operated upon by this method, with five deaths. Although the Murphy button has been frequently advocated and employed, we feel strongly that by its use there is no saving of time worth the mentioning, and the risks are decidedly grave.

The duodenal cut end may be implanted into the anterior wall of the stomach. Czerny has used both the anterior and the posterior methods, with and without the Murphy button, but does not express any preference for one over the others.

Continuous sutures (catgut for the mucous membrane and 
silk or Pagenstecher thread for the serous surfaces) with one or more interruptions are employed, as in gastro-enterostomy.

The details of the operation are as follows: An incision 4 or 5 inches in length is made in the middle line, more or less above the umbilicus, according to the position of the pylorus. After opening the abdomen, the tumour is examined carefully to determine the limits of its infiltration. Small openings are made in the greater and lesser omenta wide of the growth, to enable the clamps to be introduced. The omenta are then ligatured; if any vessels are torn, they are at once secured. When the tumour is thus isolated it is everywhere surrounded withsterile compresses. The stomach is then clamped with two Kocher's clamps, one above, the other below. The duodenum is closed with one clamp close to the growth, and a second at a distance of $I$ inch, and the gut is divided between them. The cut surface is cleansed by mopping with I in I,ooo sublimate solution, and now, and throughout the operation, care is taken to avoid soiling by the secretions of the stomach or intestine. The proximal end of the duodenum is wrapped in sterile gauze and drawn out of the wound. An assistant now grasps the stomach from above and below between the thumb and the indexfinger, and the stomach is divided between his fingers and the clamps. The cut end is dried, and any secretion which escapes is carefully mopped up. The bleeding vessels are ligatured. A continuous suture now closes the stomach incision, and includes all the coats. A second continuous Lembert suture is applied, including only the peritoneum. The assistant now seizes the stomach, and by pressure with the thumbs makes the posterior surface present to the front. The duodenal cut end is then applied to the posterior surface of the stomach, and a continuous silk suture unites its serous coat along the whole length of the posterior half to the presenting surface of the stomach. The duodenal clamp is now removed. The stomach is incised in front of the continuous suture along a distance equal to the breadth of the duodenum. After bleeding is arrested, a continuous suture unites the cut margins of the stomach and duodenum and includes all the coats, or one suture unites the muscular and another the 
mucous surface. The anterior borders of the openings are now united in the same manner as the posterior. The parts are cleansed and replaced and the compresses removed. Kocher lays stress upon three points: (I) Rigorous asepsis; (2) the employment of continuous sutures throughout; (3) the use of good clamps. He advises the use of the Murphy button when there is any tension owing to an inability to drag the duodenum forward. There is rarely any difficulty in securing easy apposition of the duodenum and stomach, for the latter has a remarkably free range of movement, and can be very easily pulled across to the duodenum. Kocher himself has removed the stomach to within two fingers' breadth of the cardia, and yet secured an apposition free from strain. An extension towards the duodenum entailing a free removal would furnish the chief difficulty to this procedure.

3. Closure of both Cut Ends-Performance of Gastró-enterostomy.-After removing the growth, the cut ends of the stomach and duodenum are completely closed by continuous sutures, of catgut for the mucous membrane or all the coats, and of silk for the serous surface. An anastomosis is then made between the stomach and the jejunum in one of the methods already described, the posterior method being that most commonly employed. The order of the operations may be reversed, as advised by Tupolske and Czerny, the gastroenterostomy being first performed, and, after the lapse of two weeks or thereabouts, the pylorectomy.* This has the advantage of lessening the demand made upon the patient's powers, and of enabling some strength to be gained by careful dieting in the interval (Fig. 23).

The following method of operation is one that we have adopted in several cases with great satisfaction (Clin. Soc. Trans., vol. xxxv.) :

The abdomen is opened about $\frac{1}{2}$ inch to the right of the middle line through the rectus muscle, the fibres of which are split. The incision must be of good length, so that adequate room is afforded for the subsequent manipulations. The stomach being exposed, a preliminary ligature is applied to its chief vessels

\footnotetext{
* See report of case on p. 115.
} 


\section{PLATE X.}

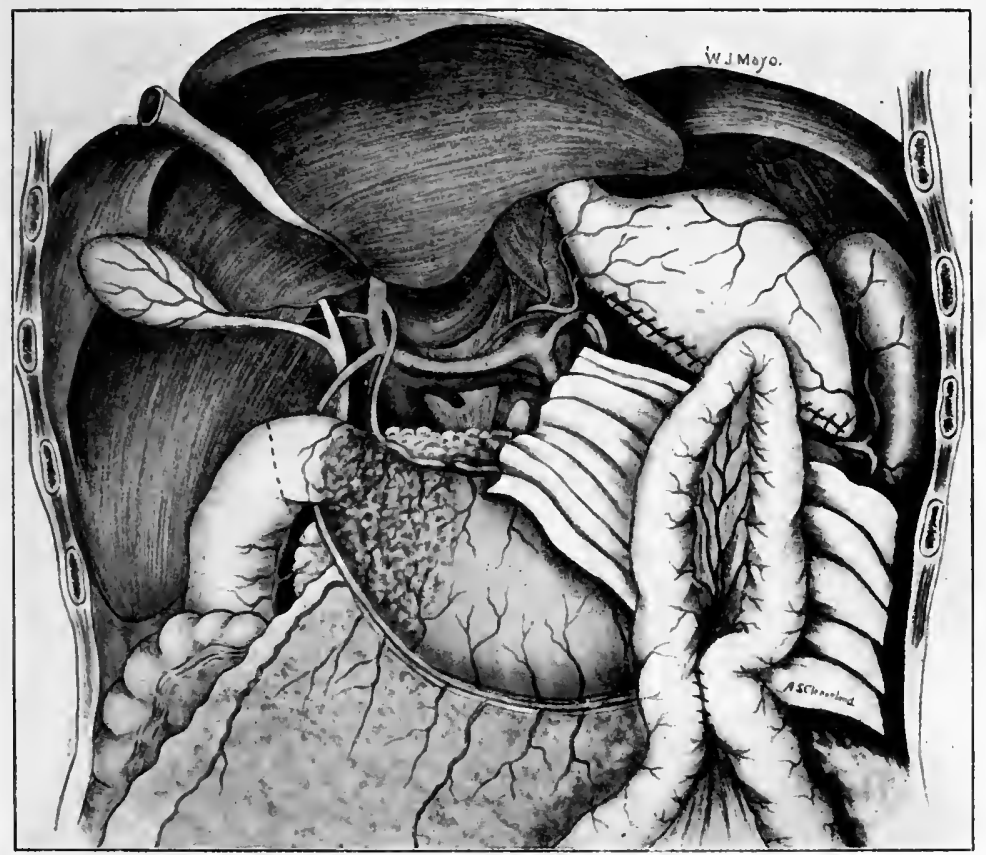

Fig. 23.-Gastro-enterostomy after Partial Gastrectomy (Mayo).

To face p. II2.] 

-the coronary, the pyloric, the gastro-duodenal, and the left gastro-epiploic. The veins running with the arteries are included in the ligatures, and in each case two ligatures are tied, and the vessels are divided between them. The coronary artery is exposed first, as it lies in the 'ligament of Huschke'-the falx coronaria -whose folds enclose it. This is readily done if the position of the artery be defined by lifting up the liver and exerting traction upon the stomach, pulling the viscus forcibly downwards and outwards until a ridge is raised up in the peritoneum by the underlying vessel. The artery and vein are then surrounded by two ligatures, passed with an aneurism needle or Greig Smith's forceps, and divided. If the vessel is easily seen it should be divided after the osophageal branches have been given off, as these offsets carry blood not only to the cesophagus, but to a portion of the fundus of the stomach.

The pyloric artery and the gastro-duodenal are best found by exposing the hepatic artery, from which they both arise, separately or by a common trunk. The hepatic can be found a little above the pylorus before it passes upwards in front of the foramen of Winslow. A gentle traction of the pylorus downwards and to the right wili render its discovery an easier matter, or the vessel may be ligatured after section of the stomach on the left, as suggested by Cunéo. The pyloric portion of the stomach, after section, is turned over to the right and dragged firmly. In the pancreatico-duodenal recess the vessels can be felt and safely ligatured. The left gastro-epiploic is ligatured at the point of section of the stoniach, at the junction of the great tuberosity with the body of the viscus. This point has to be guessed at approximately, but a little experience of stomachs in the postmortem room will enable the operator to obtain the correct point accurately. The vessels being ligatured, the omenta are separated from the stomach, and are divided at such a distance from the viscus as will insure that all the glands remain with the stomach. A few points may require clipping and ligaturing during this manipulation.

It is important to remember that the middle colic artery is readily wounded in dividing the great omentum. Its injury involves a risk of gangrene of the colon, and extreme care should therefore be exercised in dividing the omentum in its neighbourhood. The stomach and duodenum are. now free. A clamp is applied at the cardiac end, a second clamp on the duodenum, at the junction of the first and second portions, and the portion between the clamps removed. The portion taken away consists of the lymphatic areas $a$ and $b$, the first portion of the duodenum, and all the lymphatic glands along the coronary and right gastro- 
epiploic arteries. After its removal a gland or two from the head of the pancreas may have to be dissected away, and possibly a small portion of the pancreas itself may need removal, as in cases recorded by Richardson, Obalinski, Christy Wilson, and ourselves.

The anastomosis between the stomach and intestine must now be made. End-to-end approximation (Kocher's method) or a separate gastro-enterostomy with suture of the stomach and duodenal cut surfaces may be performed. In one of our cases it was found impossible to perform end-to-end suture, and we therefore closed both ends and performed a posterior gastro-enterostomy, pulling a. loop of the jejunum up through a slit in the transverse mesocolon. End-to-end approximation is the most desirable method, and has the support of Mikulicz, Krönlein, Terrier, ourselves, and others. If, however, the first portion of the duodenum is removed it will be found a matter of great difficulty, if not an impossibility, to effect an anastomosis without undue tension.

\section{Partial Gastrectomy for Cancer of the Body of the Stomach.}

The ideal operation for pyloric growth involves the removal of so much of the stomach wall that it may be properly called partial gastrectomy. There are, however, cases of mural cancer in which local excision is necessary. For example, an hour-glass contraction of the stomach may be caused by a thick ring of growth, like a napkin-ring, in or near the middle of the stomach. In such a case the growth may be removed, and the cut edges of the stomach united.

The vessels in the small and large omentum are ligatured and divided. The stomach being clamped on each side, the growth and a wide area of seeming healthy wall around it are removed. The minimum distance between the line of section and the margin of the growth must be 3 centimetres. Mikulicz lays emphasis upon the fact that the frequency of local recurrence shows that too little of the stomach is generally removed. The lymphatic glands along the whole length of the lesser curvature are removed with or without the stomach wall along which they lie. If any are found in the great omentum or along the greater curvature, they 


\section{PLATE XI.}

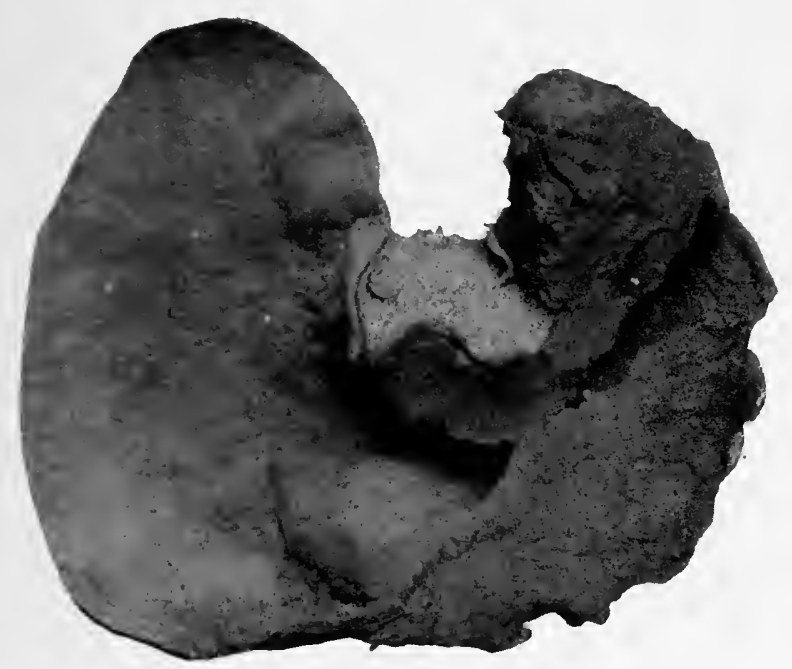

Fig. 24.-Cancer of Pylorus, producing Stenosis, in a Woman aged THIRTY-SIX.

(No. 2.4 I I a, Royal College of Surgeons' Museum.)

To face p. II 4 .] 

also must be removed. Union of the divided ends may be effected by the simple continuous suture, as in gastro-enterostomy, or by the largest bone bobbin, as in the following cases :

Hour-glass Stomach caused by Cylinder of Cancer in the Centre. Gastro-enterostomy with Subsequent Partial Gastrectomy; Recovery.

Mr. B., aged sixty-two, suffered from a movable tumour, thought to be cancer of the stomach. This had been noticed for a month, but had been preceded by stomach symptoms, chiefly pain after food and slight vomiting, for quite a year. There had latterly been some slight coffee-ground vomit, but no large amount of blood had been vomited at any time. The tumour was not tender, and could be pushed up under the left, and moved over to the right, costal margin. A stomach splash was well marked on the left of the tumour, and on distending the stomach with $\mathrm{CO}_{2}$ the lump was pushed over to the right. The vomit was acid from the presence of lactic acid, but no free $\mathrm{HCl}$ could be found.

Pylorectomy was advised, and the patient was admitted to the infirmary, being then in an extremely weak condition.

November I 5, 1900.--On opening the abdomen a mass of growth was found in the centre of the stomach, forming a ring of cancer, and leaving a cavity on its proximal and distal sides, the cardiac cavity forming the dilated stomach where the splash on succussion was felt. Dr. Seaton, who was giving the anæsthetic, advised that the patient was too feeble to bear a prolonged operation, and a colleague also confirmed the fact that the pulse could be barely felt at the wrist. A posterior gastro-enterostomy was therefore performed, a bone bobbin being employed.

$\mathrm{He}$ soon rallied and made a good recovery, gaining flesh and weight, and expressing himself as very well.

December 20.-The radical operation of removing the growth by partial gastrectomy was performed, about half the stomach being removed in order to leave a wide margin of healthy tissue between the growth and the wound.

Union was effected by suturing the cardiac and pyloric incision together over a bone bobbin, catgut being used for the mucous and celluloid thread for the serous sutures, the whole operation being completed within the hour. No glands were felt beyond the resected area. Recovery was uninterrupted, and he returned 
home within the month, eating and enjoying food, and having gained considerably in weight.

April, I901.- - He continues well and active, and has been able to resume his work.

Further History.-He lived for sixteen months, and until nearly the end took his food well.

\section{Cancer; Hour-glass Stomach. Partial Gastrectomy ; Recovery.}

Mrs. J., seen January 24, I901, with a movable tumour in the epigastrium that had been noticed three weeks previously. There had been no vomiting, although indigestion, pain an hour after food, and loss of flesh and strength, had been noticed since August, I 899 .

She weighed 7 stones, having weighed 8 stones or more previous to her illness. The tumour appeared to be the size of a tennis ball, was free from tenderness, and could be made to move across the abdomen from one side of the umbilicus to the other.

On distending the stomach with $\mathrm{CO}_{2}$, it reached nearly to the pubes on the left side, but very little distension was noticed on the right, beyond the tumour. She was admitted to the infirmary, and on January 31 , on exposing the stomach, a tumour was found involving the whole circumference a short distance from the pyloric end, so that between the pylorus and the tumour a small second stomach cavity existed, the dilatation being formed entirely by the cardiac complement. The tumour was completely excised, and the distal and proximal portions united over a bone bobbin. Glands were removed from the lesser and greater omentum. Recovery was uninterrupted, and the patient left the hospital before the month-end, having regained the weight lost during the week after operation, and having added $\frac{1}{2}$ pound over. The tumour proved to be cancer. She gained her normal weight, and when heard of in 1903 was quite well.

\section{Partial Gastrectomy, Cholecystectomy, and Partial Hepatectomy for Cancer; Recovery.}

Mrs. S., aged fifty-four, was sent to us by Dr. Fry, of Haworth, on July 28 , I goo. The previous history was rather indefinite, as the patient did not think it necessary to seek medical advice before the previous April. She said that she had been in excellent health until November, I898, when she began to suffer for a 


\section{PLATE XII.}

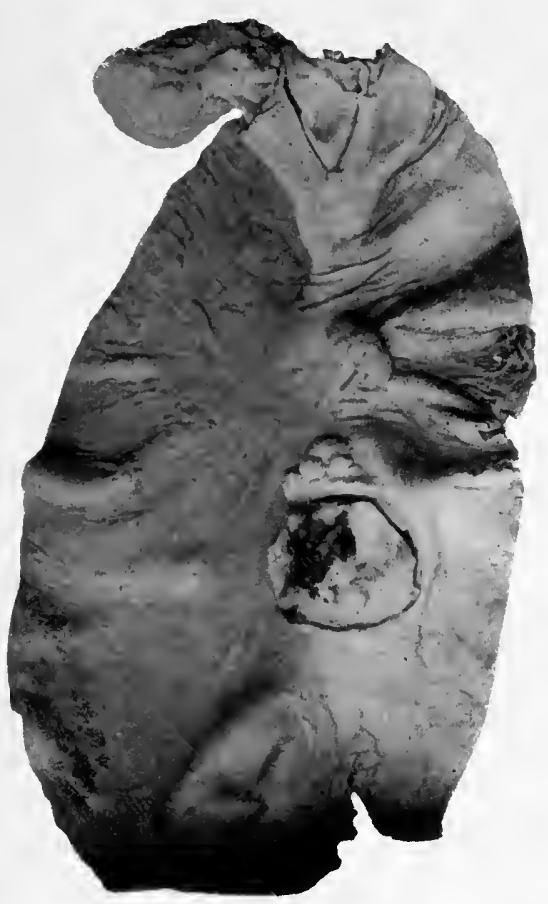

Fig. 25.-Perforation of the Stomach due to Sloughing Cancer. (No. 2,407, Royal College of Surgeons' Museum.)

To face p. II6.] 

month or six weeks from spasmodic pains over the gall-bladder region, but she was never jaundiced after the attacks. She also had pain at the epigastrium like indigestion. When Dr. Fry saw her she was suffering from a severe attack of pain in the right hypochondrium with vomiting, and was in a state of general collapse. Her general condition was then unsatisfactory, as she had lost considerably in weight, and was evidently in failing health, there being some slight swelling of the legs and general distension of the abdomen. The skin was sallow, with a slight tinge of jaundice; the liver was found to be enlarged, and the gall-bladder could be felt as a hard mass, well defined, about 2 inches wide and 3 inches long. It was tender on pressure, and was painful after any exertion.

In June and July the swelling gradually increased, and the mass extended over a larger area, approaching nearer to the umbilicus, and later a sensation of fluctuation was felt, when the deep swelling became less definite, but the surface swelling was more obvious on inspection. About the middle of July there was a sudden decrease in the size of the tumour, with some relief and temporary improvement. It was at that time that we saw her, and found a distinct tumour beneath the right costal margin, apparently adherent to the parietes. Cholelithiasis with suppuration was diagnosed, and the question of malignant disease raised. The stomach was markedly dilated. On August 9 a vertical incision through the right rectus was made on a mass of adherent viscera. An abscess was found in the sheath of the rectus, which was cleared out and purified as far as possible. The gall-bladder, which was very much thickened and adherent, was opened, when some thick purulent discharge escaped. General soiling of the peritoneum was prevented by sponge packing. A large gall-stone was removed from the gall-bladder, and another from the cystic duct. A fistula was found between the gall-bladder and the pyloric end of the stomach, through which the empyema was discharging itself into the stomach.

The pylorus was found to be much thickened and the seat of a nodular growth, which also involved the gall-bladder and adjoining part of the liver. Cholecystectomy was performed, and with the gall-bladder was removed a V-shaped portion of the liver, the gaping edges in the liver being brought together by deep chromicized catgut ligatures, the hæmorrhage being controlled by sponge pressure. A tube was placed in the cystic duct, which was then firmly stitched round the tube, so as to avoid the escape of bile. Partial gastrectomy was then performed, the cut section of the stomach being united to the cut section of the duodenum by means of two :continuous sutures around a bone ${ }_{-}$bobbin. An 
omental graft was then sewn over the junction to afford additional security. As the disease was clearly malignant and all the growth within the abdomen had been removed, the portion of rectus involved in the disease and the adjoining peritoneum were excised, a further transverse incision being necessary. To close the wound the peritoneum, aponeurosis, and muscular wall were then securely closed layer by layer, the whole operation being completed within the hour.

The patient rallied well, and convalescence was uninterrupted. Bile flowed freely through the tube until it was removed on August 22; a gauze drain was then employed until the wound healed. The fæces soon assumed a normal colour, and the slight jaundice cleared up. Microscopic section of the growth showed it to be columnar-celled carcinoma. The patient returned home within the month, and when she came to see us two months after operation she was then gaining strength, and had had no return of her symptoms. The general health had much improved, and, beyond a slight sinus where the drainage-tube had been and the scar of the wound, there was no trace of operation. The small sinus did not discharge bile, but merely a few drops of sero-pus in the day.

A report from Dr. Fry in March, igor, was to the effect that the patient was in very good health, and another in October, I903, stated that the patient continued well.

The following case came so near to being a complete gastrectomy that it may be conveniently placed here between the complete and partial gastrectomies, especially as it shows with what advantage a small portion of the dome of the stomach may be in some cases left. The interesting point about the case is that the patient remains well three years subsequent to operation.

Mr. - , builder, aged thirty-eight, was sent on March I8, I90I, by Dr. R. O. Petrie, of Bridlington, with the following history:

He had since childhood always complained of flatulence, and had suffered from indigestion, though he had only been ill for two years, during which time he had suffered from fatigue, with some loss of strength but no pain. Six months ago he began to have pain every morning, which started in the epigastrium and passed over to the right side of the abdomen. There was no pain immediately after food, but it always canie on before the next meal, when food gave relief. $\mathrm{He}$ vomited for the first time the week before seeing us. At that time he had an attack of 
diarrhœa which was thought to be due to a chill. He had never been constipated. There had been great loss of flesh during the past twelve months, amounting to 2 stones, his weight when seeing us being 9 stones I pound. He looked ill and cachectic. He was quite sure that he had never vomited blood, and that he had never seen blood in the motions.

On examining the abdomen a tumour could be easily seen and felt, occupying the epigastric region, and extending from the left costal margin nearly as far as the right. On distending the stomach with air the tumour was pushed downwards, but there did not seem to be much dilatation. The tumour had a wide range of mobility, could be made to pass to the right and left side of the abdomen, and could be pushed up under cover of the liver, and down below the margin of the ribs. During manipulation the tumour hardened under the hand, when it was very distinct, but when the stomach muscle was relaxed the growth was less prominent. There was no free $\mathrm{HCl}$ in the stomach contents. An operation was proposed and consented to, and in the presence of Dr. Petrie the abdomen was opened by an incision through the right rectus. The tumour at once came into view, and proved to be a firm nodular malignant growth, involving nearly the whole of the stomach from the pylorus to the cesophagus, the only portion of the organ apparently free being a little of the dome near the left of the cesophageal opening. There was no ascites, and no enlarged glands could be felt, nor could any secondary growths be seen.

As it was clearly useless to perform any lesser operation, and as the tumour was so mobile, gastrectomy was decided on.

The duodenum an inch beyond the pylorus was clamped by long forceps covered with rubber tubing, the lesser and then the greater omenta were divided between ligatures, and as there were no adhesions the large tumour was then drawn down, and the œsophagus and dome of the stomach were clamped by two forceps applied from the left and right side respectively. The stomach was then cut away by scissors, and after all visible vessels had been ligatured the clamps were released and a few other bleeding-points taken up; but throughout very little blood was lost. The duodenum was brought across the spine and fixed by an external celluloid thread and an internal catgut suture around a decalcified bone bobbin to the margin of the stomach remaining around the œsophageal opening. The duodenum and cardiac end of the stomach seemed to hold together with very little tension. The operation had been effected without soiling the peritoneal cavity, as the parts had been isolated throughout by sterilized gauze. The abdomen was closed in layers by means 
of continuous catgut sutures, and the patient was returned to bed in very good condition.

He was allowed to take a little liquid nourishment with Plasmon after twenty-four hours, and, after a week, light custard pudding. Nourishment of more consistency was then given, and within the month he was taking minced meat and other ordinary foods. A breaking-down hæmatoma at the week-end delayed healing for a fortnight, but otherwise recovery was uninterrupted, and he was able to return home before the end of April.

On August 27 a letter was received from Dr. Petrie to say, 'Mr. _ continues well; I saw him to-day, and he has become considerably stouter.'

In November, Igor, he called to report himself. He looked healthy and fat, and seemed to be vigorous and well. $\mathrm{He}$ had gained 2 stones in weight. $\mathrm{He}$ said that his digestion was very good if he did not attempt too large a meal. He gave the following as his ordinary diet chart.

7 a.m.-Breakfast cup of boiled milk and one tablespoonful of brandy.

Breakfast.-One egg boiled, or a little bacon, bread-and-butter, one cup of tea.

I $a . m .-B r e a k f a s t$ cup of boiled milk, with one tablespoonful of Plasmon.

Dinner.-Varying as follows: Lean of a mutton chop, little fish, chicken, or pigeon, with a little cauliflower and bread; always milk pudding, chiefly rice.

3 p.m.-Breakfast cup of boiled milk and one teaspoonful of Plasmon.

Tea.-Bread-and-butter or a little toast, one cup of tea.

8 p.m.-Cup of milk and one teaspoonful of Plasmon.

Supper.-Nearly one pint of boiled milk and bread.

In April, 1902, over a year after operation, he was seen by the matron of the surgical home where he stayed when in Leeds, and she reported him as looking in robust health and of normal weight.

The tumour removed was a nodular, softish growth, involving almost the whole of the stomach, including the pylorus, and extending from it to the cardiac end, where a small margin of healthy stomach wall remained. It weighed I pound immediately after operation. Unfortunately, the specimen, which was sent to a pathological laboratory to be mounted and reported on, was mislaid and cannot be found. It was undoubtedly malignant, and was invading the stomach walls generally. The growth was breaking down on its visceral aspect, and the stomach cavity contained some grumous material thrown off from the growth. 
The pylorus was invaded, but the line of section in the duodenum and at the cardiac end of the stomach showed a healthy appearance, and a free portion existed between the growth and the cut margin.

In 1904 he was reported to be well. The case illustrates an operation that may be performed with much greater facility and safety than complete gastrectomy.

It is a fact that the dome of the stomach is frequently free from disease, and can be safely left when the rest of the stomach is removed. This is easily explained by a reference to the diagrams of the lymphatics of the stomach, which show the dome free from glands and lymphatic trunks. Should such a state of affairs be found, it simplifies the operation very much, as the duodenum and remnant of the stomach can be made to meet over a decalcified bone bobbin very much more easily than the œsophagus and duodenum, or even the jejunum, can be directly joined, as in complete gastrectomy.

Results.-We do not think that any useful purpose can be served by quoting the statistics of the earlier cases of pylorectomy. The mortality was enormous; the experience gained, however, was invaluable. In order to obtain a fair estimate of the risks and successes of an operation so rarely performed, it is essential that we should select only those operators who publish all their cases, ignoring the isolated successful cases recorded from time to time in the journals. The continental surgeons chiefly afford us the information necessary.

Krönlein (Archiv f. Klin. Chir., I898, p. 447) records all his cases of partial excision, and his assistant Schlatter's case of total extirpation of the stomach. Of 24 cases, 5 died; of the first 4 resections 3 died (I88I-I838), of the following 20 only 2 died (I888-I898). The after-history of the I9 patients who recovered is as follows: 2 died of intercurrent disease (heart failure, pneumonia) within four months, without recurrence; 8 died of recurrent growth, 2 in the first year, 4 in the second year, and 2 in the third year. The average duration of life in these 8 cases was 507 days. Eight patients were alive in July, 1898 , without 2 recurrence 
6 in the first year and 2 in the fourth year; I case was living with a recurrent growth (Schlatter's case died thirteen and a half months after operation).

Maydl (Medical Press, October, 1899) reccrds 25 operations for carcinoma of the stomach: 4 patients died, directly or indirectly, as the result of the operation in two, three, and five days, and from embolic gangrene of right leg; 7 patients recovered from the operation and left the hospital, but suffered from a return of the growth within a short period. The average duration of life was II' 7 months. Fourteen patients were alive at the time of the report ; of these, 7 had lived for over two years, averaging four years two and a half months each, and 7 had lived for under two years.

Kocher (Korrespondenzblatt für Schweizer Aerzte, I898) has resected the pyloric end of the stomach 57 times, with 5 deaths. Eight cases are considered as cured. One, a woman, was alive ten years after operation, another five years, another three years, another two and a half years. Four patients died over three years after the operation from other causes.

Rydygier (Deut. Zeitschr. fïr Chirurgie, January, Igor) relates all the stomach operations he has performed in the last twenty years. There are 25 partial gastrectomies. Of these, 8 recovered and $I 7$ died. The causes of death are: pneumonia, 5 cases; septic peritonitis, 2 cases; shock and collapse, 5 cases; in 5 the cause is not stated. Rydygier resects a large portion of the stomach for limited tumour, cutting at least 5 to Io centimetres beyond the apparent confines of the growth.

Czerny (Beitr. zur Klin. Chir., 1899, p. 18, and Archiv f. Klin. Chir., I8g8, p. 459) has performed 29 partial gastrectomies since I $88 \mathrm{I}$. Of these I I died. Of the survivors, one has lived 7 years, one $3 \frac{1}{2}$ years, and in others life was prolonged from 3 to $3 \mathrm{I}$ months. The average duration of life after operation was 22 months.

Mr. Rutherford Morison, of Newcastle, has kindly favoured us with the details of all his cases of partial gastrectomy. There are 16 cases. Of these, 7 died within a month of operation-4 days, I 4 days, a few hours, 6 days, 29 hours, 
8 days, and I4 hours; 9 recovered from the operation, and lived 7 weeks, 37 months, 26 months, 19 months, 6 months, 2 months, and 7 months; 2 are still living without recurrence-I was operated upon in October, $I 898$, and $I$ in October, Igoo.

With regard to operations on the stomach for malignant disease, it is very manifest that the success depends altogether on an early recognition and early operation. For instance, of the partial gistrectomies performed by us before I 897 the mortality was 75 per cent., whereas since I 896 of 8 cases 7 recovered, thus yielding a mortality of 12.5 per cent., and several of these patients are remaining in good health at the present time.

\section{Complete Gastrectomy.}

This operation was first performed in 1883 by Connor of Cincinnati. His patient, a woman of fifty years of age, died on the table. In I884 Schuchardt, and in I892 Maydl, reported cases of extirpation of the stomach. In I894 Langenbuch published two cases of 'resection of the stomach'; in each only seven-eighths, not the whole of the stomach, was removed. The first successful complete gastrectomy in the human subject was performed by Schlatter of $Z$ urich on September 6, I897; the second by C. B. Brigham of Boston, U.S.A., on February 24, I898. In the former the cut end of the œesophagus was united to a loop of the jejunum, the duodenal opening being closed; in the latter the cut ends of the œsophagus and duodenum were united over a Murphy's button.

The following account is taken from Dr. Schlatter's description (Lancet, vol. i., I898) of the operation performed by him :

'After shutting off the peritoneal cavity with sterilized compresses, I isolated the stomach on its great and small curvatures, separating the great and small omentum with the aid of Péan's clamps, and ligaturing the clamped portions with silk. I then pulled it firmly downwards in order to obtain access to the œsophagus. The left lobe of the liver, which covered the field of 
operation, was held up constantly by the hand of an assistant, in which way we succeeded in applying a Wölfler's compressorium to the oesophagus tolerably high up. I applied a Stille's clamp quite close to the cardiac border of the tumour, and separated the stomach from the œsophagus just at their point of junction. The direction of the incision happened to be somewhat oblique, so that I found it to be advantageous to reduce the aperture of the œsophagus by means of a small suture. The pylorus was treated in exactly the same way. The duodenum was freed as far as possible towards the head of the pancreas, and the stomach, together with the pylorus, was separated between a "duodenum compressorium" applied as far away as possible, and a tumour compressorium applied to the duodenal region. The aperture of the portion of the duodenum which remained in the abdomen was cleansed with pads of iodoform gauze, just as had been already done in the case of the aperture of the cesophagus. An extensive portion of the continuity of the digestive tract was now cut away. I endeavoured to draw up the end of the duodenum to the end of the œsophagus, but it was only with the greatest difficulty that I could bring them into contact, so that the union of the two orifices was not to be expected. I turned in the border of the duodenum, and closed the aperture with a double suture. Starting from the duodeno-jejunal angle, I followed the small intestine downwards for about 30 centimetres (I 2 inches), drew it out at that point, brought it across the transverse colon, and applied it to the end of the cesophagus. A piece of the small intestine about ro centimetres ( 4 inches) long being held in Wölfler's compressors, the intestine was fixed to the œesophagus by suturing the serous membrane, after which it was incised for about $\mathrm{r} \frac{1}{2}$ centimetres $(0.6$ inch) in the direction of its length, and the nucous membrane of the cesophageal part was united in its whole circumference with the mucous membrane of the intestine by means of a continuous circular silk suture. A continuous suture in the muscular and serous tissue and a Lembert's interrupted silk suture were applied over the suture of the mucous tissue. The compressors on the small intestine, as well as the one in the extremity of the œsophagus, which latter had been in position more than two hours, were removed. When returned to the peritoneal cavity, the sutured parts retracted themselves upwards with some force to the place where the œesophagus traverses the diaphragm. The abdominal walls were closed. The anæsthesia (8 fluid ounces of ether) progressed quietly. The pulse after the operation was regular, tolerably full, and 96 per minute.'

The patient recovered from the operation, and lived until October 29, I898. At the autopsy the mesenteric, retroperi- 
toneal, bronchial and supraclavicular lymphatic glands were found enlarged, and secondary deposits were found in the pleuræ.

The following is an abstract of Brigham's case :

On opening the abdomen the tumour was found to comprise nearly half of the wall of the stomach; there was no marked glandular involvement or adhesions, and it was decided to remove the whole organ. The greater and lesser omenta were tied off and divided, the duodenum was clamped, and a ligature was placed around it $\frac{1}{2}$ inch above the clamp, and the tissues were divided between the two. The ends were washed in salt solution and wrapped in iodoform gauze. The same method was employed with the œsophagus and the cardiac end of the stomach. It was found that the osophagus and duodenum could be brought together, and they were united by a Murphy button to shorten the operation, as the patient was growing weak. No Lembert sutures were used, as the approximation was good. The operation lasted two and a quarter hours, and was followed by considerable shock. At the end of six weeks, when the report of the case was made, the patient was quite well, enjoyed her food, and was gaining in weight. Never after the operation was any undigested food found in the fæces.

The fourth operation was by Maurice Richardson, of Boston. The patient recovered, but died eleven months later of recurrence.

The next case is recorded by Macdonald. The patient was a man, aged thirty-eight. The duodenum and osophagus were united by a Murphy button. The patient was alive and well eighteen months after the operation.

Kocher removed the whole stomach and a portion of the transverse colon from a woman, aged forty-four. The duodenum and cesophagus were united by suture. The patient died of peritonitis.

Bernays removed the stomach and a portion of the pancreas from a man aged forty-two. Death occurred in thirty-six hours.

Chavasse operated upon a man aged sixty-four. As it was impossible to unite the osophagus to the intestine, its end was closed by suture and duodenostomy performed. The patient died in a few hours. 
Bardeleben removed the entire stomach from a woman of fifty-two. The cut ends of the esophagus and duodenum were closed by suture, and a lateral anastomosis made between the osophagus and a loop of small intestine. The patient was alive nine months later.

Harvie removed the stomach and 3 centimetres of the duodenum from a woman aged forty-six. The œsophagus was united directly to the duodenum. Recovery followed.

Dollinger. - The whole stomach was removed from a man aged thirty-seven. A loop of small intestine was sutured to the osophagus. The patient recovered, but a few days after the operation a biliary fistula formed, owing, it was presumed, to imperfect closure of the duodenum.

Herciel. - Complete removal of the stomach from a woman aged forty-two. The œsophagus and duodenum were closed, and a lateral anastomosis made between the former and a loop of small intestine. Sutures were passed between the diaphragm and the anastomosing loop, to avoid traction on the sutured line. The patient a year and ten months after the operation had gained $18 \frac{1}{2}$ kilogrammes in weight.

Rivera removed the whole stomach from a woman of thirty-six. The patient recovered, and remained under observation for fifteen months.

Gurreì $y$ Hurtado removed the stomach from a woman of forty-one. The œsophagus and duodenum were united by suture. Recovery followed.

Fedoroff performed total extirpation upon a man aged thirty-five. The œsophagus was united to the duodenum by suture. The patient died on the fifth day of peritonitis.

Vander Veer removed the whole stomach from a woman of forty-two. The duodenum and œsophagus were united by a Murphy button. The patient died the following day, and at the autopsy it was found that the attachment between the œsophagus and the duodenum had given way.

There are thus 16 cases in all. The ages range between thirty-five and sixty-six. There were 6 deaths, Io recoveries.

On reading through the records, it will be seen that the 
junction between the œsophagus and intestine has been made in the following ways:
Esophagus to duodenum
(by simple suture.
(by the Murphy button.
Esophagus to jejunum
by simple suture.
iby the Murphy button.

Esophagus and duodenum both closed by suture, and a lateral anastomosis made.

Esophagus closed by suture, the open end of the duodenum stitched to the skin = duodenostomy.

The case upon which we operated was the following:

Mrs. R., aged forty-nine, seen with Dr. Oldfield, had been ailing for four to five months. Pain after food was the symptom first noticed; this quickly became so severe that solid foods were abandoned. Gradually it was found that liquid foods began to cause distress, and eventually these could be taken only in very small quantities. If a teacupful of milk were taken, a portion of it was rejected almost at once. There had gradually been a loss of weight, but altogether only about I stone was lost. On examining the abdomen, a hard ovoid mass was found lying transversely across the lower part of the epigastrium. When an attempt was made to inflate the stomach by administering portions of the two halves of a Seidlitz-powder separately, a considerable part of the fluid swallowed was almost instantly ejected as foam from the mouth. There was no perceptible stomach resonance either before or after inflation, and a diagnosis of carcinoma, giving rise to a 'leather-bottle stomach,' was made. The condition and the risks of operation were fully explained to the patient, who elected to submit to operation, and hoped that an attempt would be made to remove the whole growth. Her condition was poor, but no evidence of other disease could be found. On June 26 , I902, the abdomen was opened. The whole stomach was seen to be invaded by growth, and the glands along both curvatures were enlarged. The stomach was free from any but the slightest adhesions, and no secondary nodules could be seen in the liver. The whole stomach was therefore removed. The steps of the operation in the early stages were similar to those already described (p. I I 2). The cut end of the duodenum was closed by suture. On the lower end of the œsophagus a small clamp was temporarily applied. An anastomosis was now made between the œsophagus and a loop of the jejunum pulled upwards through a rent in the transverse mesocolon. After the posterior layer of a continuous 
suture of fine Pagenstecher thread had been passed, the jejunum was opened. A rubber stomach tube was now passed down the wsophagus and onwards into the distal portion of the jejunum, and kept in this position while the anterior suture was applied. After the completion of the circular suture, a second line of continuous suture was passed. At intervals this was interrupted, and at each interruption a stitch was taken through the diaphragm, so as to fix the jejunum up to its under surface. The suturing was not so difficult as I had anticipated. The œsophageal tube certainly made the hold on the parts more secure. At the completion of the stitching, $\frac{1}{2}$ pint of peptonized milk was passed through the tube and 'milked' downwards in the jejunum. The patient was very much collapsed after the operation, and, despite all our endeavours, died of shock in six hours.

In addition to the cases recorded above, numbering seventeen, there are three cases operated upon by Professor von Mikulicz, the details of which have not yet been recorded.

It was at one time considered doubtful whether a complete extirpation of the stomach, even if possible, could be a legitimate procedure. It was argued that by the time so much of the stomach was involved as to make desirable its complete removal, other growths, in the liver or elsewhere, would be found which would effectively preclude any possibility of a radical operation. But an examination of many records, specimens, and cases has shown that in some cases-very exceptional, it is true - the whole stomach may be infiltrated, when even on minute post-mortem examination no secondary deposits could be seen. Two cases, at least, of our own have satisfied us of this fact: in one jejunostomy had been performed; in the other no operation had been done. In both, so much of the stomach was involved as to make a complete removal necessary if extirpation of the growth were attempted. In neither case were there complex adhesions, distant lymphatic enlargements, or secondary growths of such a character as to forbid resection, to be found after the most careful examination. In a certain very small proportion of cases, therefore, a complete removal of the whole stomach may be demanded, if resection of the growth is considered desirable. 
PLATE XIII.

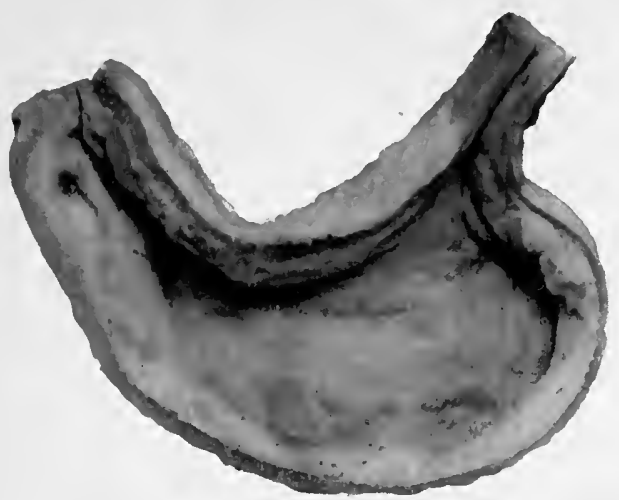

Fig. 26.-Extremely Small Stomach dependent on Neoplasm-

'Leather-bottle Stomach.'

(No. 2,408, Royal College of Surgeons' Museum.)

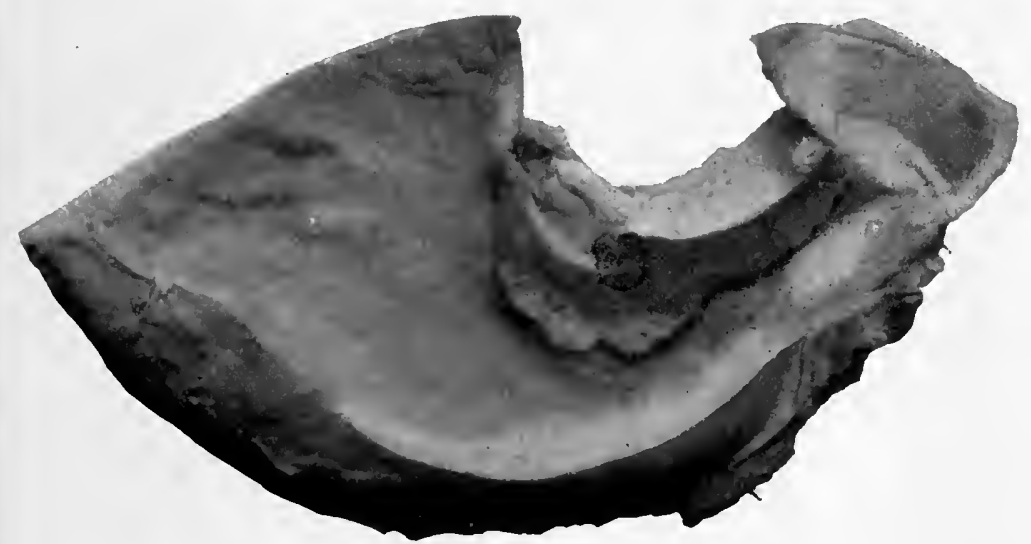

Fig. 27.-Colloid Cancer of Pylorus producing Stenosis. (No. 2,426, Royal College of Surgeons' Museum.)

To face p. I28.] 

So far as the difficulties in the operation are concerned, it may safely be said that they are less than would be anticipated. The stump of the œsophagus and its peritoneal investment might certainly be planned upon a more generous scale, but there is nevertheless enough for the purpose. The œsophagus can be freed from its opening in the diaphragm by stripping with the finger, though this is probably not desirable. There is the risk of pleural infection. In our case it seemed that the œsophagus was looser when the patient's neck was fully flexed, the chin being pressed down on to the sternum. The use of the œsophageal tube was certainly a great help, and we would urge its employment upon any who may be called upon to do the operation. Complete extirpation of the stomach is, therefore, not merely a piece of brilliant surgical gymnastics. The operation has its place in surgery.

It may not be inadvisable to enter a protest against the description of any operation as a 'complete' or 'total gastrectomy' when a larger or smaller portion of the dome of the stomach is left.

(For references to this subject, see Fedoroff, Russische Med. Rundschau, vol. i., I903.)

\section{Jejunostomy.}

Jejunostomy is an operation that can be but rarely called for. It is suitable only for those patients suffering from advanced disease, in whom, owing to the position, or extent, or character of the growth, gastro-enterostomy is deemed imprudent or impossible.

Indications for Operation.- $I$. Extensive infiltration of the stomach with carcinoma, when there is little or no healthy stomach wall that can be utilized for the purpose of gastrostomy.

2. General cicatricial contraction of the stomach, simple in character, dependent upon the swallowing of caustic fluids.

3. Neumann has suggested that in cases of pronounced hyperchlorhydria, jejunostomy should be performed in prefer- 
ence to gastro-enterostomy. He points out that the intensely acid secretion of the stomach passing into the jejunum through the new opening may produce ulceration, which will rapidly destroy the intestinal wall and lead to perforation-this is to say, a peptic ulcer of the jejunum results, in a manner precisely similar to that occurring when a peptic ulcer of the first portion of the duodenum develops. Peptic ulcer of the jejunum is recorded by Braun, Halm, Kausch, Körte, Steinthal, Hadra and Neumann, ourselves, and others. In four cases the ulcer caused death by perforation,

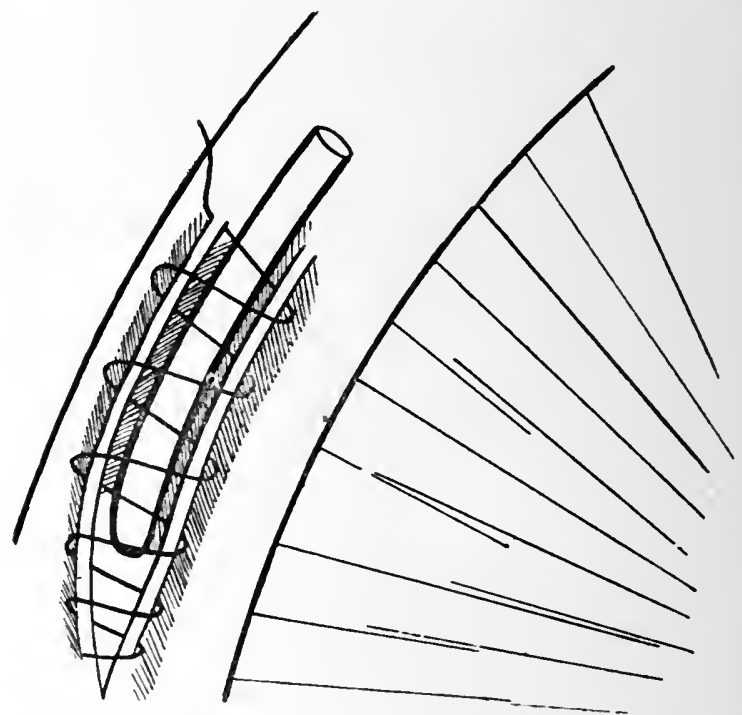

Fig. 28.-Jejunostomy-Adaptation of Witzel's Method.

and in all there was a great excess of free hydrochloric acid.

4. Cackovie and others have suggested that in cases of persistent hæmatemesis rest might be afforded to the stomach by an artificial mouth in the jejunum. This plan has received the sanction and approval of Professor Mikulicz.

Mode of Operating.-Several methods have been recommended and adopted for the performance of jejunostomy. The jejunal loop has been stitched to an abdominal incision, and opened or brought up to the surface by means of a 
bone plate placed in its lumen. There are three methods which are likely to survive. Of these, one has been suggested by Maydl, another is based upon the method of Witzel for gastrostomy, and a third is after the modification of French's operation for gastrostomy suggested by one of us.

Maydl's Method.-Maydl has performed jejunostomy in 22 cases, with 4 deaths as the result of the operation. After opening the abdomen, the upper part of the jejunum is found, and is cut completely across. The proximal cut end

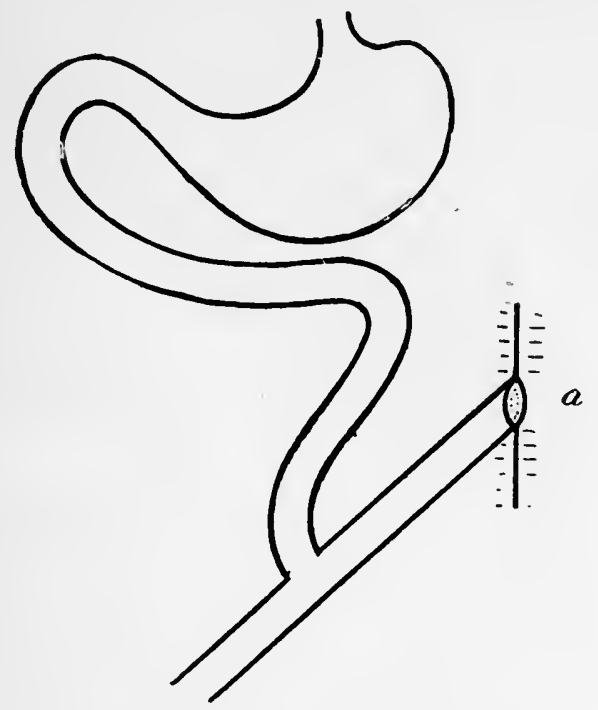

Fig. 29.-Jejunostomy-MaydL's Method.

is then implanted into the distal portion a few inches from its divided end, and the latter is stitched to the abdominal wall. The diagram will make the condition of things appreciable (Fig. 29).

In the British Medical Journal, June, r9o2, will be found an account of a method adopted by one of the authors. It is a modification of Witzel's operation for gastrostomy (Fig. 28).

'The abdomen was opened a little to the left of and above the umbilicus, through the rectus muscle, whose fibres were split. The duodeno-jejunal junction was sought, the jejunum traced. downwards for 6 or 8 inches, and a loop drawn out of the abdo- 
men. On the side of this loop furthest from the mesentery a small longitudinal incision was made, opening the bowel, and a piece of drainage-tube about the size of a No. I2 catheter was introduced, and fixed by a single catgut stitch, which included the cut edge of the bowel and the side of the tube. The tube was then laid along the bowel upwards towards the duodeno-jejunal flexure. A continuous stitch was now passed from side to side of the groove made by the tube, so that the tube, when the stitch was tightened, was buried by the overlapping of the edges of the groove. The stitch was begun about $\frac{3}{4}$ inch below the opening in the jejunum, so that any leakage there might be effectually prevented. About 2 inches of the tube was covered by the stitch, which was then tied and cut short. The line of suture in the bowel was then made fast to the anterior abdominal wall by a stitch at each extremity, and the abdominal incision was tightly closed round the tube, which projected for about Io inches.

When the patient is fed through such a tube, a funnel is fixed on to the end, and food poured in slowly. At the first only 6 ounces are introduced, but after the first few days up to a pint may be given in the space of ten minutes.

The opening in the jejunum is made, therefore, at approximately the point where Schlatter made the œsophago-intestinal anastomosis in his case of complete gastrectomy. It is clear from this case that enough food can be taken by the jejunum to enable weight to be gained and the general well-being of the patient to improve. If a jejunostomy were performed for any non-malignant condition of the stomach, the probability would be that, as in Schlatter's case, a decided increase in the capacity of the jejunum would be demonstrable, affording a reservoir for the food.

The same variety of food may be given in jejunostomy as in gastrostomy; the staple article of diet should be peptonized milk. In some cases a duodenostomy may be preferred to gastrostomy. Hartmann has recently reported an example of duodenostomy for a cicatricial narrowing of the stomach following upon the swallowing of a caustic fluid. A duodenal mouth may be considered as more efficient than one opening into the jejunum, in that the food is introduced into the bowel at a point higher than the orifice of the ampulla of Vater.

If a duodenal opening is to be made, the method that is here described is probably better than any other.

We have performed jejunostomy on five occasions. Four of the patients survived the operation for several weeks, and were benefited by it, but one patient succumbed within a week of operation. 
The following is an account of three of our cases :

Extensive Cancer of Stomach, with Inability to Retain Food. Jejunostomy; Recovery from Operation; Death from Progress of Disease at End of Two Months.

Case I.-Mrs. E. B., aged fifty-eight, residing in Lincolnshire, was sent on June I9, I89I, by Dr. Hamilton, of Crowle, on account of an abdominal tumour, accompanied by persistent vomiting and rapid loss of flesh. The patient said that she had been quite well until a year and a half before admission, when she was suddenly attacked with vomiting, which had continued ever since. She had lost weight from that time, until on admission she was worn almost to a skeleton. She had never had hæmatemesis. The vomiting took place directly after food, and was unaccompanied by nausea. At the beginning she had a stabbing pain after food in the region of the left breast, but during the six months before admission the pain had been continuous, and always worse after eating. About three months before admission she first noticed a swelling in the left hypochondrium, which had increased rapidly up to the time of admission. From the time the tumour was noticed the pain always seemed to radiate from it.

On admission the patient was extremely emaciated, and vomited everything immediately after eating. The vomit consisted simply of what she had taken, and contained free hydrochloric acid, but no sarcinæ or blood cells could be detected microscopically. Occupying the left hypochondrium, and reaching into the epigastrium, was a hard nodular tumour, moving up and down with respiration, the skin being quite movable over it. A tumour could also be felt beneath the liver, which was diagnosed as a distended gall-bladder.

Cancer of the stomach was diagnosed, and the patient was fed with small quantities of Brand's essence and peptonized foods, nutrient enemata being also administered. The pain was controlled by morphia.

At first slight improvement took place, but towards the end of the month she again lost ground, and the vomiting still persisted. After consultation, an exploratory incision was decided on, in order to see if the disease could be removed; but if it should prove too extensive for removal, as it was suspected it might, jejunostomy could be done.

On July I, I $89 \mathrm{~g}$, the A.C.E. mixture being the anæsthetic and the skin of the abdomen having been asepticized, an incision of 3 inches was made in the linea alba above the umbilicus, exposing the tumour, which was found to be occupying the whole 
of the anterior wall of the stomach, and therefore incapable of removal. There was also a distended gall-bladder, containing gall-stones.

The jejunum was then found at its fixed point on the left of the spine, and traced for about 6 inches downwards, at which spot a knuckle of the bowel was brought forward and fixed by loop sutures (after the parietal peritoneum and skin had been connected by a continuous suture), in the way suggested for gastrostomy by Mr. Greig Smith, and which a case of ours published in the British Medical Journal for June 7, 1890, proves to be a safe and efficient method.

A loop of silver wire was first inserted into the convex surface of the bowel at a spot where it would have to be opened. A round, large-eyed sewing-needle was then threaded with $\mathrm{I} 2$ inches of silk of medium thickness, and passed under the peritoneal coat of the intestine in a circle about $\mathrm{I}_{2} \frac{1}{2}$ inches in diameter, the suture being made to emerge and leave five loops at equal intervals. The loops were drawn through the parietes at about $\frac{1}{3}$ inch from the margin of the wound. As each loop was drawn up, a piece of No. 6 catheter was passed through it, and when all were in position the ends of the silk were drawn in and the loops tightened over the catheter, over which the two ends were then knotted; the silver wire was then fixed under the catheter, and a few sutures connected the bowel to the skin. The rest of the parietal incision was brought together by interrupted silkwormgut sutures in the ordinary way.

On the following day the patient felt quite comfortable, and was fed by the rectum entirely. This was continued until July 7 , when a small opening was made in the exposed bowel with a tenotomy knife, and a soft catheter was passed in, through which she was fed by peptonized food, of which she was able to take two or three pints a day. She gained flesh, and was able to return home on July 27 . Her chief trouble was that the skin around the artificial opening became irritable; but through the opening into the jejunum she continued to be able to take a fair quantity of food, which was well retained. The original disease continued to advance, and after two months she died from exhaustion.

No autopsy could be obtained.

CASE 2.-T. L., male, aged fifty-four, seen March 3, Igor, with Dr. Sykes of Cleckheaton. The symptoms were chiefly those of obstruction at the lower end of the oesophagus; a bougie passed I 8 inches. Hour-glass stomach with a small cardiac complement was diagnosed. On opening the abdomen, the stomach was found generally infiltrated with cancer ('leather- 
bottle stomach '); at the cardiac end a deep constriction separated off a small pouch from the body of the stomach. Jejunostomy was the only operation possible. Secondary deposits were found in peritoneum and liver. Death April 9. Food was taken by the new opening in fair quantity.

CASE 3.-J. G., male, aged seventy-three. The symptoms pointed to cancer of the stomach, and a hard rounded mass was felt in the epigastrium. The patient was so ill that it was thought inadvisable to give an anæsthetic, but as all food, even in spoonfuls, was vomited, it was necessary to attempt to give relief. The abdomen was opened, and cancer found affecting the whole stomach, and large nodules in the liver and peritoneum. Jejunostomy was performed. Food was taken freely, the patient gained in weight about 4 pounds, and was greatly relieved by the operation. The operation was performed on June 2, death took place on July $2 \mathrm{r}$. 


\section{CHAPTER IX}

\section{GASTROSTOMY}

THE purpose of this operation is to form an artificial opening into the stomach, to permit of food being administered when the osophagus is rendered impassable by stricture.

The operation was first suggested in I837 by Egeberg, a Norwegian military surgeon. He spoke of the possibility of making 'an opening into the cavity of the stomach, either for injecting a sufficient quantity of food or for attacking an œsophageal stricture from below.'

The cases of Voigtel and Beaumont had shown that a gastric fistula was compatible with life, and experiments performed upon dogs by Blondlot ("Traité Analytique de la Digestion,' I843) suggested the possibility of successful operations upon man. The first operation was performed on November I3, I849, by Sedillot, the patient dying in a few hours. In 1875 Sydney Jones, who had twice operated without success, performed a gastrostomy upon a patient who survived twenty days; and in 1876 Verneuil operated upon a patient who lived sixteen months. In Sedillot's first operation the stomach was opened immediately; in his second an interval of five days was allowed to elapse between the fixing of the stomach to the abdominal wall and the opening of the stomach. This, the first operation $\dot{a}$ deux temps, was performed on January $2 \mathrm{I}, \mathrm{I} 853$.

The variety of methods suggested for opening the stomach is almost bewildering. All the methods which now find favour have as their chief principle the formation of a valvular orifice, so that fluid is readily introduced into the stomach, but is unable to escape from it. The evils which 
result from the dribbling of the gastric juice on to the surface of the abdomen are intolerable. The skin is digested, is of a vivid red colour, and looks angry and florid. The pain is described as unendurable, and the patient welcomes death as a release from suffering. With any one of the modern operations a leakage should never occur.

The operations which we consider the most satisfactory are :

I. Frank's operation as modified by Mayo Robson.

2. Senn's operation.

3. Witzel's operation.

4. Kader's operation.

5. Depage's operation.
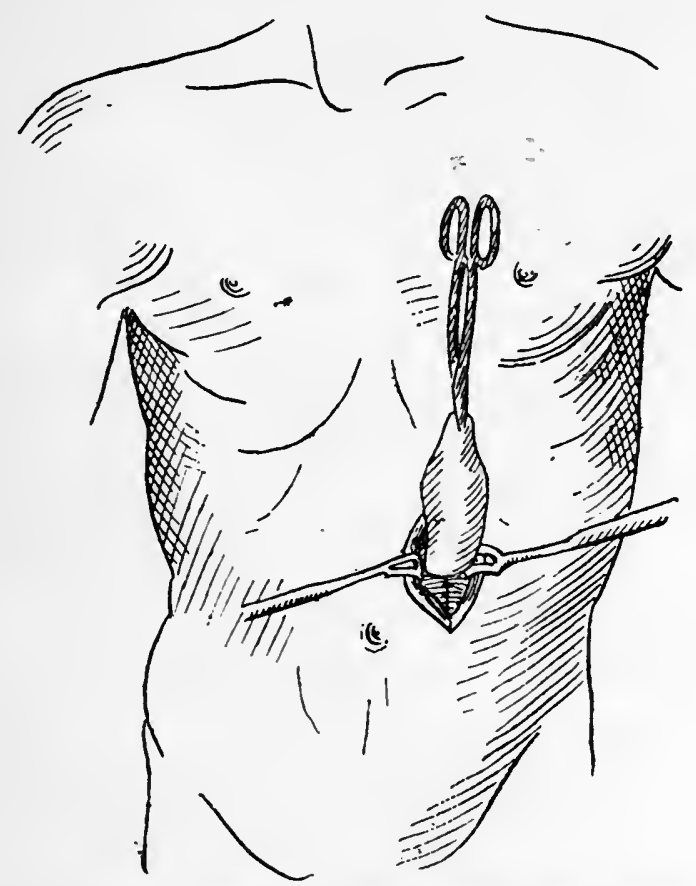

Fig. 30.-Gastrostomy. (Frank's Method modified by Mayo Robson.)

I. Frank's Method (Wien. Klin. Woch., I893-variously described as 'Ssbanajew-Frank's,' 'Albert-Frank's,' or 'Albert-Frank-Kocher's ').-We have employed this method in a large number of cases, and are eminently satisfied with 
it. When the patient is much reduced in strength, the anæsthesia of cocaine is quite sufficient, a general anæsthetic not being necessary. The following modification of the original procedure, first suggested and adopted by Mayo Robson, is employed :

A vertical incision of about $I \frac{1}{2}$ inches is made over the outer third of the left rectus abdominis, commencing $\frac{3}{4}$ inch below the costal margin; the fibres of the rectus are
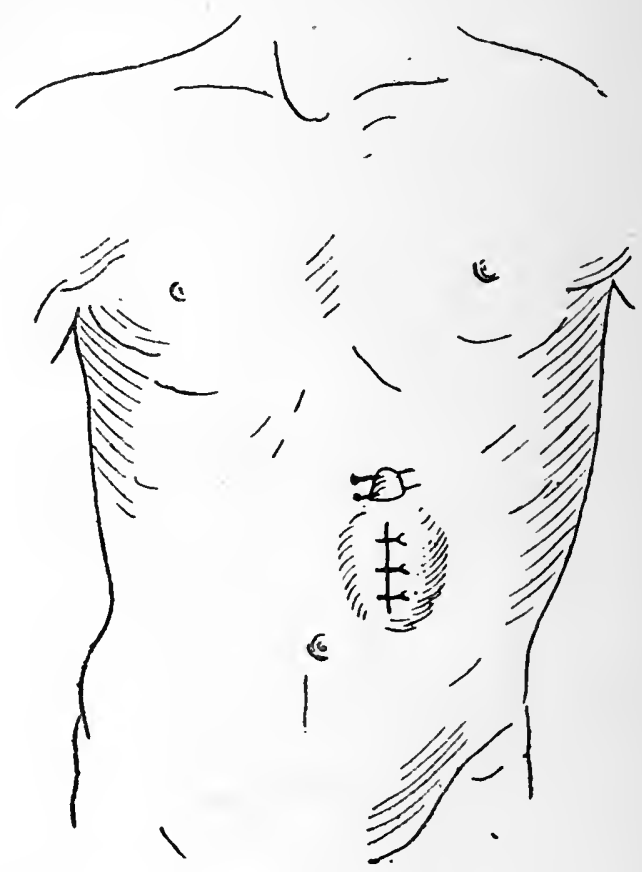

Fig. 3r.-Gastrostomy. (Frank's Method modified by Mayo Robson.)

separated, but not divided, to the extent of the incision, and the posterior part of the rectus sheath and peritoneum are divided together, the opening being an inch in length. A portion of the cardiac end of the stomach is then brought up through the wound and held forward by an assistant until four sutures are inserted into the base of the cone by means of a curved intestinal needle, so as to fix the visceral peritoneum of the stomach to the edges of the parietal peritoneum. A transverse incision of $\frac{1}{2}$ inch is then made through 
the skin I inch above the upper end of the first cut, and by means of a blunt instrument, such as the handle of a scalpel, the skin is undermined so as to connect the two openings beneath a bridge of skin and subcutaneous tissue. A closed pair of pressure forceps is introduced through the upper incision as far as the projecting part of the stomach, and made to grasp the most prominent part, which it draws up to and beyond the surface of the second opening, where it is

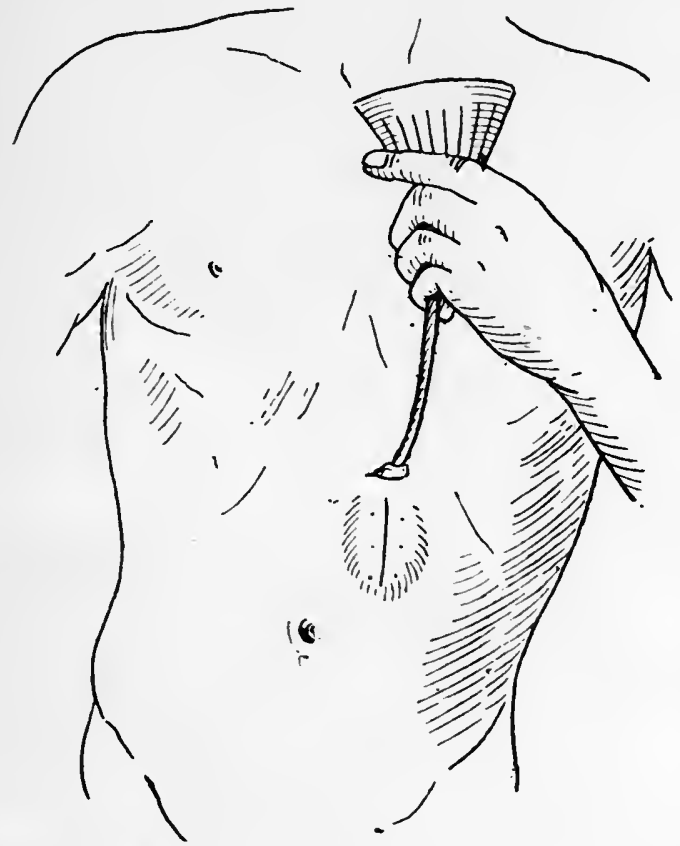

Fig. 32.-Gastrostomy. (Frank's Method modified by Mayo Robson.)

retained by means of two hare-lip pins. It should just fill the opening, and should require no sutures. The lower opening is now closed by two or three silkworm-gut sutures or by a continuous stitch, and the edges are dried and covered with collodion and gauze. If needful, the stomach can be opened at once by a tenotomy knife introduced between the pins; but, if possible, the opening should be deferred for twenty-four hours, when a barrier of lymph will have been thrown out. After opening the stomach, a soft 
catheter, from a No. 8 to No I2, is inserted, to which a piece of tubing is fixed, and by means of a funnel the patient can at once be fed with warm milk and egg, or whatever liquid may be thought desirable. The catheter may be left in position for a few days, after which it is easy to insert it whenever a meal is required (Figs. 30,31 , and 32 ).

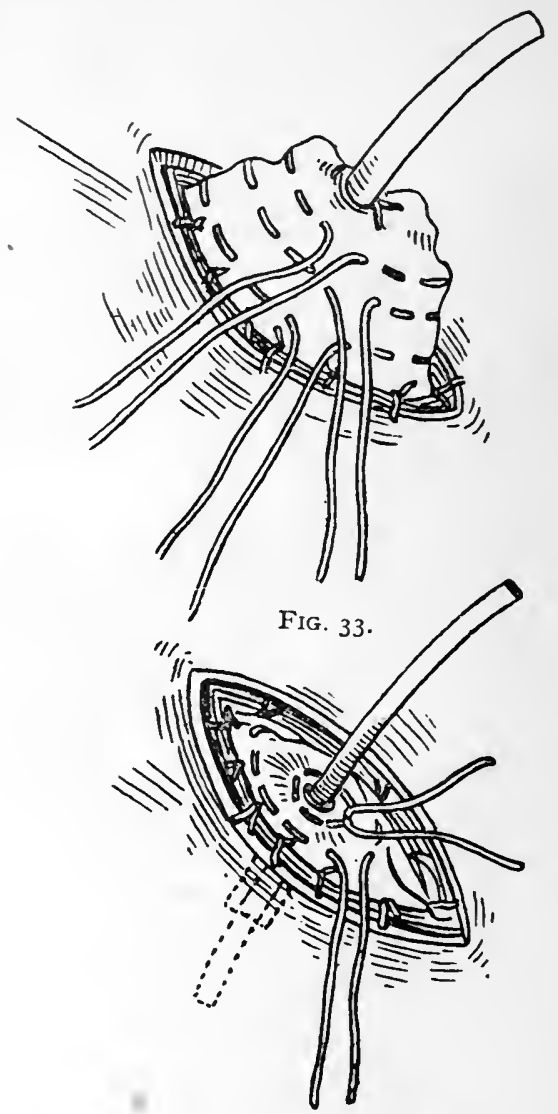

Fig. 34--Gastrostomy. (Senn's Method.)

2. Senn's Method (Journ. Amer. Med. Assoc., I896).-The stomach being exposed, an incision about $\frac{1}{4}$ inch in length is made into its cavity as near the cardia as possible, and midway between the greater and lesser curvatures. A tube equal to a No. 12 or 14 catheter is now introduced into the 
stomach, and there fixed by a suture, which includes the cut edge of the stomach and the side of the tube. In order to infold the tube in the stomach wall, a purse-string suture is passed round the tube at a distance of $\frac{1}{2}$ inch from it. The tube is pushed inwards towards the stomach cavity while the suture is tied. A second purse-string suture, and then a

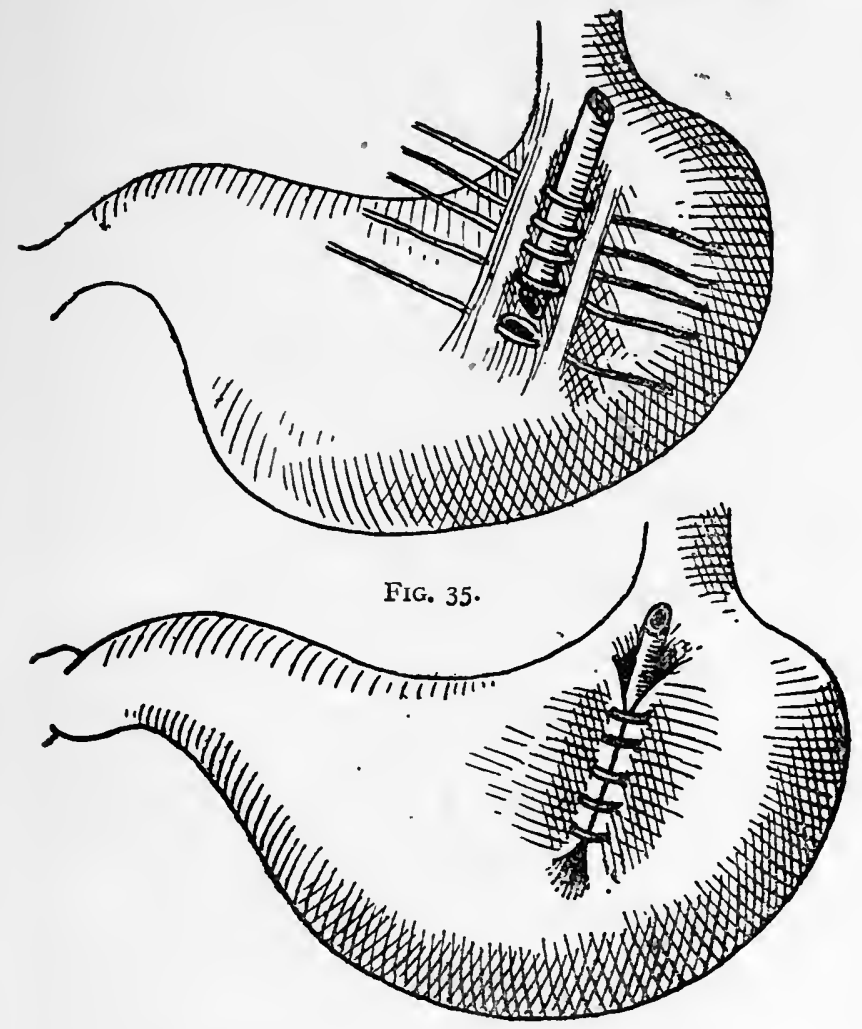

Fig. 36.-Gastrostomy. (Witzel's Method.)

third, are passed and tied in the same manner. The result is that the tube lies in a funnel-shaped inverted portion of the anterior wall of the stomach, and is there fixed by the sutures placed one above the other. The stomach is now fixed to the anterior abdominal wall by a suture above and a suture below the tube, and the abdominal incision is closed in the usual manner. The advantage of this method over Frank's, 
or its modification, lies in the fact that, as the portion of the anterior stomach wall used for the purpose of effecting a valvular action is pushed inwards instead of being dragged outwards, a larger cavity is left for the reception of food, and the area of the gastric mucosa brought into contact with the food is therefore more extensive (Figs. 33 and 34).

3. Witzel's Method (Cent. fïr Chir., r89r).-Fenger's incision, parallel to the costal margin, is made until the rectus muscle is reached. The fibres of the muscle are split vertically, and the peritoneum opened. The stomach is

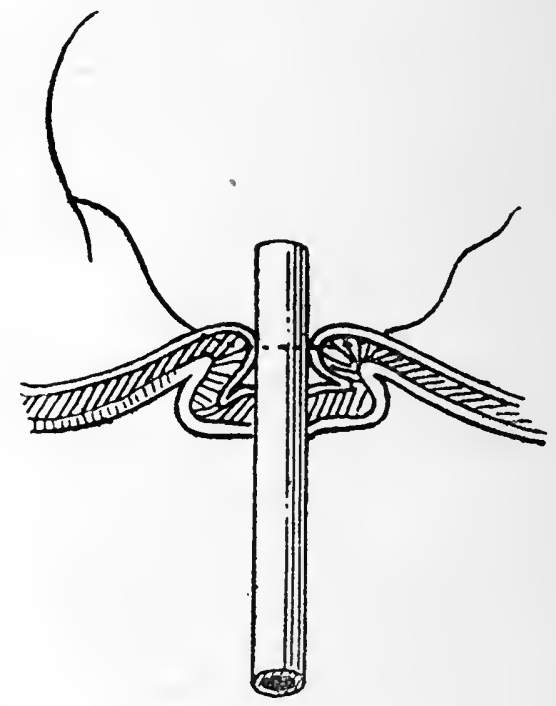

Fig. 37.-Gastrostomy. (Kader's Method.)

exposed and drawn out of the wound; a small incision is made into the stomach, a tube introduced and fixed by a single catgut suture. The tube is then laid upon the stomach wall for a distance of 2 inches or rather more, and a gutter is made for it by raising up a fold on each side and stitching the folds over the tube. The tube then opens into the stomach ' in the same manner as the ureter opens into the bladder.' The stomach is fixed to the abdominal wall by two or three sutures. Mikulicz and Helferich have shown that after the lapse of a few months the oblique passage for 
the tube becomes a direct one, the inner orifice lying behind the outer (Figs. 35 and 36).

4. Kader's Method (Cent. fïr Chir., I896).-The stomach is exposed through Fenger's incision, a cut is made into it, and a tube introduced and fixed by a single catgut stitch. Two parallel folds of the stomach are then raised up, one on each side of the tube, and their summits are sutured by two or three Lembert's sutures above, and the same number below, the tube. The sutures are cut short. Two similar parallel folds are again raised up and again stitched, and, if necessary, a third tier is added. A most efficient valve is
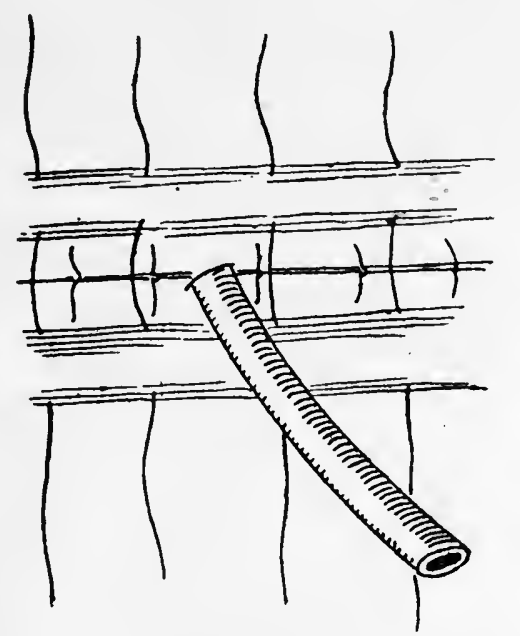

Fig. 38.-Gastrostomy. (Kader's Method.)

thus formed. The stomach is fixed by one or two sutures to the anterior abdominal wall (Figs. 37 and 38 ).

5. A. Depage (Journal de Chirurgie, November, December, Igor; Medical Review, February, I902) devised the following operation, which he successfully performed in a case of cancer of the upper part of the œsophagus when solid food could not be taken: A vertical incision 7 or 8 centimetres long is made a little to the left of the middle line and at the seat of election. After opening the abdominal cavity, a portion of the stomach is drawn out and separated from the 
peritoneal cavity by a continuous suture which unites the wall of the organ to the edge of the peritoneum. A flap

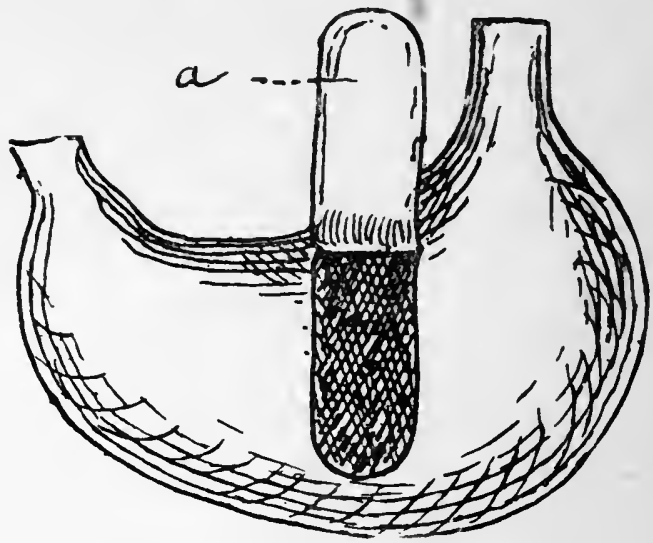

Fig. 39.-Depage's Gastrostomy.

$a$, the flap raised from the anterior wall of the stomach.

with its base upward is then cut out of the anterior wall of the stomach (Fig. 39). This can be easily done by pinching

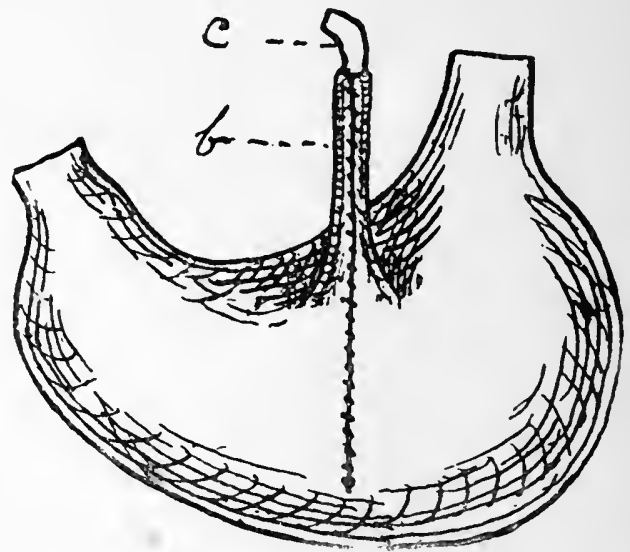

Fig. 40.-Depage's Gastrostomy.

The edges of the incision sutured together, and the flap transformed into a canal $(b)$, through which a sound $(c)$ is passed.

up a piece of the wall between pressure-forceps and cutting along the blades. The flap is then turned upward, and the 
incision in the stomach is closed by a continuous suture, carried first only through the mucous coat. The serous layer is sutured in the same way. Each of the sutures is continued on to the flap, which is in this way transformed into a canal (Fig. 40). The canal thus made is fixed to the abdominal wall, or, if long enough, it may be drawn through a parietal tunnel to near the xiphoid cartilage. The abdominal incision is then sutured, and a sound is introduced into the canal, and may be left there or inserted before each meal.

In all these methods a tube, closed by a clip or a safetypin, should always be kept in the stomach, as the openings so readily contract.

Statistics.-Up to I 896 we had performed gastrostomy on nine occasions, with six deaths; but as many of the patients were almost moribund when operated on, it is not to be wondered at that the mortality was considerable.

Since 1897 we have had our cases earlier, and have always fed them immediately, the first meal being given on the operating-table. This probably accounts for the different results, for out of thirty-four cases operated on, thirty-one have recovered. 


\section{CHAPTER $\mathrm{X}$}

\section{ULCUS CARCINOMATOSUM}

THE question of the cancerous transformation of an ulcer of the stomach was first discussed by Cruveilhier in 1839 . It is to this author that we are indebted for the first description of ulcer of the stomach, and for its differentiation from cancer. He considered it possible that a simple ulcer might show changes due to malignant growth in those of a ' cancerous diathesis.' Rokitansky in 1840 also recognised the difference between ulcer and cancer, and said that the latter might be implanted upon the former. To Dittrich, writing in 1848 , belongs the chief credit for drawing attention to the subject. He described in I6o cases of new growth six cases of cancer developing in the immediate vicinity of active or healed ulcers, two cases of the association of cancer and ulcer, and two cases in which the cancer was limited to a certain part of the margin of the ulcer, the rest remaining sound. Brinton in 1856 recognised the possibility of the grafting of cancer upon long-standing ulcer.

Lebert in 1878 considered that the cancerous transformation occurred in 9 per cent. of ulcers. Zenker in 1882 expressed a strong opinion that all cases of cancer of the stomach were secondary to ulceration. In the same year Hauser, assistant to Zenker, examined minutely a case of malignant growth in the stomach, secondary to ulcer, in a patient who had died of pneumonia, and from whom, or of whom, no clinical history could be obtained. In the following year he devoted his inaugural thesis to a further consideration of this and other cases. He attributed the cancerous degeneration in an ulcer to be due to glandular deformation 
caused by the processes of inflammation and cicatrization exciting and favouring epithelial proliferation. He called attention for the first time to the persistence of free hydrochloric acid in the stomach contents in cases of cancer grafted upon ulcer. Berthold, in $\mathrm{r} 883$, in reviewing the literature and statistics of former writers, computed the frequency of malignant changes in ulcer at 3 per cent. In I 888 Rosenheim made an exhaustive study into the condition of the mucosa in cases of primary carcinoma of the stomach. The mucous membrane was almost always in a condition of atrophy, but in one case of ulcer which had achieved malignancy the mucosa was normal throughout. In 1889 he found in fifty-six cases of cancer, four in which the malignant change was secondary to ulceration. In all of these free hydrochloric acid was present, and he therefore considered this as a sign of the first importance. He wrote: 'In cases where the signs of cancer are undoubted, the persistence of free hydrochloric acid signifies, a priori, that the cancer has developed from an ulcer.' The importance of this persistent presence of free hydrochloric acid was also mentioned by Sticker.

In $\mathbf{1} 889$ Wetzoldt recorded the case of a woman aged forty-three, who had suffered from gastric troubles, pyloric stenosis, and its consequences, since the age of twenty-eight. A gastro-enterostomy proved fatal. At the post-mortem an ulcer of the lesser curvature was found, showing malignant changes at one edge, and malignant implication of the lymphatic vessels and glands. Free hydrochloric acid was present in the stomach contents. In I 898 Duplant, in a Lyons thesis, reviewed the previous records, and gave details of eight cases seen by himself. He considered that there was no proof that the malignant change ever occurred in a round ulcer, and, for reasons which he gave in detail, he was of the opinion that the cases previously observed were, in fact, cases of cancer, in which, owing to thrombosis of the vessels, secondary ulceration had occurred.

Hayem, in I9OI, discusses the whole subject very thoroughly in two papers. He describes a condition of 'prepyloric ulcero-cancer,' in which an ulcer, situated almost invariably 
close to the pylorus, undergoes a cancerous change. The resulting growth is one of exceptional malignity.

G. Fuetterer, in I902, made an extensive research into the question of the origin of carcinoma of the stomach from chronic round ulcer. His conclusions, briefly stated, were as follows :

I. If a carcinoma develops from a chronic ulcer of the stomach, then this development occurs from those parts of the edges of the ulcer which are most exposed to mechanical irritation by the contents of the stomach.

2. In the pyloric region it is the lower pyloric margin of the ulcer which is most exposed to mechanical irritation, and from which carcinoma develops. But other parts of the edges may be the ones involved when dilatations and adhesions have changed the position of the organ.

3. Development of carcinoma from ulcers of the stomach in the pyloric region occurs with great frequency, while such a development occurs less often in other parts of the stomach.

4. If what has been said under the third conclusion is correct, then we must, in all cases in which an ulcer of the stomach or its scar narrows the pylorus, recommend an early gastro-enterostomy, to prevent the development of carcinoma. If a gastro-enterostomy has been made, then the mechanical irritation of the ulcer in the pyloric region by food is reduced, and the severe friction necessary to produce a carcinoma will probably not occur.

In I903 Audistère (from whom many of the foregoing details have been obtained) recorded examples, and made very careful examination of four personal cases. His experience is summed up in the following manner :

I. Simple ulcer of the stomach may be the starting-point of a cancerous growth, a condition of things which appears to be not infrequent.

2. This malignant degeneration affects, as a rule, the chronic ulcers, especially in the prepyloric portion. The change begins in the mucous membrane at the margin of the ulcer.

3. The transformed ulcer presents for a long time almost 
the same symptoms as a simple ulcer; the differential diagnosis is, therefore, very difficult.

4. Nevertheless, the diagnosis can be made by noting the persistence of the symptoms, the resistance to treatment, the wasting, and the progressive anæmia. The pain, as a rule, is more severe than in cases of simple ulcer.

5. In cases of cancer, apparently primary, the origin in an ulcer may be suspected if the pain is unusually severe and paroxysmal, if hyperchlorhydria is pronounced, or if hæmatemesis or perforation occur.

6. The prognosis is decidedly more grave, for the progress of cancer grafted upon an ulcer is more rapid, and bleeding or perforation may occur.

The estimates of the frequency of this malignant implantation upon a chronic ulcer vary greatly. The number of carcinomata beginning in chronic ulcer is reckoned at 3 per cent. by Häberlin, Fenwick, Plange, and Berthold, 4 per cent. by Wollmans, 6 per cent. by Rosenheim and Hauser, 9 per cent. by Lebert, and I4 per cent. by Sonicksen. Zenker, as already mentioned, believes that all, or almost all, carcinomata are secondary to ulcer.

In the majority of recorded cases, the symptoms of ulcer have existed for many years, and they merge by imperceptible degrees into those of cancer. The following case recorded by Fenwick ('Cancer of the Stomach,' p. 218) may be considered a typical example:

'A man, forty-eight years of age, was admitted into hospital under our care in 1887 for a chronic ulcer of the stomach. For more than a year he had complained of pain and sickness after meals, and on two occasions had vomited a large quantity of blood. Under treatment these symptoms eventually subsided, and he apparently became cured. In I 890 he again sought admission into hospital for severe indigestion. According to his statement, he had been perfectly free from pain for more than a year, when, after a few weeks of ill-health, he began to experience a sense of weight and oppression at the chest after meals, attended by flatulence, nausea, and want of appetite. During the last two months he had lost much flesh, and felt very weak. There had been no vomiting or hæmatemesis. On examination, he was found to be very thin and markedly anæmic The stomach was somewhat 
dilated, and pressure over the region of the pylorus gave rise to pain, but no tumour could be discovered. The temperature was subnormal, the urine healthy, and the blood showed a great reduction in the number of red corpuscles and of hæmoglobin, with slight leucocytosis. After a test meal, the contents of the stomach gave the reaction for free hydrochloric acid, but were free from lactic acid. For two or three weeks a milk diet was attended by improvement, but subsequently the discomfort after meals increased, and vomiting occurred each night. The patient continued to lose flesh and strength, and on two occasions exploration of the stomach with a tube revealed the existence of altered blood in the organ. Free hydrochloric acid disappeared about the fifth month of the disease, but no lactic acid was ever detected. Shortly afterwards he was attacked by pneumonia, to which he succumbed.

'Necropsy. - On the posterior wall of the stomach, about 2 inches from the pylorus, was a chronic ulcer the size of a five-shilling piece. Growing from its lower margin and base was a large, firm growth of grayish-brown colour, which, on microscopical examination, proved to be a spheroidal-celled carcinoma. The lymphatic glands along the lesser curvature and behind the stomach were enlarged, but there were no metastases in the liver or other organs.'

Though we have met with several cases of malignant disease of the stomach in which the clinical history pointed very strongly to the origin of the growth in a chronic ulcer, yet the only instance in which there is undoubted microscopic evidence was the following :

July, rgor, female, aged thirty-eight. At the age of seventeen she had a gastric ulcer; vomiting and hæmatemesis for several weeks. Since then has always had gastric uneasiness. Ten years ago 'an ulcer burst' after a long walk. She suddenly experienced acute pain in epigastrium, and fainted. During the subsequent night she vomited a 'chamber utensil full' of blood, and later 'coffee-grounds' material. Since this attack has never enjoyed a hearty meal. For last few months has lost flesh rapidly; for the last three weeks she has been in bed, and has not been able to take anything but milk-and-water, and has returned most of that. Gastric tetany in limbs. Free hydrochloric present.

Condition found.-Hour-glass stomach, the constriction being nearer the cardiac than the pyloric end. The stomach was adherent over an area about $2 \frac{1}{2}$ inches in diameter to the anterior abdominal wall and liver. On detaching, the stomach was opened. 
The opening was about $\mathrm{I} \frac{1}{4}$ inches in diameter, the edges were over $\frac{1}{2}$ inch thick, and the mucous membrance greatly thickened and pouting. At the point of adhesion the stomach was markedly narrowed. The ulcer and a surrounding mass of stomach was excised, and gastroplasty performed. The pylorus would only admit the passage of a director, and pyloroplasty was therefore performed. The patient seemed to be going on well until the fourth day, when the pulse rapidly failed. The examination of the excised mass showed malignant disease to be present. The lower margin of the ulcer was greatly thickened by heaped-up granulation tissue, which, on microscopic examination, proved to be carcinomatous.

The origin of carcinoma in an ulcer of the stomach is only another instance, added to many of which we have knowledge, of the effect of persisting irritation in establishing malignant changes. The origin of cancer of the tongue or lip in old fissures or ulcers, the almost constant presence of gall-stones in cancer of the gall-bladder and biliary passages, the origin of sarcomata in injured areas, are all instances which may be cited as being analogous. Ulceration of the stomach is chiefly found on the posterior surface and the lesser curvature near the pylorus. Riegel computes that the greater portions of the organ-namely, the fundus, the anterior surface, the greater curvature, and the cardia-are affected by ulcer in only one-fifth of the cases. An examination of the statistics of carcinoma of the stomach shows that these latter areas are affected only in 16 per cent. Carcinoma, therefore, occurs most commonly in those areas in which the ulcers chiefly lie. Whatever the frequency of the malignant change in chronic ulcer may prove to be, the fact of its occurrence should be an additional incentive to the earlier surgical treatment of ulcers which prove rebellious.

The symptoms of ulcus carcinomatosum may vary within a considerable range. In some the symptoms of ulcer may have persisted, with occasional intervals of freedom, for many years-fifteen, twenty, or even more. At the last recurrence the symptoms are unusually severe-anæmia is more pronounced, wasting and cachexia are soon observed, and the disease hastens to its ending. In other cases the symptoms of ulcer-pain, vomiting, hæmatemesis or melæna, 
and so forth-may appear for the first time in patients between thirty-five and forty-five years of age. The course of the disease may seem to be but slowly progressing. There is rarely, if ever, an interval during which improvement occurs. The course is steadily downward. Sometimes, according to Hayem, the symptoms (of a condition he describes as 'ulcero-cancer') may appear for the first time in a patient of forty years of age or over. There are pain, hyperchlorhydria, and often the sudden outbreak of a copious hæmorrhage. The patient, despite all treatment, wastes rapidly, anæmia is found, and within six months in the more acute cases, but always within fifteen months, the patient dies of metastases or hæmorrhage. In rare cases the symptoms from the first may be those of cancer. The ulcer has been latent throughout, and it is only on postmortem examination, or during operation, that it is possible to recognise the disease.

\section{Carcinoma of the Duodenum.}

Carcinomatous ulceration in the duodenum is decidedly uncommon. Schlesinger (Wien. Klin. Wochenschr., I898, No. Io) found that in twenty-five years in Vienna there were 42,000 post-mortems. Of these, 3,583 were cases of malignant disease. Of this latter number, 443 were examples of intestinal malignant disease, and included seven cases of primary malignant disease of the duodenum. In Perry and Shaw's classical paper in the Guy's Hospital Reports there were quoted ten instances of primary malignant disease of the duodenum, four of which were carcinoma.

Carcinoma of the duodenum is generally annular, and therefore produces local constriction, with dilatation behind the point of narrowing. Sarcoma, on the other hand, generally involves a longer segment of the gut, causes softening of the wall, and generally a passive dilatation. As a rule the growth is columnar-celled carcinoma, which may show a transition to a spheroidal-celled type, or may undergo colloid degeneration.

The symptoms resulting from carcinoma in the duodenum 
vary according as the growth is above, involves, or is below the bile papilla. The growth is generally described as ' supra-ampullary,' 'juxta-ampullary,' or 'infra-ampullary,' with reference to the ampulla of Vater. In $4 \mathrm{I}$ cases quoted by Rolleston (Lancet, vol. i., I90I), 8 occurred in the first portion of the duodenum, 5 in the first and second, 24 in the second, and 4 in the third part. Of these $4 \mathrm{I}$ patients ro were females, $3 \mathrm{I}$ males. The average age of the females was $53^{\circ} 4$ years, of the males $5^{\circ} 7$ years.

Supra-ampullary carcinoma gives rise to symptoms precisely similar to those resulting from malignant obstruction at the pylorus. When occurring in the first part of the duodenum the growth may be implanted upon an oldstanding ulcer (ulcus carcinomatosum). The infrequency of the extension of a pyloric growth along the duodenum has been frequently remarked.

Juxta-ampullary (peri-ampullary) carcinoma is the most frequent form. Rolleston says: 'Carcinoma has a special tendency to arise in the duodenal mucous membrane covering the biliary papilla.' Possibly this is due to some fotal displacement or irregular inclusion of the epithelial cells during the development of the hepatic diverticula, or the pancreatic, from the duodenum. The symptoms are jaundice and dilatation of the gall-bladder. The jaundice comes on gradually, varies in intensity from time to time, may even temporarily disappear, but on the whole becomes steadily deeper. In 25 cases related by Mathieu jaundice was present in 23. The jaundice seen in carcinoma of the head of the pancreas appears gradually, but increases persistently, and becomes permanent. The growth may ulcerate, and thereby causes septic processes to arise in and near it. An acute septic ascending cholangitis results, is followed by intrahepatic suppuration, and may speedily produce a fatal result.

Infra-ampullary carcinoma gives rise to narrowing of the gut below the ampulla of Vater. The symptoms are those of pyloric obstruction, with the important difference that bile and pancreatic juice are always present in the vomit. According to Rolleston, 'the occurrence of bile should at 
once suggest carcinoma of the duodenum below the biliary papilla, or a gastro-biliary fistula. In such case the vomit should be tested for trypsin by seeing whether fibrin is digested in an alkaline solution. In this way the diagnosis between the two conditions (pyloric obstruction and infraampullary stenosis) might be made.' 


\section{CHAPTER XI}

\section{SARCOMA OF THE STOMACH}

SARCOMA of the stomach may be primary or secondary. The former is the more frequent, and alone is of interest to the surgeon. Primary sarcoma was formerly considered an extremely rare disease-a pathological curiosity, in fact; but recent observation makes it probable that not a few cases of so-called 'cancer' are in reality examples of true sarcoma. Perry and Shaw, in their well-known paper (Guy's Hospital Reports, I892), found that four of fifty museum specimens of malignant disease of this organ were sarcomata. Schlesinger (Zeitsch. f. Klin. Med., I897) collected the records of thirty cases. Fenwick, in November, 1900 (Lancet), makes mention of sixty, and of these fifty-three were recorded at sufficient length to permit of analysis and classification.

The following pathological varieties are recognised:

I. Round-celled Sarcoma ('Lympho-sarcoma').-Thirty-three of the fifty-three are of this form. The tumour is generally found in the pyloric portion of the stomach, giving rise to a considerable local thickening and induration, which shades off into tough bands along the curvatures. The growth may project boldly upon the surface, or may form a solid stiff plate in the thickness of the stomach wall. The pyloric orifice is not narrowed, but as a rule, according to Fenwick, the rigidity of its tissues renders it patent, and the valve incompetent rather than contracted. The disease commences in the submuicosa, and extends to the muscular coat; the mucosa is stretched by the growth, thinned, and at times ulcerated. It has been shown (Redtenbacher, Jahrbuch der Weiner Krankenaustalten, 1894) that a diffuse infiltration of 
round cells in the mucosa extends some distance beyond the obvious limits of the disease.

The following are typical cases recorded in the literature :

Hadden (Pathological Society's Transactions, vol. xxxvii., I886) describes a case of lympho-sarcoma of the stomach. There was 'a globular tumour in the anterior wall of the stomach, close to the lesser curvature, rather nearer the pyloric orifice than the cardiac orifice. On the inner surface of the stomach, corresponding to the mass seen externally, a triangular ulcer, $\mathrm{r}_{4} \frac{3}{4}$ inches in its largest measurement, was found. The ulcer led by a free opening into the centre of the tumour for a distance of $I_{2} \frac{1}{2}$ inches. This excavation was roughly globular, and its external surface fairly smooth. On microscopic examination, the tumour was found to be composed of small round nucleated cells contained in a reticular stroma. In fact, the growth seemed to be a lympho-sarcoma, but its peculiarities consisted in a papillary or alveolar arrangement; and in the centre of most of these alveoli a small vessel could be seen.' This case illustrates the tendency of these growths to soften, ulcerate, and break down.

Schopf (Cent. f. Chir., I899, p. I I63).-In this female patient there was a large movable tumour of the size of a child's head in the abdomen. The abdomen was opened, numerous glands found in the small and large omenta, and a tumour occupying the greater part of the stomach. The stomach was removed two fingers' breadth from the cardia, and one from the pylorus, and the cui ends of viscus stitched together. The tumour was seen on examination to occupy the lower two-thirds of the portion of stomach removed, was hard and nodular, and microscopically was lympho-sarcoma. The patient recovered from the operation, and was well without recurrence twelve months later.

Finlayson (British Medical Journal, vol. ii., I899, p. I535) records an example of round-celled sarcoma in a boy three and a half years old. The tumour was not diagnosed during life. The chief symptom was a profound anæmia. There was a slight but continuous pyrexia. The child sank gradually, and died, the growth being found post-mortem.

2. Spindle-celled Sarcoma. - Twelve of the fifty-three are of 
this variety. This form of growth is generally seen as a circumscribed tumour near the greater curvature of the stomach; it is often pedunculated, and when growing on the posterior surface hangs in, and by its enlarging may obliterate, the lesser sac of the peritoneum. The larger the mass formed, the softer, as a rule, is the tissue composing it. Cystic or gelatinous degeneration is not seldom observed. The following are examples:

Billroth successfully removed a cystic sarcoma attached to the greater curvature and the contiguous portions of the anterior and posterior walls.

Hartley (Annals of Surgery, vol. xxiii., I896, p. 609).-The tumour occurred in a woman fifty-four years of age. Except for long-standing dyspepsia, she had been in perfect health until five years before. At that time she, without apparent cause, vomited a large amount of 'coffee-ground' material. For four years she remained without symptoms, then had another attack of vomiting of coffee-ground material and blood and clots. Pain in the back was now complained of, and a tumour was noticed in the epigastrium. On physical examination, there was felt in the left lumbar and umbilical regions a rounded tumour, hard, elastic, freely movable. A tentative diagnosis of movable kidney was made, but was, when the patient was examined under ether, considered doubtful. An incision was made in the left linea semilunaris, and the tumour was felt behind the transverse mesocolon in the left splenic region. The mesocolon was torn through, and the tumour exposed, lying in the lesser sac. The growth was seen to spring from the posterior wall of the stomach by a pedicle $I_{2} \frac{1}{2}$ inches in diameter. The stomach was pulled well forwards, grasped below the pedicle; its wall was cut through entirely around the pedicle, removing a portion of its wall with the tumour. The stomach opening was closed. The pathological report was: 'Pear-shaped tumour I $_{5} \times$ Io $\times 8$ centimetres. Its surface is smooth, but nodular. The tumour is cystic. An area of stomach wall $5 \times 4$ centimetres has been removed with the tumour. Sections of the tumour show the typical appearance of spindle-celled sarcoma.'

Cantwell (Annals of Surgery, vol. ii., I899, p. 596).-A female 
patient, aged fifty-two, complained of a large abdominal tumour presenting no signs or symptoms save those of weight and pressure. On opening the abdomen, an immense mass, covered by omentum and mesentery, presented. An opening was made through its envelopes down to the tumour proper, which was so soft as to verge on the gelatinous; from its whole surface there oozed bloody serum. It was not until the tumour was carefully enucleated and lifted from its bed that it was seen to arise from the posterior wall of the stomach down to its greater curvature. The weight had dragged the stomach down to a point several inches lower than its ordinary position. The growth with part of the stomach was cut away with scissors, and the wound closed with two tiers of sutures. On the tenth day a parotitis occurred, but subsided. The tumour weighed I 2 pounds, and 5 inches square of the mucous membrane of the stomach had to be removed. Recurrence was noticed eight months after operation.

3 and 4. Myosarcoma (five cases of the fifty-three) and Angiosarcoma (two of the fifty-three) are unusual varieties. The former are characterized by a remarkable tendency to extravagant hæmorrhage, which imperils the patient's life. An example of the latter was successfully removed by Kosinski (Deutsch. Gesellsch. f. Chir., I892).

All these varieties exhibit a marked proneness to the formation of metastatic deposits in the skin or elsewhere. A small nodule has been on several occasions noticed at the umbilicus. An example of melanotic sarcoma, with deposits in the skin, is preserved in the Hunterian Museum at the Royal College of Surgeons ; the visceral deposit is probably secondary.

\section{Symptoms.}

The symptoms of sarcoma very closely resemble those of carcinoma. One of the earliest and most striking features is a progressive emaciation with failure of physical power. Anæmia is almost constant, and is often profound, suggesting a diagnosis of 'pernicious anæmia.' There is often a trivial but continuous pyrexia, and persisting albuminuria is not 
seldom observed. In some cases-those, for example, of Baldy and Cantwell-symptoms were almost or entirely lacking. A tumour may be, but often is not, felt. Dilatation of the stomach and vomiting are infrequent. When vomiting is present, blood, either fresh or altered, is constantly noticed, and the blood may be found on careful examination of the stools. Kundrat places reliance as a diagnostic sign upon ' enlargement of the tonsils with occasional swelling and ulceration of the follicles of the tongue.' The chemical findings are similar to those in carcinoma. The $\mathrm{HCl}$ is absent from an early stage. Lactic acid and the OpplerBoas bacilli are found (Schlesinger, Zeit. f. Klin. Med., vol. xxxii.).

A study of recorded cases shows that small round-celled sarcoma is generally diagnosed as carcinoma of the stomach, whereas spindle-celled growths are only recognised at the time of operation as having their origin in that viscus. 


\section{CHAPTER XII}

\section{GASTRIC ULCER}

\section{Pathology and Diagnosis.}

Although in its earlier phases gastric ulcer comes under the care of the physician, the later stages, as well as many of the complications to which both acute and chronic ulcers give rise, demand surgical intervention, and, in fact, can be treated satisfactorily only by surgical means. This being so, it is necessary that we should consider the subject generally, before taking particular note of those conditions which specially call for surgical intervention.

For the present, passing over malignant, tubercular, and syphilitic ulcers, the special form of ulceration which produces the complications calling for surgical treatment is the simple ulcer of Cruveilhier, and to this may be added the simple erosion of the mucous membrane described by Dieulafoy, which at times leads to serious, or even fatal, hæmorrhage.

Although for clinical purposes it is necessary to distinguish two distinct forms of ulceration of the stomach, acute and chronic, which appear to be separate and characteristic, there are so many intermediate stages that it is questionable whether or no these classes are so distinct as their extreme varieties would seem to indicate.

In the classification of ordinary ulcer of the stomach, the following varieties seem to us to include the various forms :

\section{Erosions.}

Of these Dieulafoy has described two varieties :

(a) Simple Erosions, consisting apparently of mere abrasions 160 


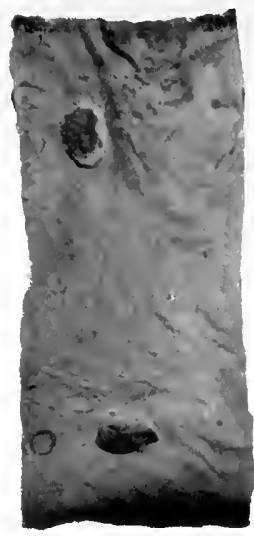

Fig. 4r.-Multiple Round Ulcers.

(No. 2,400, Royal College of Surgeons' Museum.)

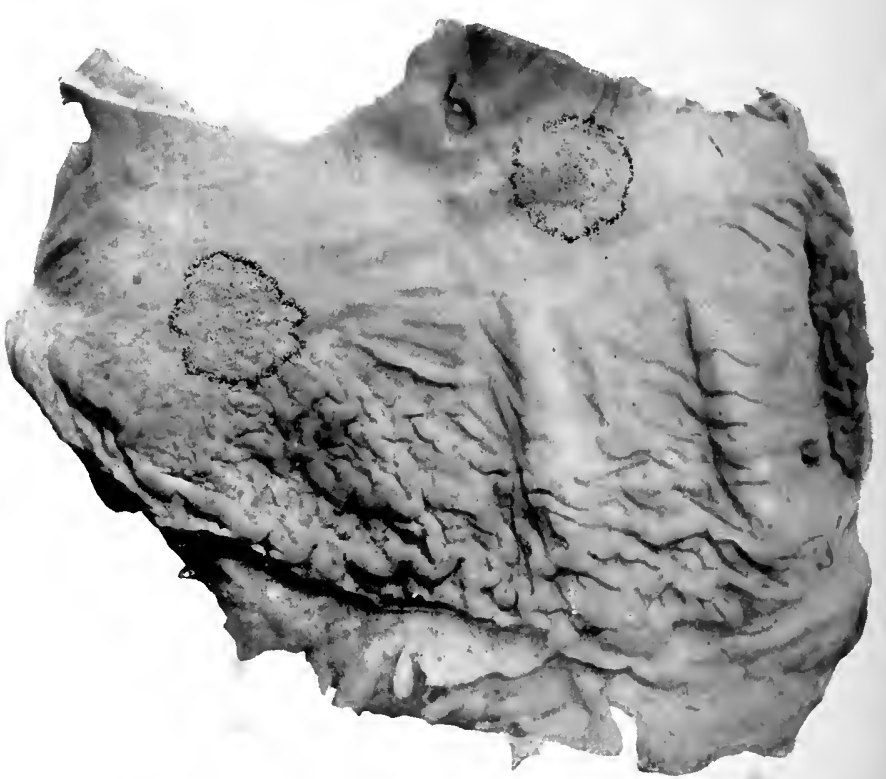

F1G. 42.-Acute Round Ulcers in the Stomach of a Woman aged TWENTY.

Death from perforation in forty hours. The perforation is very small, on the anterior wall close to the middle of the lesser curvature, and there is another ulcer nearer the cardiac orifice. (No, 2,395a, Royal College of Surgeons' Museum.)

To face p. I6r.] 


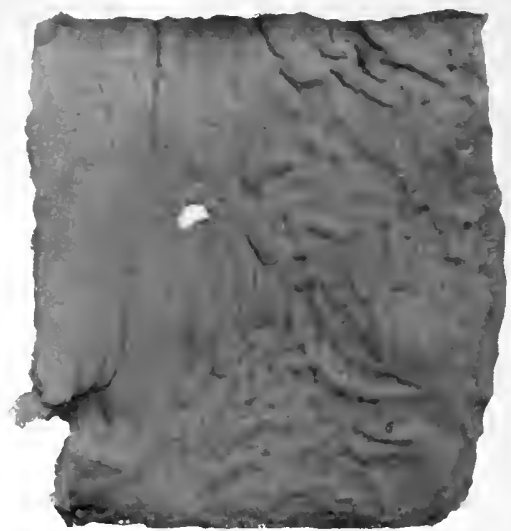

Fig. 43--'Simple Chronic Ulcer' of Cruveilhier, or the 'PerFORATING ULCER' OF ROKITANSKY.

From a girl of seventeen. The ulcer is situated on the anterior wall of the stomach 2 inches from the cardiac orifice. (No. 2,395, Royal College of Surgeons' Museum.)

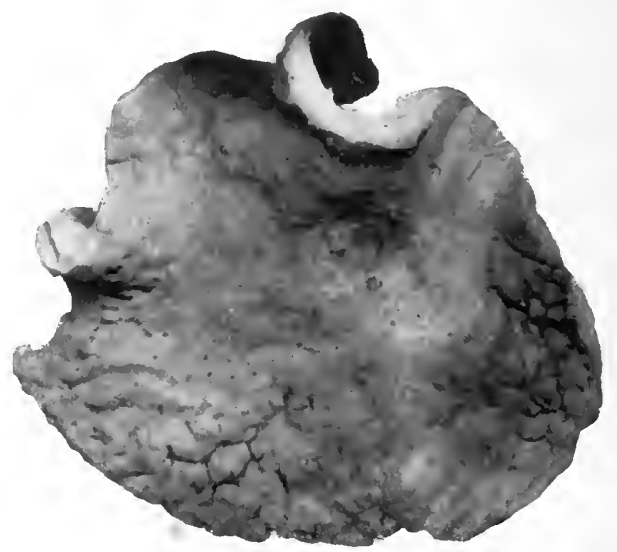

Fig. 44.-A Small, Universally Ulcerated Stomach, 5 Inches Long, 6 Inches in Widest Circumference, with the Coats Three or Four LINES THICK.

From an old gentleman of seventy, accustomed to take up to 3 ii of Colchicum wine for gout. Although he abstained for nine months before death, he had incessant pain and vomiting.

To face $p$. I6r.] 
of the surface epithelium, which, though so small as to be scarcely perceptible to the naked eye, may give rise to most alarming hæmorrhage. On the post-mortem table abrasions of this kind may be easily overlooked; but as seen when hæmorrhage is going on, the mucous membrane seems to be studded with numerous bleeding-points.

(b) Exulceratio Simplex.-In the form to which Dieulafoy applied this term the lesions are rather more extenisive, and the surface layers are removed to such an extent that the arterioles running under the muscularis mucosæ are exposed. This form of ulceration may give rise to terrible hæmorrhages that may prove rapidly fatal unless arrested by treatment.

\section{Simple Ulcer.}

The second form, described by Cruveilhier, includes:

(a) The Acute Round Ulcer, which is most frequently found in women, often in chlorotic young women, and which is apt to be complicated by profuse hæmorrhage and by perforation.

(b) The Chronic Form, irregular in outline, associated with thickening of the edges, and frequently found in menaccording to Dr. Seymour Taylor, in the proportion of 72 per cent. in males to 28 per cent. in females. This form is one which gives rise to the greater number of complications with which the surgeon has to deal.

According to our experience in the operating theatre, this chronic form of ulcer occurs very frequently in women, and, in fact, we have operated on quite as many cases in the female as in the male. Probably there is no hard-and-fast line of demarcation between acute and chronic ulcers, just as it is difficult to define the boundary between simple erosions and the 'exulceratio simplex' of Dieulafoy. Notwithstanding that there has been much investigation into the subject, and that many experiments have been made, with the view of elucidating the pathology of gastric ulceration, the question can scarcely be held as settled, nor can any of the theories at present put forward be supposed to account for all cases. It is not our intention to discuss, or even to state, all the theories supported by the different 
authorities; but we shall mention one or two which seem to afford a likely explanation for a large proportion of cases.

It is well known that in many apparently functional disturbances of the stomach slight abrasions of the mucous membrane occur, which heal readily enough under ordinary circumstances. Many experimenters have produced similar abrasions in animals, and have found them to heal equally rapidly by proliferation from the epithelial margins of the sores so formed. Quincke and Daettwyler (Correspondenzblatt für Schweitzer Aerzte, 1875, p. ror), in addition to injuring the mucous membrane of the stomach, rendered the animals experimented on anæmic by bleeding them, and then found that the abrasions did not spontaneously heal, but continued to form distinct ulcers, and in some cases even went on to perforation. Another experimenter (Silbermann, Deutsche Med. Woch., I886, No. 29) produced hæmoglobinuria, and found a similar, though less marked, result follow the production of abrasions of the gastric mucous membrane. It is possible that these experiments explain the frequency with which simple ulcer of the stomach is found in anæmic young women, while the latter series may account for those obscure cases of perforating ulcer of the duodenum following on extensive burns, since it is well known that in a fair proportion of cases of burning there is extensive destruction of red blood corpuscles, as shown by the existence of hæmoglobinuria. More recently' the recognition of the fact that in a very large proportion of all cases of gastic ulcer the gastric juice is hyperacid has led to a belief that this is the chief factor in the production of the lesion. It is quite possible that the hyperacidity may, in the presence of other conditions, determine the production of ulceration; but since in many other morbid conditions of the stomach the gastric juice may have an excess of hydrochloric acid, and yet no ulceration be induced, it can only be considered as one of the contributing causes. Moreover, well-marked cases of gastric ulceration have been put on record in which there was not only a deficiency, but even an entire absence, of hydrochloric acid, and there has 
been no evidence brought forward to show that the hyperacidity is not an effect rather than the cause of the ulceration.

None of the other theories advanced have, so far as we know, any. strong basis or support in clinical observations, but seem to be founded on the production of conditions in the laboratory which occur very infrequently, if at all, in man. Notably is this the case with the theory that ulcers mostly arise in connection with the results of embolism of the smaller arteries in the stomach ; for even if solid particles be injected into the general blood-stream in animals, the stomach is one of the organs in which they are least commonly found.

Another attempt to settle this problem has been made recently by Dr. Van Ijzeren, a Dutch physician, who claims to have produced ulcers of the stomach presenting all the characteristics which they have in man-that is to say, unity, chronicity, and localization near the pylorus. These results are said to follow division of the pneumogastric nerves below the diaphragm in rabbits. Slight paresis was followed by more or less permanent contracture of the muscular wall of the stomach, and at the end of two or three weeks in about half the cases an ulcer formed which was generally single, showed no tendency to heal, and occupied the pyloric region in the neighbourhood of the lesser curvature. An examination of the stomach contents after death did not show the presence of any notable or constant hyperacidity. By incising the muscular layer in a direction parallel to the axis of the pylorus the formation of an ulcer could as a rule be prevented (eleven times out of twelve), and the operation of gastro-enterostomy was also successful as a prophylactic, but less constantly. Both these operations caused the contracture of the stomach to disappear to a great extent. Dr. Van Ijzeren therefore attributes the production of the ulcer to the muscular spasm, which he believes acts by obliterating the vessels, thereby causing necrosis of the mucous membrane. He regards the spasm as a reflex produced by the passage of food through the duodenum and thence into the intestine, of which he thinks an indirect 
proof is afforded by the good results of gastro-enterostomy. The anomaly of contracture following division of a peripheral nerve is explained by the fact that the nerve fibres going to the gastric musculosa pass through ganglionic cells, so that the vagus nerve plays the part of a secondary neuron. It is, of course, true that gastro-pyloric spasm has often been observed in the subjects of gastric ulcer. The author recommends the operation which in his experience answered best in preventing the occurrence of his experimental ulcers -namely, an incision parallel to the pyloric axis through the muscular fibres of the pylorus, stopping at the muscularis mucosæ.

The subject has again been taken up by Dr. R. Dalla Vedova, of Rome, who has endeavoured to determine, by means of resection of the vagi and of the solar plexus (from which sources the stomach receives its nervous supply), or by the injection into these structures of an aseptic fluid such as concentrated alcohol, the effects of the suppression of nervous influence upon the nutrition of the stomach. He found that about half of all the animals operated on exhibited distinct changes in the gastric mucous membrane. This effect was not due to the toxic action of the alcohol causing acute septic peritonitis without any characteristic microscopic inflammatory changes, for no less than two-thirds of the animals survived for ten days; nor was it due to exposure of the intestines to cold or to any mechanical injury to the parts, since these were not, and need not be, touched in extirpation of the coeliac plexus. Dr. Dalla Vedova considers himself to be justified, from a careful consideration of all the circumstances, in drawing the conclusion that the necrotic ulcers of the gastric walls observed in his experiments were directly due to the lesions inflicted on the abdominal sympathetic. The ulcerative processes were noticed to affect chiefly the antrum pylori, and many drawings accompany Dr. Dalla Vedova's article showing the position of the ulcers, hæmorrhages, spots of necrosis, and cicatrices of ulcers that were seen after the death of the animals. This position of the ulcers he attributes in part to the more highly acid reaction of this segment of the stomach and in part to the 
constant irritation of the several tunics debilitated by the loss of their nervous supply owing to the passage over them of the more solid parts of chyme in the later stages of digestion.

Our own view is that gastric ulcers are for the most part septic in origin, and that oral sepsis is responsible for a large number of cases; hence the greater frequency of the disease in the poorer classes, who give little or no attention to the teeth.

Mild sepsis leads to gastritis, then to hyperchlorhydria, which in its turn provokes and keeps up ulceration.

The acute simple ulcer differs very materially in shape and general appearance from the chronic ulcer. As a rule, in the acute variety the ulcer is small, round, or oval, and has the appearance of being punched out; its walls are not-at least, post-mortem-infiltrated to any marked extent, and the base, though smooth, does not usually show much evidence of granulations, the submucous, muscular, or serous coat being laid bare. The general shape of the ulcer is that of a truncated cone, with its base towards the interior of the stomach, the layers from within outwards being successively less destroyed. This has been supposed to give great support to the theory that the acute ulcer takes origin from some interference with the blood-supply; but Dr. Soltau Fenwick has shown that, even if all the coats of the stomach are equally destroyed by cautery, the ulcer ultimately assumes the usual funnel shape. Up to the margin of the ulcer the mucous membrane is normal in all respects and quite supple. On the other hand, the chronic ulcer is not infrequently large, and is usually irregular in shape; the margins are hard, indurated, and infiltrated, and the base shows evidences of cicatricial processes. Much more frequently also in this variety the peritoneal coat is thickened, and adhesions to neighbouring viscera, especially the liver and pancreas, form, and it is not uncommon for the base to be formed by the tissues of one of these organs. In chronic ulcer the long axis is, as a rule, transversely placed, but this is by no means invariable. The mucous membrane for some space round the ulcerated area is thickened and infiltrated, and according 
to Fenwick ('Ulcer of the Stomach and Duodenum,' London, rgoo) there is not infrequently varicosity of the small venules in the immediate neighbourhood.

Acute ulcer is much more frequently multiple than is the chronic ulcer-probably half the cases of acute ulceration present more than one-while in the case of chronic ulceration it is comparatively rare to find multiple ulcers, though at times the shape of that found is suggestive of the coalescence of several ulcerated areas.

The different regions of the stomach are very variously affected in this disease. According to Brinton, in 43 cases out of roo the ulcer is situated on the posterior surface, in 27 at the lesser curvature, in 16 at the pylorus, in 6 on both anterior and posterior surfaces, in 4 on the anterior surface alone, in 2 at the cardiac end, and in 2 on the greater curvature.

Welch gives 793 cases distributed as follows : 235 on the posterior surface, 288 on the lesser curvature, 95 at the pylorus, 96 on the anterior wall, 50 at the cardia, 29 at the fundus, and 27 on the greater curvature.

Fenwick has collected I,OI 5 cases, and gives the following table showing the relative frequency of the disease in different regions of the stomach :

$\begin{array}{lllccc}\text { Pylorus } \ldots & & & \text { Cases. } & \text { Per Cent. } \\ \text { Lesser curvature } & \ldots & \ldots & \ldots & 366 & \text { 1 } 58 \\ \text { Posterior surface } & \ldots & \ldots & 254 & 36 \\ \text { Cardia } \quad \ldots & \ldots & \ldots & 80 & 25 \\ \text { Great curvature } & \ldots & \ldots & 42 & 7 \cdot 9 \\ \text { Anterior surface } & \ldots & \ldots & 82 & 4 \cdot 14 \\ \text { Fundus } \ldots & \ldots & \ldots & 33 & 8 \\ & & & & & 3 \cdot 3\end{array}$

It is noteworthy, however, that, of his own cases, the chronic and acute ulcers (which are not distinguished in the above table) markedly differ in their distribution. Of Iog cases, 70 chronic ulcers were distributed thus : 53 in the pyloric region, 7 in the middle zone of the stomach, and ro towards the cardiac end; the remaining 39, the acute ulcers, had the following distribution: I3 in the pyloric, 14 in the middle, and 12 in the cardiac region. Thus, 
75 per cent. of the chronic ulcers were in the pyloric region, while in acute ulceration all three divisions were fairly equally affected.

This distribution of ulcers generally, and especially of the chronic variety, has, as will be seen later, a most important bearing on the question of surgical intervention in cases with a long-continued history of gastric trouble.

The questions of the age and sex of the patient are of great importance in helping to a decision as to the character, and therefore the site, of the lesion, and require attention here.

Judging from clinical experience rather than from postmortem records, it would appear that ulceration of the stomach occurs about three times as frequently in women as in men. On the other hand, pathological reports would lead one to suppose that gastric ulcers were much less disproportionately distributed between the sexes; thus, Welch gives the relative proportions as 40 per cent. in men and 60 per cent. in women, while out of 2,03 I cases of open ulcer collected by Fenwick, I,227 occurred in women and 804 in men. This apparent discrepancy is probably to be accounted for by the fact that women are more liable to suffer from the acute affection, while the chronic process occurs more frequently in men, and is more often fatal.

That this is the true explanation cannot at present be definitely stated, since in most collected cases no distinction is made between acute and chronic ulcers; but an examination of 89 cases made by Fenwick points so strongly in this direction that there can be little doubt but that this view will ultimately be established. Of his 89 cases, 30 were acute, and only ro per cent. occurred in men, while 59 were chronic ulcers, and of these almost 73 per cent. were in men. Dr. Seymour Taylor gives almost identical figures in chronic ulcer, viz., 72 per cent. in men and 28 per cent. in . women.

With regard to age incidence, all authorities are agreed that gastric ulcer is much more frequently found in the third decade ; but here also it should be noted that according 
to the sex the age incidence varies, for while 75 per cent. of cases of gastric ulcer in women are found before the age of thirty, in men only about 25 per cent. occur before that age. It is notable also that, in the statistics referred to above, in which a distinction is made between acute and chronic ulcers, it was found that of the acute ulcers over 70 per cent. occurred within the first thirty years of life, while in chronic ulceration less than 7 per cent. occurred within that period.

Though it may be granted that the numbers with which this analysis deals are too few to enable one to dogmatize, yet the variations are too great to be accounted for on the supposition of mere coincidence.

It seems, therefore, fair to conclude that chronic ulceration is much more frequently present in men, and is proportionately much more fatal in its effects than is acute ulceration of the stomach; and that the former is a disease of middle or advanced age, as contrasted with the latter, which, as a rule, occurs within the first three decades. Along with this, however, must go the qualification that chronic ulcer is often a very slowly progressive affection, and that it may last for over thirty years.

Although the following complications are common to both forms of ulcer, certain of them are of greater frequency in the one than in the other. For instance, the acute round ulcer is more frequently followed by violent hæmorrhage and by perforation than the chronic ulcer, yet the latter is also liable to be followed by both. On the other hand, the chronic ulcer is more frequently followed by cicatricial contraction of the pylorus, with subsequent dilatation of the stomach, and by perigastritis leading to disabling adhesions; also by hour-glass contraction, fistula, and tumour of the pylorus or of the stomach, serious atonic motor deficiency, severe gastralgia with uncontrollable vomiting, cancer of the pylorus, tetany, acute and chronic pancreatitis, abscess of the liver, chronic hepatitis, profound anæmia (resembling the pernicious form), stricture of the bile-ducts, jaundice, catarrh of the gall-bladder, and other complications depending on invasion of the neighbouring organs. Both forms are accom- 


\section{PLATE XVI.}

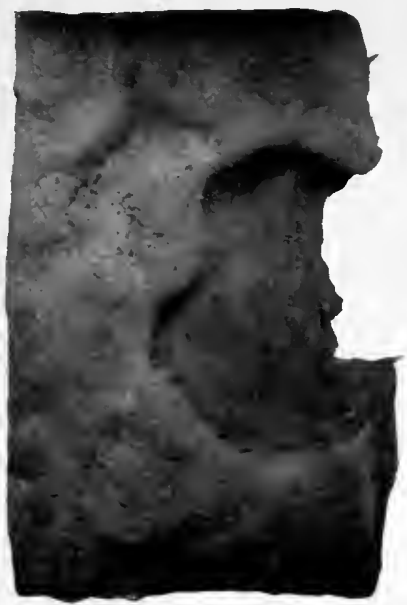

Fig. 45-Chronic Ulcer of Posterior Wall of Stomach eroding Pancreas.

(No. 2,399, Royal College of Surgeons' Museum.)

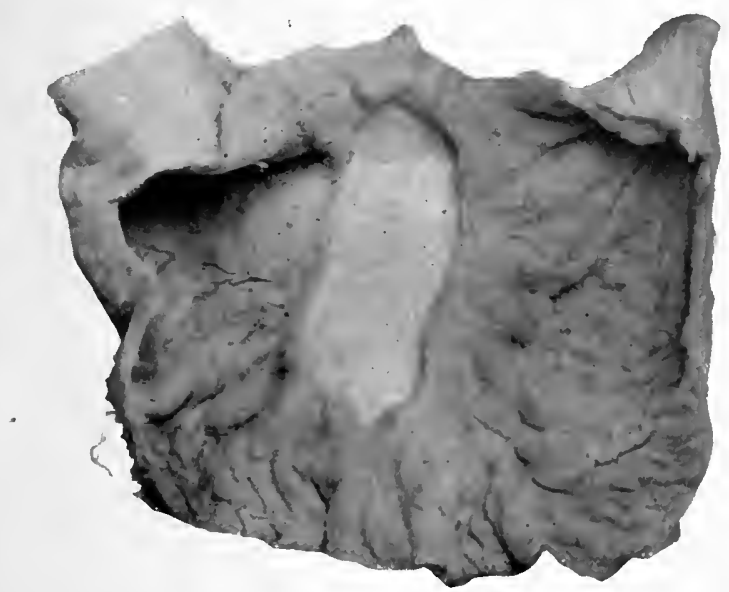

Fig. 46.-Large Chronic Ulcer, $3 \frac{1}{2}$ BY i 19 Inches, on the Posterior Wall, adherent to Pancreas and Liver.

(No. 2,402, Royal Colleze of Surgeons' Museum.)

To face p. 168.] 


\section{PLATE XVII.}

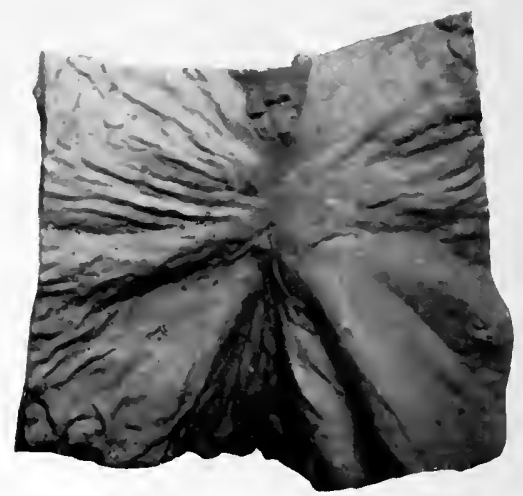

Fig. 47--Chronic Ulcer of Stomach showing Characteristic Puckering and Contraction the Resclt of Healing.

(No. 2,402a, Royal College of Surgeons' Museum.)

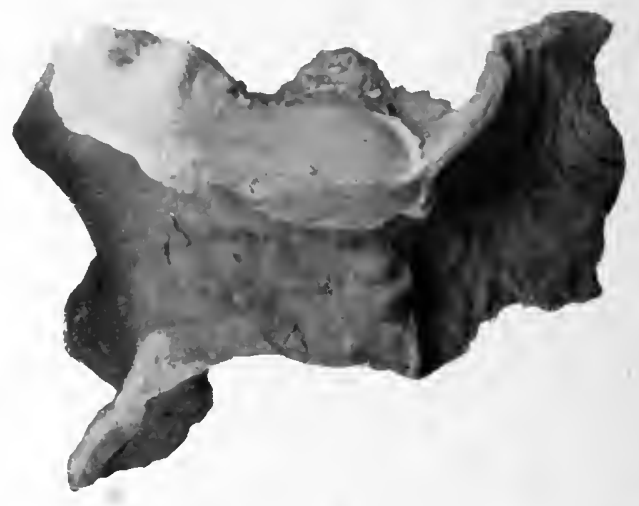

Fig. 48.- Pyloric Stenosis from Ulcer with Hy'pertrophy of Stomach.

(No. 2,4 15, Royal College of Surgeons' Museum.)

To face p. I69.] 
panied by pain, by great loss of flesh and strength, and at times by subphrenic abscess.

Thus, it will be at once perceived that surgery is intimately concerned with, and, in fact, affords the only effectual treatment in, many of these complications.

\section{Prognosis.}

According to Lebert, as quoted by Dr. Dreschfield, death occurs in Io per cent. of all ulcers, $6 \frac{1}{2}$ per cent. occurring from perforation, and $3 \frac{1}{2}$ per cent. from hæmorrhage. Habershon says that perforation occurs in 18 per cent. of all cases of ulcer of the stomach, and Brinton in I5 per cent., to which must be added the mortality from hæmorrhage, which, according to Müller, is II per cent. of all cases of ulcer, the average of all authorities being 5 per cent. ; so that, allowing $5_{5}$ per cent. to represent the mortality from perforation and 5 per cent. that from hæmorrhage, the mortality of gastric ulcer treated medically is at least 20 per cent. But Tricomi (Riforma Medica, February, I899) gives even a larger mortality as an argument in support of his views to treat all obstinate cases of gastric ulcer surgically; and Brinton gives the mortality from all causes in gastric ulcer as 50 per cent.

Leube says that one-half or three-fourths of all cases will be cured by four or five weeks of treatment, but if not cured in that time, they will not be cured by medical treatment alone.

It is probable, however, that none of the series of statistics which have been made adequately or accurately gives the mortality, since practically in none is any account taken of the large number of deaths due to the effects of those complications which run a chronic course. This underestimate is the more likely to occur, since naturally the statistics are usually compiled from the records of general hospitals; and it is unlikely that anything like a large proportion of cases, such as we refer to, would die in hospital. It is probable, therefore, that 20 per cent. is under, rather than above, the true mortality; and it is quite possible that the following table (quoted from Einhorn, 'Diseases of the Stomach'), 
cited from Debove and Rémond, adequately represents the truth :

100 cases :

$\begin{array}{llllll}\text { Perfect cure } \ldots & \ldots & \ldots & \ldots & \ldots & \text { 50 }\end{array}$

Perforation and peritonitis $\ldots . \quad \ldots \quad \ldots \quad$ I3

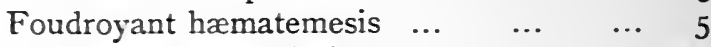

Pulmonary tuberculosis $\quad \ldots \quad \ldots \quad \ldots \quad \ldots \quad 20$

$\begin{array}{lllllll}\text { Inanition } & \ldots & \ldots & \ldots & \ldots & \ldots & 5\end{array}$

Different complications $\quad \ldots \quad \ldots \quad \ldots \quad 7$

The most recent statistics are those furnished by Dr. Bulstrode, and given in a paper by Mr. Mansell Moullin (Brit. Med. Journ., April 25, I903).

- Dr. Bulstrode collected and analyzed the statistics of all the cases admitted into the London Hospital for gastric ulcer from the beginning of 1897 down to August, I902. They were just 500 in number, 98 men and 402 women. Out of this number, no less than $+S$ (nearly Io per cent.) died from peritonitis; 13 (or $2^{\circ} j$ per cent.) from hæmatemesis; and $28(5.5$ per cent.) from other causes. The total number of deaths was 89 , or approximately I 8 per cent., taking all the cases together. Had all the slighter ones been left out, and only those reckoned in which the gravity of the symptoms suggested the possibility of operation being advisable, the rate of mortality, bad as it is, would have been far higher.

'The question of the actual rate of mortality, however, is not the only one that requires to be considered; $\$ 2$ per cent. of the cases of gastric ulcer admitted into the London Hospital were discharged as relieved or cured. It would be exceedingly interesting to ascertain what was the relative proportion of these two classes: how many were really cured; that is to say, left the hospital perfectly well, without suffering pain or inconvenience when taking ordinary diet, and, what is especially important, remained well. Such a thing, unfortunately, is not possible. Some idea can, however, be formed by ascertaining the number of patients admitted into hospital with gastric ulcer who had already been treated for and cured of similar attacks. Dr. Bulstrode has done this for us, and has calculated that out of the 500 cases no less than 2 I I ( $t^{2}$ per cent.) had suffered in this way previously. In I 6 there had been only one previous attack; in $f^{I}$ there had been two; in 15 three; and in 39 four or more. In other words, gastric ulcer recurs or relapses in at least two-fifths of the cases which are apparently cured. 
' $\mathrm{Bad}$, however, as this sounds, it is not yet the worst. These figures do not include any of the cases admitted suffering from what may be called the late sequelæ and complications of gastric ulcer. They do not take into account the cases admitted for pyloric stenosis (which Gerhardt and Warren both estimate at Io per cent.), hour-glass contraction, gastric dilatation, chronic dyspepsia, constant vomiting, perigastric adhesions, cardiac stenosis, and those numerous other disorders caused by the cicatrization of ulcers healed long since. Nor do they take into account those cases in which carcinoma has developed at the site of an old ulcer, which Hauser estimates at quite 6 per cent. When allowance has been made for all of these cases, which certainly cannot be described as cured, it must be admitted that the proportion of patients suffering from gastric ulcer of sufficient severity to require treatment in hospital, which is discharged as cured in the full sense of the term, in the sense in which a surgeon would use it after an operation, is not a very high one, not nearly so high as is generally imagined.'

\section{Symptoms.}

As a rule, in acute ulcer the symptomatology will be found fairly characteristic, though occasionally no evidence of the presence of the lesion will be afforded until alarming hæmorrhage or perforation, with peritonitis, has taken place.

Generally there will be a very marked history of distinct gastric trouble supervening on anæmia in a young person. The symptoms which are most prominent are pain, vomiting, and hæmatemesis. The pain is referred to the epigastrium, usually over a small area, but at times tending to radiate in different directions, mostly towards the left or through to the left subscapular region. Epigastric tenderness is usually well marked and associated with so much rigidity of the recti that palpation is often difficult. It is not in the early stages continuous, though it may become so later; it is originated by the ingestion of food, usually commencing shortly after a meal, and persisting until the stomach has been emptied.

Vomiting is frequent in simple acute ulcer, and often takes place within an hour of taking solid food, or the food may be immediately rejected, when instant relief occurs. Hæma- 
temesis is also a common symptom, and is much more frequently copious in acute ulceration than in the chronic form, though in the latter form a little blood may be usually found on microscopic examination of the stomach contents, and at times it is so profuse as to be rapidly fatal.

Chronic ulcer is a disease of middle or advanced age rather than of youth, though we have operated for perforation from chronic ulcer in patients as young as twenty-one years. It is frequently followed by pyloric contraction or by hourglass deformity of the stomach. If bleeding occurs, it is usually less in quantity than in the cases of the acute ulcer, but the bleeding may be severe, and even fatal.

The characteristic symptoms of gastric ulcer, pain and vomiting, may be absent until some complication supervenes, though usually they are sufficiently distinct. The pain frequently begins from one to two or more hours after food, and is generally relieved by the next meal. There is usually tenderness over the pylorus, or between the xiphoid and right costal margin. Vomiting is very irregular, but when present is characteristic. The vomit is often large in quantity, containing much mucus, and it sometimes consists largely of grumous or coffee-ground-like material; it is often fœit, and if there be dilatation there will be signs of fermentation, with sarcinæ on microscopic examination. The symptoms vary very much, and may disappear for weeks together. We have observed and operated on cases where the symptoms have been going on for five, seven, fifteen, seventeen and twenty-five years, with more or less long intervals, when the symptoms were in abeyance. Dilatation of the stomach is a frequent complication, and is dependent on pyloric contraction, the result of cicatrization, it being associated with hypertrophy of the muscular coat and with visible peristalsis. Loss of flesh is always present; it may be well marked, especially in the later stages, and in some cases is extreme, extending to half the body-weight. A tumour of the pylorus or of the body of the stomach is not infrequently present, and may resemble one due to malignant disease, except that the tumour caused by simple ulcer is usually tender on pressure, whereas in cancer there is an absence of pain on 
palpation. Anæmia is seldom absent in chronic ulcer, and it may be so severe as to resemble the pernicious form.

\section{Diagnosis.}

The well-known symptoms of pain after food and epigastric tenderness, with vomiting and bleeding, as shown by hæmatemesis and melæna, are usually sufficiently characteristic of gastric ulcer; but in some cases hæmatemesis and vomiting may be absent, and only painful indigestion and tenderness may be noticed; or even, in others, all the symptoms may be latent. The surgeon cannot afford to pass over the diagnosis, but must confirm it personally before deciding on the question of surgical treatment.

Doubtless before he sees the case it will have been settled by the physician that the patient is not suffering from simple gastralgia due to anæmia and hyperæsthesia of the stomach; owing to the absence of localized tenderness and of hyperacidity of the gastric juice, as well as from the irregularity of the vomiting and pain, which may occur quite independent of the ingestion of food. He may, however, be called on to negative cancer of the stomach by noting the length of time over which the symptoms may have extended, the absence of a tumour, the presence or increase in quantity of free hydrochloric acid in the vomit, the absence of coffee-ground vomiting and of cachexia, and the onset of the disease in youth or before middle life, though it must be borne in mind that cancer may occur even in adolescence. We have performed gastro-enterostomy for cancer of the pylorus in a young woman of twenty-one, the accuracy of the diagnosis being confirmed some months later by an autopsy. The diagnosis between gall-stones and ulcer will usually be indicated by the irregularity of the painful paroxysms, which in gall-stone colic occur quite independent of food, by the character and site of the pain, the presence of jaundice and the character of the vomiting, which in cholelithiasis usually comes on soon after the pain begins, and persists long after all food has been rejected.

In the differential diagnosis between ulcer of the duodenum and ulcer of the stomach, the occurrence of the former more 
frequently in men than in women, and after middle age, the presence of melæna often without hæmatemesis, the pain occurring from an hour to two hours after food, and the tenderness situated to the right of the middle line, will usually afford sufficiently characteristic indications.

We would here point out, as bearing on the site of the ulcer, the importance of the character and site of the pain, which in gastric ulcer is usually definitely localized and associated with tenderness on pressure. The tender area is in the upper abdominal region, the position being more or less to the right or left of the middle line, according to the site of the ulcer. For instance, an ulcer on the anterior surface of the stomach near the cardiac end is usually associated with tenderness between the left costal arch and the mid-line, and is relieved by the dorsal decubitus; but if the pylorus be involved, the tender spot will lie between the right costal margin and the middle line, and the pain is relieved by lying on the left side, and aggravated by turning to the right. An ulcer on the posterior wall of the stomach gives rise to less epigastric tenderness, and the pain is felt more in the back beneath the left scapula, or close to the spinal column, opposite the attachment of the ninth, tenth, and eleventh ribs. If adhesions have formed between the pylorus and the liver or gall-bladder, the pain may radiate toward the right infrascapular region. When the ulcer is on the posterior wall, dorsal decubitus increases the pain, which may be relieved by lying on the face, or leaning forward, or even by assuming the erect posture. In all cases of ulcer the pain is usually increased after food, but the time of the onset of pain after eating is significant. Thus, an ulcer close to the cardiac orifice of the stomach causes pain immediately after food has been swallowed, or the pain may even start as the food is being swallowed. On the other hand, in ulcer of the pylorus the pain frequently does not begin until from one to two hours after meals. If the pain is severe, vomiting usually occurs, sometimes immediately after food, at others not for an hour or two, and the act of vomiting usually gives immediate relief. With regard to hæmatemesis, which is said to occur in about 80 per cent. 
of cases of gastric ulcer, and melæna, which is usually associated with ulcer, and which sometimes occurs without hæmatemesis, they will be considered later ; for hæmorrhage is one of the important complications on which the opinion of the profession, so far as treatment is concerned, is not yet by any means crystallized.

Latent ulcers are not uncommon; Savariaud gives their proportion as 20 per cent. of all cases of gastric ulcer. It is said that such ulcers are always of the acute round variety, but our experience in three cases, where we had to operate for perforation without any previous symptoms pointing to ulceration, would lead us to form a different opinion; for in all, considerable thickening was found around the ulcers, showing that the disease must have existed in a latent form for some considerable time. It is difficult to explain why some of these ulcers should exist without producing symptoms until either perforation or violent hæmorrhage takes place. In the cases we have seen the ulcers have been near the lesser curvature of the stomach, which might possibly afford an explanation, since in this situation they would be brought less intimately into contact with food.

In certain cases of diffuse peritonitis, the point of origin of the inflammation may be doubtful, unless it is borne in mind that thoracic pain is seldom absent in ulcer of the stomach; it may be under the left scapula or between the scapulæ, or if the ulcer be at the pylorus or in the duodenum, beneath the right scapula. 


\section{CHAPTER XIII \\ GASTRIC ULCER AND ITS TREATMENT}

IN considering the treatment of ulcerated stomach, we have to consider, not only the treatment of gastric ulcer itself, but also that of its complications and sequelæ, which are no less numerous than serious.

I. Acute perforation of the stomach wall and general peritonitis.

2. Hæmatemesis and melæna.

3. Cicatricial stenosis of the pylorus.

4. Hour-glass stomach.

5. Tumour of the stomach or pylorus of inflammatory origin.

6. Dilatation of the stomach due to obstruction.

7. Fistula between the stomach or pylorus and adjoining organs or with the surface of the body.

8. Spasm of the pylorus producing intermittent stenosis (Reichmann's disease).

9. Persistent vomiting.

Io. Local peritonitis or perigastritis ending in adhesions.

II. Local peritonitis ending in suppuration and a localized abscess.

I2. Subphrenic abscess.

I3. Abscess of the liver, pancreas, or spleen.

I4. Atonic motor deficiency.

I5. Severe gastralgia.

I6. Tetany.

I7. Stenosis of the cardiac orifice.

I8. Acute or chronic pancreatitis.

I9. Profound anæmia resembling the pernicious form. 
2o. Pressure on or stricture of the bile-ducts with jaundice.

2I. Catarrh of the gall-bladder from adhesions, producing attacks like those of cholelithiasis.

22. Great loss of flesh and strength, ending in phthisis.

23. Cancer secondary to ulcer, ulcus carcinomatosum.

24. Interstitial pancreatitis or abscess of pancreas.

The treatment of gastric ulcer is at first essentially medical, and when properly carried out, and for a sufficient length of time, it is often completely successful. Leube says that onehalf or three-fourths of all cases will be cured by four or five weeks of treatment, but if not well in that time they will not be cured by medical treatment alone. Unfortunately, however, in many cases treatment is stopped as soon as relief to pain is obtained, and long before the ulcer is healed. In some cases this may be due to the uncertainty of diagnosis, or from the impatience of the patient; perhaps in others to ignorance as to how long it takes to secure the healing of the gastric ulcer. The earlier in the course of the disease that radical treatment in the shape of dieting and rest is adopted, the less prolonged will the treatment need to be, and the more likely is it to be effectual; but probably the very earliest time a patient should be allowed to be out of bed is from a fortnight to a month after all pain and tenderness have disappeared. Failing this thorough treatment, relapses will be certain to occur, and in the long-run complications will supervene or the ulcer will become chronic, when, though medical treatment may relieve in some cases, cure can only be looked for in the greater number by surgical methods.

In considering the treatment of ulcer of the stomach, it is useful to hold in view the course of an ulcer of the leg, which directly the healing stage has arrived becomes free from pain. But this neither indicates that healing is completed nor that care may cease, and should treatment be abandoned and the ulcer become chronic, though it may even be painless, it is at any time liable to become inflamed or to extend; moreover, the surrounding tissues become infiltrated with lymph, and contraction occurs, which in a hollow viscus soon ends in stricture, as in the leg it tends to drag on the surrounding skin and produce constriction of the limb. 
The medical treatment of ulcer of the stomach consists chiefly in systematic dieting and rest.

The following method of treatment, which is employed by Dr. Robert Saundby (British Medical Journal, January 20, I9oo, p. I22), is one that should be efficient in ordinary cases; but in severe cases it may be necessary to keep the stomach void of food for several days and to feed by enemata; and then absolute rest in bed may be required over a greater length of time, and abstention from solids will have to be further prolonged.

'It is a rule to which attention may well be called, that, in the management of stomach disorders, obstinate vomiting should be treated by absolute rest in bed, and the administration of the simplest food in small quantities at regular intervals. I generally prescribe an ounce of milk and limewater every hour, with the following mixture:

$\begin{array}{lllc}\text { Sulphate of magnesium } & \ldots & \ldots & 40 \text { grains } \\ \text { Sulphate of iron } \ldots & \ldots & \ldots & 2 \\ \text { Diluted sulphuric acid } & \ldots & \ldots & \text { I } 5 \text { minims } \\ \text { Peppermint-water } & \ldots & \text { to } & \text { I ounce }\end{array}$

three times a day. The milk and lime-water, if borne without pain and vomiting, as is almost invariably the case, is increased every day or every second day up to 4 ounces every hour, and afterwards the diet is gradually increased by the addition of bread-and-milk, minced chicken, and minced mutton at intervals, so that, about twenty-one days after admission, the patient is usually able to eat the ordinary house-diet of the hospital. After this has been taken for two or three days the patient is allowed to get up, and at the end of a month is sent to a convalescent home. When the anæmia is marked the mixture may be supplemented by pil. ferri, 5 grains or more, three times a day. Should the patient not be able to tolerate so much milk and lime-water in the first instance, $\frac{1}{2}$ ounce may be given, or if there be only pain without vomiting, a mixture of bismuth and soda may be substituted for that of iron and magnesia.

'In those cases which have recently suffered from hæmatemesis it is desirable to give nothing by the mouth until 
forty-eight hours have elapsed after the vomiting of blood has stopped, and during that time I feed them by the following nutrient enema, given every four hours: One egg beaten up with one teaspoonful of brandy, and made up to 4 ounces with milk. Should there be any irritability of the rectum, 20 to 30 drops of laudanum may be added. While the hæmatemesis persists, I place an ice-bag upon the epigastrium, although I am by no means certain that it does any good, and I allow the patient to suck small pieces of ice if she wishes. If necessary, to relieve pain or to keep the patient quiet, I order a hypodermic injection of $\frac{1}{6}$ to $\frac{1}{3}$ grain of morphine. It is of great importance to see that the patient is able to eat ordinary food with comfort before she leaves the hospital, and I always try to impress upon each one the importance of continuing to do this after she returns home. Many of these patients have been dieting themselves for so long a time, and have become convinced, partly as the result of injudicious advice, partly from their own experience, that they cannot eat the same food as other people, that they have suffered in health from insufficient nutrition, and have entered a vicious circle in which the anæmia is kept up by want of food, so that the predisposing cause persists, and recovery is impossible until the circle is broken; it is therefore of the utmost importance to prove to your patient that she can take ordinary food. It is also very desirable that she should continue to take iron for some time after leaving the hospital, and I may perhaps be allowed to mention that the dose of sulphate of magnesium in the mixture should be adjusted to the needs of each case, and may be very properly increased or diminished at different times as required.'

Dr. Westphalen commences with the so-called abstinence treatment, in which nothing is given by the mouth beyond a little cold tea, or Vichy water, or bits of ice. If the abstinence is long continued, nutrient enemata are used. Rost, of the Augusta Hospital, keeps food by the mouth from his patients for six days. Whether food in the rectum sets up gastric secretion or not, it is an undoubted fact clinically that in cases of gastric ulcer the painful symptoms disappeared more quickly by withholding food from the 
stomach than by any other method of treatment. In an extreme case the author continued the abstinence for sixteen days. After completion of the abstinence period he gives four glasses of milk per day, increasing the quantity by half a glass each day. After ten to fourteen days he gives soups and more solid food. He counsels a long-continued milk diet (fresh milk or whey), with the addition of cream, which reduces the hyperacidity of the stomach, the milk food keeping the motility of the stomach at a minimum. Passing over local applications, such as poultices, etc., which many would think unnecessary, he gives large doses of bismuth-a heaped-up teaspoonful in a glass of water before breakfast in the morning. In no case does he keep his patient in bed less than three weeks. Even when all active symptoms have subsided, his patients must keep to a carefully regulated diet for at least a year. Von Sohler recommends a chiefly vegetable diet, as by this the hyperacidity is favourably influenced.

The treatment of hepatic colic by olive oil internally is of ancient date, but Professor Mathieu, of Paris (Medical Press and Circular, November I9, I902), by a series of experiments, has come to the conclusion that the same treatment is highly beneficial in cases of stenosis of the pylorus and in chronic gastric ulcer where an excess of acid exists.

The olive oil possesses a considerable nutritive value, and besides does not provoke gastric fermentation, as is observed with the lactic regimen. The amount of the oil ingested daily was about 5 ounces, given in three doses. The patients seemed to take it without much reluctance, and after about a week of the treatment the painful gastric symptoms had disappeared.

Fleiner's method of treating gastric ulcer consists in washing out the stomach every morning before breakfast until the fluid returns perfectly clear, and then introducing by the stomach tube $I 5$ to 20 grammes (23I to 308 grains) of subnitrate of bismuth suspended in 200 cubic centimetres ( 7 ounces) of tepid water. The tube is then closed for five or ten minutes, while the patient lies in a position in which the bismuth is most likely to come in contact with the ulcer- 
for instance, on the right side if the ulcer is on the pylorus. The water is then allowed to return, and the tube is removed. The patient remains in the same position for thirty minutes before he has breakfast. Bismuth in large quantities forms an aseptic crust over the ulcer, to which it adheres more readily than to intact mucous membrane. After a time the treatment is given only every other day, or when symptoms of irritation recur. The results are said to be excellent. The disadvantages of the method are the practical impossibility of performance outside an institution, the reluctance with which it is submitted to by patients, and, possibly, the danger of perforation, which might be brought about directly by the stomach tube or indirectly by the rise of pressure which accompanies retching and straining.

Pariser claims that neither the stomach tube nor the prolonged maintenance of an often uncomfortable posture is essential. Matthes has shown that bismuth, though it first collects at the lowest point in the stomach, is rapidly spread over the gastric mucosa in a layer of uniform thickness, whatever position may be assumed. Fifteen to 20 grammes of subnitrate of bismuth should be taken every morning fasting, stirred up in a glass of water, and washed down with a drink of tap water. The patient should lie on his back for forty-five minutes before having breakfast. Pariser states that, as its action depends merely on its insolubility in the gastric juice, any insoluble powder is of equal value. In several hundreds of cases he has substituted a powder consisting of equal parts of prepared chalk and talc (magnesium silicate), mixed with light magnesia in the proportions of ro to ${ }_{5}$ grammes of the latter to 60 grammes of the former. If diarrhœa is produced, the light magnesia may be omitted. Of this powder one and a half heaped-up teaspoonfuls are taken stirred in water every morning before breakfast, and every evening at ten o'clock, three hours after the last meal. The results fully equal those obtained by bismuth, and the powder has the advantage of not blackening the stools and masking melæna. It should be given for from three to eight weeks, according to circumstances. The only cases not amenable to this treatment are those of acute ulcer with pyloric 
stenosis, secondary dilatation of the stomach, and stagnation of its contents, in which irrigation with the tube or operation is indicated.

In every case of gastric ulcer absolute rest in bed is essential. No diet suitable for an ulcerated stomach can furnish sufficient calorics for the slightest exertion, much less for full work. The out-patient treatment of gastric ulcer is, therefore, a mistake. The patient should not be allowed up until some days after the ulcer is healed. This is recognised by the absence of pain after food, and of tenderness, and, above all, by the behaviour of the dorsal 'pressure point,' first described by Cruveilhier. This is a circumscribed spot of tenderness situated between and somewhat to the left of the tenth and twelfth dorsal vertebræ; rarely there may be a tender spot on the right side also, but it is always less marked than on the left. The point is always present unless the ulcer is situated on the anterior wall ; this occurs, however, in only 5 per cent. of all cases. Pressure on it produces intense pain as long as the ulcer is unhealed, even though every other symptom of ulcer has disappeared. Women should rest during the next two menstrual periods. As regards diet, milk should never be given alone. It should be boiled with some farinaceous substance, such as tapioca. Eggs are allowable if beaten up or mixed with weak broth. Some preparation of casein, such as ' nutrose,' is useful. Meat juices and extracts should be avoided. After convalescence is established, all meat should be pounded, and later minced. For months all vegetables should be put through a sieve, and given as purée. Under the powder treatment complete abstinence from food by the mouth is seldom necessary, and hardly ever for longer than three days. During this time ordinary nutrient enemata are required, and it is sufficient to give injections of wine in about 8 ounces of mucilaginous fluid.

\section{The Surgical Treatment.}

The surgical treatment of intractable or relapsing gastric ulcer is, in the greater number of cases, the only satisfactory method of dealing with these refractory cases, and operation 
should be resorted to at a much earlier period than has hitherto been the custom, and always before the patient is so far reduced by pain and starvation or the supervention of serious complications that weakness and anæmia render any operative procedure hazardous.

Ulcer of the stomach is a much more serious matter than is generally recognised, for, according to various authors, it has a mortality, when treated by general and medical means only, of from 20 to 50 per cent., and the excuses of a few years ago, that there is a great responsibility in recommending surgical treatment, either from the uncertainties of diagnosis or from the risk of operation, can no longer avail, since the diagnosis of gastric ulcer, thanks to the researches of Ewald, Hemmeter, Einhorn, and others, has been brought to a greater state of perfection than exists in many other obscure diseases where radical treatment has to be adopted on much more slender foundations; and, fortunately, now that the mortality in operations for simple diseases of the stomach has been reduced in the hands of experienced surgeons to 5 , or less than 5, per cent., the risk of surgical treatment cannot be advanced even by the most ardent opponents.

Dr. Bulstrode collected and analyzed the cases of gastric ulcer admitted into the London Hospital from $\mathrm{r} 897$ down to the present time, with the view of ascertaining the results of hospital practice. The number was exactly 500, 98 men and 402 women. Of these no less than 48 , or nearly ro per cent., had died from perforation and peritonitis ; $13,2 \frac{1}{2}$ per cent., from hæmatemesis; and $28,5^{\circ} 5$ per cent., from other causes. The total number of deaths was 89 , or, approximately, i 8 per cent. These figures, however, were deceptive in that they embraced all the slighter cases on which no one ever proposed to operate. If these were left out, and only the gravest of the grave counted (such cases, for instance, as those in which operation might be contemplated), the percentage would be far higher. Dr. Bulstrode also calculated that of these 500 cases upwards of 216 , or 42 per cent., had been in hospital already for this complaint, and had relapsed. If to the I 8 per cent. that die, and the 42 per cent. that relapse, are added all those cases in which the later sequelæ 
(such as pyloric stenosis, etc.) occur, and those in which cancer develops (estimated by Hauser at 6 per cent.), it must be admitted that the results of the present method of treatment are not so successful as they are usually thought to be, and not nearly so successful as they would be if an operation were performed in the more serious cases, those which would terminate fatally.

In England we are not prepared to subscribe fully to the views of Tricomi (Riforma Medica, 1899), who draws a parallel between the treatment of hernia and that of ordinary gastric ulcer, and proposes that as hernia is treated radically with success, so gastric ulcer should be treated radically by the performance of gastro-enterostomy.

Heydenreich (Sem. Méd., February 2, r898) argues: 'The death-rate from all cases of gastric ulcer is from 25 to 3o per cent., but from gastro-enterostomy only $16 \cdot 2$ per cent.; therefore the operation has less danger than the disease. Another advantage of not waiting for complications is that the patient is in better health. At any rate, cases which do not improve with medical treatment in a reasonable time should be treated surgically.'

At the time of delivery of the Hunterian Lectures on the Surgery of the Stomach, in March, I9oo, we had been able to collect $\mathrm{I} 84$ operations for gastric ulcer (excluding those for perforation and hæmorrhage), of which 157 recovered and $3 \mathrm{I}$ died, thus giving a mortality of $16{ }^{*} 4$ per cent.

Now, although the deaths from gastric ulcer, medically treated, averaged 25 per cent., and those from even the worst and most inveterate cases of ulcer, when treated surgically, only 16 per cent., at the time of those lectures, yet the difference did not then appear so great as to make it desirable or prudent very strongly to advocate surgical treatment until the disease had become chronic or until serious complications had ensued.

To-day, however, the facts are very materially altered by the all-round improvement in operations on the stomach, and the contrast of 25 per cent. of deaths in cases treated medically and 5 per cent., or less, as shown in our latest statistics, in those treated surgically in the worst and most 
complicated cases, is so striking that we feel it incumbent to urge most strongly that, although cases of gastric ulcer should first be submitted to medical treatment, yet if such treatment fails to cure in a reasonable time, or if relapses occur on the resumption of solid food, then medical should give place to surgical treatment; for it is unfair to the surgeon to hand over to him almost moribund cases, and it is unjust to the patients to persist in dosing them with medicine or otherwise treating palliatively cases that can only be benefited or cured by surgical means.

Operative Treatment.-Before the abdomen is opened it is quite impossible to say what operation or operations will be required, and the surgeon must be prepared to adapt himself to circumstances on discovering the position of the ulcer and the conditions associated with it, especially as to the presence or absence of adhesions and other complications.

Any one of the following operations, or a combination of one or more, may be called for in each individual case: Exploratory gastrotomy; gastro-enterostomy, to secure physiological rest to the stomach and relieve the hyperchlorhydria, or, in other cases, to short-circuit a stenosis; excision of the ulcer; pylorectomy; pyloroplasty; gastroplasty ; gastro-gastrostomy ; gastrolysis ; pylorodiosis.

History.-The first operation for the cure of ulcer of the stomach was performed in I88I by Rydygier, who successfully resected a large ulcer from the posterior wall of the stomach.

A report in the Centralblatt f. Chir., No. 32, Igoo, states that a woman from whom he excised the pylorus in I88I, for chronic ulcer, is now aged forty-two and quite well, and that she has since borne five children. A similar case was treated in I88I by Van Kleef. A year later-in 1882Czerny repeated the operation successfully.

After other cases of excision, Doyen in r893 introduced the operation of gastro-enterostomy for the treatment of gastric ulcer, and at the German Congress in 1895 he related a series of cases in which he attributed the relief or cure of ulcer of the stomach by gastro-enterostomy to the rest induced by the operation. 
Czerny, Monod, Durivier, Kuster, Novaro, Tricomi, ourselves, and many others, have followed on the same lines.

The Preparation of the Patient.-It has been the custom with many surgeons to put stomach patients through a long course of preliminary treatment, such as frequent lavage of the stomach and abstention from food before operation. This, as a rule, is quite unnecessary, and certainly inadvisable in the greater number of cases-first, because the treatment is depressing and debilitating in the case of patients already exhausted by a long illness; secondly, as proved by Dr. Harvey Cushing's bacteriological investigations, the stomach contents speedily become aseptic if the mouth be cleansed and aseptic foods administered; and, thirdly, as proved by ample clinical experience, elaborate preliminary treatment is unnecessary to success.

If the stomach is greatly dilated and the contents are foul, then lavage with simple boiled water night and morning is adopted for two days before operation. The careful cleansing of the mouth and teeth and the administration of foods sterilized by boiling is advisable. The last meal is given the night before, about twelve hours; the stomach is washed out about two hours, and a nutrient enema given about an hour before operation.

In other cases no lavage is adopted, but the same care is exercised in cleansing the mouth, giving sterilized food, and administering a nutrient enema consisting of $I$ ounce of brandy, I ounce of liquid peptonoids, and Io ounces of normal saline solution. Every patient is enveloped in a suit of cotton-wool made by the nurse out of Gamgee tissue, and each has an injection of ro minims of liq. strychniæ (B.P.) administered subcutaneously before the operation is begun.

The preparation of the skin and other aseptic details of the operation differ in no respect from those observed in operations generally.

Exploratory Gastrotomy, or opening the stomach by a free incision in its anterior wall, is an operation occasionally called for in the surgical treatment of ulcer :

(a) In order to verify the diagnosis of ulcer when there is 
so much thickening of the stomach walls as to suggest the presence of cancer.

(b) When, although the symptoms have pointed to ulcer as the cause of the gastric trouble, the stomach, on exposure, betrays no evidence of puckering or other characteristic signs, and, in order to verify the diagnosis and ascertain what is best to be done, it is felt desirable to examine the interior of the organ.

(c) In certain cases of gastrorrhagia it is desirable to perform exploratory gastrotomy, in order to find and ligature the bleeding vessels, or to otherwise arrest the hæmorrhage.

(d) It necessarily forms part of any operation for the excision of ulcer of the stomach. The detailed description of the operation is given under the chapter on Gastrorrhagia and its Treatment, p. 275 .

It will be gathered, on reading the context, that, as our experience increases, the tendency is to the performance of gastro-enterostomy, without previous exploratory gastrotomy, in cases of ulcer.

As examples of exploratory gastrotomy, we may briefly refer to the following cases :

Man aged thirty-eight. Symptoms of chronic ulcer extending over several years. On exposure of stomach, no evidence on the surface to indicate accuracy of diagnosis. Exploratory gastrotomy. Discovery of large ulcer, $\mathrm{I} \frac{1}{2}$ inches by 3 inches, on posterior wall of stomach. Posterior gastro-enterostomy. Recovery'.

Acute gastrorrhagia. No evidence of ulcer on exposing the stomach. Exploratory gastrotomy. Numerous bleeding ulcers seen. Two, which were bleeding freely, were ligatured en masse. Gastro-enterostomy. Recovery.

At the time of exploration it will be generally advisable to have everything ready to follow up the exploratory procedure by whatever further operation may be called for. It may be discovered that the disease is manifestly not malignant, and that some curative operation can be done, as in the following case :

Mr. S., just over fifty years of age, who had suffered from indigestion for several years, began to have severe pain about an 
hour after food, and to lose flesh and look ill during the six months before we saw him. A doubtful tumour could be recognised, and during the attacks of pain the stomach could be felt to harden under the hand. Free $\mathrm{HCl}$ was present after a test meal. The serious nature of his ailment was brought home to the patient by his inability to continue his business.

On operating, the swelling was found to be a greatly thickened pylorus forming a distinct tumour, and about $I_{2} \frac{1}{2}$ inches from the pylorus along the lesser curvature was another tumour, which, from the enlargement of the glands, we suspected might be growth; but on opening the stomach it was found that the pyloric swelling was an enormously hypertrophied sphincter, and that the stomach tumour was thickening around a chronic ulcer. The latter was freely excised, the edges being brought together by continuous sutures. The stomach opening was closed, and a posterior gastro-enterostomy was then performed. As the gallbladder was full of thickened mucus and gall-stones it was at the same time emptied and drained. A smooth recovery has followed, much to the relief of the patient and his friends, who all suspected the disease to be cancer.

Excision of the Ulcer is, as a rule, unnecessary, but not always to be avoided, as in some cases of bleeding ulcer, and in others where the thickening and induration render it difficult to decide on the absence of malignant disease. This was the case in a man of fifty-four on whom we operated in I89I, when, finding the pylorus the seat of diffuse induration, excision of the whole indurated area was performed. A careful examination of the removed mass showed that the growth was inflammatory around a chronic ulcer. In a nother middle-aged man, where the diffuse induration was suggestive of cancer, the pylorus was opened, and a deep ulcer on the posterior wall successfully excised, the edges of the original incision, as well as the margins of the posterior wound, being brought together in a direction transverse to the axis of the pylorus over a bone bobbin.

Rydygier prefers excision of the ulcer to gastro-enterostomy, because he believes that carcinoma not infrequently develops in the scar of an old ulcer.

It is impracticable to give any specific description of the operation of excision of an ulcer other than will be given in the description of operations involving the removal of part 
of the stomach wall, seeing that the procedure will vary with the size and position of the ulcer.

The cases related below sufficiently exemplify the operations that may be necessary.

After excision of an ulcer, the bleeding from large vessels must be controlled by ligature, but the oozing from the smaller vessels will be stopped readily by the continuous suture employed to bring together the edges of the wound. If the excision involve the serous coat, a Lembert's continuous stitch, with a silk or celluloid thread suture, will be necessary. Should the excision have been near the pylorus, the line of suture must be placed transversely to the axis of the canal, so as to avoid stricture.

The following cases are examples of gastric ulcers treated by excision :

\section{Ulcer of Pylorus; Stenosis; Dilatation of Stomach. Excision of Ulcer and Pyloroplasty.}

John W. R., aged thirty-eight, admitted to the Leeds Infirmary with the history of stomach trouble for thirteen years. Pain after food and vomiting were the initial symptoms. Severe hæmatemesis occurred six years after the onset of symptoms. Great loss of flesh and weakness were followed by inability to work, although he had stomach lavage and other appropriate treatment. On admission, the patient was very thin and profoundly weak. He weighed 8 stones. An indefinite swelling could be felt below the right costal margin. The stomach reached 3 inches below the umbilicus, and there was visible peristalsis. Free $\mathrm{HCl}$ present. Operation November I 5, I900. The pylorus was found much thickened, forming a hard nodular swelling, adherent to the gall-bladder and liver, and to the abdominal wall by omental adhesions. After separating the adhesions, a small perforation was discovered in front of the pylorus, evidently the site of a perforation which his medical man, who was present, said he remembered occurring some months previously, and which was then treated successfully by rest and rectal feeding.

The pylorus was freely laid open, and found to be the site of a round perforating ulcer in front, and another in the posterior wall ; the latter had perforated into the substance of the pancreas. Both were resected, the removal thus practically constituting a pylorectomy. The edges of the posterior wound were brought 
together transversely to the axis of the stomach. The anterior wound was prolonged into the duodenum, and its edges were brought together, also transversely to the axis of the stomach, over a bone bobbin, thus leaving a capacious channel between the stomach and duodenum surrounded by healthy mucous membrane.

Recovery was uninterrupted, and he was discharged on December I2, weighing 8 stones 5 pounds.

On January 9, I90I, he returned to report himself well, and then weighed 9 stones I I pounds.

\section{Pyloric Ulcer treated by Excision of Ulcer and PyLOROPLASTY.}

Mrs. M. K., aged forty-four. Seen with Dr. Johnstone, Ilkley. Well till two years ago, when she had colic and loss of flesh. Under treatment she recovered, apparently, and regained some of the lost weight.

September I 5, I 897.-Recurrence of attacks similar to that of a year before, but with pain at the right side over the pylorus. Loss of weight and strength. The patient had for some time been an invalid, and had been continuously under medical treatment for months. Her weight was 6 stones II pounds. There was visible peristalsis toward the pylorus, which was fixed to the gall-bladder; no pain or tenderness. Liver 2 inches below costal margin, but not nodular, and no jaundice present.

July 23, I 898.-Operation. An ulcer at the pylorus adherent to the liver, which formed the base of the ulcer; stenosis of pylorus. Pyloroplasty performed after excision of ulcer, the opening being sutured transversely over a bone bobbin.

December 3, r898.- - Had gained I stone 8 pounds in weight. No digestive troubles.

December 23, I899.-Dr. Hearder reports patient as 'very well,' and of normal weight.

See p. 3I, ' Gastrotomy,' for other cases.

The operation of Pylorectomy for ulceration of the pylorus may be mentioned under the heading of excision of ulcer. It has, however, been more convenient to consider this operation in detail when describing partial gastrectomy for cancer, seeing that the operation for the two conditions is essentially the same. Dr. Rodman (Philadelphia Medical Journal, June 9, I goo) has collected from literature and personal correspon- 
dence detailed reports of forty pylorectomies, partial gastrectomies and excisions, with six deaths. As this includes cases since 188I, when Rydygier performed the first excision of ulcer, it does not show the operation under a favourable light, the mortality being 15 per cent. ; for it would be found that the later operations under improved technique contrast favourably with the earlier ones.

Nevertheless, the mortality after excision will probably always be higher than the more simple operation of gastroenterostomy, with its 5 per cent. or smaller mortality. The more severe and radical operation should therefore be reserved for cases that are not suitable for the less severe operation, or for cases in which the suspicion of cancerous degeneration is entertained, and cannot be disproved on naked-eye inspection.

The following case is an example:

\section{Tumour of Pylorus and Chronic Hamatemesis due to Ulcer. Pylorectomy.}

In $189 \mathrm{I}$ we were asked by a medical friend to see a man of fifty-four, who for six months had suffered from pain coming on an hour after food, and more recently from vomiting of blood of coffee-ground character in considerable quantities, so that he was not only reduced in flesh and strength, but had also been rendered profoundly anæmic by the loss of blood.

A tumour of the pylorus could be easily felt, and the stomach was markedly dilated.

As he was rapidly losing ground, an operation was performed, and the pylorus was found thickened and nodular, with adhesions to the liver and omentum. After separating the adhesions, the pylorus was excised, and the open end of the duodenum was fixed to the opening in the stomach by means of two lines of sutures without the use of a bobbin, the rest of the stomach aperture being closed by a double layer of sutures.

The tumour proved to be inflammatory around an open ulcer which had been the source of the hæmorrhage. The bleeding was not repeated, and the patient rapidly gained flesh, and returned home within the month.

The subsequent history of this case is interesting on account of cicatricial contraction of the new pyloric aperturc, which 
led to the invention and employment of a decalcified bone bobbin to act as a temporary splint upon which to apply the sutures, and so secure a large aperture which has little tendency to contract.

Gastro-enterostomy, in the absence of special complications, is the operation to be relied on in the treatment of ulcer of the stomach; it acts by securing physiological rest by means of drainage, thus allowing the ulcer to heal without being subjected to the irritation of acid secretion, accumulation of food, or frequent stomach movement. It also, while remedying the hyperchlorhydria, relieves pyloric spasm, and while preventing stagnation of fluids, cures or materially diminishes gastric dilatation. The posterior operation is the one we personally prefer, the junction of the posterior wall of the stomach with the first part of the jejunum being effected by two continuous sutures with or without a decalcified bone bobbin. The use of a bone bobbin not only secures a temporary protection to the line of incision, but secures an ample and immediately patent opening between the two viscera for the passage of the stomach contents. The whole operation can be easily completed in half an hour, and it may even be done in half the time.

Our experience with the posterior operation has been very favourable, not only in the rate of recovery of the patients, but in the smoothness of the recovery, many of the patients recovering without even once vomiting; and only seldom have we seen slight regurgitant vomiting of bile, which in the anterior operation is much more frequently seen, and at times becomes serious, or even leads to a fatal issue.

A detailed description of the operation of gastro-enterostomy and its modifications will be found on p. 227 .

The following cases are given as examples:

CASE I.-Pyloric ulcer treated by pyloroplasty, with subsequent contraction. Gastro-enterostony.-Mrs. W., aged thirty-two. Seen with Dr. Salter, Scarborough. Pyloroplasty during active ulceration of pylorus in December, I895. Great relief for a time, but later recurrence of dilatation, vomiting, pain, and other symptoms. Very considerable loss of flesh. Patient thin and anæmic; pulse feeble and rapid, marked dilatation, the stomach reaching well below the umbilicus. 
October 4, r898.-Operation: gastro-enterostomy (bone bobbin employed).

In October, I899, patient well and active.

February, I9oo, had gained I stone Io pounds.

Well I903.

CASE 2.-Pyloric ulcer, tumour of pylorus. Gastro-enterostomy.Mr. M. A., aged twenty-eight. Seen with Dr. Kilner Clarke, Huddersfield. Loss of weight. Two years ago weighed I 2 stones I2 pounds; now 9 stones 6 pounds. Pain two hours after food. For last two months vomiting on an average five times a weektwice coffee-ground vomit. Enormous dilatation of stomach. Pyloric tumour movable; visible peristalsis.

August 4, I 899.-Operation. Large mass at pylorus, evidently thickening, due to active ulceration; glands large, but not matted. Gastro-enterostomy (bone bobbin used).

A ugust 27, 1899.-Good recovery. Weighs 9 stones 7 pounds. September 20, I899.-Weighs Io stones I 3 pounds. Can eat anything.

Well 1903 .

CASE 3.-Extensive ulceration of stomach, with lavge tumour. Gastro-enterostomy.-Mr. D. B., aged thirty-one. Seen with Dr. Barrs and Dr. Webster, Golcar. Dyspepsia seventeen years; more severe last twenty months. Lead-poisoning discovered and treated. Good recovery. Sixteen months ago vomiting recurred, and from outset large quantities ejected, but never containing blood. Recurrence occasionally of similar attacks, always relieved by treatment.

December, I897.-Stomach reached pubes, and visible peristalsis seen. Relief followed dieting and lavage till March, I 898, since which time pain almost constant. Pain not materially worse after food nor relieved by vomiting. Loss of weight from ro stones to 8 stones $6 \frac{1}{2}$ pounds. Great feebleness.

May 6, I 898.-Operation. Large irregular tumour at pylorus and along lesser curvature; but glands, though large, discrete. Gastro-enterostomy (bone bobbin employed).

June 7, I 898.-8 stones in weight when he left the home.

August 17, I 898.-Weighs 9 stones 3 pounds.

Letter dated February I2, I900: 'My health continues perfect. I have not lost a day's work through illness since I recovered.'

CAse 4.-Miss H., thirty-two, seen with Dr. Angus, Bingley, with a history of stomach symptoms extending over several years. She had had hæmatemesis in 1892 and 1896 , since which time she had suffered from flatulency and pain after food. For two years epigastric pain constant, but increased by food. A 
year after vomited daily, then relief for a time; but for some months only milk could be retained.

Epigastric tenderness was well marked, and in distending the stomach with $\mathrm{CO}_{2}$ it reached $\frac{1}{2}$ inch below the umbilicus and far over to the right of the middle line.

October 5, 1900.-Posterior gastro-enterostomy performed, a bone bobbin being employed.

Recovery uninterrupted. Returned home within the month, taking solid food without any discomfort and gaining flesh.

On November 8 Dr. Angus wrote: 'I have seen Miss $H$. since her return, and there is every reason to be pleased with her condition. She has lost all her pain and is taking food well. Allow me to thank you for her restoration to comparative health.'

Weight February, 1901, 8 stones 6 pounds ; at time of operation, 6 stones 12 pounds.

CASE 5.-Mr. H., aged fifty-two, seen with Dr. Hearder, Ilkley, began to suffer in 1897 from symptoms of ulcer of stomach, which were relieved by restricted diet and general treatment; but in July, I899, the symptoms returned, with loss of flesh, and wellmarked dilatation of the stomach was discovered, and operative treatment advised.

Lavage and other treatment was carried out in London and in Edinburgh, but without material improvement.

When first seen by us there was visible peristalsis, with wellmarked stomach splash and a tender spot under the right costal margin. Although tall, the patient only weighed 8 stones 6 pounds, and he was extremely weak and pallid. Free $\mathrm{HCl}$ was discovered in the stomach contents.

October I 2, I g00.-Hour-glass contraction found, but the constriction was not extreme. Puckering on anterior wall of stomach, with well-marked thickening. Posterior gastro-enterostomy performed. Good recovery.

March 4, I9or.-Patient wrote from Bournemouth saying: ' I am pleased to be able to tell you that I have no return of my former complaint, and that I eat, drink, and sleep well. Have got back to my former weight.'

CASE 6.-History.-J. S., aged forty-five, residing at Batley, gave the history of two years' pain about an hour after food, with great loss of flesh. For nine months he had vomited every day, or every second day, a large quantity of yeasty material, but no blood, though he was very anæmic.

There were well-marked signs of dilatation, with tenderness over the pylorus.

June 12, 1900.-Operation. On opening the abdomen the pylorus 
was much thickened and adherent, forming a tumour, and through the centre of the mass a No. ro catheter could only be passed over a roughened, ulcerated surface. A posterior gastro-enterostomy was performed.

After-history.-An uninterrupted recovery followed. - Food was begun the second day, and solids could be taken in the second week without pain. He rapidly gained flesh and strength, and is now well.

Demoulin and Tuffier (Bull. et Mém. de la Soc. de Chiv. de Paris, October 3I, 1899, and British Medical Journal, Suppl., I 899) publish the case of a naval Lieutenant, aged thirty-two, who suffered from severe dyspeptic symptoms early in I897, aggravated by a diet of preserved meat during a cruise round the coast of Ireland. Early in 1898 a hard swelling developed in the epigastrium. Medical treatment at Vichy and elsewhere was of no avail. His weight fell to 77 pounds, the cachexia was marked, and emaciation extreme. The swelling was as big as an orange, movable transversely, and following respiratory movements. It was tough rather than hard. Directly the patient swallowed anything, even a few spoonfuls of water, great distension of the epigastrium, with severe pain, occurred, due evidently to contraction of the stomach for forcing its contents through the pylorus. Malignant tumour was diagnosed, and Demoulin operated on December 1,1898 . An incision 4 inches long was made in the middle line above the umbilicus. A soft tumour of the size of an orange lay around the pylorus and adjacent parts of the stomach and duodenum. It felt like a fatty growth, and was not adherent to any surrounding structures. A stellate cicatrix on its upper aspect showed signs of a healing ulcer. There were no enlarged glands in the neighbouring peritoneal folds; the liver and gall-bladder were healthy. The patient was in so exhausted a condition that a pylorectomy was considered impracticable. Demoulin therefore performed a Van Hacker's gastro-enterostomy (posterior and transmesocolic), leaving the tumour alone. A litre of salt solution was injected under the skin directly after the operation. The patient's life was in great peril for several days from ether bronchitis. On the third day hæmatemesis occurred, and nearly a pint of blood was vomited. By the roth the patient was able to eat fish and fowl without any bad effects. On the 2oth Demoulin for the first time palpated the epigastrium. To his surprise, the tumour could no longer be felt, its site being marked by a little thickening, which vanished within ten days. In May, I899, the patient was again on active naval service; no local symptoms remained, but he was troubled with an obstinate cough and free expectoration. 
For the complications and sequelæ of gastro-enterostomy, see p. 234 .

Pyloroplasty will be more fully described when the subject of pyloric stenosis is under consideration, but we must not fail to mention it as a method of treatment in certain cases of chronic ulcer associated with stenosis of the pylorus. The operation as a curative measure in this class of cases has certain very definite limitations, but where it is feasible it is a method of great utility, which can be performed rapidly and with very little exposure of viscera. Pyloroplasty, if the pylorus be stenosed, free from extensive adhesions, easily drawn forward, and not actively ulcerating, is a simple and short operation, and in quite a number of cases of both gastric and pyloric ulcer we have found it to answer well. It must not be relied on, however, where active ulceration of the pylorus itself is found, unless at the same time the ulcer be completely excised; otherwise cicatricial contraction will occur, and a second operation will be subsequently required. It acts in the same way as does gastro-enterostomy, by affording a free exit to the stomach contents, and thus securing physiological rest to the stomach.

The following cases exemplify the complete success which attended the operation in appropriate conditions, and also the disappointment which followed its employment in one of the earlier cases, which, owing to inexperience, was not properly selected.

Pyloroplasty is too dangerous in cases where there are extensive, severe hardening of the tissues, much peripyloritis, and adhesions to liver, gall-bladder, colon, etc., and in cases of duodenal stenosis. It is indicated in cases of spasmodic stenosis, and in slight annular stenoses from ulceration accompanied by muscular hypertrophy.

It may be that the more extensive operation suggested and carried out by Dr. Finney will be less liable to relapse, though as yet sufficient time has not elapsed to prove this. For description of this operation, see Chapter XVI., on Pyloroplasty.

Examples of cases treated by pyloroplasty : 
CASE I.-March 9, I 895.-Mrs. W., aged twenty-nine. 'Spasms' for ten years, but pain more on left side. Attacks two or three times a week ; start without apparent reason, last an hour or two, but may persist twenty-four hours, relieved by vomiting. Severe cramp in legs, loss of 2 stones in weight, no jaundice, marked constipation. Rigid right rectus; no rigidity, but tenderness to left. Dilatation of stomach well marked.

November 22, I 895.- Relief under treatment, followed by relapse; now vomiting daily. Weight 9 stones. Operation. Adhesions of pylorus separated. Active ulceration at pylorus and tight stricture. Pyloroplasty (bone bobbin).

July 24, I897.-Weight 9 stones 5 pounds, very much better. Relapse in 1898 , possibly from recurrence of ulceration. We then performed gastro-enterostomy. Quite well in I9oo, and former weight fully regained.

CASE 2.- Jamiary I 3, I897.-Mr. M. B., aged fifty-two. Ulcer of pylorus, with stricture. Pyloroplasty. Seen with Dr. Wilks, Grassington. Bad health for twenty years, with dyspepsia. Enteric fever nine years ago. Last two years much worse-pain, sickness, and vomiting two to three hours after meals, relieved by vomiting of large amounts. Never vomited blood. Loss of flesh. Weight 9 stones 3 pounds. Emaciation, dilatation of stomach. No tumour. Operation. Stricture of pylorus. Pyloroplasty (bone bobbin used).

September I9, I 898.-Dr. Wilks writes: "For some time little improvement; stomach now works well. Looks better than I have ever seen him.' Well February, I90 . Has gained normal weight. Recurrence of symptoms four years later, with tumour of the pylorus, for which gastro-enterostomy was performed.

CASE 3.-March I8, I 897.-Mrs. W., aged forty-six. Stricture, with active ulceration at pylorus. Pyloroplasty. Seen with Dr. Drake, Headingley. Gastralgia for several years, relieved by food. In November, I 894 , vomited dark fluid; since then frequent vomiting, longest interval two or three weeks. Pain in stomach, accompanied by hard lump, and often followed by vomiting. Great loss of flesh and strength.

Operation. Pyloroplasty for contraction and thickening of pylorus, passage only admitting a No. 12 catheter. Good recovery. Well I899. Considerable gain in weight.

CASE 4.-May 24, I 897.-Mr. H., aged thirty-nine. Seen with Dr. Matheson, Sheffield. Letter dated May I3, I897, to say: "During the last eighteen months I have suffered much pain, which has caused me to be bed-fast for two, three, or four weeks at a time, and it has required another month or more for me to gain strength enough to move about.' Eighteen months 
ago epigastric pain several hours after food, relieved by vomiting. Since then health never good; three and a half months ago similar attack, very severe, with collapse. Vomit contained blood. Fourteen days ago another severe attack. Normal weight Io stones Io pounds; now 9 stones $3 \frac{1}{2}$ pounds. Stomach 'weak' since childhood. Marked dilatation. No tumour.

Operation. Deep ulcer at pylorus. Extreme stricture, barely admitting ordinary director. Pyloroplasty (bone bobbin used). Complete recovery from operation, and rapidly regained normal weight. Letter dated February 16, I898, to say : 'I thought you would like to know that I am able to attend business as usual, and have done so without interruption since July 19, I897.'

CASE 5.-July I 2, I 897.-Mrs. W., aged forty-six. Seen with Dr. Johnson, Aysgarth. Said to have had ulcer of stomach twenty years ago. Since then subject to attacks of pain half to two hours after food; sometimes continuous pain. For three or four months vomiting three times a day. Lost a stone weight in that time. Leading life of an invalid, and for a long period under medical treatment without benefit. Dilatation of stomach; visible peristalsis; tenderness over stomach, especially at the pylorus. No tumour could be felt.

Operation. Stomach much dilated; thickening at pylorus. Pyloroplasty (bone bobbin used).

Good recovery. Weighed i I stones January 8, I898, a gain of over 2 stones.

Case 6.-July 27, I897.-Mr. C., aged twenty-three. Seen with Dr. Percival and Dr. Barrs. Vomiting and loss of flesh for two years. Was ro stones; now is 7 stones in weight. Dieting and lavage give only temporary relief. Emaciation, pallor, dilatation of stomach. No tumour.

Operation. Much-contracted pylorus, great hypertrophy, the walls more than $\frac{1}{i ;}$ inch thick. Pyloroplasty with bone bobbin. Good recovery.

December 23, 1897.-Weight 9 stones 2 pounds. Well.

Relapse in 1902. Tumour of pylorus due to chronic ulcer. Gastro-enterostomy. Complete recovery. Well in I903.

CASE 7.-W. F., aged fifty-two, seen with Dr. Cheesewright, Rotherham, in March, 1895. He had suffered from indigestion for two years. This, however, had not interfered much with his general health till the previous Christmas, when the indigestion was accompanied every second day by acute pain and vomiting, coming on about two hours after food. The vomited matter was in large quantity, offensive and sour, and at times coffee-ground in character. From this time the patient became extremely weak and pale, and rapidly lost flesh to the extent of $1 \frac{1}{2}$ stones in five 
weeks. He had no previous history of severe illness, nor did his family history denote hereditary tendency to disease. Patient extremely emaciated, anæmic, and weak; tongue coated and bowels constipated. He had pain on pressure over the pylorus, but no distinct tumour was felt. There was marked dilatation of the stomach, and during the attacks of pain it could be felt to harden under the hand. Heart, lungs, and urine normal. Our feeling was that, on account of the very rapid loss of flesh, accompanied by cachexia, the patient was suffering from cancer of the pylorus. Operation was advised with a view to performing pyloroplasty or pylorectomy, as circumstances demanded, since washing out the stomach and all ordinary medical means had given no relief.

On April 8, 1895, the abdomen was opened by an incision in the middle line above the umbilicus, exposing the pylorus, which formed a distinct tumour adherent to and under cover of the liver, and which, after being freed from adhesion to surrounding structures, was found to be tightly strictured, so as only to admit the passage of a No. I 2 catheter, the mucous membrane being extensively ulcerated and the walls thick and almost cartilaginous. The stricture was incised longitudinally and sutured transversely over a bone bobbin by a double row of sutures. The stomach was much dilated, atonic, and anæmic. Though the pyloric tumour gave rise at the moment to a suspicion of cancer, there was no evidence of growth and the glands were not affected. The after-progress of the case was uneventful, the wound healing by first intention. A month later he had gained 7 pounds in weight, and on October 30 , I896, he called to report himself, looking robust and well. He had gained 3 stones in weight since his operation.

Gastroplasty is an operation that we have successfully employed in a number of cases of chronic ulcer of the stomach leading to hour-glass contraction. The operation, which can be performed in several ways, will be fully described in the chapter dealing with that deformity of the stomach (see p. 349).

It consists in making a longitudinal incision through the strictured part of the stomach and bringing the edges of the wound together transversely, thus obliterating the stricture. A convenient method of performing the operation is by the use of a large decalcified bone bobbin, as described in the cases appended to the chapter on Hour-glass Contraction. 
If the strictured part of the stomach be actively ulcerating, as in a case related elsewhere, the ulcer must be excised at the same time-otherwise subsequent contraction may occur; or possibly the ulcer, already chronic, may persist, and lead to a continuance of the symptoms. In such a case, if excision be impracticable, gastro-enterostomy must be performed. (For examples see chapter on Hour-glass Contraction.)

The operation of Gastro-gastrostomy, in order to shortcircuit the constriction in hour-glass contraction of the stomach due to chronic ulcer, will be described in the chapter on Hour-glass Contraction, where the description of cases will be found.

Adhesions of the stomach to adjoining organs are so common in chronic stomach ulceration that Gastrolysis, or the detaching or otherwise treating bands and short adhesions to adjoining viscera or to the abdominal wall, is performed in by far the greater number of cases. Such adhesions are frequently the only remnants of ulcers that have healed; at other times they have been left by perforation of the stomach wall by an ulcer, from the dire consequences of which they have saved the patient. In many cases they give rise to symptoms resembling ulcer, though the adhesions may be due to causes such as gall-stones outside the stomach itself. In such cases the operation of gastrolysis may be entirely curative, but in the more severe cases it is advisable to perform gastro-enterostomy at the same time, as the adhesions are likely to re-form and again to cause trouble.

We propose to describe the operation under the chapter dealing with Adhesions, but give one case here as an example of the benefits to be derived from the opcrations of gastrolysis alone.

\section{Gastrolysis for Adhesions caused by Chronic Gastric} ULCER.

Miss M. B., aged forty-two. Seen with Dr. Holmes, Garstang.

History. - Twenty-two years ago symptoms of ulcer of stomach. Since then has suffered from vomiting attacks every week or two, and from pain after food. During the last three years symptoms 
more marked. Under medical treatment, with rest in bed, no improvement. Vomited matter large in quantity and fermenting, sometimes containing blood. Loss of weight to the extent of 3 stones. Great tenderness over stomach, especially to the left. Stomach dilated, reaching below umbilicus and well over to the right.

Operation. On anterior surface of stomach scar of an old ulcer. Lesser curvature closely adherent to the liver. Pyloric extremity and first part of duodenum attached to the gall-bladder and cystic duct.

January 2, 1900.-Operation. Adhesions separated and omentum interposed between pylorus and gall-bladder.

March 7, I900.-Perfect recovery. Can eat anything without discomfort, and is rapidly putting on flesh.

Has gained 20 pounds since her operation in January.

Some months later had gained 2 stones ro pounds.

Pylorodiosis, by which name is understood the operation of stretching the pyloric sphincter, either by means of the fingers invaginating the stomach wall, when it is known as ' Hahn's operation,' or by digital or instrumental stretching after having made an opening into the stomach, when it is known as 'Loreta's operation,' is a method of little practical value in the treatment of ulcer, and in some of the cases where we performed the operation, though the immediate results were good, relapses occurred.

The operation will be described under Pyloric Stenosis.

Treatment subsequent to 0peration.-After any operation for ulcer of the stomach, it is desirable that care in diet should be observed for some time, and that the patient should remain under the observation of his physician, so that the health may be fully restored before the ordinary mode of life is resumed.

Anæmia should be treated; oral asepsis should be obtained by the help of the dentist; any remaining gastritis should be remedied by appropriate remedies, so as to correct the hyperchlorhydria, and possibly prevent the formation of other ulcers and the development of peptic ulcers in the jejunum.

The operation should be looked on as an incident in the treatment of a chronic condition, and not, as is so often the case, as a final measure, which needs no subsequent supplemental care. 


\section{CHAPTER XIV}

\section{HYPERCHLORHYDRIA}

THE term hyperchlorhydria is used to define that condition in which there is secreted a gastric juice that contains an excessive quantity of hydrochloric acid. It is to be distinguished from hypersecretion or gastro-succorrhœa, in which the secretion of the gastric glands is poured out, not only during the time that food is present in the stomach, but also during the time of fasting. The two conditions are, without question, often associated, and in many cases the condition of excessive acidity precedes that of excessive secretion.

Gastric hyperacidity is a frequent precursor or accompaniment of many intractable diseases of the stomach, and its symptoms and significance are, therefore, worthy of close observation.

The history given by one who suffers from hyperchlorhydria is gemerally as follows :

On waking in the morning he is conscious of a sense of uneasiness in the pit of the stomach. This sensation, which may conveniently be called 'hunger pain,' is noticed always when the stomach is empty. There is a burning, oppressive feeling over the whole epigastrium; there is no localized tenderness. There is a bitter, acid taste in the mouth, and belching is frequent, yet compulsory; the patient resorts to it in the vain hope of getting relief. As the breakfast is eaten with, as a rule, a good enjoyment, the pain and discomfort gradually disappear, and by the end of the meal the condition is one of complete comfort. Such it remains for two hours or more, but generally within three hours the pain and 
epigastric discomfort return. Patients will notice that, if the hour of breakfast is punctually fixed, the time of the onset of the uneasiness can be foretold to a few minutes. As time passes, the burning feeling increases in intensity, though there may be intervals of a few minutes or even of half an hour in which complete relief is felt. When the mid-day meal is eaten, the feeling of distress again disappears, until, at its end, ease and complete content are experienced. Similarly, in two or three hours 'hunger-pain' begins once again, to be again relieved by food. And so the days repeat themselves. On each day the same sequence-fasting, hunger-pain, meal-time, relief-is renewed. In severer cases the night also may be disturbed. As a rule, the patient wakes between one and three o'clock with the accustomed feeling of distress, which is relieved only by food, or, less satisfactorily, by a copious draught of water. It is no uncommon thing for sufferers from this disease to have a store of biscuits or a glass of milk at the bedside, so that on waking their means of relief may be ready to hand. The sensation experienced in the epigastrium is rarely one of acute pain ; it is none the less intolerable. It is described as gnawing, or boring, or burning, or scraping. During its persistence intensely acid eructations are frequent, and the teeth feel like chalk, soft and rough. There is often a sensation of overdistension, almost of tightness and bursting of the stomach, which belching, imperative though it seems, fails utterly to relieve.

To explain these symptoms a brief reference to the process of gastric digestion is necessary. Food consists chiefly of three forms, proteid, starch and fat. In the mouth the starch, properly prepared by adequate cooking, which breaks up the starch capsules, is converted (or, rather, the conversion is begun) into sugar. As soon as the food enters the stomach, hydrochloric acid contained in the gastric juice comes in contact with it. When a certain acidity of the gastric contents is reached, the digestion of the starch is arrested, and the digestion of the proteids is carried on.

In order that the proteid digestion may be adequately 
maintained, fresh gastric juice, pepsin, and hydrochloric acid are poured out continuously until the affinities of the proteid food are satisfied. Beyond this point, in health, there is some further secretion of hydrochloric acid. At all but the earlier stages of digestion free hydrochloric acid can be found-that is to say, that hydrochloric acid above the amount necessary to digestion has been secreted. This amount, which is called 'free hydrochloric acid,' has a narrow range, between maximum and minimum, within which it may be considered normal. Beyond this maximum lies the condition of hyperchlorhydria, and hyperchlorhydria, therefore, implies an excess (not merely the presence) of free hydrochloric acid. When the full amount of gastric juice necessary to digestion has been poured forth, the proteid constituents of the food are changed with rapidity, and are speedily passed onwards through the pylorus. The starch, however, whose conversion has been arrested by the acid in the stomach is, probably in consequence of the hyperchlorhydria, retained in the stomach. In the stomach it acts as a foreign body, and, in precisely the same way as the glass rod in the experiments upon dogs, or the rubber tube passed into the stomach of a man, there excites a fresh output of secretion. This starch residue may be rejected by vomiting, when ease is obtained, or little by little may be passed onwards into the duodenum. The researches of Pawlow have shown that until the duodenal contents are rendered alkaline the pylorus does not relax to allow of the onward passage of food from the stomach. If, then, the food so passed onwards is excessively acid, the time of its neutralization must be greatly increased, and in this increased time the quantity of secretion in the stomach aroused by the foreign body is also increasing. In this way does hyperacidity cause pyloric spasm and gastric stasis, and probably also in this way may be caused gastro-succorrhœa, motor insufficiency, and dilatation.

The relationship existing between ulcer and hyperchlorhydria has given rise to endless speculation and much unprofitable discussion. The doubt remains as to whether 
ulcer is the cause, or the result, or is independent, of the hydrochloric acid excess.

There can be little doubt that the presence of hyperchlorhydria is intimately bound up with the existence of an ulcer, and with its perpetuation. It is possible that an ulcer may be formed when the gastric juice is normal, but the callousness of an ulcer and its chronicity must be due to an excessively acid secretion. Hyperacidity may in some cases exist without giving rise to obvious symptoms. The stomach contents of an individual who makes no complaint of gastric disorder may show at every examination during several weeks a high excess of free hydrochloric acid. On the other hand, there are cases of ulcer in which free hydrochloric acid is consistently absent. Ageron (Mïnch. Med. Woch., July 29, I902) has called attention to these recently.

The relationship of hyperchlorhydria to ulcer will, doubtless, be wholly or in part made clear by the investigations of the surgeons. When only post-mortem evidence was available, or such evidence, hæmatemesis, vomiting, etc., as to make the presence of ulcer indubitable, the question as to the existence of an ulcer in any case of hyperchlorhydria could not always - could, indeed, very seldom-be positively affirmed; but when the stomach is examined during life more frequently the surgeon will be able to furnish more abundant and more reliable evidence than is now available. Unfortunately, in our own series of cases of operations for ulcer of the stomach and its sequels, the chemical examination of the stomach has not always been made, and if made, has not been often repeated. But this we can positively affirm, that no case upon which we have operated, which showed on chemical examination of the stomach contents an excess of free hydrochloric acid, has failed to be a case of ulcer. In our experience, that is, hyperchlorhydria has never existed without ulcer in the stomach, or duodenum, or both.

In cases of duodenal ulcer the excess or free hydrochloric acid is often enormous. Indeed, we have come to the conclusion that, whatever may be the relationship existing between gastric ulcer and hyperchlorhydria, there can be no 
doubt that the latter condition is the direct cause of ulcer in the duodenum.

For the reduction in the excessive secretion of hydrochloric acid, belladonna, strychnine, carbonate of soda, with calcined magnesia and Carlsbad salts, are the most potent drugs. 


\section{CHAPTER XV}

\section{THE OPERATION OF GASTRO-ENTEROSTOMY, WITH INDICATIONS FOR ITS PERFORMANCE}

Perhaps no operation better illustrates the advance of surgery than does the one of gastro-enterostomy. It is only twenty-four years ago since Nicoladoni first suggested the possibility of making an anastomosis between the stomach and small intestine, so as to short-circuit obstruction at the pylorus, the first operation being performed by Wölfler, of Vienna, on September 27, I88I; his patient, who had cancer of the pylorus, lived for four months.

At that time, and for some years later, so little confidence was placed in this and similar operations on the stomach that patients, as a rule, were treated medically until almost moribund before being handed over to the surgeon, with the result that the mortality was appalling. This naturally retarded progress, since the deaths following on operations in moribund cases were mistaken for deaths due to the operation itself; and when we take into account the fact that out of 35 cases in which gastro-enterostomy was performed between the years I $88 \mathrm{I}$ and $\mathrm{I} 885$, there were only I2 recoveries-in other words, that the mortality was $65^{\circ} 7 \mathrm{I}$ per cent.-it is not to be wondered at that little confidence was placed in surgical methods. It rather reminds one of the beginnings of ovarictomy, in which the mortality was still higher, and it accounts for the prejudice against recommending surgical treatment that existed for many years among physicians, and that still exists among many of the older practitioners and in some of the more conservative schools. Even at the time of the Hunterian Lectures on the 
Surgery of the Stomach at the Royal College of Surgeons of England in I899, out of 1,978 cases of gastro-enterostomy collected from all sources, there had been a mortality of $36^{\circ} 4$ per cent.; not a very encouraging outlook, when one patient out of every 2.75 might be expected to die. It is, however, a very different tale that we are able to tell to-day, only four years later, when we can point to a considerable series of cases of posterior gastro-enterostomy in all classes of cases, with a mortality of only 5 per cent. This, we venture to think will be granted, brings the operation within the realm of comparative safety; and seeing that it has to be undertaken in many cases for diseases that would be otherwise inevitably fatal, it may be looked on as an operation the benefits of which not even the most conservative among us can afford to ignore.

These changes have been brought about, not only by patients being operated on at an earlier stage and under more favourable conditions--for many of our patients have been extremely ill at the time of operation-but also by greater care in technical details, by greater expedition in operating, by more careful asepsis, by the avoidance of, or greater care in the use of, irritating antiseptics, by the prevention or better treatment of shock, by care in postoperative treatment, such as early feeding subsequently to the operation, and by the careful preparation of the patient before the operation.

The following are special indications for the operation of gastro-enterostomy; but they cannot be stated in one category, for while in the first series the indications, with certain provisos, are absolute so soon as the diagnosis is established, in the second series the indications for the operation apply only after failure of general treatment.

In the first series are:

r. Simple stenosis of the pylorus.

2. Malignant stenosis of the pylorus.

3. Congenital stenosis.

4. Congenital atresia.

5. Chronic gastric ulcer, with tumour of doubtful character, too extensive or adherent for effectual removal, not necessarily associated with stenosis. 
6. Duodenal cancer or tumour causing obstructive symptoms.

7. Hour-glass contraction of the stomach not favourable for gastroplasty.

8. Perigastritis with adhesions around the pylorus producing obstructive symptoms or pain, and too extensive for gastrolysis.

9. Dilatation of the stomach dependent on pressure outside the pylorus from tumour of the pancreas, liver, or gallhladder, incapable of removal.

In the second series, after failure of medical treatment, are : Io. Acute or chronic gastric ulcer.

II. Duodenal ulcer.

I2. Hæmorrhage from gastric or duodenal ulcer.

I3. Persistent spasm of pylorus or Reichmann's disease, with dilatation of the stomach.

I4. Hyperchlorhydria.

I5. Persistent gastralgia.

I6. Tetany of gastric origin.

I7. Acute gastric dilatation not yielding to lavage and general treatment.

I8. Pancreatitis secondary to gastric ulcer.

I9. Certain cases of jaundice dependent on gastric or duodenal ulcer, leading to thickening around the common bile-duct.

20. Simple atonic dilatation of the stomach after failure of lavage and general treatment.

Although the operation of gastro-enterostomy has been performed in many different ways, there are practically only two distinct methods-one Wölfler's, in which the jejunum is fixed to the anterior stomach wall; and the other Von Hacker's, in which the anastomosis is effected between the jejunum and the posterior wall of the stomach. From a somewhat extensive experience of the two, we have no hesitation in strongly recommending the posterior where that operation is possible, for it must be granted that there are some exceptional cases where, on account of adhesions to the pancreas, involvement of the posterior wall of the stomach by growth, or from congenital deformity, the anterior method must be selected. 
Our preference for the posterior operation, whilst at first based on theoretical considerations, has been supported by ample practical experience of its advantages, and the smooth recovery, with absence of pain and vomiting, that one is accustomed to witness leads us strongly to advocate the method. It affords free drainage of the stomach into the bowel, and secures exact apposition by posture of the newlyjoined structures; hence there is little or no discomfort subsequently to operation, and feeding may be begun quite early. Moreover, as there is no loop of bowel constricting the colon, there is no fear of obstruction, and, owing to the unimpeded passage of the bowel contents, that distressing complication regurgitant vomiting of bile is avoided.

Each operation has its own advocates. We have more of late exclusively adopted the posterior operation. Mikulicz, whose experience is considerable, at one time abandoned the posterior method for the anterior, but has again given preference to the posterior; while Carle, in malignant cases, has abandoned the anterior for the posterior. The chief advantage of the posterior over the anterior is claimed to be that the opening is at a more dependent portion of the stomach, and therefore, while the patient lies upon his back. in bed after the operation, the stomach contents will the more readily drain into the intestine. When the junction is made on the anterior surface, however, the traction of the lesser bowel tends to bring the anastomosis to the lowest point of the stomach, as may be seen after the viscera are replaced, or especially when the parts are examined, if death follows some weeks or months after the operation. Under such circumstances, the opening is not seldom seen to be at the apex of a funnel-shaped portion of the stomach. It is said that regurgitation of bile and pancreatic juice is less likely to occur after the posterior operation, but the evidence on this point is not satisfactory. It is now proved beyond question that in all cases of gastro-enterostomy a certain amount of regurgitation of these juices into the stomach occurs constantly. Neither secretion in the least affects the digestion of food, as has been shown by Dastre, Oddi, Moynihan, and others.

The following cases afford examples from our experience 
of the beneficial effects of the operation of gastro-enterostomy under various conditions:

CASE I.-The patient, a man aged forty-five years, was seen at the Leeds Infirmary. He had suffered pain for two years, with vomiting for nine months. Great dilatation of the stomach was present. There were thickening and puckering near the pylorus. On June I2, Igoo, exploratory gastrotomy was performed. A large chronic ulcer was discovered on the posterior wall, as well as cicatricial stenosis of the pylorus. Posterior gastro-enterostomy was performed. Complete restoration to health resulted, and the patient was well in rgor.

CASE 2.-The patient, a man aged twenty-eight years, who was seen with Dr. G., was suffering from pyloric ulcer with tumour of the pylorus. Two years previously his weight was I 2 stones I 2 pounds; when seen it was 9 stones 6 pounds. He had pain two hours after food. For the last two months vomiting had occurred on an average five times a week, and on two occasions the vomit was like coffee-grounds. There was enormous dilatation of the stomach. The pyloric tumour was freely movable. There was visible peristalsis. Operation was performed on August 4, I899. A large mass was found at the pylorus which was evidently thickening, due to active ulceration. The glands were large, but not matted. Gastro-enterostomy was performed on the 27 th. The patient made a good recovery, and weighed 9 stones 7 pounds. On September 20, I899, his weight was Io stones $\mathrm{I}_{3}$ pounds. He could eat anything. $\mathrm{He}$ was well when seen in IgoI.

CASE 3.-The patient, a married woman aged thirty-eight years, who was seen with Dr. M., had suffered from indigestion for twenty years. The pain was constant; lately it was present one and a half hours after taking food. It was easier when lying down. She had lost I stone II pounds in weight. Posterior gastro-enterostomy was performed on January 8, 1901. Scars of ulcers were found on the pylorus and the anterior stomach wall. Stenosis of the pylorus was present, as well as enlarged glands in the lesser omentum. Recovery ensued. Her weight was 6 stones II pounds at the time of operation; two months later it was 7 stones 9 pounds. She was quite well and of normal weight when she was seen in October, Igo2.

The following cases serve to illustrate the beneficial effects of gastro-enterostomy in malignant stenosis of the pylorus incapable of removal, and we would take this opportunity of stating that we differ in toto from the opinion expressed 
lately by an eminent surgeon, who said : ' It is very doubtful if there is ever sufficient benefit to be derived from gastroenterostomy and other short-circuiting operations in malignant disease to make it worth while undergoing these operations. Needless to say, wherever the glands are not extensively involved, or there are no secondary growths, the tumour should be completely removed. In this class it might be expected that the mortality would be much higher than in simple disease, and in our first series of cases that was so, but later experience with the improved operation shows the immediate results to be almost equal to those of simple stenosis.

CASE I.-The patient was a married woman, aged thirty-seven years, who was seen with Dr. D. Cancer of the body of the stomach and pylorus with dilatation was diagnosed. Gastroenterostomy was performed on December 21, 1899. She made a good recovery, and was so well that gastrectomy was considered; but cancer of the uterus supervened and prevented further operation. She lived for nine months in comfort, and was able to take ordinary food.

CASE 2.- The patient, 'a man aged sixty-three years, had symptoms for five years, at first those of chronic ulcer, later those of malignant ulcer with tumour, associated with hæmatemesis. Gastro-enterostomy was performed on March 22, I90I. He made a good recovery. He returned home at the end of the month, and gained 4 pounds in weight during the fourth week. He ultimately gained about 2 stones, and lived for a time in great comfort ; but the growth progressed, and he succumbed to exhaustion about a year later, having been able to enjoy life for some months.

CASE 3.-The patient was a single woman, aged twenty-four years. A history was given of ulcer of the stomach, with frequent vomiting for five years, the vomit of late being coffee-ground in character. Great emaciation was present. She had been confined to bed for some months. Her weight was only 4 stones 5 pounds. The vomit contained free $\mathrm{HCl}$, with blood and sarcinæ. A tumour in the epigastrium could be easily felt and seen. Posterior gastro-enterostomy was performed on April I7, 1902. The tumour was adherent, and presented every appearance of cancer; the anterior wall of the stomach was extensively involved, the glands being largely affected. The patient made a good recovery, with a rapid gain in weight. Her medical adviser stated in October that the tumour had apparently disappeared, 
and that she had gained 5 stones, thus more than doubling her weight within six months of the operation. It remains to be seen if this is a case that should come under simple tumour simulating cancer.

The following is an example of cancer of the stomach where the patient was too ill for gastrectomy, in which a rapidly-performed gastro-enterostomy gave such relief that a subsequent radical operation was well borne:

CASE I. - The patient, a man aged sixty-three years, was seen with Dr. G., of Burnley, for stomach symptoms of a year's duration, a tumour having been noticed for a month. Gastro-enterostomy was performed on November I 5, rgoo. A central ring of cancer was found dividing the stomach into two cavities. The patient was too ill to bear gastrectomy. A good recovery was made, with a rapid gain in weight and strength, so that on December 20 the complete removal of the growth by gastrectomy was well borne, and when heard of a year later he was at work and in good health.

Chronic Ulcer with Tumour of Doubtful Character too Extensive or Adherent for Effectual Removal.-We have operated on quite a number of cases in which, after a long history of stomach symptoms, a tumour had developed which on external examination presented every appearance and the feel of cancer, and even when the abdomen was opened the tumour gave the same impression; but any enlarged glands were discrete, and there were general adhesions which precluded any attempt at removal. The following cases serve as illustrations, and to show that in some of these cases, where merely temporary relief only was anticipated, complete and permanent cure resulted from the operation:

CASE I.-The patient was a man, aged thirty-one years, who was seen with Dr. B. and Dr. W. He had had dyspepsia for seventeen years; this had been more severe during the last twenty months. Sixteen months previously vomiting began, and from the outset large quantities were ejected, but never contained blood. There was recurrence occasionally of similar attacks, which were always relieved by treatment. In December, I897, the stomach reached to the pubes, and visible peristalsis was present. Relief followed dieting and lavage until March, I898, since which time the pain had been almost constant. The pain was not materially worse after food nor relieved by vomiting. $\Lambda$ loss of weight occurred, from 10 stones to 8 stones $6 \frac{1}{2}$ pounds. 
There was great feebleness. Gastro-enterostomy was performed on May 6, I898. A large irregular tumour was found at the pylorus and along the lesser curvature, with extensive adhesions, but the glands, though large, were discrete. A good recovery was made, followed by relief of all the symptoms. When the patient left the home on June 7 , his weight was 8 stones; on August 17,1898 , it was 9 stones 3 pounds. The following is an extract from a letter received from the patient, dated February I2, I900: 'My health continues perfect. I have not lost a day's work through illness since I recovered.' He was in good health in 1903 .

CASE 2.-The patient, a man aged forty-five years, gave a history of pain for two years about an hour after food, with great loss of flesh. For nine months he had vomited every second day a large quantity of yeasty material, but no blood, though he was very anæmic. There were well-marked signs of dilatation of the stomach, with tenderness over the pylorus and the presence of a tumour. Posterior gastro-enterostomy was performed on June 12, 190o. On opening the abdomen, the pylorus was found to be much thickened and adherent, forming a hard nodular tumour, having the appearance and feel of cancer. Through the centre of the mass a No. Io catheter only could be passed over a roughened surface.

An uninterrupted recovery followed. Food was begun on the second day, and solids could be taken in the second week without pain. He rapidly gained flesh and strength, and is now well.

CASE 3.-The patient, a man aged forty-eight years, who was seen with Dr. R., was supposed to be suffering from cancer too advanced for treatment, the patient being extremely feeble, and having lost from 2 to 3 stones in weight in six months. The presence of a tumour in the pyloric region, with well-marked dilatation of the stomach and profuse coffee-ground vomiting, rendered Dr. R.'s diagnosis extremely probable, though the history of pain from one and a half to two hours after food for three years, with other symptoms of pyloric ulcer, led one to hope that the disease might be simple.

Operation was performed on May 30, 1902. An extensive tumour at the pylorus, associated with stenosis and dilatation of the stomach, with perigastritis and adhesions, together with numerous discrete, though much enlarged, glands, led us to perform gastro-enterostomy. The patient rapidly put on weight, gained strength, and lost all his distressing symptoms. When he returned to report himself in three months he was robust and well, and no trace could be found of the tumour or of the dilatation of the stomach. He is to-day in perfect health. 
These cases teach the important lesson that one ought not hastily to give a hopeless prognosis after gastro-enterostomy for tumour, for when there is any doubt whatever about the malignancy or otherwise of the growth it is well to give the patient the benefit that is derived from the hope of cure. Another lesson to be learnt is that an exploratory operation is worth performing in nearly all cases of tumour of the stomach, for, even if the growth cannot be removed, the rest engendered by a gastro-enterostomy will almost certainly give relief, and in some cases thought at first to be malignant a cure may be effected.

In cancer or tumour of the duodenum producing obstruction to the onward passage of the stomach contents, gastro-enterostomy acts exactly in the same manner as in cancer of the pylorus. As a rule, this form of disease in the second part of the duodenum is removable only with the greatest difficulty, in consequence of the important structures in the border of the lesser omentum being involved.

We were once tempted to perform such an operation, which involved removing the vertical part of the duodenum, part of the head of the pancreas, and about one inch of the common bile-duct, the passage of bile having to be made good by the performance of cholecyst-enterostomy, and of the stomach contents by gastro-enterostomy. The patient promised to do well for some days, but leakage from the duodenum ultimately led to death, from exhaustion. We shall in future be content with the performance of gastroenterostomy alone, as in a case on which we operated recently, where there was a well-marked tumour (probably inflammatory around a duodenal ulcer), which entirely disappeared after gastro-enterostomy, and the patient is now well.

Hour-glass contraction of the stomach may be due to chronic ulcer or to cancer. If the contraction be due to simple ulceration, gastroplasty is the operation of choice, and in a number of cases in which we have performed it the results have been excellent; but if the stricture be a long one, and the thickening be very extensive, then gastroenterostomy, in which the proximal cavity is united to the jejunum, is the better operation, as in the following cases, 
but great care must be observed that the junction is not made between the bowel and the distal cavity, as in at least two reported cases, otherwise no benefit will ensue. It should be the operation of choice when pyloric stenosis is associated with hour-glass contraction.

CASE I. - The patient, a man aged fifty-two years, who was seen with Dr. H., gave a history of pain, vomiting, and loss of weight and strength for three years. Epigastric tenderness was well marked. Posterior gastro-enterostomy was performed on October 12, Igoo. Hour-glass stomach was found, though the stricture was not extreme. The cardiac complement was also dilated. Recovery ensued. On March 4, Igor, a letter stated that the patient's weight was 9 stones I I pounds, and that he was in good health. At the time of operation his weight was 8 stones 6 pounds.

CASE 2.- The patient was a man who gave the history of stomach symptoms for three years. In one attack perforation occurred. Posterior gastro-enterostomy was performed on November I5, I90o. Strong adhesions to the liver and colon were found. The stomach was contracted in the centre. The patient made a good recovery, and when heard of subsequently he was in very good health.

In perigastritis with adhesions the operation of gastroenterostomy may be entirely.curative, as in many cases that we have operated on; but there are some cases where the adhesions are so extensive and the secondary dilatation of the stomach is so well marked that to rest content with simply detaching adhesions would be to court failure. If the pylorus is patent, yet embarrassed by adhesions, we always separate them, and try to avoid their recurrence by interposing the right free border of the omentum between the raw surfaces left by the gastrolysis, thus substituting a long, freely movable attachment for short binding ligaments should adhesions re-form. If, however, adhesions are very extensive and very short, dense, and firm, the operation of posterior gastro-enterostomy had better be done, and this especially if at the same time there is stenosis of the pylorus or hourglass stomach.

In December, I9oo, we saw a woman, aged fifty-seven years, suffering from constant pain at the epigastrium, intensified by 
food, so that she was unable to take any solids, and as she had been unrelieved by general and medical treatment, operation was performed, and the anterior wall of the stomach was found adherent to the abdominal wall and to the liver. The extensive adhesions were detached, and a posterior gastro-enterostomy was performed, with the result that she was entirely relieved of her distressing symptoms and has remained well.

In tumour outside the pylorus, but pressing on it and causing obstruction to the passage onward of the stomach contents, gastro-enterostomy may be required at the same time that the tumour is treated. The following is a case in which a pancreatic cyst was drained and gastro-enterostomy was performed at the same time:

A man, aged thirty-five years, after a history of ulcer of the stomach for four years, associated with pain an hour after food, and with vomiting and great loss of weight, was sent to us by Dr. G., who had tried medical treatment most thoroughly with only temporary relief. The patient's weight had gone down from I 70 to 130 pounds. From the history of the pain being relieved when the paiient turned into the prone position, and the fulness felt behind the stomach in the region of the pancreas, together with slight jaundice, it was suspected that the ulceration was in the posterior wall, and that the pancreas was becoming involved. Gastro-enterostomy was advised, but declined. The patient returned two months later in the last stage of exhaustion, and with all the stomach symptoms more marked, the stomach being now greatly dilated. There was a tense fluctuating tumour occupying all the epigastric region, and extending well over to the right side, which was diagnosed as a pancreatic cyst. Anterior gastro-enterostomy was performed on June I0, I902. A large pancreatic cyst was exposed containing four pints of grumous fluid. This was tapped through the great omentum, and then drained, the tube being fixed in the cyst by a purse-string suture, so as to make the opening embrace the tube tightly and prevent leakage. The posterior wall of the stomach and the pylorus were found to be fixed to the pancreas. The patient made a steady recovery, and rapidly put on flesh. All stomach symptoms disappeared, and he was able to return home within the month, looking and feeling well. Though the fluid collected in the pancreatic cyst three months later, it caused no return of stomach symptoms, and the insertion of a tube soon effected relief of the tumour. 
The following indications apply only after failure of systematic medical treatment:

In ulcer of the stomach, whether acute or chronic, not yielding to medical treatment, surgical treatment is in the greater number of cases the only satisfactory method of dealing with these refractory cases, and operation should be resorted to at a much earlier period than has hitherto been the custom, and always before the patient is so far reduced by pain and starvation or the supervention of serious complications that weakness and anæmia render any operative procedure hazardous. Before the abdomen is opened, it is quite impossible to say what operation or operations will be required, and the surgeon must be prepared to adapt himself to circumstances on discovering the position of the ulcer and the conditions associated with it, especially as to the presence or absence of adhesions and other complications. The following case, one out of many on which we have operated, serves to show the beneficial effects following on gastroenterostomy in intractable gastric ulcer. It acts by securing physiological rest of the stomach, and at the same time curing the hyperchlorhydria which is usually present.

The patient, a single woman aged thirty-two years, seen with Dr. A., gave a history of stomach symptoms extending over several years. She had had hæmatemesis in 1892 and 1896 , since which time she had suffered from flatulence and pain after food. For two years epigastric pain had been constant, but increased by food. For a year she had vomited daily, and for some months only milk in small amounts could be retained. Epigastric tenderness was well marked, and on distending the stomach with $\mathrm{CO}_{2}$ it reached $\frac{1}{2}$ inch below the umbilicus, and far over to the right of the middle line. Posterior gastroenterostomy was performed on October 5, Igoo. The patient returned home within the month, taking solid food without any discomfort and gaining fiesh. On November 8, Dr. A. wrote: 'I have seen Miss since her return, and there is every reason to be pleased with her condition. She has lost all her pain, and is taking food well. Allow me to thank you for her restoration to health.' Her weight in February, Igor, was 8 stones 6 pounds; at the time of operation it was 6 stones 12 pounds.

Duodenal ulcer very frequently fails to yield to general 
treatment, and from our experience of these cases we believe it is much more frequently the cause of fatal symptoms than is generally recognised.

The following out of a number on which we have operated will serve as an example:

A man, aged fifty-eight years, was sent by Dr. B. and Dr. S., of Burnley, with a history of stomach trouble for over ten years. Vomiting was not a prominent symptom, but on four occasions he had had profuse loss of blood by the bowel, though only on one occasion had he vomited blood. The attacks of bleeding had been alarming on several occasions, and not only had the motions been tarry, but much clotted blood had been passed. The last severe hæmorrhage had occurred ten weeks before we saw him, though slight bleeding had occurred since, and he was still profoundly anæmic. There was well-marked tenderness over the duodenum, with some dilatation of the stomach. Operation July I4, I902, when numerous adhesions were found around a thickened and indurated duodenum. A posterior gastro-enterostomy was performed, since which time he has remained well, and has had no further bleeding; moreover, he has regained flesh and colour.

In hæmorrhage from the stomach or duodenum, as shown by hæmatemesis or melæna, where general treatment has been tried and failed and where the bleeding is persisting or recurring after brief intervals, operation is advisable with a view to find and to secure, if possible, the bleeding-point or points, or of performing gastro-enterostomy in order to obtain physiological rest, and thus to favour natural hæmostasis.

The following are cases selected out of a number on which we have operated :

CASE I.-The patient, a married woman, aged twenty-eight years, was seen on April I4, I902, with Dr. T.s Dr. F., and Dr. R., of Nottingham. A few months previously the patient had been operated upon for varicose veins, and six weeks previous to the attack in question she had had influenza. On April 8 she was out hunting, and felt quite well. On the 9 th, at 12 noon, while in the house, she felt faint, and vomited a pint of dark clotted blood, after which she was put to bed and treated medically. She continued to improve, and had no further vomiting until April I4, when at I2.30 p.m. she suddenly felt sick, 
became blanched and faint, the pulse becoming very weak. Blood was vomited in large quantity, and when the bowels were moved by enema a large amount was parted with. In response to a telegram, I saw the patient with the three physicians, and as the bleeding seemed to have stopped, and as the pulse was improving, it was decided to give suprarenal extract, to stop all mouth feeding, and to feed by the rectum. Steady improvement again took place, but on the evening of April i 8 (the tenth day of the illness), we received a telegram saying: 'Hæmorrhage has recurred; have transfused. Come immediately, prepared to operate.' On arrival the patient was blanched, and with a pulse at times almost imperceptible. It was quite clear that the bleeding was persisting, and as medical treatment had been steadily persevered with for nine days, and there had been three separate hæmorrhages, it was decided to operate forthwith.

Dr. McGregor Young gave ether, and Mr. J. F. Dobson immediately assisted. On opening the abdomen, the stomach was found to be contracted, but the small intestine looked dark as if filled with blood. The stomach was opened and carefully explored, when, though no evidence of any large ulcer could be found, a considerable number of bleeding-points were discovered, three of which were ligatured en masse; afterwards the whole of the interior of the stomach was swabbed with a sponge saturated with tincture of hamamelis. Thinking it desirable to set the stomach at rest, the gastrotomy opening was closed and a posterior gastroenterostomy performed, using a decalcified bone bobbin. As the pulse was feeble, intravenous infusion of 4 pints of normal saline solution was performed. After this there is very little to record, except that the patient made a steady progress to recovery, and was able to be out of bed in the third week and to leave the house within the month. She rapidly regained her strength, had no further hæmorrhage, and is now quite well.

CASE 2.-The patient, a man aged forty-four years, was sent by Dr. M., of Huddersfield, on January 2, I goo, with the history that he had had pain after food, together with other symptoms of gastric ulcer for four years. Latterly he had had recurrent hæmorrhage. On his arrival at the surgical home, he was in a state of collapse, with a barely perceptible pulse. Tenderness over the pylorus, pain occurring one and a half hours after food, and melæna, left no doubt but that the ulcer was at or near the pylorus.

Operation was decided on and performed on January 4. The patient being in such a feeble condition, every precaution was taken to guard against shock. Posterior gastro-enterostomy was therefore done, a decalcified bone bobbin being used to make the 
anastomosis. The patient made an uninterrupted recovery. $\mathrm{He}$ was up at the end of three weeks, and returned home within the month. A year later he was in perfect health, and had had no recurrence of his stomach symptoms or any further bleeding.

CASE 3.-A man, aged sixty-three years, was sent by Dr. W., in March, IgoI, with a five years' history of chronic gastric ulcer and with a loss in weight of 6 stones. A year previously he had had severe hæmatemesis, which had been repeated slightly on several occasions. Within a few hours of his arrival, he vomited about 5 pints of clots and dark blood mixed with mucus, and he became so ill that death appeared imminent. Although he improved somewhat for a few days with rest, the vomiting and bleeding continued, and Dr. W. agreed that it would be better not to postpone operation. An hour before operation he vomited blood freely again. On the morning of March 22 the abdomen was opened, and the lesser curvature of the stomach was found to be much indurated, forming a tumour. There was also much puckering of the surface of the stomach, and the glands in the greater and lesser omenta were enlarged, but discrete. A posterior gastro-enterostomy was performed, a bone bobbin being used. He returned home at the end of the month, and ultimately gained about 2 stones. He had no further bleeding, and lived for a time in great comfort, but the growth, evidently cancer grafted on chronic ulcer, progressed, and he succumbed to exhaustion about a year later, having been able to enjoy life for some months.

CASE 4.-The patient, a female aged twenty-seven years, was admitted to the Leeds Infirmary on January 27, I902, for acute hæmatemesis. Beyond some indigestion, the patient had had nothing special to complain of until seven weeks before admission, when she was suddenly seized with severe pain at the epigastrium, and she vomited blood freely. The vomiting of everything taken continued for a fortnight, when she had another attack of hæmatemesis, after which she was sent to the hospital on the medical side. After her return home, vomiting recurred on her taking food again, and on January $2 \mathrm{I}$, after severe pain, she vomited about 2 pints of blood, and the vomiting continued up to her readmission on the 27 th. During the seven weeks she lost a stone in weight. She was transferred on February $I$, her pulse being on that day I 30 and respirations over 40 . On February 2, four days after the last hæmorrhage, operation was performed, and as no definite signs of ulcer could be found on the surface of the stomach, a posterior gastro-enterostomy was performed. She made a good recovery, and returned home at the end of three weeks. Though on one occasion in June she had indigestion for a day and vomited, there had been no return of the bleeding, and 
she had regained flesh and health when last seen six months later.

In persistent spasm of the pylorus, or Reichmann's disease, leading to dilatation of the stomach, pylorodiosis, or stretching the sphincter, may be effectual in relieving spasm and in producing immediate relief to the obstruction; but as it is apt to be followed by relapse, we have given up performing the operation in favour of gastro-enterostomy.

Not feeling satisfied with the calibre of the pylorus in one case after stretching it as far as thought safe, we immediately performed pyloroplasty. The condition then revealed showed why relapse often occurs, for two rather extensive lacerations through the mucous membrane quite into the substance of the pylorus were found. As these lacerations would have had to heal under the unfavourable condition of being constantly irritated by food and the acid contents of the stomach, there must have been a considerable effusion of plastic lymph, which would inevitably have led to cicatricial contraction, and so to stenosis. This, we suspect, occurs in all cases where the pylorus is fully stretched, and explains one reason for our having no faith in Loreta's operation.

A number of cases of chronic gastric ulcer, cured by gastroenterostomy, afford examples of spasm of the pylorus, but in the following case the hypertrophy was so marked as to suggest tumour of the pylorus.

Mr. S., just over fifty years of age, who had suffered from indigestion for several years, began to have severe pain about an hour after food and to lose flesh and look ill during the six months before we saw him. A doubtful tumour could be felt, and during the attacks of pain the stomach could be felt to harden under the hand. Free $\mathrm{HCl}$ was present after a test meal. The serious nature of his ailment was brought home to the patient by his inability to continue his business, and on the advice of his medical man, Dr. McGregor Young, and of Dr. Barrs, who saw him in consultation, we saw him, and supported the opinion as to exploration. On operating, we found the swelling to be a greatly thickened pylorus forming a distinct tumour, and that about $\mathrm{I}_{2} \frac{1}{2}$ inches from the pylorus along the lesser curvature was another tumour, which, from the enlargement of the glands, we suspected might be growth; but on opening the stomach it was found that the 
pyloric swelling was an enormously hypertrophied sphincter, and that the stomach tumour was thickening around a chronic ulcer. The latter was freely excised, the edges being brought together by continuous sutures. The stomach opening was closed, and a posterior gastro-enterostomy was then performed. As the gallbladder was full of thickened mucus and gallstones, it was at the same time emptied and drained. A smooth recovery has followed, much to the relief of the patient and his friends.

Hyperchlorhydria is a concomitant of ulcer of the stomach, though it may occur apart from ulceration. As a rule, it yields to medical and general treatment, but where this fails to relieve and the life of the patient is being made miserable by constant indigestion with acid eructations, the operation of gastro-enterostomy is well worth consideration, especially when in such a case it can be performed with almost no risk; for in the absence of complications such as occur in ulcer and cancer, the operation, when properly performed by an expert, ought to have no mortality. (See p. 202.)

Persistent Gastralgia.-Cases must have occurred in the practice of every physician where, although the positive signs of ulcer were absent, gastralgia of such intensity has persisted, and in case of temporary relief has recurred so regularly, that the patient is brought to the last stage of exhaustion by an utter inability to take food, because of the pain induced by even a mouthful of solids. Some of these cases are doubtless due to simple ulcer, but in others the absence of tenderness in the epigastrium, of rigidity of the recti, of regular vomiting, and of hæmatemesis, makes the diagnosis extremely doubtful. Even rectal feeding and absolute rest in bed do not always cure the condition, and the patient gradually loses weight and strength and lapses into a state of chronic invalidism, without any positive sign of organic disease. After all ordinary means have failed, gastro-enterostomy is well worthy of consideration, and, as the following case will show, it may lead to excellent results:

The patient was a single woman, aged twenty-seven years, who was seen at the Leeds Infirmary. She had suffered from pain and indigestion since she was a girl; vomiting occurred at times, though she never brought up blood. The stomach was dilated 
and tender. Free $\mathrm{HCl}$ was found in the contents of the stomach. She had lost weight, and weighed now 7 stones 6 pounds. Gastro-enterostomy was performed on March 7, I901. No scarring or other signs of ulcer were discovered. Soft solids were allowed at the end of the month. She made a good recovery, and was able to take food without pain; ultimately she was completely restored to health.

Tetany of Gastric Origin.-The prognosis of tetany occurring in gastric dilatation is undoubtedly very serious, and, according to some authorities, death occurs in 75 per cent. of the cases. The largest mortality occurs in cases where the cramps in the extremities are associated with tonic spasms in the head and trunk muscles or with clonic spasm. Our own experience of tetany and of the severe muscular spasms in association with gastric dilatation leads one to think that we may possibly take a more hopeful view if the cases be treated surgically at an early period. (See p. 408.) The following case will serve to illustrate these views:

A man, aged thirty-four years, was sent by his medical attendant in January, 1895 , with a view to having something done in the Leeds Infirmary to relieve his condition. He had been suffering for five years from pain after the ingestion of food, with attacks of vomiting. Latterly his condition had become aggravated, the pains occurring from half an hour to one hour after each meal, and being relieved only by vomiting, so that for the last six months he had had to give up his work as a printer. For some time before coming under my notice there had been very evident peristalsis from left to right in the epigastric region. Throughout the period over which his stomach symptoms extended, his bowels had for the most part been very constipated, but there were occasional attacks of diarrhœea. During this time he had attacks of what he described as 'cramps' in his limbs, and especially in his legs. He had lost very considerably in weight, as in December, 1892, he weighed 9 stones $7 \frac{1}{2}$ pounds. On examination, there was found well-marked dilatation of the stomach, but the feature of the case which presented most interest in connection with the present question was the occurrence while under observation of severe tetanic spasms affecting almost all the muscles of the body. So extreme and so widespread were these, the muscles of the trunk and of the cervical region as well as those of the limbs being affected, that on January 17 the question of possible strychnine poisoning was. 
raised. As palliative treatment of the stomach condition gave no relief, the stomach was exposed on the 24 th, and the diagnosis of cicatricial stenosis with hypertrophy of the pylorus was confirmed.

Pyloroplasty was performed, a bone bobbin being employed to insure patency of the new pylorus. Recovery was uninterrupted, and although the cramps were present up to the time of operation, he never had a threatening of cramp afterwards. On February I 5 he was able to take a mutton chop for his dínner without inconvenience, and by March 2 (five weeks after operation) he had gained 8 pounds. Two years after the operation he had quite recovered his strength, and was working as usual, his weight then being just over what it was in December, I892, and quite 2 stones above what it was at the time of operation. There had been no recurrence of 'cramps' or of muscular spasms of any kind. In a letter received at the beginning of 1899 he speaks of some stomach disorder, but he adds: "You will please understand that I am a new man to what I was when you saw me last.' A recurrence of the stenosis necessitated gastro-enterostomy, which was performed early in I902, but it is interesting to note that on this occasion, though he was much wasted, and was vomiting every day, there was no return of the tetany. He made a good recovery, and is now well and strong again.

Acute gastric dilatation is one of the most serious diseases that can be encountered. It was first described by the late Dr. Fagge, his paper being illustrated by two cases, both in men, thirty and thirty-one years of age respectively, and both ending fatally. In the Leeds Infirmary, during the past ten years, there have been at least four fatal cases. (See p. 389.)

We would suggest that in every case of this kind where stomach lavage has failed, but only then (for the stomach tube is the first indication, no matter at what stage it may be recognised, unless, indeed, the patient be actually dying), the abdomen should be opened and the stomach should be emptied and connected with the jejunum, thus providing for continuous drainage into the intestine. We believe that as yet gastro-enterostomy for acute dilatation has not been put to the test.

Pancreatitis.-In an exceptional case of ulcer of the stomach eroding the pancreas and producing pancreatitis, with abscess draining into the stomach and acute gastritis, a gastro-enter- 
ostomy apparently saved the life of the patient by draining the very foul contents directly into the intestine.

Cholelithiasis may lead to perigastritis, and the contraction of bands of lymph around the pylorus may produce obstruction or kinking, thus leading to dilatation of the stomach, at the same time that the concretions lead to obstruction of the bile-ducts. As a rule, the condition can be relieved by gastrolysis, but where the adhesions are extensive or the thickening of the pylorus is very pronounced, the operation of gastro-enterostomy may have to be performed at the same time as the cholecystotomy. Gall-stones may also lead to fistula between the gall-bladder and pylorus, and the thickening of the pyloric canal may lead to obstruction, for which gastro-enterostomy may be the most suitable operation, though in the cases that have come under my care the repair of the fistula in one case and pyloroplasty in another were sufficient when combined with cholecystotomy to remedy the disease.

In atonic dilatation of the stomach, general and medical treament, supplemented, if need be, by electrical treatment and lavage, if carried out systematically and for a sufficient length of time, usually yield good results; but in some cases, despite regular lavage of the dilated organ, well-regulated diet, and general medical treatment, the dilatation persists, and the nutrition of the patient and the general health become seriously impaired. In such cases the operation of gastrorrhaphy or gastroplication may be worth considering, though in certain cases gastro-enterostomy may possibly be performed with advantage. The indication for operation in these cases is not so distinct as in the cases of dilatation due to pyloric obstruction, seeing that the cause is a general one, nor is the recovery after operation so rapid and satisfactory. The general health requires considerable attention at the same time that the stomach is being dealt with. (See p. 430.) 


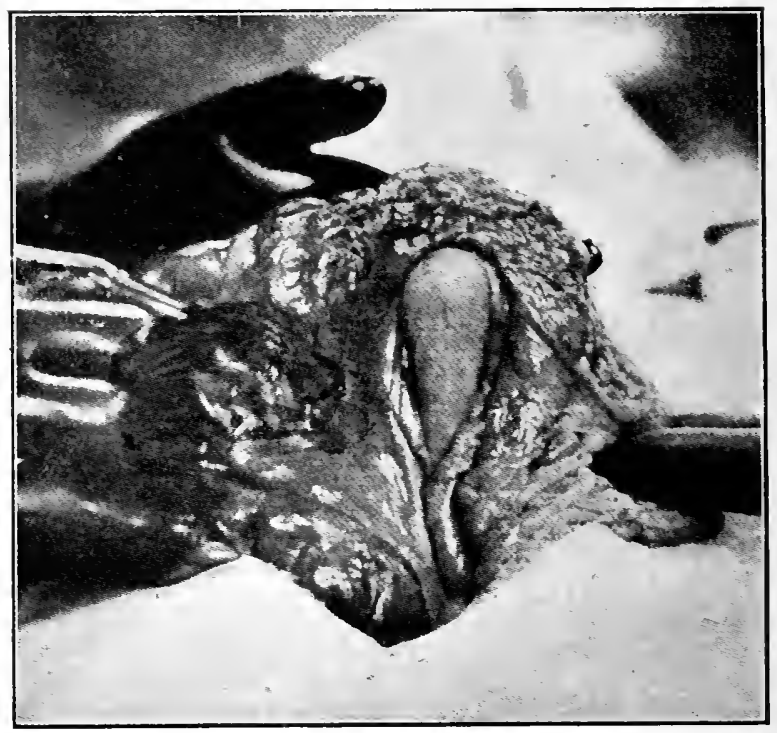

Fig. 49.-Showing the Posterior VVall of the Stomach projecting throvgh the Rent in the Transverse Mesocolon.

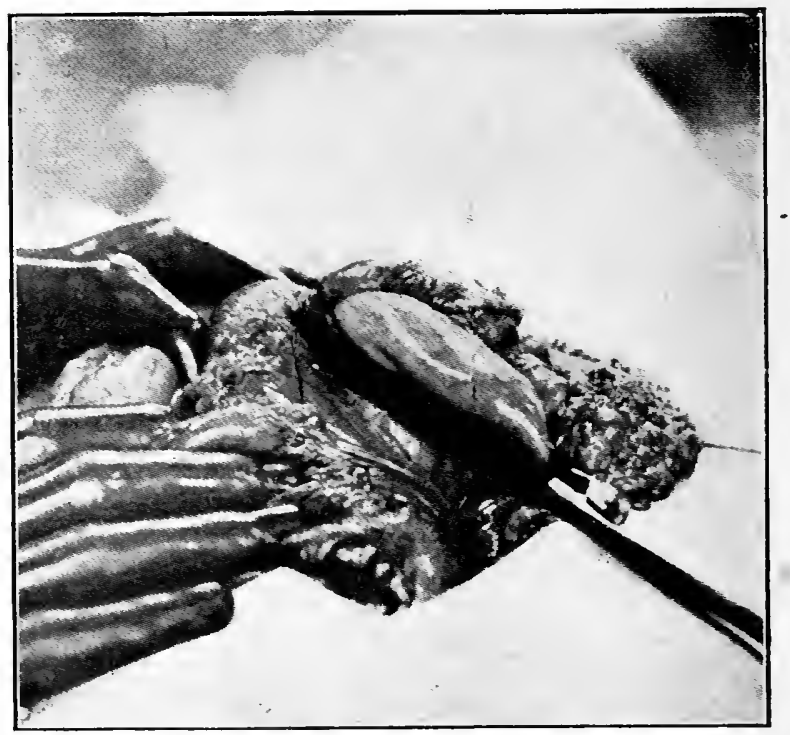

Fig. 50.-Showing the Obligue Application of the Clamp to the STOMACH. 


\section{Operation.}

The operation may be done by simple suture, by suture over an internal temporary splint in the shape of a decalcified bone bobbin, or by the Murphy button.

Operative Technique.-(a) By Simple Suture.-The abdomen is opened about $\frac{3}{4}$ inch to the right of the middle line, and the fibres of the rectus are split. After opening the peritoneum, the great omentum and transverse colon are lifted out of the abdomen and turned upwards over the epigastrium. The under surface of the transverse mesocolon is exposed, and the vascular arch formed mainly by the middle colic artery is seen. A bloodless spot is chosen, a small incision made in the mesocolon, and the finger passed into the lesser sac. The opening in the mesocolon is then gradually enlarged by stretching and tearing until the fingers can be passed through it. It is very rarely necessary to ligature any vessel. The hand of an assistant now makes the posterior surface of the stomach present at this opening; and the surgeon grasps the stomach and pulls it well through. A fold of the stomach, about 3 inches in length, is now seized with a clamp specially made for us by Messrs. Down Brothers, the blades of which are sheathed in rubber tubing. It is important that the point on the greater curvature held by the clamp should be the lowest point. This is made certain before the stomach is turned over to reach its posterior surface, by observing that point which lies lowest in the abdomen. When the posterior surface is exposed, special care is taken that this lowest point is fixed in the grasp of the clamp. The duodeno-jejunal angle is now sought, and readily found by sweeping the finger along the under surface of the root of the transverse mescolon to the left of the spine. The jejunum is then brought to the surface, and a portion of it, about 7 or 8 inches from the angle, is clamped in a second pair of clamps. The two clamps now lie side by side on the abdominal wall (Fig. 49), and the portions of stomach and jejunum to be anastomosed are well outside the abdomen embraced by the clamps. The stomach, with the exception of the part embraced by the clamp, is returned to 
the abdomen through the upper part of the incision. The whole operation area is now covered with gauze wrung out of hot sterile salt solution, the clamps, with the stomach and jejunum which they embrace, alone being visible outside the abdomen. A continuous suture is then introduced, uniting the serous and subserous coats of the stomach and jejunum. The stitch is commenced at the left end of the portions of gut enclosed in the clamp, and ends at the right. The length of the sutured line should be at least 2 inches; its average length is $2 \frac{3}{4}$ or 3 inches. In front of this line an incision is now made into the stomach and jejunum, the serous and

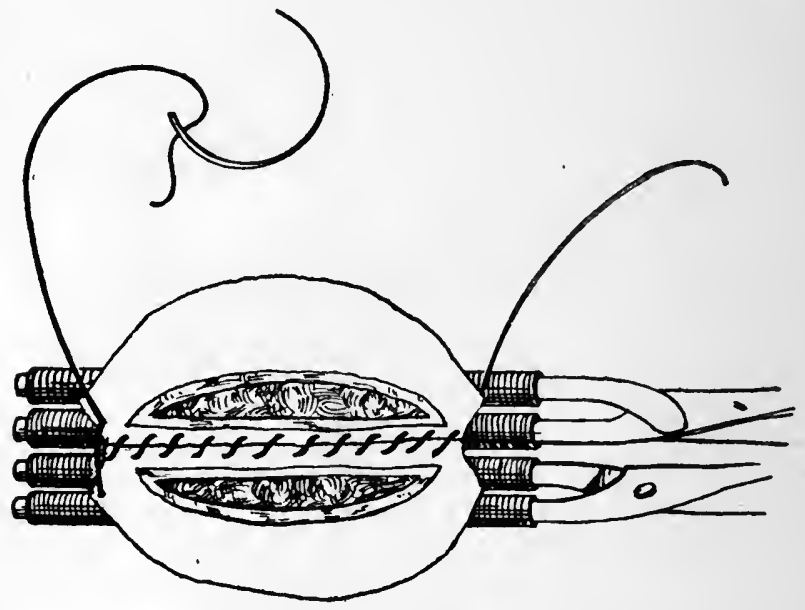

Fig. 51.-Clamps Side by Side; the First Line of Suture.

muscular layers of each being carefully divided until the mucous membrane is reached. As the cut is made, the serous coat retracts, and the mucous layer pouts into the incision. The cut edge of the serous coat is loosened all round from the underlying mucosa. An ellipse of the mucous membrane is now excised from both stomach and jejunum, the portion removed being about $\mathrm{I}_{4}^{3}$ or 2 inches in length, and rather more than $\frac{1}{2}$ inch in breadth at the centre. The stomach mucosa shows a marked tendency to retract; it is therefore seized with a pair of miniature (French) vulsella on each side. No vessels are ligatured as a rule. The cut surface of the bowel and 
stomach may occasionally ooze slightly. This can be checked at once by tightening the clamps one notch. The inner suture is now introduced. It embraces all the coats of the stomach and jejunum, and the individual stitches are placed close together and drawn fairly tight, so as to constrict all vessels in the cut edges. The suture begins at the same point as the outer one, and is continued without interruption all round the incision to the starting-point, where the ends are tied and cut short. It will be found that there is no need to

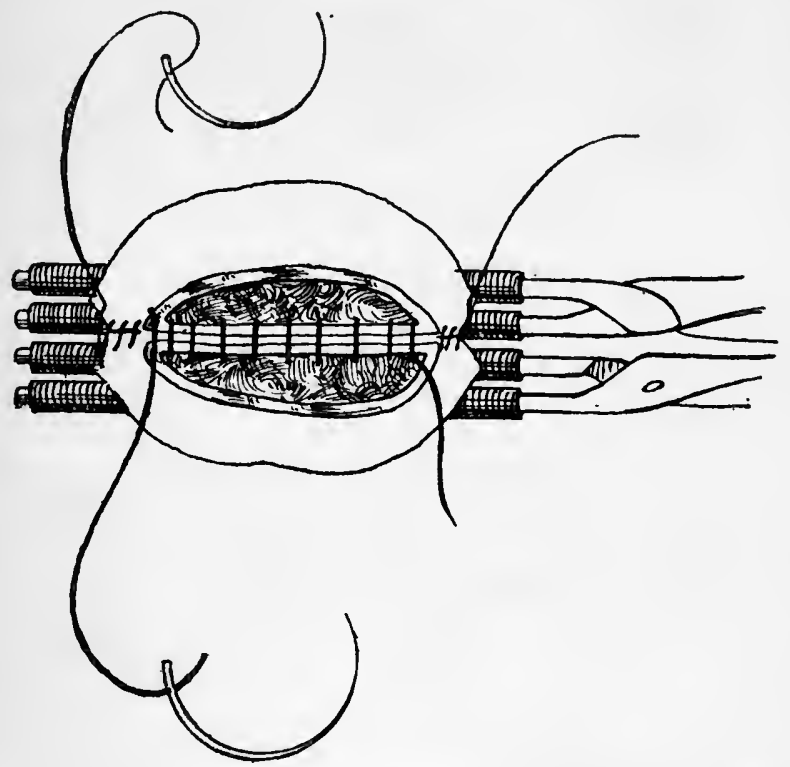

Fig. 52.-The Stomach and Jejunum opened; the InNer, Hemostatic SUTURE.

interrupt the stitch at any point, for there is no tendency on the part of the sutured edges to pucker when the stitch is drawn tight. The clamps are now removed from both the stomach and the jejunum, in order to see if any bleeding-point is made manifest. Very rarely, about once in ten cases, a separate stitch at a bleeding-point is necessary. The outer suture is now reassumed and continued round to its starting-point, being taken through the serous coat about it: inch in front of the inner suture. This outer stitch is also 
continuous throughout. When completed, the ends are tied and cut short as with the inner stitch. There are thus two suture lines surrounding the anastomotic opening : an inner,

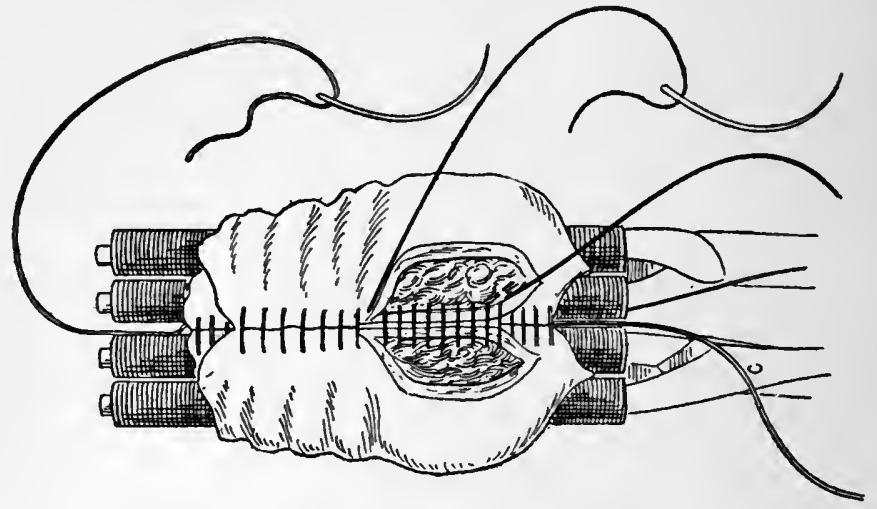

Fig. 53.-Inver Suture Complete.

hæmostatic, which includes all the layers of the gut; and an outer, approximating, which takes up only the serous and subserous coats. For both stitches thin Pagenstecher thread is employed, or the serous suture may be of celluloid thread

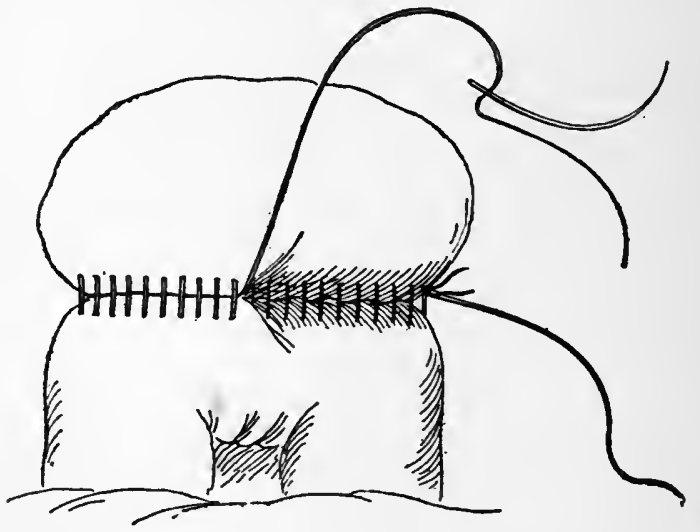

Fig. 54.-Clamps removed; Completion of Outer Suture.

and the inner suture of catgut. The needle used may be of the ordinary curved sewing-needle pattern or of a special pattern, being rather more than half a circle and having a 
slot-eye for easy threading. (These needles are made by Down Brothers, of London.) In introducing the stitches, it will be found a great advantage to draw lightly upon the thread between the needle and the last stitch. A little ridge is thus raised up both in the stomach and in the intestine, and into these ridges the needle passes quite readily. There is then no need to dip down, as it were, to pick up the gut on the point of the needle. No sutures are passed through the mesocolon and stomach. The gut is lightly wiped over with a swab wet in sterile salt solution, the stomach and omentum lifted out of the upper part of the wound, and replaced within the abdomen in their normal positions.

Other intestinal anastomoses are carried out in an exactly similar manner. The portions of gut to be united are lifted outside the abdomen, clamped, the clamps approximated, all the bowel, save that embraced by the clamps, returned within the abdomen, the operation area surrounded by swabs or gauze wrung out of hot sterile salt solution, and the stitching begun. The length of the opening made can be varied at the will of the operator.

If Roux's method of gastro-enterostomy be performed, a longer loop of jejunum is placed in the clamp. The proximal part of the jejunum is now divided about $\mathrm{I} \frac{1}{2}$ inches from the clamp, the distal limb then anastomosed to the stomach, clamped as in the ordinary operation of gastroenterostomy, and the proximal limb united to the side of the distal. The suturing of the intestine is all carried out upon the long loop as it lies with its ends gripped by the clamp.

(b) With the Bone Bobbin.--The preliminary part of the operation is the same. The stomach and intestine being placed in apposition, a continuous Lembert stitch of spun celluloid thread unites the serous surface for a distance of 2 inches or more. The line of this stitch is semi-oval, and joins the surfaces of the viscera close to the posterior border of the anastomotic opening. Incisions are then made into the viscera in front of the suture. A second continuous suture is now passed, uniting the posterior cut edges; this takes all the coats, and is of catgut. After completing the posterior 

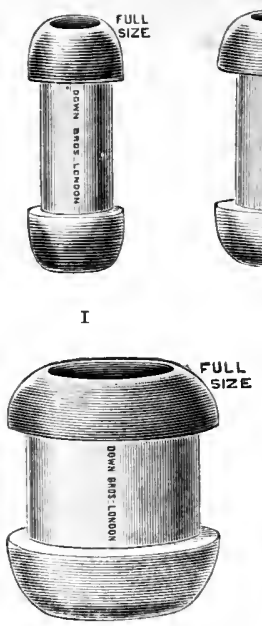

5

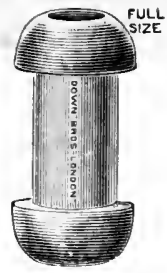

2

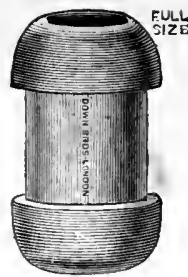

3

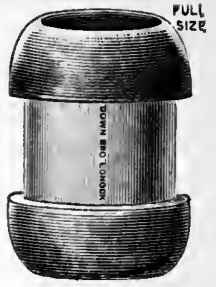

4

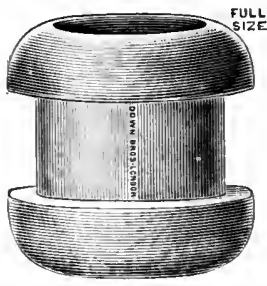

6

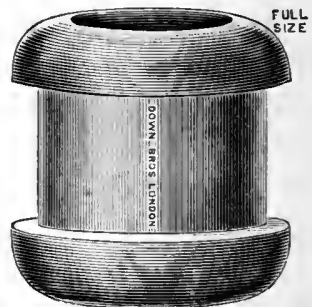

7 .
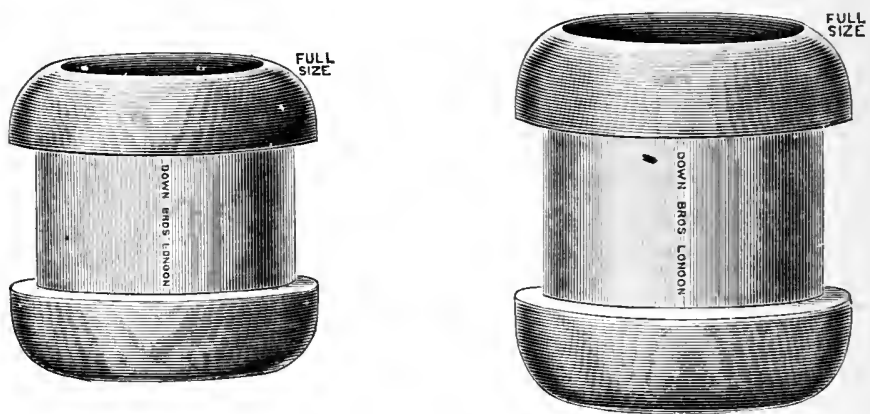

8

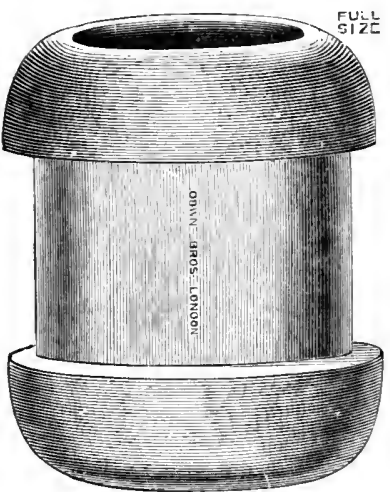

Io

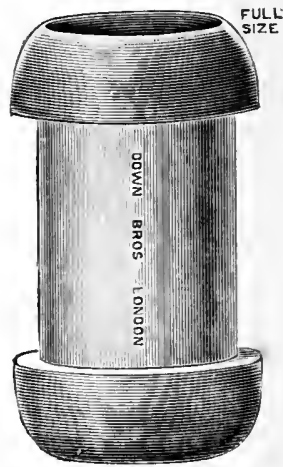

II

Fig. 55.-Mayo Robson's Decalcified Bone Bobbins (The various sizes are here shown.) 
half, the bobbin is introduced. 'The anterior semicircles of stitches are now continued, first the catgut circle to the cut edges, and then the celluloid circle to the serous coats, in precisely the same fashion as the posterior sutures were applied. The sutures are drawn firmly on to the bobbin in tying.

The bobbin, which is made by Messrs. Down Brothers, of London, in various sizes, from the small one required for cholecystenterostomy or for the union of the small viscera of children, to the large one employed for gastroplasty or for the end-to-end union of large intestine in the adult, is nothing more than a cylinder of decalcified bone with raised ends, which is placed in the new anastomotic opening, around which the sutures are applied.

The advantages claimed for the method are:

I. That it secures the opening, being of the exact size intended, and that there is no possibility of the passage being made too small by the drawing up of the sutures before the knots are tightened.

2. That it secures an immediately patent channel between the two anastomosed viscera.

3. That the bobbin protects for from twenty-four to fortyeight hours the new line of union from pressure and from the irritation of the visceral contents.

4. That it facilitates the application of the sutures and so expedites the operation.

5. That no foreign material is left in the alimentary canal, which may irritate or cause subsequent trouble, for the bobbin rapidly dissolves in the alimentary juices.

That the method has now been proved by ample experience to be rapid, easy, efficient, and safe.

For stomach operations it is used in gastro-enterostomy, in pyloroplasty, in gastroplasty, and in pylorectomy or partial gastrectomy, and in the latter operation it is unnecessary to use more than two continuous sutures for the whole operation.

(c) The Murphy Button Method.-The preliminary part is the same as in the other operations. The purse-string suture usually employed is unnecessarily cumbersome, and probably harmful, puckering up the coats of the gut around the central tube, and preventing accurate fitting of the opposed serous 
surfaces when the button is closed. The better method is to make a small incision into the viscus, and introduce the half button until the cylinder is tightly gripped. If the stitches are to the left of the button in the stomach, they may be with advantage put to the right in the jejunum, in order to secure perfect accuracy of serous coaptation. Though Murphy's button enables a rapid and effective anastomosis to be made, the remote dangers are not inconsiderable. We hardly think it likely that we shall ever use it again in performing gastro-enterostomy.

Anterior Gastro-enterostomy.-The foregoing descriptions apply to the posterior or Von Hacker's operation, but in some exceptional cases, either on account of extensive adhesions, involvement of the posterior wall of the stomach in the disease, or from congenital deformity, in the shape of a very short mesocolon, the posterior operation may be impracticable, when Wölfler's anterior method may have to be done.

In that case, a longer loop of jejunum is brought forward around the transverse colon, and the junction is effected to the lower margin of the stomach at a point about $\mathrm{r} 2$ or 13 inches from the commencement of the jejunum. In order to prevent kinking, it will be found advantageous to insert one or two sutures between the stomach and bowel on the distal and proximal side of the anastomosis.

\section{Complications.}

The complications that may follow gastro-enterostomy are:

r. Regurgitant vomiting.

2. Contraction of the new orifice.

3. Peptic jejunal ulcer.

4. Pneumonia or other chest complications.

5. Adhesions.

6. Intestinal obstruction.

7. Non-union and separation of the anastomosed viscera.

8. Hernia of the intestine through the loop in the anterior, or through the mesenteric slit in the posterior operation.

9. Exhaustion.

Io. Hæmorrhage. 
PLATE XIX.

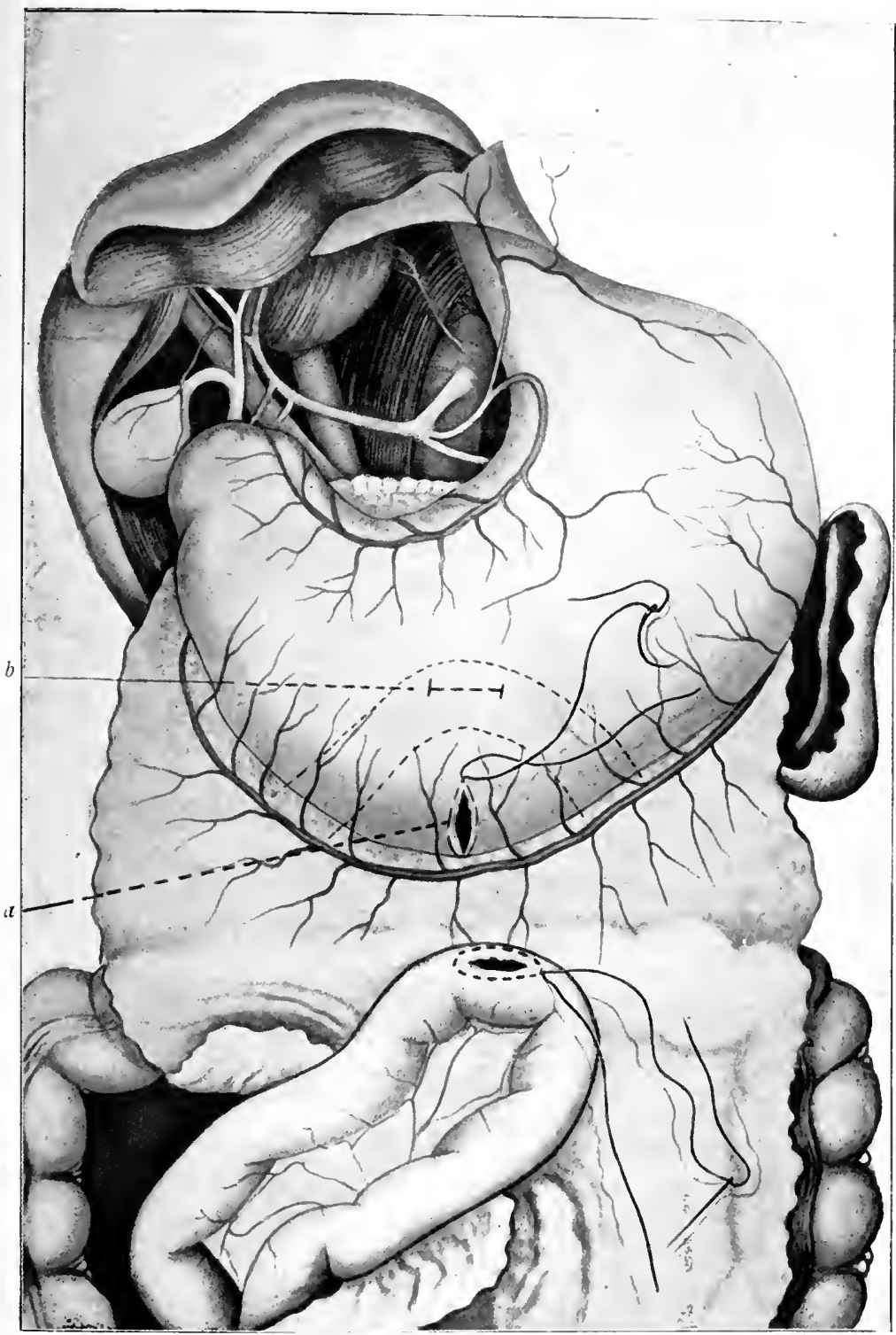

Fig. 56. - Anterior Gastro.enterostom:, showing Proper and Improper Locations of Opening. (After Mayo.)

$a$, Proper position, leaving no pouch ; $b$, usual position, forming intragastric To face $p .234$. ] pouch. 

Regurgitant Vomiting. - Regurgitant vomiting has been very much more frequently seen after anterior than after posterior gastro-enterostomy, and in our earlier cases when we performed Wölfler's operation it was not an uncommon sequel, but since adopting the posterior operation it has been very rarely seen. At times the vomiting is trivial, occurring but once or twice; at others it is most serions, and hastens or determines death.

Where the patient shows any tendency to vomit after a gastro-enterostomy, the head and shoulders should be well raised by pillows, so as to place the patient in the semirecumbent position; the stomach should then be washed out, this being repeated if necessary, and mouth-feeding should be replaced by rectal alimentation. A grain of calomel, repeated in a few hours and followed up by small doses of apenta water until the bowels are moved, will frequently stop the vomiting; but if these fail, an entero-anastomosis will be advisable, the two arms of the jejunal loop being anastomosed.

As to the cause, our own view is that regurgitant vomiting is essentially due to obstruction to the passage onwards of the duodenal contents, either from paresis of the bowel that has been handled or from kinking of the gut at the site of the insertion or lower, or later to adhesions interfering with the regular vermicular contraction of the intestine. The following case affords a good example :

A single woman, aged twenty-eight years, sent to us on December I2, I90I, gave a history of pain after food and vomiting of three years' duration, the weight having diminished from 9 stones to 6 stones. When seen she could only take sips of milk and water, and had constant epigastric pain. There was also coffee-ground vomit. The outline of the stomach on distension with $\mathrm{CO}_{2}$ was hour-glass in shape, and there was marked epigastric tenderness. After a gastro-enterostomy she lost her pain and gained weight, but three months later there was a letter to say that she had daily vomiting of bile in large quantity, and though she could take food, it did not nourish her. In June the regurgitant vomiting was so troublesome that she was admitted to the Leeds Infirmary for observation, and after a few days the abdomen was opened, and a band of lymph was found passing 
from the transverse mesocolon across the distal loop of jejunum, and so constricting it as to produce obstruction. The fistula between the stomach and jejunum was fully patent, and had not contracted in the least. The constricting band was divided, and in order to make the result more certain the two arms of the jejunal loop were connected by a bone bobbin about 3 inches away from the stomach fistula. Recovery was uninterrupted, the vomiting ceased entirely, and she returned home within the month, well and able to take normal food. The improvement has continued, and the patient is now well.

Several 'complementary operations' have been suggested in order to prevent regurgitant vomiting. Roux and Doyen cut the intestine across, implant the distal end into the stomach and the proximal into the side of the distal, and thus avoid the circulus vitiosus. Jaboulay, Braun, and Weir advise a side-to-side approximation of the proximal and distal loops.

W. J. Mayo, of Rochester, Minnesota, considers that unless the anastomosis be made at the lowest part of the stomach, at that part of the stomach nearest the most dependent portion of the greater curvature, a reservoir will be formed below the opening. Into this will be poured all the bile and pancreatic juice entering the stomach, and these, being stagnant, will set up vomiting. This explanation we believe to hold good for some cases.

In the earliest days of gastric surgery attention was called to the dire results of regurgitation after short-circuiting operations between the stomach and the small intestines. Tavel, of Berne (Rev. de Chir., December, Igor), relates in full a case operated upon by several surgeons in which the difficulties are pointedly illustrated. The following is a brief extract :

M. S., a medical man, born in 1863 . When twenty-seven years of age he began to suffer from stomach disorder, atony, slight dilatation, hyperacidity, etc. He consulted many physicians of great ability-Leube, Kussmaul, Müller, and Van Noorden, and was advised by the last-named to submit to gastro-enterostomy. This was performed in November, 1896 , by Dr. Sick, of Hamburg, after the method of Kocher. Immediately after the operation 
there was an intense dislike of food, a bitter taste in the mouth, fetid eructations, anachlorhydria, and apepsia. His condition became insupportable, and he therefore consulted Professor von Mikulicz, who operated. The anastomosis was found to be of the diameter of a lead pencil. Entero-anastomosis was performed, and the opening of the gastroenterostomy was enlarged. There was no improvement, and Professor Kocher was consulted. He performed a third operation, pleating the afferent loop after the method of Von Hacker. For a time the patient did well, and gained 8 pounds in weight. After a few weeks, however, the old symptoms began to return. A fourth operation was then undertaken by Tavel, of Berne, who divided the first part of the duodenum and sutured the cut ends. At a fifth operation, conducted by the same surgeon, the afferent loop between the point of

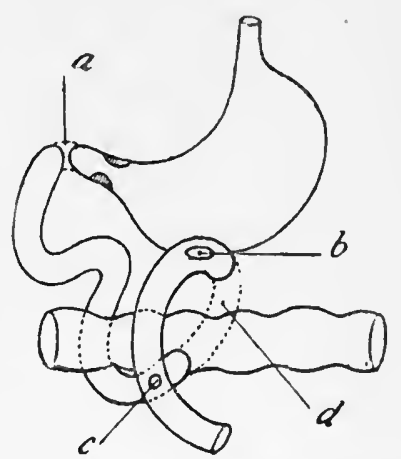

FIG. 57.-Showing THE VARIOUS OPERATIONS PERFORMED. entero-anastomosis and the gastro-enterostomy was completely excised (Fig. 57).

Many theories have been put forward to explain the occurrence of regurgitant vomiting after gastro-enterostony. The formation of a 'spur' at the point of anastomosis has undoubtedly some influence, and has been demonstrated by Chlumskij in certain cases operated upon by Mikulicz. It was formerly thought that the sole cause was the presence in the stomach of bile and pancreatic juice. Riegel, Malbranc, and Weil related cases where a reflux of bile into the stomach resulted in grave symptoms. Billroth remarked upon the serious import of bile regurgitation after gastro-enterostomy. Claude Bernard and others, founding their opinion upon laboratory experiments, considered that bile inhibited gastric digestion. Dastre, in dogs with gastric fistulæ, introduced bile at all stages of digestion, and concluded that the alkalinizing effect of the bile was swiftly negatived by a copious outflow of gastric juice. No ill effects were noticed either on the digestive powers or on the general health. Oddi, experimenting upon dogs, obliterated the common 
bile-duct and united the gall-bladder to the stomach. All the bile consequently flowed at once into the stomach, with the result that the animals gained in weight and suffered not at all. Max Wickhoff, Angelberger, and Terrier have performed cholecysto-gastrostomy for obstruction in the common bile-duct, and recently Perrin (Thèse de Lyon, Igor) has suggested the routine performance of cholecysto-gastrostomy in occlusions, presumably complete and irremediable, of the common duct. These records all show that the mere presence of aseptic bile alone is insufficient to induce vomiting. Chlumskij has suggested that the regurgitant vomiting is due to the presence of pancreatic juice in the stomach. To settle this point, Steudel undertook a series of experiments upon dogs. He divided the intestine completely across at the duodeno-jejunal flexure, closed the duodenal end, and implanted the jejunal into the anterior wall of the stomach. The dogs lived and thrived for a time, but died from perforation of the duodenal loop by fragments of bone, which had been eaten, and had passed from the stomach through the pylorus.

In the British Medical Journal of May, Igor, was recorded by Moynihan the case of a boy, aged six, upon whom he performed gastro-enterostomy for complete rupture of the gut at the duodeno-jejunal flexure. The torn end of the duodenum was sutured, and, after resection of a few damaged inches, the jejunum was joined to the stomach. As a result, the whole of the bile and the pancreatic juice passed into the stomach. The boy made a perfect recovery, and remained in splendid health until the Io4th day after the operation. He then became suddenly collapsed and died in a few hours, death being due to the perforation of the bowel by the Murphy button that had been used for the anastomosis. This case proves beyond dispute the ineffectiveness of bile and pancreatic juice as sole agents in producing regurgitant vomiting.

There are four varieties of misdirected current after gastroenterostomy (Fig. 58) :

I. Regurgitation of duodenal contents through the pylorus.

2. Escape of fluids from the stomach into the afferent loop. 
3. Escap z of fluids from the afferent loop into the stomach.

4. Regurgitation of the contents of the efferent loop into the stomach.

Any one of these establishes the circulus vitiosus. Of these four, the third, the escape of duodenal contents from the afferent loop into the stomach, is generally considered the most frequent and the most grave.

Chlumskij (Beit. z. Klin. Chir., Bd. 20), in discussing the causes of regurgitant vomiting, suggests the following:

I. Formation of a spur (Fig. 59). This is certainly the most frequent. When the anastomosis is affected the two

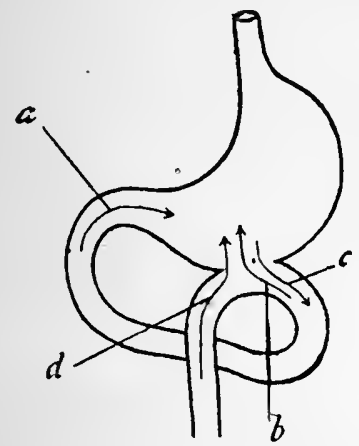

Fig. 58. - Showing THE VARIETIES OF MISDIRECTED Current after GastroENTEROSTOMY.

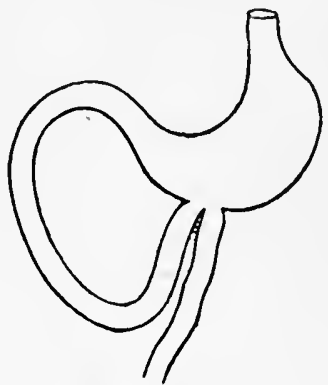

FIG. 59.-ShowING Sper FORMATION.

limbs of the loop hang downward, and a sharp kink, with spur-formation, results at the point of junction.

2. The jejunal displacement may cause a kink at the duodeno-jejunal junction, and thereby produce an acute obstruction of the duodenum.

3. The mucous membrane of the stomach may form large pouting valves which obstruct the afferent opening.

4. Closure of the anastomotic opening, if the muco-mucous stitch is improperly applied.

5. Compression of the efferent branch of the loop by the colon (Doyen).

6. Steudel related a case, operated upon by Czerny, in 
which the opening in the transverse mesocolon had narrowed and constricted the efferent loop.

7. In the original operation of Wölfler the anti-peristaltic implantation of the jejunum favoured regurgitation (Fig. 6o).

Of the frequency of regurgitant vomiting the following figures give some indication: In 65 cases operated upon by

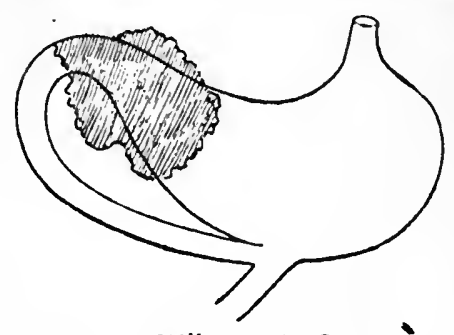

Fig. 6o.-WÖlfler's Originàt, Procedure.

The operation was suggested by

Nicoladoni, who was acting as assistant.

those of Steudel by Petersen, there was not a single case of reflux vomiting. In $2 \mathrm{I}$ cases of anterior gastroenterostomy, an entero-anastomosis was necessary in 3 .

A very large number of modifications of the operation of gastro-enterostomy have been suggested with the hope of preventing reflux. In Wölfler's original operation (Fig. 6o), performed September 28, I88I, the jejunum was united to the stomach with its proximal end nearest to the pylorus. Von Hacker, in I885 (Figs. 6I and 62), suggested that the jejunal anastomosis should be made, not on the anterior, but on the posterior surface of the stomach, which could be reached by tearing through the transverse mesocolon. The point on the jejunum to be utilized for the anastomosis was placed by Von Hacker 20 to 25 centimetres from the duodeno-jejunal flexure, by Kappeler 40 to 60 centimetres, by Chlumskij jo centimetres, by Kader 6o to 80 centimetres. Petersen emphasized the importance of the anastomosis being as near to the flexure as possible (Fig. 62). In 1883 Courvoisier performed a 'gastro-entérostomie rétrocolique postérieure transmésocolique,' an operation attended by no satisfactory results, but memorable as containing the germ 
of the idea which Von Hacker utilized in his method. In I892 Jaboulay suggested, and in 1894 performed, the operation of gastro-duodenostomy (Fig. 63); Kümmell, in I895,

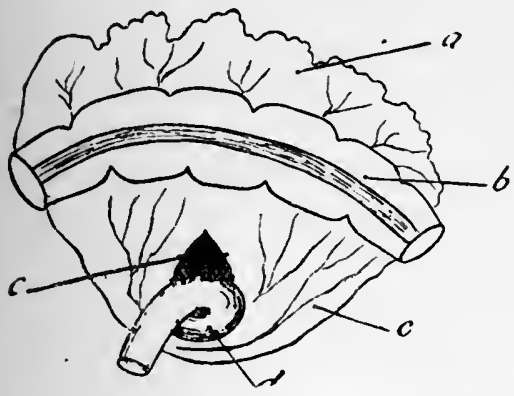

Fig. 61.-VON HaCker's Operation.

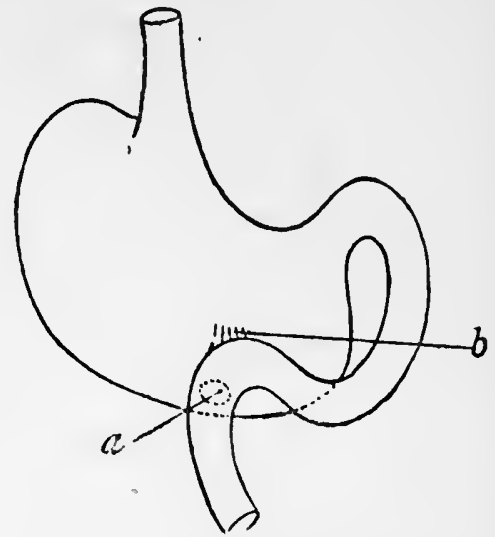

Fig. 62.- VoN Hacker's Operation seen from Behind. (After Petersen.)

independently suggested the same procedure. Mikulicz and Villard strongly commend the method. Kümmell divided the duodenum completely, closed the proximal end, and implanted the distal into the stomach (Fig. 64). Jaboulay

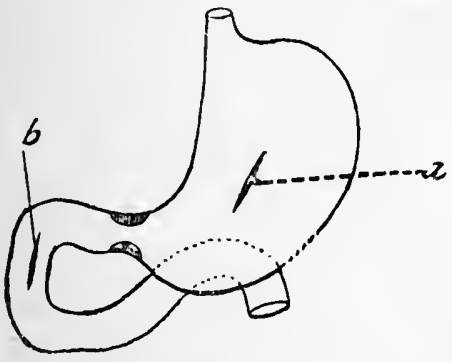

Fig. 63.-Gastro-duodenostomy. (JABOULAY.)

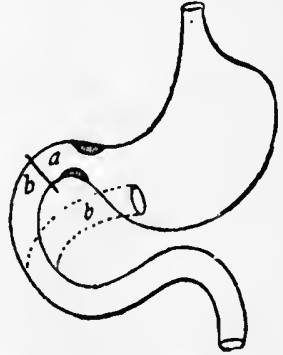

Fig. 64.-Gastro-duodenosTOMY. (KÜMMELL.)

united the anterior wall of the duodenum to the anterior wall of the stomach, folding the duodenum forward over a hinge formed by the pylorus. Villard described his method under the title 'gastro-duodénostomie sous - pylorique.' 
Instead of folding the duodenum over on to the stomach, he anastomosed the adjacent surfaces of the stomach and duodenum (Fig. 65). Brenner introduced a retrocolic, anterior gastro-enterostomy. A loop of the jejunum was

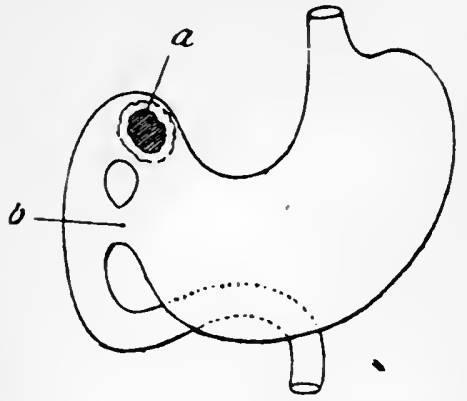

Fig. 65.-Gastro-DUodenostomy.

(VILlaRD.) passed through a rent in the transverse mesocolon, and then pulled forwards through the omentum just below the greater curvature of the stomach, and united to the anterior wall.

In 1887 Rockwitz drew attention to the fact that in the operation of Wölfler the current in the stomach was opposite in direction to that in the duodenum. To insure an iso-peristalsis he suggested that the proximal end of the loop should be fixed towards the cardiac end and the distal towards the pyloric end of the stomach (Figs. 66 and 67). In 1898 Roux, of Lausanne,

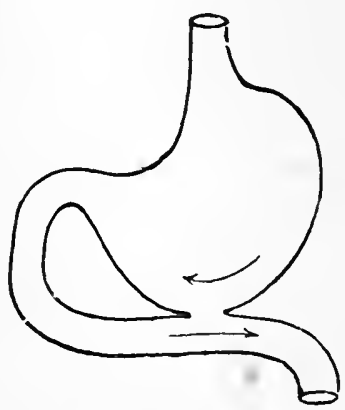

Fig. 66.-ANTi-PERISTALtic FixATION.

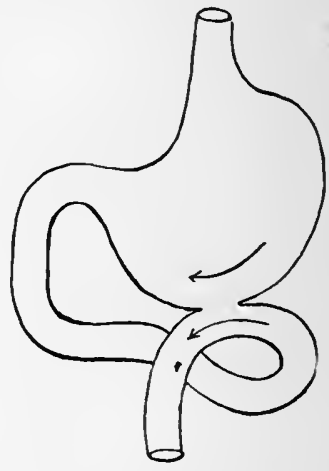

Fig. 67.-Iso-PERISTALTIC Fixation.

performed his method of 'gastro-enterostomy in Y,' adapting to this operation a method that had been practised in 1892 by Maydl for jejunostomy. Roux divided the jejunum completely across, implanted the distal end into the stomach, and the proximal into the side of the distal (Fig. 68). 
The operation of entero-anastomosis was first suggested in I 890 by Lauenstein (Fig. 69), who advised that the afferent loop should be united to a neighbouring coil of intestine. In

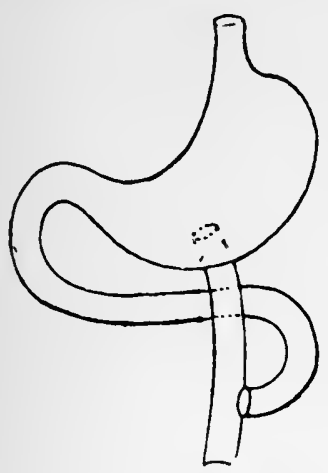

Fig. 68.- GASTRO-ENTEROSTOMY in Y. (Roux's MEтHOD.)

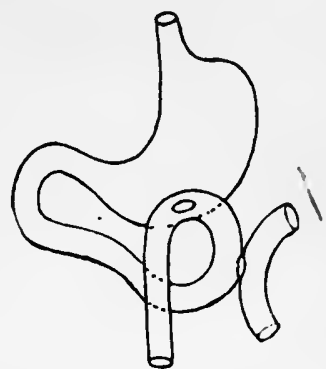

Fig. 69. -ENTERo-ANASTOMOSIS AS SUGGESTED BY LaUenstein.

I892 Braun and Jaboulay performed entero-anastomosis between the afferent and efferent loops (Fig. 70). Doyen performs a gastro-enterostomy in $\mathrm{Y}$ which is similar to that adopted by Roux, but is anterior instead of posterior, and

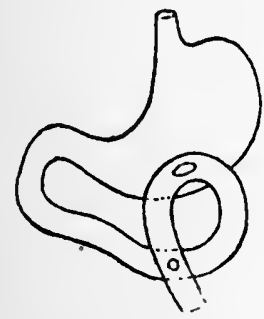

Fig, 70. - ENTERoANASTOMOSIS BETWEEN AFFERENT AND EFFERENT LOOPS. (BRAUN AND JABOULAY.)

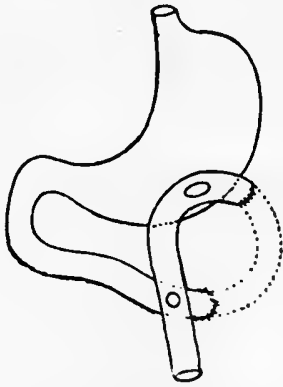

Frg. 7I.-Doyen's OPERATION.

the approximations are made side to side and not end to side. His method is considered by Lücke to be the best of any method yet suggested. It is practically a gastro- 
enterostomy, an entero-enterostomy, and a resection of the afferent loop combined (Fig. 7I).

Several methods of narrowing the afferent loop with the hope of preventing its filling with stomach contents have been suggested. Wölfler surrounds it with a circular stitch, Chaput packs round it with iodoform gauze, and Von Hacker makes a series of longitudinal pleats in it. Tavel has shown that in his patient after a few weeks no trace of a fold could be discovered.

Several methods of preventing misdirection of the stomach have been carried out; the methods are usually described as 'gastro-enterostomy by invagination.' Faure invaginates a cone of the stomach into the distal segment of the anas.

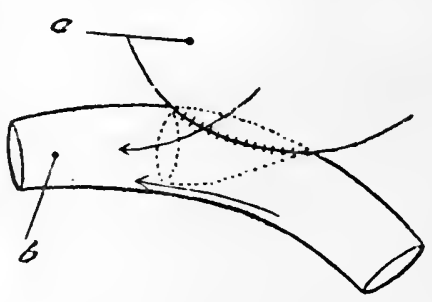

Fig. 72.-Fatre's Operation.

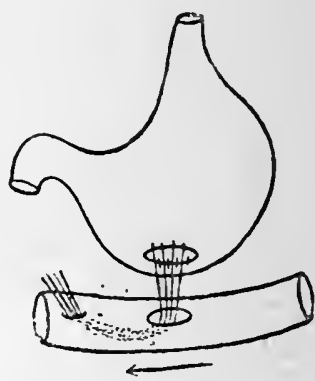

Fig. 73.-SONNENBURG'S Operation.

tomosed loop (Fig. 72). Sonnenburg sutures the edges of the stomach incision, leaves the threads long, passes them into the jejunum at the opening for the anastomosis, and out by a second opening a couple of inches distal to this. By drawing now upon the sutures the stomach is pulled well into the jejunum (Fig. 73). Rutkowsky, Witzel, and Kader have combined the operations of gastro-enterostomy and jejunostomy, passing the tube into the stomach and through the gastro-enterostomy opening into the distal segment of the duodenum (Fig. 74).

The attempted formation of a valve at the new opening, suggested by Wölfler in I883, has been carried out most effectively by Kocher, who makes a semilunar incision in 
both stomach and intestine, the convexity being upwards (Fig. 75).

Hadra, in $189 \mathrm{I}$, was the first to suggest that the loop of the bowel united to the stomach should be attached to it

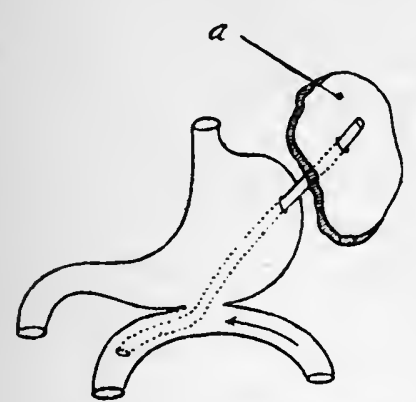

Fig. 74.-Gastro-enterostomy and Gastrostomy.

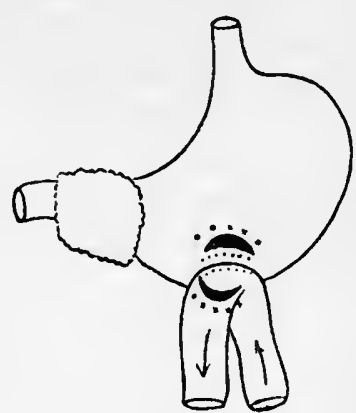

FIG. 75.-KOCHER'S OPERATION.

both above and below the opening, so that it should be suspended from the stomach by a wide attachment. In this manner the formation of a spur would be effectually prevented (Fig. 76). Lauenstein, in I896, suggested the suspension of the gut only on its proximal side (Fig. 77).

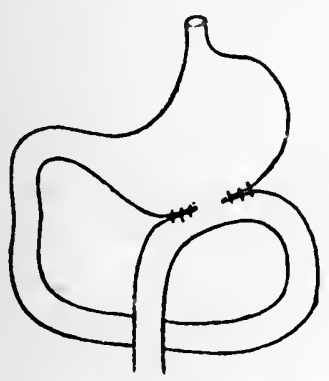

Fig. 76.-Suspension of LOOP. (HADRA'S METHOD.)

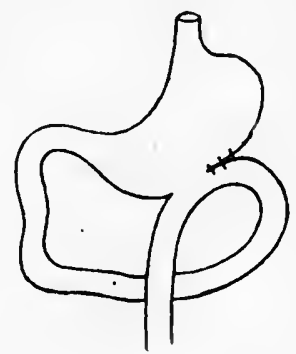

Fig. 77.-SUSPENSION OF LOOP. (LAUENSTEIN'S METHOD.)

Subsequent contraction of the new orifice between the stomach and intestine must occur to some extent in all cases when the dilatation of the stomach present at the operation undergoes progressive diminution, but this contraction, if moderate, 
need cause no inconvenience. If the pylorus be patent, and the operation be performed simply for setting the stomach at rest for a time, as in gastric or duodenal ulceration, there is always probably a tendency for the new anastomotic opening to close; and if the need for drainage has passed there will be clearly an advantage, though if the anastomosis has been performed because of stenosis of the pylorus or duodenum the closure of the new channel will be a catastrophe that can only be remedied by a further operation.

We have reason to believe that the substantial opening made when the bone bobbin is employed has little tendency to contract, as in the cases where we have had the opportunity of examining the anastomosis months after the original operation, and in one case over three years later, the passage remained satisfactory in size, and the mucous lining between the two channels was continuous.

The great advantage of the method of simple suture cannot be overlooked. If, in making an anastomosis, we have to deal with an inordinately large stomach, the opening in the stomach and the jejunum may be made 3 or even 4 inches in length. When the contraction of the orifice has reached its furthest limit there will still be left an opening as large as, or probably larger than, that originally made by any other method. The removal of the mucous membrane from the stomach and jejunum at the anastomotic openings has also a tendency to do away with the risk of subsequent narrowing of the orifice.

One of us has recorded one case in which he performed a second operation because of closure of the orifice, made with the help of Laplace's forceps, and has operated upon another in which a colleague had three years previously performed gastro-jejunostomy with the aid of Murphy's button. In this latter case the opening had completely closed. Czerny records a case where the Murphy button was used in which the opening narrowed from a diameter of 5 centimetres to 8 millimetres. As bearing on the closure of an anastomotic opening, we have found the opening in a cholecystenterostomy made by a Murphy button to be closed three months later. Mr. W. H. Brown related in the Lancet, 
July 7, I900, a case of closure of an anastomotic opening in which Senn's plates were used in a woman, sixty-two years of age, for pyloric stenosis due to tumour, a recurrence of symptoms occurring a fortnight after the original operation. An exploration through a gastrotomy incision showed the opening to be closed by a membrane, which the operator tore through with his finger, the ultimate result being excellent.

Dr. W. J. Mayo, who has favoured the use of the Murphy button, related his experience as follows:

' Gastro-jejunostomy, if the pylorus be unobstructed, is far from satisfactory. In a paper read before the American Surgical Association, June, Igo2, I reported four cases in which contraction at the site of the anastomosis took place, and we have reoperated upon four similar cases since that time. In six of these cases we did a secondary entero-anastomosis between the limbs of the loop. Four times the enteroanastomosis was effected with the Murphy button, and two of these patients died from sudden separation of the anastomosed area at the end of the first week. This did not take place in two suture operations. In all of these cases the proximal limb of jejunum from the point of anastomosis to its origin looked enlarged and thickened, a condition that might be called water-logged, and in marked contrast to the bowel immediately distal to the anastomosis. In this condition of the afferent loop lay the reason for the failure of the plastic union after the button, and merely illustrates the well-known danger of setting up pressure necrosis in damaged tissues. Primary entero-anastomosis with the button is safe, but not so secondary operations. If the obstruction at the pylorus is complete, this condition of the jejunum above the gastrointestinal anastomosis has not been found. A large number of cases of benign affections of the stomach without pyloric stenosis require operation. This is particularly true in ulcer, and relapse after this operation has been frequent. Our observations would seem to show the following course of events: After the operation there is at least temporary healing of the ulcer. The pylorus begins to functionate normally, and the unnecessary gastro-intestinal fistula 
contracts. There is renewed irritation from retained secretions, followed by reopening of the ulcer, return of pyloric spasm, and failure of the operation to effect a permanent cure. In some cases the double stomach drainage seems to give rise to unpleasant symptoms without contraction of the fistula. In twenty-eight cases of gastro-jejunostomy with open pylorus, eight came to secondary operation from contraction of the gastro-intestinal opening, while in all cases with permanent obstruction at the pylorus there were no cases of secondary operation.'

Ulcus Pepticum Jejuni.-The occurrence of peptic ulcer in the jejunum after the operation of gastro-enterostomy was first recorded by Braun in I899. In this case the ulcer perforated and caused death. The ulcer may be solitary, or two or more may be present. In all, I4 cases are recorded. Of these, there was hyperacidity in Io, in 3 the gastric juice was normal, in I, a case of Kocher's in which Roux's gastro-enterostomy in $\mathrm{Y}$ was performed, there was lessened acidity. All the operations were undertaken for benign conditions. In ro of the I4 cases the anterior operation was performed, in 2 the posterior, in I the operation of Roux, and in I there is no mention of the method. There can be no doubt that peptic ulcer of the jejunum is due to a digestion of the mucous coat by the acid gastric juice; it is produced, therefore, in precisely the same manner and by the same causes as ulcer in the duodenum.

Neumann has suggested that a jejunostomy should, in cases of hyperchlorhydria, be performed at the same time as the gastro-enterostomy, so that the patient may be fed through a tube passed into the jejunum until such time as the gastric juice becomes normal.

Dr. S. H. Watts, of Baltimore, has recorded an example of peptic ulcer in the jejunum of the dog. The following table, showing all the recorded cases occurring in man, is copied from his article in the Johns Hopkins Hospital Bulletin, vol. xiv., No. I48: 


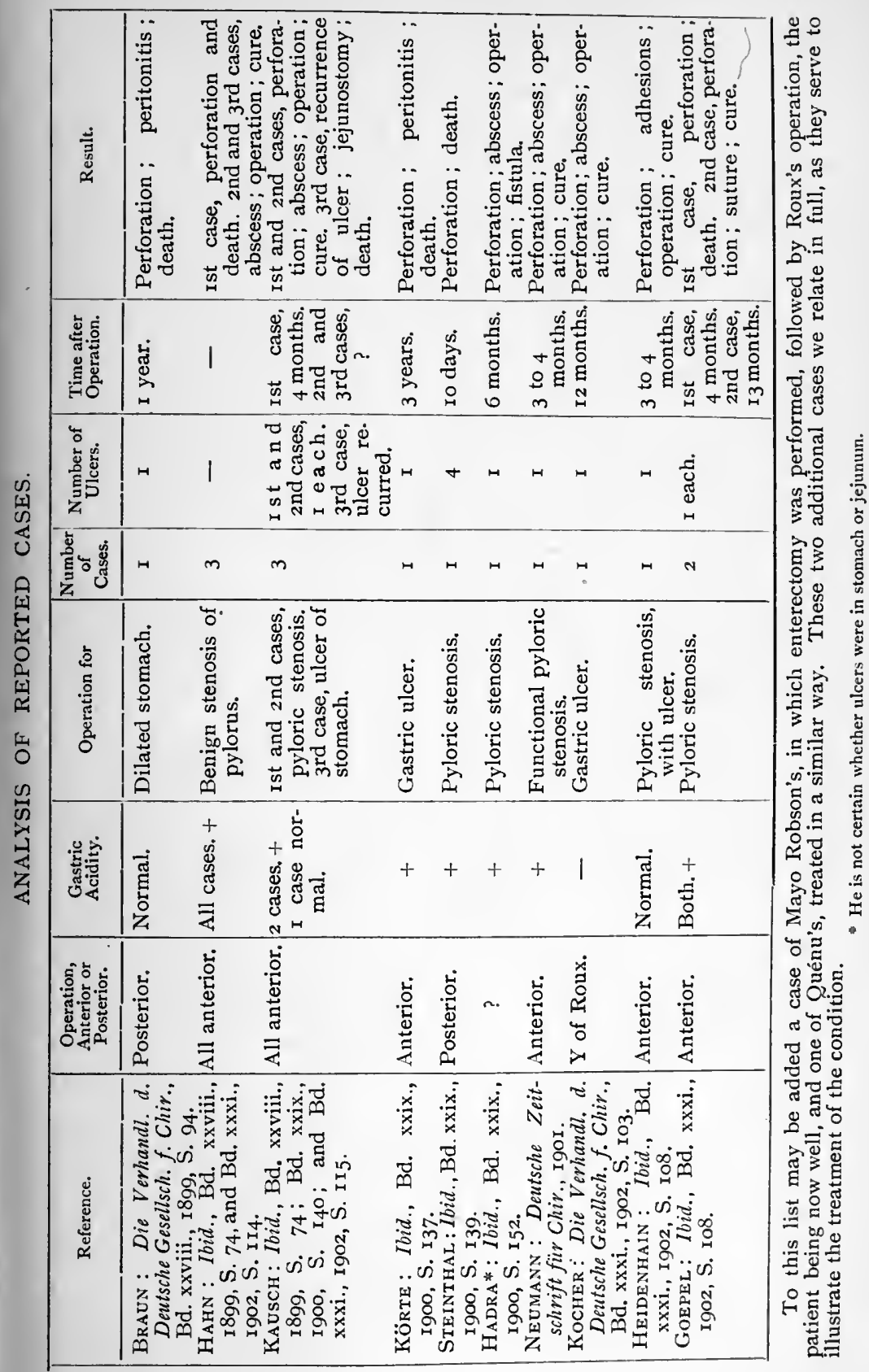


Peptic Ulcer occurring in Jejunum Three Years and Four Months after Gastro-enterostomy, treated by ENtereCtOMy aNd Roux's Operation. Recovery.

Mr. C., aged forty-four, was sent by Dr. Peter Macgregor, of Huddersfield, on January 2, Igoo, with the history that he had had pain after food, together with other symptoms of gastric ulcer, for four years, though his first attack of vomiting blood occurred in May of the previous year. The bleeding was rather profuse, but under medical treatment and rest he recovered, and was able to resume his occupation. A week before he was sent there had been a recurrence of the hæmorrhage, which was repeated three days later.

On his arrivakat the surgical home he was in a state of collapse, with a barely perceptible pulse. There was well-marked dilatation of the stomach, with tenderness over the pylorus. The history of the pain occurring one and a half hours after food, together with the site of the tenderness and the presence of a large amount of blood in the motions, left no doubt but that the ulcer was at or near the pylorus. As the bleeding had recurred twice in the week, and was persisting and passing into the bowel, operation was decided on. This was done on January 4, I900. As the patient was in such a feeble condition, every precaution was taken to guard against shock, and it was decided only to perform gastro-enterostomy, as it was quite clear that he would not stand any prolonged search for the bleeding-point. An anterior gastro-enterostomy was done, a decalcified bone bobbin being used to make the anastomosis, the opening being made close to the lower border of the stomach. The pylorus and first portion of the duodenum were very much thickened, evidently the result of ulceration. His temperature and pulse were never above normal, and he made an uninterrupted recovery. He was fed by nutrient enemata for the first few days, only taking sips of water by the mouth; but at the end of that time he was allowed to take milk and soda water, and then gradually to have stronger food. $\mathrm{He}$ was up at the end of the third week, and returned home within the month.

The patient remained well for two years, had neither pain after food nor vomiting. He gained his normal weight, and was able to do his work. After this he began to have pain at the epigastrium. The pain seemed to bear no relation to food, and was unaccompanied by vomiting. There was marked tenderness at the epigastrium, especially on the left of the middle line. At times the pain was so acute as to cause him to roll on the floor and to perspire profusely. At first the attacks were not very 
frequent, only coming on at intervals of a month or two, but recently they had been more frequent, almost daily, and during the week before we saw him dark blood had been noticed in the motions on several occasions. He was sent by Dr. James Hall on May 5, 1903, when a well-marked tumour was found occupying the epigastrium and decidedly tender to pressure, but there was no vomiting, and no sign of stomach dilatation could be discovered. Tarry matter was seen in the motions.

The diagnosis of thickening around a chronic ulcer and perigastritis was made, and an exploratory operation advised. The abdomen was opened by an incision just to the right of the middle line, and the stomach was found adherent to the anterior abdominal wall, one inch to the left of the old scar. On separating the adhesions, it was found that ulceration had occurred at the point of junction between the bowel and the stomach, and that it had involved the whole circumference of the jejunum at that point, the ulceration having completely extended through the intestinal wall at the front; but this must have occurred after adhesion, so that there had been no acute symptoms of perforation. Through the opening left when the adhesions had been separated the finger was passed into the stomach, when it was found that the pylorus had quite closed as a result of the ulceration three years before, so that nothing could be passed through it. On passing the finger into the intestine, extensive ulceration and thickening were discovered. The ulcerated portion of the bowel was completely excised, and then separated from the opening in the stomach, the edges of which were pared; the distal end of the jejunal loop was implanted into the old opening in the stomach, the union being effected over a bone bobbin. The proximal end was implanted into the jejunum about 4 inches lower. This union was also effected over a bone bobbin.

The accompanying drawings show the site of the perforation, the base of the ulcer taken from the anterior abdominal wall, the portion of intestine excised, and the intestinal anastomosis that was adopted.

The patient made a steady recovery, so that on June 2 the following note was made: 'The patient is now quite well, and is entirely free from his old pain. The wound has completely healed by first intention.' He returned home on June 2. When seen by his medical man at home on June 4, he was smoking a cigar, and expressed himself as very well. Soon afterwards he had a little pain in the region of the gall- 
bladder, and on June 9 had a free hæmorrhage, as shown by melæna, after which he made a good recovery and remained well. A report from Dr. Hall in November, I9o3, says that he is in good health.
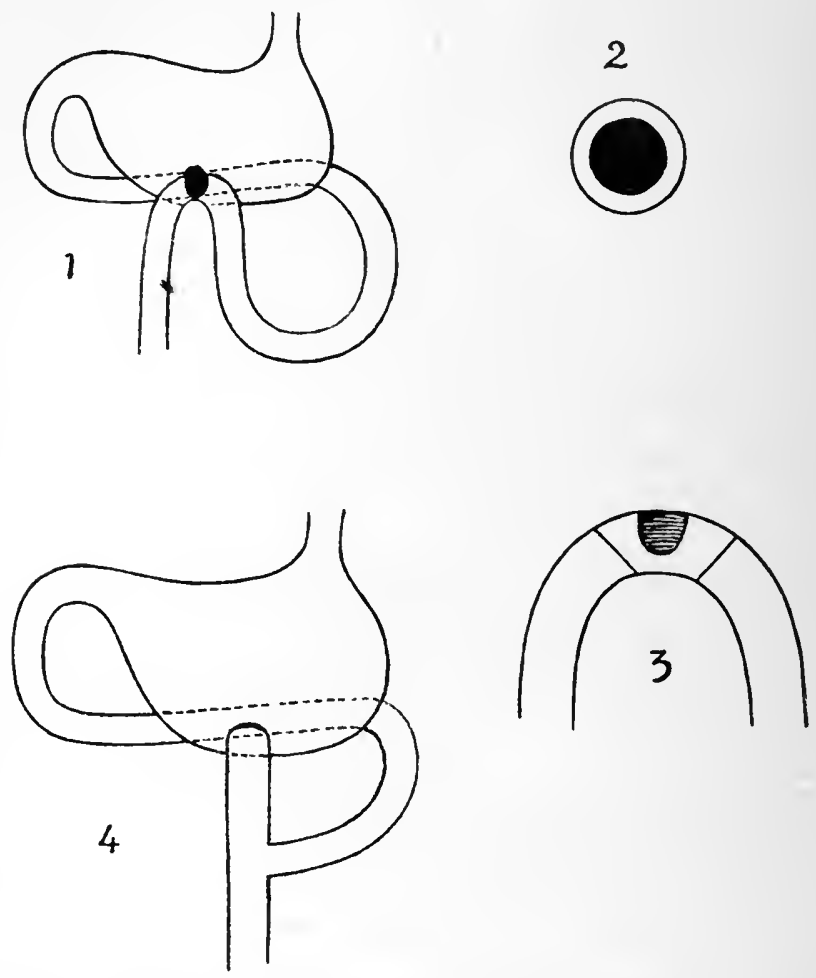

Fig. 78.-I, Diagram of Condition found on separating Adhesions, May, i903, showing Perforation. 2, Diagram of Portion of Ulcer Adherent to, and Detached from, Anterior Abdominal Wall. 3. Diagram of Portion of Intestine Excised. 4, Diagram of Anas. tomosis Performed after the Enterectomy.

Quénu (Bull. ct Mém. de la Soc. de Chir. de Paris, No. 7 , I902) puts on record a case of repeated gastro-enterostomy on the same patient which presents several points of special interest. 


\section{Plptic Ulcer occurring Four Years after Gastro- ENTEROSTOMY.}

The patient, a male, aged twenty-nine, had during eight years before the first operation, which was performed in July, I897, suffered from severe gastric troubles, caused by cicatricial stenosis of the pylorus and enormous dilatation of the stomach. This operation was followed by complete relief, which, however, did not last long, since after an interval of eighteen months decided symptoms of gastric ulcer were presented. After these symptoms had been removed by rest and medical treatment, there was a long period of relief; but in June, Igor, four years after the first gastro-enterostomy, it was found necessary to perform this operation again on account of ulceration of the anastomosed loop of small intestine, this ulceration having involved nearly the whole thickness of this portion of small intestine and,the corresponding portion of the abdominal wall. The ulcerated loop of intestine was resected, and then the jejunal end of the divided intestinal canal was sutured to the orifice in the stomach, the margin of which remained sound, and finally the duodenal end was implanted into the jejunum. The patient made a speedy recovery, and is now quite well. The history of this case, it is pointed out, proves that gastro-enterostomy does not insure subsequent freedom from gastric ulcer, unless the patient be very careful as to diet and submit to proper niedical treatment. The rare result of ulceration of the anastomosed loop of intestine is attributed to the fact that the descending limb of the loop constitutes a substituted duodenum, which may be freely exposed to the corrosive action of a hyperacid gastric fluid.

\section{Treatment.}

Though well recognised, it has not been sufficiently grasped that the essential cause of peptic ulcers is of a septic nature, and in many cases the source of the trouble is oral; hence, probably, the greater frequency of gastric ulcer among the poorer classes, the sequence of events being septic gastritis, hyperchlorhydria, and ulceration.

In the cases of gastric ulcer that have failed to yield to medical treatment and in which gastro-enterostomy has been performed, we have not properly grasped the fact that the operation is, though an important one, still only an incident in the treatment, which ought to be continued on general 
lines for some time longer, or until good health is again established. Greater attention to oral asepsis and to the gastric condition of hyperchlorhydria subsequent to operation is advisable and in this way the very serious complication of peptic ulcer in the jejunum might be prevented.

In all the cases reported, perforation, associated with acute, subacute, or chronic symptoms, has occurred, as will be seen by referring to the reported cases; hence there can be no question as to the desirability-nay, as to the absolute necessity - of operation, which ought not to be delayed too long.

When the abdomen is opened, the treatment will depend on the condition found. If perforation has occurred into the general peritoneal cavity, the condition will be one of the utmost peril, and only capable of relief by immediate cleans. ing of the peritoneum and closure of the opening or excision of the ulcer, with subsequent suture.

Though peptic ulcer of the jejunum is less frequent after posterior gastro-enterostomy, only two cases having been recorded, when it does occur it is more likely to be acute and not to lead to adhesions.

If, as in the greater number of cases, adhesions have formed, the condition will be less acute, although very distressing, from the associated pain due to perigastritis and adhesions. It will be necessary to detach adhesions and to repair the perforation, but probably in the greater number of cases an excision of the portion of intestine involved and the performance of a Roux's operation will give the best results.

Chest Complications.-Pneumonia or Pleurisy is said to have followed operations on the stomach with greater frequency than other operations on the abdomen, the reason given being the fixation of the ribs and the consequent difficulty in expanding the lungs subsequently to operation. Our experience does not bear out this, for we have not found chest ailments to follow after operations on the stomach with greater frequency than is the case after any other major operation.

Dr. W. J. Mayo writes (Annals of Surgery, August, I902) :

'There have been many explanations as to the frequency of lung complications following operations upon the stomach. It was thought to occur as a result of general anæsthesia, but experience 
has shown that it is relatively as frequent after the use of a local anæsthetic. The situation of the incision in the epigastrium, preventing coughing and expectoration, is thought to be an element in causation, yet similar incisions in the gall-bladder region have no such effect. The latest theory is that some of the venous blood returning from the stomach does not pass through the portal vein, and in this way infected emboli are carried directly into the circulation and pass at once to the lungs. In two of the three cases a chronic bronchial cough was present at the time of operation, and the patients were in bad general condition: In one case material was aspirated through the trachea from the osophagus, causing an aspiration pneumonia. It is difficult by means of the stomach tube to thoroughly cleanse and empty the greatly dilated stomach in debilitated subjects. In this case, on elevating the stomach out of the abdominal incision, some of the fluid contents gravitated into the osophagus. This should be avoided in these cases by elevation of the head and thorax at this time. The recumbent posture has also an evil influence on some cases, and we now encourage the old and feeble to sit up early. It is evident that there is as yet no entirely adequate explanation for the production of the pulmonary complication. There are probably several contributory causes in most cases.'

A want of union in the newly-joined viscera is an accident of a very serious and probably almost universally fatal character that used to be less rare when only moribund patients were operated on, but which is now seldom seen except when the Murphy button has been used, in which case there is nothing to prevent extravasation if union be delayed beyond the first few days, whereas when the union is effected by a double line of sutures delayed union, if not too prolonged, is not serious. We have only seen the complication on one occasion in recent years, and that was after the use of the metal button. Dr. W. J. Mayo, in a paper in the Amals of Surgery for August, 1902, speaks of having had two fatal cases from separation of the anastomotic openings-one on the seventh day in malignant disease, and one on the ninth day following an epileptic seizure; in both the Murphy button had been employed. He states:

'In the two cases in which the anastomosed intestine was detached, causing death from leakage, one took place on the 
seventh day after gastro-enterostomy for malignant pyloric obstruction. There was a small amount of free fluid present in the abdomen at the time of operation, which would usually contraindicate a plastic procedure, such as gastro-enterostomy. In the second case, detachment on the ninth day followed an epileptic seizure. This was in a patient with benign obstruction, who had up to that time done unusually well. $\mathrm{He}$ had suffered from epilepsy for years, and the aura began in the epigastric region. In a violent contraction of the stomach such a detachment might easily take place. Chlumsky's experiments on presumably healthy - animals went to show that after five days the union was perfect. That this is not true as to diseased states in the human subject is shown by these two cases.'

Perigastritis with adhesions of various kinds is so common in the condition such as ulcer and cancer, which call for the operation of gastro-enterostomy, that it would seem not unlikely that any further disturbance of the stomach might increase the number of adhesions; yet we believe that perigastritis the result of a carefully-executed operation is uncommon. The case described on p. 235 is, however, an example in which such adhesions led to constriction of the distal jejunal loop, and so to regurgitant vomiting, some months after the original operation.

In another case of extensive gastric ulceration with pyloric stenosis, the adhesions, the result of previous perigastritis, were so extensive that it was impossible to perform the posterior operation, and even difficult to find sufficient free stomach to effect an anterior gastro-enterostomy. Anterior gastro-enterostomy was performed, with an immediate successful result; but a few months later the symptoms returned, and although the gastro-intestinal opening was patent, adhesions had re-formed and evidently prevented the passage onwards of the stomach contents. A Roux's operation was performed, with an immediately successful result, and the patient left within a month. A report received some months later was to the effect that the pain and vomiting had recurred, and though we did not see the patient again, we believe that she ultimately died from exhaustion, the result of pain and vomiting. 
Internal Herniæ.-(a) Passage of small intestine through the loop formed by the junction of the jejunum and stomach is probably only possible, certainly only probable, after the anterior operation, and the only case that we know of was one reported by Dr. Mayo in the paper previously referred to, which occurred in his hands a year after an anterior gastro-enterostomy.

The following is an account of the case :

Anterior Gastro-enterostomy; Secondary Operation for the Relief of a Twist at the Anastonotic Opening caused by Small Intestine passing through the LoOp.

R. N. S., aged forty-one years, American, barber, was admitted to St. Mary's Hospital, January I, Igor, with the following history: For several years has suffered from attacks of burning pain in the epigastric region, lasting for a few minutes at a time, but recurring at intervals of several hours. Much worse when at work at his trade. 'These 'cramps' would last in this way for several weeks at a time, after which there would be an interval of weeks or months of good health. For several months has had more or less stomach trouble, and occasionally vomited up the contents of the stomach. 'The distress has caused him to eat sparingly, and he has lost 25 pounds in weight. He had an attack of appendicitis, with an abscess, some years ago; the latter had been incised, but the appendix was not removed. He has had a right inguinal hernia for many years.

Examination.--A spare man, 6 feet and I inch in height; emaciation noticeable. With the exception of the stomach, no feature of interest. Painful point in epigastrium. Free acid. Greater curvature of stomach 3 inches below the umbilicus.

Diagnosis.-Pyloric obstruction from ulcer. January 2, anterior gastro-jejunostomy, Murphy button, appendectomy, and Bassini operation on hernia. An ulcer existed at the pylorus extending to the lesser curvature, irregular contour, size of last phalanx of forefinger. Evidently partially cicatrized and obstructing pylorus. Fourteenth day symptoms of intestinal obstruction lasting forty-eight hours. Condition relieved by gastric lavage and rectal feeding. Button passed on the sixteenth day, evidently cause of symptoms. Discharged January I8; gained rapidly in weight and strength. For a year remained in good health, although complained that if he stood erect he had a 'drawing feeling' in his stomach. From this time to May 14, 1902, when 
he was readmitted to the hospital, he had slowly developed all of the former symptoms of obstruction at the outlet of the stomach, and had a constant pain in the abdomen, centring below the umbilicus. May I5, abdomen opened. Gastro-jejunal orifice nearly obliterated and stretched to an inch in length. Jejunum twisted at the site of anastomosis one half turn from the left to the right. Somewhat more than one half of the small intestine had passed through the loop of jejunum, between the origin of the jejunum and the attachment to the stomach. The point of entrance was on the right side beneath the transverse colon. The traction weight of the intestines upon the mesentery at the inferior margin of the loop had caused the volvulus. The mesentery at this point was much thickened; the intestines were replaced, the gastro-jejunal fistula divided, and the opening into the stomach closed. The opening into the jejunum was enclosed by a purse-string suture, and the half of a Murphy button was introduced and a posterior gastro-jejunostomy made. The pyloric stricture was nearly complete, the ulcer evidently cicatrized. It is probable that the part of jejunum immediately below the anastomosis passed through the loop first, producing the twist which was so prominent a feature on opening the abdomen. As to when this happened it is hard to tell, probably not for some months after the operation. When the process once began, it might be expected to continue until such an amount of intestine travelled over the loop as to pull the mesentery taut, the symptoms increasing as the condition gradually developed. It is possible that at the time the juncture was effected a slight twist might have occurred.

(b) Passage of intestine through the slit in the mesocolon made for the anastomosis.

The following case has been observed by us :

The patient, a male aged twenty-eight, was seen on October 30 , 1902. For fourteen months he had suffered from indigestion. At the onset he had an acute attack of indigestion, lasting five days; occasional vomiting then, but no blood. He fainted several times, and had tarry motions. During the last three months he had become progressively worse, and lost over a stone in weight; had constant pain after food and occasional vomiting. He could take only fluids, and was 'wearing down' fast. The stomach was moderately dilated. Free $\mathrm{HCl}$ present. Old blood was noticed in the stools in infirmary almost daily. At the operation duodenal and gastric ulcers were found; duodenal ulcer in first portion about the size of a threepenny-piece, very hard, and 
slightly adherent. A scar was found on the posterior surface of the stomach, near the pylorus. The patient died on the tenth day of acute intestinal obstruction. At the post-mortem examination it was found that there had been a hernia of almost the whole of the small intestine through the rent made in the transverse colon, and that the herniated bowel was tightly strangled at the opening.

Death from Asthenia is now seldom seen, as feeding can be begun immediately. Moreover, the operation is seldom deferred until the patient is almost moribund, as was at one time the case, and greater care is exercised before, at the time of, and subsequent to, operation in guarding against shock.

Hæmorrhage as a cause of death after gastro-enterostomy is not likely to occur as a result of the operation itself, as the continuous suture applied through the whole thickness of the margins of the anastomotic opening acts as an efficient compress to the vessels. It may, however, recur from an ulcer or growth as an accident, just as hæmatemesis or melæna may result from the disease apart from any operation. In such case the stoppage of feeding by the mouth, the use of rectal nutrient enemata, the giving of suprarenal extract, as advised by Fenwick, and the administration of calcium chloride, will usually arrest the bleeding; but should it persist the question of further operation for discovering the bleeding-points may have to be considered.

Conditions Subsequent to Operation.-Fantino (Archiv für Klinische Chirurgie, Band xlvi., S. I, 2) examined Carle's cases of gastro-enterostomy as regards the following points : (I) Changes in the peristalsis of the stomach ; (2) the ability or non-ability of the new sphincters to close the outlet; (3) the capacity of the stomach; and (4) the secretion of hydrochloric acid.

In the cases examined the operation immediately improved the peristaltic power of the stomach, though it did not render it normal. The stomach could generally empty itself, but did so gradually. Systematic examinations of the stomach contents were made after test meals, etc., and showed that after an irregular period the stomach regained completely its 
power of emptying itself; in fact, as a rule, after gastroenterostomy the stomach would be found practically empty in from three to five hours after a meal. Generally it was found that the stomach decreased in size soon after gastroenterostomy, so that the formerly distended organ became normal in size. Examination of the stomach by means of distension with carbonic acid and by other methods showed that a sphincter was developed at the new opening, and that its power increased with time. The secretion of hydrochloric acid after operation was studied. In cases where there was formerly hyperacidity this condition was lost, and though the degree of acidity in an individual case varied from time to time, yet these variations did not depart from physiological limits. In the same examinations it was found that regurgitation of bile into the stomach took place, but it was of no importance so long as the outlet from the organ was sufficient. Cases of hypo- and an-acidity showed no change in their gastric juice after operation, showing clearly that this condition is not dependent on obstruction, but on previous changes in the mucous membranes, these changes being probably in the nature of an atrophy of the peptogastric glands.

Changes in the Stomach.-If dilatation as the result of obstruction has been present before the gastro-enterostomy, a certain lessening in the size of the stomach may be expected to occur as soon as a fresh outlet is formed. The extent of this lessening depends entirely upon the condition of the muscular tunic of the stomach. If the stomach has dilated gradually during many months or years, and if the dilatation has been extreme, there will be little or no rebound; the dilatation will undergo little or no perceptible diminution. On the other hand, if dilatation has been rapid in its oncoming, and has never attained a supreme degree, the return to the normal size of the stomach will be almost, or wholly, complete. In cancer of the stomach or in injury resulting from swallowing acids, etc., where the latter conditions are fulfilled, the stomach rapidly lessens in size after operation; in simple stenosis, where the former conditions are found, the return is always trivial, if, indeed, there be any lessening 
at all. The motor insufficiency of the stomach is, according to Hartmann and Soupault, a permanent matter. A return to the normal was never observed in any of their cases, even when the examination was made after one year. Gastric digestion is prolonged beyond the normal limits. Mint $z$ and Rosenheim have shown that there is a marked delay in the evacuation of the stomach contents. Carle and Fantino, on the other hand, assert that the emptying of the stomach after a full meal is not only accomplished within the period of physiological limit, but is actually accelerated. They add : 'This is often observed immediately after the operation, and always, or almost always, in the months that follow.'

Mortality.-No operation shows so clearly the improvements that have come about in surgical technique during late years; for between the years I88I and 1885 the mortality was $65^{\circ} 7^{\mathrm{I}}$ per cent., between $\mathrm{I} 886$ and I 890 it was 47 per cent., from $189 \mathrm{I}$ to $\mathrm{I} 89633^{\circ} 9 \mathrm{I}$ per cent., and of the I, 878 cases that we were able to collect from all sources when giving the Hunterian Lectures at the Royal College of Surgeons in I9oo, the mortality was no less than 36.4 per cent.

The mortality for all classes of cases is thus seen to be a gradually diminishing one. There is, however, a striking difference between operations in cases of cancer and in cases of simple stenosis or ulceration. Haberkant calculates a mortality of $43^{\circ} 5$ per cent. in malignant and of $25^{\circ} 5$ per cent. in simple cases ; Chlumskij $4 \mathrm{I}^{\circ} 6$ per cent. for malignant and $2 \mathrm{I}^{\circ} 4$ per cent. for simple cases ; Goffe (quoted by Barker), for English and American cases, $4 I^{\circ} 5$ per cent. and $36^{\circ} 3$ per. cent. respectively.

The results during recent years have in the hand of several surgeons shown a very much better record than any of the above quoted. Drs. W. J. Mayo and C. H. Mayo, of Rochester, Minnesota, whose brilliant work we have both had the opportunity of witnessing, published the results of their experience in gastric surgery in the Annals of Surgery, July, I903. In simple cases, of 89 gastro-jejunostomies, 82 recovered, equal to a mortality of 7.8 per cent. Of 28 gastro-duodenostomies, 27 recovered, equal to a mor- 
tality of 3.5 per cent. In malignant cases, of 43 gastroenterostomies, 24 recovered, equal to a mortality of $29^{\circ} 4$ per cent.

Our experience of the anterior operation, both for simple and malignant disease, was given in the first edition of this work, published in I9or. It was the operation performed in the earlier cases so far back as I8go, many of the patients being almost moribund at the time of operation, the mortality therefore being necessarily considerable. Since that time we have done the anterior operation only on six occasions, in cases where extensive cancer or perigastric adhesions prevented the posterior operation being done; out of these, four patients recovered.

Of posterior gastro-enterostomy, which we have performed exclusively since June, Igoo, there have been 2 I 8 operations for simple diseases of the stomach, of which 2 II recovered. The cases of hour-glass stomach are not included, as they are given in detail in Chapter XIX. Of the malignant cases, there were 29 operations with 26 recoveries. It will thus be seen that the mortality is $3^{*} \mathbf{2}$ per cent. for simple disease, and to per cent. for malignant disease. 


\section{CHAPTER XVI}

\section{PYLOROPLASTY}

PyloRoplasty was first performed in 1886 by Heineke, next in 1887 by Mikulicz. The stomach being exposed, the pylorus is drawn into the wound and protected well with sponges. The stricture is then divided transversely by an incision which must extend at the least I inch on each side of it. This incision may be commenced from the stomach side, and prolonged towards and into the duodenum either by scissors or by a scalpel guided along a director passed through the constriction. Nibbed forceps are then applied to the middle of the upper and lower lips of the incision. On pulling these upwards and downwards the transverse incision becomes longitudinal, and in this position it is sutured. Two layers of stitches are applied, both continuous. The mucous membrane is stitched with catgut, and the peritoneum with silk or celluloid thread.

The modification of the operation by the use of an internal splint in the shape of a bone bobbin, over which to apply the sutures, is a method that one of us has employed in all his operations, and one which offers several advantages. For instance, only two continuous sutures are required for the mucous and peritoneal margins respectively.

The bone tube secures an immediate and thoroughly patent channel. It affords protection for from twenty-four to fortyeight hours to the line of sutures, by which time union should be established, and it prevents the new channel being inadvertently made too narrow. Although the bone bobbin originally invented for pylorectomy and enterectomy answers quite well, a recent modification, in which the barrel of the 
bobbin is lengthened, facilitates the operation. This modification of pyloroplasty has been followed by Rushton Parker (British Medical Journal, December I4, 1895) and other

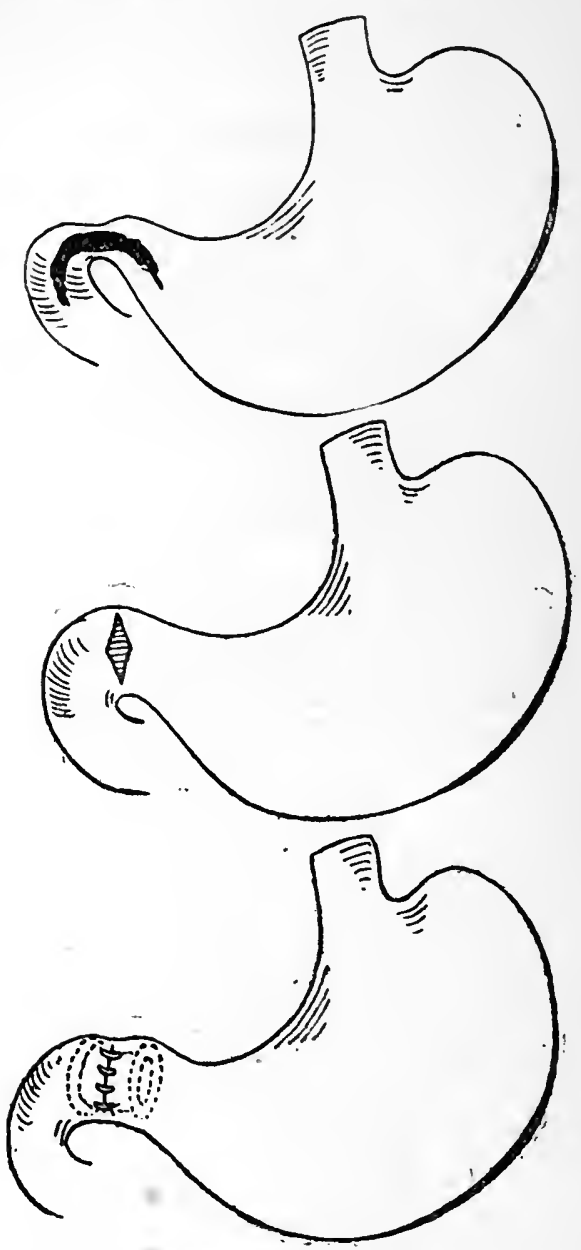

Fig. 79.-Pyloroplasty as Modified by the Use of a Decalcified Bone BoBbin.

surgeons with success. Illustrative cases have been given on p. 197 .

The important point in the operation, necessary for success, is the making of the dividing cut of ample length. 
The cut, when represented diagrammatically, is always drawn as a short straight line; it should, however, be, as Mikulicz has pointed out, drawn in the shape of a horseshoe, with the convexity upward, and the limbs extending downwards into the stomach and duodenum. As our experience of pyloroplasty increases, we are inclined to place less reliance upon it as a therapeutic measure. In certain of our own cases, and in those of our colleagues, cases which have been brilliantly successful in their immediate results have shown, after the lapse of eighteen months, two years, or longer, a tendency to relapse. In none of the cases has there been a complete return to the original condition, but symptoms of the same kind, though less in degree, have been observed. For examples, see p. I97.

Pyloroplasty must not be performed where there is active ulceration or moderate or extensive induration, or adhesions the result of peripyloritis. In those cases where narrowing of the outlet occurs after pyloroplasty, it is probable that the operation has been contra-indicated from the first, though experience to guide us at the time was lacking. As the prohibitive restrictions we have mentioned are present in the majority of cases, it necessarily follows that the rôle of pyloroplasty is a limited one. Though this opinion is contrary to that of many surgeons, we feel convinced that it will be shared by all who can watch for years the careers of their patients. Berg (British Medical Journal, August, I9oo) has abandoned pyloroplasty for gastro-enterostomy in cases of pyloric stenosis on account of this tendency to recurrence of the stricture. Pyloroplasty will be chiefly of service in cases of string-like narrowing or in spasm of the pylorus.

Professors Carle and Fantino (loc. cit.) compare the operations of gastro-enterostomy and pyloroplasty. Out of fourteen cases in which the latter operation was performed, only one died. The results of pyloroplasty, as regards function, have been little noticed in literature. To the authors' fourtcen cases, three may be added where the operation was by tearing, but the results were the same. In all the seventeen cases the results were excellent-in thirteen of them perfect and permanent, as it is now from thrce to seven years since 
operation. In these the condition of the secretions and of the peristaltic power of the stomach were the same as after gastro-enterostomy for non-malignant stenosis. Diminution in size of the stomach was not so marked as would be expected in the presence of such remarkable recovery of the general health and of the stomach's power to empty itself. In all cases, with one exception, the gastric capacity was more or less diminished, but in no case did it become normal in size.

A few cases must be expected where operation was performed for hyperacidity, with gastric atony. In these, four to five months after operation, there was delayed evacuation of the stomach and a feeling of weight. Although the general improvement was considerable, yet the authors were persuaded that a posterior gastro-enterostomy would have given better results. In one of the cases a subsequent gastro-enterostomy gave a perfect recovery.

In cases where there was hyperacidity before the operation, there was a rapid return to the normal, but not to below normal, as we found after gastro-enterostomy. The authors believe that the rapid and great diminution in hydrochloric acid after the latter operation is due to the very rapid evacuation of the stomach after a meal, and do not deny the possible influence of a regurgitation of bile into the stomach. Both these conditions are absent after pyloroplasty; hence the difference in secretion.

In cases of hypo- and an-acidity, operation produced no change in this particular, and yet health was restored. The results of pyloroplasty may be summarized :

I. Regurgitation of bile into the stomach is prevented.

2. Secretion of hydrochloric acid, when it has been excessive, becomes normal.

3. If the secretion of hydrochloric acid has been diminished or absent before operation, it remains in statu quo after operation.

4. If there has been primary gastric atony, peristalsis is but little improved.

5. This function improves rapidly or reaches perfection if the muscular contractility has been normal or increased, and 
when the obstruction was due to fibrous stenosis or pyloric spasm.

6. In all such cases evacuation of the stomach is accomplished in its physiological period. Only in rare cases, and these only in the first months after operation, may it be delayed.

7. The capacity of the stomach always decreases, but rarely becomes as small as normal.

8. The pylorus recovers tone.

Points of difference between the results of pyloroplasty and gastro-enterostomy are :

I. The absence of regurgitation of bile, and hence the absence of any possible biliary influence on the gastric secretions.

2. The evacuation of the stomach is not accelerated; hence the difficulty the stomach has in reaching its normal size.

3. The slight or negative result obtained by pyloroplasty in obstruction from primary gastric atony compared to the positive results from posterior gastro-enterostomy.

Koeppelin (Lyon Médicale, September 24, r899, and British Medical Journal, Suppl., January 6, rgoo) reports a modification of the operation known as 'submucous pyloroplasty.' The horizontal wound along the pylorus is made through the two outer coats, serous and muscular, of the bowel, and through the cicatrix, if there be one, and the exposed and unopened mucosa at once bulges like a hernia. The divided coats are then united vertically, as in ordinary pyloroplasty. Three reported cases of the submucous operation have proved successful. The first patient was a girl, aged twenty-two years, who was subject to spasm of the pylorus. The second patient was a woman, aged sixty years, who had suffered from cancer. Marked relief followed the operation. Eighteen months later the second patient underwent gastro-enterostomy with temporary benefit. Jaboulay of Lyons performed the third submucous pyloroplasty in June, I899. The patient was a man, aged forty-two years, who had suffered eight years previously from symptoms of gastric ulcer, followed later by evidence of pyloric obstruction. He at length vomited incessantly, suffered from intense pain which necessitated large doses of morphia, and became very 
thin. At the operation cicatricial stricture of the pylorus was discovered. The cure was complete. Koeppelin maintains that this modification is safer than the older pyloroplasty. The advantage lies in the avoidance of opening the stomach cavity. As lessening the danger of sepsis, the modification may be occasionally employed, but it should certainly never be performed in the presence of ulcer or cancer of the pylorus.

If, owing to the cicatrization of the ulcer, there be extensive hypertrophy of the pylorus, with a large amount of thickening, pyloroplasty is insufficient, as in such cases contraction will be likely to recur. Here pylorectomy may be performed, as in cases already related; or, better still, gastro-enterostomy, which is a simpler, quicker, and safer operation. Numerous and firm adhesions, active ulceration, and the presence of new growth, are also contra-indications for pyloroplasty.

Dr. Maurice Richardson (Boston Medical and Surgical Journal, November 30, r899) advocates partial excision of the stricture where there is extensive thickening and well-marked stricture, as in the following case in a man aged fifty-four years. The pylorus was extensively thickened and tightly constricted. The longitudinal incision was made for 3 inches through the stomach, the stricture, and the pylorus. The incision was converted into a lozenge shape or a broad $V$ by removing a considerable portion of the cicatrix of the anterior wall of the pylorus. By bringing together the opposing surfaces of the duodenum and the stomach, a wide lumen was procured. The mucous membrane was first united by a continuous suture, and the peritoneum by an interrupted silk Lembert's suture. The patient was in good health five months later. This pyloroplasty with partial excision seems to be the right practice in some cases of bad organic stricture and in active ulcer of the pylorus.

The dense tissue being cut away, the lozenge-shaped incision can be readily sutured so as to become transverse; while if a simple longitudinal incision is made through the tissues of a dense stricture, it is impossible to convert it safely into a transverse line of sutured wound owing to the great tension if the two ends be made to meet in the middle. 
A case of ours reported under Excision of Ulcer is a good example of this method of performing pyloroplasty.

Statistics.-In the Hunterian Lectures we collected 3 I 8 cases of pyloroplasty from all sources, of which 269 recovered, which equals a mortality of $15^{\circ} 4$ per cent. As in the earlier operations many were performed on cases that would be now treated by gastro-enterostomy, the mortality in properly selected cases, we think, should not exceed 5 per cent. at the outside estimate; and of the 27 cases operated on by us since 1897 , there is only one fatality to record.

A very important modification of the operation of pyloroplasty has been made by Dr. J. M. T. Finney, of Baltimore. The operation, though described by Dr. Finney as a form of pyloroplasty, would, in our judgment, be more aptly classed as a gastro-duodenostomy.

For the following account of the procedure we are indebted to Dr. Finney.

'The operation is as follows: Divide the adhesions binding the pylorus to the neighbouring structures, also free as thoroughly as possible the pyloric end of the stomach and first portion of the duodenum. Upon the thoroughness with which the pylorus, lower end of the stomach, and upper end of the duodenum are freed depends in large measure the success of the operation, and the ease and rapidity of its performance. I wish to emphasize this as one of the most important points in the operation. Frequently at first sight the pylorus may seem hopelessly bound down, when after a little patient toil and judicious use of the scalpel and blunt dissector it is found that it can be freed with comparative ease. A suture, to be used as a retractor, is taken in the upper wall of the pylorus, which is then retracted upwards. A second suture is then inserted into the anterior wall of the stomach, and a third into the anterior wall of the duodenum, at equidistant points-say about I2 centimetres from the suture just described in the pylorus. These second sutures mark the lower ends of the gastric and duodenal incisions respectively. They should be placed as low as possible, in order that the new pylorus may be amply large. Traction is then made upwards on the pyloric suture, and downwards 
in the same plane on the gastric and duodenal sutures. This keeps the stomach and duodenal walls taut, and allows the placing of the sutures with greater facility than if the walls remained lax. The peritoneal surfaces of the duodenum and stomach along its greater curvature are then sutured together as far posteriorly as possible. For this row I would recommend the use of the continuous suture, as it is more easily and quickly applied, and it can be reinforced after the stomach and duodenum have been incised. After the posterior line of sutures has been placed, an anterior row of mattress sutures is taken, which are not tied, but left long, in the manner indicated in the diagrams. These sutures, after they have been placed, are retracted vertically in either direction from the middle of the portion included in the row of sutures. Then, after all the stitches have been placed and retracted, the incision is made in the shape of a horseshoe. The sutures should be placed far enough apart to give ample room for the incision. The gastric arm of the incision is made through the stomach wall just inside the lowest point of the line of sutures, and is carried up to and through the pylorus, and around into the duodenum, down to the corresponding point on the duodenal side. Hæmorrhage is then stopped. It is well to excise as much as possible of the scar tissue upon either side of the incision, in order to limit, as far as possible, the subsequent contraction of the cicatrix. This procedure I carried out in two of my cases with great satisfaction, and I should strongly recommend it in all cases where the walls of the pylorus are much thickened and there is much scar tissue present. It is well, too, to trim off with scissors redundant edges of mucous membrane, in order to prevent the formation of a valve-like fold of mucous membrane at the new pylorus. A continuous catgut suture is now taken through and through all the coats of the intestine on the posterior side of the incision. This reinforces the posterior line of sutures, secures better approximation of the cut edges of the mucous membrane, and prevents the reunion of the divided intestinal walls. The anterior sutures are then straightened out and tied, and the operation is complete, unless one wishes to reinforce the mattress sutures 
$$
\text { . }
$$ 
PLATE XX.
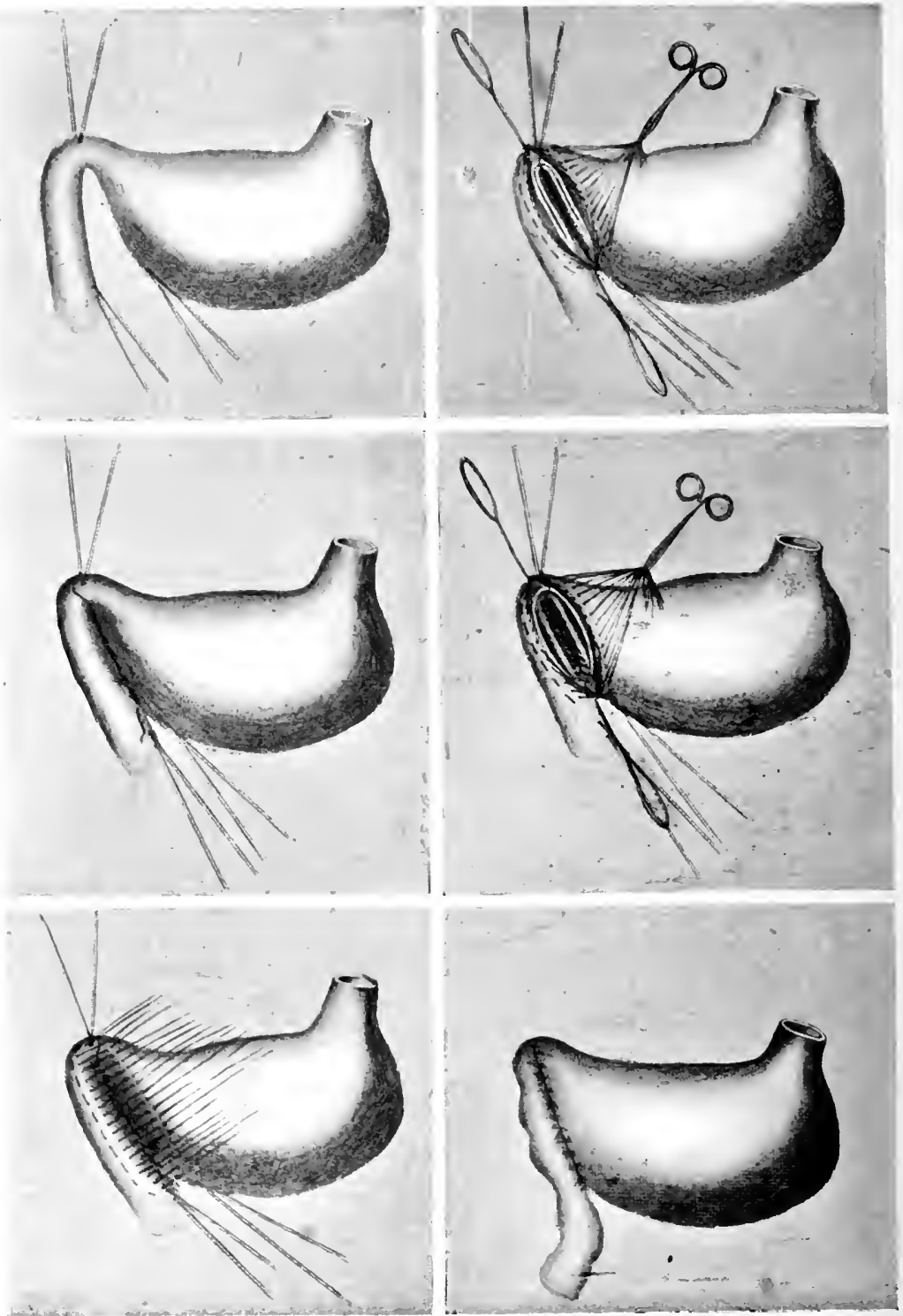

Fig. So.-Finner's Operation

To face p. 271.? 
with a few Lembert stitches. This procedure, as is readily seen, gives the minimum of exposure of infected surface. All the stitches are placed and the posterior row tied before the bowel is opened, and it remains open just long enough to control the hæmorrhage. The size of the newly-formed pyloric opening is limited in this operation only by the mobility of the stomach and duodenum and the judgment of the operator. In all of my cases the incision has been about I2 centimetres in length, and could have been made longer had I chosen to make it so. Unless the stomach is very much dilated or has descended to an unusual extent, the lower limit of the new outlet is at or near the level of its most dependent portion.'

In a paper published in the Medical Record, March 7, Igo3, Dr. Finney had been able to collect twenty-nine cases operated upon by this method, with a mortality of 6.8 per cent. The advantages of this method were: (I) The ease and facility with which it could be performed; (2) its low mortality, 6.8 per cent.; (3) its success in relieving at once the disagreeable symptoms; (4) its applicability, even in the presence of dense adhesions about the pylorus ; (5) except in cases of extreme dilatation it furnished effective drainage, placing the outlet at or on a level with the most dependent portion of the stomach; (6) it prevented absolutely spur formation and the vicious circle; (7) it placed the new pylorus in approximately its proper place. The disadvantages which had developed so far were theoretical rather than practical. In one case the mesentery was so short that the apposition of the stomach and duodenal walls was rather difficult. It was conceivable that the adhesions around the new pylorus might cause in time a second obstruction, but there was as yet no evidence that this ever occurred. The operation admitted of the excision of a large portion of the anterior gastric wall as well as of the pylorus, and the opening could be made so large as to greatly lessen the fear of subsequent cicatricial contraction. There had been a singular freedom from post-operative nausea, vomiting, and pain in his cases. 


\section{Gastro-duodenostomy.}

This operation was first suggested in 1892 by Jaboulay, and performed by him in I894. He has since related four cases, three of them successful. The operation has been warmly approved by Mikulicz and Villard. The anastomosis is made between the pyloric end of the stomach and the anterior surface of the duodenum. Each viscus is opened by a vertical incision, and union may be effected either by the button, the bobbin, or by simple suture. The operation is most easily performed when there is a largely dilated stomach and a mobile duodenum. In one case reported by Spencer any other gastro-intestinal anastomosis was impossible by reason of extensive adhesions which affected all but the pyloric portion of the stomach. When the pyloric region is adherent or invaded by growth the operation is difficult, or may be impossible.

The advantages of this procedure over gastro-jejunostomy are claimed to be the easier emptying of the stomach at an orifice near the natural outlet and the absence of bile regurgitation, owing to the fact that the new opening in the intestine is placed above the bile papilla.

Professor Kocher has recently suggested that the duodenum should be freed by stripping up the peritoneum to the right of the vertical (second) portion, so that an anastomosis between the stomach and the duodenum may be more readily performed. The following description is given by Professor Kocher (Scott. Medical and Surgical Journal, October, I903, p. 3II) :

' The most suitable incision is one similar to that which we recommend for exposing the gall-bladder-viz., an oblique incision two fingers' breadth below and parallel to the right costal margin, beginning at the middle line. After dividing the skin and fascia, the rectus muscle is cut through as far as the broad abdominal muscles. The posterior layer of the rectal sheath, the fascia transversalis, and the peritoneum are divided. In muscular subjects the transversalis muscle is split parallel to its fibres, which are then firmly drawn apart. Should any adhesions exist between the gall-bladder and the colon, they must be divided. The liver is drawn upwards, the stomach to the left, and the 
transverse colon and the descending limb of the hepatic flexure downwards. The duodenum is then brought into view, and its outer border is clearly defined. A pad of gauze is placed against the under surface of the liver, and the latter is then drawn well upwards with a suitable retractor. Gauze compresses are also employed to push aside the stomach and colon.

'The delicate layer of parietal peritoneum covering the kidney is divided vertically $1 \frac{1}{2}$ inches external to the second part of the duodenum, and the incision is then continued vertically downwards through the upper layer of the transverse mesocolon (which is held on the stretch) as far as the larger branches of the vessels. The fingers are then introduced behind the left edge of the incision through the peritoneum, and the duodenum is separated from the vertical column, the vena cava, and the aorta, until it can be brought forward and pressed against the pyloric portion of the stomach, which, in its turn, is compressed against the left edge of the wound in the abdominal wall, so as to shut off the general cavity of the stomach and prevent escape of its contents. Both stomach and duodenum are now compressed above and below between the fingers of an assistant, and the lateral anastomosis is effected in the usual manner by two rows of sutures.

' To one who has once convinced himself how easily and securely this lateral gastro-duodenostomy can be performed-provided the duodenum can be rendered sufficiently movable-it will be evident how admirably the operation fulfils the indications for treatment in stenosis of the pylorus. Unlike the other surgeons who have performed gastro-duodenostomy, we do not limit the operation to special cases; on the contrary, we regard it as the normal procedure, and we are of the opinion that it will take precedence over all the previous methods of gastro-enterostomy and pyloroplasty.

'The method is subject to only one contra-indication-viz., the presence of such extensive adhesions to the under surface of the liver that the duodenum cannot be sufficiently freed. This difficulty of adhesions can, however, often be overcome, as we have proved in three of our cases; but the fact of having to perform the suturing inside the abdomen is apt to interfere with the security of the stitching, especially in difficult cases. It is on this account that subpyloric gastro-duodenostomy did not meet with universal acceptance. The subpyloric portion of the duodenum cannot be drawn out of the wound on account of its connection with the gastro-hepatic omentum and the important structures contained within it. This fixation to the under surface of the liver may be so firm that only the lower two-thirds, or only the lower part, of the vertical portion of the duodenum, together with the inferior flexure, can be brought in contact with the stomach. 
'We therefore propose that, instead of Villard's subpyloric gastro-duodenostomy, the name lateral gastro-duodenostomy be given to this operation, to distinguish it from our method of inserting the divided duodenum into the posterior wall of the stomach after resection of the pylorus. The great difference between Villard's subpyloric gastro-duodenostomy and our procedure is that we render the descending portion of the duodenum, the inferior flexure, and a considerable portion of the third (transverse) part so movable that the parts to be sutured can readily be raised up and surrounded with gauze, so that the sutures can be introduced extraperitoneally with comfort and security.

- We intend in future to perform lateral gastro-duodenostomy in all cases of stricture of the pylorus, and only to have recourse to gastro-jejunostomy in cases rendered unusually difficult by firm adhesions. We have performed the operation on five occasions, four times for cicatricial stenosis and once for malignant stricture. The results have been convincing. Regurgitation of bile either does not occur at all or only at first, when a large, gaping opening has been made. It is advisable to make the anastomotic opening as high as possible, and not too large. The patient with carcinoma was at once relieved of all her discomfort. Only one patient complained subsequently of very severe pain, and he had a simultaneous cholecystotomy performed for gall-stones. That a dilated and sacculated stomach is not so well emptied as by our inferior gastro-jejunostomy is obvious. If gastro-duodenostomy be performed in such a case, it is advisable to occasionally wash out the stomach.' 


\section{CHAPTER XVII \\ THE COMPLICATIONS OF GASTRIC ULCER}

\section{GASTRORRHAGIA.}

THE opinion held by many practitioners of medicine, ' that bleeding from the stomach the result of ulceration rarely proves fatal,' requires carefully reconsidering, and the facts marshalling and looking at also from the surgical point of view.

Just as in other internal hæmorrhages, bleeding from the stomach was until quite recently a subject that, so far as treatment is concerned, only came under the notice of the physician. It is only since the advances in surgery have demonstrated the feasibility and safety of exploring the abdomen that the question of surgical treatment of hæmatemesis has been a subject that can be discussed with advantage.

We shall confine our remarks entirely to the treatment of hæmatemesis or melæna arising from gastric or duodenal ulcer, and to that form of gastrorrhagia, occurring for the most part in young women, where the question of ulcer is still sub judice, leaving out of consideration the vomiting of blood from cirrhosis of the liver, aneurism, heart disease, and other general conditions which are only amenable to medical treatment. From the point of view of treatment, it is convenient to classify the subject under two divisions, which are well marked clinically :

I. The sudden severe attacks of hæmatemesis or melæna, occurring usually in young anæmic women, threatening life immediately and frequently occurring without any or with only slight preliminary symptoms; and in this class may be 
conveniently considered the hæmatemesis said to be due to vicarious menstruation, and the form known as post-operative hæmatemesis, for these three forms are all related in the fact that after death the stomach lesions seem altogether inadequate to explain the serious nature of the bleeding.

2. The cases associated with serious well-marked ulceration of the stomach, pylorus, or duodenum. This class of cases may be conveniently divided into two, both characterized by preliminary symptoms pointing to serious organic changes and by pathological conditions which adequately explain the complication of hæmorrhage. These are $(a)$ the serious acute hæmorrhages often occurring at longer or shorter intervals, and preceded by, or associated with, symptoms of ulceration; and $(b)$ the frequently-repeated slighter hæmorrhages, where, though no large amount of blood may be lost at one time, the steady bleeding in the shape of coffee-ground vomit or blood in the stools leads to profound anæmia and other complications, these being associated either with chronic ulcer or malignant disease.

The conditions coming under this second class are associated with obvious organic disease, usually ulcer of the stomach or duodenum of a kind generally only amenable to operation; and in advocating surgical treatment for such of these cases as have bled seriously, one of the strongest arguments in its favour is that at the same time that the complication causing immediate anxiety is being treated the disease giving rise to it will be cured. Although we have operated on a large number of cases coming under both varieties of the second class, we have only operated on two of the first class of cases while the bleeding was in progress, and in both successfully; but so small a number of cases only demonstrates that operation can be performed even in extreme conditions with a fair chance of success, and by no means proves that it should always be done, though we feel firmly convinced that both of those patients would have died had the operations not been performed. A third case on which we operated while the bleeding was in progress we shall mention under the second class, as it was associated with obvious ulceration. In none of these cases was operation 
performed during the first or even second attack of bleeding, and we do not know of any surgeon who has advocated operation during a first attack until after failure of ordinary treatment.

CASE I.-On July 6 we were asked by Dr. Humphrey, of Armley, to see a patient, aged thirty-three years, who had been seized with violent hæmatemesis a week previously, the attack having recurred four days later, and again on the day that we saw her. The patient had been anæmic before operation and had had some slight indigestion, but otherwise did not consider herself ill, and she had been married only three months. On our arrival, we found her quite blanched and with a very rapid pulse; but as the bleeding had resisted ordinary remedies, and as she was apparently going to die if unrelieved, though it seemed a rather desperate procedure, we decided to give her the chance of relief by operation. She was removed by ambulance to a surgical home, and as during the journey the bleeding began again, we operated without delay. A pint of saline fluid containing I ounce of brandy was administered by the rectum, and ro minims of solution of strychnia were administered just before the operation, the patient being completely enveloped in cotton-wool. On opening the abdomen, there were no indications on the surface of the stomach as to the situation of the bleeding ulcer. We therefore made an incision in the axis of the stomach, which enabled us to explore the interior. We counted no less than seven bleeding-points. As two of them were bleeding freely, we took them up by artery forceps and ligatured the mucous membrane en masse; the other points stopped on exposure to air. We then rapidly closed the wound in the stomach and performed posterior gastro-enterostomy, using one of the bone bobbins as a splint over which to make the anastomosis. We had a little anxiety on account of shock for the first twenty-four hours, but by administering saline fluid by the bowel and injecting it freely into the subcutaneous tissue, together with the administration of several injections of liquor strychniæ, the difficulties were tided over, and the patient's further progress towards recovery was uninterrupted. She was able to take solid food in the second week, and when she left at the end of the month she could eat any ordinary food with. out discomfort. A year later she had had no recurrence of symptoms, and was well in every respect.

CASE 2.-The patient, a married woman, aged twenty-eight years, was seen on April I4, I902, with Dr. T., Dr. F., and Dr. R., of Nottingham. A few months previous the patient 
had been operated upon for varicose veins, and six weeks previously to the attack in question she had had influenza. On April 8 she was out hunting and felt quite well. On the gth, at I 2 noon, while in the house, she felt faint and vomited a pint of dark clotted blood, after which she was put to bed and treated medically. She continued to improve, and had no further vomiting until April I4, when at I2.30 p.m. she suddenly felt sick, became blanched and faint, the pulse becoming very weak. Blood was vomited in large quantity, and when the bowels were moved by enema a large amount was parted with. In response to a telegram, we saw the patient with the three physicians, and as the bleeding seemed to have stopped, and as the pulse was improving, it was decided to give suprarenal extract, to stop all mouth feeding, and to feed by the rectum. Steady improvement again took place, but on the evening of April i 8 (the tenth day of the illness) we received a telegram, saying: 'Hæmorrhage has recurred; have transfused. Come immediately, prepared to operate.' On our arrival, we found the patient blanched and with a pulse at times almost imperceptible. It was quite clear that the bleeding was persisting, and as medical treatment had been steadily persevered in for nine days and there had been three separate hæmorrhages, it was decided to operate forthwith. On opening the abdomen the stomach was found to be contracted, but the small intestines looked dark, as if filled with blood. We opened the stomach and carefully explored the interior, when, though we could find no evidence of any large ulcer, we found a considerable number of bleeding-points, three of which we ligatured en masse, afterwards swabbing the whole of the interior of the stomach with a sponge saturated with tincture of hamamelis. Thinking it desirable to set the stomach at rest, we rapidly closed the gastrotomy opening and performed a posterior gastro-enterostomy, using one of the decalcified bone bobbins. As the pulse was feeble, intravenous infusion of 4 pints of normal saline solution was performed. After this there is very little to record, except that the patient made a steady progress to recovery, and was able to be out of bed in the third week and to leave the house within the month. She rapidly regained her strength, had no further hæmorrhage, and is now quite well.

Now, it will be noticed that in neither of these cases was any distinct ulcer to be seen, though bleeding was occurring from a number of distinct and separate points, some of which were ligatured. The condition has been described by Dieulafoy, who gives two varieties : (I) Simple erosions, con- 
sisting apparently of mere abrasions of the surface epithelium, which, though so small as to be scarcely perceptible to the naked eye, may give rise to most alarming hæmorrhage. On the post-mortem table abrasions of this kind may be easily overlooked; but as seen when hæmorrhage is going on, the mucous membrane seems to be studded with numerous bleeding-points. (2) Exulceratio simplex. In the form to which Dieulafoy applied this term the lesions are rather more extensive, and the surface layers are removed to such an extent that the arterioles running under the muscularis mucosæ are exposed. This form of ulceration may give rise to terrible hæmorrhages that may prove rapidly fatal unless arrested by treatment. Dr. F. J. Smith (Medical Press, November I9, IgO2) has recently described what appears to be still another variety of erosion, small ulcers no larger than a pin's head, not spreading superficially and discoverable only on close scrutiny, but when found leading directly into a bloodvessel, this in several cases related having led to fatal hæmorrhage.

Dr. Donald Hood, in a paper read before the London Medical Society, February I5, I892, pointed out that the violent hæmatemesis occurring in early adult female life is of less significance than that occurring in later years, and that in many cases it is not associated with organic disease of the stomach, but is probably dependent on a derangement of the circulation due to the anæmic condition.

Dr. W. Hale White (British Medical Journal, Igoo, vol. ii., p. $16 \mathrm{r}_{3}$ ) has also raised the question in a very able paper, supported by a series of clinical and post-mortem observations, 'Are not some patients said to be afflicted with gastric ulcer really suffering from a different disease?' and this question cannot be summarily dismissed by a negative answer, for the paper is supported by careful observations by the author himself, as well as by other able pathologists. The important point is that, as stated by Dr. Hale White and many other physicians, some of the patients die directly from the bleeding, and what we surgeons have to try to prove is, Can we save life by supplementing medical treatment by surgical methods where medical means alone have failed? The two cases just related afford a small contribution 
in that direction. Possibly all these varieties are merely incipient stages of the true simple round ulcer of the stomach so well known, but this question cannot be settled by discussion, and can only be cleared up by a number of careful clinical and pathological researches. It is certainly a fact that in a large percentage of cases the bleeding ceases under rest in bed, abstinence from food, and general treatment, and the treatment adopted for the bleeding frequently leads to healing of the erosions; but in certain cases, as in those related, where there are repeated hæmorrhages, and where medical treatment has failed, are we to stand idly by and to allow the patient to die from bleeding which, if it were on the surface, we could arrest in a moment? We think not. Erosion is the most likely cause of hæmatemesis in those cases in which bleeding comes on without warning or after only a short duration of symptoms in young anæmic women, and from the fact that medical and general treatment is successful in arresting hæmatemesis in a considerable percentage of cases, and that it is difficult in the present state of our knowledge to say at first that the bleeding is not occurring from capillaries or small arterioles, it necessarily follows that medical treatment should always have a fair trial in every case of acute hæmatemesis. The very fact of medical treatment being so often successful in cases of apparently alarming hæmatemesis goes to show that capillary oozing or bleeding from arterioles is much more common, and accounts for many more cases of gastric hæmorrhage than has hitherto been supposed; but while thoroughly believing that we must also not close our eyes to the experience we have in general surgery of blceding from mediumsized arteries, such as the radial or ulnar, which we know would rapidly bleed a patient to death if only perforated on one side and surrounded by warm compresses, a condition that practically applies in all cases of hæmatemesis where the larger vessels are eroded. We must also bear in mind that in some cases the ordinary round ulcer may progress even to perforation without preliminary symptoms, just as it may erode a large vessel and lead to violent hæmatemesis. This we can vouch for from our own experience alone. If, 
therefore, medical treatment and rest properly carried out are not successful in arresting the bleeding, or if after being arrested it recurs, we must be driven to the conclusion either that a large vessel is perforated and requires direct treatment, or that even if the bleeding be due to multiple erosions further treatment should be considered; and if a surgeon has not been previously asked to see the case we would say emphatically that a surgical consultation ought to be held with a view to considering the question of operation and immediate arrest of the bleeding by direct treatment or by gastro-enterostomy if the patient is in a fit condition to bear it, just as would be the case if an ovarian artery were bleeding in ruptured extra-uterine gestation or a brachial or radial after an accident.

Before resorting to operation, the patient will have had a full trial of complete rest in bed, abstention from mouthfeeding, the use of rectal alimentations, and the application of an ice-bag to the epigastrium; but besides these general measures, Tripier's treatment by high rectal injections of hot water at a temperature of from $\mathrm{I} I 2^{\circ}$ to $\mathrm{I} 20^{\circ} \mathrm{F}$., repeated, if necessary, several times, may be well worth trying, or the use of suprarenal extract, as advised by Dr. Soltau Fenwick, (British Medical Journal, November 30, I9or).

The plan usually adopted was to administer ro fluid ounces of a freshly-prepared decoction containing 2 grains of the desiccated gland to the ounce as soon as possible after an attack of hæmatemesis, and to repeat the dose at the end of two hours. In two instances, where melæna was the sole symptom, three doses were given in four hours. In no case were any unpleasant symptoms observed, though occasionally the first dose produced an inclination to vomit. The cases which afford the most immediate and striking results are those in which the ulcer is comparatively recent, and is situated near the cardiac end of the stomach, as, for example, in the acute disease of young adults, and in that which sometimes complicates cirrhosis of the liver and valvular disease of the heart. In chronic ulcers of the pyloric region, on the other hand, where the eroded vessel is often of large size and so firmly embedded in fibrous tissue as to be in- 
capable of contraction, experience will probably show that suprarenal extract, in common with other hæmostatics, is of little value; but even in such cases an effort should be made to irrigate the stomach with a strong solution through a soft tube.

Although capillary hæmorrhage may supervene on other conditions, such as congested portal circulation, enlargement of the spleen, and engorgement of the venous system, when general treatment will usually be all-sufficient, yet there are two other special conditions in which the bleeding may be so free as to raise the question of surgical treatment; these are vicarious hæmatemesis at the menstrual period and postoperative hæmatemesis. The former condition is well recognised, and usually yields to medical treatment. Petersen's experience of surgical treatment in three cases of vicarious hæmatemesis terminating fatally after operation would not encourage surgical intervention, unless the circumstances of the case were very exceptional.

Post-operative Hæmatemesis.-In the Hunterian Lectures attention was drawn to post-operative hæmatemesis, of which we had seen several cases-four fatal. The cases occurred after operations for intestinal obstruction, tuberculous peritonitis, herniotomy, ovariotomy, and gall-bladder operations. Other cases have since been reported - one by Dr. Winslow (Lancet, October 20, I900, p. I900), in a stout woman, aged sixty-five years, after operation for umbilical hernia, where death occurred from profuse gastrorrhagia on the third day; and at the post-mortem examination, though the stomach and intestines contained a large quantity of blood, no vascular lesion could be discovered; and another by Mr. C. W. Mansell-Moullin (Lancet, October 20, I900, p. I900), in a young man, aged twenty-three years, who died from profuse hæmatemesis within forty-eight hours of a simple exploratory incision in the left iliac fossa. Post-operative hæmatemesis was neither generally recognised nor well understood, but since that time several papers have appeared on the subject both at home and abroad, and, though now fully recognised, its cause remains obscure. Professor von Eiselsberg recorded eight 
cases at the German Surgical Congress, I899, and tried to explain it as a result of injury to the omentum from several cases observed in the Königsberg Klinik after operations involving ligature of the omentum, after proctectomy for cancer, and after torsion of the omentum. Although this explanation may serve as a cause, it does not account for all cases, as proved by one of ours in which a malignant tumour of the common bile-duct causing jaundice and distended gall-bladder was operated on under cocaine, the distended gall-gladder being simply exposed through a small incision, stitched to the parietes, and drained, without in any way exposing any other viscera; yet violent hæmatemesis set in the day after operation, and proved fatal in three days. This case also disproves two other theories that have been advanced-one, that general anæsthesia is the cause, and another, that sepsis affords an explanation, the latter being a theory advanced by Dr. Rodman in his oration before the American Medical Association in June, rgoo. That sepsis was not the explanation in the cases in which the opportunity has occurred of observing the condition after death is undoubted, for in each there was no sign of peritonitis or other septic trouble ; and, moreover, there was no gross evidence of ulcer or other injury to any vessel of sufficient size to be visible to the naked eye. The theory of sepsis is also negatived by recovery in the greater number of cases, and by there being no sign of distension or any of the other usual concomitants of septicæmia when it occurs after abdominal operations. The only explanation that seems at all feasible is that the hæmorrhage is dependent on a reflex nervous influence, and it is quite possible that this same explanation applies to vicarious hæmatemesis at the menstrual period; but it should also be remembered that slight erosion of the mucous membrane of the stomach, sufficiently deep to cause severe gastrorrhagia, may be almost imperceptible to the naked eye, even when searched for carefully on the post-mortem table. Though serious, it is not necessarily fatal, and we have known several cases yield to general treatment, such as high injections of hot water at a temperature of from $I 12^{\circ}$ to $116^{\circ} \mathrm{F}$., as advocated by Tripier, free purgation by calomel, the stopping of 
all mouth-feeding, and the application of ice to the epigastrium, etc. In post-operative hæmatemesis operation can be seldom feasible or advisable, and Reichard's experience of operations followed by death in two cases would lead one in the future, as in the past, to rely on general rather than on surgical treatment.

An interesting paper was published in the Edinburgh Medical Joumal, March, I9o2, by Dr. Robert Purves, who had collected 37 cases, of which he had obtained particulars in 29. Twenty-four followed abdominal operations-including hernia, 8 cases; gall-bladder, 3 ; appendicitis, 6 cases; the others being after operations for ovariotomy, tuberculous peritonitis, intestinal obstruction, exploration for stab of abdomen, and exploratory incision in the iliac fossa. Of the 5 other cases, 2 followed excision of the rectum; I, suprapubic lithotomy; and I, removal of cancer of the palate. He then goes on to report II cases from the wards of the Royal Infirmary, Edinburgh, in which the mortality was $72^{\circ} 5$ per cent., and of the 29 cases previously recorded, 69 per cent. died.

Class 2.-We have next to consider the second class of cases, where the bleeding is associated with ulccration of the stomach or duodenum, which may be conveniently divided clinically into acute and chronic hæmorrhages.

Surgical treatment in this class of patients serves two purposes : the arrest of bleeding and the cure of the ulceration that has led to it.

When it is borne in mind that gastric ulcer occurs in 5 per cent. of the community, and that, according to Brinton, Habershon, Muiller, Dreschfeld, Lebert, and others, the mortality from all causes in all cases of ulcer is from to per cent. to $5^{\circ}$ per cent., it must be at once allowed that the consideration of the subject is a matter that should claim the attention of the profession to a greater extent than it has hitherto done. When it is fully grasped that many of the complications can only be dealt with successfully by surgical means, and that even in the mildest cases. of gastric ulcer some of the most serious complications, such as perforation and hæmorrhage, 
may suddenly supervene, we think that all will agree with the opinion that physicians and surgeons should, in the treatment of these serious cases, be in a closer touch and have a more perfect understanding, so that valuable time may not be lost when surgical interference is required. At present we have no hesitation in expressing a conviction that the treatment of gastric ulcer, especially among the working classes, is altogether too careless and unscientific, and that the out-patient treatment of such cases, whether in hospital or private practice, is absolutely wrong and attended with danger, either of sudden catastrophe from rupture or bleeding, or of the transformation of an acute into a chronic disease, which can then only in the greater number of cases be set right by a surgical operation. As soon as gastric ulcer is suspected, the patient should be told the serious nature of the disease, and the uncertainty of cure without rest in bed and long-continued care in diet under proper medical supervision. If, then, from business or other reasons the advice of the medical attendant is ignored, the blame rests on the patient, and not, as at present is so often the case, on the slipshod method of aiming at relief and calling it a cure. Fortunately, the surgical treatment of chronic and recurring gastric ulcer and its complications is becoming more and more advocated, and with increasing experience more and more perfect, so that to-day the tale is very different from that of a few years ago.

The complication of hæmorrhage from the stomach, which, according to different authorities, occurs in a greater or less degree in from 50 to 80 per cent. of all cases of gastric ulcer, is, it is said, fatal when medically treated in from II to 3 per cent. (Müller, II per cent.; Welch, from 3 to 5 per cent.; Brinton, 5 per cent.; Debove, 5 per cent. ; Steiner, 6.36 per cent.; Lebert, 3 per cent.; Dreschfeld, $3 \frac{1}{2}$ per cent.; and Rodman, 8 per cent.). We believe that from 3 to 5 per cent. of deaths in cases of gastrorrhagia from ulcer will not be far from the truth. We are therefore dealing with a very serious and common accident.

The most recent evidence we have concerning the result of medical treatment in cases of gastric ulcer is from the 
London Hospital, which we referred to in Chapter XII. (p. I7o). Out of the I 8 per cent. of patients that died, $2^{\circ} 5$ per cent. succumbed to hæmorrhage.

The bleeding may be arterial, venous, or capillary, and, curiously, capillary hæmorrhage may be so free as to render it difficult to say that some large vessel has not given way; yet after death a careful examination may fail to discover any gross vascular lesion.

Venous hæmorrhage, if from perforation of one of the large trunks, such as the coronary, splenic, or portal, may cause rapid death; but, owing to the diminished pressure in the veins and the more languid circulation, a rapidly fatal result is less likely than in arterial bleeding, and should the hæmorrhage persist or recur, such cases would be likely to benefit by surgical treatment.

Arterial bleeding is mostly responsible for the serious and fatal hæmatemesis due to gastric ulcer. The bleeding may be from the small arterioles which radiate between the peptic glands, from the trunks along the curvatures (the coronary being the most frequently affected), or from extrinsic arteries, the splenic alone being responsible, according to Brinton, for 55 per cent. of the deaths in fatal hæmatemesis.

The following list of 54 fatal cases of hæmatemesis is taken from M. Savariaud's thesis: Ulcerations of the splenic artery, I7 cases ; ulcerations of the coronary artery, 6 cases ; ulcerations of the pancreatico-duodenal, 7 cases; ulcerations of the gastric arterioles, Io cases; branches of the coronary vein, 2 cases; other veins, 2 cases; vessel not determined, 2 cases; no vascular orifice visible, 4 cases; vessel not mentioned, 4 cases. The pancreatico-duodenal and right gastro-epiploic are the arteries chiefly affected in pyloric and duodenal ulcers. The peculiarly fatal nature of hæmorrhages from medium-sized arteries depends on the fact that an ulcer makes a lateral opening into the vessel which prevents natural hæmostasis by retraction and contraction of the artery. The whole force of the heart is then exerted on the clot, which tends to form during the syncope that usually occurs when a large quantity of blood is suddenly poured out. 


\section{PLATE XXI.}

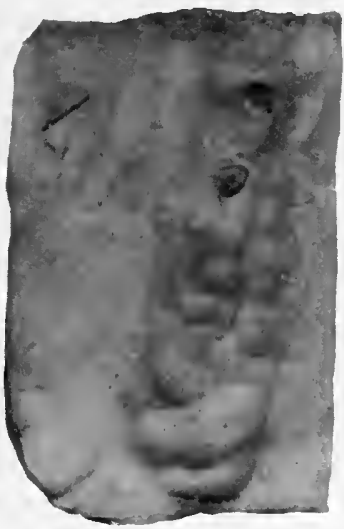

Fig. 8I.-Stomach shoiving Large Varicose Veins.

Perforation occurred at two places, and led to fatal hæmorrhage.

(No. 2,402, Royal College of Surgeons' Museum.)

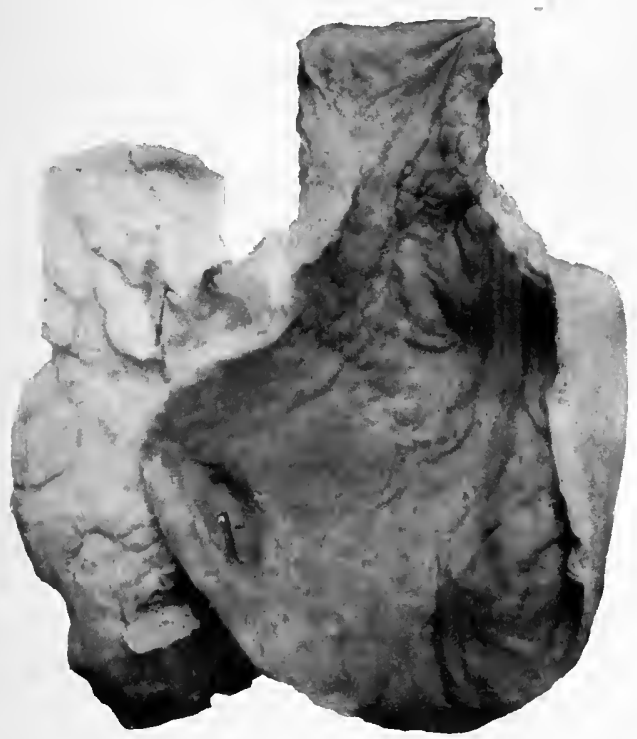

Firs. 82.-Ulcer Midway between Cardiac and Pyloric Orifices, near Lesser Curvature, eroding Splenic Artery, on which is a Small Aneurysmal Dilatation.

From a man of fifty-six. Death from hæmorrhage. (No. 2,401a, Royal College of Surgeons' Museum.)

To face p. 286.] 

The interval between the first onset of bleeding and death is of great importance surgically, and is well shown in the table from M. Savariaud's thesis, which is therefore worthy of note:

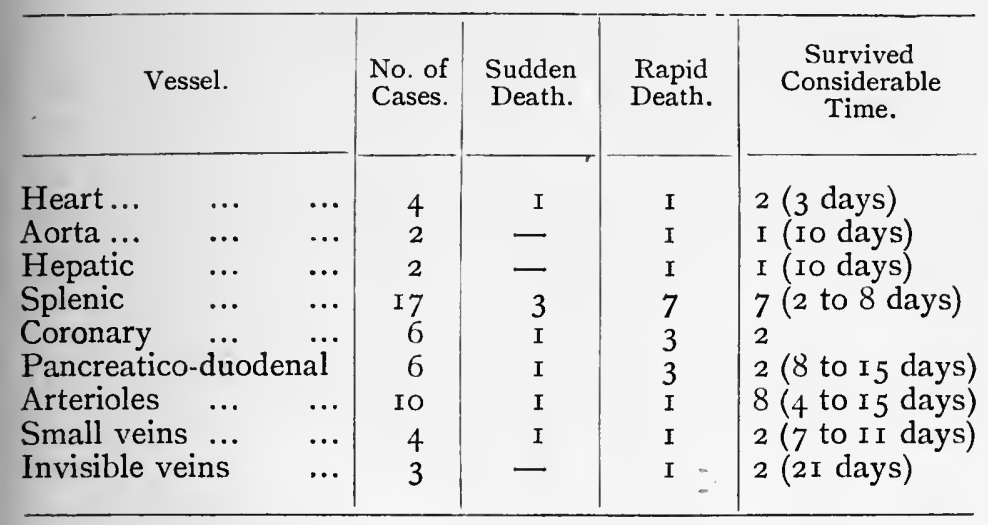

It shows that in the present state of our knowledge it is impossible to diagnose the size of the vessel perforated, either from the amount of blood lost or the length of survival.

A probable diagnosis of the site of the bleeding may, however, often be arrived at in hæmatemesis from ulcer by a careful study of the previous history, especially with regard to the site of pain, the time of onset after food, and the influence of posture, as well as by the direction in which the pain radiates. The situation of the tender spot, the presence or absence of tumour, and of dilatation or contraction of the stomach, also afford assistance.

As will be seen later, the diagnosis of the site of the ulcer, and therefore presumably of the bleeding, is of great importance from a surgical point of view, and will influence both the advice given as to treatment and the method of procedure when the abdomen is opened. 


\section{Treatment.}

From the fact that medical and general treatment is successful in arresting hæmatemesis in 95 to 97 per cent. of cases, and that it is difficult in the present state of our knowledge to say at first that the bleeding is not occurring from capillaries or small arterioles, it necessarily follows that medical treatment should always have a fair trial in every case of acute hæmatemesis. The very fact of medical treatment being so often successful in cases of apparently alarming hæmatemesis goes to show that capillary oozing or bleeding from arterioles is much more common and accounts for many more cases of gastric hæmorrhage than has hitherto been supposed; but while thoroughly believing this, we must also not close our eyes to the experience we have in general surgery of bleeding from medium-sized arteries, such as the radial or ulnar, which we know would rapidly bleed a patient to death if the vessel were perforated on one side and surrounded by warm compresses, a condition that practically applies in all cases of hæmatemesis where the larger vessels are eroded.

If, therefore, medical treatment and rest properly carried out are not successful in arresting the bleeding in a few hours, or if after being arrested the bleeding recurs, we should be driven to the conclusion that a large vessel is perforated; and if a surgeon has not been previously asked to see the case, we would say emphatically that a surgical consultation ought to be held with a view to considering the question of operation and immediate arrest of the bleeding by direct treatment if the patient is in a fit condition to bear it, just as would be the case if an ovarian artery were bleeding in a case of ruptured extra-uterine gestation, or a brachial or radial after an accident.

An analysis of the table of M. Savariaud of 54 cases showing the prolongation of life in various fatal cases of hæmatemesis demonstrates that in 8 out of 54 -that is, in I 4.8 per cent.--death occurs so rapidly that there is barely time to consider the question of surgical treatment, and that in I9 out of the 54 , which equals $35^{\circ}$ I per cent., death occurs 
within from twenty-four to thirty-six hours after the first appearance of hæmorrhage. Now, although at present these cases are often allowed to die without operation, yet if the bleeding were from a radial or tibial, or any accessible artery, they would all be saved by the simple operation of direct ligature of the bleeding vessel, an operation which would also cure the bleeding stomach artery could it be readily found. The difference between the two is, however, not only one of accessibility, but also one of diagnosis; for if we are able to diagnose capillary hæmorrhage, the case will be generally one for medical treatment alone, but if the time arrives when we can diagnose with great probability arterial hæmorrhage from a large vessel, the case will, we believe, be considered one for the adoption of surgical measures. Our efforts, both as physicians and surgeons, must therefore be directed towards making a diagnosis of the size of the damaged vessel, for if it can be asserted that in all probability a large artery has given way, an operation could be undertaken in an early stage of the case, before exhaustion and profound anæmia have supervened, with great probability of success.

Where there have been distinct signs of gastric ulcer preceding the hæmorrhage, and where a sudden hæmatemesis has occurred, with great loss of blood, accompanied by an attack of syncope, a large vessel will usually be found to be the source of the bleeding. In all such cases not speedily yielding to medical and general means, surgical treatment will probably in the future be carefully considered, and in some it will be followed out; for there can be no absolute rule formulated that will apply to every case, and each must be considered on its merits. The present condition of the patient, the previous history, the surroundings, the possibility of skilled surgery and of good nursing, and other like circumstances, will all help in the decision.

Although both surgical and medical treatment in cases of fulminating hæmorrhage have so far yielded disappointing results, in the remaining $5^{\circ}$ per cent. of fatal hæmorrhages, where repeated bleedings occur, and the interval between the first seizure and death varies from a few days to two or three 
weeks, medical treatment will have been fully tried and failed, and there can be no question as to the advisability of surgical procedures being adopted.

At present, with the exception of Dieulafoy, who advocates operation during the first bleeding if as much as $\frac{1}{2}$ litre of blood is lost, all other surgeons who have written on the subject agree that general means ought to be relied on during and after a first attack, as in from 95 to 97 per cent. of cases such treatment succeeds, and until our means of diagnosis as to the size of the vessel injured is rendered more reliable we must assent to this rule; but after a second bleeding we have no hesitation in advising operation, even during the course of the hæmorrhage if the patient can bear it, or as soon after as his condition will permit the operation to be done; for experience tells us that further hæmorrhages are almost certain to occur unless preventive measures be adopted.

Doubtless the desirability of stopping bleeding from an ulcer of the stomach by surgical means must have occurred to many minds before being put into execution. To Mikulicz, however, belongs the claim of having been the first to operate for hæmorrhage from a gastric ulcer, on February I3, I887. Roux of Lausanne was the first to perform a successful operation in a case of hæmorrhage from the coronary artery, in which he excised the ulcer and ligatured the artery at both ends.

The following cases serve to illustrate the operative treatment in hæmorrhage associated with gastric ulcer :

CASE I. - The patient, a man aged forty-four years, was seen with Dr. M., of Huddersfield, on January 2, 1900, with the history that he had had pain after food, together with other symptoms of gastric ulcer, for four years. Latterly he had had recurrent hæmorrhage. On his arrival at the surgical home he was in a state of collapse, with a barely perceptible pulse. Tenderness over the pylorus, the pain occurring one and a half hours after food, and melæna left no doubt but that the ulcer was at or near the pylorus. Operation was decided on, and performed on January 4. The patient being in such a feeble condition, every precaution was taken to guard against shock. Gastroenterostomy was therefore done, a decalcified bone bobbin being used to make the anastomosis. The patient made an uninter- 
rupted recovery. He was up at the end of the third week, and returned home within the month. A year later he was in perfect health, and had had no recurrence of his stomach symptoms or any further bleeding.

CASE 2.-A man, aged sixty-three years, was seen with Dr. W. in March, Igor, with a five years' history of chronic gastric ulcer, and with a loss in weight of 6 stones. A year previously he had had severe hæmatemesis, which had been repeated slightly on several occasions. Within a few hours of his arrival in Leeds he vomited about 5 pints of clots and dark blood mixed with mucus, and he became so ill that death appeared imminent. Although he improved somewhat for a few days with rest, the vomiting and bleeding continued, and Dr. W. agreed that it would be better not to postpone operation. An hour before operation he vomited blood freely again. On the morning of March 22 the abdomen was opened, and the lesser curvature of the stomach was found to be much indurated, forming a tumour. There was also much puckering of the surface of the stomach, and the glands in the greater and lesser omenta were enlarged but discrete. A posterior gastro-enterostomy was performed, a bone bobbin being used. He returned home at the end of the month, and ultimately gained about 2 stones. He had no further bleeding, and lived for a time in great comfort; but the growth, evidently cancer grafted on chronic ulcer, progressed, and he succumbed to exhaustion about a year later, having been able to enjoy life for some months.

CASE 3.-The patient, a female aged twenty-seven years, was admitted to the Leeds Infirmary on January 27, I902, for acute hæmatemesis. Beyond some indigestion, the patient had nothing special to complain of until seven weeks before admission, when she was suddenly seized with severe pain at the epigastrium, and she vomited blood freely. The vomiting of everything taken continued for a fortnight, when she had another attack of hæmatemesis, after which she was sent to the hospital on the medical side. After her return home vomiting recurred on her taking food again, and on January $2 \mathrm{I}$, after severe pain, she vomited about 2 pints of blood, and the vomiting continued up to her readmission on the 27 th. During the seven weeks she lost a stone in weight. She was transferred to our care on February $\mathrm{I}$, her pulse being on that day $\mathrm{r} 3 \mathrm{O}$, and respirations over 40. We operated on February 2, four days after the last hæmorrhage, and as we could find no definite signs of ulcer on the surface of the stomach, we simply performed a posterior gastro-enterostomy. She made a good recovery, and returned home at the end of three weeks. Though on one occasion in 
June she had indigestion for a day and vomited, there had been no return of the bleeding, and she had regained flesh and health when we saw her six months later.

CASE 4.- The patient was a man, aged thirty-eight years, who had vomiting and pain after food for five years. In 1899 , while at work, he vomited 2 pints or more of blood and fainted. In January, IgoI, he again vomited blood freely, and after this hæmatemesis occurred in small or large quantities at various intervals up to our seeing him. There had been very considerable loss of weight, and he was profoundly anæmic. No tumour could be felt, but there was some dilatation of the stomach. On June 27 exploration showed a small cicatrix of an old ulcer on the anterior surface of the stomach near the pylorus, and the pyloric end of the stomach was thickened and firmly fixed by adhesions to the head of the pancreas, which was also indurated. Gastroenterostomy was therefore performed. Recovery was uninterrupted, and he was able to return home on July 27 , on which date he expressed himself in gocd health, and able to eat anything without pain. The improvement continued, he had no further vomiting or loss of blood, and he is now well.

CASE 5--At the end of June we were asked to see a patient, aged forty-four years, who had suffered from symptoms for nine years, but during the last four years he had had several attacks of hæmatemesis, sometimes followed by melæna for eight or ten days after the attacks. At first the loss of blood was limited in amount, but in the later attacks hæmorrhage had been very free. We advised operation. At the beginning of October, I902, we saw the patient again, and in the meantime, beside lesser attacks, he had had two rather severe hæmatemeses. As the last attack was subsiding under abstinence from food and rectal feeding, we advised that he should wait a week and then come to a surgical home for operation. Although on October I I there was a little vomiting of blood, he was moved to the home on the I2th, and we operated on the ${ }_{4} 4^{t h}$, when we found a rather extensive tumour of the pylorus, which, though nodular and associated with enlargement of the glands in the vicinity, was apparently due to thickening around a chronic ulcer. As it was adherent to the parts in its neighbourhood, we thought it better not to attempt removal, and simply performed a posterior gastro-enterostomy. From this time the vomiting ceased, and recovery was uninterrupted. He returned home at the end of the month. He has had no further hæmorrhage, and is now in good health.

CASE 6.-A man, aged fifty-eight years, was seen with Dr. B. and Dr. S., of Burnley, with a history of stomach trouble for over ten years. Vomiting was not a prominent symptom, but on four 
occasions he had had profuse loss of blood by the bowel, though only on one occasion had he vomited blood. The attacks of bleeding had been alarming on several occasions, and not only had the motions been tarry, but much clotted blood had been passed. The last severe hæmorrhage had occurred ten weeks before we saw him, though slight bleeding had occurred, and he was still profoundly anæmic. There was well-marked tenderness over the duodenum, with some dilatation of the stomach. We operated on July I4, I902, when we found numerous adhesions around a thickened and indurated duodenum. We performed a posterior gastro-enterostomy, since which time he has remained well and has had no further bleeding; moreover, he has regained flesh and colour.

CASE 7.-A female patient, aged forty-eight years, was seen with Dr. C. on January 29, 1902, with a history of symptoms of gastric ulcer, which had persisted for some months, and the loss of 2 stones in weight. The epigastrium was very tender, but no tumour could be felt. On January $3 \mathrm{I}$ exploration revealed the scars of several ulcers, with peritoneal adhesions at the cardiac end of the stomach and on the posterior wall. A posterior gastroenterostomy resulted in a rapid recovery, entire cessation of the hæmorrhage, and all untoward symptoms. She returned home at the month-end well, eating any ordinary food without discomfort, and has remained well since.

It would be easy to give the history of other cases (some have been reported in the Lancet, March Io, I900, p. 67I) in which hæmatemesis was the prominent symptom for which we operated. We must, however, mention one case more on account of the after-history which bears on the treatment.

CASE 8.-A married woman, aged forty-two years, was seen with Dr. T., of Scarborough, on March 7, 1902, with a history of stomach symptoms for fifteen years, and for some years pain from one to two hours after food. There had been five attacks of severe and alarming hæmatemesis, as well as loss of blood by the bowel. A tumour of the pylorus, which appeared to be fairly movable, and was distinctly tender, could be readily felt. We operated, and found the following conditions as taken from notes written the same day: "The tumour, which was hard and nodular, was on the lesser curvature, extending to the pylorus and duodenum and producing stenosis; but whether it was growth or inflammatory thickening around an ulcer it was difficult to say, judging from naked-eye appearances alone, though it had the 
appearance of cancer.' There were so many enlarged glands that, had it been malignant, the removal of the tumour would have been useless. We therefore performed gastro-enterostomy. Recovery was as smooth as possible, no vomiting occurred, and when the bowels were moved the motions were free from blood. The wound healed by first intention, and we took leave of the patient in the third week, when she appeared to be in excellent health, and was enjoying her food. At the end of the month, while sitting up, and in the presence of her husband, who was reading to her, she suddenly became faint and collapsed, and died within a quarter of an hour. The cause of death was evidently a profuse hæmorrhage into the bowel, for blood oozed from the anus directly after death. Unfortunately, no necropsy could be obtained. We think that in all probability the disease was carcinoma grafted on to ulcer, and invading either the portal vein or the vena cava, and that only a removal of the growth at an earlier period could have prevented the catastrophe.

The operations that may be performed for gastrorrhagia are: (I) Gastro-enterostomy; (2) exploratory gastrotomy; (3) ligature en masse of the mucous membrane; (4) excision of the ulcer; (5) pylorectomy or partial gastrectomy ; (6) pyloroplasty; (7) pylorodiosis, or Loreta's operation ; (8) cauterization of the ulcer and of the bleeding surface; (9) ligature in continuity of the principal arteries of the stomach; and (IO) ligature of the bleeding vessel in situ. It will be seen that the operation we prefer is gastroenterostomy, which can be performed expeditiously and without severe shock; it acts by draining the stomach into the jejunum, relieving the hyperacidity of the stomach contents, and giving physiological rest to the stomach. Even if some other operation, such as ligature of the vessels or excision of the ulcer, be performed, rest should still be secured by the performance of gastro-enterostomy. That gastro-enterostomy alone may occasionally fail we must admit, but as a rule gastro-enterostomy will succeed not only in arresting the bleeding, but in curing the disease on which the bleeding depended. In operating for hæmatemesis, if there be no sign of ulceration on the stomach wall, we would advise gastro-enterostomy alone; but if there be distinct evidence of a chronic ulcer on the anterior wall, or where it can be safely, reached, then excision of the ulcer should precede 
gastro-enterostomy. If there be a tumour of the pylorus or evidence of a chronic ulcer and adhesions are not extensive, pylorectomy will be the better operation. If the ulcer be adherent to or eroding the pancreas, it is better to be content with gastro-enterostomy and not to meddle with the ulcer, and this applies if the ulcer be in any region not readily accessible, where the removal of the ulcer would involve a difficult and prolonged operation. Although both pyloroplasty and pylorodiosis act by giving the stomach physiological rest, we would not advise either as a rule, for they are apt to be followed by relapse; moreover, gastro-enterostomy is much more efficient and a safer operation. Ligature of the principal arteries of the stomach would seem to be an uncertain method, and, though quite easy of application, we should only think of performing the operation as an accessory to other treatment. Ligature of the bleeding vessel in situ, which seems to be very simple, has been tried, and failed on all occasions on account of the friability of the tissues, unless, as in the cases we have recorded, the mucous membrane has been taken up and ligatured.

Technique of Exploratory Gastrotomy for Gastrorrhagia.On account of the fear of exciting fresh hæmorrhage, it is undesirable to wash out the stomach before operating on a bleeding ulcer; and as the patient is usually anæmic, and will therefore bear shock badly, it is desirable to have him enveloped in cotton-wool or otherwise kept warm on a heated table, and to give him a subcutaneous injection of strychnine before operation. If it be unnecessary to infuse saline fluid before the operation, an assistant should be ready to perform that duty while the abdominal operation is being proceeded with, should it be called for. After the abdomen is opened, the stomach may be emptied by pressing the contents into the duodenum, as suggested by M. Terrier. This saves much time, and rapidity is of great importance. The surface of the stomach should then be carefully examined, as very frequently a puckering of the surface or thickening of the coats will be noticed at the site of the ulcer, or a difference in colour may sometimes indicate its site. Should the operator be fortunately able to find 
these indications, much time may be saved; but if no indications of this sort be obtainable, the stomach must be drawn well forward and opened, either by a vertical incision, which gives less hæmorrhage, or by an incision in the long axis of the stomach, which affords a much better view of the interior. In order to avoid soiling the peritoneum, thin, flat sponges should be placed within the abdomen, and it is well to have a perforated sterilized gauze pad covering the

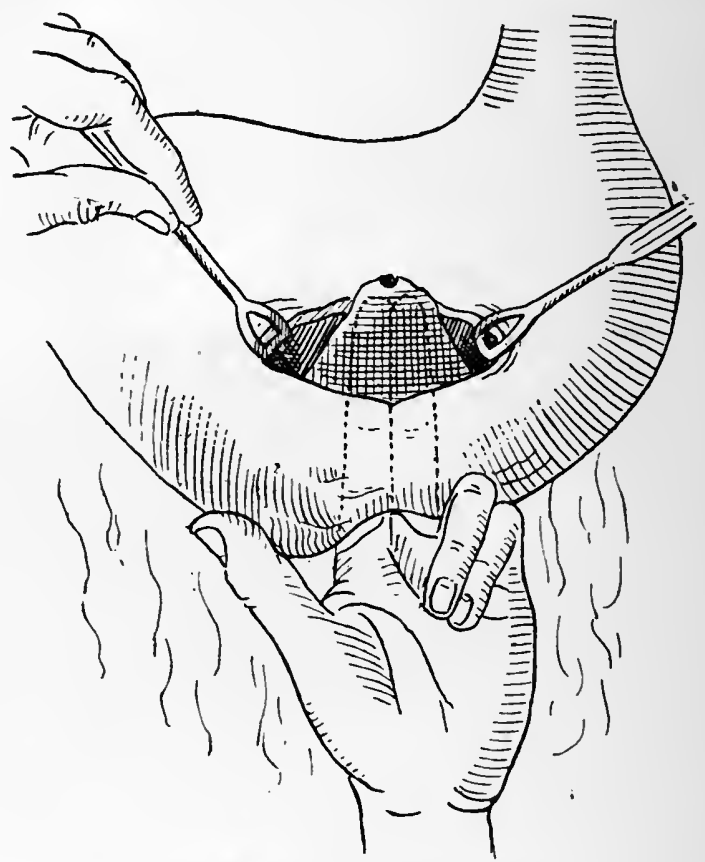

Fig. 83.-METHOD OF EXPOSURE OF UlCER.

stomach, the opening to correspond with the opening in the stomach. The edges of the stomach should then be held open by forceps, one above and below, and one at each end.

The interior of the stomach must now be systematically examined-first the anterior and posterior surfaces, then the upper and lower curvatures, and lastly the pyloric and cardiac orifices, all of which can be done by the naked eye, or by the aid of an electric cystoscope or a reflected light. 
If the posterior wall of the stomach cannot be sufficiently examined, a slit may be made in the omentum, and two fingers, or even the whole hand, pushed through it, and employed to invaginate the posterior wall. If no ulcer can be found on the wall of the stomach, the duodenum should be explored, not only by the finger, but by invagination. If no ulcer be found anywhere, and the bleeding proves to be capillary or from small undiscoverable ulcers,

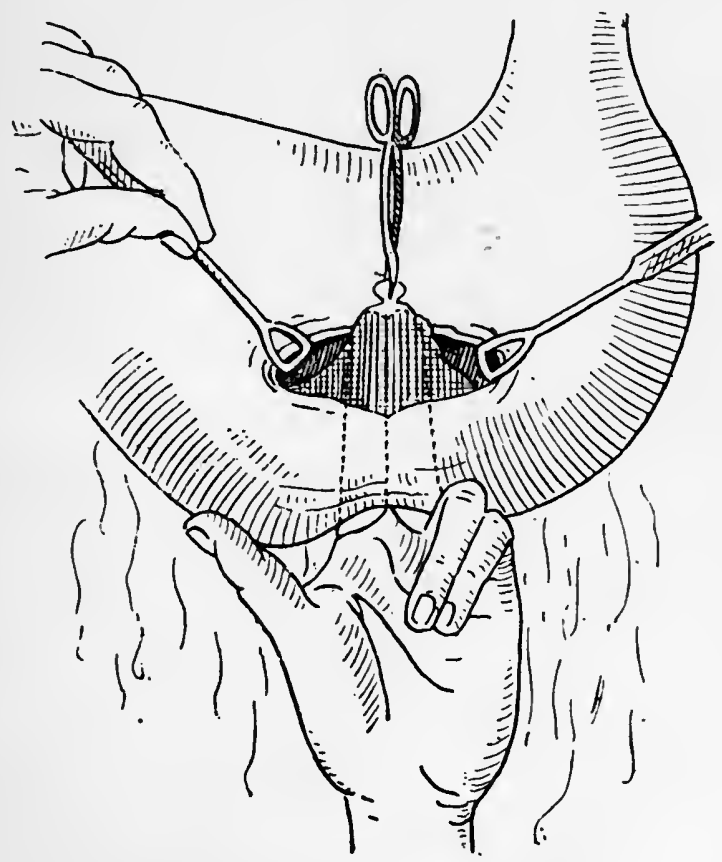

Fig. 84--Ligature of Bleeding-Point.

gastro-enterostomy should be performed; but if an ulcer be discovered, and it be possible to excise it, that operation should be done, as it undoubtedly offers the likeliest method of cure. After excision of the ulcer and ligature of the bleeding vessels, the edges of the wound are brought together by sutures, of which one or two layers may be employed. Should the excision involve the peritoneal coat, it is desirable to apply Lembert's sutures, and if it be on the posterior wall 
the operation may present some, though not insuperable difficulty. Should the ulcer be discovered in some region where excision is impracticable - for instance, near the cardiac end-the method adopted by Mikulicz, in his first operation, in $\mathrm{I} 887$, of applying the cautery, may be employed. This seems to have answered well in several cases, and where death occurred afterwards it was not from a recurrence of the bleeding. In a case of this kind gastroenterostomy is also advisable, by way of setting the stomach at rest. If any superficial bleeding-points be discovered, they may be caught up by forceps and ligatured; but this is more easily said than done, as the mucous membrane is very friable, and a ligature, if drawn at all tightly, tends to cut its way through. Moreover, forceps easily tear the mucous membrane, and may produce a larger bleeding area. The method of ligature $c n$ masse may be employed if the ulcer is small; it has proved serviceable in several cases-for instance, in Cases I and 2 (see pp. 277 and 278) -and it is easy of application when the ulcer can be reached.

The method has been tested by experiments on animals by Dr. Andrews and Dr. Eisendrath (Annals of Surgery), and has been shown to endanger perforation if the serous coat be taken up, except when supported by external sutures. Ligature of the main arteries of the stomach was suggested theoretically by Savariaud when no bleeding ulcer could be discovered; it does not, however, commend itself as being safe or likely to be efficient. Should the bleeding be from an ulcer at the pylorus, the operation of pyloroplasty may be performed, the ulcer being excised, and the edges of the gap left by the excised ulcer, as well as the edges of the incision, being united, so that the line of sutures shall be transverse to the axis of the pylorus. We do not, however, advise pyloroplasty when there is active ulceration of the pylorus, unless the ulcer can be completely excised; for in three cases of this kind there have been subsequent disappointments owing to contraction in healing. In one case the patient made a good recovery from the operation, but subsequently suffered from stenosis, for which a further operation had to be done. In a second case recovery took place, and the 
patient regained his lost weight; but subsequently a tumour of the pylorus developed, which was thought to be cancer, and for which pylorectomy had to be performed. In the third case perforation subsequently occurred. We think, therefore, that in cases of ulcer of the pylorus, where a clean excision of the pylorus cannot be done, it is better to perform gastro-enterostomy or pylorectomy, whichever at the time may appear to be the easier.

Wherever the pylorus is adherent, as it is in so many cases of ulcer, we should certainly prefer gastro-enterostomy; but if the pylorus be extensively ulcerated and free from adhesions, pylorectomy can be almost as quickly performed as gastro-enterostomy, seeing that the pylorus is already in the hand.

Although the operative treatment of parenchymatous or capillary hæmorrhage by any surgical method would seem to be of doubtful value [for, according to a report by Petersen (Medical Press, I899) from Czerny's clinic, of three cases operated upon for severe parenchymatous hæmorrhage from vicarious menstruation, operation entirely failed to relieve, and of other two cases of post-operative parenchymatous hæmorrhage by Reichard (Medical Press, January 3, Igoo) both died], yet if such cases, owing to persistence, seem to call for operation, then gastro-enterostomy, by securing complete physiological rest, would seem to offer the best chance of success.

In performing gastro-enterostomy our recent experience has led us to prefer the posterior operation, which may either be performed by simple suture, or may be modified by using a decalcified bone bobbin as a hollow splint over which to apply the double continuous suture, uniting the mucous and serous margins of the openings between the viscera, thus securing an immediately patent channel between the stomach and jejunum, and a temporary protection to the line of union.

The conclusions we would urge concerning the treatment of hæmorrhage from the stomach or duodenum are as follows :

I. Prevention.-All cases of acute uncomplicated gastric 
ulcer should be submitted to thorough medical treatment in the shape of long-continued rest and attention to diet, and not dismissed from observation until a sufficient time has elapsed to show that they are well.

2. In acute hæmatemesis of the first variety further accuracy in diagnosis is urgently needed; and the co-operation of the physician and surgeon is advisable in such cases if relief be not obtained by medical and general treatment, so that surgical means may be adopted if the bleeding is believed to occur from a large vessel, or is recurring or continuing and risking life; but seeing that capillary hæmorrhage is usually capable of relief by medical means alone, medical should always precede surgical treatment.

3. In profuse recurring, as in all chronic, hæmatemeses associated with gastric ulcer or other organic disease surgical treatment is decidedly called for. 


\section{CHAPTER XVIII}

\section{THE COMPLICATIONS OF GASTRIC ULCER (continued).}

\section{PERFORATION OF GASTRIC OR DUODENAL ULCERS.}

THE perforation of a gastric or duodenal ulcer is one of the most serious and most overwhelming catastrophes that can befall a human being. The onset of the symptoms is sudden, the course rapid, and unless surgical measures are adopted early, the disease hastens to a fatal ending in almost every instance.

Perforation of the stomach is usually described as being of two varieties, acute and chronic; but there is an intermediate class of cases, not embraced by either of these terms, which is best described as subacute.

In acute perforation the ulcer gives way suddenly and completely. A larger or smaller hole results, and through this the stomach contents are free to escape at once into the general cavity of the peritoneum.

In subacute perforation the ulcer probably gives way almost as quickly as in the acute form, but, owing to the small size of the ulcer, or to the emptiness of the stomach, or to the instant plugging of the opening by an omental flap or tag, or to the speedy formation of lymph, which forms, as it were, a cork or lid for the ulcer, the escape of fluid from the stomach is small in quantity, and the damage inflicted thereby is less considerable. The symptoms at their onset may be as grave as those in acute perforation, but on opening the abdomen the ulcer may be seen to be plugged, and no further escape of fluid is occurring.

In the subacute form of perforation there is always a 
complaint of greater discomfort for several days preceding the rupture. Vague general or localized pains have been felt in the abdomen, or a sharp spasm or 'stitch' when the patient turned quickly or attempted to laugh. One girl, a housemaid, felt the pain down her left side, especially when reaching up to her work; another said that it hurt her to bend, as her side felt stiff. These premonitory symptoms are important, and if recognised they should enable us to take measures to prevent the occurrence of perforation. They doubtless have their origin in a localized peritonitis, and the stiffness is due to the unconscious protection of an inflamed area by a muscular splint.

In chronic perforation the ulcer has slowly eaten its way through the stomach coats, and a protective peritonitis has had time to develop at the base. The escape of stomach contents is, therefore, local merely; barriers of lymph confine the fluid to a restricted area, and a perigastric abscess may form. A chronic perforation occurs more frequently on the posterior surface of the stomach, and the perigastric abscess occasioned thereby is recognised as 'subphrenic.' The acute and subacute forms of perforating ulcer are more common on the anterior surface.

\section{Acute and Subacute Perforation.}

I. Frequency.-The frequency of perforation in cases of gastric ulcer has been variously estimated by different writers. The extreme assessments are 6.5 and 28.5 per cent. The mean of all recorded cases is not an accurate index to the general liability, for a very striking difference is observed in the statistics collected in various countries. Tinker, Finney, and others have remarked on the immunity which exists in the United States. Lebert noticed perforation in I2 per cent. of his own cases. In Fenwick's own series of II 2 cases, perforation was observed in 28.5 per cent., and in a series of 678 autopsies in 22.8 per cent. Brinton states that perforation occurs in from 13 to 15 per cent., and Habershon in 18 per cent. In England the percentage may be stated to be approximately $\mathrm{I}_{5}$ to 20 . 

2. Site of the Ulcer.-The treatment of perforation of a gastric ulcer by operation necessitates as accurate an estimate as possible of the locality of such ulcers. Pariser and Lindner state that, of 200 cases of gastric ulcer, rgo will be on the posterior wall, and of these 4 will perforate; Io will be on the anterior wall, and of these $8 \frac{1}{2}$ will perforate. Brinton estimated that 70 per cent. of all perforations occur on the anterior surface of the stomach, 2 I per cent. on the lesser curvature, and only 9 per cent. on the posterior wall. Ulceration is more frequently found near the pylorus, but perforation is more common near the cardiac extremity. Fenwick, from a study of operation cases and post-mortem records, remarks that "whereas the acute disease usually perforates the comparatively thin coats of the stomach in the cardiac half of the viscus, close to the lesser curvature, and on the anterior surface, the chronic form of the complaint is most prone to perforate in the pyloric portion of the organ on the posterior aspect near the upper margin.' The greater liability of an ulcer of the anterior wall may be due to the fact that it lacks the support derived from viscera that is accorded to the posterior wall. Protective adhesions are therefore less likely to form.

The ulcers which lead to perforation may be multiple. In 20 per cent. of the recorded cases there were more perforations than one. Rarely a perforation of a gastric and duodenal ulcer may occur together.

3. Sex.-Perforation is seen more frequently in women, especially young women, and among them in young servantgirls, than in men. Allowing for the greater frequency of ulcer in women, we find that relatively perforation is more frequent in men, especially in men over the age of forty. Dr. Porter Parkinson has recorded the perforation of an ulcer in a child aged two.

4. The Size of the Perforation may vary widely. In some cases the opening is hardly larger than a pin-prick, in others it readily admits two fingers. The importance of this variation is probably considerable. A small perforation, especially when the stomach is empty, will permit the least trickle of fluid, and will become readily and completely blocked by an 
omental plug. A large perforation occurring when the stomach is full leads to the flooding of the cavity with a torrent of fluid and the sudden overwhelming of the peritoneum. The swiftness and suddenness of the onset and the severity of the symptoms are not improbably measured, therefore, by the size of the perforation and the state of repletion of the stomach.

There can be no doubt that recovery by medicinal treatment alone is possible both in the acute and in the subacute forms of perforation. We have had several cases under our care in which a diagnosis of perforation had been made by competent medical men; but an operation was impossible, as no skilled help was available until the urgency of the symptoms seemed to have passed off. Upon operating many months later, the evidences of peritonitis completely surrounding the stomach were undeniable.

Though patients may recover, their recovery cannot be urged as a reason for the delay or withholding of surgical help in all cases. For the possibility of spontaneous recovery, though not denied, is yet so remote as to make it imperative to adopt operative treatment at the earliest possible moment. The risk of operation is definite, the hazard of delay is immeasurable. There are times when the diagnosis may be difficult. If morphin has been administered to still the intolerable pain, the patient's condition becomes placid and comfortable. It may be almost impossible then to recognise the extreme urgency of the case. In such circumstances we have, however, placed great reliance upon a continued hardness and rigidity of the abdominal muscles. Even when the patient expresses herself as free from pain, when the aspect has become natural, and when the pulse has returned to the normal, the abdominal rigidity remains. In the case of I. S., a girl aged seventeen, upon whom an operation was performed for a perforated duodenal ulcer, the medical man who sent her to the infirmary had diagnosed a perforated gastric ulcer, and had told the patient and her parents that immediate operation alone could save her life. Having obtained consent to operation, he despatched the girl to the infirmary, and gave a hypodermic injection of $\frac{1}{4}$ grain 


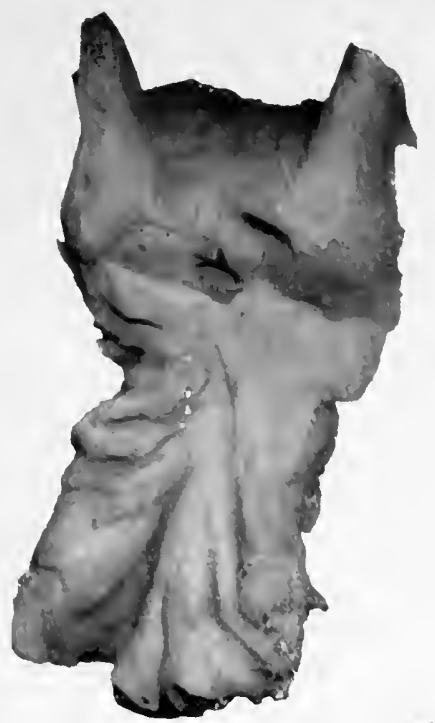

Fig. S7.-Piloric Ulcer showing Small Perforation, and Anothek Causing Contraction of Prlorus.

(No. 2,398, Royal College of Surgeons' Museum.)

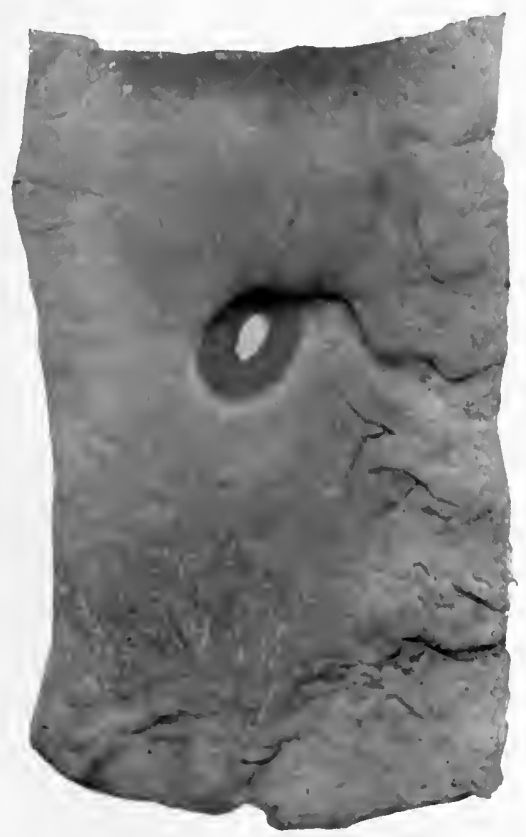

Fig. 88. -A Perforating Round Ulcer causing Death in a Lady Twenty-two Years of Age.

(No. 2,396, Royal College of Surgeons' Museum.)

To face $p$. 304.] 

morphin to lessen the distress of the journey. When seen shortly after her arrival, she looked in perfect health; she had no suffering, and her pulse and respirations were normal. The abdomen, though not distended, was absolutely rigid and immobile, and an operation was therefore performed at once. In any uncertain case we should incline to operation rather than to indefinite postponement to solve the diagnosis.

\section{Symptoms.}

Acute Perforation.-The perforation of a gastric ulcer may or may not be preceded by the symptoms and signs of ulceration. Symptoms are often lacking when an ulcer is situated on the anterior wall of the stomach; and as such an ulcer is more liable to perforation, the absence of characteristic symptoms is readily understood. If a history suggestive of ulceration can be obtained, the diagnosis of perforation in any abdominal catastrophe becomes simpler. In a certain, though small, proportion of cases (about 8 per cent.) no history of antecedent gastric disablement can be elicited.

The initial symptom is almost constantly a sudden, sharp, stabbing pain, altogether intolerable and overwhelming, in the epigastrium. Not seldom the patient remarks that something ' has given way' in the abdomen, and a feeling as of the trickling or gushing of fluid may be remarked. The pain may be limited in area, or may radiate to the shoulders or interscapular region; rapidly it invades the whole abdomen. There is immediately, or within a very few moments, a condition of collapse of varying profundity, but generally most severe. The face is drawn, and the expression tells of agony and anxiety and of the fear of present or impending disaster. The surface of the body is pale, and cold, and clammy, the lips livid, the face blanched, and there is a tinge of blueness in all the skin. The respiration is shallow and hurried; the pulse rapid, thin, or almost imperceptible. Vomiting, with or without bleeding, is an inconstant symptom. Finney finds it recorded in 40 per cent. of cases ; Fenwick in 29 per cent. Probably its occurrence depends upon the amount of fluid in the stomach, the 
size of the ulcer, and the depth of the collapse. Traube explains its infrequency as being due to the ease with which the contents escape through the gap made by the ulcer, an explanation which is in no degree convincing. Thirst is urgent, distressing, and unquenchable; the urine is scanty, and may, indeed, be wholly suppressed. At the first, the abdomen is hard and retracted, the muscles tight and fixed as a protecting and unyielding splint, insuring rest. Gradually, however, the abdomen fills with gas and fluid, and a bulging of its walls is seen. Liver dulness is then generally absent, but its presence or absence is void of any significance, and is unreliable as an aid to diagnosis. The whole abdomen, but more especially the upper half, is tender, and the patient shrinks from the contact of the surgeon's hand. Death may occur during the stage of collapse (in 4 per cent.-Fenwick). In rare cases death may seem almost instantaneous. If the patient rallies, the symptoms and the signs are those of general peritonitis. The severity of the instant shock and of the subsequent peritoneal reaction varies within wide limits, and is doubtless dependent upon the size and position of the perforation, the character and quantity of food in the stomach, and the severity of the vomiting. The sudden incidence of a marked leucocytosis is considered an important diagnostic sign by Finney. That it is not always reliable has been shown in several cases. Its precise worth and significance must be determined by future observation. Among the rare complications of the perforation of a gastric ulcer general emphysema must be mentioned. Demarquay, in 1866 , was the first to call attention to this remarkable occurrence. In one case related by Korach the gas that escaped into the peritoneal cavity was combustible.

Subacute Perforation.-Subacute perforation probably occurs when the stomach is empty or nearly so, or when a few filmy adhesions have formed which check the rapidity of the peritoneal invasion. The symptoms are of the same type as those described, but they are less brusque in their onset and throughout less vivid, and there is often a premonitory symptom, aching or stiffness, or a 'stitch' in the side for a few days before the acute onset. There is pain, sharp and 
stabbing, followed by sickness, faintness, and a degree of collapse. All these are much less marked than in the acute form, though they may, at the end of two or three days, develop rapidly, and end the patient's life in a few hours. Subacute perforation implies a less extensive, as well as a less acute, infection of the peritoneum. In some cases there may be a gradual merging into those classed as 'chronic,' where, inflammation being limited in area, a localized abscess forms.

\section{Differential Diagnosis.}

The conditions likely to be mistaken for acute perforating ulcer of the stomach are those which result from sudden intraperitoneal disasters. Such are acute perforating ulcer of the duodenum, ruptured tubal gestation, acute intestinal obstruction, acute hæmorrhagic pancreatitis, thrombosis of the superior mesenteric vein, and acute perforative appendicitis. When ulcers of the pyloric antrum or of the duodenum perforate, the visceral contents will run downwards to the right iliac fossa, and there setting up peritonitis, may cause symptoms which mimic with remarkable success those resulting from acute appendicitis. Other conditions which may resemble perforating ulcer in their symptoms are oncoming pneumonia, with its sudden pleuritic pain, acute poisonings, acute dilatation of the stomach, and acute inflammatory conditions in the gall-bladder. A minute history will enable a correct diagnosis to be made in nearly all the cases. The fact that in 92 per cent. a previous history of gastric ulceration can be obtained is helpful, but may be misleading.

We have seen a difficulty in diagnosis arise, and three cases are known to us in which negative exploration had been performed, when the patient was a woman at the commencement of a menstrual period who showed all the signs and symptoms of peritonism. From some unexplained and indeterminate cause a sharp attack of abdominal pain, followed by vomiting, distension, prostration, and collapse, had occurred in all, and had caused a confusion in the diagnosis. In the case under our own observation a history 
of previous similar, though less severe, attacks at the menstrual epoch and the absence of any marked abdominal stiffness or tenderness, though the belly was obviously distended, enabled us to negative the question of perforating ulcer of the stomach.

A difficulty may also arise in the diagnosis of a perforated duodenal ulcer. In a paper published in the Lancet in December, Igor, attention was drawn to the fact that in eighteen cases out of a total of forty-nine recorded, a diagnosis of appendicitis had been made, and an operation had been undertaken for that condition. The symptoms and signs in all these instances had been limited to the right iliac region, or had been more accentuated there. This is due to the fact that, owing to a hillock in the transverse mesocolon under the pyloric end of the stomach, extravasated fluids are directed downward and to the right into the right renal pouch, and thence to the right iliac fossa.

The two following cases may usefully be contrasted and compared. In the first a perforating gastric ulcer resembled in its symptoms a case of extra-uterine gestation, and in the second an extra-uterine gestation had to be distinguished carefully from a perforating gastric ulcer :

On January 18, I899, Mrs. P., aged twenty-nine, was suddenly seized at the railway-station with acute abdominal pain, followed immediately by collapse, in which condition she was removed to the hotel.

Her last regular ' period' had occurred seven weeks previously, and having missed the next 'period,' and thinking herself to be pregnant, she took an ecbolic five days previously, which brought on severe gastro-intestinal disturbance and fainting. Three days later-that is, two days before the attack in question-she had had some uterine hæmorrhage, but no stomach symptoms were complained of; and it was only on close inquiry later that any history of indigestion was obtained. Dr. Macrae saw her four hours after, and found liver dulness present. No abdominal distension; no rigidity of recti ; some fulness in left loin and tenderness over left ovary. On vaginal examination, there was fulness in Douglas's pouch and an indefinite fulness in the left fornix. When seen later the pulse was I30, and she was pallid and collapsed. A diagnosis of rupture of an extra-uterine gestation was made, and the abdomen opened above the pubes, when 
odourless gas escaped and a little fluid. The small suprapubic incision was therefore closed, and one made above the umbilicus, when a small perforation was discovered in the centre of a large chronic ulcer near the cardiac end of the stomach, close to the upper border on the anterior surface. The edges of the ulcer were refreshed and brought together by sutures over the line of incision. The abdomen was wiped clean after the evacuation of a quantity of turbid fluid from the right renal pouch and the space between the liver and the diaphragm. A small gauze drain was employed, but, as the sequel proved, was needless, and it was removed the next day. Recovery was uninterrupted, and she returned home February I6. She is now, and has been since, quite well.

In the following case, under the care of Dr. Johnstone, of Ilkley, the symptoms of perforation of gastric ulcer were mimicked to a degree which rendered diagnosis difficult :

Mrs. C. D., aged twenty-two, married twelve months, was seized suddenly with acute abdominal pain and collapse. There was a history of pain after food, and occasional vomiting, which might have been due to gastric ulcer or early pregnancy. A 'period' due three weeks before the sudden attack had been missed. The shock was combated with strychnine and digitalis. Dr. Johnstone, diagnosing a ruptured tubal gestation, called Dr. Scott and Mr. Jessop in consultation. The problematical history of gastric ulcer, a long-standing anæmia, the general distension of the abdomen, with tympany, and the absence of liver dulness, were considered suggestive of perforating gastric ulcer. An incision was made above the pubes, however, and a ruptured tube discovered and removed.

\section{Treatment.}

There can, we feel sure, be no doubt that acute perforating ulcer of the stomach does occasionally heal spontaneously. We have on three or four occasions at least, in operating for various pathological conditions of the viscus, found ample evidence of such in the guise of old peritonitis around and upon the stomach. Dense and widespread adhesions to the liver or abdominal wall are found, and on separating these the stomach may be opened at the site of an old ulcer in the anterior wall. In these cases, on subsequent inquiry, we could elicit a history of a catastrophe that doubtless occurred at the time of perforation, and probably ended happily only 
because of the empty condition of the stomach at the moment of rupture. Pariser has collected fourteen cases, and Hall six cases, of apparent perforation ending in recovery. But though such cases do exist, they form but a trifling proportion, and have been computed at the most at 5 per cent. of the whole number. Their occurrence, therefore, is no argument against the law of invariable and immediate operation in all diagnosed cases.

There are cases in which, owing to an absence of clearlycut and unmistakable symptoms and signs, a doubt may exist in the mind of the surgeon as to the diagnosis. A perforation may be suspected, but a little hesitation is felt in proceeding at once to operation. In such a dilemma resource may be had to the 'lesser abdominal section,' the exploratory incision. This procedure has been strongly advocated by many experienced surgeons, and should be carried out under cocaine anæsthesia. An incision large enough to admit the finger is made, and is ample for purposes of investigation and diagnosis. If the exploration prove negative, no harm is done, no shock is felt, and the patient is none the worse; if the exploration prove positive, the incision may be extended under cocaine anæsthesia, or ether may be given.

Operation.- The first surgeon to advise and adopt operative intervention in perforation of a gastric ulcer was Mikulicz, then Billroth's assistant, in I88o. The first successful operation was performed in 1892 by Kriege (Berl. Klin. Woch., December, 1892). In England the first surgeon to appreciate the possible achievements of surgical treatment in this disease was Mr. Nelson Dobson, of Bristol (Bristol Med. Chir. Journ., December, I883).

An incision 4 inches long is made in or near the middle line of the abdomen above the umbilicus. This may later need enlargement upwards, downwards, or obliquely, in order to permit free access to the ruptured point. As soon as the abdomen is opened, a rapid survey of the whole stomach surface is made. It is well to begin at the cardiac end, for it is here that perforation is most frequent. The left costal margin is well dragged upwards by an assistant, 
and gentle traction on the stomach is made in order to bring the fundus well into view. If the anterior surface is sound, the great omentum immediately below the greater curvature is torn open to an extent sufficient to admit the index-finger, which then explores the posterior surface; or the great omentum is lifted up and the transverse mesocolon torn through, so as to open up the lesser sac and bring into view the posterior surface of the stomach. A massive outpouring of lymph on the surface of the stomach affords generally a useful guide to the perforation. These flakes and bands are protective in character, and are densest in the area where they are most needed. On gently breaking through them the ulcer will be exposed. When found, the perforation must be closed as speedily as possible. The ulcer may or may not be excised. There is probably no advantage in removing it, and a little time is wasted in so doing. The orifice, if small, may be closed by a purse-string suture. If more than a puncture, it should be closed by at least two layers of continuous sutures. If the walls of the stomach are, as is not seldom the case, easily lacerable, an interrupted suture, or Halsted's mattress suture, is preferable. There are, though rarely, cases where, owing to excessive destruction of the wall or inaccessibility of the ulcer, the perforation cannot be stitched up. In such the omentum may be used to close the gap. Enderlen's experiments and those of Sundholm show that the omentum not only acts efficiently, but permits a subsequent restoration, apparently complete, of the stornach wall. In place of omentum, an adjacent coil of intestine may be stitched along the gap, and will effectually close it. If neither of these methods can for any reason be adopted, the best course is to drain the cavity freely by passing strips of gauze or an indiarubber tube, or both, down to the ulcer.

After the perforation has been dealt with, the general peritoneal cavity must be cleansed as thoroughly as time will permit by sponging or hot flushing.

In the previous edition of this book we inclined to the advocacy of a free flushing of the peritoneal cavity with hot sterile salt solution, in order to insure that all the hidden 
recesses of the abdomen were cleansed of their impure contents. A riper experience and further thought have, however, convinced us that a washing out of the abdomen is rarely necessary. Much depends upon the time after perforation that the operation is begun-that is, upon the extent to which there has been extravasation of the contents of the stomach. If the soiling is limited to the area immediately surrounding the stomach, then a gentle sponging with damp swabs will suffice to cleanse away all impurity. If, however, the whole peritoneal cavity is drenched with the fluids and particles of food escaped from the stomach, then a free lavage will be needed. As the question of flushing is decided by the extent and severity of the soiling of the cavity, so also, and equally, is the question of drainage. In the cases of slight contamination, readily cleansed away, there is no need of any drain, but if the soiling is widespread, a drain down to the stomach and a second drain into the pelvis from a separate suprapubic wound may both be needed.

If general septic peritonitis has developed, the following plan, advocated by Finney, may be adopted: One or more incisions, free enough to allow an examination of the entire peritoneal cavity, should be made; through these the intestinal coils are withdrawn sufficiently to allow the operator to evacuate any peritoneal exudate, and thoroughly cleanse with warm salt solution and gauze pledgets all recesses and folds. The intestinal coils, which should be kept covered by an assistant with warm, soft sponges or towels during this manœuvre, should then be cleansed as far as possible from exudate and lymph by irrigation and wiping with pledgets of gauze, and replaced in the abdominal cavity. Too much time should not be consumed in the cleansing, nor should the intestines be handled roughly, but it is difficult to exercise too much care in the peritoneal toilet.

As perforation in 20 per cent. of the recorded examples has occurred at two or more points, the surgeon should not be content with suture of the first ulcer discovered, but should satisfy himself that the stomach elsewhere is sound. 


\section{Prognosis.}

The prognosis in ruptured ulcer of the stomach will largely depend upon :

r. The condition of the stomach, whether full or empty.

2. The time elapsing between perforation and operation.

3. The size and number of the perforations.

4. The presence or absence and the severity of the vomiting

I. The Condition of the Stomach, whether Full or Empty.-If the stomach has been recently filled, the food will be in solid particles of varying size, and will contain a variety in number and character of micro-organisms. Under such circumstances a perforation will probably be large, and will permit an escape of material of a kind best calculated to cause an intense peritoneal reaction. Symptoms will be of the acutest character, shock will be profound, and life will be seriously imperilled. If, on the other hand, the stomach is empty, there will be an escape of a little gastric secretion only, and the perforation will almost certainly be small. A little local peritonitis may alone result, symptoms will be less aggressive, and recovery will follow. Such are probably the circumstances in cases like those already mentioned by Pariser and Hall.

2. The Time elapsing between Perforation and Operation.This is an element of conspicuous importance. The study of a large number of cases proves beyond the possibility of question that the shorter the period between the perforation and operation, the greater the chances of success. The operations performed in each succeeding period of twelve hours after perforation have a gradually increasing mortality, as shown in the accompanying table:

\begin{tabular}{|c|c|c|c|c|c|c|}
\hline & & & $\begin{array}{c}\text { Total } \\
\text { Cases. }\end{array}$ & $\begin{array}{l}\text { Recov- } \\
\text { ered. }\end{array}$ & Died. & $\begin{array}{l}\text { Percentage } \\
\text { of Deaths. }\end{array}$ \\
\hline $\begin{array}{l}\text { Under I } 2 \text { hours ... } \\
\text { From I } 2 \text { to } 24 \text { hours } \\
\text { From } 24 \text { to } 36 \text {, } \\
\text { From } 36 \text { to } 48 \text { ", } \\
\text { Over } 48 \text { hours ... }\end{array}$ & $\begin{array}{l}\cdots \\
\cdots \\
\cdots \\
\cdots \\
\cdots\end{array}$ & $\begin{array}{l}\cdots \\
\cdots \\
\cdots \\
\cdots \\
\cdots\end{array}$ & $\begin{array}{r}49 \\
33 \\
16 \\
2 \\
33\end{array}$ & $\begin{array}{r}35 \\
12 \\
2 \\
16\end{array}$ & $\begin{array}{r}\text { I4 } \\
2 I \\
\text { I } 4 \\
2 \\
\text { I } 7\end{array}$ & $\begin{array}{r}28 \cdot 5 \\
63 \cdot 6 \\
87 \cdot 5 \\
\text { IOO*0 } \\
5 I \cdot 5\end{array}$ \\
\hline
\end{tabular}


The fact that the mortality from operation diminishes in those dealt with more than forty-eight hours after operation is readily explained. In such cases the perforation will almost certainly have been subacute, for the ordinary acute case is always moribund at the end of forty-eight hours.

3. The Size and Number of the Perforations.-If there is but one perforation, and that a small one, the condition is, symptomatically, subacute. If there are more perforations than one, and if any of these are large, the symptoms are the acutest conceivable.

4. The Presence or Absence and the Severity of the Vomiting.Vomiting is present in 29 per cent. (Fenwick) or 40 per cent. (Finney) of the cases. At each act of vomiting the stomach contents are ejected not only through the osophagus, but through the perforation also. The more severe the vomiting, the greater the amount of the stomach contents thrown into the peritoneal cavity, and, concomitantly, the more severe the peritoneal reaction thereby induced.

\section{Results.}

There are few matters so difficult to deal with statistically as this. It is probable that the vast majority of successful cases are recorded, for each one is a triumph of diagnostic acumen and surgical skill; each one indisputably means a life saved. On the other hand, in operating upon an unsuccessful case the surgeon feels that he has merely made a powerless interference in the course of a certainly fatal disaster, and the record is consigned to oblivion.

In our last edition we computed from a large series of cases, approximately 500 in number, that the mortality was $55^{\circ} 4$ per cent. Owing to the greater attention which has been given to the study of gastric diseases during the last two years, there can be no doubt that the death-rate has lessened. The cases are recognised at an earlier stage, and surgical treatment is more often and more speedily adopted. Of our last seven cases six have recovered, and in one of these there were two perforations. It is not too much to claim now an average recovery of approximately 60 per cent. 


\section{PLATE XXIV.}

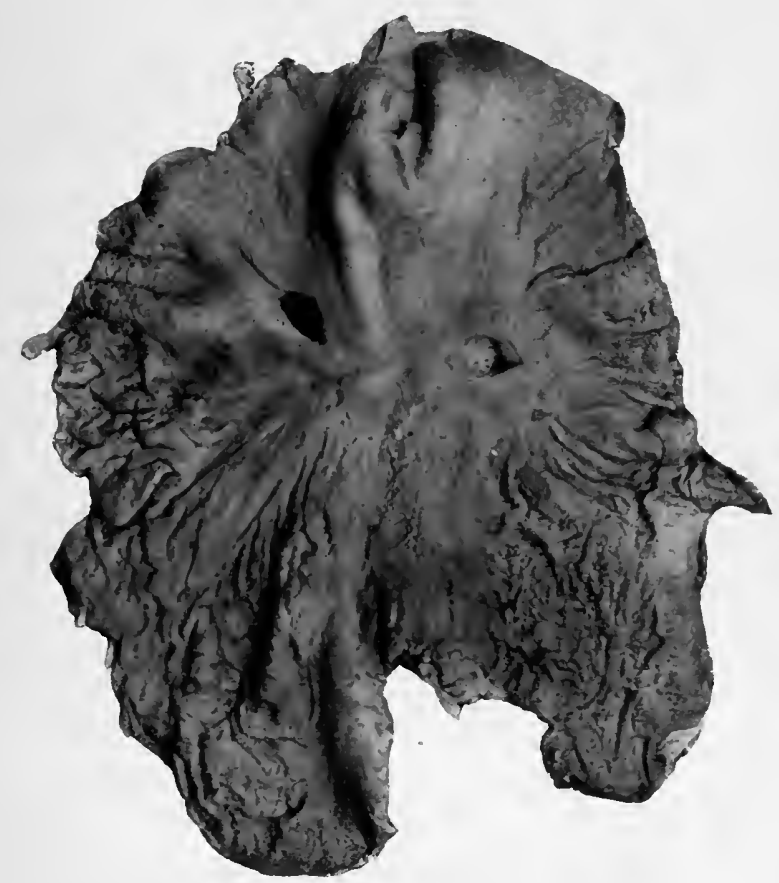

Fig. 89.--Stomach showing Two Perforating Ulcers: one on the Anterior, one on the Posterior, Surface.

To face $\left.p \cdot 3^{1} 4 \cdot\right]$ 



\section{Perigastric Abscess.}

Perigastric abscesses result chiefly from perforating ulcers of the stomach. They may in occasional examples be due to causes originating elsewhere than in the stomach, but such matters do not now concern us.

Ulceration of the stomach leading to perigastric abscess may be of varying degrees of activity. In by far the great majority the ulcerative process has been dilatory, and has permitted the formation of limiting adhesions; but acute

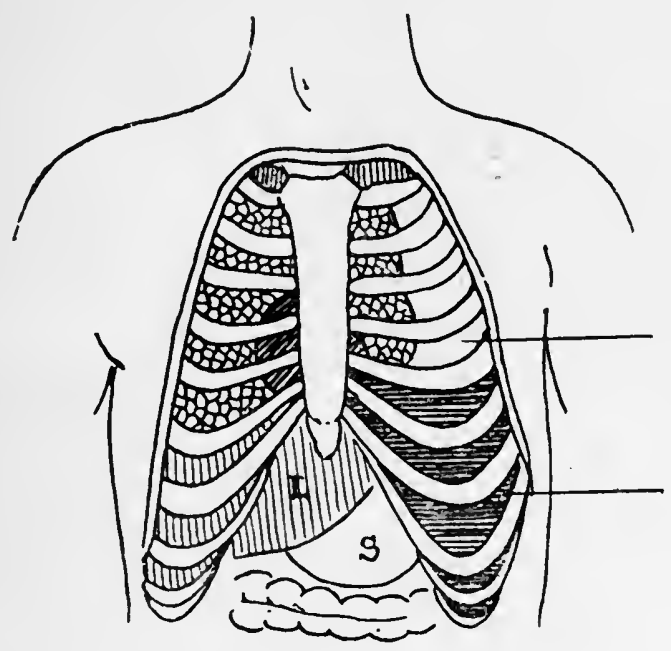

Fig. 90.-PyopNeumothorax.

perforation, subacute perforation, and acute perforation operated upon but imperfectly drained, may all give rise to localized abscess.

Perigastric abscesses so arising may have any conceivable relation to the stomach, and may vary considerably in intensity and acuteness. In a minority of cases a circumscribed swelling results, situated most commonly in the left. hypochondriac region, or to the left of the middle line, in the epigastrium. As such an abscess slowly enlarges, it may soften the skin and cause it to break down, or rupture may take place into one of the hollow viscera in the abdomen, 
or through the diaphragm into the pleural or pericardial cavities.

Owing to the frequency with which perforating ulcers of the stomach are found in definitely localized situations, the abscesses therefrom resulting are localized also in wellrecognised areas. The commonest of all positions is beneath the diaphragm, when a subphrenic abscess - the 'pyopneumothorax subphrenicus' of Leyden-forms. In 179 cases of subphrenic abscess collected by Maydi, 20 per cent. were due to perforation ulcers of stomach and duodenum. The clinical and pathological conditions resulting from subphrenic abscess vary so widely that it is almost impossible to present a clearly-defined symptom group as characteristic of the condition. Godlee (British Medical Journal, rgoo) says: "Think only of such a case as that of a very chronic ulcer of the stomach which has caused extensive adhesions between the viscus and the diaphragm; an abscess may form amongst these adhesions, and burrow about by complicated and narrow tracks until it points perhaps through an intercostal space. Then contrast it with one in which a sudden perforation of a stomach ulcer has occurred, but in which the suppuration is to some extent localized and contains air. It is difficult to trace a family resemblance between these two conditions.'

A subphrenic abscess may be defined as any collection of pus, or gas and pus, which as a whole or in part intervenes between the diaphragm and the structures normally in contact with it. Such abscesses are anterior, resulting from perforation of the anterior wall, and posterior.

Anterior abscesses may form between the right lobe of the liver and diaphragm, to the right of the falciform ligament, or between the left lobe and the diaphragm, to the left of the falciform ligament. The lower boundary in both cases is formed by adventitious adhesions, the result of a massive outpouring of lymph.

Posterior abscesses are contained within the limits of the lesser sac of peritoneum; or, having caused adhesion of the opposing layers of the sac, pus may accumulate in the retroperitoneal tissue. 


\section{Symptoms.}

The following account may be held to portray with general accuracy an average case of this kind. At the first there are the warnings of chronic perforation: Pain sudden and sharp, faintness, short and hurried and painful breathing. Tenderness is noticed on the upper part of the abdomen, with irregular, but generally constant, elevations of temperature, and occasionally rigors. The case may now assume the aspect of an acute thoracic condition, and pleurisy,

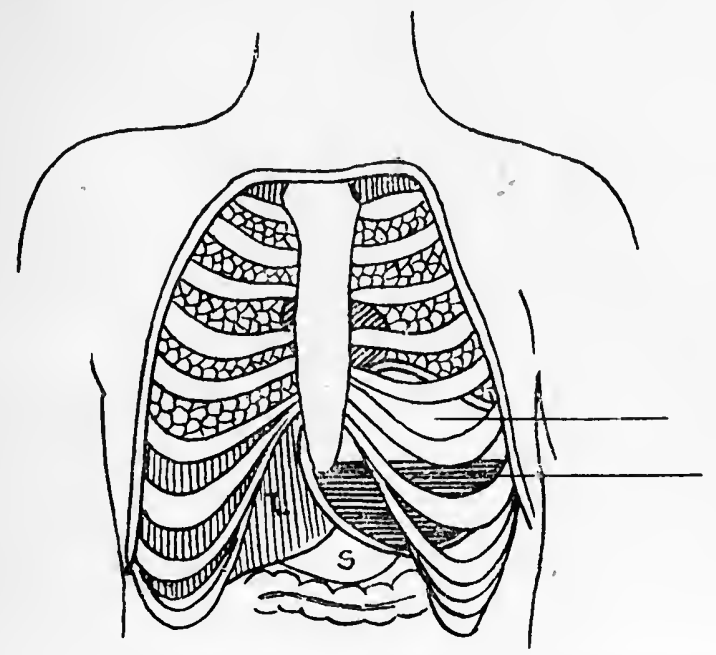

Fig. 91.-' Pyopneumothorax Subphrenicus': Subphrenic Abscess.

pericarditis, or empyema, may develop and speedily terminate the patient's life. In the absence of these complications, the symptoms may quietly continue. There will be occasional vomiting, frequent attacks of coughing; dyspnœea will be persistent, and at times distressing; there will be loss of flesh, sweating at night, and all the symptoms of a persisting septic absorption. The pus, gradually increasing in quantity, collects, and alone, or mixed with gas, bulges outwards the lower part of the chest, and pushes upward the wing of the diaphragm. So the heart may be tilted, and its apex beat felt a couple of inches higher than the normal. The lung 
also is compressed, and the percussion-note over it may be dulled. The resemblance to a pleuritic effusion may be striking and misleading, but the following points should enable a distinction to be made: (I) The movements of the chest are not impaired on the affected side; (2) the upper limit of dulness is not so sharply defined; and (3) breath-

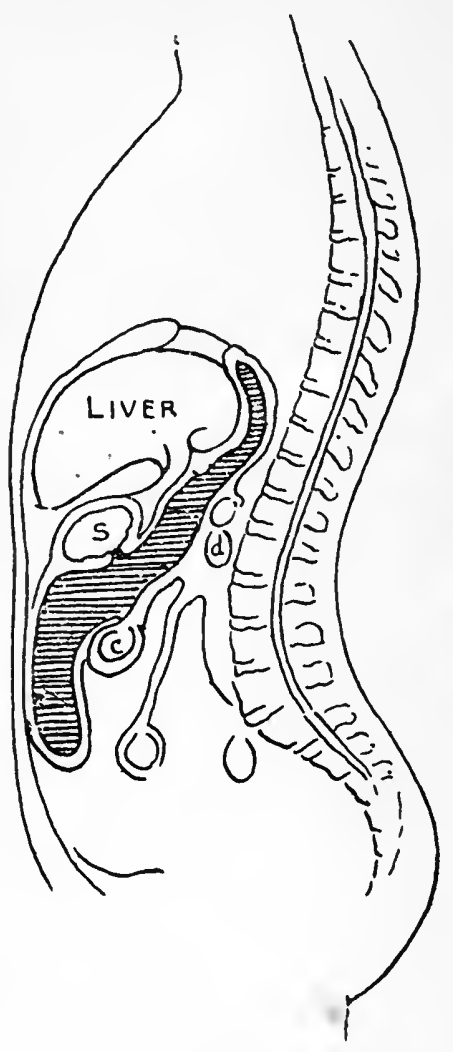

Fig. 92.-Posterior Perigastric Abscess.

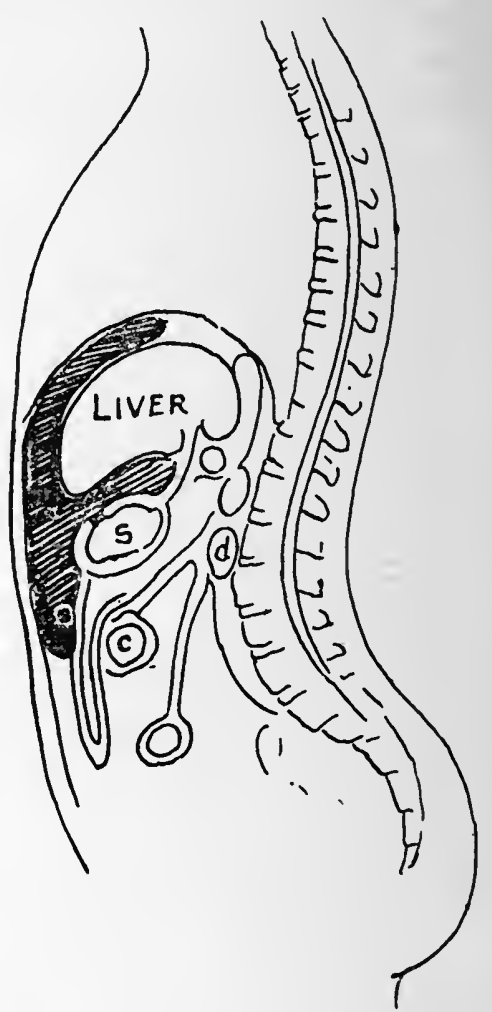

Fig. 93.-Anterior Perigastric Abscess.

sounds may be heard below the level of the dulness, and if a deep inspiration be made, the line at which the breath-sounds and vocal resonance are heard, and at which vocal fremitus is felt, is distinctly lowered. (See Figs. 90 and 9r.)

The 'diaphragm phenomenon,' to which much attention has been paid, is the existence of a shallow depression which 
moves with respiration and crosses the intercostal spaces of the left side as the diaphragm ascends and descends. On palpation of the upper region of the abdomen a collection of fluid may be felt. Greig Smith drew especial attention to the significance of a line or band of induration and resistance, felt through the abdominal wall, moving with respiration. This band is due to the presence of adhesions which limit the abscess cavity below. In the assured absence of tubercular peritonitis, Greig Smith believed this sign to be pathognomonic of subphrenic abscess. When gas is present in the cavity, a tympanitic note will be obtained over the front of the left side of the chest, extending as high up as the fourth rib, or even higher, the whole cardiac area being resonant. On auscultation the vesicular murmur will be heard in the upper part of the chest; below the fourth rib, or thereabouts, there will be amphoric breathing. At the upper part of the abdomen metallic tinkling can be heard, and a loud succussion splash is produced by jerky movements of the body. Over the area of hyper-resonance the bruit d'airain may be heard. When the perforation is on the posterior wall of the stomach, the lesser sac of the peritoneum may be filled with the extravasated contents. Pus rapidly forms, and the foramen of Winslow is closed by plastic adhesions. A case of this kind is recorded by Chiari (Wein. Med. Woch., I876) in which the pancreas, sequestered by the inflammatory process, floated in the pus of the abscess cavity. In such a case physical signs may be wholly lacking, or a tender swelling may be noticed in the umbilical region and lower epigastric, which is dull on deep percussion, and has a varying area of resonance over it. The extremely fœtid contents of such an abscess may be vomited, and in the vomit may be recognised shreds of dead tissue.

The abscess, after its definite localization and its hemming in by adhesions, may assume an acuter aspect and burrow upwards or downwards, bursting into the lung, into the pleura (causing pyopneumothorax), into the pericardium, or, rarely, into any of the hollow viscera of the abdomen. Surface perforation is occasionally seen, the abscess pointing and bursting through the abdominal or thoracic walls. In 
this manner the gastric listulæ, internal or external, are formed. If a retroperitoneal abscess form, the pus may burrow widely, and an abscess may point in the loin, or groin, or anterior abdominal wall, as in the following :

\section{Retroperitoneal Abscess due to Chronic Gastric Ulcer.}

We saw a case in August, I 899, in a woman of thirty-three in which there was an abscess in the retroperitoneal cellular tissue coming after a protracted illness, accompanied by digestive disturbances and occasional vomiting.

The abscess certainly communicated with the stomach, as, when it was opened and drained in the left iliac region, pus of the same odour and the same character in every respect was vomited. At the same time, there was dulness over the lower part of the thorax on the left side, and some pleurisy, though the fluid in the pleural cavity cannot have been pus, as it gradually disappeared after the abscess was drained.

The patient improved very much after the drainage of the abscess, and in about six weeks the tube was left out.

She died in the eighth week, when apparently doing well, she having been seen only an hour before death, which occurred from sudden heart failure.

Unfortunately, an autopsy could not be obtained, so that there must necessarily be a doubt as to whether the abscess was dependent on chronic ulcer perforating the stomach gradually after adhesions had formed between the peritoneal layers in the lesser sac, or whether the abscess burst into the stomach by ulceration from without.

The diagram shows the course of the pus in this case to have been similar to that of a duodenal ulcer bursting posteriorly into the retroperitoneal tissues, only in this case it was on the left side.

A perigastric abscess the result of a gastric ulcer may burst secondarily into the stomach, as is exemplified in the following.

Chronic Gastric Ulcer; Abscess of Pancreas bursting into Stomach; ACute Gastritis; Vomiting; Impending Death. Posterior Gastro-enterostony.

On November I2, I900, we were asked by Dr. Mercer, of Bradford, to see Mr. R., who was extremely ill and supposed to be suffering from pancreatic disease.

On arrival we found the patient, aged thirty-five, extremely 
emaciated, lying in a typhoid condition, vomiting extremely offensive, dark-coloured pus and mucus mixed with blood. He was extremely feeble and had a rapid pulse. A tumour could be felt above the umbilicus, which was tender to pressure. On distending the stomach with $\mathrm{CO}_{2}$, great pain was produced, and vomiting followed. 'The stomach was found to be dilated, reaching on the left side to the level of the umbilicus. There had been an elevated temperature for a week or two, but this had become subnormal after the vomiting of pus. Pain after food and indigestion had existed for some months, during which time there had been steady loss of flesh; but recently, especially during the past month, the wasting had been very considerable. There was a little sugar in the urine and a trace of albumin, and the fæces contained free fat. No medicine had done any good, either in relief of the vomiting or in controlling the horrible odour which permeated the whole house. A diagnosis of chronic gastric ulcer of the posterior wall of the stomach, with secondary ulcerative pancreatitis and abscess of the pancreas, was made, and gastroenterostomy proposed as the only means likely to produce any chance of relief, but even that seemed almost hopeless. He was removed to a surgical home by ambulance, and the stomach was washed out carefully by Dr. Stevens. As showing the nature of the stomach contents, both the attendant nurses were made sick by the smell of the material evacuated by the tube.

On November I8, after enveloping the patient in cotton-wool and subcutaneously administering strychnine, posterior gastroenterostomy was performed, a bone bobbin being used. The tumour felt before operation was found to be formed by stomach and pancreas firmly fixed together towards the pyloric end, but leaving the dilated portion free at the cardiac end of the stomach, so that no difficulty was found in doing a satisfactory operation, which was completed in twenty-five minutes. Saline subcutaneous injections and rectal injections were given and strychnine was freely administered, but for two days we had a great fight with death, apparently due to poisoning from the foul stomach contents. Hot water was freely given to induce vomiting on the second day, as the patient could not bear the stomach tube being used. This gave relief, and afterwards progress to recovery was uninterrupted. He rapidly gained strength and put on flesh, returning home in five weeks after operation. His friends, who had despaired of his recovery, were astonished to find him so well. In April he was in such good health that he married. 


\section{Treatment of Subphrenic Abscess.}

The only treatment that can be counted upon to do good in cases of subphrenic abscess is incision, with subsequent drainage. There are unquestionable cases of abscess which recover spontaneously, either from the rupture of the abscess cavity in one of the directions already mentioned or from gradual drying up of the contents. The most exemplary instance of this which we have met with is the following :

\section{Symptoms of Perforation of Gastric Ulcer, with Signs of Left Subphrenic Abscess and Peritonitis. Re- COVERY WITHOUT OPERATION.}

Early in Igoo we were asked to see a young lady of twenty-one, with Dr. H. She had been anæmic for several years, and had for some months shown signs of gastric ulcer. One evening during dinner, at which she apparently had not taken any food, she was seized with acute pain in the upper abdominal region, and became faint and extremely ill. She was put to bed immediately, and all food by the mouth was stopped, as rupture of a gastric ulcer was suspected.

When we saw her later there were well-marled signs of peritonitis in the upper abdomen, and the recti were tense, especially the left. The lower ribs were fixed and rigid, and there was a distinct prominence beneath the left costal margin. From the seventh rib downwards there was an entire absence of breathsounds, and percussion showed hyper-resonance with tension, as if gas were compressed in a limited space. The temperature had been raised, but was subsiding, and as the pulse, which had been rapid, was quieting down, we decided to wait and watch the case, feeding by the bowel and keeping the stomach free from food. Recovery was gradual, but complete, and we hear that the patient is now well.

According to Lang, who has collected I 76 cases from surgical literature, after operation the percentage of recoveries is $47^{\circ} 9$ per cent., and of the recoveries without operation I2.3 per cent. Beck found six spontaneous recoveries in I 46 cases. The incision should be made, as a rule, over the most prominent point of the abscess. This may be in the abdominal wall, below the costal margin in front, or behind in the lumbar region, or in the thoracic wall. 
The incision is carefully made into the abscess cavity, the contents emptied, and the cavity flushed with sterile solution, or, and this is better, carefully sponged until as clean as possible. Flushing may lead to rupture of protective adhesions, and if adopted should be carefully supervised. If, after so cleansing the cavity, the ulcer which has perforated can be recognised, and is easily accessible, it may be stitched up. Drainage by means of large tubes with gauze wicks is adopted.

When the thoracic wall has to be incised, the pleural cavity is opened by resection of one or more ribs. The parietal and diaphragmatic layers of the pleura are then stitched together and the diaphragm is incised. Drainage is adopted in the manner above described.

\section{Subphrenic Abscess from Perforating Gastric Ulcer dis-} Charging through Lung. Drainage; Recovery.

Miss Isabel P., aged twenty-four, was seen on June I, Igoo, with Dr. Densham, of Stockton, with the history of three years' indigestion and vomiting, ending in perforation of gastric ulcer and peritonitis in November, 1899. She was extremely ill and for many weeks in a dangerous state, but gradually improved. A month after the perforation there were signs of suppuration with some dulness at the base of the left chest, and two months after the perforation an abscess burst through the left lung and a large quantity of offensive pus was coughed up, and continued to be coughed up, until she reached the Leeds Infirmary, where the purulent expectorations measured about $\frac{1}{2}$ pint daily. She was then extremely feeble and suffering from a hectic temperature. There was dulness over the lower part of the left lung. On examining the offensive material expectorated, muscular fibres were found in it, but no elastic tissue, thus showing the source of the pus.

An exploring needle used in the ninth left intercostal space found pus. A portion of the ninth rib was therefore dissected, and an incision made through the thoracic wall and diaphragm into the abscess cavity, which was freely drained. Recovery was rapid, and the foul expectoration ceased at once.

The opening leading from the stomach to the abscess closed spontaneously, and the tube was left out in about a month, after which the wound closed. She was sent to a convalescent home, and returned home in three months, well. 
The following are the recorded results of perforation of the stomach the result of ulceration :

I. Acute Perforation.-Acute septic peritonitis; secondarily, perigastric or subphrenic abscess.

2. Chronic Perforation.-The ulcer may be supported on the outer side by adhesions to--

Omentum,

Abdominal wall, leading to external gastric fistula,

Diaphragm,

Solid viscera, liver, spleen, pancreas, lymphatic glands.

The ulcer may perforate into the lesser sac, the foramen of Winslow being closed.

The ulcer may perforate, a perigastric abscess forming and bursting into-

Pleura,
Pericardium,
Heart,
Bronchus,
Mediastinum ;

or into hollow viscera, forming internal gasíric fistula :

Intestine, large or small,

Stomach,

Gall-bladder,

Common bile-duct,

Pancreatic duct;

or into peritoneum or subperitoneal tissue, causing emphysema of subcutaneous or subperitoneal tissue, or leading to the occurrence of gas in the veins and arteries (Jürgensen).

(The gas found in general emphysema is said to consist chiefly of hydrogen, and to burn with a blue flame.)

The following case of perforation into the left ventricle is recorded by Thue (Norsk. Mag. f. Laegevidenskaben, I903, p. 185 ).

A male, aged forty-eight years, had complained of gastric pains for several years. On admission to the hospital there was pleurisy on the right side, and cough with copious expectoration (without tubercle bacilli). There was also distinct retention and hyperacidity. He improved under treatment, and then death occurred suddenly from hæmorrhage. The autopsy showed marked dilatation of the stomach, which was adherent to the diaphragm. 
There was a small perforation through the diaphragm, which communicated with the left ventricle by an opening to the left border of the mitral valve.

\section{The following is a list of our cases :}

CASE I.-This was the first case operated on in Leeds, and we believe it was one of the earliest, if not the first, operated on in England.

Miss E. W., aged seventeen, was admitted to the Leeds Infirmary on October I8, I888. Unfortunately, the operation was not performed until the third day, when peritonitis was general. The abdomen was flushed out and drained by a glass tube in the pelvis. The perforation was the size of a shilling in the anterior surface of the stomach, and in a most favourable place for treatment, which was unfortunately adopted too late. Death occurred a few hours later.

Case 2.--Miss S., seen with Dr. Kebbel, Flaxton, on October Io, I892, at a farmhouse some miles away from a railway-station, suffering from general peritonitis following on perforation of gastric ulcer three days before. The patient was extremely ill, and on opening the abdomen a large quantity of pus mixed with food was found free in the peritoneal cavity; the lesser sac of the peritoneum was also full of fluid. An opening was found near the cardiac end of the stomach, and another close by on the posterior wall. These were closed, and the abdomen was purified as far as possible by lavage, after which drainage was adopted. The patient died of exhaustion two days later, so that life was probably a little prolonged by the operation.

CASE 3.-On May I6, I897, we were asked by Dr. F. H. Mayo to see Miss __-, a nurse, aged twenty-four, who, while nursing an elderly lady, was suddenly seized with acute pain in the upper abdominal region without any preliminary symptoms except indigestion, to which she had been subject for some years. Her symptoms were very acute, and within a few hours her abdomen became distended, and there was evidence of fluid in the flanks and in the pelvis. The attack came on immediately after breakfast, when the stomach was full of tea, bread-and-butter, etc.

Operation at ro p.m., sixteen hours after rupture. Chronic ulcer with perforation the size of a sixpence found near lesser curvature on the anterior surface near the cardiac orifice.

Edges of ulcer refreshed and brought together by sutures; omental graft applied.

Lavage of abdomen with hot saline solution. Suprapubic drainage for several days.

Recovery delayed by an attack of left-sided pleurisy, but ulti- 
mately perfect, and she is now and has since been quite well, and has performed her duties as a nurse ever since her recovery.

CAse 4.- Miss T. B., admitted to the Leeds General Infirmary on July 10, I897, suffering from peritonitis, the result of perforation of gastric ulcer forty-eight hours before. She was immediately transferred to the surgical side, and the abdomen was opened, when a small perforation was found on the anterior wall of the stomach near the lesser curvature. The opening was closed by sutures, and further secured by an omental graft, after which the abdomen was washed out and a suprapubic drain inserted. The patient made a recovery from the operation, and was transferred to the medical side, as, although the wound was healed, she did not gain strength, and appeared to be weak and anæmic. On August I 3 a small abscess was found in the left iliac region, when about 2 drachms of pus were evacuated by a colleague, after which her condition did not improve materially, and she continued to lose flesh and strength, and died from exhaustion on August 28, seven weeks after the first operation and fifteen days after the second.

CASE 5.-See p. 308.

CASE 6.-Described on p. 323. The diagram (Fig. 93a) shows the site of the abscess and the position of the incision for its relief.

Case 7.-Mr. M., seen with Dr. Barrs and Dr. G. Myrtle, suffering from peritonitis due to perforation of a duodenal ulcer twenty-four hours previously. The patient was extremely ill and feeble, and had had symptoms of duodenal ulcer for a considerable time. Operation, I2/3/rgor. The abdomen was opened on the right side, and a large quantity of very offensive pus and débris of food were evacuated, but he was too ill to bear any extensive operation, and drainage was simply adopted. The patient died from a continuance of the peritonitis on the second day.

CAse 8.-Mr. F., aged thirty-eight, seen with Dr. Hebblethwaite six hours after a sudden abdominal attack, which had been preceded by symptoms of indigestion for several years. The attack had occurred after breakfast. On being seen, there was an absence of liver dulness. Abdominal section was performed on October Io, Igor, by an incision through the right rectus, and on opening the peritoneum air immediately escaped, together with a thin flaky fluid. A small perforation was found on the duodenal side of the pylorus. Some adhesions were detached, and the pylorus was brought well into view. The margins of the ulcer were pared, and the edges brought together by sutures, the wound being covered by an omental graft. The abdomen was washed out with saline fluid, and the pelvis drained by a glass tube. Recovery was uneventful, and the patient is now well.

Case 9.-Mr. M., aged forty-two, seen with Dr. Tunnicliffe. 
The patient had had symptoms pointing to duodenal ulcer for ten years, and recently the attacks of pain and indigestion had become much more urgent. On July 5, I902, he was seized with violent pain beneath the right costal margin. This was followed by collapse and severe vomiting. The pain and collapse were so severe that morphia had to be administered. He was removed to a surgical home on July 6 , and the abdomen was opened through the right rectus, when a quantity of fluid was found surrounding the first part of the duodenum, there being a minute aperture in

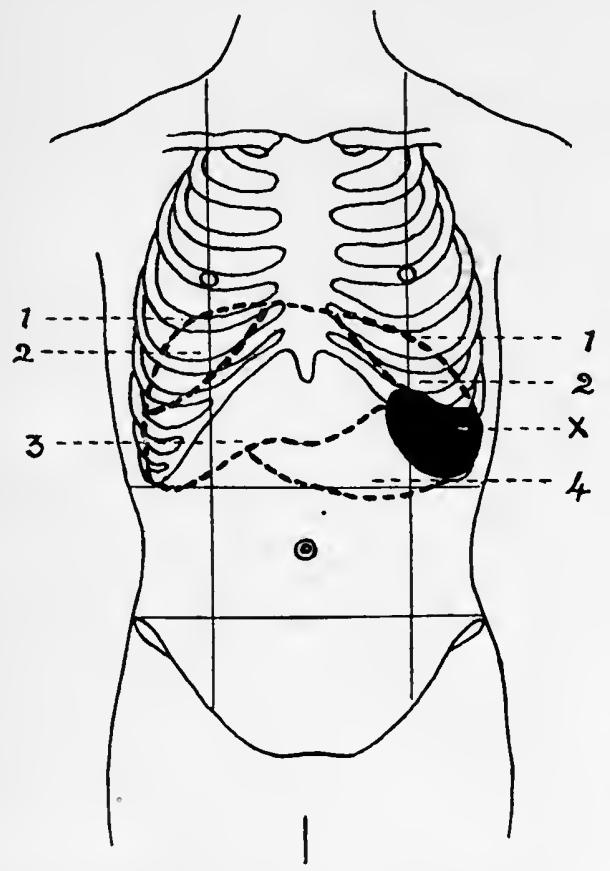

Fig. 93a.-Illustrating Site of Abscess in Case 6, and Position OF INCISION FOR ITS RELIEF.

I, Diaphragm; 2, lungs; 3, liver ; 4 , stomach; $x$, site of incision into abscess.

the duodenum just beyond the pylorus. The edges of the ulcer were pared, and as the pylorus was stenosed it was laid open over a director, pyloroplasty being performed. The abdomen was purified by sponging, and a drainage tube was left in the right kidney pouch. The patient had a good deal of pain for the first forty-eight hours, after which recovery was uninterrupted. $\mathrm{He}$ was quite well up to December, when he began to have pain over the pylorus, relieved by food, but it always returned before the next meal. There was decided tenderness in the duodenal 
region, though there was no dilatation of the stomach. He was generally relieved by resting, but the pain returned on exertion. On March 5, 1903, as the painful symptoms were continuing, the abdomen was opened, when very firm adhesions were found connecting the duodenum to the liver and to the parietes. The thickening along the first and second portions of the duodenum pointed to a continuation of the ulceration. A posterior gastroenterostomy was therefore performed, from which the patient made a good recovery and remains well.

CASE I0.-A woman, aged thirty-nine years, was admitted on April 30, I897, with a history of acute onset of severe abdominal pain, chiefly in the epigastric region. There was faintness, followed in about half an hour by vomiting. There was no hæmatemesis. The abdomen gradually became distended, and the pulse gradually became quicker and feebler. The abdomen was opened, and a very small gastric ulcer near the lesser curvature was found to have perforated. There was free effusion, and some curdled milk was seen in the peritoneum. Suture of the ulcer, lavage, and drainage were performed. Recovery ensued.

CASE I I.-The patient, a woman aged twenty-seven years, was seen on May 22, 1897. During the previous year she was treated by Dr. W. E. Bennett for gastric ulcer. Since then she had constantly had dyspepsia, pain in the back, and epigastrium, and occasional vomiting, but not recently, of blood. On May 22, at 2.30 a.m., she was suddenly roused from sleep by intense epigastric pain; she felt faint, and vomited. The collapse continued, and soon distension and tenderness occurred; the vomiting was persistent. The abdomen was generally distended, the upper part especially. The pulse was very tense, and only just perceptible. She had cold, clammy hands and livid skin. Operation was performed at Ir.25 p.m. There was a large perforation $\frac{1}{2}$ inch in diameter just below the lesser curvature nearer the cardia. Death ensued.

CASE I2. - A woman, aged twenty years, was seen on November I6, I898. She had been under the care of Dr. T. Johnstone some months previously for indigestion. With dieting, etc., the symptoms abated. She had had no discomfort recently. On the I 8 th, at 5.30 p.m., while at tea, she had sudden pain, overwhelming, localized beneath the ribs on the left side, 'as though a knife were run in.' The abdomen gradually became distended. The patient remained collapsed for several hours, but improved after morphine. There was no liver dulness. The tenderness was over the appendix region chiefly. Operation was performed on November 20 at I 2 noon. There was a large ulcer, easily admitting the middle finger, about 3 inches from the 
cardia on the anterior surface. There were general peritonitis and a large collection of thick turbid fluid and lymph. Death occurred in twenty-three and a half hours.

CASE I3.-The patient, a man aged forty-four years, was seen on April 26, Igoo. Symptoms had been present for eighteen months. The chief of them was pain two, three, or four hours after food. Blood had been observed when the patient vomited. Vomiting was frequent but irregular. There was no meiæna. On the twenty-fifth, while in the infirmary, the man became suddenly worse. Pain came on acutely in the whole abdomen. Distension and rigidity were soon observed. Collapse was pronounced. The respirations were 28 and the pulse was I28. A diagnosis of perforating ulcer was made and the abdomen was opened. The ulcer was found at the beginning or the second part of the duodenum; its diameter was about $\frac{3}{4}$ inch. After stitching the ulcer up the gut seemed narrowed to at least half its diameter. A gastro-enterostomv, with the aid of a Murphy's button, was therefore performed. The patient never rallied from his collapse, and death ensued.

CASE I 4.-The patient, a woman aged twenty-eight years, was seen on April I2, I90I. She had had symptoms of indigestion for eight years. On April I I she was suddenly seized with acute abdominal pain about three hours after breakfast. On admission to the infirmary she was found to be in very acute pain, the abdomen, especially in the upper half, being much distended and very rigid. The epigastric region was excessively tender. An ulcer equal in size to the end of a lead-pencil was found near the lesser curvature towards the cardia. The stitches held imperfectly until taken very wide of the ulcer. To give security an omental flap was stitched over the ulcer. The early course was satisfactory, but later the temperature ran up, and a subphrenic abscess and multiple suppuration of the abdomen resulted, and the patient died on May 7 .

CASE I5.-The patient, a man aged twenty-three years, was seen on May 24, Igor. He had had symptoms of gastric ulcer for several months. He was a policeman, and had recently been on night duty. Frequently he had vomited during the night. On the morning of May 23 he had a sudden acute attack of pain, and vomited when going on duty. He returned to bed, felt ill and cold all day, and at night first sent for a medical man. The abdomen was then distended, rigid, and tender; the pulse was I24, thin, and feeble. An ulcer on the anterior surface near the pylorus had perforated. There were masses of lymph between the liver and the stomach. Fluid was found everywhere in the peritoneum. The ulcer was of about the size of a sixpence. The patient died, 
CASE I6.-A man, aged twenty-five years, was seen on June 18 , IgOI. The patient, a sturdy, robust labourer, stated that for about four weeks before admission he had suffered from indigestion and vomiting. On June 18 , while climbing a ladder, he was suddenly seized with intense abdominal pain. He was seen at once by a medical man who happened to be near and sent to the infirmary. He was then profoundly collapsed, his breathing was quick and short, his pulse was $\mathrm{I} 28$, and the abdomen was rigid and unyielding. A diagnosis of perforated ulcer was made. A perforation equal in diameter to a No. 8 or No. 9 catheter was found in the duodenum $I$ inch from the pylorus. The ulcer was stitched, and the abdomen was cleansed and drained. Recovery ensued.

CASE 17.-The patient, a female aged twenty-four years, was seen on March I3, I902. She had been married eight weeks previously. She had suffered slightly before that from indigestion, but never severely. Thirty-six hours before operation she was suddenly seized with acute intolerable pain underneath the left costal margin. There was no vomiting, but faintness and collapse were pronounced. Gradually the abdomen began to distend and the pulse to fail. On examination the abdomen was found to be distended uniformly, and was everywhere tender. In the left upper quadrant, beneath the left rectus, there was a specially tender area. An ulcer near the cardia had perforated. The edges for at least an inch round were solid and hard, and the stitches cut through when tightly tied. An area of stomach was infolded, and an omental flap was stitched like a lid over the line of suture. There were large masses of thick lymph for a few inches round the ulcer. Recovery ensued.

CASE I8.-A girl, aged seventeen years, was seen on April 20, 1902. For several weeks she had had slight indigestion and epigastric pain, but not in sufficient severity to send her to a medical man. On April I9, at 9 p.m., she had a sudden attack of acute epigastric pain. Morphine was given. When seen at 7 a.m. the abdomen was very rigid and rather tender, especially over the gall-bladder. The patient vomited once. The pulse was I 12. There was very shallow respiration. An ulcer which had perforated was found on the anterior surface of the duodenum about $\frac{3}{4}$ inch from the pylorus. There was some fluid above the stomach. The ulcer was closed by suture, and the peritoneum was cleansed by wiping with swabs wet with sterile salt solution. There was no lavage and no drainage. The patient recovered.

CASE 19.-A woman, aged twenty years, was seen on October II, Igo2. In March, IgoI, she had a very severe attack of 
hematemesis, and afterwards symptoms of indigestion, but never severe. She remained anæmic for several months. In November, IgoI, she had a second attack of hæmatemesis. She had had symptoms of indigestion since up to three months ago. Latterly she had been quite well, better than for years, and had suffered no discomfort from food. On October ro, at $.5 \mathrm{p} . \mathrm{m}$., she had her tea ; at 9 p.m. she had some broth, and at II p.m., while undressing, she had a sudden attack of pain under the ribs on the left side, 'catching her breath.' She was coiled up in bed groaning with pain, and was slightly collapsed up to 2 p.m., when morphine was given. She had been comfortable since. When seen at 3 p.m. the abdomen was tight, but not distended, moving very little. It was tender to the left of the umbilicus, especially just beneath the costal margin. The pulse was i22. There was no vomiting. Perforation was diagnosed on an almost empty stomach. A perforation was found close to the lesser curvature on the anterior surface equal in size to a No. Io catheter. There was an indurated area around this equal in size to at least a florin. A single stitch was put through the ulcer to close it, and then the ulcer was buried by a double continuous suture. A flap of omentum was turned over like a lid. There were many masses of lymph between the stomach and the liver, and there was free effusion of turbid fluid into the peritoneum. This was wiped with wet swabs and then dried. The patient died.

CASE 20.-The patient, a girl aged eighteen years, was seen on November 8, I902. For the last few weeks she had had some slight pain after food just beneath the left costal arch. The pain had been worse when she laughed and when she stretched her left arm upwards. There was a sudden onset of symptoms about 8 p.m. on November 7-pain, collapse, shallow breathing, etc. The abdomen was intensely rigid and immobile. The perforation was about equal in size to a lead-pencil, and was situated near the lesser curvature towards the cardia. There were some flaky adhesions. The ulcer was sutured, and an omental flap was turned over it. There was no drainage. The patient recovered.

CASE 2I.-A woman, aged twenty-three years, was seen on March 5, I903. She had had indigestion for several months. On March I, when in London, she had a sudden attack of pain beneath the left costal margin. She felt faint and prostrate, and vomited. She gradually felt better, and on the 3 rd travelled from London to Bawtry, and ate a good luncheon on the journey. The side then felt 'stiff,' and hurt her if she laughed or turned quickly. On the fourth, after breakfast, she had a sudden extremely severe pain, with collapse and vomiting. The abdomen had gradually distended, and was now blown out and tympanitic. 
A fluid wave was easily felt. The patient looked very ill. The pulse was I56. Two perforated ulcers were found, both on the anterior surface and in the cardiac half of the stomach near the lesser curvature. They were distant about $I_{\frac{1}{2}}$ inches from each other. One opening was of the diameter "of a leadpencil, and the other of a knitting-needle. From both fluid gushed out. Both were closed by suture, and the stomach was folded over. The omental lid afterwards covered both in. Drainage was effected by a split tube and a gauze wick at the upper part of the incision and through a separate suprapubic incision. Recovery ensued.

CASE 22.-The patient, a man aged twenty-eight years, was seen on March 26, 1903. He had been quite well up to 5 p.m. the night before. At that time he was straining heavily at work, and suddenly felt a pain in the upper part of the abdomen, which caused him to feel faint. The pain lessened considerably in about an hour, but he then felt ' as if he had been winded' by a blow on the epigastrium. The abdomen became slightly distended and very rigid; tenderness was especially noticed in the upper part on the right side and downwards towards the appendix. There had been no premonitory symptoms of indigestion. A diagnosis of duodenal perforation was made. The abdomen was opened through the right rectus. There was a large quantity of loose flocculent lymph surrounding a perforation in the first part of the duodenum. The ulcer was stitched up by two continuous sutures. Time after perforation, thirty-one hours. Subphrenic abscess followed on the twentieth day and was opened. The patient recovered.

A case of perforated gastric ulcer followed by hepatic abscess is related by Dr. H. Hollis (Lancet, December 5, I903). The following is his account, with comments:

The patient, a female aged twenty-four years, who had no important history of stomach trouble, fell from her bicycle and hurt her 'chest' at the end of August, 1903. On September 6 she ate her usual breakfast, but only had French beans for dinner. In the evening, after walking for a quarter of a mile, she was sick, and brought up a small quantity of blood, and was sick again after some brandy had been given. She was seen half an hour after the first attack of vomiting, and, being in a state of collapse, was removed to the cottage hospital. There was great pain in the epigastrium, the face was blanched, breathing was thoracic, the pulse was 120 , the abdomen was retracted and hard, and liver dulness was absent, Mr. W, E. Audland and Dr, W. I, Watson 
agreed with me that the patient probably had a perforation of the stomach. Four hours after the initial attack her abdomen was opened, and a hole $\frac{1}{3}$ inch in diameter was soon found in the centre of a roughly quadrilateral, grayish, sloughy-looking patch $I$ inch long and $\frac{3}{4}$ inch broad, situated on the anterior of the smaller curvature of the stomach. The patch was inverted, the stomach wall was sewn over it, and the abdomen, which contained a quantity of greenish fluid and several partlydigested French beans, was thoroughly washed out. After the operation there was no sickness. The temperature was normal thirty hours after, and it continued so for nine days: On September I 3 the wound was dressed and all the stitches were removed. On the $I^{\text {th }}$ the temperature began to rise and the pulse-rate to quicken without any obvious cause or any discomfort. After a day or two there was thought to be increased dulness above the liver. The temperature now varied from $99^{\circ}$ to $105^{\circ} \mathrm{F}$., and on five days, between September 15 and October 9, she had a rigor. On September 23, 27, and 30 a needle was put in the dull patch with negative result. On October 2 there was some hardness in the abdomen below the ensiform cartilage, so the upper end of the operation incision was reopened, and an abscess in the liver was found, the contents of which were most offensive. A tube was put in, and the wound was well packed with gauze. For two or three days the patient improved, then she gradually became worse, and on October 9 she died.

' Post-mortem the liver was found to be adherent to the upper part of the abdominal wound and to a small patch of the diaphragm. In the left lobe there was an abscess cavity, and a small abscess as large as a Barcelona nut was found in the spleen. The wound in the stomach was quite healed, and, indeed, was difficult to find. In the right lobe of the liver were two small dark patches at the site of the exploratory punctures. There was no peritonitis, and all the other organs were healthy.

'It seems difficult to account for the abscess, as it does not appear to have been part of a general pyæmic condition, and the late onset, nine days after operation, and the absence of peritonitis, do not point to any connection with the ulcer. As the patient had never had any vomiting or hæmatemesis, can it be possible that the fall from the bicycle was the cause of both lesions?'

The following case, in which two perforations of a gastric ulcer occurred within a period of five months, is recorded by Dr. Aitken (Brit. Med. Journ, vol. i., I904, p. 665) : 
History.-B. S., female, aged twenty-nine. Had suffered from symptoms of ulcer of stomach for years, and had been treated medically. She had not had hæmatemesis. At 5 p.m. on February 20, 1903, she ate a 'parkin,' and soon afterwards complained of great pain in the upper part of the abdomen, and became collapsed. She was admitted to the Blackburn and East Lancashire Infirmary at i I a.m. next morning.

State on Admission and Operation.-The diagnosis was clear, and, considering the fact that seventeen hours had elapsed since the perforation, she was in good condition for operation. The abdomen was rapidly opened for about 6 inches in the middle line above the umbilicus. The perforation, which was near the middle of the anterior surface of the stomach, was easily found, as the stomach contents were seen escaping as soon as the edges of the wound were retracted. There was a considerable amount of thick lymph round the perforation, which was surrounded by an indurated area of stomach wall. This area was doubled in by eight or ten Lembert sutures without difficulty. The abdominal cavity was filled with hot saline solution after a suprapubic opening had been made. The flushing was continued till the returning fluid was clear, the hand being meanwhile moved about in the abdomen to insure thorough distribution of the fluid. The greater part of the fluid was then removed by sponging. Large rubber drainage tubes, with a wick of wet gauze in each, were then inserted into each loin and to the seat of suture, and another through the suprapubic opening to the bottom of the pouch of Douglas. The operation lasted under an hour, most of the time being taken up in cleaning the peritoneum.

Progress.-There was no great shock after the operation. The tubes were removed on the third day and replaced by gauze drains. The patient seemed to be doing well, except that a parotid abscess formed about the seventh day. On March I4twenty-one days after operation-pneumonia of a septic type developed, and, getting rapidly worse, the case seemed hopeless. The pulse-rate at one time was never below I 40 for four consecutive days. After fourteen days the condition cleared up, and the wounds entirely healed at the end of six weeks. She went to a convalescent home, and came back able to eat any ordinary food.

Second Admission.-On Saturday evening, July I8, I903, the same patient was readmitted, and as she had had $\frac{1}{2}$ grain morphine hypodermically a short time before, the diagnosis was not at all apparent. She had not had pain in the stomach, but had been feeling out of sorts generally for about a week. Acute pain came on at 5 p.m. on July I8, after which she walked a mile and drank some hot soda-water on getting home. I saw the patient 
ar I I a.m. on the following day, and could not be quite certain that a perforation had occurred. The temperature was $97^{\circ} \mathrm{F}$., pulse 108 and respirations 33. I saw her again at 6 p.m., when she was evidently rapidly getting worse, and I decided to operate at once. It was then twenty-five hours after the supposed time of perforation.

Second Operation.-The operation resembled the first in the ease with which the perforation was found, but the application of the sutures meant at least fifteen minutes' work, as, even after cutting the left rectus across, the perforation was very difficult to reach, the chest being flat, and having a very acute epigastric angle. The perforation was smaller than the first, and the stomach contents which had escaped seemed to be mostly mucus. Thick flakes of lymph were adherent to the adjacent stomach and liver. The character of the extravasated material decided me on sponging instead of flushing the abdomen, and as several ounces of dirty fluid were swabbed from the suprapubic opening, I put in drainage tubes as before, and finished the operation as quickly as possible, with serious misgivings as to the result. Very soon they were relieved, and without a bad symptom the patient recovered. The wounds were finally healed on the twenty-ninth day.

\section{Duodenal Ulcer.}

The perforation of a duodenal ulcer pathologically and clinically presents some points of difference from a gastric ulcer, and is therefore worthy of special description.

A duodenal ulcer may perforate at once and acutely into the peritoneal cavity, or may slowly destroy all the coats of the bowel and lead to the formation of a localized encysted abscess. If the former, the fluid escaping from the viscus is free to run at large in the peritoneal cavity. In many of the cases, as is seen from a study of the records, a well-defined path is taken. The fluid (generally mucus more or less tinged with bile) escapes on to the upper surface of the transverse mesocolon to the right of the hillock, which is formed by the fitting in of the transverse colon to the greater curvature of the stomach. It therefore tends to run to the right to the hepatic flexure, and then to descend along the outer side of the ascending colon to the iliac fossa. There collecting, it may cause symptoms strongly suggestive of appendicitis. From the iliac fossa the fluid drains to the 
pelvis, and, filling that, overflows into the left iliac fossa. If an abscess forms, it may be bounded by lymph, by the liver, or by intestines. Meunier describes a local abscess in his case as being bounded by the quadrate lobe of the liver, the gall-bladder, and the transverse mesocolon. Lennander relates a case where an abscess was hemmed in on all sides by intestine. The perforation of such an abscess may lead to acute septic generalized peritonitis, as recorded by Planchard. Perforation of the upper portion of the duodenum may lead to subphrenic abscess. Seven cases of this kind are related in Maydl's monograph. An ulcer may destroy all the coats of the bowel, and its base be formed by the liver (Keyl, Herzfelder, Collin), or by the gall-bladder (Krauss, Moynihan). An ulcer may destroy all the coats of the bowel and perforate a hollow viscus, forming an 'internal duodenal fistula.' Rokitansky describes a case of gastroduodenal fistula, Hoffman and Gross cases of cholecystoduodenal fistulæ, and Dudensing a case where the ulcer had perforated the pancreatic duct. An ulcer may destroy all the coats and lead to the formation of an abscess, which bursts upon the surface of the body, forming an 'external duodenal fistula,' as recorded by Lumeau and Bucquoy. The duodenal wall may be ulcerated through by an abscess arising from without, as in a case of lumbar abscess bursting into the duodenum close to the biliary papilla, under the care of Davies-Colley.

\section{Symptoms.}

At the first the symptoms of a perforated duodenal ulcer are precisely similar to those seen in cases of perforated gastric ulcer, but after the first shock has passed the symptoms and signs of gastric and duodenal perforation differ in their development. When the ulcer is in the stomach, the signs are those of general peritoneal involvement; when the ulcer is duodenal, the course taken by extravasated fluids leads to a more acute and an earlier involvement of the peritoneum on the right side and in the right iliac fossa. The clinical picture of appendicitis is copied with such accuracy that in forty-nine recorded cases, in eighteen the first incision was 
made over the appendix, after a diagnosis of acute appendicitis had been made. In the final stage, the peritoneal infection is universal.

When the abdomen is examined even within the first three or four hours, a greater resistance and a more marked tenderness may be found upon the right side, and the tenderness over McBurney's point may be exquisite.

It is interesting to note that in many of the recorded cases of duodenal perforation, and in most of our own cases, there was an entire absence of any previous history of pain after food, vomiting, hæmatemesis, or other symptoms indicative of a serious lesion in or near the stomach. In some instances the patients are unusually hearty, and the only complaint made is of a heaviness or burning after a large meal taken to appease an excessive appetite. 


\section{CHAPTER XIX}

\section{THE COMPLICATIONS OF GASTRIC ULCER (continued)}

\section{HOUR-GLASS STOMACH}

BY 'hour-glass' stomach is understood that condition of the stomach in which the viscus is divided into two cavities. The cavities are generally of unequal size. The terms 'hourglass contraction of the stomach,' 'double stomach,' and 'bilocular stomach' have been adopted by various authors. Hour-glass stomach may be described as congenital and acquired.

\section{Congenital Hour-glass Stomach.}

Congenital hour-glass stomach is commonly asserted to be the more frequent variety. Thus Fenwick writes: 'In about 45 per cent. of the cases which have been recorded neither ulcer nor scar could be detected in the stomach, while in the great majority of the cases where an ulcer was present it was obviously of more recent formation than the stricture.' And again: 'That the deformity is a rare result of ulceration is proved by the fact that only one case of the kind is mentioned in the records of the London Hospital for forty years, whereas several instances of the congenital form of the disease were encountered during the same period of time.' Similar statements have been made by Carrington, Roger Williams, Hirsch, and, in fact, every writer who has dealt with the subject.

We consider that the evidence of the existence of hourglass stomach as a congenital deformity is insufficient to be convincing. It cannot be denied that congenital cases may exist, for, as a fact, they are not improbable when we contemplate the circumstances in congenital stenosis of the pylorus, but we are sceptical that any of the recorded cases 
of congenital deformity are in reality of such an origin. It is a noteworthy fact that in many of the so-called congenital cases, if not in all, ulceration was present in some part of the stomach. This is true of Carrington's cases, of Hudson's, Saundby's, Williams's, and Hirsch's. In Watson's first case it is said that the mucous membrane at the point of constriction and on each side of it 'is thrown into abnormally heavy ridges,' which implies that a process of narrowing from a larger bulk has here occurred. In certain of the museum specimens labelled 'congenital,' it is quite clear that no adequate examination of the specimen has ever been made. Indeed, such an examination necessitates a so free handling of the specimen as to spoil it entirely for decorative purposes. The simple aspect of a pathological constriction can be most deceptive. One of the cases related below was an exemplary instance of this. The isthmus uniting the cardiac and pyloric cavities was barely the size of one's little finger; it was perfectly soft, smooth, and everywhere supple. There was no trace of any thickening or puckering of the surface. On invaginating a finger into the cavity on each side of the narrowing, no orifice could be felt leading from the one to the other. To all appearance no pathological process had ever existed there. On slitting the stricture up, as a preliminary to performing gastroplasty, the following condition was found: The passage was equal in diameter to a No. 4 or 5 catheter; most of its circumference was natural in appearance, but at the upper and anterior part was a small dead white area, clearly cicatricial, and from near this there ran, on the pyloric side, vertically down to the opposing mucous surface a column clad completely in mucous membrane, and $\frac{1}{2}$ inch in diameter. The column was removed between two ligatures, and it was then found to be built up of dense fibrous tissue. There was, in fact, a 'bridle stricture' in the stomach. That it was pathological admits of no question, yet nothing short of a division of the stricture could have revealed its inflammatory origin. There can be no doubt that if the whole stomach had been obtained from a post-mortem examination, the specimen would have been proclaimed an admirable example of congenital hour-glass stomach. 
Hochenegg on a similar case performed the operation of gastro-gastrostomy. No careful examination of the narrow stricture was made, but the case is quoted as an example of 'congenital' deformity. Such a case is quite inadmissible. Doyen's case, referred to in the list, is christened 'congenital.' At the point of narrowing there was an ulcer adherent to the anterior abdominal wall. On separating the adhesions a gastric fistula was exposed, showing unmistakably that a localized perforation of the ulcer, with anchoring, was responsible for the warping of the stomach.

Williams (Journ. Anat. and Physiol., r883) describes ten cases of 'congenital contraction of the stomach.' The account of one of the cases is based on the examination of a wax model, of another on the inspection of an 'inflated dried' specimen, and a third on the appearance of a 'dried stuffed' specimen. It is doubtful whether one of the examples can be accepted as an 'hour-glass' stomach. In all the rest pathological conditions, ulceration, puckering, thickening, adhesion to pancreas, were present. It is improbable that any of the stomachs was the seat of a congenital deformity. The same criticism applies to Carrington's cases and to Maier's.

The facts that pathological changes producing marked changes in the contour of the stomach may be inconspicuous, that ulceration in association with 'congenital' deformity is not infrequent, and that in many of the examples no purposeful examination of the specimen has been made, warrant us in saying that congenital hour-glass stomach is certainly rare and not improbably mythical.

In 20 examples of this supposed variety collected by Watson, the stricture was situate in the middle in 7 , at the junction of the upper and middle thirds in 3 , at the junction of the lower and middle thirds in 4 , and was not noted in 6.

\section{Acquired Hour-glass Stomach.}

Acquired hour-glass stomach may be caused by :

I. Perigastric adhesions.

2. Ulcer, with local perforation and anchoring to the anterior abdominal wall. 
3. Circular ulcer, with subsequent cicatricial contraction and induration.

4. Cancer.

I. Perigastric Adhesions.-These may be the result of many causes, among them being perforation of a gastric ulcer. The adhesions chiefly concerned in producing the condition may be a thick cord running downwards from the liver, and sharply pressing into the anterior wall of the stomach. In one of our cases such an adhesion caused the cardiac end of an hour-glass stomach to be itself divided into two, thus forming a 'trifid stomach.'

2. Ulcer with Local Perforation and Anchoring of the Stomach to the Anterior Abdominal Wall.-In this form a chronic ulcer of the anterior wall of the stomach makes its way in languid fashion through the coats of the organ." As it nears the serous coat adhesions are formed, binding it to the parietal peritoneum, which solders the base and prevents a general leakage. The stomach being then firmly anchored at this one point, a sagging of the cavity on each side, but chiefly in the cardiac side, occurs, and this, with the cicatricial contraction occurring in the ulcer, results in an hour-glass stomach. Excellent examples of this are referred to below.

Similar cases are recorded by Steffan and Finney.

Cases of general perforation into the peritoneal cavity are recorded by Siewers, W. H. Brown, Thomsen, Leonard Bidwell and ourselves.

3. Circular Ulcer, with subsequent Cicatricial Contraction and Induration.-Such an ulcer extends transversely to the long axis of the stomach, and in its contraction while healing must inevitably cause a high degree of narrowing (see Fig. 85). The simple round ulcer is also fully competent to produce a stricture, though probably not with the same completeness as the former. The conditions found in some of our cases suggest very forcibly the likelihood of the ulcer having perforated into the general peritoneal cavity.

The stricture may be placed at any part of the stomach; it is generally near to the middle, or, rather, to the pyloric side of the middle. The calibre of the orifice may vary from that of a No. 4 or 5 catheter to that sufficient to allow of the 
passage of three fingers. The greater curvature is generally puckered up towards the lesser, but the reverse is recorded in one case by Schwarz, and in another seen with the late Mr. Jessop the same condition was found.

Klein has recorded one example of hour-glass stomach resulting from the contraction of an ulcer, which had been caused by the drinking of hydrochloric acid with suicidal intent. Syphilis of the stomach may result in ulcer or gumma, and in consecutive warping of the viscus.

The amount of induration found around a chronic ulcer may be so considerable and its density so marked that a mistaken diagnosis of malignant disease of the stomach may be made. This happened in one of our cases. A large densely hard, immovable mass, adherent to the pancreas, was found in the stomach walls between the two loculi. The appearance of malignant disease was accurately simulated. The mass could not be removed, and the cardiac pouch could not be reached with sufficient ease to allow one to perform a gastro-enterostomy, and it was, therefore, only possible to dilate' the constriction between the two pouches. After considerable pressure the little finger was invaginated through the isthmus, which was slowly dilated until three fingers could be passed through. It was hoped by so doing to lessen the distress of vomiting, which had been almost continuous. The patient speedily recovered, and now after two years is perfectly well; she has gained $2 \frac{1}{2}$ stones in weight, is ruddy and healthy in appearance, and the tumour, readily palpable before the operation, has entirely disappeared.

One point which is deserving of especial emphasis is the frequency with which in cases of hour-glass stomach due to chronic ulcer a narrowing of the pylorus is also found. The constriction in the middle of the stomach hinders the passage of food from the cardiac to the pyloric pouch; the narrowing at the pylorus makes difficult the emptying of the pyloric sac, which, in consequence, undergoes dilatation. This double constriction is an illustration of the fact we have verified in operations upon the stomach-the frequency of multiple ulcers in the stomach, or in the duodenum, or in both. If the cardiac loculus alone is obstructed, the pylorus being 


\section{PLATE XXV.}

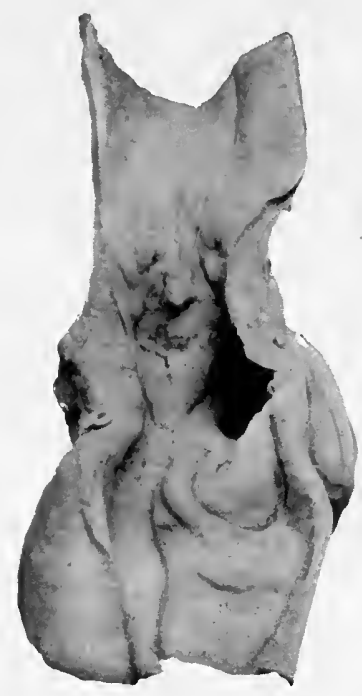

Fig. 94.-Cancer of the Cardiac End of the Stomach causing the Fundus to be partially shut off, so as to form a Large Pouch. (Modification of Hour-glass Stomach.)

From a woman of fifty, who had had symptoms for three years. 'Ulcus car. cinomatosum.'

To face p. 342.] 


\section{PLATE XXVI.}

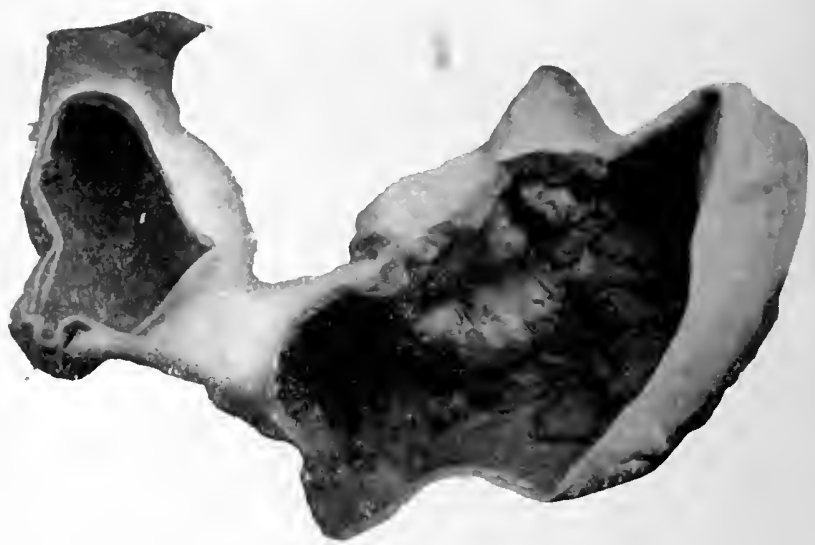

Fig. 95.-Hour-glass Stomach; possibly Congenital, with Growth rouxd Cardiac Orifice.

(No. 2,416, Royal College of Surgeons' Museum.)

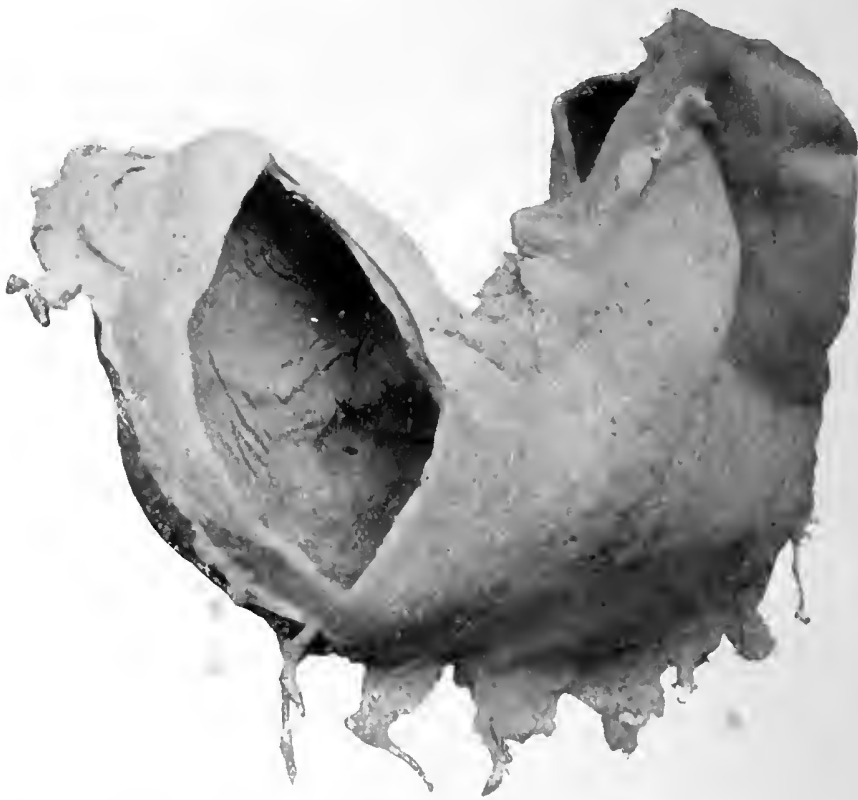

Fig. 96.-Cancer of Anterior IVall of the Stomach producing Hourglass Contraction.

Man, aged sixty, with four years' history of vomiting, and other signs of ulcer. This is an example of 'ulcus carcinomatosum.' (No. 2,408c, Royal College of Surgeons' Museum.)

To face p. 343.] 
free, the walls of the former are much thicker than those of the latter. Lunnemann, in such a case, found the circular muscular fibres 2 to 2.5 millimetres thick on the cardiac side and only $I$ to $x^{\circ} 5$ millimetres on the pyloric. It is possible that the contraction found at the pylorus may be the result of a long-continued spasm, set up by the ulcer whose healing has caused the hour-glass shape of the stomach. Frequent spasm would cause hypertrophy of the muscular coats, and fibrous transformation would occur in the overdeveloped muscle. In two cases under our care a double constriction had been formed in the stomach, and three pouches had thereby resulted-one is the only recorded example of trifid stomach due to ulceration, the other had a double cause, one constriction being due to the contraction of an ulcer, the other being dependent on adhesions due to perigastritis.

4. Cancer.-Cancer of the stomach is a not infrequent cause of hour-glass stomach. In some of the specimens the cancer has clearly been implanted upon a chronic ulcer (ulcus carcinomatosum). In others the cancer, originating in a localized area of the stomach, and there producing the constriction of the hour-glass, has in its later growth involved the greater part or even the whole of the stomach. The stomach then is thickened in all its coats' by a diffuse growth, and the condition described as 'leather-bottle' stomach results. Fig. 96 is an example of hour-glass stomach due to cancer.

\section{Symptoms.}

The symptoms of hour-glass stomach are oftenest those of dilated stomach supervening upon chronic ulcer of the stomach. In most of the cases recorded, a diagnosis of ' dilated stomach' has been made, and in those dealt with surgically operative interference has been undertaken for the relief of supposed pyloric stenosis. In certain cases, however, the symptoms and signs are clear and pointedly characteristic. In four of our cases a confident diagnosis was made thereon. Two of the most helpful signs were pointed out by Wölfler. They are :

I. These phenomena were observed upon washing out the 
stomach: The fluid introduced into the stomach seemed to disappear altogether, 'as though it had flowed through a large hole,' and is not returned through the tube. In such a circumstance the fluid passes, it is obvious, from the one compartment to the other.

2. It was further noticed that when the stomach was washed out until the lotion returned clear a sudden, unlooked-for gush of foul, or even putrid, fluid occurred; or if, after gentle lavage until the stomach seemed clean, an interval of a few minutes was allowed to elapse and the tube again passed, foul or dirty fluid at once returned. This is due doubtless to the reflux of the contents of the pyloric cavity through the stricture.

Jaworski (Wein. Klin. Woch., r898) noticed in one instance that, after apparently emptying the stomach by passing the tube, a splashing sound could still be obtained by palpation over the gastric area. The pyloric cavity, which is not drained by the stomach tube, causes this suggestion of dilated stomach. The condition has been appropriately described as 'paradoxical dilatation.'

Eiselsberg remarked in one of his cases that upon injecting a quantity of fluid into the stomach there was a bulging and distension of the left side of the epigastrium; after a few moments this gradually sank and subsided, and simultaneously a swelling of the right side of the epigastrium slowly developed.

A sign to which Eiselsberg also called attention in this case was well exhibited in two of our cases. On distending the stomach with $\mathrm{CO}_{2}$, the bubbling and gushing of fluid through a narrow chink could be heard with the stethoscope, an observation that was repeated in Watson's case. If the area of gastric resonance be marked out before and after distension, a marked increase, even to doubling, is noticed in the resonance of the cardiac complement, while the pyloric complement may seem to mimic the appearance of a dilated stomach. Investigation in this manner would have revealed this sign very clearly in Pollard's case and in Childe's case, recorded in the list. There can be no risk in distending the stomach with $\mathrm{CO}_{2}$, and therefore this method should be 
adopted in all doubtful cases. It seems not unlikely that the sign will be most helpful in making an accurate diagnosis. In two cases seen by Schmidt-Monard and Eichhorst a distinct sulcus separating two dilated cavities was seen on inflation with $\mathrm{CO}_{2}$. This appearance was observed in a patient seen in private at separate consultations by both of us. In slighter cases of constriction a notch may be recognised in the lower margin of the stomach.
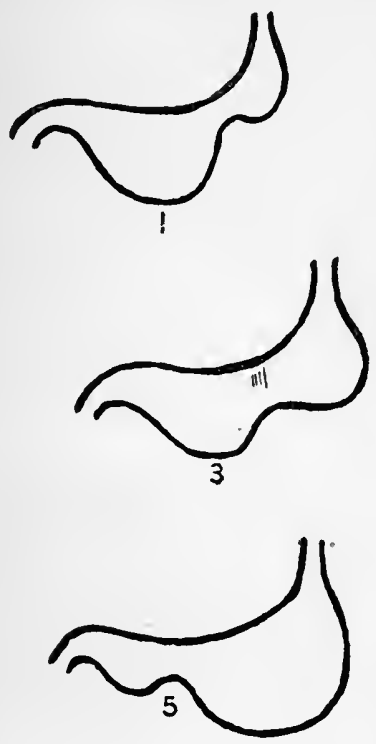
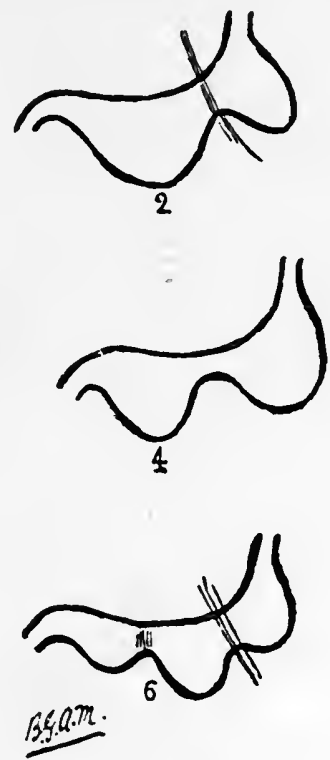

Fig. 97-Types of Hour-glass Stomach.

I, Constriction near the cardia (simulates esophageal obstruction); 2, cardiac complement hidden by adhesion (thorough examination of the whole stomach is necessary); 3 , constriction with much induration; 4 , a narrow channel connects the two pouches; 5 , constriction near the pylorus (simulates pyloric obstruction); 6, hour-glass stomach (the cardiac complement subdivided by an adhesion), ' trifid stomach.'

The symptoms resulting from a narrowing in the stomach will naturally depend upon the part of the viscus affected. If the contraction be near the pylorus the cardiac pouch will distend to almost any capacity, and the clinical picture will be that of dilatation of the stomach. If the stricture be placed but a little way from the cardiac, the symptoms will suggest œsophageal obstruction. The differential diagnosis 
can, however, be achieved by noting the distance which the œesophageal bougie passes. In one case seen with Dr. Sykes, of Cleckheaton, a correct diagnosis was made, and a seemingly accurate estimate of the capacity of the cardiac complement made by observing the following symptoms: When the patient took a tablespoonful of milk he retained it; if an ounce or two were drunk at a gulp, the greater part was returned. The stomach tube passed 18 inches without difficulty. On attempting to wash the stomach out, I ounce or a little more of fluid passed in, but no more. We administered a seidlitz-powder in separate halves. The first half was retained; on administering the second the patient was overwhelmed by a gush of foam, which burst from his mouth and nostrils. The diagnosis of hour-glass stomach with a smaller cardiac and a larger pyloric segment was made. At the operation this condition was found; it was the result of malignant disease, which had already caused secondary deposits in the parietal peritoneum and ascites.

The characteristic signs, then, are :

I. Disappearance of fluid introduced through the stomach tube, 'as though it had flowed through a hole' (Wölfler).

2. After cleansing of the stomach by lavage a sudden gush of putrid, sour, ill-digested food, etc. (Wölfler).

3. 'Paradoxical dilatation'; succussion splash in pyloric cavity after siphonage of the cardiac (Jaworski).

4. Distension of cardiac loculus, its gradual subsidence, and concomitantly the distension of the pyloric loculus (Eiselsberg).

5: During this period a gurgling, forcing sound heard over or near the middle of the stomach (Eiselsberg).

6. On distension with $\mathrm{CO}_{2}$ a large increase, even to a doubling, in the thoracic area, tympanitic on percussion, and a slight distension, clearly demarcated, of the pyloric loculus.

7. Rarely a sulcus may be seen on inflating with $\mathrm{CO}_{2}$.

\section{Differential Diagnosis.}

The two conditions for which an hour-glass stomach is liable to be mistaken are obstruction in the lower part of the œsophagus and pyloric stenosis. If the constriction in the 
stomach is within an inch or two of the cardiac orifice, the upper loculus of the stomach will be very small in size, and capable, therefore, of holding only small quantities of food. Food, when swallowed, may be regurgitated within a few minutes almost unaltered, and the patient may tell the same story of difficulty in 'getting the food down' as is told by one whose œsophagus is obstructed. A correct diagnosis can be made by introducing the œsophageal bougie; if the bougie passes over I6 inches from the teeth, the obstruction does not lie in the osophagus.

If the constriction be near the pylorus, the cardiac complement will be dilated, and will present the same appearance and signs as a dilated stomach. Wölfler's two signs ( $\mathrm{I}$ and 2 in the list given) will generally enable a correct diagnosis to be achieved.

If the obstruction should lie at any point between the two mentioned, there should be no difficulty in making a correct diagnosis.

Bouveret (Lyon Médical, March 16, I902, and British Medical Journal, Suppl., May 31, I902) records a case in which the dilated cardiac pouch of a bilocular stomach was mistaken first for hydatid cyst of the spleen, and afterwards for dilatation of the whole stomach consequent upon pyloric stenosis.

The patient, a woman forty-five years of age, had suffered at intervals for fifteen years from pain after food and vomiting. For two years and a half she had known of the existence of a swelling in the left flank, which enlarged slowly. For eight days she had vomited everything taken. On examination, the left flank was found to be occupied by a large tumour, which caused the ab. dominal wall to project from the thorax to the iliac crest. Pressure upon the thoracic organs was shown by difficulty of breathing and rapidity of the pulse. The tumour was uniformly dull to percussion, and evidently contained fluid. The tumour was diagnosed as a unilocular hydatid of the spleen, and as it was thought to be on the point of rupture, was tapped. The fluid obtained resembled the vomited matter, was grayish in colour, contained hydrochloric acid, and under the microscope showed the presence of yeast, starch granules, and epithelial cells. The patient improved rapidly after the puncture. The tumour reappeared a month later, and was again confined absolutely to the 
left side. The use of the stomach tube caused it to disappear, but insufflation reproduced it, the gas passing on into the intestines in a few minutes. The patient declined operation. She was readmitted into hospital some weeks later in a moribund condition, and at the necropsy a bilocular stomach was seen, the mediogastric narrowing allowing very little communication between the two halves of the stomach.

Bouveret concludes that the stomach tube should be used before making a definite diagnosis of cystic tumour of the upper part of the abdomen.

\section{Treatment.}

The following operations have been practised in cases of hour-glass stomach :

I. Gastroplasty, with or without resection of the ulcer.

2. Gastro-gastrostomy, or gastro-anastomosis.

3. Gastro-enterostomy.

4. Partial gastrectomy.

5. Digital dilatation of the constriction.

Frequently, owing to the existence of adhesions, gastrolysis has to be performed in addition to the operations mentioned.

The choice of an operation will be determined by the conditions found at the operation. It is essential that a careful and complete examination of the whole stomach be made before the curative procedure is adopted; for in some of the cases related below it will be noticed that the lack of adequate and precise knowledge has led to futile measures and fatal results.

If the constriction be near the middle of the stomach, if there be little induration and no active ulceration, and if the pylorus be free, a gastroplasty will prove successful.

If the constriction be in any part of the middle third or half of the stomach, if the pouches on each side are 'sagging' and free from adhesion, and if the pylorus is free, a gastro-gastrostomy will suffice to effect a cure.

If, however, the stomach narrowing be associated with pyloric stenosis, the existence of which must be suspected if the pyloric segment is dilated also, no single operation will 
suffice. A gastroplasty and a pyloroplasty, or a gastroplasty and a posterior gastro-jejunostomy, must both be performed.

It is possible in some cases-such as that of Schwarz, where the lesser curvature is dragged down to the greaterthat the incision dividing the constriction to the stomach might be utilized for the purposes of an anterior gastrojejunostomy.

If on separating parietal adhesions a gastric fistula be found, as in Doyen's case and in two of ours, the ulcer may be excised, or its edges refreshed, and a gastroplasty be performed.

If the constriction be due to new growth, a partial or complete gastrectomy may be performed. If these prove not feasible, a palliative jejunostomy may be necessary.

Various operators have expressed individual preference for certain methods. Thus, Eiselsberg prefers gastro-enterostomy. He has performed operations upon eight patients : three of the patients died as a result of the operation, and of the five survivors one suffered from a recurrence of the symptoms at the end of nine months. Eiselsberg's objections to gastroplasty is, that recurrence of the stenosis is probable. The same objection has been brought by Berg and others against pyloroplasty. The probability is that while the process of ulceration is still active, or induration or adhesions are present, it is unwise to perform a gastro- or pyloro-plasty.

It is especially important to observe the condition of the pyloric portion of the stomach. If the isthmus be a narrow one, the pyloric segment should be small and empty. If the pyloric cavity be dilated, as is expressly noted in Jaboulay's case and in Watson Cheyne's, there is a strong presumption in favour of the existence of a pyloric narrowing. If such narrowing be present and a gastroplasty alone be performed, the operation, though 'successful' so far as recovery is concerned, cannot be expected to relieve the patient of all disabilities, and a second operation may be imperative.

r. Gastroplasty is an adaptation to the body of the stomach of Heineke's operation for pyloric stenosis. It was performed by Bardeleben in 1889 , Kruckenberg in 1892 , and later by Zeller and Doyen and others. 
A transverse incision, 4 inches in length at least, is made, dividing the stricture. The incision is then stitched up vertically. Two continuous layers of sutures are introduced -one for the mucous membrane only, or all the coats, and the other for the serous or subserous coats alone.

One of us employs a large bone bobbin, over which the sutures are applied (Fig. 98).

2. Gastro-gastrostomy, or Gastro-anastomosis, was first performed by Wölfler in 1894. The incisions made into the

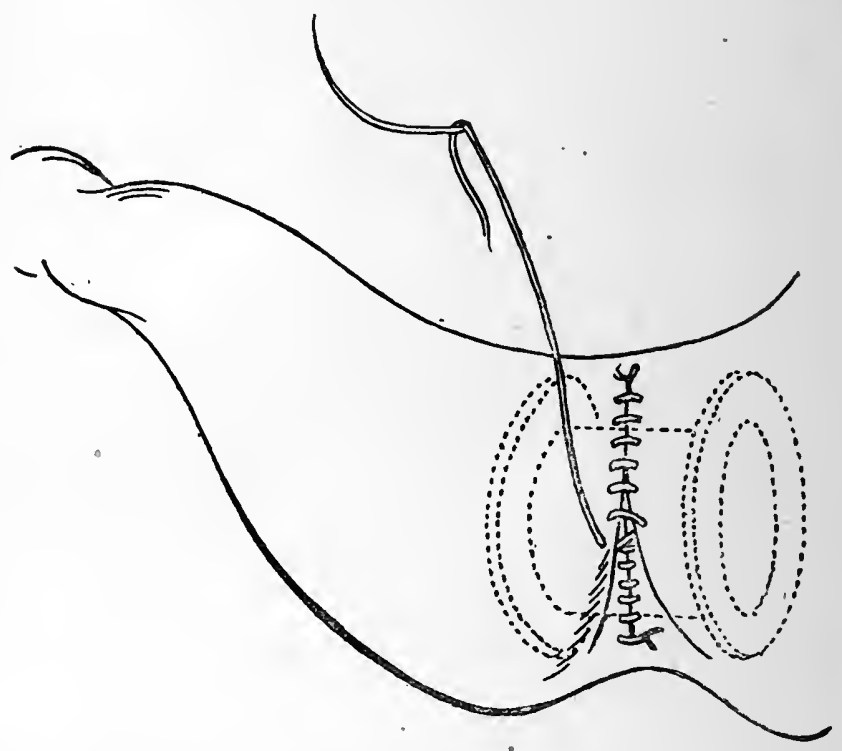

Fig. 98.-Gastroplasty with the Aid of the Decalcified Bone Bobbin.

dependent pouches on each side of the constriction were vertical and 7 centimetres in length. An adequate anastomosis between the two halves was thus made.

Sedgwick Watson, in I895, successfully performed gastroanastomosis in a different manner. The incisions in the two segments of the stomach were transverse. The pyloric portion of the viscus was folded over the cardiac, with the constricting isthmus as a hinge, and the two stitched together with an elliptical line of sutures before being opened. The anastomosis was then made by incising the wall of the 
pyloric compartment, which now lay anterior and through the opposite surface, making the anastomosis. This method is unnecessarily cumbersome, and possesses no potential advantages over Wölfler's method.

3. Gastro-enterostomy is not generally applicable; for if the pyloric pouch be united to the jejunum, as in Pollard's case and in Childe's, the operation will avail nothing, for the disability is due to the fact that food cannot enter the pyloric portion of the stomach. If the cardiac complement be united to the intestine, the pyloric pouch is undrained, and symptoms will persist and necessitate a second operation, as in Tuffier's case. Weir and Foote (Medical Neres, 1896) advise that a double anastomosis between both pouches and the jejunum be made at the same operation, a method that would probably prove satisfactory.

4. Partial Gastrectomy.-In cases of cancer always, and in certain cases of simple disease, a partial gastrectomy may be the operation of choice. In cancer a wide excision should be made; in simple disease a removal of the constriction will suffice. In both an end-to-end suture is desirable. In the chapter on partial gastrectomy will be found reports of two cases of hour-glass stomach, due to cancer of the centre of the viscus, which were treated successfully by excision of the growth and direct union of the cardiac and pyloric portions of the stomach.

A method, which is a modification of Finney's operation for pyloroplasty, has been described by Dr. F. Kammerer as follows :

The patient was a man of forty-six years, who had suffered for four years from symptoms referable to ulceration of the stomach; lately he had shown signs of pyloric obstruction. He had lost 20 pounds in weight during the last three years. On opening the abdomen under ether, a typical hour-glass contraction of the stomach presented itself. The larger part of the stomach was on the cardiac side, the capacity of this portion being about double that of the compartment on the pyloric side. The stomach was adherent posteriorly at the point of contraction, and could not be raised from the abdominal cavity, although the dependent vertical portions could be nicely approximated. The contracted part, it seemed, would not have admitted the index-finger. Beginning 
at the lowest point of the constriction, a running suture was applied through the serous and muscular coats, bringing the vertical edges of both compartments of the stomach into close approximation along their posterior margins. An inverted U-shaped incision was now made through the entire thickness of the stomach wall, about $\frac{1}{4}$ of an inch to either side of the Lembert

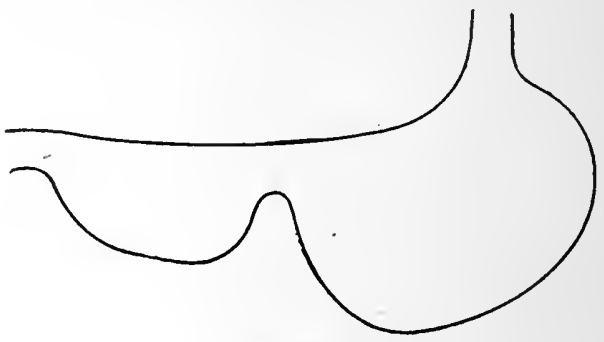

FIG. 99.

suture. The posterior wound edges were now brought together with another running suture from within, the same procedure being then applied to the anterior edges from without. The final act of the operation consisted in re-enforcing the anterior suture with a running Lembert stitch, and placing a few extra sutures at the lowest point of the stomach through the serous and muscular coats, where tension would naturally be greatest. When the stomach was first incised, a deep ulcer, just admitting the index-

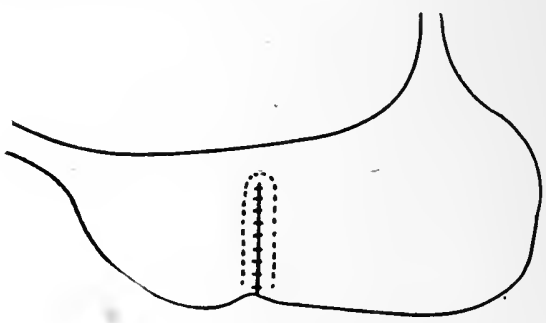

FIG. Ioo.

finger for about an inch, was discovered on the posterior wall of the stomach, immediately above the point of constriction, near the lesser curvature. This had evidently perforated all the coats of the stomach, but was enclosed within firm adhesions. The latter had evidently prevented the delivery of the stomach upon the abdominal walls at the beginning of the operation. The patient made an uninterrupted recovery, and has gained 22 pounds in weight since the operation last June. 
Divulsion of Stricture.-The operation of divulsion of the stricture may occasionally prove useful where, on account of its proximity to the cardiac orifice or from adhesion, a more complete operation cannot be performed with safety, and in such cases a gastro-enterostomy will usually be necessary in order to secure healing of the ulcer. The following is an example :

Hour-glass Contraction near Cardiac Orifice treated by Divulsion.

Dr. Joseph A. Blake presented to the New York Surgical Society, November 12, 1902, a woman, thirty-five years of age, who was admitted to Roosevelt Hospital on July 18, 1902. At the time she was nursing an infant twelve months old. Her family and previous history was negative, except that for several years she had had slight indigestion. She had not vomited until four months before admission, when vomiting commenced and was incessant, all her food, as she says, being vomited soon after eating. Vomiting persisted until her admission. She never vomited blood nor food that had been in her stomach for any length of time.

Two months before admission she began to have severe cramplike pains in the epigastric region, accompanied with tenderness. In four months there was a loss of 20 pounds in weight, and she became sallow. The bowels were constipated. No history of blood in the stools was obtained.

Upon admission she was emaciated, skin was dry and sallow. Her temperature was $99^{\circ} \mathrm{F}$. ; pulse, 92 ; respiration, 22.

The abdominal walls were flaccid. Two inches to the left of the median line and just below the costal arch a hard, irregular mass about 2 to 3 inches in diameter could be felt moving with the diaphragm. No dilatation of the stomach could be made out, but distending the stomach made the tumour more evident. Analysis of the gastric contents showed a marked increase in free hydrochloric acid and no organic acids.

Operation.-Nitrous oxide ether anæsthesia. Incision 5 inches long in linea alba above umbilicus. The stomach was found to be embedded in adhesions, which bound it to the liver and pancreas, especially in the neighbourhood of the cardia. Two inches from the cardia it was constricted and indurated, the constriction forming an isthmus 2 inches long, uniting a cardiac and pyloric division of the stomach, the latter being more than double the size of the former. On account of the adhesions, their exact 
disposition could not be made out until a tube had been passed and the stomach inflated. It was then found that they could not be opposed unless unwarrantable force was employed.

The pyloric division was then explored through an incision in its anterior wall, the finger being passed into the constriction, which admitted it easily. The lower wall of the constriction was covered with a soft tissue feeling like granulation tissue. No indurated ulcer was felt. The constriction was stretched until it would nearly admit two fingers.

Inasmuch as it was impossible to get at the cardiac division on account of the adhesions which had obliterated the lesser peritoneal sac, and therefore bound the stomach to the pancreas, and as the two divisions could not be approximated, gastro-enterostomy by Von Hacker's method was performed with the pyloric division, by means of Murphy's buttons, with the idea of improving the ulcer. Entero enterostomy was also done. The anterior wound was used to introduce the stomach button, only the inner cylinder being passed through a short slit in the posterior wall of the stomach. The wound in the anterior wall of the stomach was then closed.

The subsequent results have been marvellous. The patient has not had a gastric symptom since the operation. She eats everything, and has gained 30 pounds in weight.

The explanation of this improvement may be that the gastroenterostomy, by affording physiological rest and neutralizing the hyperacidity, has brought about the healing of the ulcer. The buttons have not been passed.

The following is a complete list of all recorded cases up to IgOI :

Case i : Bardeleben, I889.-Hour-glass stomach. Gastroplasty. Recovery and relief. (Klemperer, Bevliner Klinische Wochenschrift, 1889.)

CASE 2: KruckenberG, I892.-Thickened scar on the anterior surface. Gastroplasty with excision of ulcer. Recovery with complete relief. (Schmidt-Monard, Münchener Medicinische Wochenschrift, I 893 , No. 19.)

CASE 3: Zeller, I893.-Hour-glass stomach, the result of healed ulcer; cicatrix. Resection of cicatrix of healed ulcer. Death from septic peritonitis, result of perforation of another ulcer. (Centralblatt fïr Chivurgie, I 894.)

Case 4: Doyen, I 893.-Constriction at middle of stomach, due to ulcer adherent to the anterior abdominal wall. On separating adhesions a gastric fistula was found. Scraping of ulcer and 
inversion; gastroplasty. (This case is called congenital.) Recovery. ('Traitement Chirurgical des Affections de l'Estomac,' Paris, 1895 , p. 308.)

CASE 5: Wölfler, 1 894.- - Stomach constricted into two equal portions by scar of ulcer. Gastro-gastrostomy. Recovery; at the end of three months quite well, and had gained 9 kilogrammes in weight. (Beiträge zuv Klinischen Chiruvgie, I895, No. 13.)

CASE 6: EiselsberG, I894.-Cicatrix of old ulcer. Gastrogastrostomy. Death; sutures introduced into indurated area had cut through. Septic peritonitis. (Archiv für Klinische Chirurgie, Band 1.)

CASE 7: Watson, I895.-Constriction at junction of middle and lower thirds, the result of old ulcer. Gastro-gastrostomy. Recovery; complete relief; gained 42 pounds in six months. (Boston Medical and Surgical Journal, 1896.)

Case 8: Lauenstein, i 89.5.-Constriction due to cicatrix, from which adhesions ran to liver. Gastro-gastrostomy. Recovery; in three months gained 35 pounds. (Münchener Medicinische Wochenschrift, 1896, No. 43.)

Case 9: Langenbuch, I 896.-Hour-glass stomach. Gastroplasty. Recovery without relief. (Berliner Klinische Wochenschrift, $\mathrm{x} 896$; Thèse de Lyons, r896.)

CASE io: VAN Noorden, I896.-Perforation of the stomach by ulcer. Establishment of fistula. Death on fifty-sixth day. (Miinchener Medicinische Wochenschrift, 1896.)

Case il : Hofmeister, I896.-Ulcer and cicatrix. Excision of ulcers. Gastroplasty. Recovery. (Beiträge zur Klinischen Chirurgie, 1896.)

CASE I2: Schwarz, I 896.-Constriction near middle, due to adhesions extending to diaphragm, liver, etc. The lesser curvature was drawn down to greater. Gastro-gastrostomy; gastrolysis at second operation. Symptoms not improved until after second operation. (Wiener Klinische Wochenschrift, June, I896.)

CASE I3: EiselsBerg, I 896.-Large ulcer on greater curvature, near fundus; adhesions to anterior abdominal wall. Gastroplasty. Recovery; return of symptoms after nine months. (Archiv für Klinische Chivurgie, 1899.)

CASE I4: Jaboulay, I896.-Constriction very narrow in pyloric third of stomach. Both cavities dilated. Cicatrix near lesser curvature; adhesions to the left lobe of the liver. Gastroplasty. Recovery; relief of symptoms. (Archives Provinciales de Chirurgie, I 896.)

CASE I5: TUFFIER, I897.-Hour-glass stomach; both cavities dilated. Gastro-enterostomy between cardiac pouch and intestine; symptoms not wholly relieved. At a second operation a 
gastro-enterostomy between pyloric pouch and intestine. Recovery from both operations; complete relief after the second. (Bulletin et Mémoive de la Société de Chivurgie, 1897.)

Case 16: Watson Cheyne, 1897.-Constriction permitting passage of a crow-quill only in middle of the stomach. Both parts of the stomach dilated. Gastroplasty. Recovery. (Lancet, March I9, I 898, p. 785 .)

Case i 7: Eıselsberg, I 898.-Constriction admitting little finger in pyloric third of organ; adhesions to liver and pancreas; perigastric abscess. Gastroplasty. Death in ten hours. (Archiv fïr Klinische Chirurgie, Band lix.)

CASE I8: EiselsberG, I 898.-Constriction in middle of the stomach; adhesions to posterior abdominal wall and liver, and erosion of pancreas by open ulcer. Gastroplasty, jejunostomy. Death in twelve hours. (Archiv für Klinische Chirurgie, Band lix.)

Case i9: Eiselsberg, i 898.-Constriction admitting the little finger in the pyloric third of the stomach. Gastroplasty; twelve months later gastro-enterostomy. Recovery from both operations. Symptoms recurred nine months after the first operation. (Avchiv fïr Klinische Chirurgie, Band lix.)

CASE 20: ErselsberG, I898.-Circular constriction nearer cardiac than pylorus. Three operations-two gastroplastic, one gastro-gastrostomy; intervals were four, nine, and two months. Recovery from operations, but recurrence of symptoms after all. (Archiv fiir Klinische Chinurgie, Band lix.)

Case 2I: Eiselsberg, I899.--Constriction admitting - the passage of one finger, due to circular cicatrix and adhesions to liver. Gastroplasty. Recovery with entire relief. (Archiv fïr Klinische Chirurgie, Band lix.)

CASe 22: Eiselsberg, I899.-Circular constriction nearer cardiac than pylorus; dense mass of adhesions from constricted portion of stomach to liver. Gastro-enterostomy. Recovery and complete relief. (Archiv für Klinische Chirurgie, Band lix.)

CASE 23: Von Unge, I 898.-A circular constriction admitting forefinger 8 centimetres from pylorus. Gastroplasty. Recovery. (Centralblatt fïr die Grenzgebiete der Medicin und Chirurgie, May, I 899.)

CASE 24: VON UNGE, I 898.-A circular constriction admitting forefinger ro centimetres from pylorus; a mass of adhesions. Gastroplasty. Recovery. (Centralblatt für die Grenzgebiete der Medicin und Chirurgie, May, 1899.)

Case 25: Hastings Gilford, i898.-Hour-glass stomach; the constriction barely admitted one finger. On the cardiac side of the constriction an ulcer had perforated and fornied an adventitious cavity. Gastroplasty. Died one month after operation 
from hæmatemesis. The wound in the stomach was partially unhealed at the point of thickest induration. (Guy's Hospital Reports, vol. liii., I 898.)

Case 26: Courmont, I898.-Hour-glass stomach. Gastroplasty. Recovered. (Hochenegg, Wiener Klinische Wochenschrift, I898.)

C.ASE 27: Cumston, I898.-Constriction at the junction of the middle and lower thirds; adhesions to surrounding structures. Gastroplasty. Recovery; well eight months after. (New York Medical Journal, I899.)

CASE 28: Hochenegg, 1898.-Constriction at junction of middle and pyloric thirds, admitting little finger; cardiac portion immensely distended. Gastro-gastrostomy. Recovery. (Wiener Klinische Wochenschrift, I 898.)

CASE 29: Bier, I898.-Female, aged fifty-one. 'Ten years' history of stomach trouble, loss of weight, pain, vomiting; never hæmatemesis. Gastroplasty. Ulcer near lesser curvature; the cardiac pouch much dilated. Recovery.

CASE 30 : Bier, I 898.-Female, aged twenty-nine. Stomach troubles since ten years old. Hæmatemesis; constant pain. Stomach dilated to three fingers' breadth below umbilicus. Narrowing of pylorus found at operation; gastro-duodenostomy; death. At the post-mortem an hour-glass stomach, the pyloric complement of which had been mistaken for the whole stomach.

CASE 3I: Bier, I898.-Female, aged thirty-nine. Stomach trouble since childhood-vomiting, pain, etc. Above the umbilicus an area of resistance and tenderness; the greater curvature descends I inch below the umbilicus. At the operation a tumour the size of a hen's egg found in the middle of the stomach adherent to the anterior abdominal wall; tumour excised, and found to consist of induration round a chronic ulcer. Gastroplasty. Recovery.

CASE 32 : BIER, I 898.-Male, aged fifty-six. Since twelve and a half years of age stomach trouble. In the epigastrium a tumour the size of a man's fist ; found on exploration to be due to adhesion of stomach and œdema of abdominal wall. An ulcer in anterior wall between curvatures, which in its contraction had caused an hour-glass stomach. Excision of ulcer; gastroplasty. Death. (Cases 29 to 32 are related in a thesis by Eduard Asbeck. Vier Fälle von Sanduhrmagen, Kiel, 1898.)

CASE 33: Morse.-Male, aged sixty. Pain in stomach for twenty years. Hour-glass stomach with large cardiac pouch; adhesions between liver and constriction. Gastrolysis; gastroplasty. Recovery. (Lancet, May, r 899.)

Case 34: MacGillivray,-Woman, aged twenty-four. For 
several years continuous indigestion, severe pain and romiting, once hæmatemesis. Sudden seizure, pain and collapse. On opening abdomen an hour-glass stomach, with perforation of an ulcer near lesser curvature. Gastroplasty. Recovery. '(Scottish Medical and Surgical Journal, July, I 899.)

CASE 35: Watson, I900.--Perforating ulcer, with perigastric abscess and a mass of adhesions at the junction of the middle and lower thirds; stricture admitting one finger. Ulcer inverted and sutured; gastrolysis. Death on fourth day from general septic peritonitis. (Annals of Surgery, July, I goo.)

CASE 36: KLEIN, 1900.-Hour-glass stomach, the result of cicatricial contraction following the drinking of corrosive fluid. Gastro-duodenostomy. Recovery and entire relief. (Wiener Klinische Rundschau.)

Case 37 : Sidney Martin and Bilton Pollard, 1900.-Hourglass contraction and stenosis of pylorus. At the first constriction there was a small perigastric abscess. The second compartment was much larger than the first. Pylorus very stenosed, and peripyloric abscess found, due to perforating ulcer just beyond pylorus. Gastro-jejunostomy. The second compartment of the stomach united to the intestine. Death on fifth day. (British Medical Journal, vol. ii., I 90o.)

CASE 38: Childe, I901.--Hour-glass stomach. The cardiac portion lay under cover of the ribs, and was not noticed until the necropsy. The pyloric portion was supposed to be the whole stomach, and 'was not abnormally large.' Gastro-enterostomy between pyloric segment and jejunum. Death on fifth day. (British Medical Journal, vol. ii., I901.)

The following is a complete list of all our cases :

Hour-glass Stomach due to Simple Disease.

CASE I.-Mrs. S. W., aged forty-five, admitted to the Leeds Infirmary on May Io, I 899 , with a nine years' history of stomach trouble. The first attack was accompanied by severe pain after food and by vomiting, so that she was unable for three weeks to retain anything in the stomach. After a period of almost complete starvation, she recovered, and was comparatively well for six years, when a second period of illness occurred. On both occasions a swelling in the epigastrium with great tenderness was noticed. In September, I898, a third attack occurred, since which time she had never been free from pain, and the swelling and tenderness in the epigastrium had persisted. The patient was very much reduced in strength and weight.

Operation, May I8, I 899.--Very firm adhesions were discovered 
between the anterior stomach wall and the parietes, on separating which a perforation was found in the centre of a cicatricial mass, placed in the middle of the stomach, which had divided the organ into two cavities, the proximal one being the larger. The stricture was divided longitudinally by a very free incision, and the edges were brought together transversely over a decalcified bone bobbin. She was able to leave for home on June 6 , when she weighed 6 stones 7 pounds, this being a gain in weight. On July 29 a report was received saying that she weighed 7 stones 2 pounds, and that she was well.

Case 2.- In May, I 899, Miss P., aged twenty-six, was seen with Dr. Duncan, of Clay Cross. She had been ill for fifteen years with stomach symptoms, characterized by pain after food and vomiting. For some years the vomit had been coffee-ground in character and streaked with bright blood. It varied from a small quantity to several pints; but for the past eight years no blood had been vomited, the vomit being sour, watery fluid in large quantities. Flesh had been steadily lost, until the patient was reduced almost to the state of a living skeleton.

Operation, June 6, I899.-On opening the abdomen, the stomach was found to be trifid, owing to a double constriction, the proximal one being formed by an adhesion passing from the liver across the greater curvature of the stomach, constricting it ; the distal cavity was caused by a stricture about $3^{\frac{1}{2}}$ inches from the pylorus, it being dependent on a cicatricial contraction due to chronic ulcer. The proximal cavity was the most dilated, and this gave rise to the stomach splash and to the well-marked signs of stomach dilatation. On dividing the firm band of adhesion forming the first constriction, a sulcus was left in the stomach. On opening the second stricture, a chronic ulcer was exposed, and the channel was so narrow that it would only allow the passage of a No. I2 catheter. Gastroplasty was performed by one of the largest-sized decalcified bone bobbins. The patient made a good recovery, and returned home within the month. A report received on August 6, I 899, stated that she had gained 2 stones in weight and was quite well. Reported in good health January, I904.

CASE 3.-Mrs. E. M., aged twenty-three, was admitted to the Leeds Infirmary with a history of two years' pain after food and vomiting, 'with great tenderness in the epigastrium and loss of 3 stones in weight during the past two years. A well-marked stomach splash could be made out, but no tumour was manifest, and the patient could with difficulty bear palpation, on account of great tenderness.

Operation, July 20, I899. - On opening the abdomen, the stomach was found to be hour-glass in shape. A constriction was 
found 2 inches from the pylorus, just admitting the tip of the little finger. Gastroplasty was performed, the suturing being carried out over the largest-sized bone bobbin. The patient made a good recovery from the operation, and returned home on September 6 .

CASE 4.-Miss D., aged forty-eight, seen with Dr. Hindle, Askern. Indigestion and flatulency for twenty years; free hæmatemesis two years ago. Great loss of flesh. Dilatation of the stomach well-marked, and epigastric tenderness present.

Operation, July 28, I 899.-Gastroplasty performed. Extreme contraction one - third distance from the pylorus discovered. Stricture would just admit tip of little finger. Patient made a good recovery from the operation, and there was an increase of weight on November I.

The patient was quite well a year later, but socn after began to complain of pain dependent on perigastritis, for which an operation was subsequently performed. (See Case I6.)

CASE 5.--In August, I 899, Miss S., aged fifty-two, was seen with Dr. Walker, of Huddersfield. The patient gave a history of stomach trouble for twenty-six years. At first the pain had occurred about two hours after food, and vomiting was only occasional. During the first twenty years there had been periods of comparatively good health between the severe seizures. In I 894 she was told by an eminent physician that she would never be cured, but might be relieved by dieting. For a year she had been sick every third day, vomiting large quantities, and during that time she had lost 2 stones in weight, her weight being reduced to 5 stones Io pounds in her clothes. Dilatation of the stomach was well marked; peristalsis could be felt, and what was taken to be the pylorus could be felt to harden under the palpating hand.

Operation, September I, I 899.-The abdomen was explored, and an hour-glass contraction of the centre of the stomach was discovered, the stricture only just admitting a No. 7 catheter. The proximal cavity of the stomach was enormously dilated, and there was great thickening and induration around the stricture. Gastroplasty was performed by means of a bone bobbin, and the patient made a good recovery. On November I, I 899, she had gained 26 pounds in weight, at Christmas she had gained 4 stones in weight, and in December, I903, we had a note to say that she was still gaining weight and that her digestion was perfect.

CAse 6.-Mrs. A. P., aged thirty-six, was admitted to the Leeds General Infirmary on September 28, I899, with a history that for ten years she had had stomach trouble, and during that time she had lost 3 stones in weight. The symptoms had been intermittent, but never completely absent during the whole of the time, and for many months she had only been able to get relief 
after vomiting, which occurred every day. The physical signs were very distinct; peristalsis was well-marked, and much increased by the use of the stomach tube. When the stomach was washed out, after the first cavity had been thoroughly cleansed, the patient would suddenly retch and vomit a large quantity of foul material by the side of the tube, and as this contained no bile, it clearly came from the second cavity. The second sign elicited was by filling the stomach with water, when the outline of the first cavity could be easily percussed. A third sign was found by filling the stomach with air, when the lower limit of the first cavity could be readily felt in the left hypochondrium; it became lost in the middle line, and the stomach resonance could again be discovered to the right of the umbilicus. The stomach splash could always be elicited. Free $\mathrm{HCl}$ was found in the stomach contents.

Operation, October 5, I 899.-Numerous adhesions were found between the stomach and abdominal wall, when two cavities were discovered separated by a long narrow isthmus 21 inches in length, this being surrounded by dense cicatricial tissue. The pylorus was also much narrowed and thickened, it being also cicatricial. Gastroplasty was performed with some considerable difficulty, on account of the great length of the stricture, and after this had been completed pyloroplasty was also carried out; but in neither case did one feel satisfied with the sutures, which were applied under tension. The patient was fairly comfortable after the operation, and beyond a little regurgitation, which was relieved by lavage on the third day, no untoward symptoms followed, and a week later a note was made to say that the patient was doing well and altogether comfortable. At io o'clock on the eighth day after operation she was suddenly seized with violent pain, and the pulse rose to I20. In the absence of the operator, Mr. Lawford Knaggs was summoned, and after consultation with Mr. Littlewood it was decided to reopen the abdomen. The wound was found to be firmly healed, but on opening the peritoneal cavity air and bile-stained fluid escaped, due to perforation at the pylorus, some of the tense stitches having ulcerated through. Gastrorrhaphy was performed, and the abdomen was washed out. Unfortunately, however, the shock was too great, and the patient succumbed within a few hours.

Case 7.-Miss W., aged twenty-seven, seen with Dr. Ellis, Halifax. Eight years' history of stomach symptoms. Pain two hours after food and vomiting; had lost a stone in weight in two years.

Operation, March 2I, I900.-On opening the abdomen and exposing the stomach, it was found to be hour-glass in shape, owing to a cicatricial stricture 3 inches from the pylorus, just 
admitting the tip of the little finger. Gastroplasty was performed by means of a bone bobbin. The patient made a good recovery, and later there was a report to say that she was several pounds heavier than before the operation. On December 19, I903, Mr. Ellis wrote to say that she was in good health, looked well, weighed 9 stones, and was able to digest her food satisfactorily.

CASE 8.-Miss E. B., aged twenty-nine, waitress, had suffered from chlorosis due to overwork for years before the beginning of her stomach troubles four years ago, since which time she had had pain after food and occasional vomiting, with hæmatemesis and melæna. Very thorough medical treatment had been followed out on several occasions in the shape of rest and rectal feeding, but without any benefit. There had been great loss of flesh.

Operation, March 22, 1900.-On opening the abdomen, omental adhesions were discovered, these covering a puckered ulcer on the front wall of the stomach, 3 inches from the pylorus, where was an hour-glass contraction, which would just admit the tip of the little finger. Pyloroplasty was performed, a bone bobbin being used. The patient made a good recovery, and returned home on April 30. On May 9 she had gained several pounds in weight.

CASE 9.-Mr. L., aged eighteen, was seen on February 24, I900, and gave the history that he had never been strong since an attack of pneumonia four years before, and that he had had pain after food for two years, the pain usually coming on from one to one and a half hours after meals. Although close on 6 feet in height, his weight was only 9 stones 8 pounds. Though not absolutely an invalid, he was unable to carry out any of his ordinary duties, and was getting worse rather than better. The stomach was dilated, the lower border reaching midway between the umbilicus and the pubes. A stomach splash was very evident, and there was well-marked tenderness over the pylorus. After two months' careful general treatment he had lost 5 pounds in weight and felt weaker.

Operation, April 24, I900. - Hour-glass contraction of the stomach was found 4 inches from the pylorus, there being a scar on the surface of the stomach at that point, showing that the cause was chronic ulcer. The stricture would only admit the tip of the little finger. Gastroplasty was therefore performed, the largest-sized bone bobbin being used. He made a good recovery from the operation, but in November the stomach splash could easily be elicited, as if the muscular power had not been regained. Lavage of the stomach and the administration of strychnine were advised. In December, I903, we had a report to say that he was able to attend to his business every day, though he had still to take care in his diet and not to get overfatigued. 
Case I0.-Miss I., seen with Dr. Angew, Bradford. Patient in a state of great exhaustion, with extreme emaciation, the history being that she had had symptoms of gastric ulcer for fifteen years, and that for six years she had lived on liquid food in small quantity. She had been for a long time completely bedridden.

Operation, April 30, 1900.- The stomach was found extensively ulcerated and scarred, there being hour-glass contraction 4 inches from the pylorus, just admitting the tip of the finger. At this point there were signs of an old perforation that had been covered by omentum, which was extensively adherent. The stomach was so bound down by adhesions that anterior gastro-enterostomy only was possible. The patient rallied well from the operation, and progressed satisfactorily up to the twelfth day, when, after a good night, she expressed herself as feeling very well. About mid-day she complained of abdominal pain, suddenly became collapsed, and died a few hours later, apparently from perforation, though an autopsy could not be obtained to verify this.

CASE II.-Mr. D. M, aged forty-four, was admitted to the Leeds General Infirmary on May 31, I900. He gave a history of stomach trouble for twenty-three years, with occasional periods of good health, though recently the healthy intervals had been very short. Coffee-ground vomiting was a marked symptom. There was considerable loss of weight.

Operation, June 7, 1900.-Large cicatricial surface, 2 inches in diaineter, was discovered on the front wall of the stomach, forming an hour-glass contraction $2 \frac{1}{2}$ to 3 inches from the pylorus. On incising this longitudinally, the mucous membrane was found ragged and ulcerated for 2 inches on either side of the incision. Gastroplasty was performed over a bone bobbin. In this case no free $\mathrm{HCl}$ could be discovered in the stomach contents. The proximal cavity was the larger, the distal being comparatively small. The patient made a good recovery, and returned home on July 25 .

CASE I2.-Early in October, Igoo, Mr. H., aged fifty-two, was seen with Dr. Hearder, Ilkley. There was a three years' history of pain and vomiting, with epigastric tenderness and loss of flesh. On examination, the stomach was found to be markedly dilated.

Operation, October 12, I900.--An hour-glass contraction was discovered near the centre of the stomach, but the stricture was not extreme. Posterior gastro-enterostomy was performed, and the patient made a good recovery. On March 4, Igor, he wrote to say that he was quite well, and that he had gained I stone 5 pounds in weight.

CASE 13.-Miss D. B., aged twenty-five, was admitted to the . General Infirmary on November 5, 1900, with a history of 
stomach symptoms and pain and vomiting for three years. During part of the time she had been in hospital and under thorough medical treatment, but without material benefit, except of a temporary character.

Operation, November I 5, I900.-A large constriction was found in the centre of the stomach, forming a characteristic example of hour-glass contraction, both cavities being dilated, the proximal being larger than the distal cavity. Numerous adhesions had to be separated, especially around the pylorus, and others between the stomach and colon. A posterior gastro-enterostomy was performed, from which the patient made an uninterrupted recovery. She returned home on December II. On April 9, I 90 I, she returned to the Infirmary, on account of having had some recurrence of pain and vomiting, but under rest and careful dieting the symptoms completely passed off in three weeks and she was sent home, weighing 7 pounds more than she did when in the hospital on the former occasion. During the three weeks when under observation she had no sickness, and during the latter part of the time she was able to take ordinary food without discomfort.

CASE 14.-Mr. J. H., aged fifty-three, was admitted to the Leeds General Infirmary on November 29, 1900, with a two years' history of pain an hour to an hour and a half after food. Vomiting was not a marked symptom until a few weeks before admission. His ordinary weight was I 4 stones, but on admission it was Io stones Io pounds. There were no signs of dilatation of the stomach, and free $\mathrm{HCl}$ was present.

Operation, December 6, I900.--An hour-glass contraction of the stomach was found, 3 inches from the pylorus, there being considerable thickening of the stomach wall at that point; the proximal cavity was the larger. Posterior gastro-enterostomy was performed. He made a good recovery from the operation, and returned home on January 7 , I901.

CASE I 5.-On February I 3, Igor, Miss I: was sent to me by Dr. Mackenzie, Burnley, with a history of stomach trouble extending over several years. The attacks had come on about four times a year, lasting from six to eight weeks, when, after starvation with rectal feeding, she was relieved for a time. The stomach reached to the level of the umbilicus, but after distension with $\mathrm{CO}_{2}$ the greater curvature was $2 \frac{1}{2}$ inches below. The stomach splash was still well marked four hours after a meal. On distension, the outline of the stomach appeared to be bilocular, there being some irregularity in the centre. The patient was very thin and feeble.

Operation, April 2, I901.-A cicatricial contraction of the body 
of the stomach was found within an inch of the cardiac orifice, the stricture extending obliquely across the stomach. In this case the cardiac complement formed the smaller sac, the distal pouch forming the bulk of the dilatation felt before operation. There was spasm, but no stricture, of the pylorus. Gastroplasty was performed, and the patient made a good recovery from the operation. Reported well January, I904.

Case I6.-Miss D., aged forty-nine, seen with Dr. Hindle, Askern, with gastric symptoms for twenty years. Gastroplasty had been performed on July 28, I899, for hour-glass contraction, the constriction being at a point one-third of the stomach length from the pylorus. She had made a good recovery and had increased in weight, so that at the end of the year she was quite well. Soon afterwards she began to complain of pain on movement and on straightening herself, as well as of discomfort and pain after meals, with flatulency, though she had no vomiting. When she rested in bed she completely lost her symptoms, and could take food well, though directly she resumed the erect posture the pain recurred. There was tenderness and an indefinite swelling to the left of the middle line, with a well-marked stomach splash on succussion. A diagnosis was made of perigastritis, with adhesions to the anterior abdominal wall. After general treatment had been carried out for some time without any benefit, an operation was decided on.

Operation, January 24, 1901.- - On opening the abdomen, an old scar with a firm adhesion was found between the anterior stomach wall and the parietal peritoneum, just above the umbilicus to the left. This firm adhesion produced little discomfort when the patient was recumbent ; but when the body was erect, it re-created the old hour-glass deformity, with a difficulty in emptying the proximal pouch, which was markedly dilated. This adhesion was separated, and on invaginating the stomach wall so as to pass the fingers through the part that had been formerly stenosed, the channel was found to have a wide calibre, so that the gastroplasty had been quite effective. It was found, however, that there was narrowing of the pylorus, though no absolute stenosis. A posterior gastro-enterostomy was therefore performed. The patient made an excellent recovery, and left the surgical home at the end of three weeks. She was then $5 \frac{1}{2}$ pounds heavier than at the time of operation, and a report was received from Dr. Hindle on May 14, I903, to say that Miss D. was quite well in every respect.

CASE I7.-Miss C., aged twenty-nine, seen with Dr. Bryson, York. She had been ill with epigastric pain for ten years. The pain had been constant, with exacerbations, and had been increased after food, though this was not a marked symptom. She had 
never vomited blood, but had passed blood freely from the bowel on several occasions. She said that all her trouble dated from typhoid fever ten years before. She was extremely weak and very thin, only weighing 6 stones II pounds with her clothes on. On examination, just above the umbilicus there was a wellmarked tender swelling that seemed bound down by adhesions. It was feared that the tumour might prove to be malignant.

Operation, October 24, I901.-The abdomen was opened by an incision I inch to the right of the middle line above the umbilicus, when a large tumour was discovered in the centre of the stomach, there being a dilated portion of the stomach on each side of this. The communication between the two cavities was a small channel surrounded by an indurated chronic ulcer. The induration extended along the lesser curvature of the stomach, where enlarged glands were felt. There were many adhesions between the stomach, liver, gall-bladder, and colon. Posterior gastro-enterostomy was performed. She made a good recovery, and returned home. We had a letter on the last day of December to say that she was progressing favourably. On December I9, I903, we received a report to say that she was quite well, and that she had not had a bad symptom since her operation.

CASE 18.-Miss R., seen with Dr. Mercer, Ripponden, on account of wasting associated with pain after food and vomiting, the patient having had stomach symptoms for over two years. She was very thin, only weighing 5 stones Io pounds, and was feeble and anæmic. The stomach was markedly dilated, and there was a hard swelling in the centre of it. She had tried medical treatment very thoroughly for the greater part of the time since the first attack, but had never been long free from pain.

Operation, March 7, 1902.- The abdomen was opened, and an hour-glass stomach was found, with well-marked, hard cicatricial tissue about the centre. As the narrowing of the channel was not extreme and the pylorus was patent, a posterior gastro-enterostomy was done by connecting the first cavity of the stomach to the jejunum. She made a good recovery, and when she left the surgical home had gained weight. After her return home there was some recurrence of pain, but she was able to return to her duties; and when we last heard of her, Dr. Mercer said that he had not seen much of her for the last two months.' A subsequent report received in December, I903, was to the effect that the patient had completely relapsed. From the history, the recurrence of trouble is probably due to adhesions, but the patient is unwilling to have anything more done.

CASE I9.-Mrs. C., aged forty-one, seen with Dr. T. Robinson, Kensington. 'The patient gave a seven years' history of stomach 
trouble in the shape of painful indigestion, though after the first two years the symptoms had clearly pointed to gastric ulcer. She had been relieved by medical treatment up to eleven months ago, when there was a recurrence of pain after food, with loss of flesl. At Christmas she had a free hæmatemesis, and a second attack occurred a week later. On June 7 , 1902, a swelling of the epigastrium could be felt about the size of a walnut, it being movable and tender. On May 18 a third attack of hæmorrhage occurred, and from that time onwards she had been steadily losing flesh, so that her weight had diminished from 9 stones 4 pounds to 7 stones. When she was seen there was a well-marked, tender tumour in the epigastrium. The patient was complaining of great weakness, and she was profoundly anæmic. There was no ascites, and the legs were not œdematous.

Operation, November 21, I903.-The abdomen was explored through an incision to the right of the middle line above the epigastrium, when the stomach was found to be adherent at the front over an area about the size of a five-shilling piece. On separating this, a small aperture was discovered, where perforation had occurred. The centre of the stomach was occupied by a hard cicatricial mass, dividing the organ into two cavities. The indexfinger could barely be passed with difficulty from one cavity to the other. The glands along the lesser curvature were enlarged but discrete, and other glands were found at the back of the stomach and in the great omentum. The proximal cavity was connected to the jejunum by posterior gastro-enterostomy, a bone bobbin being employed. On account of the great induration of the tissue surrounding the point of perforation, it was impracticable to apply sutures. An omental graft was therefore detached from the great omentum and stitched into position, so as to close in the perforation. Recovery was uninterrupted, and the wound healed by first intention. She was able to take food from the first day, and soon began to put on flesh. When she left the nursing home on the twenty-fourth day she was looking well, and expressed herself as feeling better than for two years.

CASE 20.--August, I 899, female, aged thirty-nine. Ten years ago the patient had an illness characterized by profound anæmia. Seven months ago there were clear symptoms of ulcer of the stomach, but neither then nor at any time any acute illness suggestive of perforation. Now vomited after all ordinary food, and more often than not even after small quantities of fluid food. Pain after food was exceedingly severe. On examination of abdomen, a dilated stomach could be felt. At one point, a little to the left of the middle line and slightly below the ensiform cartilage, was an area 2 inches in diameter, which was markedly 
tender, and offered increased resistance on palpation. This area was found at the operation to correspond precisely with the area of stomach adhesion. The patient had lost weight and deteriorated seriously in general health during the last few months. At the operation the condition found was hour-glass stomach, the narrow constriction being near the middle of the stomach, and adherent to the anterior abdominal wall over an area equal to a crown piece. On each side of this the stomach dilated, and seemed to be anchored by the adhesion. On separating the stomach from the abdominal wall, an opening into the viscus was exposed, and the stomach contents escaped. This opening was enlarged transversely, and the wound and fistula were stitched up vertically. An omental graft was brought to cover in the sutured area, in order to guard against future anchoring. The patient recovered. For a month after the operation there were pain at times and loss of appetite. Since then she has been free from pain and in excellent health. Appetite and digestion are good. Patient was seen with Mr. Bailey, Horsforth. In February, Ig02, her condition was still perfectly satisfactory.

CASE 2I.-April, I900, female, aged twenty-seven. There was no history of gastric ulcer. The patient had complained of irregular attacks of pain in the epigastric region for eleven months, always associated with the taking of food, and coming on about one hour after a meal. Vomiting was occasional and irregular, in rather large quantities; there was no blood. On examination, the stomach was distended and splashy; contractions were seen when distended. Medical treatment had proved unavailing. At the operation the condition found was hour-glass stomach. The constriction was about 4 inches from the pylorus; the passage between the two sacs equalled a No. 12 catheter in diameter. There were marked induration and puckering, but no adhesion to the abdominal wall or elsewhere. The strictured neck was divided by an incision about $2 \frac{1}{2}$ inches long in the transverse direction, and stitched up vertically, the mucous membrane with a continuous catgut suture, and the serous with a continuous silk suture. Two additional interrupted sutures were applied at each end of the wound. The patient recovered. There was vomiting (bloodstained) for five days after the operation. Since then she has been perfectly well. The patient was seen with Dr. Waugh, Skipton. In March, I902, she was reported to be in complete health.

CASE 22.- January 24, IgoI, male, aged forty-five. Patient had been ailing for three years. Pain in the epigastrium radiating to the left chest. WVas worse after food, coming on 'within half an hour,' and lasting for from two to four hours, unless relieved by vomiting. Frequently vomited about an hour after food ; never 
any blood. Lately the pain had been almost constant, aggravated by food, and eased by vomiting. Had lost 2 stones I 2 pounds. Dilated stomach. On distending with $\mathrm{CO}_{2}$, a notch was noticed at the upper border of the stomach, and a tentative diagnosis of hour-glass stomach was made. Free $\mathrm{HCl}$. A trace of lactic acid and a few rod-shaped bacilli. At the operation the condition found was hour-glass stomach. The scar of the ulcer was equal in size to a florin, was situated close to the lesser curvature, and nearer the cardiac orifice than the pyloric. There were much puckering and induration of the surface. There were no adhesions. The index-finger entered but could not pass the stricture. An incision $3 \frac{1}{2}$ inches long was made transversely, and stitched longitudinally in two layers. A slender adhesion of gall-bladder to the pylorus was divided. Gastroplasty, gastrolysis. The patient recovered. Weight before operation was 7 stones 6 pounds; has gained I stone $8 \frac{1}{2}$ pounds. The patient was seen with Dr. Trevor Pritchard, Retford. In March, I902, he was reported to be quite well.

CASE 23 -March 6, Igor, female, aged twenty-eight. Symptoms of gastric ulcer for more than five years. Patient was under treatment at beginning of illness at the infirmary. Had been gradually getting worse. Vomited now after every meal; pain and vomiting came on from about half an hour to one hour after food. Vomit was very 'sour.' On examination, hour-glass stomach. On distending with $\mathrm{CO}_{2}$, the cardiac half increased considerably, forming a very large lymphatic area. The pyloric half distended but little; the division was clearly seen. On auscultation, a forcing, gurgling sound was distinctly heard. At the operation the condition found was hour-glass stomach. The cardiac side of constriction was much dilated, and larger than a normal stomach. Rather beyond the middle of the organ was a constriction that would just admit the forefinger ; round constriction were much induration and many adhesions ; especially noticeable was one thick, cord-like one coming from the liver. This was divided between the ligatures. The liver was slightly torn on separating widespread adhesions along the lesser curvature. Below it on each side the stomach sagged, and between the cavities an anastomosis was made, which readily admitted three fingers. Gastro-gastrostomy, gastrolysis. The patient recovered. March, I902, perfect result. Eats well and heartily, and has no discomfort. Patient seen with Dr. Waugh, Skipton.

CASE 24.-March 9, I90I, male, aged fifty-five.-Stomach troubles for sixteen years. At first pain after food and occasional vomiting. Five years ago an acute attack of hæmatemesis, melæna, and general swelling of the body, which it was feared 
might prove fatal (? perforation). No ease in the stomach since then; constant vomiting, pain after every meal, and great wasting. Now looked thin, pinched, and shrivelled. For three weeks had had no food, only sips of water and nutrient enemata. On examination, dilated stomach. Free $\mathrm{HCl}$. At the operation the condition found was hour-glass stomach. At first it was thought to be a dilated stomach. On tracing the stomach toward the pylorus, an extremely narrowed isthmus, barely as thick as the little finger, was come to, which was supposed to be narrowed pylorus; but on tracing beyond it, stomach was still found. On invaginating a finger from each side of constriction, no opening could be felt. Many adhesions were separated until all was clear and free. Then incision into stomach or cardiac side of the isthmus, and a probe director was passed through constriction, which it fitted snugly, the opening being no bigger than a No. 4 or No. 5 catheter. Gastroplasty was performed. On the pyloric side of the stricture was a column of mucous membrane, forming a bridle stricture. This was ligatured, divided at both ends, and removed. The patient recovered. This patient was in the infirmary under Mr. Littlewood, and was operated upon by one of us in his absence.

CASE 25.-April, igor, male, aged forty-six. Symptoms for twelve months; pain, heaviness, discomfort after meals ; loss of weight and general deterioration in health. At Christmas had an attack of acute pain and hæmatemesis, and was very ill for several days; since then has never taken any food, solid or liquid, without pain. Has noticed on several occasions that the stools were 'black as ink.' Vomits now every day. At the operation the condition found was: two ulcers near the pylorus, one on gastric, one on duodenal side. A mass equal in size to a walnut found at the pylorus. Pylorus very narrow; adherent to liver and gallbladder by dense bands. At the middle of a hugely dilated stomach a constriction that would admit four fingers. Posterior gastro-enterostomy to pyloric pouch. At the post-mortem two ulcers (duodenal and gastric) were found, the former very adherent to the gall-bladder; the latter had perforated into a mass of adhesions, its base being partly formed by the pancreas. The patient was sent by Dr. Crawford Watson, Harrogate. All went well for three days; then a large prolapse of rectum (from which he had previously suffered) came down during the night, and was not discovered for four hours. When we saw him the prolapse was livid and œedematous, and could not be reduced until ether had been administered. From this time the patient became gradually worse ; the temperature ran up to $104^{\circ}$ and $105^{\circ}$, and he died four days later. The following is an abstract from 
the post-mortem record, written by Dr. Maxwell Telling: 'The stitches are quite sound, the opening good, and surgical technique perfect. No peritonitis. The hæmorrhoidal and inferior mesenteric veins are full of disintegrating clot. Septicæmia.'

CASE 26.-May 24, I901, male, aged forty-eight. For many years has vomited after food. Pain comes on about one and a half hours after food, and is eased by vomiting. In November, I 899 , and May, 1900, had severe hæmatemesis. Three years ago was in the infirmary, and a diagnosis of 'cancer' was made. On examination, an irregular, dilated stomach. On inflation, a distinct notch seen in the lower border; just above this a very loud, forcing, gushing sound heard. Hour-glass stomach diagnosed. Signs I, 3, 5, and 6 present. At the operation the condition found was hour-glass stomach. The cardiac pouch much dilated, pyloric small and not dilated. The constriction was about 3 inches from the pylorus. At the upper border of the isthmus a hard mass equal to a walnut in size, with puckering of stomach and lesser omentum. Posterior gastro-enterostomy. Recovery. The patient was sent by Dr. Isaac Taylor. His weight before operation was 8 stones 2 pounds; his weight in June, Igo2, was ro stones.

Case 27.-May, rgor, female, aged fifty. The patient had been ailing for many years, the symptoms dating from an illness in which 'gastric ulcer' was diagnosed. A diagnosis of hour-glass stomach was made by Dr. McGregor Young. Signs I, 3, and 6 present. At the operation, the condition found was hour-glass stomach, the isthmus being about $2 \frac{1}{2}$ inches from the pylorus. The stomach and all the intestines were thin and translucent, and seemed almost devoid of muscle. On exposing the posterior wall of the cardiac pouch, a second ulcer was seen. Posterior gastroenterostomy was performed. After the operation, regurgitant vomiting of such severity and persistence that a second operation was undertaken, and the proximal and distal segments of the jejunum loop were united. Jejuno-jejunostomy. The patient, who was seen with Dr. McGregor Young, recovered. The result of the operation has been most satisfactory; the patient is much better than she has been for many years.

CASE 28. - June, I9or, male, aged thirty-nine. Stomach troubles for nine or ten years. Then began quite suddenly to have great pain after food, and to vomit two or three times a day ; no hæmatemesis. The pain usually came on about one and a half hours after food. Seen four years ago by a consulting physician, who diagnosed dilated stomach, and advised lavage, which has been done regularly since. Relief at first very great, but lately a serious relapse, and no relief from washing. Hour-glass 
stomach diagnosed. Signs I, 2, 3, and 6 present. At the operation the condition found was hour-glass stomach. The constriction just admitted the end of the forefinger, was situated about 3 inches from the pylorus, at a point where loud gushing sounds were heard before operation. The cardiac complement was enormous, the pyloric a little dilated. Posterior gastro-enterostomy. Pylorodiosis. Death. The patient was sent by Dr. Ellis, of Halifax. After the operation the patient had suppression of urine. In four days secreted only $\mathrm{I}_{2} \frac{1}{2}$ ounces, tinged with blood. Pulse became very rapid and of poor volume. Five pints of saline solution infused. Urine was secreted almost at once. Gradual improvement then for nine days; then suppression for forty-eight hours and death. Both kidneys diseased, the left small, granular and cirrhotic, the right granular. Operation area normal. The urine before operation had been frequently examined, and nothing abnormal discovered.

CASE 29.-June, Igor, female, aged twenty-five. For three years had suffered from vomiting, pain after food, inability to take ordinary diet. Has never vomited blood, but has seen vomit which 'resembled tea.' Vomiting now almost continuous. Has lost 3 stones in weight. On examination, a hard mass above and to the left of the umbilicus. On distending the stomach with $\mathrm{CO}_{2}$, a distinct swelling on each side of tumour. Hour-glass stomach clearly seen. At the operation the condition found was hour-glass stomach. A large mass, about 4 inches in diameter, found at the junction of the two pouches, densely hard, immovable, and adherent firmly to pancreas. Several enlarged glands. Supposed to be malignant. Stricture between the two pouches dilated freely. Recovery. The patient was sent by Dr. Crawford Watson, Harrogate. Weight before operation, 5 stones. Vomiting ceased at once, and food was freely, almost greedily, taken. The tumour quickly disappeared. Looks ruddy and well. Weight now 7 stones 7 pounds.

CASE 30.-April, I901, male, aged forty. About six months ago was suddenly seized with acute, intolerable pain in upper part of abdomen. Constant troubles since; pain, weight, 'dragging sensations.' Has lost flesh quickly. On examination, a smooth globular swelling between mid-line above umbilicus and left costal margin: soft and fluctuating. At the operation the condition found stomach of hour-glass shape ; the constriction was adherent to the anterior abdominal wall. On separating the adhesion, the stomach was opened and gastroplasty was performed. The tumour pushed the stomach forward. The great omentum below stomach was torn through, and the finger passed into the lesser sac. A smooth globular cyst found projecting into the cavity, 

PLATE XXVII.

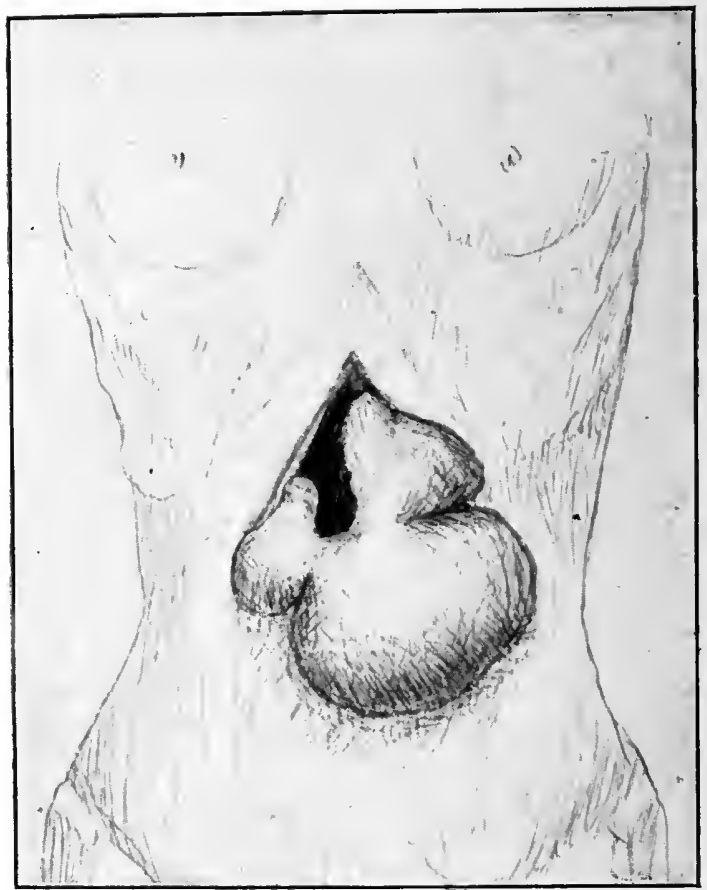

Fig. ioi.-Semidiagrammatic Representati d of the Trifid Stomach. To face $p$. 373.] 
and springing from the pancreas. Recovery. The patient was sent by Dr. J. Exley, Wortley. A pancreatic fistula remained for twelve months. Health and weight fully regained.

CASE 31.-July, r9or, female, aged twenty-eight. Six years' history of increasing indigestion, dating from an illness described by the doctor as ' ulcerated stomach.' Pain comes on one hour after food, worse when lying on the right side. Vomits after nearly every meal. Is getting steadily thinner. Signs I, 2, 3, 4, 5,6 , and 7 all present. At the operation the condition found was hour-glass stomach and very tight pyloric obstruction. We performed gastroplasty and posterior gastro-enterostomy into the distal pouch. Death. The patient was sent by Dr. BaxterTyrie, of Keighley. Death from pneumonia on the fifth day. The anæsthetic was badly taken, lividity and rigidity of abdominal muscles being present throughout.

CASE 32.-April 22, I902, female, aged forty-two. Chronic indigestion. The symptoms came on gradually; pain after food, vomiting, and constipation. Eighteen months ago had hæmatemesis, and since then the symptoms have been greatly increased. Signs I, 2, 3, 5, and 6 present, and a diagnosis of hour-glass stomach made. At the operation the condition found was hourglass stomach - a perfect example. The constriction in the body admitted the little finger snugly. In the narrowed isthmus, on posterior surface, a densely hard, indurated area, at least $\frac{3}{4}$ inch in thickness; the lesser and greater omenta both puckered up. Gastroplasty. A scar of the ulcer seen on mucous surface of posterior part. A very tight stricture found at the pylorus, the pyloric pouch being dilated also. Posterior gastro-enterostomy from pyloric pouch. Recovery. The patient was sent by Dr. H. H. Greenwood. A very rapid pulse for four days after operation. Has since done very well. Has gained about I I pounds, and takes food heartily. No vomiting.

CASE 33.- November 29, I902, female, aged twenty-seven. Nine years' history. Pain after food and vomiting were the symptoms first noticed. Soon all solid food caused pain, and was rejected. The food became 'sour,' and the gas belched up smelt putrid. On several occasions blood has been vomited. The signs and symptoms of hour-glass stomach were elicited, and a confident diagnosis made. The two pouches of an hour-glass could be distinctly seen on inflation. Signs I, 2, 3, 5, and 6 were present, and an hour-glass stomach diagnosed. At the operation the condition found was hour-glass stomach. The stomach was traced up to the cardiac orifice, and another stricture and another pouch were discovered. There were thus two annular constrictions in the stomach and three pouches. The 
first pouch was the largest, the pyloric the smallest, being only the size of a lemon. Gastro-gastrostomy and gastro-enterostomy were performed. The stomach was scarred almost universally. The patient, who was sent by Dr. Knowles, Newstead, Halifax, made a most excellent recovery. She has gained 2 stones in weight, and from a chronic invalid has become an active, healthy, and happy woman. The diagram opposite p. 373 represents the condition (Fig. IOI):

Case 34.-January 20, 1903, female, aged thirty-four. Nine and a half years ago vomited a 'great deal' of blood. Dr. Mackenzie then diagnosed ulceration of the stomach. Since then has always been ailing, especially after a moderate meal. Five years ago was very ill; had acute abdominal pain, severe vomiting, and hæmatemesis. Was in Manchester Royal Infirmary with 'ulcer of the stomach.' One and a half years ago hæmatemesis. For several years has taken no solid food, and has never had an ordinary meal. Has lived on milk, custards, porridge. On examination, an hour-glass stomach was diagnosed. Wölfler's two signs; paradoxical dilatation; increase in subcostal tympany; gurgling, rippling sound at left end of stomach-all well marked. At the operation the condition found was an hour-giass stomach and an hour-glass duodenum. Two large stomach pouches united by a narrow isthmus at the lesser curvature. Tight constriction at the pylorus; the first portion of the duodenum was dilated to form a sac the size of a lemon; beyond this another constriction. The pyloric pouch of the stomach was more densely scarred than any stomach we have seen; its texture was almost wholly fibrous. On its posterior surface also many scars were seen. Gastrogastrostomy and posterior gastro-enterostomy to the pyloric pouch. Recovery. The patient was sent by Dr.' Mackenzie, Burnley. Within three weeks of the operation she ate solid food heartily, and had taken all the vegetables in season. In October, 1903, she was reported to be perfectly well, able to eat anything, and she had gained $\mathrm{I} \frac{1}{2}$ stones in weight.

CASE 35.- July I 4, I903, female, aged thirty-five. For the last six years has had abdominal pain, to the left side of the epigastrium chiefly, especially after food. Has vomited every week during this time, and six years ago, three years ago, and in February, I903, had hæmatemesis. Since February has had constant pain and vomiting, is unable to take any solid or semisolid food, and but little fluid. Is wasting rapidly, and looks thin, haggard, and very feeble. At the operation an hour-glass stomach was found. A deep pucker on the anterior wall and upper surface, pulling the lesser curvature down to the greater. From the centre of the dense indurated, stenosing ulcer a thick 
band and a web of adhesions passed up to the liver. The posterior surface of the stomach also very adherent, so that a posterior gastro-enterostomy was performed with very great difficulty. Recovery. The patient was seen with Mr. Jessop.

\section{Cancerous Hour-glass Stomach.}

Case 36.-Mrs. T., aged thirty-six, was sent to us by Dr. Chamberlain, of Rawdon, on September 2I, rgoo, and was admitted to the Leeds General Infirmary. There was a history of daily vomiting for four months, and as the vomiting always occurred within an hour of meals, the patient was reduced to the last degree of weakness. A tumour had recently appeared, and was readily palpable on the right of the umbilicus. As she was so weak, she was put on rectal feeding for a few days, but without improvement, and operation was decided on as a last resort.

Operation, September 28, 1900. - An annular cancer of the stomach was found 3 inches from the pylorus. It had considerably encroached on the lumen of the stomach, so that the stricture would barely admit the passage of the finger. The proximal cavity was very much dilated, but the cavity between the growth and the pylorus was not dilated. An anterior gastro-enterostomy was performed, as there were some extensive adhesions, and it was thought that time might be saved by the anterior method. Regurgitant vomiting followed and persisted until the third day, bringing her almost to the verge of death. Food and stimulants were then given in large quantities, and from that time there was no more trouble with vomiting; but on October 7 (the ninth day) she became collapsed, and died in the evening.

Case 37.-Mrs. J. was sent to us by Dr. Forster, of Dalton-inFurness, January 24, 1901, with a movable tumour in the epigastrium that had been noticed three weeks previously; there had been no vomiting, although indigestion, pain an hour after food, and loss of flesh and strength, had been noticed since August, I 899.

She weighed 7 stones, having weighed 8 stones or more previous to her illness. The tumour appeared to be the size of a tennis-ball, was free from tenderness, and could be made to move across the abdomen from one side of the umbilicus to the other.

On distending the stomach with $\mathrm{CO}_{2}$, it reached nearly to the pubes on the left side, but very little distension was noticed on the right, beyond the tumour. She was admitted to the infirmary, and on January $3 \mathrm{I}$, on exposing the stomach, a tumour was found involving the whole circumference a short distance from the pyloric end, so that between the pylorus and the tumour a small 
second stomach cavity existed, the dilatation being formed entirely by the cardiac complement. The tumour was completely excised, and the distal and proximal cavities united end to end over a bone bobbin. Glands were removed from the lesser and greater omentum. Recovery was uninterrupted, and the patient left the hospital before the month end, having regained the weight lost during the week after operation, with an additional $\frac{1}{2}$ pound. The tumour proved to be cancer. A report was received in 1903 to say that the patient was in good health and well nourished.

CASE 38.-Mr. B., aged sixty-two, was sent to us by Dr. James Gardner, of Burnley, suffering from a movable tumour, thought to be cancer of the stomach, which had been noticed for a month, but which had been preceded by stomach symptoms, chiefly pain after food and slight voniting, for quite a year. There had latterly been some slight coffee-ground vomit, but no large amount of blood had been vomited at any time. The tumour was not tender, and could be pushed up under the left and moved over to the right costal margin. A stomach splash was well marked on the left of the tumour, and on distending the stomach with $\mathrm{CO}_{2}$, the lump was pushed over to the right. The vomit was acid from the presence of lactic acid, but no free $\mathrm{HCl}$ could be found. Pylorectomy was advised, and the patient was admitted to the infirmary, being then in an extremely weak condition.

Operation, November I 5, I 900.-On opening the abdomen, a mass of growth was found in the centre of the stomach, forming a ring of cancer, and leaving a cavity on its proximal and distal sides, the cardiac cavity forming the dilated stomach, where the splash on succussion was felt. Dr. Seaton, who was giving the anæsthetic, advised that the patient was too feeble to bear a prolonged operation, and a colleague also confirmed the fact that the pulse could be barely felt at the wrist. A posterior gastro-enterostomy was therefore performed, a bone bobbin being employed. $\mathrm{He}$ soon rallied, and made a good recovery, gaining flesh and weight, and expressing himself as very well. For further history, see the following case.

CASE 39.-Mr. B. had had gastro-enterostomy performed on November 15, 1900. He seemed to be so well and had gained strength so rapidly that it was felt desirable to give him the chance of cure by completely removing the growth; and after fully explaining to him his condition, he gave consent.

December 20, 1900. - The radical operation of removing the growth by partial gastrectomy was performed, about half of the stomach being removed, in order to leave a wide margin of healthy tissue between the growth and the wound. Union was effected by suturing the cardiac and pyloric incision together over a bone 
bobbin, catgut being used for the mucous and celluloid thread for the serous sutures, the whole operation being completed within the hour. No glands were felt beyond the resected area. Recovery was uninterrupted, and he returned home within the month, eating and enjoying food, and having gained considerably in weight. He lived for sixteen months, and for nearly the whole of the time took his food well, and until January retained his normal weight.

CASE 40.-March 3, I90I, male, aged fifty-four. Vomiting of fluid if taken in quantities exceeding an ounce. At the operation the condition found was hour-glass stomach. Narrowing close to cardia due to malignant disease, which had invaded the stomach everywhere. Jejunostomy. The patient was seen with Dr. Sykes, Cleckheaton. Recovered from the operation, but only survived until April 9.

CASE 4I.-July, I9oI, female, aged thirty-eight. At the age of seventeen had a gastric ulcer; vomiting and hæmatemesis for several weeks. Since then has always had gastric uneasiness. Ten years ago 'an ulcer burst' after a long walk. She suddenly experienced acute pain in epigastrium, and fainted; during the subsequent night she vomited a 'chamber utensilful' of blood, and later coffee-grounds material. Since this attack has never enjoyed a hearty meal. For last few months has lost flesh rapidly; for the last three weeks she has been in bed, and has not been able to take anything but milk-and-water, and has returned most of that. Gastric tetany in limbs. Free $\mathrm{HCl}$ present. At the operation the condition found was hour-glass stomach, nearer the cardiac than the pyloric end. The stomach was adherent over an area about $2 \frac{1}{2}$ inches in diameter to the anterior abdominal wall and liver. On detaching adhesions stomach opened. The opening was about $1 \frac{1}{4}$ inches in diameter, the edges over $\frac{1}{2}$ an inch thick, and the mucous membrane greatly thickened and pouting. At the point of adhesion the stomach was markedly narrowed. The ulcer and a surrounding mass of stomach were excised, and gastroplasty performed. Pylorus would only admit the passage of a director, and pyloroplasty was therefore performed. Death. The patient was sent by Dr. Cheetham, Guiseley. She seemed to be going on well until the fourth day, when the pulse rapidly failed. The lower margin of the ulcer was greatly thickened by heaped-up granulation tissue, which, on microscopic examination, proved to be carcinomatous.

Statistics of our Operations for Hour-Glass Stomach.-Simple cases, 35 operations with 30 recoveries; malignant, 6 operations with 4 recoveries. 


\section{CHAPTER XX}

\section{THE COMPLICATIONS OF GASTRIC ULCER (continued)}

\section{DILATATION OF THE STOMACH.}

THOUgh gastrectasis is by some authors only treated as a symptom, it is one of such importance that in many cases it may truly be said to be a disease in itself; this is certainly the case in that serious and often fatal form known as 'acute dilatation.'

A medical friend, whose practice lies among mill-operatives, told us that a moderate degree of dilatation was almost a constant symptom among them, owing to their living so much on tea and farinaceous food; and it is generally known that well-marked dilatation is usually seen in gross feeders, or in those living almost solely on bulky farinaceous food, such as potatoes. The capacity of the stomach cannot therefore be taken as a guide in estimating the dilatation that should come under the notice of the surgeon, and, in fact, it is only in the well-marked cases that a surgical opinion is sought. Unfortunately, the aid of surgery is too frequently put off until extreme emaciation has ensued and the patient is almost moribund.

The causes of dilatation of the stomach are:

I. Mechanical obstruction at the pylorus, or near it, or in the duodenum-(a) from cicatrization of a simple ulcer at or near the pylorus; $(b)$ from cancer of the pylorus; $(c)$ from perigastritis leading to stricture or to kink of the pylorus; (d) from hypertrophy of the pylorus and spasm as a sequel of ulceration, which may continue long after the original ulcer has disappeared; (e) from fibroid thickening of the pylorus ; 
$(f)$ from polypus; $(g)$ from congenital stenosis; $(h)$ from tumour outside the pylorus; (i) from pressure on the duodenum by chronic pancreatitis or growth, when the head of the pancreas is embracing the duodenum; $(j)$ from pressure of mesenteric vessels as they cross the duodenum; $(k)$ from gallstones ulcerating through the gall-bladder into the pylorus; (l) from kinking of the pylorus, due to the dragging of a movable right kidney; $(m)$ from kink due to gastroptosis (q.v.).

2. Dilatation from atony from various causes not mechanical, but persisting after the original cause has ceased to act.

3. Acute dilatation from causes not yet well understood.

\section{Diagnosis.}

Although in cases brought before the notice of the surgeon the diagnosis is usually already made, he will need to verify its correctness for himself, and if possible to arrive at the probable cause. Where there is great dilatation, the stomach soon becomes also displaced downwards. Dilatation is always accompanied by diminished passage of the stomach contents onward, and is usually associated with vomiting, the vomiting differing from that which occurs in any other condition. For instance, it does not occur after meals, nor even every day, unless the stenosis is extreme, but usually every second or third day, and more frequently at night than in the day; and when the vomiting occurs, it is, as a rule, in large, or even in enormous, quantity, the vomit consisting of fermented material with mucus, and sarcinæ and yeast cells are usually present. When the dilatation is dependent on ulcer, the vomiting may be very acid, owing to the presence of excess of free hydrochloric acid. If, however, the dilatation is dependent on cancer, the acidity is due to lactic acid, and hydrochloric acid will be in small quantity or absent. Though patients with dilated stomach do not, as a rule, vomit after food, they complain of a sense of heaviness and discomfort, and have flatulent eructations, these symptoms becoming more and more intense as the stomach contents accunulate, until relief is obtained by vomiting. As a rule, the first sign noticed in stomach dilatation is the splash on 
succussion of the abdomen, this being the more marked the greater the capacity. If the patient has previously vomited, the stomach splash may be absent, but it is usually obtained by allowing him to drink freely of warm water. Constipation and diminished secretion of urine are nearly always present. Where the obstruction is mechanical, visible peristalsis from left to right can frequently not only be felt, but observed through the abdominal wall; and if visible peristalsis of the stomach be present, it is always indicative of considerable pyloric obstruction, which will probably only yield to surgical treatment. Loss of flesh, diminution of body temperature, coldness of the hands and feet, and general feebleness, are characteristic of the later stages. Tetany is sometimes present, and if well marked may even lead to a fatal termination; but before this stage is reached, cramps in the muscles of the forearm and drawing inwards of the thumb-otherwise a tetanoid condition-are usually noticed. The amount of dilatation may be ascertained by distending the stomach with air introduced through a tube and pumped into it by means of a Higginson's syringe. This can be done with very little discomfort and without danger. A simpler method which we frequently employ is to give a dose of tartaric acid in water, immediately after one of carbonate of soda, when, if the patient is recumbent, inspection, palpation and percussion show the increase in size of the organ.

As to the cause of dilatation, the history materially helps. If it be simple, the history is usually a question of years, but if malignant, of months only ; but in the latter case a tumour is much more frequently present than in the former, and an examination of the stomach contents after a test-meal is also of service. In dilatation from ulcer, there will be usually a long history of ulceration, possibly with vomiting of blood, but it is quite possible for an ulcer of the pylorus to pursue a quiet course and for a long history to be absent. That peculiar form of dilatation known as acute may apparently occur as a result of spasm without organic pyloric obstruction. It is highly probable that nearly every case of extreme dilatation of the stomach has some mechanical explanation, and can only be efficiently treated by surgical means. We know that 
this view is one which will raise some criticism, but we think time will prove that the statement is correct. We do not, of course, refer to those cases of moderate dilatation which are so commonly seen, and which are associated with atonic conditions and with chronic catarrh, but to the cases of dilatation producing the characteristic symptoms just mentioned.

\section{Treatment.}

The treatment in any form of gastrectasis will at first be medical and general, but if in a short time weight and strength be not definitely gained and maintained, with relief to pain and discomfort, then time should not be wasted by persisting with lavage and medical treatment for months, until the vital powers have become sapped and the patient reduced to the last extremity of exhaustion, before surgical treatment is advised.

Surgical Treatment. - If the contents are offensive, the stomach should be washed out for a day or two before operation; and during twelve or twenty-four hours rectal injections may with advantage supplement light stomach feeding. The last lavage should be a few hours before operation. In cases where there is profound debility it may be undesirable to delay operation even for a day, and in no case do we now advise a long preparation of the stomach. It is desirable to give the patient sterilized food for twentyfour hours before operation, and to have the mouth and teeth well washed with some antiseptic solution for a day or two before the stomach is opened. A subcutaneous injection of to minims of liquor strychninæ (B.P.) may with advantage be given just before the operation, so as to forestall shock, which may also be further lessened by giving a pint of saline solution by the rectum, and by operating on a heated operating-table. After exposing the stomach by a vertical incision I inch to the right of the mid-line above the umbilicus, a diagnosis of the cause will be speedily made by inspection and palpation of the stomach and pylorus. If there be stricture of the stomach itself, either from adhesions caused by perigastritis or from ulcer, leading to hour-glass 
contraction, it must be treated in one of the ways previously described. If the dilatation be dependent on adhesions of the pylorus, and these can be detached, leaving the pylorus free, they should be dealt with as described (see Gastrolysis). If there is a stricture of the pylorus, it must be treated in one of the several ways to be mentioned immediatelypylorodiosis, pyloroplasty, gastro-enterostomy, or pylorectomy-according to its nature.

Pylorodiosis. - If the narrowing of the pylorus be due to spasm or hypertrophy of the circular muscular fibres, pylorodiosis, or dilatation of the pylorus, may be indicated. This is known as Loreta's operation, since it was he who first employed the method as a definite operative procedure in I 884 and brought it before the profession. But in I88I Richter had dilated the pylorus by jougies, and Hahn had also done the same. Hahn also advocated rapid dilatation by invagination through the stomach wall. Loreta, however, practised and strongly advocated dilatation of the pylorus through an incision on the stomach side of the pylorus. Through the opening in the stomach he introduced first the right and then the left index-fingers into the pyloric orifice, and stretched it forcibly until the diameter was approximately 8 centimetres, after which the stomach wound was closed by sutures. Bull also advocated the use of bougies, and Barton employed a uterine dilator instead of the fingers.

Jaboulay, in a case of cancer, dilated the pylorus by invaginating the stomach wall by means of a finger, great relief to the patient resulting. This all sounds very simple, and when we are told of Loreta having had twenty-nine cases all successful, it would seem that the operation does all that can be desired; - but there is another side to the picture, when we hear that, in the hands of many able surgeons, this apparently simple operation has such a high rate of mortality as 40 per cent., and that Loreta himself confessed to having had several deaths from hæmorrhage and peritonitis. Moreover, recurrence of the stricture seems to have occurred in so large a number of cases that the operation is scarcely likely to hold its own in competition with the more exact and safer operation of gastro-enterostomy. It probably has some 
use in that very limited class of cases where the stenosis is simple and dependent on spasm of the sphincter, which may either be caused by ulceration or occur independently of such a condition. A case has been related by Paul (British Medical Journal, 1896) :

The patient was a man, aged twenty-one years. $\mathrm{He}$ was admitted into the Royal Infirmary, Liverpool, under the care of Dr. Caton, in November, $\mathrm{J} 895$, for pain in the epigastric region and a lump. The lump proved to be a largely dilated stomach, in which splashing sounds could be easily produced. He was treated by careful dieting and washing out of the stomach; but not having materially improved in January, 1896 , the case was transferred to me. The patient was anæmic, and decidedly of neurotic type. On January 6 the abdomen was found to be dilated, the pylorus was thick but not fibrous, and upon invaginating the anterior wall upon the forefinger into the pyloric orifice abnormal resistance was met with. This was gradually overcome by continued steady pressure, and ultimately the opening was overdilated without splitting by passing three fingers through it. No cut was made in the organ. The patient healed well, but was subsequently attacked with acute mania, and had to be sent to the county asylum, where he remained for five months before he got his discharge. He had no recurrence of the gastric symptoms.

We have had several cases similar to the above in which dilatation was successfully done by invagination. This is, however, the operation suggested by Hahn, and not by Loreta. Under the heading Gastroplication will be found reports of two cases in which Hahn's method of dilating the pylorus was adopted successfully at the same time that the dilated stomach was diminished in volume by gastroplication. In one of these cases, however, a gastro-enterostomy had to be performed some time later. In the chapter on Congenital Stenosis is also reported a case of Hahn's operation which was followed by relapse, and in that case a pucke:ing of the pylorus seen at the second operation showed that the stretching had been followed by ulceration and stenosis (vide pp. 52 and 54 ).

The late Greig Smith held a favourable opinion of Loreta's operation, but many surgeons have told us that they have 
given up pylorodiosis, and our own feeling decidedly accords with that view.

Statistics of Loreta's Operation.-In the Hunterian Lectures we collected a series of 78 operations, of which 47 recovered, this giving a mortality of $39^{\circ} 7$ per cent.

Pyloroplasty.-The principle of the operation consists in obtaining an increase of the calibre in a stricture, by dividing the narrow passage longitudinally and closing the wound transversely.

The operation was performed first by Heineke in March, I886, and his patient recovered. Mikulicz performed his first pyloroplasty in $\mathbf{1 8 8 7}$, but the patient died; in his second operation in the same year the patient recovered. Of the first seventeen operations eleven recovered.

If the pylorus be free from adhesions the operation is of the simplest, as the pylorus can be brought outside the abdomen and surrounded by gauze pads or sponges, so that this heavy mortality of $35^{\circ} 3$ per cent. must have been due either to an improper selection of cases or to defective technique, as later results show a very different rate; for of twenty-five pyloroplasties that we have performed since 1896 for simple stenosis, all have recovered. It is desirable to adopt all the usual precautions against shock, and the stomach should, if practicable, be washed out some little time before operation, so as to avoid the danger of soiling the peritoneum. The incision through the pylorus must be longitudinal, commencing on the stomach side of the sphincter, and being prolonged through it either by scissors or by the knife over a director. Blunt hooks or catch forceps placed in the wound convert the horizontal incision into a transverse one, and it is then closed by a double line of sutures. For details of the operation and its various modifications, see p. 263 .

If on opening the stomach or pylorus a polypus be found as a cause of the obstruction, it must be removed, and, if necessary, the operation can be completed by a pyloroplasty.

Gastro-enterostomy is the operation which we strongly advocate in dilatation of the stomach which fails to yield to general treatment. 
It is much more certain than pyloroplasty or pylorodiosis, and is not attended with greater risk. The operation is fully described on p. 227, where are also mentioned the indications and contra-indications, as well as the complications which at times follow the operation.

Dilatation dependent on Pressure outside Pylorus.-If the dilatation be dependent on an obstruction outside the stomach -as, for instance, a tumour of the pancreas, liver, or gallbladder-relief may be given by removing the tumour, or, if that be impracticable, by gastro-enterostomy. The instance reported by Dr. Ewart and Mr. Jaffrey (Lancet, October 28, I899, p. II55) is a case in point, where vomiting, incapable of relief by medical means, was dependent on an aneurism flattening out the pyloric end of the stomach and causing obstruction. The following is an account of the operation, with remarks by Mr. Jaffrey :

Dr. Ewart asked me to see this case in consultation with him on August 26. There was a swelling midway between the umbilicus and the ensiform appendix, which appeared to me to pulsate more freely than one would expect from a growth of the stomach lying over the aorta. However, taking into consideration the symptoms and the swelling, we agreed that an exploratory laparotomy should be done. This I did on the following day. I made an incision from 4 to 5 inches long above the umbilicus. The stomach presented in the wound, and on examination it was found to be quite healthy, though dilated. On pulling the stomach out of the wound, there appeared to be between from $\frac{1}{2}$ pint to I pint of fluid collected in the most dependent part of the fundus. The colon appeared to be normal, and the walls of the stomach did not appear to be materially thinned. I attempted to press some of the fluid into the duodenum, but did not succeed in getting much to pass, and on further examination the swelling which we had felt through the abdominal walls proved to be a large fusiform aneurism of the abdominal aorta. It commenced about $\frac{1}{2}$ inch below the aortic opening of the diaphragm, and extended to the level of the umbilicus. The pyloric end of the stomach was flattened over the convex surface of the aneurism, so much so that it caused obstruction to the passage of the contents of the stomach into the duodenum. This seemed to me to be partly due to the weight of the dilated stomach and its contents, and of the colon and omentum. I deemed it advisable to pull as much as possible of the stomach over to the right side 
of the aneurism, so as to relieve this pressure. As the stomach seemed inclined to remain in its new position, I did not think it necessary to fix it in any way. The patient rapidly recovered from the operation, the wound was dressed with dry cyanide gauze, and the sutures were removed on the eighth day. There was slight vomiting, frequently recurring for several days, in connection with nausea, excited by the smell of brandy. The alarming symptoms disappeared, and the patient slowly improved, and became able to take solid food and to walk for short distances. She complained of burning sensations in the epigastric region, and had general hyperæsthesia of the abdomen, but the pulsating mass was less easily felt ; this was no doubt due to the fundus of the stomach being placed between the aneurism and the abdominal wall.

\section{Dilatation due to Obstruction by Cancer of the Pylorus.-If} cancer of the pylorus be found to be causing the dilatation, pylorectomy or partial gastrectomy may be indicated if the disease be limited and there be no secondary infection; but if it be too extensive for removal, gastro-enterostomy will be the only treatment likely to afford relief (see p. 2II).

Dilatation due to Pyloric Tumour the Result of Ulcer.-In some cases of chronic ulcer with great thickening, pylorectomy may be the best treatment when it is a matter of doubt as to the disease being cancer, but in all cases where the disease is thought to be of a simple character gastro-enterostomy will be the operation of choice.

A case related on p. I93 is a good example of a large tumour - of the stomach and pylorus producing gastric dilatation, in which we performed gastro-enterostomy, thinking the disease to be cancer. The entire disappearance of the tumour and the present well-being of the patient, however, prove that the disease was inflammatory thickening around an ulcer.

Other cases of dilated stomach due to tumour of the pylorus the result of ulceration, treated by pylorectomy, pyloroplasty, and gastro-enterostomy, are related elsewhere, under the headings of these various operations.

Dilatation of Stomach due to Cholelithiasis and Consequent Perigastritis around the pyloric end of the stomach is so common a sequence of events that we have come to look on 
it as a concomitant ailment ('Diseases of Gall-bladder and Bile-ducts,' 3rd edit.).

Several cases out of many that we have had that were successfully treated by gastrolysis are related in the chapter on Perigastritis. A case is also related in the chapter on Fistula, where severe stomach symptoms, characterized by dilatation and vomiting, were remedied by detaching the gall-bladder from the pylorus and closing the fistulous opening.

Terrier ('Chirurgie de l'Estomac') records two cases of pyloric obstruction due to gall-stones ulcerating through the gall-bladder into the pylorus. In the chapter on Pylorectomy for Cancer is recorded a case of cancer starting in the gall-bladder, extending to the pylorus, and producing dilatation of the stomach, in which at the same time partial hepatectomy, cholecystectomy, and pylorectomy were performed successfully on August Io, I900, the patient being now in good health, more than three years after operation.

Pyloric Stenosis of Biliary Origin.-In a paper recently read before the Lyons National Society of Medicine, Villard reported a case in which pyloric obstruction was cured by the performance of ideal cholecystotomy and choledochotomy (Lyon Méd., May I8, 1902).

The patient, fifty-eight years of age when operated upon on March 5 this year, enjoyed excellent health until $189 \mathrm{I}$, when she first suffered from hepatic colic and jaundice. The colic returned from time to time during the succeeding nine years. In I90o there was a series of hepatic crises, together with pronounced and persistent icterus. At this time delay in digestion and vomiting after food were first noticed. The patient afterwards became rapidly exhausted and anæmic. Examination before the operation showed undoubted signs of pyloric obstruction and dilatation of the stomach. A double tumour was felt in the right side of the abdomen. The lower part of this was the right kidney; the upper, situated in the position of the gall-bladder, was hard, tender, movable transversely, and bilobar. Cancer of the pylorus was considered as pussibly present in addition to the gall-stones. After the abdomen had been opened, the pylorus was found tightly bound by firm adhesions to the under surface of a thickened gall-bladder. Behind the pylorus was a second hard tumour, which had given rise to the suspicion of a cancer, but 
which turned out to be the common duct distended by calculi. An attempt was made to press back these calculi into the gallbladder for removal, but this having failed, incisions were made both into bladder and duct, and the stones removed through both openings. $\Lambda$ fter the division of the adhesions the pylorus was found perfectly patent, without any sign of thickening of its sphincter. The wounds in the gall-bladder and duct were closed without drainage by muco-mucous and sero-serous sutures, partly because there was no sign of infection and the wounds were easily accessible, and partly because the drainage from a biliary fistula would have been a great disadvantage in a patient already exhausted. Recovery was uninterrupted and rapid. 

PLATE XXVIII.

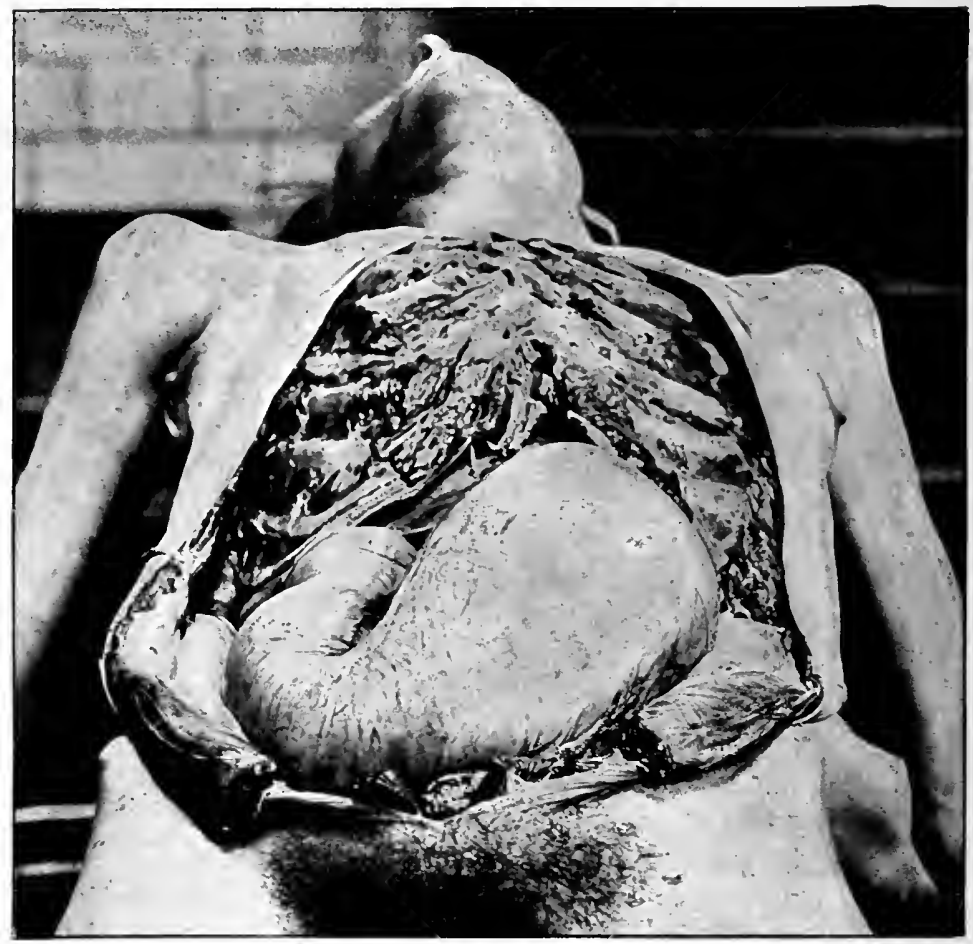

Fig. 102.-Acute Dilatation of the Stomach complicating Pneumonia and Pleurisy. (Campbell Thomson.)

To face p. $3^{89 .]}$ 


\section{CHAPTER XXI}

\section{ACUTE DILATATION OF THE STOMACH}

ACUTE dilatation of the stomach was first carefully described by Hilton Fagge (Guy's Hospital Reports, I872-1873). He recorded four cases, two of them obseryed by himself; these two both occurred in men, and both terminated fatally. The first patient was aged thirty; he suffered from acute dilatation of the stomach, which proved fatal after three days. The chief symptoms were incessant vomiting and anuria. At the post-mortem examination the stomach was found enormously dilated; all the other organs were normal. The second patient was aged eighteen. $\mathrm{He}$ had been in poor health for about two weeks, but had never suffered from any illness referable to the stomach. On the first day of his illness he complained of severe abdominal pain, and vomited then and on the subsequent day. For the next few days his condition improved, but gradually the symptoms returned. During the last two days his condition became extremely grave; he vomited enormous quantities of greenish fluid, and had diarrhoa. The urine was scanty. At the postmortem a greatly dilated stomach was seen, and a retroperitoneal abscess communicating with the duodenum was found. Several isolated cases were subsequently recorded, and in I892, Kelynack (Medical Chronicle, vol. xvi.), after recording the occurrence of a case in a female, aged nineteen, in whom a dilatation of the stomach and the first part of the duodenum was found, gave reference to all the previously published cases.

At the Leeds General Infirmary there have been four fatal cases, and three cases in which recovery has taken place, 
during the last few years. The following are brief notes of one of these, under the care of Mr. W. H. Brown: The patient, a man aged fifty-five years, said to be suffering from intestinal obstruction, was admitted into the hospital. $\mathrm{He}$ was in his usual good health up to forty-eight hours previously, when suddenly he was seized with intense pain in the abdomen, accompanied by incessant vomiting; the romiting continued until three hours before admission. On admission the hands were cold, the pulse was small and thready, and the face was pinched and sunken. The abdomen was distended above the pubes, but flattened at the sides and epigastrium ; it was resonant, except between the umbilicus and pubes, where it was dull. Fluctuation could be felt, and a succussion splash was obtained. The temperature was subnormal. Earlier in the day the bowels had been moved slightly, but no urine had been passed since the onset of the illness-i.e., for forty-eight hours. The abdomen was not tender, but the patient was in great pain, turning from side to side constantly, and asking for relief from his agony. A catheter was passed, and about a drachm of bloody urine was withdrawn. An exploring syringe was passed into the fluctuating swelling midway between the umbilicus and pubes, and withdrew about 2 drachms of thick greenish fluid, with a curious smell, recalling the contents of a pancreatic cyst. As the general aspect of the case presented unusual difficulties with regard to diagnosis, and as it seemed certain that the man would die shortly if unrelieved, and that the pressure of the fluctuating tumour was the cause of his suffering, it was decided to operate. Ether was given, and the abdomen was rapidly opened over the most prominent part of the swelling. A tense, thinwalled, rounded cyst was found, which contained a dark liquid with some gas. As no colon could be seen or felt, and as the tissues of the cyst wall seemed to be more like peritoneum than any viscus, Mr. Brown drew it to the surface, opened it, and let out about 3 pints of dark-green, viscid fluid, closely resembling that of a pancreatic cyst. The walls were stitched to the skin, and the wound was closed. The patient rallied from the operation, and expressed himself as 
being relieved, but he died five hours later. At the necropsy the cyst was found to be a dilated stomach, which would easily hold 5 pints; there was no pyloric obstruction. The kidneys showed marked signs of nephritis, and were saccular; all the other organs were healthy.

Mr. Jessop's case occurred in a female, aged twenty-six, who was convalescent after an excision of the hip, performed a month before. Vomiting, copious and uncontrollable, set in after eating an apple. Death occurred on the fourth day. At the post-mortem there was great dilatation of the stomach and of the duodenum to a point 6 inches below the pylorus.

Our own case came on ten days after a duodeno-choledochotomy for the removal of a gall-stone impacted in the common duct; the patient was doing well in every respect until twenty-four hours before her death, when she suddenly began to vomit, the abdomen became enormously distended, the urine became suppressed, and the patient died in a state of collapse. At the post-mortem examination, beyond dilatation of the stomach, nothing was found to account for death. It is interesting that in this case there were adhesions of the pylorus to the liver and gall-bladder, which we thought might probably have been the cause of the trouble. We were, unfortunately, out of town at the time of the catastrophe; otherwise we would certainly have operated, though probably the result would have been the same, for when the gravity of the condition was recognised, and a colleague was called in to see the patient, she was pulseless and cyanosed.

The fourth case was under the care of Dr. Barrs. The patient was ill with ulcerative endocarditis, and suddenly developed enormous abdominal distension, which proved rapidly fatal. There had been no previous stomach symptoms, and at the autopsy nothing was discovered to account for the condition.

But that the condition of acute gastric dilatation when recognised early is capable of being successfully treated the following three cases coming under our care distinctly prove:

In one, a woman of thirty-five recovering smoothly from cholecystotomy, which had been performed a week previously, was suddenly seized with pain in the epigastrium, followed by vomiting, 
which soon became ineffectual in emptying the stomach. Rapid dilatation ensued, and the stomach not only formed a large swelling, filling up the superior abdominal region, but also extended well below the umbilicus towards the pubes. This, owing to pressure on the diaphragm, and through it to pressure on the heart and lungs, led to great shock, quick and oppressed breathing, and a rapid, feeble pulse, with signs of lividity and imperfect blood aeration. The urine was scanty, and, in fact, almost suppressed.

Strychnine was freely given subcutaneously, but no relief came until the stomach tube was used, when a large quantity of gas and several pints of brownish fluid were evacuated; the lavage was repeated several times during the next two days, during which time alimentation was entirely rectal. It was interesting to note that the pulse and temperature were paradoxical, the former running up to 150 and the latter down to $97^{\circ} 3^{\circ}$. All the symptoms passed off under treatment almost as quickly as they came, and in a week the stomach had returned to the normal size.

In another case, a patient of twenty-nine, who had had abdominal hysterectomy for a large myoma, and whose after-progress had been most satisfactory, the wound having healed by first intention, and the temperature and pulse having been normal throughout, was seized with pain over the stomach a fortnight after operation; this was followed by vomiting of frothy mucus with a few brownish streaks in it, and by distension of the abdomen, beginning in the epigastrium. On percussion the stomach was found to be down to the pubes, and on succussion a well-marked splash was easily obtained; the pulse became rapid and the face pinched, so that within thirty-six hours of being quite well the patient presented every appearance of impending death. The urine was very much diminished, and for twenty-four hours almost suppressed. Strychnine was given subcutaneously, and rectal feeding was adopted, but no relief was obtained until the stomach was washed out, after which relief was immediate and the patient steadily improved, though no food was given by the mouth for three days. Within a week the stomach had returned to the normal size. Here also the temperature and pulse were paradoxical, the former being subnormal and the latter very rapid. The only cause that could be assigned was the eating of a raw apple, but that may have had nothing to do with the condition. The patient said that she had always been subject to a weak digestion since childhood, but at the time of operation nothing abnornal was noticed in the stomach.

The third case was seen in a woman of fifty-eight, who had been operated upon for the removal of a large abdominal tumour, 
which proved to be a parovarian cyst. After operation matters progressed smoothly for twenty hours; then vomiting set in, and the stomach was found to be much distended, producing a prominent bulging in the epigastric and umbilical regions, which contrasted very strikingly with the emptiness of the rest of the abdomen. The patient was rolled over on to her face, vomited three times within half an hour, the quantity on each occasion being over a pint, but almost immediately expressed herself as relieved. Vomiting ceased and did not recur, and the patient made a good recovery.

In a paper read before the Royal Medical and Chirurgical Society, and subsequently published in pamphlet form, Dr. C. Thomson collected the records of forty-four cases, including five cases in which he had performed the postmortem examination. Able and thoughtful papers have also been published by Dr. C. R. Box and Mr. Cuthbert Wallace.

\section{Symptoms.}

The symptoms of this disease appear, as a rule, with suddenness, and progress rapidly to a fatal termination. The patient complains, as a rule, of the sudden onset of an intolerable pain, limited at the first to the epigastrium, but spreading swiftly over the whole abdomen. In exceptional cases, as in Fagge's second case and in one related by Hughes Bennett, there were pain, discomfort, and a feeling of something different from the usual for several days. Vomiting appears early, and, as a rule, remains, with barely any intermission, till the end. The fluid vomited is often greenish in colour and turbid, and its quantity is often enormous. In one case it has been likened to the contents of a pancreatic cyst. In later stages of the disease the fluid may be brown in colour and horribly offensive. The act of vomiting is not often distressing; matters are brought up, as H. Morris says, 'in large gulps without straining.' The stomach, however, despite the incessant ejection of fluids, never empties. If the stomach tube be passed and a huge volume of gas and a large quantity of fluid be evacuated, the stomach begins almost at once to refill, and within half an hour or an hour its distended form may be recognised on 
inspection of the abdomen. In a case of Sir W. Broadbent's 8 pints of fluid were removed by the siphon; but at once the stomach began to refill, and it rapidly regained its former dimensions. On examining the abdomen the outline of the stomach can often be seen. The distension is gigantic. The bulging of the abdominal wall is mostly to the left and in the lower half; the right hypochondrium by contrast has been said to be flattened or excavated. In other cases, though the abdomen is large, tense, and resisting, no special bulging at any point can be seen, while in yet others the abdomen may be, as in Henry Morris's case, retracted. In this instance, at the post-mortem examination, although the stomach was enormously dilated and occupied the greater part of the abdomen, its anterior surface was said to be flattened.

The whole abdomen is, as a rule, hyper-resonant on percussion. This is due to the fact that in the stomach there is present a very large volume of gas. When the stomach tube is passed, there is, at the moment that the tube enters the stomach, a loud, gushing sound, sometimes almost a report, and a large volume of quite inoffensive gas escapes, and the prominence formed by the stomach can be seen to subside. On palpation a very loud succussion splash can be obtained. Peristalsis has only once been observed, by Schultz. Campbell Thomson writes, "The absence of peristaltic contraction opposes the theory that the dilatation is due to pyloric obstruction,' a statement which will not bear examination, for peristalsis in any part of the intestinal canal is never seen in acute obstruction unless it supervenes upon a chronic impediment to the outflow of fluids. In several instances there has been an easily recognised fluctuation.

The general condition of the patient is recognised as being serious from the first. The respirations are frequent and shallow; the pulse is rapid, thready, and of poor quality; the aspect of the patient shows that his tissues are starved of water; he is pinched and careworn and old in appearance, and the limbs are often cold and clammy. Thirst is intolerable and unquenchable; the patient is restless, and at times 


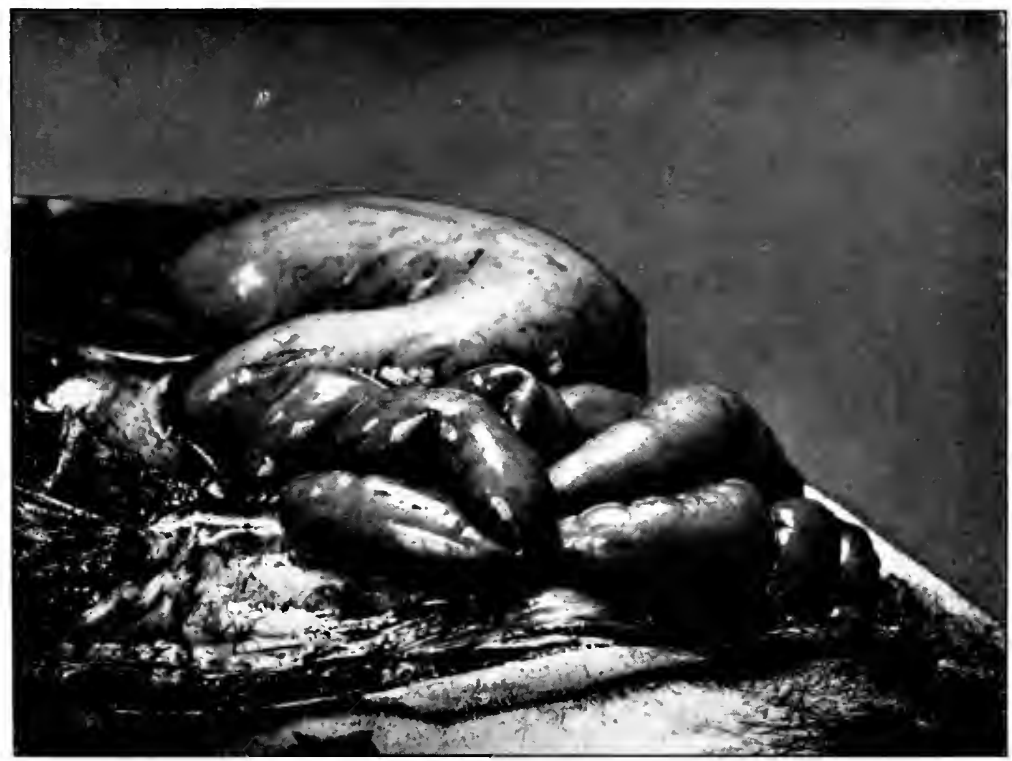

Fig. ioz.-Acute Dilatation of Stomach and Upper part of INtestine. (Campbel, Thomson.)

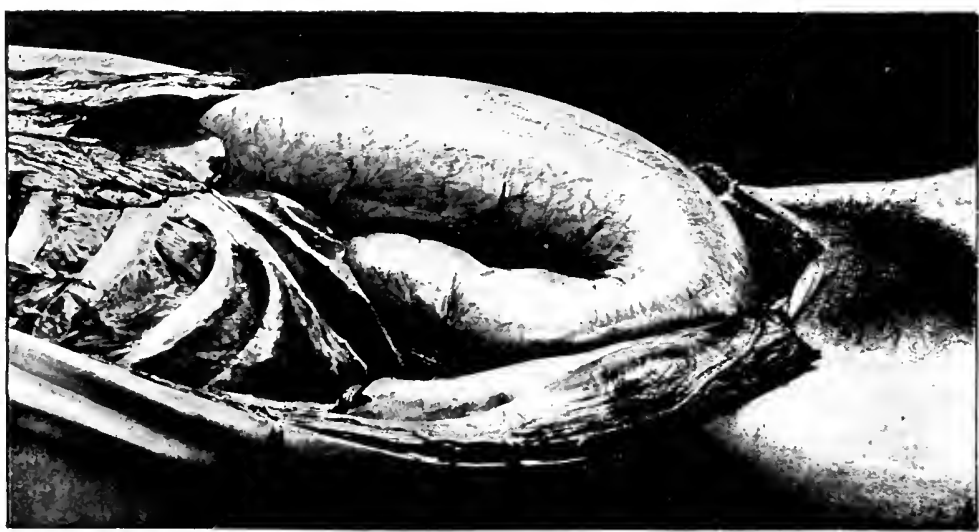

To face p. 394.]

Fig. IO4--Side View of the same Case. 

irritable and weary. In one case only, related by Sir W. Broadbent, was gastric tetany seen. Diarrhœa has been occasionally observed. The amount of urine in nearly every instance in which special attention has been given to the point has been very considerably reduced in quantity, or the secretion entirely abolished.

\section{Etiology.}

No satisfactory explanation of the origin of this condition has hitherto been suggested. In the great majority of the instances no cause for the condition has been found at the necropsy, and the origin of the gastric dilatation has remained undisclosed. In some cases the acute-dilatation has supervened upon a chronic stenosis of the pylorus, as in one case of Campbell Thomson's. In others there have been associated conditions, such as retroduodenal abscess, which may have played some part in the causing of the distension.

In Thomson's series of forty-four cases no fewer than twelve followed surgical operations. Four of the operations were upon the abdomen, two upon the kidney, and six upon the extremities; of the four abdominal operations three were in connection with the bile-passages, and the fourth was an ovariotomy. In all the cases the patients were, as it seemed, progressing rapidly to convalescence. In five cases an indiscretion in diet, an overeating or the eating of some extremely indigestible or unusual food, has been noted.

Kirch (Dentsche Medicinische Wochenschrift, Igoo) reports a case in a youth, nineteen years of age, who made a supper of soup and beer, which was followed by pain and uncontrollable vomiting. The stomach was washed out, and $5 \frac{1}{4}$ pints of grayish-green fluid were evacuated. The next day another $2 \frac{1}{2}$ pints were removed by the stomach tube, but the patient died in the evening from heart failure, four and a half days after the beginning of his illness. At the post-mortem examination the stomach was found to have lost its normal shape, and to have the form of a long dilated tube reaching from the left hypochondrium to the true pelvis, and then turning sharply upwards to the abdomen. 
Wiesinger (Deutsche Medicinische Wochenschrift, February, I90I, and Philadelphia Medical Journal, March, I90I) has related a case of acute dilatation of the stomach in which mechanical blocking of the orifices of the viscus by rotation seemed to be responsible for the distension.

The case occurred in a man of forty-one, who was taken ill immediately after a dietetic indiscretion, with the clinical appearances of intestinal obstruction associated with enormous distension of the epigastrium and left hypochondrium. There were attempts at vomiting, but nothing was brought forth. The distension increased, and operation was undertaken on the fourth day while the patient was in extremely bad condition. The large mass in the epigastrium proved to be the stomach. It was suspected at first that this was merely pressed forward by a cyst (possibly pancreatic) lying behind the stomach, as the mass felt like a cyst. The stomach was punctured and the contents drawn off, and it was found that the whole mass consisted of the tensely distended stomach. The pancreas itself was found to be normal, excepting for, perhaps, some enlargement. There were widespread areas of fat necrosis. The stomach was found twisted at an angle of about $I 80^{\circ}$, and fixed in this position. The cardia and pylorus were completely closed. The patient recovered completely, and had subsequently no digestive disturbances.

The case was notable for the complete cure of the fat necrosis. The latter condition was probably due to pressure upon the pancreas and pancreatitis set up by the enormously distended stomach. The occurrence of volvulus of the stomach was attributed to the displacement of the colon above the stomach resulting from the abnormal length of the mesocolon. After a partial volvulus had occurred, this was increased by the enormous secretion which took place in the stomach. Four cases followed an injury; two appeared rapidly after an abdominal injury, and one occurred as a later complication of an injury to the lower dorsal region of the spine.

Appel (Philadelphia Medical Journal, August I2, I899) has reported a case where acute dilatation of the stomach super- 
vened on an abdominal injury. The abdomen was opened for what was supposed to be intestinal obstruction, but threequarters of the abdominal cavity were occupied by a dilated stomach. The stomach was opened, giving vent to a quantity of gas and fluid, the incision being afterwards closed; but the second night after the operation the abdomen became distended as before, and death rapidly ensued. At the necropsy nothing was found abnormal, except the distended and dilated stomach. It was suspected that the great sympathetic abdominal ganglia might have been injured, but nothing was found wrong with them on examination. Dr. Box and Mr. Wallace (Lancet, June 4, I898, p. 1538) report a case in a boy, sixteen years of age, following on a blow on the epigastrium. Abdominal section disclosed a dilated stomach, which was incised and emptied, but in that case also death followed.

The following case related by Valentin is quoted by Peebles and Campbell Thomson. The patient was a youth, aged twenty, of a delicate constitution, and when attempting to climb a tree he fell, and received severe contusions on the back and loins. In a few days all food, both solid and liquid, was vomited, and in a short time he was obliged to take to his bed from exhaustion. He was shortly afterwards attacked with a fever, and, as this assumed a mild character, great distension of the abdomen occurred, accompanied by constant vomiting of a serous, blackish fluid. Death took place two months after the first appearance of the fever. After death the stomach was found to be enormously distended, the other viscera being normal.

\section{Pathology.}

The appearances presented on post-mortem examination are curiously similar in almost all cases. The stomach is enormously distended, and is sharply bent upon itself, so as to form a gigantic U-tube, whose distal limb is rather shorter and less thick than the proximal. At the lesser curvature there is, therefore, a sharp kink. The stomach seems to occupy the greater part or even the whole of the abdomen, cutting 
off from sight and severely compressing all the small and large intestine. The walls of the stomach are tightly stretched and thinned, in one case seeming no thicker than a single layer of peritoneum. In Brown's case the tumour was mistaken for a thin-walled pancreatic cyst. The thinning of the walls is not equally distributed; very thin patches are sometimes seen, the greater part of the stomach remaining normal, or nearly so. In twelve cases out of forty-four, the dilatation was not limited to the stomach, but involved the duodenum also, and in one case the upper few inches of the jejunum. Box and Wallace have shown that the condition found in acute dilatation of the stomach can be exactly reproduced on the cadaver. They write: 'We have found by actual experiment on the cadaver that the stomach can be enormously' distended by water pressure, with the jejunum cut right across and lying patent in the abdomen. Moreover, the stomach remains thus distended. The same result can be attained after the superior mesenteric vessels and the peritoneal folds in their neighbourhood have all been divided. If, however, by introduction of the finger well behind the distended stomach a little to the left of the mid-line of the spinal column, the fundus and posterior wall of the stomach be gently raised, the excess of fluid will at once flow freely away from the stomach through the cut jejunum. If the part of the duodenum which lies on the right side of the spine, behind the peritoneum, be first incised, the tense distension of the stomach cannot be produced. We therefore feel justified in assuming that the tense distension is due to actual pressure of the stomach on the part of the duodenum which crosses the third and ascends by the side of the second lumbar vertebra to end in the jejunum.

'We would suggest, therefore, that in producing the train of symptoms met with in acute dilatation of the stomach two factors come into play. There is, first, a paralytic condition of the viscus which leads to distension, and then, at a certain stage, the distended stomach actually produces obstruction by pressing on the duodenum on the front and to the left of the spinal column' (the Lancet, November 9, I90 I, p. I260).

A large number of more or less ingenious hypotheses have 
been suggested to explain the incidence of acute gastric dilatation; but none of them are completely satisfying, another example of the fact that where there are many theories there is little truth.

By Fagge and others an excessive secretion of the stomach was considered the primary factor, the organ being 'paralyzed from overdistension, and unable to rid itself of its burden.' Henry Morris, who considered the excess of secretion to be an important determining case, suggested the name 'gastrosuccorrhœa' for this disease. In several cases, however, the distension of the organ is not by any means wholly or even chiefly due to the fluid contents; for when the stomach is emptied of gas by a stomach tube, and before any fluid has escaped, the viscus may return almost to its normal size.

Campbell Thomson writes: 'It is, of course, a difficult matter to establish precisely the relationship which exists between the distension and the secretion, but it seems probable that they must be looked upon as two distinct processes.

' The most likely explanation seems to be that the stomach wall becomes paralyzed - the possible causes of which will be discussed further on-and then, later on, the organ becomes distended by gas or excessive secretion. Moreover, the fact that excessive secretion is not present in every case makes it impossible to consider it as the primary cause; in some cases there is very little fluid found after death, the stomach being almost entirely distended with gas.'

Pepper and Stengel suggest that the immediate cause of the dilatation is a spasm of the pylorus; but if this were so, the not infrequent implication of the duodenum would be still to explain. Wiesinger, already quoted, considers that there is a volvulus of the stomach, and that the distension is similar to that found in a twisted sigmoid flexure.

Albrecht was the first to suggest that the constricting agent was the superior mesenteric artery, which, owing to the downward dragging of the intestines, presses upon and obstructs the third portion of the duodenum. Kundrat, Ewart, and others have offered evidence in support of this 
view, and there is little doubt that in some cases at least the explanation is satisfactory. Albrecht points out that if the descending portion of the duodenum be opened and the finger passed onwards into the transverse portion, and with the other hand traction be made upon the mesentery by pulling the intestines towards the pelvis, the constricting power of the superior mesenteric artery will be clearly recognised. In Jessop's case and in others the distended duodenum was abruptly narrowed at or near the point of crossing of the artery; but in other cases, and these form a majority, there was no constant point at which the stricture was found.

The view which seems to be most highly favoured by the majority of writers is that the paralysis of the muscular wall of the stomach is primary. Carrion and Hallon (Semaine Médicale, August 2I, I895) have shown that the section of the pneumogastric nerves in the dog leads to dilatation of the stomach, and to symptoms resembling in many cases those of uræmia. It is clear that in some instances as soon as the stomach has reached a certain size it is almost impossible for it to recover, either because there is some kinking or rotation at the pylorus or because pressure is exerted, as Box and Wallace suggest, upon that part of the duodenum which crosses the third and ascends by the side of the second lumbar vertebra in the left side.

\section{Treatment.}

In the great majority of cases the issue has been fatal, and all treatment has been unavailing. But the disease is by no means necessarily lethal, and we have, in our own experience, three cases to record in which the symptoms have subsided and the patients progressed to recovery.

In the early stages recourse should be had at once to lavage of the stomach. If the organ rapidly fills after it is emptied, the tube may be left in for several hours. The position of the patient must be altered, so that he lies prone in bed, with a pillow under the pelvis and the lower part of the abdomen. In the severer instances it may be necessary 
to open the stomach and drain, performing, in fact, a gastrostomy; or recourse may be had to gastro-enterostomy, whereby the stomach is drained continuously into the intestines. The latter operation has never been put to the proof, but on theoretical grounds, and from our experience in other diseases of the stomach, it would seem likely to be of service. The fact that gastrostomy has always proved fatal need not deter the surgeon, for an examination of the recorded cases shows that all patients upon whom it was practised were moribund or almost in the last extremity.

\section{Acute Post-operative Dilatation of the Stomach.}

It seems not improbable that some of the cases of ileus after abdominal operations may be caused by acute dilatation of the stomach, which, when once initiated, tends to persist and get worse, owing to the distended stomach dragging on and kinking the duodenum, thus leading to shock by pressure on the heart, without there being any sign of sepsis. Hence, in all cases of ileus after operation the use of the stomach tube should not be neglected.

Attention has been specially drawn by P. Müller (Deut. Zeit. f. Chir., August, Igoo) to dilatation of the stomach following upon abdominal operations. In some of these cases the gastric distension is only a part of a general involvement of the intestinal canal, due to peritonitis. The septic condition induces a paresis of the bowel walls, and distension of the gut rapidly follows. In other cases, and it is these to which Müller draws particular attention, the dilatation of the stomach is due to the pressure upon the duodenum of the superior mesenteric artery. When a large ovarian tumour or a fibroid tumour of the uterus is removed, the intestines, compressed for many months or years to the upper part of the abdomen, sink down into the pelvis, and so drag upon the superior mesenteric artery as to compress the duodenum in the manner already described.

We have for several years adopted the practice, in all abdominal cases where vomiting is severe or long-continued, of washing out the stomach. A single washing almost 
always suffices to relieve the patient and to check the vomiting, but in some few cases a daily lavage for two, three, or four days may be desirable. In all abdominal cases in which vomiting is a symptom threatening to persist, an examination of the abdomen, with the object of discovering any dilatation of the stomach, is desirable. 


\section{CHAPTER XXII}

\section{ATONIC DILATATION OF THE STOMACH}

Dilatation from Atony is due to various general and local causes, and may persist after the original cause has disappeared. As we mentioned when speaking of dilatation of the stomach generally, pure atonic dilatation, apart from some degree of stenosis or some mechanical cause, is probably rare. The condition is due to a weakened state of the muscular coats of the stomach, so that there is deficient peristalsis, and the contents are not pushed towards the pylorus sufficiently rapidly for the stomach to empty itself in a normal time.

In these cases, even in the morning before breakfast has been taken, a stomach splash may be obtained on succussion, and if the contents be siphoned off, particles of food, with fluid, will be found-not necessarily coarse particles of food, as are found in dilatation due to true stenosis of the pylorus, but digested food that would easily pass the pylorus if normal peristalsis were present.

\section{Symptoms.}

The appetite is poor as a rule, though it may be abnormally increased. Thirst is increased, and there is a feeling of dryness in the throat almost constantly present. Flatulency, relieved by eructations, is usually met with, and the expelled gas may have an unpleasant or disagreeable odour. There is a constant sense of oppression, with fulness at the epigastrium, and frequently palpitation will be complained of. Vomiting occurs in advanced cases, and may be repeated 
daily or once or twice a week, the vomited matters being offensive, and containing yeast cells, sarcinæ, and other organisms. Free $\mathrm{HCl}$ will usually be found. Emaciation is usually present, and as the disease advances it may become extreme.

There are certain cases of dilated stomach of the atonic variety in which the chief symptoms depend on the great bulk of the organ. As descriptive of the amount of paralytic distension that is possible, Bamberger mentions an extreme case, in which the stomach held 70 pints of fluid (Gould. and Pyke, p. 630). As these cases are frequently dependent on chronic catarrh or on some general cause not mechanical, it is not necessary in a surgical work to enter very fully into the etiology, except to say that the myasthenic variety of dilatation may be dependent on $(a)$ fatty or colloid degeneration of muscle, $(b)$ destruction of muscle fibres by ingested poisons, by ulceration, fibrosis, and cancer, and on (c) paresis of the nerves of the stomach.

General and medical treatment, supplemented, if need be, by electrical treatment and lavage, if carried out systematically and for a sufficient length of time, usually yield good results; but in some cases, despite regular lavage of the dilated organ, well-regulated diet, and general medical treatment, the dilatation persists, and the nutrition of the patient and the general health become seriously impaired. In such cases the operation of gastrorrhaphy or gastroplication may be worth considering, and in certain cases gastroenterostomy may be performed with advantage.

\section{Gastroplication.}

Bircher performed the operation, and reported three cases in $\mathbf{1 8 9 0}$, and Weir, of New York, published a paper on this subject in 1892 . The reports show that the operation is a most beneficial one if the cases be well selected; but before performing gastroplication it should be clearly ascertained that there is no mechanical obstruction, either at the pyloric orifice or in the duodenum.

The following diagrams illustrate the method of folding 
and diminishing the volume of the stomach adopted by various operators. In the slighter cases, probably, Bircher's operation would do all that is required, but in the more severe cases a method first described by Moynihan in the Lancet, 1898 , is the more efficient. The operation is neither difficult nor attended with serious risk, since there is no danger of sepsis, as the cavity of the stomach is not opened;
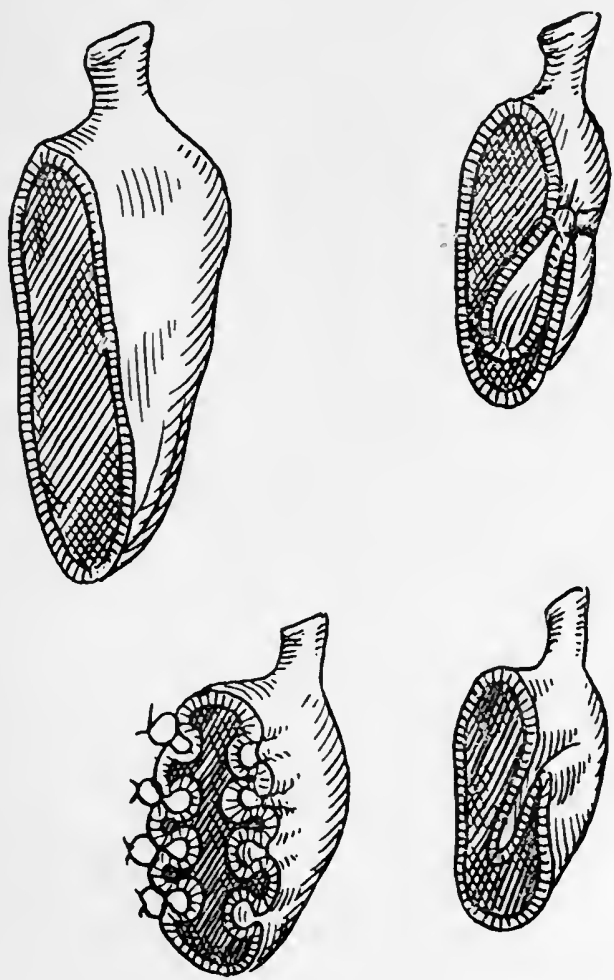

Fig. I05.-Gastroplication.

and as the stomach can be drawn out of the wound to be sutured, the general peritoneal cavity is saved from long exposure.

In the Hunterian Lectures we collected all the reported cases, twenty-eight in number, showing a $7^{\circ} \mathrm{I}$ per cent. mortality. 
We have only seen two cases, in addition to the one referred to, that we thought suitable for gastroplication.

One patient, IV. R., aged thirty-five years, who had been invalided for eighteen months, but who had had stomach symptoms for seven years, and who had tried lavage and other medical treatment for several years without benefit, expresses himself as cured of all his former symptoms, and he certainly looks well,
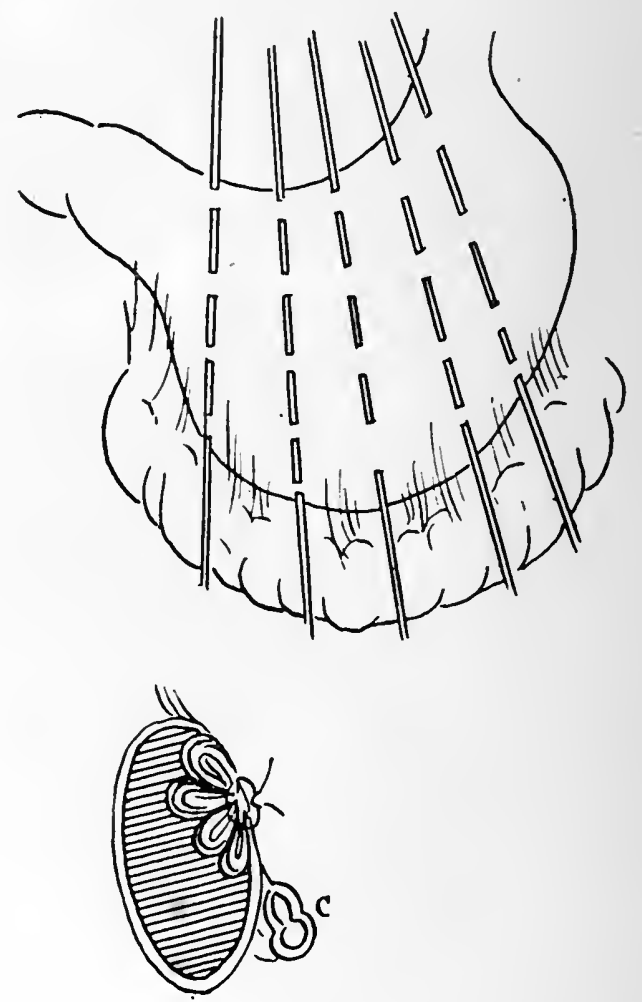

Fig. io6.-Gastroplication.

and seems able to take his food without any difficulty. The operation was performed on January 24, I900, and at the same time the pylorus, which was thought to be narrow, was stretched by invaginating the anterior wall of the stomach, and pressing first the index, then the middle finger, then the thumb, and lastly two fingers, through it, until a freely patent channel was obtained. 
In another case, a Mrs. R., aged fifty-six, was sent by Dr. West of Morley, with an enormously dilated stomach, reaching to the pubes, and with a history of stomach symptoms extending over several years. Recently she had been entirely confined to bed, had lost all desire for food, and had become much emaciated. Gastroplasty and dilatation of the pylorus by Hahn's method were performed on April 23, 1900, with immediately brilliant results, and in two months the patient had gained over a stone in weight.

The after-history of this case is both interesting and important ; for six months later, although she could take food and digest it well, she began to suffer from severe pain at the epigastrium, which, after a full meal at first, and later after any solid food, became so distressing that she had to resort to living on milk and liquid foods.

We were again asked to see her, and found her in considerable distress from pain at the epigastrium; associated with great tenderness, rigid recti, and an obscure feeling of swelling, as if a tumour were present. On distending the stomach with $\mathrm{CO}_{2}$, it reached an inch below the umbilicus.

Vomiting occurred at times, but was not a marked symptom. She weighed a stone more than when she was operated on, though Dr. West and her friends said that she had lost weight considerably since the pain began.

Lavage of the stomach, rest, and dieting giving no relief, it was decided to perform gastro-enterostomy, which was done on December 5, I goo.

On opening the abdomen, very extensive adhesions were found, clearly following on the gastroplication, as the anterior wall of the stomach, especially towards the pyloric end, was fixed to the liver and to the abdominal wall; and it was at this part where her pain had always been, and where the tenderness was constantly felt. The gastroplication had produced obliteration of the anterior wall, the greater and lesser curvatures being close together; but the posterior wall had remained stretched, and was only limited by the capacity of the lesser peritoneal sac, which it filled completely.

The pylorus was quite patent. The adhesions to the abdominal wall were separated; but as a large raw surface was necessarily left, it was felt that the adhesions would probably re-form. A posterior gastro-enterostomy was therefore done. Recovery was uneventful, and the patient left the 'home' within the month quite free from symptoms and feeling well. She could take solids and enjoy her meals. The improvement has continued. 


\section{CHAPTER XXIII}

\section{GASTRIC TETANY}

THE occurrence of tetanoid spasms in association with certain diseases of the stomach, though mentioned by Morgagni, was first prominently recorded by Kussmaul in 1869 . His observations referred to cases of dilatation of the stomach in which convulsive attacks had been noticed. It is now generally recognised that tetany is an occasional complication of several diseases implicating the gastro-intestinal tract. There are, however, widely divergent views as to the frequency and as to the seriousness of the disease, and an attempt has been made to limit the term 'gastric tetany' to cases in which there are increased mechanical excitability of the muscles, increased mechanical and electrical irritability of the motor and sensory nerves, a profoundly serious constitutional disturbance, and often a fatal termination. Indeed, it has almost been made a criterion of the disease that it should end fatally. Frankl-Hochwart, for example, in discussing the records of forty cases, disallows all but eleven, and of these ten terminated fatally. To restrict the term ' gastric tetany' to cases of this type seems to us to preclude deliberately a large number of cases presenting similar, though less serious, manifestations-cases precisely similar in kind, though slighter in degree. The distinction between gastric tetany and tetanoid spasms associated with, and dependent upon, gastric dilatation is one which is arbitrary and irrational. It must, we think, be conceded in the future that the term 'gastric tetany' shall include all cases of tetany and tetanoid spasms associated with, and directly due to, diseases of the stomach. 
The occurrence of tetany in gastric disease is more common than is generally known. In the slighter degrees the symptoms are often inconspicuous, and are in no way associated in the mind of the patient with the more urgent stomach trouble; it is then not until some leading question is put that he will refer to the 'cramps' or 'tingling' in the extremities.

In the early stages the patient generally complains of a peculiar numbness, or prickling or tingling, or a feeling of 'pins and needles' in the extremities. There may be also a feeling of coldness or heaviness in the limbs, and drowsiness, or a feeling 'as if the head were swelling' has been remarked. More than one patient has spoken of a feeling of fatigue, of infinite weariness in the muscles about to be affected.

The formication frequently follows closely upon the act of vomiting or upon the washing out of a laden stomach. In not a few cases, indeed, the first onset of tetany has been observed after the stomach has been emptied of its contents by the stomach tube. In the severer forms, and in almost all the fatal cases, a history is given of severe and uncontrollable vomiting immediately preceding the attack. As the formication increases, a tetanic contraction of the muscles occurs. In the hand the fingers and the thumb are stiff and slightly flexed, the thumb is strongly adducted, and the position of the 'accoucheur's hand' is reproduced; the hollow of the hand is deepened, and the thenar and hypothenar eminences exaggerated. The wrists are, as a rule, strongly flexed, but may be extended; the forearm is usually flexed upon the arm, but all the muscles of the forearm and upper arm feel tense and rigid in contraction. During a tetanic seizure some alteration of the position of the limb may gradually occur : the wrist may from a condition of rigid extension pass into one of flexion, or one of pronation into supination. Or slow, writhing movements may be performed. Occasionally, when the patient feels a tonic spasm beginning in the hand, forcible voluntary movements of the fingers will cause the attack to pass away. As a rule, the spasms are symmetrical ; but, as Albu pointed out, the one side is almost constantly more profoundly affected than the other. The 
muscles of the feet and legs may be also, though more rarely, affected. The toes are drawn up into the sole; the ankles and the knees are flexed. The muscles of the face and jaws, of the neck and of the abdomen are all involved at times. The facial expression is greatly altered, a frowning, anxious, or 'snarling' appearance being remarked. The muscles of the eyeball are not seldom affected, and the sight may be lost for a time; the patient calls out that he cannot see or that he is blind. In one of Trousseau's cases the sight did not return for three days. During the paroxysm the patient may mutter or shout unintelligibly, almost as if in delirium ; words and phrases are run together unmeaningly. In all the well-marked attacks the signs of Trousseau, Chvostek, Erb, and Hoffmann are present.

Trousseau's symptom is described as follows: 'So long as the attack is not over, the paroxysms may be produced at will. This is effected by simply compressing the affected parts, either in the direction of their principal nerve-trunks or over their bloodvessels, so as to impede the venous or arterial circulation.'

Chvostek's symptom is shown by the very great increase in the mechanical excitability of the motor nerves of the part affected-a facial spasm, for example, being produced by tapping over the facial nerve near its exit from the stylomastoid foramen.

Erb's symptom is shown by the great increase in the electric irritability of the nerves and muscles, especially to anodal stimulation.

Hoffmann's symptom has reference to the heightened excitability of the sensory nerves, the slightest pressure on which may cause paræsthesia in the region of distribution.

In some cases of gastric tetany, but more especially in one case in an old man who had gastric dilatation, the result of duodenal ulcer, we have noticed during the tetanic seizure a dusky lividity of the parts involved. The face and neck look leaden and suffused, and the forearms and arms, swollen and turgid, have the same appearance as would be produced by the light application of a tourniquet.

As the spasm passes off, a return of the tingling in the 
hands and feet is often noticed, and the patient will rub the hands together or rub the parts against the bedclothes to obtain relief.

We have almost always found that the agony of the spasm may be relieved by forcibly bending the fingers, flexing or extending the wrist or forearms, or by reproducing passively those movements which would be caused by the active contraction of the muscles involved. Though the first passive movement will often cause an excruciating pain, yet a gentle persistence in the act will cause the spasm gradually to resolve, and will afford the patient great relief.

Intermissions during an attack are often observed. A seizure in which the wrists are flexed may slowly subside, to be presently followed by one in which the wrists are extended. And so the posture of the limbs may vary from time to time. The muscles of the abdomen are affected in the severer cases, the abdominal wall feeling hard, tense, and absolutely unyielding.

The general effect produced upon the patient by an attack varies greatly according to the severity and the duration of a seizure. In many cases the tingling or numbness, though frequently noticed, is nothing more than an unpleasant sensation whose significance is never appreciated. Cramps in the extremities, noticed from time to time soon after the patient gets to bed, are not attributed to their real cause until the surgeon makes pointed inquiry as to their existence. When, however, there is a wide involvement of muscles, and especially when the attack is unduly prolonged, the physical suffering is intense, and the most profound prostration may be witnessed. The pulse at the wrist may be quite imperceptible, though the tensely-contracted vessel can be rolled beneath the finger. Consciousness may be lost for a time, or the patient's mental balance gravely disturbed by the intensity of his suffering. A severe and prolonged seizure of gastric tetany is then almost as serious an ordeal as any patient may experience and live. It is these severer cases which furnish the large mortality recorded by Frankl-Hochwart, Müller, and others. 


\section{Theories as to the Causation.}

Three chief theories have been put forward to explain the occurrence of gastric tetany:

I. Kussmaul, to whom we owe the first description of the disease, attributed the tetany to the general desiccation of the tissues of the body. He considered that the loss of fluid to the body was occasioned in part by the copious and frequent vomiting, and in part by the absence of adequate absorption of fluid from the alimentary canal. Though Kussmaul himself subsequently disclaimed this theory, there have been several who have accepted it, or have thought that the deprivation of fluid might be one among several causative factors. Thus Fleiner (Miinch. Med. Woch., March 10, 1903, No. Io) states that there is some evidence of inspissation of the blood, and that a fall of blood-pressure is probable, and Jürgensen (Deut. Archiv f. Klin. Med., I8g8) gives support to the theory. Albu thinks also that the loss of fluid, if not the final or sole cause, is probably an important predisposing condition. In one case, related by Blazicek (Wien. Med. Woch., I894), investigation failed to show any loss of fluid to the blood. With few exceptions this theory has now been abandoned by all observers.

2. Germain-Sée, Berlizheimer, and others have suggested that the tetanic spasms are due to a reflex action aroused by the stimulation of the sensory nerves of the stomach. The frequency with which the seizure is immediately preceded by an act of vomiting or by the passage of a stomach tube, and the fact that a spasm can be elicited by flicking or percussing the stomach, have each lent some support to this reflex theory. It is, indeed, not to be denied that, whatever may be the ultimate cause making possible the tetanic condition, the stimulus necessary to start the seizure may be derived reflexly from the gastric mucosa. Albu quotes the partly analogous cases of tetanus and of strychnine-poisoning in the frog, in both of which toxic conditions a convulsion is instantly caused by the slightest peripheral irritation. Though this theory has been practically discarded by recent 
writers, it is, in our judgment, one that we cannot afford wholly to ignore.

3. The third theory is the theory of auto-intoxication. It is one that has received the weightiest measure of support from recent authorities, and is that which is most in accord with the facts. The poison or poisons are generated in the stagnant and putrefying contents of a dilated stomach. Bouveret and Devic separated from the stomach contents of a patient with hyperchlorhydria and gastric retention a poison, soluble in alcohol, which produced convulsions in animals. In their opinion, hyperacidity is a condition to which considerable importance should be attached. The frequency of its occurrence is attested by the fact that in many of the records it is especially obseryed that the vomited matters were intensely acid or sour. If this theory of autointoxication were correct, it would naturally be expected that the injection of the untreated stomach contents into animals would produce some convulsive seizures resembling those occurring in man. All such experiments have, however, entirely failed. Carnegie Dickson, who has very fully and very ably recorded a most interesting case of gastric tetany, quotes Müller, Von Taksch, Berlizheimer, Blazicek, Landsteiner, Gumprecht, and Albu as all having failed to obtain results. In Carnegie Dickson's case no results were obtained from a series of inoculations into rabbits and guinea-pigs. Subcutaneous injections were made of the following solutions:

(I) Filtrate of unaltered stomach contents.

(2) Filtrate of stomach contents evaporated to dryness in vacuo over strong sulphuric acid at a temperature of $40^{\circ} \mathrm{C}$., and extracted with normal saline solution.

(3) Filtrate of stomach contents evaporated as in No. 2 ; extracted with alcohol; again evaporated to dryness, and then extracted with normal saline solution.

No result whatever, either of a paralytic or of a convulsive nature, followed these inoculations, though none were made by the intravenous method recommended by Bouveret and Devic. The auto-intoxication theory, therefore, though plausible and attractive, lacks any proof from the experimental side. 
We believe, as pointed out in a paper published by us (Lancet, November 26, I898), that in the second and third theories jointly the true solution may be found. A poison formed in the stagnant contents of the dilated stomach may be absorbed, and both invoke the tetanoid convulsion and make more responsive to peripheral irritation the nerve centres in the brain and spinal cord.

It is worthy of emphasis that in many instances an excess of free hydrochloric acid has been present in the stomach contents. To this, as already mentioned, Bouveret and Devic attach considerable importance as a factor in the etiology. Gumprecht and many others have found this condition of persisting hyperchlorhydria; but Blazicek, in one case, was unable to discover any trace of the free acid.

The relationship which may exist between gastric tetany and the condition of the kidneys is in need of further investigation. In many of the recorded cases albuminuria has been present; in some of these no naked-eye existence of kidney disease has been obtained at the autopsy; in others advanced chronic nephritis has been found. If the tetany be due to auto-intoxication, it is possible that the elimination of the toxin may have a damaging effect upon the kidney, and be the cause of an inflammatory change. Hemmeter has recorded three cases of nephritis which he thought owed their origin to auto-intoxication. The single case of albuminuria in gastric tetany that we have had under our own care was in the person of an old alcoholic.

\section{Pathology.}

In almost all the recorded cases of gastric tetany dilatation of the stomach has been a pronounced feature; indeed, only one authority, Albu, has admitted the existence of tetany without dilatation. The dilatation is due to stenosis at or near the pylorus, and is associated with thickening and muscular hypertrophy of the stomach walls. Carnegie Dickson observed in his case that in the height of the seizure the size of the stomach was greatly increased. As a rule, the peristaltic waves can be seen passing across the stomach, and we observed in one of our cases that 'the exacerbations of 
the tetanic state were always associated with a painful contraction of the stomach, and that when the wave of contraction had reached the pylorus, the latter, which was previously incapable of being felt, formed a distinct, hard tumour. The abdominal pain then became very intense, and the tetanic cramps in other muscles came on, or, if already present, became intensified.'

The ulcer at the pylorus may extend into the duodenum or be associated with separate duodenal ulcers (as recorded by Leube, Neumann, and others), or duodenal ulcer may alone be found (as recorded by Bamberger, Reuvers DujardinBeaumetz, and others). In Blazicek's case the gastric dilatation was due to the pressure of an enlarged stonecontaining gall-bladder upon the duodenum. Berlizheimer assigned the dilatation in his patient to the pressure of a pancreatic cyst on the duodenum. In one case, related by Müller, tetany occurred in connection with an hour-glass stomach. In fewer instances the pyloric obstruction may be caused by malignant disease, as recorded by Trevelyan and others, and in fatal cases a condition of ulcus carcinomatosum has been found by Bouveret and Devic and Riegel. In Trevelyan's first case malignant disease of the duodenum was found. In three cases out of a total of five under our own care submitted to operation, duodenal ulcer, associated in all with gastric ulcer, was found. In one of these there was well-marked chronic pancreatitis. An examination of the recorded cases has certainly seemed to show a large proportion of instances of duodenal ulceration. The conditions most frequently found associated with gastric tetany are pyloric stenosis, dilatation and hypertrophy of the stomach, and enduring hyperchlorhydria. The stenosis is due to the partial or complete healing of a chronic ulcer of the stomach or duodenum. Of the existence of this ulcer there is ample evidence in the history of prolonged indigestion, vomiting, and other characteristic symptoms.

\section{Treatment and Prognosis.}

The medical treatment of gastric tetany consists in frequent and thorough lavage, rigid dieting, and the administration 
during the height of an attack of sedatives, bromides of potassium or ammonium, or, in the severer crises, of morphine hypodermically. It has been said, and with some show of reason, that the washing out of the stomach excites the occurrence of tetany. The lavage is distinctly teasing and irritating, and if the cleansing of the stomach be inadequately done, an ample quantity of putrid fluid may be left to provide a toxin for absorption-absorption which is rendered easier by the washing away of mucus from the lining of the stomach. But the lavage-and the point needs emphasis-should be very thoroughly done, and the surgeon must not desist until the fluid returns absolutely clean. In one patient of our own no less than 40 quarts of fluid were used before the return water was clear, and the washing out took sixty-five minutes. The process of gastric lavage is too often performed in a hasty and very perfunctory fashion; but carefully and patiently done it cannot be in itself the cause of gastric tetany. As Clifford Allbutt says, 'Careful washing should rather cleanse and prevent evil than be itself the cause of tetany and convulsions.' Carnegie Dickson states that in the case related by him the feeling of gastric discomfort and the tendency to vomiting were considerably allayed by the following method, which Professor Greenfield has for several years adopted with very marked benefit in many cases of gastric dilatation:

After washing out the stomach with a solution of phosphate of soda, and previously, if necessary, with weak Condy's fluid or boric acid solution, a certain quantity-say ro ounces - of boiled milk, containing sodium phosphate in the proportion of I drachm to $\frac{1}{2}$ pint, is left in the stomach diluted with hot water.

Intravenous infusion of saline fluid may be adopted, or copious hot-water enemata may be given.

Surgical Treatment.-The final and the appropriate treatment in all cases of gastric tetany should, however, be surgical, as was first suggested and pointed out by one of us in a paper published in the Lancet for November 26, 1898 , where several cases are given as examples of successful surgical treatment. From what has been said as to the pathology of 
the condition, it is clear that in almost all cases there is a grave mechanical obstacle to the onward passage of the food. It is this obstacle which causes the dilatation and the hypertrophy of the stomach. To relieve this obstruction and to do away with the stagnation of the stomach contents surgical measures are necessary. In simple cases pyloroplasty or gastro-enterostomy will be the methods of choice; in malignant cases a partial gastrectomy or gastro-enterostomy, as circumstances dictate. In hour-glass stomach, gastrogastrostomy combined with gastro-enterostomy performed in the distal pouch will, as a rule, be the suitable operation.

Prognosis.-Death occurring as the result of gastric tetany may occur in the first seizure or after a long series of attacks. Marten's case terminated fatally in the first attack four hours after its onset. Trevelyan's patient, who had carcinoma of the duodenum, was first attacked with tetany on the morning of July 8,1898 , and died at 2 p.m. the same day. In other cases, as in Kussmaul's and in C. Dickson's, there may be intervals of perfect health between severe attacks.

The records given by many writers show that gastric tetany is an extremely serious and often fatal disease. Thus, in II cases accepted by Frankl-Hochwart as genuine, Io died; Bouveret and Devic record I 8 deaths in 23 cases; Albu, 3I deaths in 40 cases; Riegel, I6 deaths in 27 . But almost without exception such cases are of old standing. The chronic gastric ulcer, the stenosis which it causes, the dilated and hypertrophied stomach, have all been sources of trouble for months or for many years. It is only in the late stages of such cases that severe gastric tetany is seen. And such late stages are preventable. By treating all such cases surgically - and there is no other rational treatmentgastric tetany of the lethal type will soon be a forgotten disease. Even the slighter manifestations which we have mentioned-prickling, tingling, or numbness of the hands, occasional and transient cramps in the muscles of the extremities-even these are only seen in cases of long standing. The earlier adoption of surgical measures in these disabling conditions of the stomach-conditions in which there is grave mechanical impediment to the free working of the organ- 
will banish altogether that class of patient which furnishes us with the last desperate forms of gastric tetany.

Our own experience of tetany and of the severe muscular spasms in association with gastric dilatation leads us to think that we may take a more hopeful view if the cases be treated surgically at an early period. The following cases will serve to illustrate our views. In the first case the tetany was so pronounced that the question of strychnine-poisoning crossed our mind. In the second and third cases, though there were well-marked tetanic spasms, the cramps were limited to the extremities and the abdomen.

CASE 1.-A man, aged thirty-four years, was sent to us by his medical attendant in January, 1895 , with a view to having something done in the Leeds Infirmary to relieve his condition. $\mathrm{He}$ had been suffering for five years from pain after the ingestion of food, with attacks of vomiting. Latterly his condition had become aggravated, the pains occurring from half an hour to one hour after each meal, and being relieved only by vomiting, so that for the last six months he had had to give up his work as a printer. For some time before coming under our notice there had been very evident peristalsis from left to right in the epigastric region. Throughout the period over which his stomach symptoms extended, his bowels had for the most part been very constipated, but there were occasional attacks of diarrhœea. During this time he had attacks of what he described as 'cramps' in his limbs, and especially in his legs. He had lost very considerably in weight, as in December, 1892 , he weighed 9 stones $7 \frac{1}{2}$ pounds.

On examination, there was found well-marked dilatation of the stomach; but the feature of the case which presented most interest in connection with the present question was the occurrence while under observation of the severe tetanic spasms, affecting almost all the muscles of the body. So extreme were these and so widespread--the muscles of the trunk and of the cervical region, as well as those of the limbs, being affectedthat on January I 7 the question of possible strychnine-poisoning was raised. As palliative treatment of the stomach condition gave no relief, the stomach was exposed on January 24 , and the diagnosis of cicatricial stenosis, with hypertrophy of the pylorus, was confirmed. Pyloroplasty was done, a bone bobbin being employed to insure patency of the new pylorus. Recovery was uninterrupted, and, although the cramps were present up to the 
time of operation, he never had even a threatening of cramp afterwards. On Febriuary is 5 he was able to take a mutton chop for his dinner without inconvenience, and by March 2 (five weeks after the operation) he had gained $8 \frac{1}{2}$ pounds. Since then he has done very well, and two years after the operation he had quite recovered his strength, and was working as usual, his weight then being just over what it was in December, I892, and quite 2 stones above what it was at the time of the operation. There had been no recurrence of any 'cramps' or of muscular spasms of any kind. In a letter received at the beginning of 1899 , he speaks of some stomach disorder, but he adds: 'You will please understand that I am a new man to what I was when you saw me last.'

CASE 2.-A man, aged twenty-four years, was seen by us on October 5 , I897, in consultation with his private medical attendant, on account of severe painful cramps of the extremities and of the abdomen, with persistent vomiting. He gave the history of having had pain after food for several years previously, and of having vomited blood, since which time he had never been well and had gradually lost flesh. For some little time before we saw him he had vomited every day, unless the stomach was washed out, and from weighing over Io stones he had diminished to a little over 8 stones. There was well-marked dilatation of the stomach, but no pyloric tumour could be felt, and simple stricture of the pylorus was diagnosed. He was admitted to the infirmary in October, and on the 2 Ist pyloroplasty was performed, as the pyloric orifice would only permit the passage of a No. Io catheter. The longitudinal incision in the pylorus was sutured vertically over one of the bone bobbins. Recovery was uninterrupted, and from that time the cramps and vomiting ceased. He returned home within a month. On October 23,1898 , the following letter was received from him: "It is with a grateful heart I write to thank you for the good received at your hands twelve months ago. I am thankful to say I have never had any trouble with my stomach since. I feel it my duty to acknowledge the good I received, and also to thank you for your extreme kindness.'

CASE 3.-A woman, aged twenty-nine years, was sent to us from the East Coast, in the spring of 1895 , suffering from severe pain in the abdomen, associated with vomiting and loss of flesh. There was well-marked dilatation of the stomach, and the pain always started on the left side of the abdomen and passed to the right, on which side, just below the ninth rib, there was wellmarked tenderness, with rigidity of the right rectus muscle. Operation was declined, and we did not see the patient until nearly the end of the year, when she said that the pain in the abdomen was excruciating and recurred every day. She said it 
was associated with severe cramp in the limbs, especially in the legs and thighs, and that at night she was kept awake by the pain. Vomiting of large quantities occurred daily, and she was steadily losing weight and strength. Her symptoms began in I 888, and she had lost 2 stones in weight between that year and 1895. On December I4 we opened the abdomen, and found active ulceration of the pylorus, which was adherent to the gallbladder, liver, and abdominal walls, and was so much thickened as to form a distinct tumour. Pyloroplasty was performed after the adhesions had been separated. Recovery was uneventful and satisfactory in every way, and she returned home within the month. She had never any return of the tetanic symptoms, and a report sent in March was to the effect that she had gained flesh and was 'well.' In July, I 896 , we saw her again for stomach symptoms, but without much loss of flesh. In 1897 and I 898 the vomiting, loss of flesh, and well-marked stomach splash showed that the pyloric trouble had recurred, and as a distinct tumour of the pylorus was felt, which was believed to be simple inflammatory induration, gastro-enterostomy was performed in October, I 898 . She made a good recovery, and has been quite well since. A letter in December, 1899 , states that she is quite well and has fully regained her weight.

The interesting point in this case is that there was no return of the painful cramps in the limbs after the first operation.

Stenosis of the Pylorus due to Chronic Ulcer; Great Dilatation of the Stomach. 'Tetany, almost Fatal, PERSISTING FOR FOUR DAYS AFTER OpERATION.

CASE 4.-Mr. G., aged fifty, was brought on June 6, I903, to a surgical home in a state of profound exhaustion and almost pulseless. He gave a six years' history of illness. At first he had had pain from one to two hours after food, and on one occasion he vomited blood. The pain had continued off and on up to the time of our seeing him, but he had been able to continue his work until three months before, though he had had frequently to leave off to go and vomit. At first the vomiting occurred once a week, afterwards twice a week, and latterly every day. For three months he had been existing on what could be absorbed between the time of his taking it and vomiting, or recently between his taking food and having the stomach washed out in order to obtain relief, this scanty nourishment having been supplemented by occasional rectal enemata, so that he had been slowly starving, and when we saw him he looked like a living skeleton His 
extremities were cold and blue and his tongue dry. He was so weak that the motions were passed in bed unconsciously. For a few weeks tetany had been well marked, it being associated with painful peristalsis, but lavage of the stomach had given relief both to the peristalsis and the tetany. The urine had a specific gravity of I025, and contained a considerable amount of albumin and a trace of bile. The stomach was found to be markedly dilated, but no tumour could be felt. A diagnosis was made of chronic ulcer with pyloric stenosis. He was so ill that at first operation seemed hopeless, but as he was manifestly going to die unless operated on, a posterior gastro-enterostomy was performed early on the morning of June 3. The jejunum was joined to the posterior wall of the stomach by a bone bobbin. Immediately on his recovery from the anæsthetic champagne was given, and feeding carried out every half-hour. Needless to say that for a few days his progress to recovery was tardy and attended with considerable anxiety.

A curious point in his case was that the tetany persisted for four days after operation, and on the third day was very trying to the patient, as the cramps in the arms and legs were most painful. They were relieved by securing evacuations of the bowels by means of calomel and apenta water, and by the administration of subcutaneous saline injections containing glucose. After this improvement was steady, though slow, and he was able to return home at the end of the fifth week. Four months later he wrote to say that he was very well, had regained his normal weight, and was able to perform his duties as usual.

Though tetany occurs apart from gastric dilatation-for instance, in children during teething, when it is frequently associated with gastro-intestinal disorders, and not infrequently merges into general convulsions-and in other conditions possibly apart from stomach disorders, yet tetany or painful muscular spasms of a serious 'character are so frequently associated with gastric dilatation that one cannot ignore the association and pronounce it merely accidental. It is curious that we have seldom seen the symptoms in other than dilatation due to simple causes, such as adhesions of the pylorus to the gall-bladder or liver or stricture of the pylorus due to the cicatrization of ulcers. We have seldom seen it in simple unobstructive dilatation or in dilatation due to cancer of the pylorus, though Dr. E. F. Trevelyan gives single examples of its association with both in his paper; 
and in one of our cases of cancer tetany was well marked and very distressing. In the case of children suffering from tetany, which is usually accounted for by the irritation of teething, it may be advisable to examine the size of the stomach as a routine measure; for we suspect that in many cases it will be found that there is dilatation due to congenital stenosis or to spasm of the pylorus. We have shown elsewhere that pyloric trouble in children and young adults is much more common than has been generally thought.

In the case of adults suffering from painful muscular spasm and cramps in the arms and in the gluteal muscles, it may also be well to remember the frequent association of gastric dilatation and tetany; for on several occasions recently where we have been consulted for these conditions we have found well-marked stomach dilatation, previously quite unsuspected.

\section{Tetany in Association with Dilatation of the Small Intestine.}

It is certainly not generally recognised that tetany may occur in association with dilatation of the small intestine, though in the case of a male patient, aged sixty-two, whom we saw in November, Igo3, with Dr. Goodhart and Dr. Prichett, the symptom was well marked. The case was at first thought to be one of dilatation of the stomach due to pyloric stenosis, but after gastric lavage it was clearly proved that the dilatation was in the upper part of the small bowel and associated with stenosis of the jejunum.

That the condition may become serious, or even fatal, the following case, occurring in the Romford Union Infirmary, under the care of Dr. Dudley Greenfield, and reported in the Lancet, October ro, Igo3, will prove :

A married woman, aged thirty-three years, was admitted into Romford Union Infirmary, under the care of Dr. Greenfield, on August 29. She had had three children, the youngest of whom was nine months old. About twelve days before admission she had been seized with vomiting, and had been steadily getting worse. She had previously had no serious illnesses, but for some weeks she had been badly fed and cared for. The bowels had been relieved twice during this time on the administration of an 
enema. On admission the patient had a very worn appearance, and complained of lack of sleep owing to the vomiting. She had no acute pain at any time. The vomiting was distressing and very frequent. The vomit was distinctly fæcal in odour, and in appearance it was very like a characteristic typhoid stool. The abdomen was considerably distended and tympanitic on percussion, the normal areas of dulness being preserved. It moved fairly well on respiration, no pain was caused on palpation, and no tumour could be felt. The abdominal wall was not in the least rigid, and no peristalsis was visible. The bowels had been relieved two days previously after an enema, and the patient's general condition was fairly good. A soap enema was administered, and a very large evacuation of clay-coloured fæces resulted, and about half an hour afterwards the bowels again acted freely. The patient expressed great relief, and said that she 'felt a different woman.' The distension of the abdomen was considerably less. She was given albumin water only by the mouth and plain water when thirsty. Five grains of calomel were given, but she vomited shortly after. In the evening she was more comfortable, the vomiting was less, and the bowels were moved slightly after an enema. The tongue was moist, the pulse was good, and the abdomen was only slightly distended.

On August 30 the patient had passed a good night, and felt much better, as she had not slept for several days. The vomiting was only occasional. The vomit had lost its fæcal odour and was only bilious. There was a good result from an enema, the fæces being yellowish and loose, and no longer clay-coloured. Another 5 grains of calomel were given without result. The abdomen was said by the patient to be of about the natural size. Albumin water was still the only food given, and she had also ro grains of salol every four hours. In the evening she had two typical attacks of tetany, involving the hands, the feet, and the face, and lasting about half an hour on each occasion, giving the patient much alarm. The marked abatement of symptoms, the disappearance of the fæcal odour from the vomit, the four actions of the bowels, and the approach to normal of the last motion, all seemed to negative a diagnosis of intestinal obstruction other than that due to impacted fæces. The possibility of a gastro-intestinal fistula was considered, and some disease of the pancreas was suggested by the whitish fæces, but nothing ${ }_{j}$ could be felt on deep palpation of the abdomen. On the 3 Ist the vomiting had ceased entirely, and the bowels acted freely after an enema. The pulse was good, about 9o, and the temperature was slightly subnormal. She had another attack of tetany lasting about forty minutes. On September I the patient had slept well after an injection of 
morphia given fur the attack of tetany on the previous evening. There was no return of the vomiting, and she took plenty of albumin water and fluid by the mouth. She was also given nutrient enemata of starch, eggs, salt, and milk to 8 ounces every six hours, but the bulk had to be reduced owing to a bad tear of the perineum (not recent) and difficulty in retention. The bowels acted when the rectum was washed out with saline solution preparatory to the administration of the nutrient enema. The only adverse symptom at this time was the subnormal temperature, but the pulse was good and the extremities did not feel cold. On the and the bowels were opened after the wash-out of the rectum in the morning. The albumin water. was well retained, as were also the nutrient enemata. In the evening she became very cold and collapsed, but improved again when some stimulant was administered. On the $3^{\text {rd }}$ the patient was better again. The fond given by the mouth was increased, and the nutrient enemata were administered only twice daily. She took I $\frac{1}{2}$ pints of peptonized milk, 2 ounces of pounded beef, some essence of beef, beside I pint of albumin water, and retained it

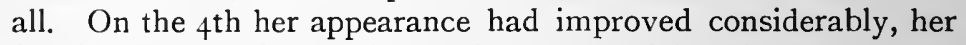
face being much fuller. The pulse was good, but the temperature was still slightly subnormal. Slight peristalsis was visible in the morning, and in the afternoon the vomiting returned. On the $5^{\text {th }}$ the patient had vomited frequently in the night, small quantities of bilious material being brought up. The bowels were moved several times quite naturally; the fæces were loose but normal in appearance, no blood or mucus being present. Peristalsis was again seen in the morning, and her friends were advised that an operation would probably be required. In the afternoon, however, she became rapidly worse, and died unexpectedly.

Necropsy.-A partial examination was permitted. On opening the abdomen some very distended coils of small intestine lay in front, matted together by some tough adhesions. These proved to be the upper part of the jejunum, the lower half of the jejunum and the ileum being empty and contracted. The junction of the distended and contracted portions was matted firmly down behind, close to the root of the mesentery. A finger passed down from above easily went through the point of apparent obstruction, and that hefore the positions of the parts were much disturbed. On unravelling the coils no cause of obstruction could be determined. There was no fibrous stricture, and no evidence of dragging or strangulation could be demonstrated. There was very slight capillary injection of the lowest end of the distended portion, but there was no extravasation of blood, no lymph, or any sign of recent peritonitis. The pancreas was apparently healthy, and 
the peritoneum showed no hæmorrhages or fatty masses. The stomach was not dilated, and was free from any sign of ulceration. The rest of the abdominal viscera and the heart were quite normal in appearance.

Remarks.-From the post-mortem examination it is clear that the primary trouble was acute dilatation of the upper part of the small intestine. No obstruction could be discovered, although the patient had old peritoneal adhesions, and seeing that the bowels were opened well on each day that she was in the infirmary (eight days in all), and that on the last two days the bowels were opened naturally several times, no obstruction was probably present. Was this, then, a paralytic distension? The occurrence of tetany was very interesting in a case of this sort. It was fortunate that no operative interference was attempted, as the utmost that could have been done was to have relieved the distended bowel, and any attempt to arrive at the point of apparent obstruction would have been useless and impracticable owing to the dense bands of fibrous tissue. The post-mortem examination proved that the suspicion of pancreatic disease as a cause of these conflicting symptoms was unfounded.

The subject is mentioned here, as it has a very direct bearing on the differential diagnosis of gastric tetany.

\section{REFERENCES.}

Albu, Volkmann's Sammlung, I 897-I 9oo, p. I 499.

Bouveret and Devic, Rev. de Med., I892, vol. xii., p. $4^{8}$.

Blazicek, Wien. Klin. Woch., I894.

Berlizheimer, Bevl. Klin. Woch., I 897 .

Carnegie Dickson, The Practitioner, vol. i., I903.

Fleiner, Arch. fïr Verdannngskr., I 895, vol. i., p. 243; and Mïnch. Med. Woch., 1903, vol. i., p. 853 .

Frankl-Hochwart, Nothnagel Spec. Path. u. Ther., I 897, Bd. 2.

Gumprecht, Cent. f. inn. Med., I 897.

Kussmaul, Arch. f. Klin. Med., I 869 .

Loeb, Deut. Arch.f. Klin. Med., I 890.

Marten, Lancet, vol. i., 1887 , p. 74.

Müller, Charité Annalen, I 889, vol. xiii., p. 309.

Neumann, Deutsche Klinik, I869.

Riegel, 'Diseases of the Stomach,' English Edition, p. I63.

Trevelyan, Lancet, vol. ii., I 899, p. 791.

Mayo Robson, Lancet, vol. ii., I 898, p. I392. 


\section{CHAPTER XXIV \\ PERIGA S TRITIS}

AdHesions of the stomach and pylorus to neighbouring organs are a common result of perigastritis, the causes of which may be extrinsic or intrinsic. Among the extrinsic causes are gall-stones, tuberculous peritonitis, local peritonitis from any cause, such as intestinal ulceration, or from malignant growths in adjoining organs, typhoid fever, and possibly other fevers. Among the intrinsic causes, ulceration is by far the most frequent, though cancer, tubercle, and syphilis of the stomach must aiso be included.

Though, at the time, adhesions the result of perigastritis are conservative, and may frequently result in the saving of life by limiting the peritonitis, the result of perforation or of bacterial infection due to ulcer, yet later such adhesions may lead to great functional disturbance by interfering with the motor activity of the stomach and causing pain, disability, and other ailments. As we shall hope to show, these disturbances caused by adhesions may not be merely of a functional nature, producing dyspepsia and so-called visceral neuroses; but they may, and frequently do, cause dilatation of the stomach, vomiting, severe pain, and great loss of flesh.

In I893 one of us read a paper before the Clinical Society of London on the subject of pyloric adhesions, and related several cases which had been cured or relieved of longcontinued stomach symptoms by their division.* This was, we believe, the first time that attention had been drawn to

* Transactions of the Clinical Society, 1893 : 'Dilatation of Stomach and Severe Pain dependent on Pyloric Adhesions,' by A. W. Mayo Robson. 
the importance of adhesion around the pylorus as a cause of dilatation of the stomach and other digestive troubles. Since that time our experience on this subject has considerably extended, and we can now point to a very large number of cases (over a hundred) of pyloric or other stomach adhesions, caused in some cases by gall-stones, in others by gastric ulceration, where the adhesions, though not the original disease, were of the first importance in producing the symptoms for which operation was undertaken, and where gastrolysis led to their relief or cure. Though in some cases the adhesions may be due to a perforating ulcer, it is by no means necessary that ulceration need be near perforation to lead to perigastritis; for in many cases, long before the peritoneum is reached by the ulcer, a local peritonitis occurs, leading to the pain and well-known local tenderness which characterize ulcer of the stomach, and then lymph is thrown out which may fix the inflamed region to contiguous organs.

Adhesions are found around the stomach in about 5 per cent. of all necropsies, and about 40 per cent. of all cases of ulcer of the stomach are associated with adhesions. Our experience in a very considerable number of abdominal sections, over $\mathrm{I}, 000$ of which have been in the upper abdominal region, shows that pyloric adhesions are very much more common than is usually supposed, and that their importance is greater than is generally recognised even at the time of operation. This is especially the case in cholelithiasis, where we are accustomed to find gastric dilatation as a regular sequence of gall-stones, and to look for it as an ordinary concomitant sign. In such cases it is nearly always due to adhesions of the pylorus to the gall-bladder and liver.

Sometimes the adhesions may be strictly limited to the pylorus and gall-bladder, leading to a characteristic train of symptoms. In a case under our care 'acute dilatation' of the stomach came on suddenly and ended fatally within thirty-six hours, apparently due solely to pyloric adhesions, as a post-mortem examination revealed no other cause for the obstruction (see p. 39I). At other times adhesions may 
extend from the pylorus along the lesser curvature of the stomach, fixing the upper border of the stomach firmly to the gall-bladder, cystic duct, and under surface of the liver. When the pylorus is tied up in this abnormal position, not only are the natural movements of the stomach interfered with, but the food has to be forced out at a higher level, and when the pylorus is alone fixed a kink forms, leading soon to well-marked dilatation with flatulence, chronic dyspepsia, and loss of flesh. Where perigastritis is due to ulceration, the adhesions arc only found over the site of the ulcer, and if this be at the pylorus a double difficulty is presented, for not only is there fixation of the pylorus at an abnormally high level, but the pyloric orifice itself becomes stenosed from the contraction due to the healing of the ulcer. If the ulcer happens to be on the anterior wall of the stomach, it may lead to fixation of the stomach to the anterior abdominal wall and to hour-glass contraction, as in cases related under that heading.

The symptoms vary according to the site and cause of the disease, from mere dyspepsia to serious apepsia, and the brief descriptions of cases given at the end of this chapter are selected as illustrating the different causes and effects of gastric adhesions.

Although cancer is a well-recognised cause of perigastritis in every region of the stomach, the symptoms from adhesions are merged in the more serious ailment; it is, therefore, unnecessary that we should further consider them at this moment, except to say that they seriously add to the difficulty and danger of pylorectomy or partial gastrectomy. The cases mentioned can leave no doubt in the minds of our readers as to the serious effect of pyloric and gastric adhesions, although it is a curious fact that many recognised authorities on stomach diseases barely mention them. The remarks that were made by several eminent physicians at the reading of the original paper on this subject before the Clinical Society of London showed that, although the subject was not unknown, its importance was certainly underrated. That we are not exaggerating its importance is shown by reports of cases by other observers. For instance, Hart- 
mann and Soupault (Revue de Chirurgie, I899, p. 340) relate cases where adhesions were the only apparent cause of gastric dilatation. Terrier, in his work on 'Stomach Surgery' (I899), relates a case of a woman, aged sixty-two years, whose gastric symptoms were relieved by breaking down adhesions.

Bireto related a very instructive case, resembling one above described, where a band was found attached to the lesser curvature of the stomach, about $\mathbf{I}_{\frac{1}{2}}$ inches from the pylorus, which ran obliquely downward to the anterior abdominal wall on the right of the umbilicus. When the stomach was full, the band dragged the upper border downwards, and produced a kink which led to stenosis. A case related by Mr. H. W. Page (British Medical Journal, January 23, I899) is a good example, and many others can now be found scattered through medical literature.

In a case that we saw recently in a young married man of twenty-nine, who had signs of dilatation of the stomach, with dyspepsia, loss of flesh, and general malaise, and who had been treated off and on without benefit for fourteen years, we found the great omentum adherent to the pelvic brim and the pylorus fixed firmly to the liver, with the result that when the stomach was full the pylorus was being dragged on, and when empty the lower border of the stomach was in a state of tension. The condition was remedied by gastrolysis combined with gastro-enterostomy.

The diagnosis in these cases rests on a careful consideration of the previous history, together with the presence of well-marked signs and symptoms, so that it is not generally difficult. There will usually be a history of gall-stones, typhoid fever, ulcer of the pylorus, or some local peritonitis in the pyloric region, months or years previously. This will have been followed by indefinite dyspepsia and flatulence, which later takes a very definite shape, consisting of discomfort and pain after food, especially after a full meal, so that the patient prefers to take several small repasts rather than one or two large meals. The pain and discomfort are at once relieved by the recumbent posture, so that a necessity to lie down or rest after every meal is suggestive. Relief is 
frequently found by the wearing of a belt, and this is usually discovered by the patient before advice is sought. The pain is often of a dragging character. At times it may be acute, and it is increased or brought on by lifting or reaching, say, to adjust a picture. Walking or any active exertion is shunned, and inability to perform ordinary duties is complained of. When dilatation of the stomach is well marked there may be loss of flesh and vomiting every few days, often in large quantity.

Besides the ordinary signs of dilatation of the stomach and the tenderness between the umbilicus and the ninth costal cartilage, as shown by the rigid right rectus, there is an important physical sign to be obtained by distending the stomach with gas, when, in case of pyloric adhesions, the cavity of the stomach will be found to extend further to the right and higher than normal. An entire cessation of pain and freedom from dyspeptic troubles if the patient be confined to bed is suggestive, for it will be seen that under these circumstances the higher level of the pylorus is done away with, and the recumbent posture prevents the dragging of adhesions; but, after all, an exploratory operation is the only way of setting doubt at rest, and if these distressing symptoms have resisted long-continued medical treatment, a surgical operation is clearly justifiable, though doubtless in some of the less severe forms careful medical treatment may lead to an amelioration of symptoms, and may give such marked relief that an operation will not be called for. Relief may be obtained by advising the patient to take small meals, to avoid bulky and fermentable foods such as potatoes and bread, to rest after meals, and to wear a well-fitting abdominal belt.

In some cases where relief ensues without operation, it is probably due to the stretching of adhesions and to their absorption, if the exciting cause has fortunately been removed; but in the more severe cases, where the adhesions are almost universal, such a fortunate termination of symptoms is scarcely to be hoped for, and in some cases the adhesions are so extreme that nothing short of gastroenterostomy can prove of any avail. 
Gastric adhesions may give rise to no symptoms, but usually they interfere with the motor functions of the stomach. Adhesions may be suspected $(a)$ when there is a widespread tenderness in the epigastrium, extending beyond the stomach area, and assuciated with rigidity of both recti ; (b) when lavage and other treatment fail to relieve the deficient motor functions; and $(c)$ when after treatment for gastric ulcer the pain and tenderness persist.

\section{Gastrolysis.}

We have heard it asked, "What is the use of simply detaching adhesions?' Fortunately, our experience in this class of cases is sufficient for us to give a, very direct answer to this query, for we can point to many cases of cure where men and women, formerly invalided by pain and dyspepsia, are now leading active and useful lives. Slight adhesions we are accustomed to separate with the fingers, firmer bands to divide between ligatures, going carefully to work until the pylorus is quite free. Where the omentum is available, we usually bring the right border upwards, and leave it between the pylorus and the gall-bladder and the liver, so that, should any adhesions form again, they will be in the form of a loose mesentery, and not binding, like adhesions to fixed organs.

The operation of gastrolysis, where adhesions are solely responsible for the stomach trouble, is a perfectly safe one, and in a large series of operations we have had no fatality. In the Hunterian Lectures we collected from all sources 77 operations, the rate of mortality being 2.5 per cent. Of these, 47 were personal cases without a death; but this series has been extended to over a hundred cases.

Wherever the adhesions are very firm, and especially where they are extending over a wide area, great care must be exercised in separating them, and when separated, in examining the stomach carefully to see if there is a fistula left, for in no less than seven cases we have had to close perforations under such circumstances; and in several of them there was a clear history of perforation of gastric ulcer, 
with subsequent recovery, though the symptoms of ulceration had persisted and necessitated operative treatment.

A case of stomach and gall-bladder fistula seen in a lady of fifty-four, due to perigastritis of extrinsic origin, is described in the chapter on Fistula. When the adhesions were separated there was a passage into the stomach readily admitting a No. 6 catheter, which was easily closed by a purse-string suture, the gall-bladder opening being used for drainage, the patient making a good recovery.

In another case, that of a middle-aged lady, seen with Dr. Johnstone of Ilkley, on separating the pylorus from the liver, an opening was found the size of a threepenny-piece leading into the stomach, the base of the ulcer being formed by indurated liver tissue. The case is more fully described under Pyloroplasty.

Curiously, on one day while operating at the Leeds Infirmary, we had two consecutive cases where recovery from perforation had occurred, leaving very firm adhesions. In one case, that of a middle-aged man, a patient of Dr. Robinson's of Low Moor, there were very firm adhesions of the pylorus to surrounding organs and to the omentum, and on separating the latter in order to perform pyloroplasty the round opening of a perforation was quite distinct. Dr. Robinson said that he recollected the date of the accident several months previously, and that he had prepared the patient for operation then, but under rest, opium, and abstention from food, he recovered. The ulcer was excised and pyloroplasty performed, the patient making a very good recovery. The next case was one of hour-glass contraction due to chronic ulcer, and here the history of perforation was also distinct, the patient at the time having been treated by rectal feeding for a week and having been kept in bed for a month. Excision of the ulcer and posterior gastro-enterostomy were followed by recovery.

In another case, of a middle-aged woman, a very firm adhesion to the anterior abdominal wall was evidently the site of a perforation, the history of which was very clear, and suspected by her medical man at the time, though the patient recovered. On separating the adhesions there was an open- 
ing admitting a No. 5 catheter leading into the stomach. It was readily closed by inversion of the edges and the use of a purse-string suture.

We have in the same way found adhesions to the large and small intestine, pancreas and diaphragm.

The lesson to be learnt is that due care must be taken in separating firm adhesions, and the parts must also be carefully examined subsequently, not only for the arrest of hæmorrhage, but also to see that there is no fistula left.

Where gastric adhesions the result of perigastritis are present and not producing symptoms, being only discovered accidenially during the performance of other operations, we would not always counsel their division unless it can be readily done, since they undoubtedly do exist at times without serious detriment to the patient. If the pylorus is patent, yet embarrassed by adhesions, we always separate them, and try to avoid their recurrence by interposing the right free border of the omentum between the raw surfaces left by the gastrolysis, thus substituting a long, freelymovable attachment for short, binding ligaments, should adhesions re-form. If, however, adhesions are very extensive, very short, dense, and firm, the operation of posterior gastro-enterostomy had better be done, as in some of the cases related below, and this especially if at the same time there is stenosis of the pylorus or an hour-glass stomach.

M. Merklen (Société Médicale des Hôpitaux, Paris, January, I899) related the case of a man, aged forty, who, having had for three weeks crises of epigastric pain which radiated into the retrosternal, and even submaxillary, regions, was on November 10, I896, suddenly seized with acute pain in the left flank and all the signs of gastric perforation. The peritonitis was limited by previous adhesions, and a subphrenic gaseous abscess formed. On December 2 nearly a litre of pus was evacuated. The patient seemed to have perfectly recovered. But in January, 5897 , severe pains again occurred. They came on five minutes after taking nourishment, and were increased by standing and walking. They originated in the pit of the stomach, and radiated behind the sternum and into the arms to the little fingers, thus resembling angina pectoris. Each attack lasted from thirty to forty-five minutes. After fourteen months of suffering, an operation was performed in March, 1898 . 
The stomach was surrounded by adhesions, which were divided, except the principal one, which was very dense and connected the stomach and liver. The patient appeared to be relieved, but the crisis recurred four weeks later with the same localization and the same radiation. But they differed in not being provoked by food, appearing solely when the patient was standing or walking. Thus, gastric peristalsis was not painful, but the dragging on the gastro-hepatic adhesion in the upright position, especially after food, was. There was a hard and painful induration in the epigastric region. But for the history, cancer might have been suspected.

In June the part of the liver adherent to the stomach was removed with the thermo-cautery, and the wound was closed with catgut sutures. The gastric ulcer was resected in the same way. Permanent relief followed.

As to the diagnosis of perigastritis, M. Merklen argued that persistence of pain in spite of treatment was an indication, and the epigastric induration a proof. The pains were of two kinds-those produced by food and those produced by standing. The latter were explained by the position of the lesion, which was usually gastro-hepatic. Treatment consisted in dividing the adhesions or in resecting the ulcer and inflammatory new growths.

In the case of Mrs. R. (see p. 407) a second operation was necessitated by the pain solely caused by extensive adhesion following on the operation of gastroplication ; for, although the stomach had been diminished in volume by the previous operation and the pylorus had remained patent, the adhesions had produced so much pain and distress during peristalsis and on exertion that the patient was entirely incapacitated and bedridden. Gastrolysis was performed, but as the adhesions were so very extensive, in order that the relief might be permanent and complete, it was considered advisable at the same time to perform posterior gastro-enterostomy. The patient made a good recovery, lost her pain, and is now well.

The following are illustrative cases : 
Ulcer of Stomach; Dilatation; Perigastritis. Gastrolysis.

Mrs. A. H., aged forty-one, seen September, 1898. Fourteen years ago the patient first noticed pain and tenderness at the epigastrium, which had been worse since January, and were increasing in severity. The pain was paroxysmal, not worse or specially present after food, though occasionally food caused pain. Since January she had vomited frequently. It was years since any blood was brought up. No certainty of loss of weight. On two or three occasions fæces had been blood-stained. She had never been jaundiced. No fulness of epigastrium. Visible peristalsis. General tenderness of abdomen on palpation, but especially over epigastric region. Splashing sounds obtained.

September 28, 1898.-Operation. Extensive adhesions found between gall-bladder, liver, and pylorus. A cicatrix found in the wall of the stomach. There was no stricture of pylorus, but it was found kinked owing to the dilated stomach dragging on the adhesions. Omentum placed between surfaces to prevent further adhesions.

Recovery satisfactory, and patient able to take food well. Later reports satisfactory in every respect.

Pyloric Ulcer; Perigastritis ; Adhesions. Gastrolysis.

Mrs. M. H., aged forty-one, seen with Dr. McGregor Young. For months pain beneath left clavicle and indigestion, with loss of flesh. Recently noticed tumour in upper abdomen. Dilated stomach ; movable painless tumour in upper abdomen attached to liver.

Operation. Dilated stomach. Perigastritis, with adhesions of pylorus to gall-bladder and liver. Tumour was abnormal projectiơn from left lobe of liver. Adhesions separated. Very good recovery.

1900.-Health regained.

\section{Perigastritis ; Adhesions.}

Dr. L., aged thirty-three. Ten years ago obstruction of bowels, lasting a week, with vomiting and abdominal colic. Similar attack a few months later; since then subject to bilious vomiting. For two years dyspepsia and flatulence with constipation, but no marked jaundice. For two years pain in right hypochondrium, relieved by taking food. Never vomited blood. Loss of flesh and increasing debility. Well-marked tenderness over gallbladder and pylorus, with dilatation of stomach. 
December 4, 1895. - Operation. Dilated stomach; pylorus tucked up to liver and kinked. Adhesions round pylorus separated. No gall-stones present.

Good recovery. By March 3, 1896, had gained a stone in weight.

Igoo.-Well, and has been engaged in full, active work as a medical man in a large country practice ever since he recovered from his operation.

\section{Perigastritis following Cholelithiasis.}

Mrs. M. Z., aged thirty-six. 'Spasms' for twenty years. Cholecystotomy in Durban, Natal, ten months ago. Four gallstones removed. After healing of wound, in five weeks return of pain. Pain always after food, and at times vomiting; never vomited blood. Losing flesh rapidly. Dilatation of stomach. No tenderness over gall-bladder.

July 6, I 897.-Operation. Adhesions between pylorus and gall-bladder and liver broken down, and omentum interposed.

July 6, I898.-Patient writes: 'I now feel a different person, and enjoy perfect health.'

\section{Perigastritis.}

Miss M. S., aged twenty-five, seen with Mr. F. H. Mayo, Headingley. Dyspepsia without vomiting for sixteen months; some pain after food. Latterly pain and dragging continuous, chiefly in epigastrium. Loss of flesh. Dilated stomach. Dieting and rest gave no relief. Small ovarian tumour found on pelvic examination under ether.

July 25, I 899.-Operation. Pylorus adherent by long band to right anterior abdominal wall. Adhesions separated. Ovariotomy for tumour performed at same time.

Well February, Igoo. - Had lost previous troubles and had gained weight.

\section{Perigastritis ; Cholecystitis.}

Dr. D. A., aged forty-one, seen with Dr. Turner, York. Ill since February, I 898 , when he had influenza, followed by scarlet fever in March; in same year repeated similar seizures. At beginning of 1899 was jaundiced for six weeks. Latterly gastric symptoms very prominent. Pain and dragging after food; occasionally vomiting. Lost 2 stones 3 pounds in weight in last sixteen months.

Examination. Rigid right rectus; tenderness below ninth costal margin. No jaundice; dilatation of stomach. 
September 22, 1899 .- Operation. Extensive adhesions of pylorus and pyloric extremity of stomach to liver and gall-bladder, which was contracted. Gastrolysis and cholecystotomy. Adhesions separated and gall-bladder drained.

Well January, rgoo. Regained lost weight, and able to take active service in South Africa.

\section{Perigastritis.}

Dr. M. D., aged forty-nine, seen with Dr. McGregor Young, Leeds. Dyspepsia for two years, with progressive loss of weight, to the extent of 30 pounds within the last year. No history of ulceration. Questionable cholecystitis following influenza.

Examination. Rigid right rectus, with little tenderness. Stomach dilated. Patient thin, but not emaciated.

December 7, 1899-Operation. Many small bands, fixing the pyloric extremity of the stomach to the gall-bladder, and causing a kink in the first part of the duodenum. Separation of adhesions.

Good recovery. Gained I stone within two months.

\section{Adhesions after Abdominal Operations.}

The subject is cne of such importance to the surgeon that any exact record of clinical study or experimental work merits attention. Vogel (Deut. Zeit. fïr Chir., Bd. 63, 296) made a number of experiments on animals, and studied five cases in the human subject, of which four died owing to recurring adhesions causing obstruction.

He gives the following as causes:

I. Hæmorrhage from intraperitoneal wounds, in so far as the injury hinders absorption, causes clotting, and by means of the clots occasions broader adhesions than would arise from the injury per se.

2. Mechanical irritation and injury.

3. Sloughs which are sufficient to hinder primary union.

4. Chemical irritants insufficient to hinder union.

5. Foreign bodies.

6. Infection.

7. Quietude of the intestine markedly favours the formation of adhesions.

He recommends aseptic instead of antiseptic technique; the avoidance of unnecessary injury to the peritoneum 
(especially such as is caused by the use of sharp hooks); avoidance of the cautery, and careful suturing (wire being better than silk).

He says Müller's method of using normal saline solution and the employment of gold-beater's skin are not effectual in preventing adhesions re-forming after operation, but that gum arabic solution acts well (gum arabic, I; normal salt solution, 2, filtered and sterilized). It is applied as follows: After almost complete closure of the wound, a drainage tube is passed into the abdomen; through this the solution is injected, the tube removed, and the suture completed. To regulate peristalsis, subcutaneous injections of atropin, and, still better, of salicylate of physostigmin, were used. In one case after appendicectomy the latter drug was used in 0.0004 dose, from two to four times daily, with good effect.

R. T. Morris (Medical Record, May 3, Igo2) speaks well of Cargile's method of applying a thin variety of gold-beater's skin from the peritoneum of the ox over roughened peritoneal surfaces. In rabbits experimented on the results were satisfactory. 


\section{CHAPTER XXV}

\section{GASTRIC FISTULA}

GASTRIC fistula, a sequel of perforation, may be found occasionally on the surface of the abdomen, very rarely in the loin, in both of which cases it may be termed external gastric fistula; but not infrequently fistulous communications occur between the stomach and adjoining organs: in such cases they are known as internal gastric fistulæ. Gastric fistula may be pathological or traumatic. The following is an etiological classification :

Pathological.-Extrinsic: Cancer of the gall-bladder or bowel; gall-stones; empyema of the gall-bladder; abscess of the liver; abscess of the pancreas; ulcer of the bowel-simple, malignant, or tuberculous; subphrenic abscess bursting into the stomach. Intrinsic: Ulcer of the stomach or pylorus; cancer of the stomach or pylorus; foreign bodies in the stomach ulcerating into neighbouring organs; subphrenic abscess of gastric origin.

Traumatic.-(a) From injury, either by a wound inflicted from without inwards, or from perforation and escape of a foreign body previously swallowed ; and $(b)$ from operationgastrostomy and gastro-enterostomy.

Ulcer of the stomach is by far the most frequent cause of fistula. It may lead to perforation of the diaphragm, and thence to perforation of the pleura and the lung; to perforation of the pericardium and the heart; to general emphysema, owing to perforation and entrance of air into the mediastinum; as well as to the more ordinary cases of perforation of the skin, leading to surface fistulæ, to perforation of the colon and gastrocolic fistula, to gastroduodenal, gastrogastric, and other fistulæ. 
The symptoms vary according to the cause and the site of the fistula. If it be between the stomach and upper bowel, the symptoms may be slight or absent; but if it be between the stomach and the large bowel, the food will pass too rapidly through the alimentary canal, and be parted with before absorption is adequate for nutrition; and, on the other hand, gases from the colon entering the stomach will give rise to fotid eructations, at times to feculent vomiting, and always to chronic dyspepsia.

In a case of fistula between the gall-bladder and stomach, dependent on gall-stones, in a woman of fifty years of age who came under our observation, there was great loss of flesh, with frequent vomiting of bile; for though it is known that healthy bile may pass through the stomach without serious harm to nutrition, the pathological products from an inflamed gall-bladder, together with the infected bile associated with cholelithiasis, seemed in this case to have a very deleterious effect. After opening the abdomen, the fundus of the gall-bladder was found to be firmly fixed to the stomach near the pylorus, and on separating the adhesions the openings into the gall-bladder and stomach were found well within reach. The stomach fistula was closed by two layers of sutures, and then the gall-bladder was brought to the surface and a tube inserted for drainage. The patient made a very satisfactory recovery, and is now quite well, having gained considerably in weight.

In another case of abscess between the liver and pylorus, the stenosis caused by the ulcer produced such serious symptoms of itself that it was difficult to estimate the part played by the abscess. The ulcer was excised, and the wound stitched up transversely to the pyloric axis, the cavity in the liver being scraped out and purified. The patient is now in excellent health.

In a case of fistula that we found between the stomach and pancreas there was dyspepsia, with pain after food, vomiting, and great loss of flesh; but in this case the stenosis of the pylorus and consequent dilatation of the stomach seemed to account for the greater part of the trouble. 
At the Gesellschaft für Innere Medizin, Berlin, Igo3 (Medical Press, April, I 903), Hr. Eisner reported a case of gastrocolic fistula. As a result of a gastric ulcer a communication was formed from the stomach, not into the small intestine, but directly into the colon, so that the food went from one into the other without passing through the intervening small intestine. The food was therefore not digested, and it acted as an irritant on the colon. The result was that the patient's nutrition suffered seriously; she became much emaciated, and a persistent intestinal catarrh rendered the condition still worse. The patient, who was operated on three years previously, vomited blood when she was nineteen, and suffered from severe gastric pains, and the case was then treated as one of ulcer ventriculi. Nineteen years later, i.e., when the patient was aged thirty-eight, the signs of gastrocolic fistula developed with violent symptoms, with persistent vomiting and diarrhœe. The patient was so much emaciated that she weighed only 82 pounds. Examination showed that the vomited matter looked and smelt like fæces. Raw scraped flesh passed away in the evacuations almost unchanged. An operation was performed by Eugen Hahn, who found the fistula on the posterior wall of the stomach near the cardiac end and opening into the left flexure of the colon. As the fistula was not accessible, an anastomosis was made between the transverse colon and the duodenum. The result was excellent, the patient regaining her health from the date of the operation. Only one similar case was on record, one of Unruh's, who carried out a similar operation in a similar condition of affairs.

External fistulæ, if small, may cause little loss of health; but if extensive, the leakage from the stomach of food and gastric juice leads to great distress from the soreness of the skin around the fistula, and to loss of flesh from the waste of food.

Kronheimer (Deut. Zeitschr.f. Chiv., October, 1899) reported a case, verified by post-mortem examination, after an unsuccessful operation. The patient, aged forty-five, was subject to anæmia for nine years; she had never been pregnant, and the 'periods' ceased for two months. Dyspeptic pains had lasted for some time, and fixed pain was at length localized to a point a little below the left hypochondrium. At length violent vomiting and paroxysmal aggravation of the fixed pain took place. The pain was trifling when the patient fasted. A hard swelling developed at the site of the pain. Five months before admission into hospital it grew red, then soft, and burst a fortnight before; dark 
fluid and pieces of food began at once to escape, and the pain diminished. Emaciation was advanced; the integuments were intensely irritated. Fluids swallowed by the patient escaped in jets through the fistula, and very little nutritive material was digested; hence feeding through the mouth or through the fistula was impracticable. Nutrient enemata were given, but the patient clearly could not be kept alive for long by such means. Hermes undertook an operation for the excision of the fistula, but the patient took the anæsthetic badly, and the heart's action was very weak; there was also double bronchitis. He therefore, having made a median incision above the umbilicus, did an enterostomy, opening the jejunum directly after its transition from the third part of the duodenum. His aim was to feed the patient through the jejunal fistula till her strength would allow of a more complete operation. This was practicable, but peritonitis set in, and the patient died on the ninth day. A large oval chronic ulcer was found in the anterior wall of the stomach, corresponding to the fistula.

Case under the Care of Mr. C. F. M. Althorp (Medical Press, February 6, I901).- The patient was a woman, aged fifty, who for fifteen months before admission had suffered from abdominal pain and vomiting. On examination, she was pale and emaciated. The abdomen was flat. Just below and to the left of the umbilicus was a tender swelling, about 3 inches by 2 inches, in the abdominal wall, the further connection of which could not be determined. In the course of a week an abscess formed at this spot. An incision was made on October 3, I9oo, under an anæsthetic, and a quantity of thin, fœetid pus evacuated. Even at this time it was not obvious what was the cause of the abscess. The wound in the abdominal wall did not heal, and there was found, after a time, a second collection of pus to the left of the former incision. On November 28 this was incised, and found to be rather deeper than the first abscess, but outside the peritoneal cavity. In the course of three days the discharge from the second wound was found to consist largely of gastric contents. In a day or two practically all the food taken escaped by the fistula, and it was thought this indicated some stenosis of the pylorus. The patient was rapidly losing ground.

On December 7 the patient was placed under ether, and the stomach washed out through the fistula, which easily admitted the finger. The peritoneal cavity was opened by an incision above and to the right of the fistula, and a broad pedicle, $3 \frac{1}{2}$ inches by $1 \frac{1}{2}$ inches, was found passing from the stomach to the abdominal wall. The portion of abdominal wall to which the stomach was adherent was isolated by an oval incision, and 
sterilized gauze inserted to protect the peritoneal cavity from being soiled. This portion of abdominal wall was then cut away from the stomach. The fistula was found to be the centre of a large ulcer on the greater curvature, near the pylorus. There was no stenosis of the pylorus. The edges of the fistula were freshened, and the opening closed by three rows of silk suture, which, however, had to be passed through greatly indurated tissue. The omentum was drawn over the spot, and fixed there by several points of suture. The abdominal wound was closed by silkworm.gut sutures passing through the whole thickness.

There was great collapse after the operation. Rectal feeding and subcutaneous injections of saline fluid were given with good effect. On the third day small doses of brandy and meat essence were given by the mouth. The patient died on the sixth day, with symptoms pointing to pneumonia.

Autopsy.-Pneumonia of the right lung was found. The abdominal wound was dry, and there had been no escape of gastric contents into the peritoneal cavity. There was, apparently, no attempt at union in the stomach wound, and probably, had the patient survived, a perigastric abscess would have formed. The pylorus was healthy, and not narrowed by the ulcer. There was no dilatation of the stomach. The ulcer was found to be as described at the operation. The omentum was thickened greatly, so as to simulate malignant disease. The report of the microscopic examination showed that there was no evidence of carcinoma.

There are several specimens in the Hunterian Museum. No. 2,53I is a specimen of a cancerous ulcer between the stomach and the colon. The disease probably commenced in the colon. It occurred in a woman of fifty-five years of age. Death was due to exhaustion. There was no vomiting. No. $2,426 c$ is a specimen of cancer of the pylorus involving the transverse colon. It is from a woman thirty-nine years of age.

The following case, taken from the British Medical Journal, May 24, I902, is probably unique. It was reported by Harold H. Cotman.

Case of Omental Cyst communicating with the Stomach, FOLLOWING INJURY.

F. P., aged twenty-one, was sent to St. Bartholomew's Hospital, Rochester, on December 23, I90I, said to be suffering from strangulated bowel. 
History.-On September 2 I the patient received a severe blow in the abdomen from the shaft of a cart while riding a bicycle. This accident kept him away from work for five weeks. He then returned to work, but sometimes complained of ' a lump in his stomach.' Nothing further was noticed till December I, when he suffered from acute rheumatism (third attack). He remained under treatment, but from December i 8 until the day of admission (December 23) the bowels were never moved, and he was therefore sent to hospital.

State on Admission.- The patient was very thin and very sunken about the eyes, and somewhat collapsed. The abdomen was distended, and to the right and I inch above the umbilicus was an oval, slightly resonant swelling, fairly freely movable. The heartsounds were abnormal, there being a double aortic and a mitral murmur. The urine contained a fair quantity of albumin. The patient was anæsthetized with ether, and under the anæsthetic the swelling to the right disappeared, but a definite dull area appeared in the left hypochondriac region, slightly movable, and apparently somewhat near the surface of the abdomen. There was nothing to be felt per rectum. A catheter was passed, and about I ounce of albuminous urine was drawn off. There was evidently no obstruction of the bowels, and as the nature of the swelling was very doubtful, it was decided not to operate, and the patient was sent back to bed. On examining the abdomen that night we were surprised to find that the swelling, which was now definitely to the left of the middle line, was pulsating with a true expansile pulsation, and a doubtful diagnosis of abdominal aneurism was made. The patient was given castor oil $3 i$., and the bowels moved well next day. On December 25 the patient vomited three times; the tumour, which still lay to the left, but could be pushed to the right, was still pulsating, and seemed larger, but varied in size from time to time; it was never smaller than an orange. The urine was passed normally and in normal quantity. The temperature never rose above $99^{\circ} 2^{\circ} \mathrm{F}$, and was usually normal, as were the pulse and respirations. The patient was kept on a milk diet. On December 28 the tumour was clearly increasing in size. On December 31 the patient vomited once, and the tumour was larger, but had less pulsation. On January 2 the tumour was much larger, with less pulsation, and he had a very bad day owing to the vomiting, which took place eight times, and on each occasion the quantity ejected was large in amount, the vomit being like that of a dilated stomach. On January 4 it was decided to explore, owing to the persistent vomiting.

Operation.-The patient was anæsthetized, and an incision of 
about 6 inches was made in the middle line, with the umbilicus as mid-point. A large tumour presented, which pulsated freely. On examining it, it turned out to be stomach pulled down with the omentum curled up under it. On putting the hand into the abdomen, a definite tumour with well-marked free borders was felt. The stomach was then pulled up into its normal position and an attempt made to free the tumour, but in so doing it was ruptured, and a lot of clear fluid escaped. The fluid was glairy and of an acid smell, resembling vomit. As the tumour was emptying a few small pieces were seen to come away, resembling gastric contents. An examination was therefore made to see if there was a fistulous opening between the stomach and the tumour, and on the posterior wall of the stomach, about the middle of the surface and 2 inches from the pylorus, was found an irregular slit-like opening, the diameter of a sixpence, and parallel with the long axis of the stomach, which opened into and communicated with the tumour. Its edges were rounded and smooth, and easily admitted the tip of the finger. The opening in the stomach was closed by Lembert sutures, the edges having been freshened. This was accomplished with great difficulty, owing to the friability of the tissue. The tumour was then explored, and found to be a blood cyst between the layers of the omentum into the lesser peritoneal cavity. As the patient was becoming collapsed, there being a good deal of bleeding from the cyst wall, the aodomen was well flushed with saline solution, and it was decided to pack the cyst and tie the walls of it up to the abdominal wound. This was done, and the wound closed, a large plug being left in the cyst. The patient was put back to bed and rallied fairly well, but died at 2.45 next morning.

Necropsy.-The cyst was fully examined, and found to be of a size to take the closed fist. "The walls were thick and deeply pigmented. The other abdominal viscera were healthy, but vegetations were found in the aortic valves, and the strong pulsations of the aorta were no doubt due to the existing valvular defects.

Remarks. - Two theories as to the causation of this remarkable condition may be suggested. One is that a previous gastric ulcer existed, and that perforation of the stomach occurred from this cause, resulting in a peritoneal cyst instead of diffuse peritonitis ; the other is that when the injury occurred one of the vessels in the wall of the stomach was ruptured and a blood cyst formed, and that subsequently, owing to the damaged condition of the viscus at this spot, its coats were eroded and a communication was set up between the stomach and the cyst. The entire absence of any symptoms pointing to previous gastric ulceration 
is in favour of the latter theory, and, whatever may have been the reason of its existence, the presence of a cyst of this nature is, so far as I have been able to ascertain, quite unique.

\section{Diagnosis.}

As a rule, except in surface fistulæ, diagnosis can only be made after opening the abdomen; but if unaltered or slightly altered food be seen in the fæces after a history pointing to simple or malignant ulceration of the stomach, a fistula between the stomach and colon would be suspected, especially if fœtid eructations or feoulent vomiting occurred from time to time. The inability to distend the stomach with air pumped through an œsophageal tube would help in the diagnosis. The persistent nausea and vomiting of bile was a marked symptom in the gall-bladder stomach fistula described above.

\section{Treatment.}

In simple internal fistulæ, exploration by abdominal section and closure of the two openings may be done, as in the cases previously referred to. In malignant disease partial gastrectomy and enterectomy might possibly be performed if the disease could be discovered in time; but such a communication would probably mean advanced disease and secondary infection, and would therefore, as a rule, be beyond operative treatment. Surface fistulæ can be closed readily by plastic procedures, if they do not close spontaneously under careful dressing and rest. Such fistulæ may be closed by carefully dissecting, without opening the peritoneal cavity, and then invaginating the margins of the opening in the stomach, and suturing them accurately together with two layers of superimposed sutures, afterwards closing the muscular and aponeurotic layers of the abdominal wall by separate sutures, and lastly the skin. This operation is sometimes necessary in closing a gastrostomy opening that has been made for temporary use.

The first attempts to close a gastric fistula were by means of the cautery, and if the fistula be small such means may be 
quite efficient. In 1859 Middledorpf introduced a simple plastic operation for the purpose.

In I877 Billroth cured a patient by completely separating the stomach from its adhesions to the abdominal wall, and then closing the fistula. Esmarch also resected a portion of the stomach wall around a fistula, and closed the opening by sutures. We found in the two cases mentioned, of intervisceral fistulæ, that the radical method of separating the fistula from the surrounding adhesions, and then either excising or inverting the raw. edges, was attended with satisfactory results. In all cases of surface fistulæ where the fistula is not very small, in which case the cautery will probably not cure it, and where it is producing serious or troublesome symptoms, resection should be done, as anything short of this is very likely to fail. 


\section{CHAPTER XXVI}

\section{GASTROPTOSIS}

Gastroptosis, or a downward displacement of the stomach, forms one of the prominent characteristics of a disease, often dependent on a congenital deformity, and first described by Glénard of Lyons in 1885 , after whom the disease has been named.

In this ailment the ligaments or mesenteries attaching the various organs to the parietes are lengthened, thus allowing the liver to descend below the costal margin, the stomach below the umbilicus, and the intestines, kidneys, and other viscera to be more movable than occurs under normal conditions.

Owing to the displacement, kinking of the pylorus or of intestinal loops is apt to occur, leading in the one case to pyloric, in the other to intestinal, obstruction, partial or complete.

Gastroptosis may or may not be accompanied by displacement of the other organs, but whether it occurs alone or as part of a general abdominal slackness, it can readily be recognised by distending the stomach with $\mathrm{CO}_{2}$, and by palpating or percussing the outline of the displaced viscera through the abdominal walls.

The accompanying diagram shows the situation of the stomach in the case, of which brief notes are given to illustrate the symptoms that may be expected and the surgical treatment that may be adopted.

The frequency of gastroptosis may be estimated from the publication of Steele and Francine, to the effect that sixtyfive cases were observed and treated during two years in one 448 
of the medical dispensaries of the University Hospital. Glénard observed the disease 400 times in 1,300 patients.

The etiology of gastroptosis, like that of floating kidney and retrodisplacement of the uterus, is not definitely known. However, two sets of causes are generally believed to exist -predisposing and exciting. The former are congenital weakness and hereditary predisposition, the patient with gastroptosis representing an almost characteristic type of individual. The exciting causes are thought to be tightlacing, rapid emaciation, and changes in the intra-abdominal pressure, as due to constricting bands, or relaxation of the abdominal walls and diastasis of the recti muscles.

The upper curvature of the stomach, through inflation by means of the stomach tube and auscultatory percussion, is outlined in the more pronounced cases midway between the xiphoid cartilage and umbilicus.

The supporting ligaments of the stomach, the gastrohepatic and gastrophrenic ligaments, are lengthened or stretched out, and proportionate to the degree of displacement. As a result of the ptosis, there is frequently a secondary dilatation of the stomach, particularly of the pyloric end. In some instances, also, it is believed a primary dilatation of the stomach occurs, with secondary gastroptosis.

The symptoms are those of gastromotor insufficiency, brought on by gastric and intestinal atony, by the mechanical disturbances caused by the descent of the organ, and neurasthenia.

The patient comes to the physician complaining of pressure and fulness in the epigastric region, abdominal distension and flatulence, pain (gastralgia) coming on at irregular intervals, and independent of the digestive action or the quantity or quality of food. Eructations are usually, and - nausea and vomiting frequently, complained of. There are numerous pains during bodily exertion. Intense lumbago is a frequent symptom. The patient is usually, and often profoundly, neurasthenic. Nausea and vomiting in the more severe cases occur several times each day, little food is retained, the patient becomes more and more emaciated, neurasthenic, feeble, anæmic, even bedridden, and a con- 
firmed invalid. The picture, in a few words, is that of chronic severe atonic dyspepsia, with neurasthenia and increasing emaciation.

The medical treatment consists in the administration of strychnine and other tonics ; regulation of the bowels; lavage; the selection of a suitable nourishing diet; massage, electricity, cold-water applications, and abdominal gymnastic exercises to strengthen the abdominal muscles and increase the intra-abdominal pressure; the avoidance of tight-lacing or wearing of any garment which restricts the waist.

The mechanical treatment consists in applying a properly selected, adapted, and accurately fitting abdominal bandage to increase the intra-abdominal pressure and relieve the splanchnic congestion and symptoms of weight. When there is great weakness the rest cure is a most efficient means of treatment. Alternating cold and hot douches are often efficient in restoring partial tonicity to the muscular wall.

The medical and mechanical treatment, when skilfully and persistently applied, in a large proportion of the cases, gives much symptomatic relief, and the patient is able to live on in more or less comfort. The application of an abdominal binder, according to some authorities, may succeed in elevating the stomach $\mathrm{x}$ or 2 inches with more marked relief. However, the patient remains constantly under observation, and is never restored to good health.

There is a considerable percentage of cases associated with severe symptoms in which this medical and mechanical treatment gives little or no relief; the dyspeptic symptoms continue severe, vomiting persists, the neurasthenia is profound, the emaciation and illness continue, and the patient seems destined to chronic invalidism.

Duret of Lille was, we believe, the first surgeon to perform gastropexy, which he did by fixing the pylorus and lesser curvature to the abdominal wall. He made an incision in the middle line above the umbilicus, leaving the peritoneum and transversalis fascia undivided at the upper end of the incision. Sutures were then passed fixing the lesser curvature of the stomach to this undivided fascia, thus correcting the excessive mobility of the organ. 
Should there be pyloric stenosis and dilatation, such an operation would be insufficient, and pyloroplasty or gastroenterostomy would be necessary; moreover, the fixation of the stomach to the abdominal wall would probably bring on a train of symptoms such as one is accustomed to see in perigastritis from ulcer or from other causes.

Stengel and Beyer (American Journal of Medical Science,

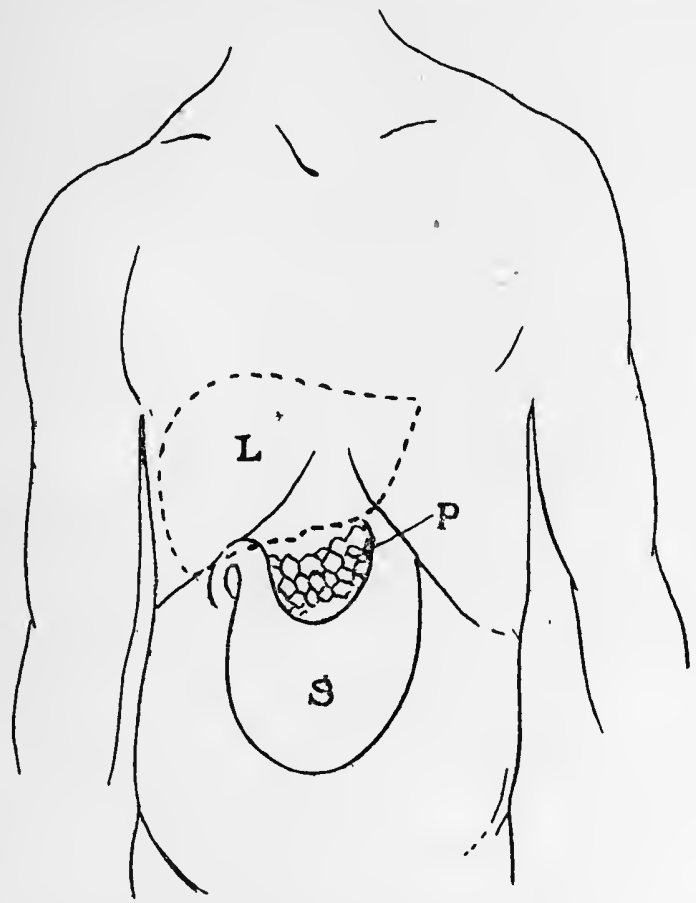

Fig, io7,-Gastroptosis, the Pancreas showing above the Lesser Curvature.

June, I 899) record a case of Glénard's disease treated by operative procedure.

The patient was a young unmarried woman, without history of injury, abdominal distension, or emaciation. At the time of observation she had already undergone nephrorrhaphy, which was followed by some general improvement. It was found, however, that the gastric symptoms remained much as before. Examination of the abdomen showed some sinking in at the epigastrium 
and a protuberance at the umbilicus, which was found to be the stomach, the lesser curvature extending from $I \frac{1}{2}$ inches above the umbilicus to a point two-thirds the distance from the umbilicus to the pubes, behind which was the greater curvature. The right border extended to the right of the umbilicus $I_{\frac{1}{2}}$ inches, the pylorus being slightly to the right of the middle line and a little above the umbilicus. No evidence of growth could be found. The digestive processes seemed slow. There was no lactic acid reaction. It was decided to operate, and the following was the procedure adopted: An incision was made through the linea alba midway between the xiphoid and umbilicus, exposing a small portion of the upper curvature and cardiac end of the stomach, the gastrohepatic omentum, the gastrophrenic ligaments, and the lower portion of the liver. At the beginning of the operation the patient's shoulders were slightly raised, but at this stage the position was reversed to allow the stomach to fall back into position. The gastrohepatic omentum and gastrophrenic were exposed, separated from underlying structures, and interrupted sutures were introduced to shorten the gastrohepatic omentum in the following manner: The first suture caught the gastrophrenic ligament above at a point as near as possible to the diaphragm (a distance of about 2 inches from the diaphragm), and below just above the gastric vessels. After this suture had been placed, the gauze sponges which had displaced the stomach were removed and the suture temporarily tightened, so as to determine the height to which the stomach could be brought by this manner of suturing, and the degree of support which would be furnished to the stomach. This having been determined to be satisfactory, the stomach was again displaced out of the wound and the suturing continued. The second suture was introduced through the gastrohepatic omentum opposite, and about $\frac{1}{4}$ inch from the first one, followed by a row of eight or ten others introduced in the same way, to include the left three-fourths of the gastrohepatic omentum. In order to be sure of gaining a wide surface of adhesion, particularly in relation with the gastrophrenic ligament, three or four more sutures were introduced, which included the peritoneum above and below those already placed. The gauze sponges were again removed, and the first and then the second row of sutures were tied, forming a tuck in the gastrohepatic omentum and gastrophrenic ligament. After this had been accomplished, the stomach was seen to occupy what was thought to be a normal position. All gauze sponges were then removed from the abdominal cavity, and the table lowered until the patient was in a horizontal position. The patient made an uninterrupted recovery, leaving the hospital in two months and 
ten days from the time of the operation. Some months later examination showed that the lesser curvature was still well supported, and that the greater did not extend more than $1 \frac{1}{2}$ inches below the umbilicus. Eleven months after the operation the woman was found better in every way; she had gained considerably in weight, was able to eat freely without discomfort, appetite and energy being distinctly good.

In a further paper (Univ. of Pennsyl. Med. Bull., February, I903), which is abstracted in the Brit. Med. Journ., March 2I, IgO3, Beyer reports the results of the operation in four cases, in three of which the symptoms were completely relieved, while the fourth was still in hospital, only having very recently been operated upon. Four cases are mentioned in which Bier, of Greifswald, Germany, operated in a somewhat similar manner; but in addition the first suture secured the serosa and muscularis of the pyloric end of the stomach to the capsule of the liver, and in two of them also the serosa of the lesser curvature was attached to the left lobe of the liver. Thus a fixation of the stomach is practised in these cases, but the procedure is not surgically ideal, since abnormal adhesions are formed, which may interfere with physiological mobility and function, though such interference would be less marked than in those cases where adhesions to the abdominal wall were obtained. Including Bier's cases, eight cases have been operated upon by the application of the gastrohepatic and gastrophrenic ligaments, and in all (except the one still recovering from operation) lasting and complete relief of symptoms and gain in weight has resulted. The complete restoration to health, the simplicity of the operation, and its comparative freedom from danger, are strong recommendations for its adoption in all cases of gastroptosis where the suffering is severe and cannot be relieved by general treatment.

R. C. Coffey (Med. Rec., October I8, I902) describes two of these cases.

A Method of Suspending the Stomach in a Hammock made of the Great Omentum.

The first patient was a woman, aged forty-four years. Examination showed the abdomen so tender that a thorough ex- 
amination could not be made, and a digital examination per vaginam revealed a retroflexed uterus, with an adherent ovary back and to the left side of it. A blood examination showed 20 per cent. diminution in hæmoglobin. A satisfactory diagnosis was possible only under an anæsthetic, or more probably an exploratory incision would be necessary. This proved to be true, and when the incision was made no small intestines were visible. The entire pelvic cavity was tented over by the omentum, which had become adherent by strong adhesions to the peritoneum lining the anterior brim of the pelvis. The lesser curvature of the stomach was almost 2 inches below the umbilicus. The appendix and cæcum were included in the adhesions. The stomach was fixed, and could not be brought out of the wound until the omental adhesions had been separated from the pelvic peritoneum. The stomach was then pushed up out of the way. The ovary, which was adherent in the cul-de-sac, was removed, the uterus stitched to the abdominal wall, and the appendix removed. The problem then arose how it would be possible to protect the stomach from again being drawn into the pelvis by a re-adhesion of the omentum, for the tendency of the stomach was to come down into the wound, even though the adhesions had been broken up and the patient was still in the recumbent posture. Finally, the omentum was stitched to the abdominal peritoneum, about an inch above the umbilicus, by passing chromicized catgut sutures through the omentum about an inch below its attachment to the stomach. Three sutures were placed in this case about an inch apart, the line of sutures running transversely. In this way the stomach was out of the way and firmly in place. The abdomen was closed and the patient put to bed, with the lower extremities elevated. She was kept in the recumbent position, with her head lowered, for nearly five weeks, feeling almost entirely well. She went home during the eighth week. A bandage was fitted, and the patient was instructed to lie down an hour after each meal, and exercise very cautiously. Within a few weeks she could eat anything she desired without distress, and she felt that her condition was absolutely cured. She is now doing her own housework. 


\section{CHAPTER XXVII}

\section{T U B ER CLE-S Y P H I I IS}

\section{Tuberculosis of the Stomach.}

THE insusceptibility of the mucous membrane of the stomach to become affected with tubercle presents a great contrast to that of the intestinal lining, for primary tubercular ulceration of the stomach is extremely rare, and even secondary tuberculous ulceration is so rare that Dr. M. Simmonds (Mïnch. Med. Woch., March 6, I899, p. 317) only found eight specimens among 2,00o tuberculous subjects.

Gastric disorders, however brought about, must be considered among the predisposing causes of tubercular disease, and especially of tubercular disease of the mucous membrane. The ulcers, which may be solitary or multiple, are usually small, with undermined and ragged edges, owing to infiltration and breaking down of the submucous tissue, like tubercular abscess in the small intestine.

The following are brief notes of a case on which an operation was performed on account of pyloric stenosis and vomiting due to tubercle affecting the pylorus:

On August 22, I895, we were asked by Dr. J. Lewis Owen, of Llangefni, to see Miss T., aged nineteen, who gave a history of pain after food with occasional coffee-ground vomiting for the preceding four years, though the loss of flesh hal only been very pronounced during six months, during which time she had been able to take very little food on account of sickness and pain. Four months before we saw her, dilatation of the stomach due to pyloric stricture had been diagnosed, and lavage of the stomach had been followed out for a month without benefit. She was extremely pale, and so thin that the skin was tightly stretched over her bones. She only weighed 4 stones ro pounds. Tuber- 
cular glands were felt in the neck, and there was a little swelling of the abdomen, due to free fluid in the peritoneum. The stomach was markedly dilated, and the pylorus could be felt thickened and movable. As she was clearly going to die unless relieved, operation was performed on August 27, 1895. On opening the abdomen some fluid escaped from the peritoneum. There were tubercular nodules on the stomach and omentum, and the mesenteric glands were found to be full of caseous material and pus. The pylorus was nodular and thickened, and was itself the seat of tubercle; the stomach was enormously dilated.

Pyloroplasty was performed, but before closing the wound in the pylorus the finger was passed into the duodenum, as a constriction could be seen an inch beyond the pylorus. Here was found a stricture that would admit the little finger. Through this a Weiss' dilator was passed, and it was dilated freely until the thumb would readily pass. After the pyloroplasty had been completed the abdomen was closed. The after-progress appeared to be satisfactory, and the wound healed by first intention, the stitches being removed at the sixth day; but no real gain of strength was made, and the patient died exhausted in the second week.

It is the only case of the kind that has come under our care, but Patella (British Medical Journal, SuppI., 399, I900, p. 82), at the Congress on Tuberculosis recently held at Naples, said he had seen three cases of stenosis of the pylorus caused by fibrous peripyloritis developing in individuals who, at least three years previously, had presented symptoms of tuberculous lesions of the lung, which had passed into the phase of obsolescence. In two of the cases gastro-enterostomy had been performed by Golzi and De Pauli, with the result that the patients, who were in a state of extreme marasmus, regained weight and strength. In these cases, in addition to thickening of the pylorus, there were found nodules of fibrous appearance; examination showed that these were not of tuberculous nature. The author affirms that the conditions found gave no ground for the belief that the peripyloritis was tuberculous; he thinks that they are examples of slow sclerosis dependent on tuberculous intoxication, the effect of which may, according to Potain and his school, become manifest in various parts, especially where (as at the pylorus) there is considerable 
movement. Whatever be the interpretation of the condition, he urges that in such cases surgical intervention should not be delayed.

Tuberculous ulcers may attain a great size (though this is exceptional), as in one of Dr. Simmonds' cases (Medical Review, rgoo, p. 463), where the ulcer measured 8 inches by 4 inches, its true nature only being recognised on microscopical investigation. It is curious that this ulcer produced no symptoms during life. The occurrence of tuberculous ulcers has been described by Eppinger, Prag. Med. Woch., 188I, Nos. 5I, 52 ; by Litten, Virchow's Arch., B. 67, p. 6r5 ; by Musser, Philad. Hosp. Rep., r89o, vol. i., p. I70; and Blumer, British Medical Journal, Suppl., I898, par. 504.

Petruschky's views as to the tuberculous character of chronic gastric ulcers is not supported either by the clinical experience of these cases treated surgically or by postmortem evidence. He claims to have diagnosed tubercular ulcers of the stomach by the reaction obtained with tuberculin, which in one case proved curative and in the other palliative. This claim will have to be supported by much clearer evidence than has yet been advanced before it can be received as distinctly proven.

As complications of tuberculous gastric ulcer, hæmatemesis and perforation have been described, but from the chronicity of the ulceration, as well as from the infrequent occurrence of tubercular ulcers, such accidents are extremely rare. Miliary tubercle may affect any of the coats of the stomach, and in this form tubercular disease is by no means uncommon. It was well marked in the case we have described, and is commonly seen in tubercular peritonitis, as well as in other general tuberculous diseases.

Batsère collected (Thèse de Toulouse, Igor) a series of thirty-five observations, in which he points out the diagnostic features of gastric tuberculous ulceration. Such ulcers, he says, are generally multiple, even numerous. They are situated for the most part close to the pylorus and on the greater curvature. They are round in contrast to the shape met with in the intestine. The symptomatology is extremely variable, at times absent, though occasionally well-marked 
signs may be present. The most reliable symptoms are pain, vomiting, and hæmatemesis. The former is most frequently absent. It is caused by the ingestion of food, as in other cases, and when occurring corresponds with the taking of food. Pain may be extremely severe and lancinating. Usually speaking, it disappears gradually within a couple of hours after taking food, and its most frequent site is the epigastric area. Vomiting frequently follows pain, and when hæmatemesis occurs it points to a diagnosis. The progress of such ulceration is generally fatally progressive; rarely is there any remission. Some cases at the same time have shown a transitory subsidence of the symptoms, and one is led to suppose that the ulcers have healed. There may then occur a very copious hæmorrhage, which generally brings about a fatal termination.

\section{Syphilis of the Stomach.}

Syphilis of the stomach may occur under the form of ulcer, stricture, or tumour. Some writers regard syphilis as an important factor in relation to gastric ulcer, one author giving the frequency of gastr.c ulcer as high as 20 per cent. in syphilitic subjects, and others as from Io to I5 per cent.

Dr. Fenwick ('Ulcer of Stomach,' p. 93), while stating that zo per cent. of cases of chronic ulcer of the stomach had suffered from syphilis at one time or another, remarks that it is extremely probable that in at least one-half of such cases where the two diseases coexist in the same patient there is no direct relationship between them. Lesions due to syphilis, having no clinically distinctive features, can only be recognised by the history, the presence of syphilis in other regions, and the result of the treatment.

Fenwick, in his later work, 'Cancer and Tumours of the Stomach,' p. 3I6, writes: 'So far as our own experience goes, these cases chiefly differ from the simple variety of the disease in three particulars, the first of which is the extreme severity of the pain and vomiting, the second the infrequency of hæmorrhage, and the third their obstinacy to ordinary treatment and their great tendency to relapse. We do not, 



\section{PLATE XXX.}

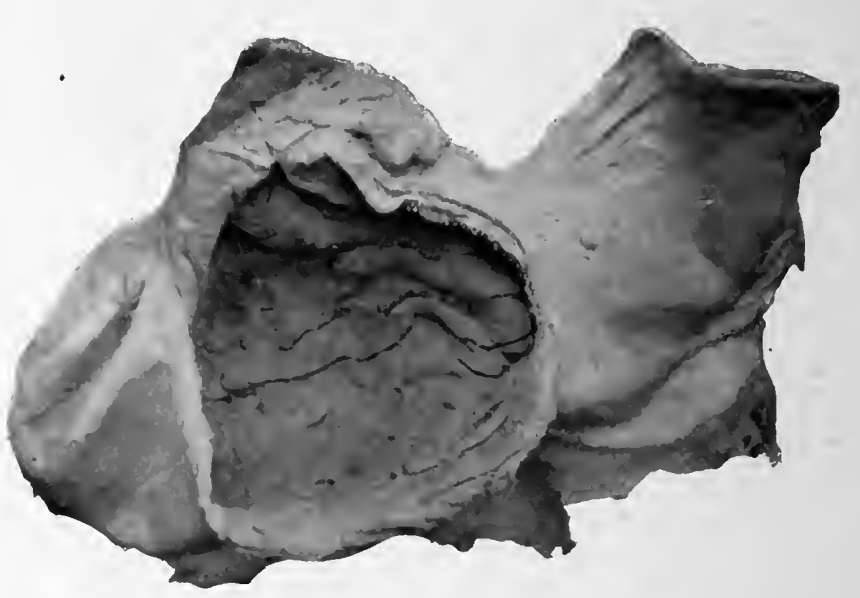

Fig. io8. - Cardiac Orifice of Stomach obstructed by a Gumma, which Also involves the Adjoining PORTion of the Liver.

From a gentleman, forty-six years of age, who died of starvation.

To face p. 459.] 
however, think it possible to draw a clear distinction between simple and syphilitic ulcer, though it is well to bear in mind the undoubted existence and the possible frequency of the latter condition.'

In the Philadelphia Medical Journal, February 3, I900, p. 262, Max Einhorn gives examples of ulcer, gumma, and pyloric stricture, all of which yielded to treatment by mercury or iodide of potassium. The cases are of sufficient interest and importance to be worth quoting.

\section{ULCER.}

CASE I.-A woman, aged thirty, had for three months suffered from violent pains immediately after meals, and occasional vomiting. The ordinary treatment of gastric ulcer had failed. She had acquired syphilis three years previously, and had recovered under mercurial inunction.

There were violent pains after taking even fluids, such as milk, bouillon, etc. The pains lasted for one or two hours, and were sometimes attended by vomiting. There were pains in the lower limbs, which were more marked at night, and the tibiæ were tender. Syphilitic ulcer of the stomach was diagnosed. Mercurial inunctions were employed, and iodide of potassium was given. After fourteen days the pains disappeared, and solid food could be taken. Recovery was complete at the end of six weeks.

CASE 2.-A woman, aged thirty-three, had acquired syphilis at twenty-six. Pains after eating had existed for two years. Profuse hæmatemesis had occurred. Under treatment for gastric ulcer she recovered, except that she did not regain her former health, and complained almost constantly of dyspeptic symptoms. A short time before she came under observation, a second severe attack of hæmatemesis, accompanied by melæna, occurred.

The patient was extremely anæmic and confined to bed. There were constant pains in the stomach, which radiated towards the back, and were increased even by liquid foods. During six weeks fluid diet and bismuth were prescribed. The vomiting ceased, but the pain persisted. Iodide of potassium was then given. In a few days the pains diminished; after three weeks they had disappeared, and ordinary diet could be taken. Permanent recovery ensued.

\section{Gastric Tumour.}

CASE I.-A woman, aged thirty-five, had contracted syphilis at thirty-three. For six months she had had pains after meals, and 
occasional vomiting. A nodular tumour about the size of a goose-egg was felt under the left costal margin. It was beneath the abdominal wall, and moved on deep inspiration. Below the tumour a splashing noise could be elicited. The gastric region was tender. In the back a tumour of bony hardness extended from the third to the sixth dorsal vertebræ. The presence of this tumour and the history led to the diagnosis of gumma of the stomach, although the nodular gastric tumour was suggestive of cancer. Under inunction and iodide of potassium improvement took place, and in a few weeks both tumours disappeared.

CASE 2.-A man, aged fifty, had acquired a chancre at thirtytwo, and for eighteen years had had severe gastric troubles, anorexia, and sleeplessness. Three years previously abdominal pains began, and he lost 15 pounds in weight. In the epigastrium a slight oval resistance was felt over an area the size of a hen's egg. Under iodide of potassium he regained weight, and the tumour disappeared.

\section{Pyloric Stricture.}

A man, aged thirty-seven, had acquired syphilis at thirty, for which he was treated for several months. After four years dyspeptic symptoms began. He was attacked with pains some time after eating, and occasionally with vomiting. During the last year he had lost 30 pounds in weight, the symptoms became much worse, and he lived chiefly on liquid food. He had to wash out his stomach two or three times daily, otherwise the pains became unbearable.

He was weak and emaciated. The stomach was dilated, extending to the symphysis pubis. Gastric peristalsis was visible. An oval tumour larger than a.pigeon's egg was felt in the mammary line under the right costal border. Under fluid diet, resorcin, and bismuth, the pain and vomiting, after fourteen days, diminished, but the tumour remained unchanged. Iodide of potassium, Io grains, three times a day, was then given. After another fourteen days the tumour had diminished, the pains had almost completely disappeared, and the vomiting no longer occurred. A month later the tumour could not be felt, and the patient's condition was improved. He was soon able to take solid food, and in four months he gained 20 pounds in weight.

Dieulafoy (Bull. de l'Académie de Méd., I898, p. 578) published a case in which the symptoms of ulcer of the stomach resisted all ordinary methods of treatment for eighteen months, and then yielded rapidly to biniodide of 
mercury. G. Andral, in 1834 , described two cases (Medical Review, March, I900, p. I54). In one, a woman, aged twentynine, lost appetite, was tormented with pains after meals, and frequently vomited. During three months all kinds of treatment failed. Pains in the neck on swallowing were then complained of, and a number of ulcers of syphilitic appearance were discovered in the pharynx. The question then arose, Were there similar ulcers in the stomach? Under mercury recovery took place in a few weeks.

Virchow, Klebs, Birch-Hirschfeld, Dalgleish, and others, have also described isolated cases.

Dr. Simon Flexner (American Journal of Medical Science, October, 1898 ) contributes a valuable article on this subject. The literature is very meagre; he could find only fourteen reliable cases, of which thirteen were German and one French. According to him, there is no instance recorded in the English language. This is scarcely correct. In the British Medical Journal, r89ı, vol. ii., p. 696, Dr. J. Keser has recorded the case of a young woman treated at the French Hospital, the subject of secondary syphilis, who had all the symptoms of gastric ulcer. She was treated with bismuth and nitrate of silver, and fed by the rectum; but only temporary improvement took place, and the hæmatemesis continued. Various drugs were tried without success. The constitutional disease was then discovered, and the patient at once improved under specific treatment. The subject has recently attracted the attention of clinicians. M. Dieulafoy discussed the subject at the Académie de Médecine on May I7. He concluded that gastric syphilis is not so uncommon as is supposed, and that, as the symptoms differ in no way from those of simple ulcer, a history should be sought in every case. In La France Médicale, July I, I898, Dr. Dubec has published the case of a man, the subject of tertiary syphilis, in whose stomach an indurated plaque could be felt, which diminished rapidly under mercury and iodide of potassium. The Transactions of the Pathological Society of London do not contain a single reference to the subject. Of the fourteen cases, five were of the inherited, and nine of the acquired, form. The lesions found were 
gummata, ulcers, and cicatrices, and were usually accompanied by syphilitic disease of other viscera, and sometimes by intestinal ulcers. All the acquired cases appear to have been in the tertiary stage, but some of the congenital occurred in new-born infants. Chiari paid special attention to gastric syphilis. In 243 necropsies where syphilitic lesions were found there were two cases of gastric syphilis.

Dr. Flexner records the case of a man, aged fifty-two, whose illness extended over three years. He had severe vomiting, following on a drinking bout. The spleen was enlarged, ascites appeared, and then anasarca of the legs and scrotum. He was repeatedly tapped; $3 \frac{1}{2}$ gallons of fluid were withdrawn on the first occasion. The liver was hard, but there was no increase of the area of dulness. Before death hesuffered from intense abdominal pain and tympanites. The necropsy showed old adhesions between liver, stomach, spleen, and pancreas; the liver gummatous and its capsule thickened; perforating ulcer of the stomach; the spleen enlarged and its capsule cartilaginous.

It will be seen that from a surgical point of view the subject is of great importance, since it would be easy to mistake a gummatous tumour of the stomach or pylorus for cancer, and perform gastrectomy when simple medical treatment would do all that is required. The lesson to be learnt is that inquiries with regard to syphilis should always be made in ulcer or tumour of the stomach, and antisyphilitic treatment should be given a trial before resorting to more radical surgical means.

But while the question of syphilis is to be borne in mind, it must not be forgotten that gummata of the stomach are extremely rare even in cases of congenital syphilis, and that too much time must not be lost in medical treatment in a doubtful case, lest the disease advance too far for surgical treatment to be of service. 


\section{CHAPTER XXVIII \\ PHLEGMONOUS GASTRITIS}

By the term 'phlegmonous gastritis' is understood a condition of suppurative inflammation of the stomach wall, having its chief seat in the submucosa, but extending also into the muscular coat and at times even to the mucous and serous surfaces.

The disease has been described under a great variety of names by different writers. As, for example, "inflammation of the submucosa' by Rokitansky; as 'gastritis submucosa' by Dittrich, Klebs, and others; as 'phlegmon ventriculi' or 'gastritis phlegmonosa' by Lebert and Bamberger; as 'linitis plastica et suppurativa' by Brinton; as 'gastrite interstitielle suppurative' by Auvray; as 'submucous suppurative phlegmon' by Krause, etc. The most apt term in English is 'phlegmonous gastritis.'

The first description of the condition was given by P. Bevel in 1656 .

There are two distinct forms of the disease-the circumscribed and the diffuse. Of 50 cases tabulated by Jacoby, 3I were of the diffuse variety, I9 of the circumscribed. Males are more frequently affected than females, in the proportion, almost exactly, of 4 to $\mathbf{I}$. The youngest patient was aged eleven (Löwenstein), the oldest seventy-six. Glax (Berl. Klin. Woch., I879, p. 565) arranges the cases recorded in the following table of age:

\begin{tabular}{|c|c|c|}
\hline & Years. & Cases. \\
\hline ror & Io to 20 & $\cdots$ \\
\hline & 20 to 30 & $\ldots$ \\
\hline & 30 to 40 & $\ldots$ \\
\hline & 40 to 50 & $\cdots$ \\
\hline
\end{tabular}

\begin{tabular}{cccc} 
Years. & \multicolumn{3}{c}{ Cases. } \\
From 50 to 60 & $\ldots$ & 4 \\
$"$ & 60 to 70 & $\ldots$ & 0 \\
$"$ & 70 to 76 & $\ldots$ & 7
\end{tabular}


The disease is primary or secondary-primary when it originates in the stomach from ulcer, growth, or, as in several recorded cases, from direct injury; secondary when it occurs in the course of some acute infectious disease, puerperal fever, typhoid, pyæmia, or small-pox. In all cases of the primary form, and perhaps in many of the secondary, there is a superficial denudation of the mucosa, which permits the entrance of the pyogenic organisms.

\section{Symptoms.}

(a) Of the Diffuse Form.- The symptoms are those of an intensely acute gastritis. The course of the disease is rapid, the general disturbance is profound, and the end is apparently inevitably fatal. There is generally a sudden onset of severe epigastric pain, attended by faintness or even collapse. Vomiting is almost constant, commencing early and lasting long, the vomited matters being bile-stained. It is said, however, by more than one writer that the cases due to malignant disease are distinguished by the absence of vomiting. There is never any pus in the vomit. Thirst, intolerable and unquenchable, is present, and often nausea, and occasionally hiccough. The epigastric pain is violent and unending, a source of constant complaint and of restlessness. There is sometimes very great tenderness in the epigastrium and over the whole upper abdomen; but, strangely enough, in other cases both the pain and the tenderness are slight or even absent, and a free handling of the body is permitted. The edge of the liver or the lower pole of the spleen can be palpated without causing discomfort. The temperature is always high, liable to a considerable range of variation, rising to $103^{\circ}, 104^{\circ}$, or even $105^{\circ}$, and falling to $99^{\circ}$ or $100^{\circ}$. The pulse is always bad. Its rate varies from IIo to 150 , the quality is poor; a thin, thready, feeble, rapid pulse may be present for days, and then some irregularity may be observed.

Mint $z$ distinguishes two stages of the disease-a first stage in which the above symptoms and signs are present, and a second in which peritonitis is the prominent condition. Though this subdivision may possess convenience, yet there 
are many cases which never show any evidence of peritoneal involvement. The serous coat is not often affected, though perforation, with the extravasation of pus into the peritoneum, has been recorded. In the later stages the abdomen may be very distended and tympanitic, and the bowels may become constipated, neither fæces nor flatus passing. In a few instances diarrhœa has been present. The general condition of the patient is desperate from the first. He gives the impression of being profoundly ill, death seems inevitable and imminent, and yet no satisfactory diagnosis may be attainable. Riegel says that, as a rule, the patients are apathetic, lying quietly in bed and gradually falling into a stupor that is interrupted by delirium. Death then comes in coma and collapse. In some cases, however, the patient is in full possession of his faculties, and remains perfectly clear mentally until within a few moments of death. In approximately one-fourth of the cases it is remarked that the patient was addicted to alcohol, a drunkard, or alcoholic.

It will be seen from this description that there is nothing specially characteristic in the symptom complex. The clinical presentment is that of a patient acutely ill from some febrile disease, with irregular elevations of temperature, very feeble and rapid pulse, vomiting, constant pain in the abdomen, referred generally to the epigastrium, and slight tenderness on deep palpation. It is therefore not a matter of surprise to learn that a positive diagnosis of phlegmonous gastritis has never been attempted. By the time that general peritonitis is recognisable the patient is nearing the end.

At a meeting of the American Medical Congress in May, Igoo (British Medical Journal, Suppl., I9oo), Kinnicutt of New York showed a specimen from a case of acute phlegmonous gastritis.

The patient was a man, aged forty-one years. There was no history of syphilis, but the patient was an alcoholic. The disease followed a drinking spell, and was ushered in by vomiting, first of food, and later of a brownish fluid. There was abdominal pain, constipation, dry tongue, shallow respiration, $4^{8}$ per minute, regular pulse, 108 per minute, restlessness, and an anxious 
expression. The temperature was $\mathrm{IOI}^{\circ}$ in the rectum. The physical examination was negative, except for a slight hypertrophy of the left ventricle. The abdominal walls were rigid and deep; palpation was sensitive in the epigastric and left hypochondriac regions. There was crepitation beneath the left costal cartilages. The urine contained casts. The patient grew progressively worse, and surgical intervention was deemed inadvisable. At the necropsy the organs were normal, except for the hypertrophy of the left ventricle of the heart, before alluded to. The peritoneum presented a slight sero-fibrinous exudate. The stomach wall was thick, especially at the pyloric end. The thickening was due to a purulent infiltration of the submucous coat, with a yellowish exudate. There was a linear cicatrix on the posterior wall of the stomach, near the pylorus, on which was a necrotic area. The endothelium was desquamated on the serous coat. The muscle was invaded by mononuclear leucocytes. The submucosa was swelled to four or five times its normal thickness, and contained leucocytes and micro-organisms. The lymphatics contained bacteria. The necrotic area in the scar had nearly exposed the submucosa, and was possibly the point of infection. Streptococcus was found throughout the stomach. The bacilli in the lymphatics were possibly of post-mortem origin.

In the discussion, W. H. Welch stated that he had seen a similar case, in which there was a fibrino-purulent peritonitis. The patient was an alcoholic. The pus was found principally in the submucous coat of the stomach. E. G. Janeway had seen three primary cases and two secondary cases. One of the patients was not a drinking man, and the two secondary cases followed sepsis, and were local in their areas of involvement.

(b) Of the Circumscribed Form.-The symptoms present in the circumscribed form are the same in type as, though less in severity than, those present in the diffuse form. They are intense epigastric pain and vomiting, appearing suddenly, but gradually lessening in their acuteness. There is always tenderness in the upper part of the abdomen, and when an abscess is present a distinct localized tumour may be felt. The patient loses all appetite, and liquid food, taken unwillingly, increases the pain. An abscess having slowly formed in the submucosa may be easily palpable. Lieutaud, 
Deininger, and Sand all record such cases; in the one the tumour was the size of a fist, and was not tender to pressure. As it increases in size, the abscess may burst : if into the general cavity of the peritoneum, the patient may speedily die, having experienced a sudden overwhelming pain in the upper abdomen, as recorded by Albers ; if into the stomach, the patient may vomit blood-stained pus and recover, as recorded by Deininger, Glax, Beckler, and Kurschmann. The latter cases, however, can hardly be unhesitatingly accepted, for a perigastric abscess, wherever arising, may rupture and discharge all its contents into the stomach. There are in the museum at Erlangen two specimens mounted by Dittrich in which hard cicatricial nodules were found in the submucous tissue of the stomach, and it has been very generally thought by Leube, Oser, and others that this is evidence of the possibility of healing of interstitial abscesses.

In one remarkable instance related by Callow the patient vomited a pint of pus in the presence of the surgeon; pus was found also in the stools. At the autopsy 7 pints of pus were discovered in the peritoneum, and were traced to a large abscess cavity in the wall of the stomach, which had developed painlessly and without symptoms.

The clinical course run by cases of the circumscribed form may be protracted. The patients may linger on for many weeks, in constant pain, unable to take food, and rapidly losing weight. Death, whenever it comes, finds them in a condition of collapse.

\section{Pathology.}

In the diffuse form of phlegmonous gastritis the whole stomach may be affected, or the disease may be limited to one part alone of the organ. The pylorus then is very much more commonly affected, an evidence of some value, showing that the infection takes place in an open ulcer. The whole wall of the stomach is greatly thickened, but the stress of the disease has fallen mainly upon the submucosa. This is intensely inflamed, sodden with inflammatory exudation, or showing patches, larger or smaller, of purulent infiltration. In later stages the whole submucous layer has disappeared, 
a pus-containing channel alone remaining. The mucous membrane is thickened and mottled by hæmorrhagic patches. In some specimens a necrotic area has been seen, at the point, in all likelihood, at which the infection has occurred. In others the appearances of the inflamed area, viewed from the mucous side, are said to have resembled a sponge or a sieve, from whose openings pus could be expressed. In those cases in which the disease has definitely followed upon injury, no laceration of the mucous coat has been recognised. Ulcers are occasionally found. In cases recorded by Gläser and Mint $z$ there was carcinoma, and in one recorded by Hemmeter and Ames ulcus carcinomatosum. The muscular layer is generally only a little involved, but a small-celled infiltration may be found between the fibres, and the fibres themselves may be swollen and distorted. The serous and subserous coats are unaffected in the majority of cases, though there may be some œdema of the latter, and the former may have lost its sheen.

In the circumscribed form an abscess is found in the submucous coat. There may be one large abscess cavity or several smaller ones. The largest is that recorded by Callow. Leube mentions one the size of a goose's egg, Lieutaud one the size of a fist. When multiple abscesses are present they are generally small, equal to or smaller than a hazel-nut. The abscess may be making its way to the outer or inner surface of the stomach, and so the mucous or the subserous and serous coats may be involved.

\section{Treatment.}

Up till the present no case has been recognised sufficiently early to allow of any appropriate treatment. In the acute diffuse form some benefit might perhaps accrue from gastrostomy or gastro-enterostomy, and drainage thereby of the stomach. In the circumscribed form the opening and draining of the abscess cavity would be advisable.

The following case occurred in one of Mayo Robson's beds at the Leeds General Infirmary, and was under the care of Mr. Lawford Knaggs during his absence. Mr. Knaggs has kindly furnished the notes. 
Case i.-Acute Phlegmonous Gastritis; no Operation; Death.

Mr. F. A. D., aged thirty-nine, attended some wedding festivities on Saturday and Sunday, and had an 'ague-like attack' and vomited on Monday at noon. In the afternoon he was struck in the region of the stomach by a railway van door. He felt sick, but walked home afterwards. He was admitted to the infirmary on Tuesday, June I5, I898. There was tenderness on pressure in the epigastric region, some sickness and pyrexia $\left(100^{\circ}\right.$ to $\left.102^{\circ}\right)$; but the most noticeable features of his illness were an increasing weakness and rapidity of pulse, a general lividity which deepened to a dark purple, an absence of pain or discomfort, and the preservation of complete intelligence to the end. So indeterminate were his symptoms so far as the abdomen was concerned that purulent pericarditis was suspected. Death took place at noon on the Wednesday.

Post-mortem.-There was purulent peritonitis, pus lying above and behind the stomach and amongst the coils of the small intestine and in the pelvis. No cause for the peritonitis could be found till the stomach was examined. This was dilated, and its walls markedly thickened. Its peritoneal and mucous surfaces appeared normal, but on section the thickening was seen to be due to coagulated purulent material uniformly distributed through the middle coat of the stomach (muscular and submucous layers), with a well-defined limit at the pylorus, but extending to the fundus. The appendix was normal. There was no other disease.

The following cases of acute gastritis, though not strictly of the phlegmonous type, may be conveniently placed in this section :

Case 2.-Chronic Gastric Ulcer eroding Pancreas; PanCreatitis; Abscess of Pancreas bursting into Stomach ; Acute Gastritis; Vomiting; impending Death. Posterior Gastro-enterostomy ; Recovery.

On November I2, I900, one of us was asked by Dr. Mercer, of Bradford, to see Mr. R., who was extremely ill, and supposed to be suffering from pancreatic disease.

Condition when First Seen.-The patient was found to be extremely emaciated, lying in a typhoid condition, vomiting extremely offensive, dark-coloured pus and mucus mixed with blood. He was extremely feeble, and had a rapid, weak pulse, and a slight icteric tinge in the conjunctivæ. A tumour could be felt above the umbilicus, which was tender to pressure. On distending the stomach with $\mathrm{CO}_{2}$ great pain was produced, and vomiting followed. The stomach was slightly dilated, reaching on the left side to the level 
of the umbilicus. There was a little sugar in the urine, with a trace of albumin, and the fæces contained free fat.

History.-There had been an elevated temperature for a few weeks, but this had become subnormal after the vomiting of pus. Pain after food and indigestion had existed for some months, during which time there had been steady loss of flesh; but recently, especially during the last month, the wasting had been very considerable. No medicine had done any good either for the relief of the vomiting or in controlling the horrible odour, which permeated the whole house.

Diagnosis. - A diagnosis of chronic gastric ulcer of the posterior wall of the stomach, with secondary ulcerative pancreatitis and abscess of the pancreas, was made, and gastro-enterostomy proposed as the only means likely to produce any chance of relief, but even that seemed hopeless. He was removed to a surgical home by ambulance, and the stomach was washed out carefully. As showing the nature of the stomach contents, both the attendant nurses were made sick by the odour of the material evacuated by the tube.

Operation.-On November I8, after enveloping the patient in cotton-wool and administering strychnine subcutaneously, posterior gastro-enterostomy was performed, a bone bobbin being used. The operation was done as quickly as possible in order to save shock. The tumour felt before operation was found to be formed by stomach and pancreas firmly fixed together towards the pylorus, but leaving the cardiac end of the stomach free, so that no difficulty was found in doing a satisfactory operation, which was completed in twenty-five minutes.

After-History.-Saline subcutaneousinjections and rectal enemata were given and strychnine was freely administered, but for two days we had a great fight with death, apparently due to poisoning by the foul stomach contents. Hot water was freely given to induce vomiting on the second day, as the patient could not bear the stomach tube being used. This gave relief, and afterwards progress to recovery was uninterrupted. $\mathrm{He}$ rapidly gained strength and put on flesh, returning home five weeks after operation. His friends, who had despaired of his recovery, were astonished to find him so well. In April he was in such good health that he married. A year later he was in perfect health.*

* Although this case is reported under Perigastric Abscess, we repeat it here as it illustrates both conditions. 
Case 3.-Acute Gastritis; Posterior Gastro-enterosiomy ; DEATH.

The following case, seen with Dr. Mitchell at Whitby on December 26, 1902, was clearly one of acute gastritis, though the cause was obscure.

The patient was a woman, aged thirty-five, who had suffered from indigestion for some time before the onset of acute stomach symptoms three weeks before our seeing her. These symptoms were characterized by persistent vomiting, great pain and epigastric tenderness. Nothing could be retained in the stomach, and at times blood or mucus tinged with blood was vomited. The rigid recti prevented accurate palpation, but the stomach was apparently swollen and an indefinite tumour could be felt. There was obstinate constipation, intense depression of spirits, amounting to melancholia, and the patient had a fixed idea that she was going to die, and, in fact, wished for death. The pulse was rapid and feeble, and the respirations were catchy and irregular. A provisional diagnosis of gastric ulcer with subacute peritonitis was made and operation advised.

On exposing the stomach it was thick, hard, and leathery, the vessels crossing over and around it being very much congested. The pylorus was contracted, apparently due to spasm. A posterior gastro-enterostomy was performed in order to try to bring about relief by resting the stomach. On opening the stomach the wall was found to be thick and inflamed, and the mucous membrane at once attracted attention by its black-red appearance, showing not only deep congestion, but staining by extravasated blood.

After operation there was decided relief, and all vomiting ceased. Mouth-feeding was carefully carried out, but alimentation was chiefly rectal.

The melancholia and the fixed idea of death never left the patient, though the pulse improved and the general condition appeared to be mending up to the fifth day, when she suddenly collapsed, without any sign of peritonitis, and nothing to account for death except her determined wish to die.

No autopsy could be obtained.

The following is a complete list of all recorded cases :

CAsE 1.-Coachman, aged twenty-eight.

Presumed Cause and Chief Clinical Symptoms.-Due to a chill. Pain in abdomen. Frequent vomiting. Wandering talk. Collapse.

Duration.-Two days. 
Post-mortem Appearances.-Stomach wall about six times usual thickness. Purulent infiltration between mucous and serous membranes. Muscular wall completely destroyed.

Reference.-Heyfelder, Schmidt's Jahrbïcher, I837, Bd. xvi., S. 192.

CASE 2.--Artisan, aged sixty.

Presumed Cause and Chief Clinical Symptoms.-Icterus. Pain in stomach. Stomach neighbourhood hard and swollen. Distension; vomiting of quantities of bile. Obstinate constipation.

Duration. - Fourteen days.

Post-mortem Appearances.-Purulent peritonitis. Stomach small. Wall six times usual thickness.

Reference.-Herzog, Kaspar's Wochenschrift, I839, S. 813.

CASE 3.-Joiner, male, aged twenty-four.

Presumed Cause and Chief Clinical Symptoms. - First entered hospital at beginning of 1842 with symptoms of gastritis or chronic ulcer; had attacks of vomiting of black substances. Improved temporarily, but bad symptoms returned. Readmitted January, I 843. Pale, emaciated; epigastric pain, frequent and produced on pressure; fever and vomiting at night of mucus or mucus mixed with curdled milk; habitual constipation. From January 25 symptoms became aggravated. On February I erysipelas of nose appeared, next day continuous hiccough, persisting till death. Erysipelas spread over face, pain in back and kidneys became violent. A pseudo-membranous stomatitis appeared on lips, cheeks, and tongue; patient sank into a state of somnolence, from which he was wakened by pain and shaking caused by hiccoughs, which came on in fits. Died on February 9.

Duration.-Between five and six weeks, dating from second admission to hospital.

Post-mortem Appearances. - Three abscesses the size of cob-nuts occupied the large curvature of stomach, situated between the two folds of the gastro-intestinal epiploon; another was situated in the gastrosplenic epiploon. On raising stomach there was found a collection of creamy pus in posterior cavity of the epiploon ; considerable destruction of peritoneum covering posterior wall of stomach. Peritoneum lining walls of posterior cavity showed hardly any injection and no false membrane. Pus seemed not to have been produced at this place, but, occurred outside the peritoneum like the abscesses found at the large curvature, then to have burst its capsule and caused ulceration on posterior aspect of stomach. Stomach was strongly adherent by its small curvature and a portion of its anterior surface, to the concave aspect of liver. On incision in direction of large curvature, a cicatrix was found near pylorus at small curvature, formed of dense, thick fibrous tissue fixed to the concave surface of liver. Around this cicatrix mucous tissue formed some folds, and pyloric opening was much 
stenosed. Mucous membrane was studded with small gray points and raised at places by small collections of pus, the largest the size of a small nut. Small intestine stenosed, and its mucous surface covered with a multitude of vesicles with gaseous contents only.

Reference.-Mayor, Bulletin Soc. Anat. de Paris, i 840, 290.

CASE 4.-Hospital nurse, female, aged forty.

Presumed Cause and Chief Clinical Symptoms.-For a fortnight had suffered from pains in limbs and back, and for a few days in stomach and chest. Abdominal tenderness subsequently much increased. Anorexia and constant vomiting of dark-coloured, bitter fluid, with intense thirst. Death preceded by restlessness and stupor.

Post-mortem Appearances.-Peritoneal cavity contained a quantity of yellow, opaque fluid of puriform consistence, very offensive. At pyloric third of greater curvature of stomach was a firm mass measuring $4 \frac{1}{2}$ inches by $3 \frac{1}{2}$ inches. On opening the stomach a small quantity of greenish fluid escaped. Mucous membrane dotted over surface with points of ecchymosis. Irregular darkbrown patch about size of a shilling found near pylorus, corresponding with centre of thickened mass. When the peritoneal and muscular coats were divided a collection of pus was found between fibres of submucous cellular tissue. Pus not liquid.

Reference.-Habershon (S. O.), Guy's Hospital Reports, London, I 855 , p. I I 5 .

CASE 5.-Male, aged fifty-four.

Presumed Cause and Chief Clinical Symptoms.-Chill and heat. Pain in neighbourhood of stomach, heart, and left thorax. Loss of appetite, malaise, vomiting of quantities of bile. Cough. Dyspnœa. Restlessness. Delirium. Collapse.

Duration.-Four and a half days.

Post-mortem Appearances. - Stomach wall enormously thickened, especially in neighbourhood of cardiac orifice and in fundus; the submucous membrane infiltrated with pus in its whole extent; mucous membrane in neighbourhood of cardia with sieve-like perforation; serous and subserous membranes dulled and infiltrated; on the left a sero-fibrinous pleuritic exudation. Effusion in peritoneal cavity.

Reference. - Klaus, Beit. zur Kenntniss der Magenkrankheiten, Inaug. Diss., Erlangen, 1857 .

CAsE 6.-Soldier, aged twenty-four.

Presumed Cause and Chief Clinical Symptoms.-Intemperance. Pain in head and depression. After one day patient went of his own accord to hospital. Pain in neighbourhood of stomach.

Duvation.-Some days.

Post-mortem Appearances,-Double pleurisy. Peritonitis. Stomach 
walls thickened from 6 centimetres to 8 centimetres, especially near the cardiac orifice, at the pylorus and at the base. Mucous membrane perforated like a sieve, submucous membrane with purulent infiltration. Serous and subserous membranes with serous exudation dulled and thickened.

Reference.-Schmidt's Jahrbiicher, $\mathrm{r} 85^{8}$, Bd. xviii.

CASE 7.-Male, aged seventy-six.

Presumed Canse and Chief Clinical Symptoms.--Loss of appetite and colicky pain. Sudden vomiting. Severe seizure. Ileo-cæcal and epigastric pain; constipation, diarrhœa later.

Post-mortem Appearances. - Mucous membrane of stomach granulated, grayish-red, four abscesses of from the size of a hazel-nut to a walnut, one at the pylorus, three in the direction of small and one in that of the large curvature. Stomach walls flabby and thickened.

Reference.-Auvray (L. A.), 'Étude sur la Gastrite phlegmoneuse,' Thèse de Paris, 1866.

CASE 8.-Coachman, aged forty-seven.

Presumed Cause and Chief Clinical Symptoms.-For four days cough, pain in head, thorax, and stomach. On next day vomiting of quantities of bile, pain in epigastrium, cold perspiration, rapid pulse. Death through falling out of window.

Duration.-Six days.

Post-mortem Appearances. - Serous exudate from peritonitis. Stomach wall thickened from accumulation of pus.

Reference.-Mazet, Bulletin de la Soc. Anat., r86r ; Auvray, 'Étude sur la Gastrite phlegmoneuse,' r866, p. I 7.

CASE 9.--Peasant, male, aged twenty-five.

Presumed Cause and Chief Clinical Symptoms.-Due to intemperance. For several months loss of appetite. Restless condition. Periodical pain at base of heart. . On some occasions face swollen. Malaise, vomiting. Region of stomach slightly distended. Severe pain in stomach. No fever.

Duration.-Three days.

Post-mortem Appearances.-Turbid yellow flocculent serum in peritoneal sac, a little grayish-red mucus. Stomach wall at the pylorus thickened to extent of $1 \frac{1}{3}$ centimetres, towards cardiac orifice to extent of I centimetre. The submucous membrane alone thickened, whitish-yellow, evacuates pus on pressure. Spleen large.

Rejerence.-Asverus (H.), ' Ein Fall von Gastritis phlegmonosa,' Jenaische Zeitschrift f. Med. Natur ; Jena, r866, Bd. ii., S. 476-482.

CASE ro.-Porter, male, aged forty.

Presumed Cause and Chief Clinical Symptoms.-Due to excess in drinking. Abdomen distended and sensitive to pain in neighbour- 
hood of stomach. Pain not increased on pressure. Vomiting, diarrhœa two days previous. Wandering talk, pupils dilated, not reacting to light. Convulsions.

Duration.-Three days.

Post-mortem Appearances.-Meninges injected; in ventricles of brain from 2 to 3 tablespoons of serous exudate. Purulent peritonitis. Walls of stomach thickened from $\frac{1}{2}$ centimetre to I centimetre. Mucous membrane in places hyperæmic, and in two places ulcerated. Submucous membrane with purulent infiltration.

Reference.-Cornil ; see Auvray, loc. cit., p. 20.

CASE I r.-Conductor. Male, aged forty-four.

Presumed Cause and Chief Clinical Symptoms.--Due to malnutrition. Malaise, bilious vomiting. Abdominal pain, diarrhœa, alternating with constipation. Small quick pulse. Delirium, collapse.

Duration.-Fourteen to fifteen days.

Post-mortem Appearances.-All the abdominal organs covered with pus. Stomach walls thickened to extent of I centimetre. At the great curvature was found a tumour size of chestnut, according to Auvray dependent on local hyperplasia of the tissue of the submucous membrane.

Reference.-Lasège; see Auvray, loc. cit.

CASES I2 and I 3 .

Presumed Canse and Chief Clinical Symptoms.-Apparently no symptoms on the part of the stomach during life, because at the post-mortem the stomachs were only examined as a part of the routine.

Post-mortem Appearances. - The mucous membrane of the stomach in its whole extent raised by a thick layer of pus infiltrating the submucous cellular tissue.

Reference.-Kurze, 'Sections befunds von Andra und Cruveilhier'; Reinking, loc. cit., p. r.

Case 14.--Soldier.

Presumed Cause and Chief Clinical Symptoms.-Vomiting, severe pain in region of the stomach. Fever, delirium.

Duration.-Some days.

Post-mortem Appearances. - The walls of the stomach in their entire extent covered with a purulent infiltration.

Reference.-Bamberger, Henoch's Klinik der Unterleibskrankheiten, Bd. ii., S. 196.

CASE 15.-Idiot, aged twenty-two.

Presumed Cause and Chief Clinical Symptoms.-Pleuritic symptoms. Duration.-Fairly long time.

Post-mortem Appearances. - Wall of stomach thickened, principally 
at the pylorus. The mucous membrane of the stomach locally ecchymosed. Purulent infiltration of submucous membrane.

Refevence. - Mascaral, Bulletin de la Société Anat., t. xv., p. I 73- 175 .

CASE 16. - Female.

Presumed Cause and Chief Clinical Symptoms.-Due to puerperal fever. No stomach symptoms.

Post-mortem Appearances.-Pneumonia in the second stage, purulent peritonitis and pericarditis. The submucous membrane of the stomach purulently infiltrated.

Reference.-Manoury, ibid., t. xvii., p. 185 .

CAse r 7.- Male.

Presumed Cause and Chief Clinical Symptoms.-Sudden onset malaise, depression, pain in back and head, with vertigo and singing in the ears. Green, bilious vomiting. Pain in epigastric. and left hypochondriac regions, but only present on pressure. Rest of abdomen soft and painless. Vomiting one of first symptoms, and repeated from time to time. Considerable fever present on second day, and intermitted. Pulse small and frequent. Rigor, intense headache, singing in ears, vertigo, great prostration and anxiety.

Duvation.-Hardly two days.

Post-mortem Appearances.-Stomach wall thickened, more than 2 centimetres near the pylorus. The mucous membrane injected and softened, ecchymosed in places and easily detached. Submucous coat infiltrated in nearly all its extent with white, creamy pus.

Reference.-Gaudy, Archives Med. Belg., I 863, t. xxxi.,pp. 459-464 (tabulated by Leith).

CAse i 8.

Post-mortem Appearances.-A sero-purulent infiltration under the mucous membrane of the stomach. Mucous membrane thickened.

Reference.-Caudmont, ibid., t. xxxiii., p. 273 ; see Reinking, loc. cit., p. I3.

CASE I9.-Workman.

Presumed Canse and Chief Clinical Symptoms.-Due to defect in diet. On the first day frequent sickness, during the next days vomiting and pain in the stomach. On the fourth day the patient left his bed and went to a public-house, where he drank warm beer. Immediately after wandered in his speech and became deathly pale. Died suddenly on being taken to the hospital.

Duration.-Four days.

Post-mortem Appearances. - A sero-purulent fluid in the abdomen. Stomach contained turbid fluid. Mucous membrane swollen. Purulent infiltration of the submucous tissue. 
Reference.-Thungel, Virchow's Avchiv, i865, S. 406-408.

CASE 20.-Workman, aged forty-two.

Presumed Cause and Chief Clinical Symptoms.-Brought to the hospital moribund. Dead in a few hours. Nothing of importance could be ascertained of his circumstances in life.

Post-mortem Appearances. - Stomach smaller than normal. Serous membrane opaque, in parts wrinkled, and in the region of the small curvature, as well as in the upper section of the anterior wall, covered with a fine layer of fibrin. On the inner surface of the stomach numerous ulcers, both large and small. The walls of the stomach thickened over the extent and in the neighbourhood of the abscesses. In the veins of the submucous membrane, as in the larger subserous veins of the posterior wall of the stomach, deposits of a purulent, fibrous nature.

Reference.-Ackermann, Virchow's Archiv, I869, Bd. xlv., S. 39.

CASE 2I.-Workman, aged twenty-six.

Presumed Cause and Chief Clinical Symptoms.-? Due to poisoning. Sudden severe pain in epigastrium. Vomiting, chill, hot and cold sweats. Pains increased on pressure on the epigastrium. After two days symptoms of the algid form of cholera set in-namely, cyanosis, thready pulse, retention of urine, intense thirst.

Duration.-T Two days.

Post-mortem Appearances.-Sero-purulent effusion in the peritoneal cavity. Thickening of the walls of the stomach. In the whole extent of the pylorus up to the cardiac orifice of the stomach effusion of pus between the mucous membrane and the serous membrane. Submucous membrane and muscular coat destroyed. The mucous membrane ecchymosed at the fundus.

Reference.-Guyot; see Auvray, loc. cit., p. 34 .

CASE 22.-Female, aged ten.

Presumed Cause and Chief Clinical Symptoms.-Friday, January 3I, I868, taken ill and vomited everything. Came under treatment Sunday, February 2. Pulse rapid and hardly perceptible, extremities cold. Headache. Temperature $96.50^{\circ} \mathrm{F}$. Vomiting still continued, nothing retained, substance mixed with greenish fluid. Remained conscious to end, and died Monday, February 3, at 5 a.m.

Duration.-Three and a half days.

Post-mortem Appearances.-Stomach walls fully $\frac{1}{2}$ inch in thickness, consisting of mucous lining and peritoneal investment, with intervening laying of purulent deposit. Milky fluid pressed out from cut surface, responding to chemical and microscopical tests for pus. Inflammatory granular corpuscles seen under microscope mixed with pus corpuscles. Purulent inflammation of connective tissues extended over whole circumference from 
cardiac to pyloric orifice of stomach. Line of division between gastric and duodenal mucous membrane marked by abrupt ridge, caused by submucous purulent exudation. Gastric mucous membrane deeper colour than usual. Peritoneal coat injected, but not inflamed.

Reference.-Hun (E. R.), N. Y. Med.Journ., I 868, vol. viii., p. I 8. CASE 23.-Male, aged twenty-five.

Presumed Cause and Chief Clinical Symptoms.-Due to intemperance. CEdema of face, want of appetite, vomiting. Singultus.

Post-mortem Appeavances.-Hæmorrhagic effusion in the pericardium. Discharge of pus between the mucous membrane and submucous membrane, the latter intact.

Reference.-Morel, Bulletin de la Société Anat., I865; see Auvray, loc. cit., p. 38.

CASE 24.-Female, aged twenty-four.

Presumed Cause and Chief Clinical Symptoms.-Due to immoderate diet. Continued vomiting. Abdominal pains did not increase on pressure. After some days the above symptoms disappeared, but began again with increased violence on the fifth day. Feeling of repletion. Abdominal distension. Sudden death. Patient reported to have twice suffered from jaundice, the consequence of gall-stones.

Post-mortem Appearances. - Recent peritonitis. Gall-bladder gangrenous, and perforated into the peritoneal cavity. Walls of the stomach were symmetrically thickened. Purulent infiltration of the submucous membrane. The mucous membrane was injected and thickened.

Reference.-Stewart, Jahresberichte von Virchow $u$. Hirsch, I868, Bd. ii., p. I25.

CAse 25.-Doctor.

Presumed Canse and Chief Clinical Symptoms.-Due to trauma. Abdominal pains, bilious vomiting, rapid, weak pulse. Typhoid condition, symptoms of peritonitis. Delirium.

Duvation.-Four days.

Post-mortem Appearances.-Recent peritonitis. The mucous membrane of the stomach, especially at the pylorus, thickened. Fibrino-purulent discharge between the mucous membrane and muscular coat. Mucous membrane in parts injected.

Reference.-Loomis; Med. Rec., N. Y., February I 5, 1869.

CASE 26.-Workman, aged forty-two.

Presumed Cause and Chief Clinical Symptoms._Delirium, cyanosis, especially on lips. Labial herpes. Violent shivering at every movement. Temperature 40 to $4 \mathrm{I}^{\circ} 2^{\circ} \mathrm{C}$. Diagnosis set down as typhus.

Post-mortem Appearances. - The mucous membrane of the stomach 
coloured light gray, with a spotted injection ecchymosis, especially in the fundus. Towards the region of the cardiac orifice of the stomach severe infiltration of pus.

Reference. - Ebert, Zur Kenntniss der Mykotischen Prozesse, Bd. xxviii., p. 2 I.

CASE 27.-Blacksmith, aged thirty-seven.

Presumed Cause and Chief Clinical Symptoms.-Due to chill. Rigor, heat, pain in the epigastrium and in the region of the kidney. Blood and cylindrical casts in the urine. Temperature normal during last days. The last two days blood and mucus in stools, and tenesmus.

Duration.-Eighteen days.

Post-mortem Appearances.-Cdema of brain and lungs. Acute nephritis and peritonitis with purulent exudation. S. Romanum with croupous inflammation. Infiltration of the stomach walls by pus. Submucous membrane thickened. In the mucous membrane four little cavities from 3 to 4 millimetres in diameter.

Reference.-Malmsten and Key, Jahresberichte von Virchow und Hirsch, I87I, Bd. ii., p. I49.

CASE 28.-Workman, aged thirty-six.

Presumed Cause and Chief Clinical Symptoms.-Due to trauma. Rigor, nausea, vomiting, pain in the epigastrium. Abdomen swollen. Temperature $38^{\circ} \mathrm{C}$. Cessation of vomiting. Collapse.

Duration.-Eleven days.

Post-mortem Appearances.-Pericarditis, pleuritis. In the peritoneal cavity $\frac{1}{2}$ litre fluid resembling pus. Walls of the stomach thickened at the pylorus to $I_{2} \frac{1}{2}$ centimetres, at the fundus to I centimetre, caused by infiltration of the submucous membrane by pus. Some abscesses in the mucous membrane. Mucous membrane hyperæmic. On the walls of the stomach a network of lymph vessels filled with pus.

Reference.-Belfrage and Bedenius, Schmidt's Jahvbiicher, I872, Bd. CLIV.

CASE 29.-Female, aged forty-nine.

Presumed Cause and Chief Clinical Symptoms.-Abdominal pain Uncontrollable vomiting. Fever, coma.

Duvation._Four weeks.

Post-mortem Appearances.-Abdominal cavity filled with fluid, illsmelling pus. At the small curvature behind and to the left a perforation of 15 millimetres diameter. Mucous and other membranes destroyed or changed into a soft mass consisting of detritus and blood elements.

Reference.-Feroci, Ann. Univ. di med. e chir. Milano, 1873.

CASE 30.-Mason, aged thirty-four.

Presumed Causes and Chief Clinical Symptoms.-Intemperance. 
Loss of appetite. Cough. Hoarseness after four days. Oppression in stomach, vomiting, chill, high temperature $\left(40^{\circ}\right.$ C.). Delirium; two weeks previously delirium tremens.

\section{Duvation.-Thirteen days.}

Post-mortem Appearances. - Stomach considerably distended. Mucous membrane thickened. Submucous membrane at pylorus infiltrated with pus.

Reference.-Rakowak, Wienter med. Presse, I873, No. xxv.

CASE 3I.-Girl, aged seventeen.

Presumed Cause and Chief Clinical Symptoms. - Rigor, fever, headache, vomiting, obstinate constipation. Abdomen swollen and tender.

Post-mortem Appearances. - In the body large quantity of a thin brownish-yellow coloured fluid. The mucous membrane of the stomach in places injected; near the pylorus the mucous membrane showed a distinct état mameloné. In the circumference of the small curvature was a phlegmono-purulent infiltration, in parts spotted, in parts diffuse. Purulent peritonitis and right pleurisy. Pharyngitis.

Reference. - Krause, 'Ueber submucose phlegmonose eitrige Magenentzündung,' Inaug. Diss., Kiel, 1874.

CASE 32.-Boy, aged eleven.

Presumed Cause and Chief Clinical Symptoms.-Pyæmia stated as cause. Constant sickness. Loss of appetite. Abdomen much swollen and painful. Constipation. Bilious vomiting. Wandering. Collapse.

Duvation.-Four days.

Post-mortem Appearances.-Purulent peritonitis, enlargement of the spleen, swelling and dulness of liver. Dulness over the kidneys. Edema of lungs. Stomach walls thickened at the pylorus to $\mathrm{I} \cdot 3$ centimetres, caused by purulent infiltration of the submucous membrane. Mucous membrane swollen, pale and opaque, tumid; muscular coat similarly swollen and tumid. Cocci proved to be present.

Reference.-Löwenstein, ' Ueber Phlegmonose Gastritis,' Inaug. Diss., Kiel, I 874.

CAsE 33--Builder, aged twenty-three.

Presumed Cause and Chief Clinical Symptoms. - According to necrotomy, pyæmia. Death eight days after operation. On the two last days pain and sensitiveness to pressure in abdomen. Frequent vomiting. Loss of appetite.

Duration.-Two days.

Post-mortem Appearances. - Purulent peritonitis, chronic nephritis. Stomach wall much thickened. Mucous membrane pale, flaccid, 
turgid, loose. Submucous membrane infiltrated with cloudy serous fluid.

Reference.-Löwenstein, loc. cit.

CASE 34.-Male.

Presumed Cause and Chief Clinical Symptoms.-Extraordinary tendency to vomit.

Post-mortem Appearances. - Granular nephritis. Stenosis of pyloric opening in consequence of adhesive inflammation of submucous tissue.

Reference.-Budd, 'Diseases of Stomach,' p. 94.

CASE 35.-Male, aged twenty-five.

Presumed Cause and Chief Clinical Symptoms.-Trauma assigned as cause. Violent abdominal pain. Vomiting. On seventh day vomited $\frac{1}{2}$ pound of pure pus. Immediate alleviation, and then recovery.

Duration.-Seven days.

Reference.-Kurschmann, Wien. Med. Wóchenschrift, I880, No. I4. CASE 36.-Male, aged twenty-five.

Presumed Cause and Chief Clinical Symptoms.-Loss of appetite. Malaise, vomiting, violent pain in neighbourhood of stomach. Sudden death.

Duration.-Five days.

Post-mortem Appearances.-Stomach distended with gas. Walls thickened, especially at pylorus, to extent of I inch. Purulent infiltration of submucous membrane, and to a less extent also of the muscular coat. Gland epithelium intact, closely beset by pus corpuscles.

Reference.-Hilton Fagge, Jahvesbericht von Virchow $u$. Hirsch, I 875, Bd. ii., Heft 225.

CASE 37.

Presumed Cause and Chief Clinical Symptoms.-In the presence of the physician 20 ounces of pure pus were vomited. Pus in stools.

Post-mortem Appearances.-More than 7 pounds of pus in abdomen, proceeding from an enormous abscess of stomach which had developed up to the time of the fatal catastrophe (perforation) painlessly and without symptoms.

Reference.-Callow, 1824, Ziemssen's Handbuch f. Spec. Path. u. Therapie, I876, Bd. vii. (2), Heft 50.

CASE 38.- Tanner.

Presumed Cause and Chief Clinical Symptoms.-Alternate rigor and heat sensations. Intense pain in neighbourhood of stomach, heart, and left thorax. Vomiting of quantities of bile. Temperature considerably increased. Abdomen distended by meteorism. Rapid pulse, severe thirst, collapse. 
Duration.-Five days.

Post-mortem Appearances.-Diffuse sero-purulent infiltration of submucous membrane, with general extension to the outer membranes. Peritonitis. Pleurisy. General blood dissolution.

Reference. - Dittrich, Ziemssen's Handbuch f. Spec. Path. u. Therapie, 1876, p. $5^{\circ}$.

CASE 39.--Male, aged fifty-three.

Presumed Cause and Chief Clinical Symptoms. - Intemperance assigned as cause. Rigor, high fever, rapid pulse, loss of appetite, vomiting, pain in neighbourhood of stomach. Abundant diarrhœa. Collapse. Delirium. Death in coma.

Duration.-Nine days.

Post-mortem Appearances.-Mucous membrane of stomach swollen, with sieve-shaped perforations. Walls thickened to extent of $1 \frac{1}{2}$ centimetres. In submucous membrane were some small layers of pus. Gastro-intestinal mucous membrane chronically inflamed. Trace of peritonitis.

Reference.-Deininger, Dentsches Avchiv f. klin. Med., Bd. xxiii., I 876, S. 624 .

CAse 40.-Female, aged thirty-two.

Presumed Cause and Chief Clinical Symptoms.-Rigor. High temperature. Violent pain in stomach. Neighbourhood of stomach distended, very sensitive to pressure. Diarrhœa. Gradual development of a tumour as large as the fist, and accentuation of all symptoms. On nineteenth day vomiting of large quantity ( $\frac{1}{4}$ pound) of pus pervaded with blood-streaks. Disappearance of tumour. Convalescence.

Duration.- Nineteen days.

Reference.-Deininger, loc. cit.

CASE 41.-Soldier, aged nineteen.

Presumed Cause and Chief Clinical Symptoms.-Cause assigned, bad diet. Stomach neighbourhood sensitive. Oppression in stomach. Regurgitation, vomiting of quantities of bile. Headache, rigor, high temperature, collapse.

Duvation.-Six days.

Post-mortem Appearances.-Stomach wall at cardiac orifice and great curvature thickened to extent of $\mathrm{I} \frac{1}{2}$ centimetres. Submucous membrane infiltrated with pus. Mammillated condition of mucous membrane. Purulent inflammation of lowest portion of œesophagus.

Reference.-Chvostek, Wiener Med. Presse, I877, Bd. xvii., S. 693 .

CASE 42.-Corporal, aged twenty-four.

Presumed Canse and Chief Clinical Symptons.-Cause assigned, bad diet. Loss of appetite. Malaise. Abdomen distended and 
sensitive. Vomiting; constipated. Stomach neighbourhood very sensitive.

Duration.-Nine days.

Post-mortem Appearances.-Purulent peritonitis. Stomach contains fluid. Submucous and mucous membranes infiltrated with pus. Mucous membrane covered with blood coagulum.

Reference.-Chvostek, Wiener Med. Presse, 1877.

CASE 43.

Post-mortem Appearances. - Short description of an acute phlegmonous gastritis with chronic nephritis.

Reference.-Flint, Phila. Med. Times, August 8, 1878.

CASE 44.

Post-mortem Appearances.-Mentions description of a case of gastritis phlegmonosa in which pus was found between the walls of the stomach 2 inches distant from the pylorus.

Reference.-Andral, S., Albers' 'Erlauterungen,' iv. 5, I8.

CASE 45--Woman.

Presumed Cause and Chief Clinical Symptoms.-Long-standing cardialgia and shortness of breath.

Post-mortem Appearances.-Between the coats of the stomach large quantities of pus collected.

Reference.-Lieutaud, 'Historia Anatomica-médica,' vol. i., p. 35. CASE 46.-Woman, aged fifty-four.

Presumed Cause and Chief Clinical Symptoms.-Pain in the stomach neighbourhood, which at first became worse after eating, at last continual loss of appetite. Sudden darting pain in the left side of breast. Shortness of breath.

Duration.-One day and a half.

Post-mortem Appeavances. - Large abscess in the small curvature of the stomach near the cardiac orifice, which extended as far as the softened and reddened muscular coat, lay between the two folds of the peritoneum which form the lesser omentum. The contents of the abscess, on pressure, moved towards the diaphragm by an opening through which the pus passed into the cavity of the left breast. The mucous membrane at the seat of the abscess red and slightly swollen, also the muscular coat. The entire wall of the stomach rich in blood.

Reference.-Albers, 'Erlauterungen,' iv., S. I8.

CASE 47.-Male, aged fifty.

Presumed Cause and Chief Clinical Symptoms.-Violent pains in neighbourhood of stomach and continuous vomiting. Pain in epigastrium, increased upon pressure. On the day preceding death the vomiting ceased. Collapse.

Post-mortem Appearances. - Brownish-red fluid in stomach. Mucous membrane black-violet colour. Stomach wall thickened 
to extent of I centimetre. Sero-purulent infiltration of submucous membrane.

Reference.-Lewandowski, Berl. klin. Wochenschr., I 879, No. $3^{6 .}$

CASE 48.- Wheelwright, aged twenty-nine.

Presumed Cause and Chief Clinical Symptoms.-Convalescing after typhus. Vomiting, pain, anorexia. Improvement after lavage of stomach, deterioration again later. Death.

Post-mortem Appearances. - Submucous membrane of stomach thickened to extent of $\mathrm{I}$ inch, this caused by purulent infiltration. Muscular coat also somewhat infiltrated. No peritonitis.

Reference.-Garel, I879.

CASE 49.-Workman, aged fifty-two.

Presumed Cause and Chief Clinical Symptoms.-Due to intemperance. Rigor. Violent vomiting, severe abdominal pain, high temperature and rapid pulse. Collapse.

Duration.-Seven days.

Post-mortem Appearances. - Exudation in peritoneal cavity. Peritoneum injected to great extent. Stomach wall much thickened, flaccid and loose. Purulent infiltration chiefly at fundus and pylorus. Mucous membrane pale, in places ecchymosed.

Reference.-Glax, Berl. klin. Wochenschr., I879, No. 38.

CASE 50.-Male, aged seventeen.

Presumed Cause and Chief Clinical Symptoms.-Violent fever. Pain in stomach; complete anorexia. Malaise and pain in neighbourhood of stomach. Frequent vomiting of slight quantities of tenacious pus. Afterwards remission of fever. Recovery after four weeks.

Duration.-Four weeks.

Reference.-Glax, Berl. klin. Wochenschr., I 879, No. 38.

CASE 5I.-Male, aged forty.

Presumed Cause and Chief Clinical Symptoms.-Pain in neighbourhood of stomach, violent vomiting. Neighbourhood of stomach distended. Constipation. Temperature increased. Collapse.

Duration.-Five days. Post-mortem examination forbidden.

Reference._Glax, Berl. klin. Wochenschr., 1879, No. $3^{8 .}$

Case 52.- Soldier, aged fifty.

Presumed Cause and Chief Clinical Symptoms.-Violent fever. Severe pain in epigastrium. Malaise, vomiting, sensation of anxiety. Collapse.

Duration._-Four days.

Post-mortem Appearances. -Stomach greatly enlarged. Walls thickened to extent of $\mathrm{I} \frac{1}{2}$ centimetres. Purulent infiltration of submucous membrane. Liver enlarged.

Reference.-Petersen, St. Petersburger Med. Woch., I879, S. 288. 
Case 53--Soldier, aged twenty-two.

Presumed Cause and Chief Clinical Symptoms.-Frequent vomiting, high temperature (to $39^{\circ} 6^{\circ} \mathrm{C}$.), violent abdominal pain.

Post-mortem Appearances. - Purulent infiltration of submucous membrane of stomach and of the upper part of duodenum.

Reference.-Chvostek, Wiener Med. Blätter, I88I. Nos. 27, 30, and 32 .

CASE 54--Male, aged fifty-four.

Presumed Cause and Chief Clinical Symptoms.-After an operation (gastrostomy) for an impassable œsophageal stricture due to epithelioma. Began with rise of temperature and sickness. Pain intense in region of stomach on twenty-first day after operation. This was increased later, and exaggerated on pressure. Vomiting - many attempts, which ceased some hours before death. Temperature $101^{\circ} \mathrm{F}$. on first day of acute attack, and rose to $103^{\circ} \mathrm{F}$. before death. Rapidly sank and collapsed, and then death.

Duration.-Thirty-six hours after onset of untoward symptoms.

Post-mortem Appearances. - Submucous and mucous coats everywhere infiltrated with pus, which flowed freely, less marked in region of wound, which was quite healthy. Recent peritonitis found.

Reference.-Silcock (A. Q.), Trans. Path. Soc., I 882-1883, xxxiv., p. 90 (tabulated by Leith).

CASE 55-Male, aged thirty-five.

Presumed Cause and Chief Clinical Symptoms.-Chill, loss of appetite. Malaise, vomiting of bile, abdominal pain, small pulse, high temperature. Death in coma.

Duration.-Four days.

Post-mortem Appearances. - Parenchymatous inflammation of liver, spleen, and kidneys. Stomach walls, especially at pylorus, thickened to extent of $\mathrm{I} \cdot 2$ centimetres through purulent infiltration of submucous membrane. In the neighbourhood of the pylorus an abscess the size of a hen's egg.

Reference. - Treuberg Vratch, I883, No. 23.

CASE 56.-Female, aged thirty-two.

Presumed Cause and Clinical Symptoms.-Commencing typhoid state, then persistent vomiting, in which pus was shown to be present. Severe inanition, but gradual convalescence followed.

Reference.-Beckler, Bayer. Aerztl. Int. Bl., No. 37.

CASE 57.- Male, aged forty-five.

Presumed Cause and Chief Clinical Symptoms.-Painfulness in neighbourhood of stomach. No fever. Vomiting. Sarcinæ demonstrated as present in vomited contents.

Post-mortem Appearances.--In the submucous membrane near the pylorus on the posterior wall was found a circumscribed abscess, 
length I 3 centimetres, breadth 8 centimetres. Mucous membrane attenuated over its summit. Stomach much dilated, although pylorus was not stenosed.

Reference.-Testi, ' Un raro caso di ascesso dello stomaco,' Annali Univ. di Med., December 9, 1883, pp. 523, 547.

CASE 58.-Joiner, aged forty-seven.

Presumed Canse and Chief Clinical Symptoms.-Cause stated to be deficient nutrition. Rigor, fever, continuous vomiting.

Post-mortem Appearances. - Stomach abnormally enlarged. An ecchymosed patch at pylorus. Stomach walls thickened to extent of $\mathrm{r}_{2} \frac{1}{2}$ centimetres, due to collection of pus between mucous membrane and muscular coat. Streptococci in evidence.

Reference.-Sebillon, Thèse de Paris, 1885.

CASE 59.-Coachman, aged seventy.

Presumed Cause and Chief Clinical Symptoms.-Fever, pain in neighbourhood of stomach. Vomiting before being received into hospital. Death in coma.

Post-mortem Appearances.-In pelvis small amount of fibrinous, purulent fluid. Stomach walls in whole extent, from the pylorus to the cardiac orifice, infiltrated with pus. At the small curvature, some centimetres distant from cardiac orifice, was found an undecomposed gastric cancer.

Reference.-Gläser, Berliner klin. Wochenschrift, I883, No. $5^{\mathrm{I}}$.

CASE 60.-Maidservant.

Presumed Cause and Chief Clinical Symptoms.-Rigor. Temperature $40^{\circ} \mathrm{C}$. Violent pain beneath the left costal arch.

Post-mortem Appearances. - At the small curvature was a circular gastric ulcer. Stomach walls thickened to extent of $I_{\frac{1}{2}}$ centimetres. At the non-infiltrated places were some superficial defects.

Reference.-Gläser, Berliner klin. Wochenschrift, I 883, No. $5 \mathrm{I}$.

Case 6I.-Female, aged forty-nine.

Presumed Cause and Chief Clinical Symptoms.-Pain due to coming down of old femoral hernia. Pain in epigastric region, and later spreading to abdomen. Vomiting of small quantity of bilious material continued till death. Temperature reached $104^{\circ} \mathrm{F}$. on last day. Died comatose.

Duration.-Six days.

Post-mortem Appearances.-Submucous membrane at pyloric end infiltrated with pus. Some pus also between muscular and serous coats. Mucous membrane pale.

Reference.-Brit. Med. Journ., I884, i. 896 (tabulated by Leith).

CASE 62.-Ostler, aged sixty.

Presumed Cause and Chief Clinical Symptoms.-Vomiting. Collapse. Violent abdominal pain, especially on pressure; this sensitiveness allayed by morphia injection. Much thirst. Wine and water 
tolerated: Suddenly vomited brownish fluid (some spoonfuls), became worse, and died.

Post-mortem Appearances. - Stomach seen from outer aspect shows cicatricial stricture in middle of small curvature. Stomach largely filled with gas, contained, nevertheless, large quantities of thin brownish fluid mixed with sediment resembling coffee-grounds. Pylorus approaches cardiac orifice at distance of about $5 \frac{1}{2}$ centimetres. Large transverse cicatrix corresponding to change in serous membrane. On anterior surface of stomach were four patches of pseudo-membranous layer of a yellowish-gray, partly reddish colour, two on each side of cicatrix, without coming into contact with it. These could only partially be removed. These areas were necrosed at the surface, and invaded by an enormous number of bacilli. The submucous and muscular coats were similarly invaded by bacilli, but not the serosa. Nature of bacillus not determined. Proved not to be anthrax bacillus by culture experiments, and patient had been exposed to no anthrax infection.

Reference.-Nasse (D.), Archiv fïr Path. Anat. (Virchow), I886, Bd. civ., S. 584 .

Case 63.-Labourer, male, aged sixty-three.

Presumed Cause and Chief Clinical Symptoms.-Due to intemperance. Rigor and fever all over body fourteen days before admission to hospital. Pain in joints of arms and legs, which continued. Vomiting absent. Appetite fair, tongue dry, covered with white fur. Temperature $1024^{\circ} \mathrm{F}$, pulse 92 on first day. Temperature $98.8^{\circ}$ and $99^{\circ} 2^{\circ}$ on second day. Temperature $104^{\circ} 2^{\circ}$ and $106.2^{\circ}$, and pulse 132 to 144 on third day, i.e., day of death. Anxious look, confused and delirious on second day. This increased, and on third breathing stertorous; coma, and death. Symptoms generally resembled rheumatic fever.

Duration.-Two and a half days after last acute onset.

Post-mortem Appearances.-Stomach wall greatly thickened all round greater curvature and pylorus, where it was about I inch thick. Submucosa greatly infiltrated with pus, showing loculation in places. Vegetations in aortic cusps.

Reference. - Lancet, I887, ii. I I66 (tabulated by Leith).

Case 64.-Female, aged sixty-six.

Presumed Cause and Chief Clinical Symptoms.-Apparently due to pus being swallowed. After extraction of tooth pain in head. Neuralgia in left upper arm and indigestion. Gum of lower jaw was swollen and covered with superficial ill-smelling pus secreted from ulcers. After ten days sudden oppression in stomach neighbourhood. Vomiting. Considerable fever and great feeling of weakness. Collapse. Death.

Duration.-Four days. 
Post-mortem Appearances.-Diffuse peritonitis. Mucous membrane somewhat hyperæmic. Membrane generally much thickened. Submucous membrane, muscular coat, and serous membrane with purulent infiltration. Thickening advancing into duodenum.

Reference.-Lindemann, Mnüchener Med. Woch., I887, No. 25.

CASE 65--Student, aged twenty-five.

Presumed Cause and Chief Clinical Symptoms.-Abdomen distended and sensitive. Vomiting, obstinate constipation. Collapse.

Post-mortem Appearances. - Purulent peritonitis. Stomach distended by gas. Walls thickened. Submucous tissues contained numerous streptococci. Mucous membrane with cellular infiltration; in the centre necrosed.

Reference.-Reinking, loc. cit.

CASE 66.-Office servant, aged fifty-nine.

Presumed Cause and Chief Clinical Symptoms.-Unhealthy conditions of dwelling. Pain in neighbourhood of stomach. Loss of appetite, constipation. Vomiting. Sensitiveness to pressure on epigastrium. Fever. Area of dulness extended over whole abdomen and over one hand-breadth to the left, behind and below on the thorax; petechix of a bluish-red colour over the whole body, some the size of a hazel-nut. Severe icterus of the conjunctivæ and of the skin.

Duration.-Ten days.

Post-mortem Appearances. -Slight purulent exudate in the left pleural cavity. Diffuse sero-purulent peritonitis. Multiple abscess formation between the mucous and serous membranes of stomach. Parenchymatous nephritis.

Reference.-Lewin, 'Zur der Gastritis Phlegmonosa Idiopathica.' Berliner klin. Woch., I884, Bd. xxi., S. 83 .

CASE 67.-Journeyman tanner, aged thirty-three.

Presumed Cause and Chief Clinical Symptoms.-Chronic arsenic poisoning. Violent abdominal pain. Stomach neighbourhood very sensitive to pressure; considerable ascites. Vomiting. Among vomited substances were found starch granules, yeast fungi, fat crystals, muscular fibres and sarcinæ. Abdomen much dilated.

Post-mortem Appearances.-Sero-fibrinous exudate in abdominal cavity. Stomach wall thickened more than I centimetre; this due to purulent infiltration. Mucous membrane at base hyperæmic and yellowish, at other places pale. Mucous surface with sievelike perforations; from the perforations pus exudes on pressure. At the great curvature, at the distance of a finger-breadth from the pyloric valve, a carcinomatous ulcer was present.

Reference.-Mintz, Deutsche Archiv f. klin. Med., Bd. xlix., S. 487. 
CASE 68.-Coloured cook, male, aged forty-eight.

Presumed Cause and Chief Clinical Symptoms.-Came on suddenly with colicky pains and vomiting after light dinner. Much colicky pain in abdomen, vomiting frequent. Collapse.

Duration. - Two and a half days.

Post-mortem Appearances.-Stomach showed an infiltration of its submucosa and almost its entire wall with pus cells. The mucosa was normal, and the serosa showed an early peritonitis.

Reference.-Smith, New York Med. Record, October I2, I 889 (tabulated by Leith).

CASE 69.-Labourer, male, aged thirty-five.

Presumed Cause and Chief Clinical Symptoms.-Followed a week after fracture of finger; pain in stomach and vomiting. Pain violent in region of stomach and continued. Vomiting of bloody material, not pure blood. This was frequently repeated. Slight jaundice Pulse small and excited. Symptoms generally resembled those of ulcer of stomach.

Duration.-About two and a half days after first stomach symptoms.

Post-mortem Appearances.-Mucous membrane intensely injected and swollen. Several fresh excoriations of mucous membrane. Numerous air bubbles varying in size in wall of stomach. Similar appearances in duodenum. No pus seen; immense numbers of bacilli resembling those of anthrax in and around the walls of the gas cavities; not found in any other part of the body.

Reference.-Fraenkel, Archiv f. Path. Anat. (Virchow), I889, cxviii., S. 526 (quoted by Leith).

CASE 70.-Female, aged sixty.

Presumed Cause and Chief Clinical Symptoms.-Two violent attacks of severe gastralgia ; on second occasion with ulcerous inflammation of stomach neighbourhood. Recovered temporarily, but died later from hæmatemesis.

Reference.-Thoman, Allg. Wiener Zeitung, I89 I, No. го.

CASE 71.-Engineer, aged forty-one.

Presumed Cause and Chief Clinical Symptoms.-Commenced with violent vomiting of mucus and bile, which persisted for two or three days. This was followed by diarrhœea. Pain first in stomach and then in neighbourhood of liver. Became much worse on seventh day. CEdema of lungs. Death.

Duration.-Eight days.

Post-mortem Appearances.-Spleen enlarged. Duodenal membrane hyperæmic, in places ecchymosed. In ductus choledochus, as well as in gall-bladder, dark-green tenacious bile. Liver tissue intensely injected. Numerous ecchymoses in fundus of stomach ; in neighbourhood of cardiac orifice was a red area, in middle of 
which was a sharply-limited stria. On section were found two long cavities ( 2 centimetres in length) filled with thick pus, between mucosa and muscularis, partly within latter. The corresponding peritoneal surface was similarly beset with pus.

Reference.-Meyer, St. Petersburger Med. Wochenschrift, I892, No. 40.

CASE 72.-Soldier, aged forty-six.

Presumed Cause and Chief Clinical Symptoms.-Swallowed small quantity of turpentine seven years before. Two years after irregular attacks of dysphagia came on, associated with vomiting. No distinct pain before admission nor until two days before death. Could only take liquid food, which was soon vomited unaltered, due to fibrous stricture $\frac{1}{2}$ inch above termination of gullet. Temperature normal, except ten days before death, when it reached $\mathrm{IOO}_{4} \mathrm{~F}$., and fell again immediately, and on day before death, when it was again $100^{\circ} \mathrm{F}$. Emaciation great. On day before patient's death condition did not appear serious; got rapidly worse next day, became collapsed and died.

Duration.-A About seven weeks.

Post-mortem Appearances.-Abscess in the submucous coat of œsophagus 3 inches above stricture, and below it the submucous coat was infiltrated with pus. Stomach walls much thickened, due chiefly to a sero-purulent infiltration of submucous coat. Mucosa congested and swollen. Considerable numbers of bacilli like the coli communus.

Reference.-Kelynack, Lancet, i896, i. (March I4, tabulated by Leith).

CASE 73.-Pipemaker, male, aged forty-nine.

Presumed Cause and Chief Clinical Symptoms.-Irregular meals. Much vomiting, which was controlled by bismuth mixture. General peritonitis diagnosed. Temperature $102^{\circ} \mathrm{F}$. Exploratory incision made. Abdomen washed out. Slight improvement after operation, but severe symptoms soon reappeared, and he died in collapse about seven hours after operation.

Duration.-Five days.

Post-mortem Appearances. - Serous coat of small intestine intensely injected where coils were in contact with parietal peritoneum, 2 to 3 ounces of blood-stained serous fluid in abdominal cavity, but no pus. Peritonitis probably arose from thin layer of purulent lymph at lesser curvature $\mathrm{I} \frac{1}{2}$ inches from pylorus. Mucous surface of stomach swollen, submucous coat greatly thickened; no pus exuded from it on pressure or after section. Muscular coat slightly swollen, serous coat slightly injected. Microscopic examination showed changes in coat of stomach (infiltration with small round cells densely packed), indicating 
acute process of short duration. Bacteriological examination showed immense numbers of streptococci (Streptococcris pyogenes and erysipelatous); separate cocci and several groups of staphylococci, without formation of pus, were noteworthy.

Reference.-Leith (R. C.), Edinburgh Hospital Reports, I896, vol. iv. $5^{\text {I-II } 4}$ (his case, pp. $5^{\text {I-62). }}$.

Case 74.-Coloured cooper, aged forty-nine.

Presumed Cause and Chief Clinical Symptoms.-History of pain intensified by eating. Had vomited considerably; vomited matter containing. blood. First treated December, 1895. Put on gastric ulcer treatment, and improved. Had another attack February, I8g6, with more vomiting. Again improved. Admitted December I to Maryland General Hospital. Given a double test-meal. There had been no hæmatemesis for ten days. Improved up to December II, and was exhibited to clinic. Temperature for two months never rose above $98^{\circ} \mathrm{F}$. Bacterial cultures from vomit showed growth of streptococci and of OpplerBoas bacillus, also presence of pus in traces. Lavage employed. On January 18 temperature fell from $98^{\circ}$ to $94^{\circ} \mathrm{F}$., pulse became feeble, respiration 14 to 16 per minute February $\mathrm{I}$.

Duration.-Two. months in hospital; fifteen days from last attack.

Post-mortem Appearances.-Peritoneum pinkish colour and roughened, its vessels being engorged with blood. Covered with fibrin. Mesentery in same condition, stomach displaced downwards, serous coat generally inflamed same as peritoneum and mesentery. Peritonitis due to small opening in anterior surface of stomach not far from pylorus and lesser curvature. On section of stomach near pylorus an old, nearly healed ulcer was found, which had perforated after fresh ulceration. Pylorus stenosed. Microscopical examination showed that mucous coat was infiltrated with cells in neighbourhood of ulcer ; the same condition prevailed in the corresponding neighbourhood in the muscularis mucosæ, the cells here indicating presence of small miliary abscesses. Submucosa consisted of dense, firm fibrous tissue about base of ulcer. Little infiltration with small cells, but beneath muscularis mucosæ were groups of cell clumps similar to those in mucous membrane. There were similar groups of cells in the same position in the muscular layer. On the serous layer were minute elevated points visible to naked eye. These, examined microscopically, proved to be cancer cells. From the ulcerated area was obtained-(I) a bacillus agreeing with Oppler-Boas lactic acid bacillus, often associated with gastric carcinoma; (2) a micrococcus; (3) a yeast-like organism.

Reference.-Hemmeter, New York Med. Record, I897. 


\section{CASE 75.}

Presumed Cause and Chief Clinical Symptoms. - Treated for chronic pharyngitis and constipation on June 24, I898. Dull spot over right lung, but no family history of phthisis. Improvement, but on July I 3,3 a.m., severe attack of vomiting, with severe attack of pain in stomach and abdomen, not localized. Improved on bismuth, creosote, morphine, and milk diet. On July I4 vomited curdled milk; some pain in epigastrium. July 16 , temperature IO ${ }^{\circ} \mathrm{F}$., pulse Ioo. July I 8 , much belching, but no abdominal pain or distension. Headache, nausea, and diarrhœa absent. Vomited white of egg in milk, so this was omitted from milk, which agreed well by itself. In the evening condition worse. Temperature I03. $2^{\circ} \mathrm{F}$, pulse I 20 . Abdomen slightly tender all over. Difficult breathing, tongue dry, great thirst. Vomit analyzed; liquid reddish, solid reddish-brown. Microscope showed many oval round cells, few larger epithelial cells, some blood corpuscles, dark-coloured granular matter (? necrotic). On July I9 patient vomited same matter again, became slightly delirious, and died in ten minutes. Extremities cold, pupils dilated. Temperature under $\operatorname{arm} 107^{\circ} 4^{\circ} \mathrm{F}$. No autopsy allowed. An ulcer diagnosed to have broken on July I 3 at boundary of submucosa and muscularis, thus setting up diffuse process of suppuration in submucous coat, this causing moderate infiltration of small round cells in mucosa and rapid extension into muscularis. Muscularis gradually in turn affected serosa, and on evening of 18 th pus entered peritoneal cavity over large area, thus causing peritonitis and death.

Duration.-About four days from onset of acute symptoms. No post-mortem.

Reference.-Baerecke, Ncw York Med. Record, i898, liv. 949.

CASE 76.-Male, old.

Presumed Cause and Chief Clinical Symptoms.--Had suffered from hypertrophy of prostate, severe purulent cystitis, and purulent epididymitis.

Post-mortem Appearances.-Extensive purulent infiltration of the stomach wall in the neighbourhood of the cardiac orifice, at the small curvature, and at the fundus. Section showed the wall to be infiltrated and permeated with pus. In this, as well as in the blood, were streptococci in abundance.

Reference.-Simmonds, Dent. Med. Woch., I 890 (abstracted in Centralblatt der Verdaunngs Krankh., I90 I, ii. 533).

CASE 77.-Male, aged forty-one.

Presumed Cause and Chief Clinical Symptons.--Patient an alcoholic. Disease followed drinking spell, and was ushered in by vomiting, 
first of food, later of brownish fluid. Abdominal pain, dry tongue, shallow respiration, 48 per minute, regular pulse, Io 8 per minute, restlessness and anxious expression. Temperature IOI $^{\circ}$ by.rectum. Slight hypertrophy of left ventricle on physical examination. Abdominal walls rigid, and on deep palpation sensitive in epigastric and left hypochondriac regions. Crepitation beneath left costal arches. Urine containing casts. Patient grew progressively worse, and surgical intervention deemed inadvisable.

Post-mortem Appearances.-Organs normal except for hypertrophy of left ventricle. Peritoneum presented slight sero-fibrinous exudate. Stomach wall thick, especially at pyloric end. Thickening due to purulent infiltration of submucous coat with yellowish exudate. Linear cicatrix on posterior wall of stomach near pylorus, on which was a necrotic area. Endothelium desquamated on serous coat. Muscle invaded by mononuclear leucocytes. Submucosa swelled to four or five times its normal thickness, and contained leucocytes and micro-organisms. Lymphatics contained bacteria. Necrotic area in scar had nearly exposed submucosa, and was possibly point of infection. Streptococcus found throughout stomach. Bacilli in lymphatics possibly of postmortem origin.

Reference.-Kinnicutt, Trans. Assoc. Amer. Phy's., xv., I 900, reported in Philad. Med. Journ., I 900, v. 989.

Case 78.

Presumed Cause and Chief Clinical Symptoms.-Recovered without operative treatment through spontaneous vomiting of pus. This case is noteworthy because pus in the vomited contents is of somewhat rare occurrence, especially in such quantities $(0.3$ to 0.4 litre) as in this case.

Reference.-Rubner (J.), Orvosi Hetilap, No. 42 (abstracted in Archiv f. Verdauungs Krankheiten, I9oI, vii. 294).

CASE 79.-Female, aged seventy-five.

Presumed Cause and Chief Clinical Symptoms.-Patient, who had been treated for a long time for chronic bronchitis and emphysema, was attacked one day before her death with high fever and violent abdominal pain. Basing his conclusion on the postmortem results, the author is inclined to connect the phlegmonous gastritis etiologically with the bronchiectasis, as he supposes that the former takes its origin either by way of the metatases or through the bronchial secretion containing streptococci being swallowed, and thus directly infecting the mucous membrane at one point of lesion that can no longer be demonstrated.

Duration.-One day from onset of severe symptoms. 
Post-mortem Appearances.-Autopsy showed, besides changes in the lungs (chronic bronchitis, emphysema, bronchiectasis), special changes in the stomach. The stomach wall in the third contiguous to the pylorus was considerably thickened in consequence of an infiltration of the submucosa with a cloudy serous and purulent fluid. The mucous membrane was similarly swollen, pale, but without visible lesions. On section through the wall of the stomach there were found an extraordinary number of streptococci, that could also be traced through the muscularis to the submucosa; the same streptococci could also be cultivated from the blood of the vena cruralis and the pus of the bronchiectasis.

Reference.-Simmonds, 'Ueber Gastritis Phlegmonosa,' Miinchener Med. Woch., I90I, xlviii. 440 (abstract in Centralb. $f$. Staffwechsel u. Verdaunngs Krankheiten, I901, ii. 223).

CASE 80.-Male, aged eighteen.

Presumed Cause and Chief Clinical Symptoms._-Presented clinical picture of perforated gastric ulcer. Operation. On the abdomen being opened in the middle line, the parietal portion of the peritoneum was found to be infiltrated with gelatinous substance ; stomach was distended, its anterior wall much thickened, covered with fibrinous layers, and rigid. In the abdomen were some turbid serous contents; the neighbouring coils of intestine were inflated and somewhat injected. The inflammatory focus was not enclosed by adhesions. The attempt to draw out the stomach failed, as it formed a thick rigid lamina. On the anterior surface of the stomach there was a uniformly bright-red portion of wall, with cedematous infiltration about the size of the palm of the hand, and within this area two yellowish-gray maculæ of irregular border, but about the size of a 'mark piece' (or shilling). A perforation of the stomach was not found. Repeated lavage with warm saline infusion; iodoform gauze plugging over the inflamed area ; in addition, three sterile gauze tampons inserted in abdominal cavity. Later there was left pleural effusion, which had to be aspirated. Recovery. In spite of the fact that an anatomical proof of the phlegmon was not to be adduced by the operation, the symptom complex appeared to the operator certainly sufficient to demonstrate that the case was solely that of phlegmonous inflammation of the stomach. The operator was Von Mikulicz, and the reporter (Dr. Lengemann) asserts that all hitherto published cases of diffuse phlegmonous gastritis have ended fatally.

Reference.-Lengemann, Mittheil. aus d. Gren. d. Medicin u. Chirurgie, Bd. ix., Heft 4 u. 5 (abstract in Centralblatt $f$. Chirurgie, I902, xxix. 1003). 
CASE 8I.-Domestic servant, female, aged twenty-six.

Presumed Cause and Chief Clinical Symptoms.-Previous health good; no cause could be assigned. On November I3, I90I, attacked with cold shivers, slight sore throat, severe headache, pains in abdomen and limbs. Continued work till i 8 th, when took to bed. Severe vomiting, with abdominal pain, commenced on I 7 th. Bowels constipated; dark offensive motions after dose of castor-oil. Admitted to hospital afternoon of Igth. Pulse I32. Temperature $104.6^{\circ} \mathrm{F}$. Abdomen somewhat distended, especially in epigastric region; tender on palpation. Next day showed all signs of acute septic peritonitis, abdomen now being greatly distended, motionless, and very tender; was collapsed. Temperature fallen to IOI' $2^{\circ}$. Died next day (2Ist).

Duration.-Eight days from commencement of symptoms.

Post-mortem Appearánces.-General purulent peritonitis. Stomach dilated, coats thickened, serous covering injected. Submucous and, to less extent, muscular and subserous coats infiltrated with pus; mucous membrane appeared intact. Change most marked over pyloric third of stomach. Bacteriological examination. Stained cover-glass specimens made from purulent exudation of submucous coat of stomach showed number of bacilli which did not stain by Gram's method, and resembled morphologically B. coli communis. Few short chains of streptococci also present, staining by Gram's method. Culture-tubes inocculated from spleen juice and from purulent exudation in submucous coat of stomach. Tubes inoculated from spleen juice remained sterile, except from accidental contamination. From purulent exudation pure culture of $B$. coli communis was obtained. From streptococcus seen in exudation not being recovered in culture, it is supposed that streptococcus infection was probably primary, and that of $B$. coli communis secondary.

Reference.-Cayley (W.), Trans. Path. Soc., I902, liii. 282.

Case 82.-Male, aged seventy-three.

Presumed Canse and Chief Clinical Symptoms.-Had been in hospital on several occasions, suffering from retention of urine, cystitis, pyelonephritis. Readmitted in an extremely feeble condition. The abdomen was distended and painful, but not tender. Pulse i Io, temperature $100^{\circ}$.

Post-mortem Appearances.-Cystitis, nephritis. The stomach was affected by diffuse phlegmonous gastritis. An examination showed also a round ulcer and diffuse chronic gastritis.

Reference.-Huguenia, Rev. Med. de la Suisse Romande, vol. xxiii., 1903.

CASE 83.-Acute phlegmonous gastritis, ending in death with- 
out operation, reported here for the first time. (Mayo Robson and Lawford Knaggs.)

CASE 84.-Chronic gastric ulcer eroding pancreas ; pancreatitis ; abscess of pancreas bursting into stomach ; vomiting ; impending death. Posterior gastro-enterostomy; recovery. (A. W. Mayo Robson.)

CASE 85--Acute phlegmonous gastritis ; posterior gastro-enterostomy ; death. (A. W. Mayo Robson.) 


\section{LIST OF AUTHORITIES}
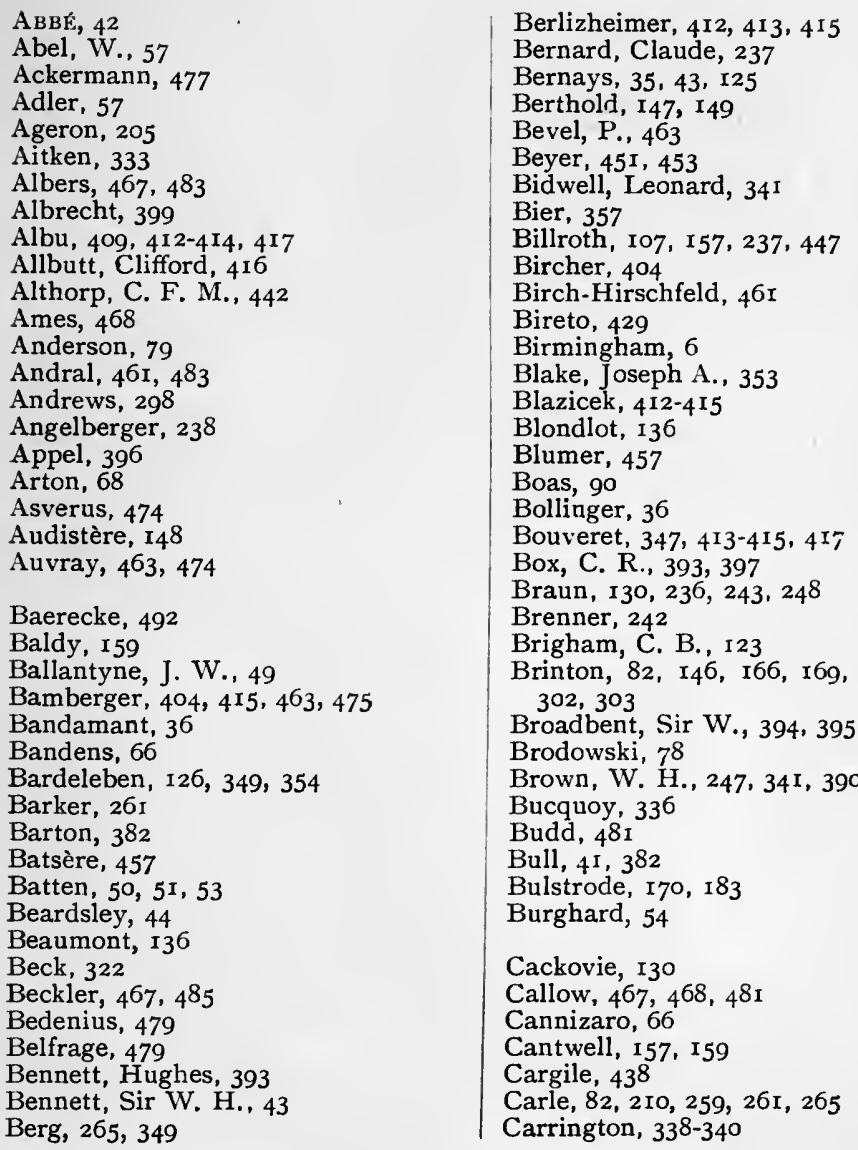

Brinton, 82, 146, 166, 169, 284, 285. 302,303

Broadbent, Sir W., 394, 395

Brodowski, 78

Brown, W. H., 247, 34I, 390

Bucquoy, 336

Budd, 48I

Bull, 4I, 382

Bulstrode, I70, 183

Burghard, 54

Cackovie, 130

Callow, 467, 468, 48I

Cannizaro, 66

Cantwell, 157, I59

Cargile, 438

Carle, 82, 2 10, 259, 26r, 265

Carrington, $338-340$ 
Carrion, 400

Cathelin, 36

Cautley, 46-48

Cayley, 495

Chaput, 43, 76, 244

Chavasse, 125

Chiari, 319, 462

Childe, 35I, $35^{8}$

Chlumskij, 237-240, 256, 26 I

Chvostek, 4 10, $482-484$

Clarke, 69

Clayton, 60

Coffey, R. C., 453

Collin, 336

Collins, 68

Connor, 122

Cornil, 475

Cotman, Harold H., $4+3$

Courmont, 357

Courvoisier, $24^{\circ}$

Crede, 36

Crile, 4

Cruveilhier, $146,160,161,182$

Cullingworth, $8_{4}$

Cumston, 357

Cunéa, 82, 108

Cunningham, 12

Cushing, Harvey, I86

Czerny, 86, 95, 107, 122, I85, 186, 239, $240,246,299$

Daettwyler, I62

Dalgleish, 46I

Dastre, 210, 237

Davies-Colley, 336

Dawosky, 44

Debove, I70, 285

Deininger, $67,4^{82}$

Demarquay, 306

Demoulin, I95

Dent, $46,47,54$

De Pauli, 456

Devic, 4I3-4I5, 4I7

Dickson, Carnegie, 413, 4I4, 4I6

Dieulafoy, I60, I6I, $278,290,460$, $46 I$

Dittrich, I $46,463,467,482$

Dobson, Nelson, 3 Io

Dollinger, I26

Doyen, $185,236,239,243,340,349$, 354

Dreschfield, I69, 284, 285

Dubec, 46I

Dudensing, 336

Dujardin-Beaumetz, 4 I5

Dumeny, 62

Duplant, 147

Duret, 450

Durivier, 186

Ebert, 479

Edmunds, 4I
Egeberg, I 36

Ehret, H., 93

Eichhorst, 91, 345

Einhorn, 26, 169, 183, 459

Eiselsberg, Von, 36, 78, 282, 344, 346. $349,355,356$

Eisendrath, 298

Elsner, 44I

Enderlen, 70, 3II

Eppinger, 457

Erb, 4 IO

Ericker, 36

Erlach, Von, 78

Esmarch, 447

Ewald, 2, 25, 26, 183

Ewart, 385, 399

Fagge, 225, 389, 399, 481

Fantino, 82, 259, 261, 265

Faure, 244

Fedoroff, 126, 129

Fenwick, Soltau, I49, I 55, I65-167, 259, 281, 302, 305, 306, 314, 338, $45^{8}$

Feroci, 479

Finkelstein, 48, 53

Finlayson, I 56

Finney, J. M. T., 269, 302, 305, 312 , 3 I 4,34 I

Fleiner, 180, 4I 2

Flexner, 46I, 462

Flint, $4^{83}$

Foote, 35I

Forgue, 63, 66

Förster, 78

Fraenkel, 489

Francine, 448

Frankl-Hochwart, 408, 4II, 4I 7

Franks, Kendal, 4I

Friedenwald, 34, 36

Fry, II 6

Fuetterer, 148

Fulton, 68

Furnivall, 8I

Gaudy, 476

Garel, 484

Gerhardt, I 7 I

Germain-Sée, 4 12

Gilford, Hastings, $35^{6}$

Gissler, 4 I

Glaessner, K., 25

Gläser, 468, 486

Glax, $463,467,484$

Glénard, 6, 448, 449

Godlee, 3 I 6

Goffe, 26I

Golzi, 456

Greenfield, 416, 422

Greig Smith, I34

Gross, 336 
Guinard, 64

Gumprecht, 4I 3, 4I4

Gussenbauer, 80, 83, 107

Guyot, 477

Haberkant, 261

Häberlin, 149

Habershon, 169, 284, 302, 473

Hacker, Von, 240, 244

Hadden, 156

Hadra, I 30, 245

Hahn, 4I, 52, 382, 44I

Hall, 313

Hallon, 400

Halm, I30

Ham, C. E., 24

Hammer, $5^{8}$

Hammerschlag, 90, 9I

Hampeln, 88

Hartley, 157

Hartmann, 89, 132, 261, 429

Harvie, 126

Hauser, 146, I49, I7 1, I 84

Hayem, 76, 147, 152

Heidenhain, 91

Heineke, 263, 384

Helferich, 142

Hemmeter, 90, 92, I83, 414, 468, 491

Henschel, 49

Herczel, 126

Herhold, 178

Herzfelder, 336

Herzog, 472

Heubner, 53

Hey, Wm., 40

Heydenreich, 36, 184

Heyfelder, 472

Hinds, 76

Hirsch, 338, 339

Hirschsprung, 44, 45

Hochenegg, 340, 357

Hoffmann, 336, 4 10

Hofmeister, 355

Hollis, H., 332

Hood, Donald, 279

Hudson, 339

Hugenia, 495

Hun, 478

Hunsy, 5I

Hurtado, Gurreà y, 126

Hutchinson, J., 79

Ijzeren, Dr. Van, 163

Irving, 52

Jaboulay, 236, 24I, 243, 267, 272, 355,382

Jacobsen, 36

Jacoby, 463

Jaffrey, 385
Janeway, E. G., 466

Jaworski, 344, 346

Jeanbrau, 63,66

Jessop, 39I

Jones, Sidney, I36

Jonnesco, 8, 9

Jürgensen, 4I2

Kader, 108, 240, 244

Kaiser, 107

Kammerer, 35I

Kappeler, 240

Kaufmann, 92

Kausch, I 30

Keen, 33

Keetley, C. B., 72, 74

Kehr, 51, 57

Kelynack, 389, 490

Keser, 46r

Key, 479

Keyl, 336

Kinnicutt, 465, 493

Kirsch, .395

Klaus, 473

Klebs, 46I, 463

Kleef, Van, 185

Klein, $342,35^{8}$

Knaggs, Lawford, 468, 496

Kocher, 86, 122, 125, 244, 248, 272

Koeppelin, 267

Kooyker, 36

Kopfstein, 70

Kops, De Bruyn, 45

Korach, 306

Körte, I30

Kortmann, 33

Kosinski, ${ }_{5} 8$

Kränzle, 34

Kraske, 86

Krause, 463, 480

Krauss, 336

Krehl, I 06

Kriege, 3 ro

Krokiewicz, 88, 96

Kronheimer, 44I

Krönlein, 97, Io9, II 4, I 2 I

Kruckenberg, 349, 354

Kümmell, $24 \mathrm{I}$

Kundrat, 159, 399

Kunze, 77

Kurschmann, 467, 48I

Kurze, 475

Kussmaul, 408, 4I2

Kuster, I 86

Labbé, 37

Landsteiner, 4I 3

Lang, 322

Langenbuch, I23, 355

Lasège, 475 
Lauenstein, 243, 245, 355

Lebert, 80, 146, 149, I69, 284, 285 , 302,463

Leith, 487,491

Lengemann, 494

Lennander, 336

Leube, 169, I77, 415, 467, 468

Levy, 106

Lewandowski, 484

Lewin, 488

Leyden, 316

Lieutaud, $466,468,4^{8} 3$

Lindemann, 488

Lindner, $83,85,89,303$

Litten, 457

Loomis, 478

Loreta, 35, 41, 42

Löwenstein, 463,480, $48 \mathrm{I}$

Lücke, 243

Lumeau, 336

Lunnemann, 343

Lyman, C. B., 43

McArdle, 83

McCrae, 88, 95

Macdonald, 125

MacGillivray, 357

MacLeod, J. J., 24

Maier, 50, 340

Malbranc, 237

Mall, 9I

Malmsteon, 479

Manoury, 476

Marten, 4I7

Martin, Sidney, $35^{8}$

Mascaral, 476

Mathieu, I53, I80

Matthes, I8I

Matthis, 3 I

Maydl, I22, I 23, I31, 242, 316, 336

Mayo, C. H., 26r

Mayo, W. J., 236, 247, 254, 255 . $257,26 \mathrm{I}$

Mayo, 106

Mayor, 473

Mazet, 474

Meltzer, 57

Merklen, 433, 434

Meunier, 336

Meyer, 490

Micaëlis, 62

Middledorpf, 447

Mikulicz, 94, 97, Іог, 106, 108, 109, I I4, I28, I30, I42, $210,237,24 \mathrm{I}$, $263,265,272,290,298,310,384$

Mintz, 261, 464, 468, 488

Monnier, 57

Monod, I86

Morel, 478

Morgagni, 78, 408

Morison, Rutherford, Io9, I22
Morris, H., 393, 399

Morris, R. T., 438

Morrison, Jas., 56

Morse, 357

Moullin, Mansell, I70, 282

Moxon, 77, 79

Moynihan, 210, 238, 336, 405

Müller, I69, 284, 285, 40I, 4II, 4I3. 415,438

Murrell, $3^{8}$

Musser, 457

Nasse, 487

Neumann, I 29, I30, 248, 4I5

Newton, Pitt, 45

Nicoladoni, 78,207

Nicoll, $5^{8}$

Noorden, Van, 355

Norman, 77

Novaro, 186

Obalinsky, I07, II4

Oddi, 210, 237

Ogston, 19

Oser, 467

Osler, $44,84-88,90,9$ r, 94

Page, H. W., 429

Pariser, 181, 303, 313

Park, Roswell, 66

Parker, Rushton, 264

Patella, 456

Paton, E. P., 36

Paul, 383

Pauli, De, 456

Pawlow, 204

Péan, 107

Pepper, 399

Perrin, 238

Perry and Shaw, 80, I52, I 55

Petersen, 240, 299, 484

Petruschky, 457

Pilliet, 96

Pinel, 62

Pitt, 77

Planchard, 336

Plange, I49

Pollard, 35I

Pollard, Bilton, $35^{8}$

Potain, 62, 456

Potter, 36

Purves, Robert, 284

Quénu, 252

Quincke, I62

Rakowak, 480

Read, H., 79

Redtenbacher, I 55

Rehn, 6I 
Reichard, 284, 299

Reinking, 488

Remond, I70

Rendu, 6r

Reuvers, 4I 5

Richardson, Maurice, 40, 107, I1 4 , I 25, 268

Richter, 4I, 382

Riegel, 88, 92, I5I, 4I5, 4I7, 465

Rindfleisch, 86

Robson, A. W. Mayo, 52, 426, 495

Rockwitz, 242

Rodman, 190, 283, 285

Rokitansky, 82, I46, 336, 463

Rolleston, I 53

Rose, E., 6I

Rosenheim, 90, 147, 149, 261

Rosenthal, 34, 36

Rost, I79

Roux, 236, 242, 243, 290

Rubner, 493

Ruprecht, 78

Rutherford Morison, I09, I 22

Rutkowsky, 73, 244

Rydygier, 107, 122, I 85, I88, I9I

Sand, 467

Sangalli, 78

Saundby, Robert, I78, 339

Savariaud, $175,287,298$

Schiff, 9 I

Schlatter, 99, 121, I 23

Schlesinger, 92, 152, I55, I59

Schmidt, 474

Schmidt-Monard, 345

Schneider, 90

Schopf, 36, 156

Schrieber, 36

Schuchardt, I 23

Schultz, 394

Schwartz, 342, 355

Seaton, I 15

Sebillon, 486

Sedillot, 136

Senator, 53

Shufflebotham, F., 24

Siewers, 34I

Silbermann, I62

Silcock, $4^{85}$

Silhol, 89

Simmonds, 455, 492, 494

Smith, 489

Smith, Greig, I34, 3 I9, 383

Smith, F. J., 279

Smith, T., 66

Socin, 65

Sohler, Von, I79

Sonicksen, I 49

Sonnenburg, 5I, 57, 244

Soupault, 26r, 429

Spencer, Walter, $3^{8}$
Steele, $44^{8}$

Steffan, 341

Steiner, 78, 285

Steinthal, 130

Stengel, 399, 45 I

Stern, 57

Steudel, 238, 239

Stewart, 478

Sticker, I 47 .

Stiles, Harold, 54

Still, 53

Strauss, 88

Sundholm, 3 I I

Sutton, 75

Swain, Paul, 35, 39

Taksch, Von, 4 I 3

Tavel, 236, 244

Taylor, Seymour, 161, 167

Terrier, II $4,238,295,387,429$

Testi, 486

Thoman, 489

Thomsen, 34I

Thomson, C., 393-395

Thomson, John, 44, 45, 48, 49

Thue, 324

Thungel, 477

Tinker, 302

Traube, 306

Trevelyan, 4I 5, 4I7, $42 \mathrm{I}$

Tricomi, I69, I84, I 86

Tripier, 28I

Trousseau, 410

Tuffier, I95, 35I, 355

Tupolski, I 2

Unge, Von, $35^{6}$

Unruh, 44I

Valentin, 397

Van Ijzeren, I63

Van Noorden, 355

Vedova, R. Dalla, I6 4

Veer, Van der, I 26

Velder, Von der, 90

Verneuil, 136

Villard, 24I, 272, 387

Virchow, $77,78,46 \mathrm{r}$

Vogel, 78, 437

Voigtel, 136

Von Erlach, 78

Wallace, Cuthbert, 393, 397

Warren, I $7 \mathrm{I}$

Watson, 340, 355, $35^{8}$

Watson, Sedgwick, $35^{\circ}$

Watts, S. H., $24^{8}$

Weil, 237

Weir, 236, 351, 404

Welch, 84, 166, 167, 285, 466

Westphalen, I79 
Wetzoldt, 147

White, W. Hall, 279

Wickhoff, Max, 238

Widerhofen, 84

Wiesinger, 396, 399

Wilks, 77,79

Williams, Roger, 338-340

IVilliamson, 44

Wilson, Christy, I07, II4

Winiwarter, 83, 107
Winslow, 282

Witzel, 73, 244

Wölfler, 207, 244, 343, 346, 350, 355 .

Wollmans, 149

Woolsey, G., 70

Zeller, 349, 354

Zenker, I 46, 149

Ziegler, 79

Zweifel, 9 I 


\section{N D E X}

Abdominal belt, in perigastritis, 430 injury, 28

Abstinence treatment of gastric ulcer, I79

Acute gastric dilatation, 225, 427 etiology, 395

pathology, 397

symptoms, 393

treatment, 400

Acute perforation of gastric ulcer, 305

Acute post-operative dilatation of stomach, 401

Adeno-carcinoma, 8o

Adenoma, 43, 75

Adhesions after abdominal operations, 433

Adhesions around stomach, 427

Albert-Frank's method for gastrostomy, 137

Albert-Frank-Kocher's method for gastrostomy, 137

Analysis of reported cases of gastroenterostomy, 249

of stomach contents, 24

Angiosarcoma of stomach, $15^{8}$

Anterior gastro-enterostomy, 234

Antrum pylori, 8, II

Appendicitis and perforating ulcer, 308

Asthenia, death from, 259

Atonic dilatation of stomach, 16, 226, 403

symptoms, 403

treatment, 404

Atresia, congenital, of the pylorus, $5^{8}$

Bacillus filiformis, 93

Billroth's method of pylorectomy, rog

Billroth, 'the fatal suture angle of,' Iog

Bilocular stomach, $33^{8}$
Blood, in carcinoma, 88

in vomited matters, 22

Blood-supply of stomach, 3

Boas's breakfast, $9 \mathrm{I}$

Bobbin, decalcified bone, I09, 232

Bougies, œsophageal, 20

Bridle stricture, 339

Calcium chloride after gastro-enterostomy, 259

Carcinoma, 8o the blood in, 88

Cargile's method for preventing adhesions, 438

Caustic fluids, swallowing of, 7 I

Chest complications after stomach: operations, 254

Cholelithiasis, 226

Chvostek's symptom in gastric tetany, 4 Io

Cirrhosis of liver, 22

Cocaine anæsthesia, 138

Coin-catcher, Hey's, 40

Collapse in injury of the stomach, 60

Complete gastrectomy, I 23

Complications of gastric ulcer, 176

Congenital atresia of the pylorus, $5^{8}$ carcinoma, 84

gastric spasm, 49

hour-glass stomach, 338

Curette for cancerous growths, 43

Cylindrical carcinomata, 8o

Cylinder-epithelioma, 8o

Cysts, 75, 78

Cyst, omental, 443

Decalcified bone bobbin, 109. 232

Defective secretion, 90

Depage's operation for gastrostomy I 43

Destructive adenoma, 80 
Diagnosis : auscultation, IS

bimanual examination, 16

electric illumination, 2 r

exploratory incision, 26

foreign bodies, 2 I

gastro-endoscope, 22

inspection, I4

instrumental aids to, 20

knee-elbow position, I7

massage, 15

pain, 19

palpation, I5

percussion, 17

Röntgen rays, 2 I

test breakfast, 23

vomited matters, 22

Diaphragm phenomenon in perforation, 318

Differential diagnosis in perforation, 307

Dilatation, abnormal, 17

amongst mill operatives, 378

diagnosis, 379

treatment, $38 \mathrm{I}$

Greenfield's method of, 4 I 6

Dilatation, acute, 389

etiology, 395

pathology, 397

post-operative, 4or

symptoms, 393

treatment, 400

atonic, I6, 226, 403

symptoms, 403

treatment, 404

Double stomach, $33^{8}$

Doyen's hysterectomy clamps in pylorectomy, 108

Drainage after perforated gastric ulcer, $3 \mathrm{I} 2$

Duodenal ulcer, $2 \times 8$ perforation, $301,335,336$

Duodenostomy, 73, I05

Duodenum, carcinoma of, ${ }_{52}$

Dupuytren's lithotomy dilator in Loreta's operation, 42

Egagropiles, 35

Electric illumination in diagnosis, 2 I

Empyema, rupture of, 23 of gall-bladder, 23

Entero-anastomosis, 243

Epithelial lining of stomach, 3

Epithelioma, cylinder, 8o

Erb's symptom in gastric tetany. 4 10

Erosions in gastric ulcer, 160

Evelyn's Diary, 3r

Ewald's method of estimating motor activity of the stomach, 26

Examination of stomach contents, 89

Excision of gastric ulcer, 188
Experimental production of gastric ulcer, 162

Exploratory gastrotomy, 186 incision, 26, 102

Exulceratio simplex, 161

Feeding after gastrotomy, 33

Fenger's incision in gastrostomy, 142

Fibro-myoma, 75, 77

Fleiner's method of treating gastric ulcer, r8o

Flushing peritoneal cavity, 3 I I

Foreign bodies in stomach, 2I, 33 gastrotomy for, 33

Frank's method for gastrostomy, 137

Gall-bladder, empyema of, 23

Gas, distension by, 17

Gastralgia, persistent, 223

Gastrectasis, 378

diagnosis, 379

treatment, $3^{81}$

Gastrectomy, IO4 complete, I23

partial, 96, 107, I14

Gastric dilatation, acute, 225, 427

fistula, 439

diagnosis, 446

etiology, 439

treatment, 446

spasm, congenital, 49

tetany, 408

Chvostek's symptom, $4^{\text {ro }}$

Erb's symptom, 4 10

Hoffmann's symptom, 4 ro

'Trousseau's symptom, 4IO

pathology, 4I 4

prognosis, 4 I 7

references, 425

theories, $4^{12}$

treatment, 415

ulcer, 16,160

abstinence treatment of, I79

acute perforation of, 305

complications, 176

erosions in, 160

excision of, $\mathrm{I} 88$

experimental production of, I 62

exulceratio simplex, I6r

Fleiner's method, 180

medical treatment, I 77

perforation, 169, 30r, 305.

See Perforation

septic origin of, 165

sequelæ of, 176

surgical treatment, I 82

treatment of, 177

Gastrite interstitielle, 463

Gastritis, phlegmonous, 463

phlegmonosa, 463 
Gastritis, submucosa, 463

Gastro-anastomosis, $34^{8}, 35^{\circ}$

Gastro-duodenostomy, 269, 272

Gastro-endoscope in diagnosis, 22

Gastro-enterostomy, 57, 74, 102, I92, $207,348,384$

analysis of cases, 249

anterior, 234

by invagination, 244

calcium chloride in, 259

complications, 234

mortality, 207, 26I

Murphy's button in, 233

non-union in, 255

operation, 227

results, 259

Roux's method, 231

Von Hacker's method, 209

Wölfler's method, 234

Gastro-gastrostomy. $348,35^{\circ}$

Gastro-jejunostomy, 247

Gastrolysis, 200, 427, 43 I

Gastroplasty, 199, 21 5, 348, 349

Gastroplication, 383, 404 mortality, 405

Gastroptosis, 448

Gastrorrhagia, 59, 275 gastrotomy for, 43 operations for, 294 statistics of, 285 technique of operation for, 295 treatment of, 288

Gastrostomy, 73, 136

Albert-Frank's, I37

Albert-Frank-Kocher's method, 137

Depage's method, I43

Fenger's incision, I 42

Frank's method, 137

Kader's method, 143

Mayo Robson's modification, 137

Gastro-succorrhœea, 202, 399

Gastrotomy, exploratory, 3I, 186 for foreign bodies, 33,40

feeding after, 33

Glandular enlargement, 88

Glénard's disease, $6,44^{8}$

Gluteal spasm, 422

Greenfield's method in gastric dilatation, 416

Gunsberg's test for free hydrochloric acid, 24

Gunshot wounds, 28, 62-7 I

Hæmatemesis, 22

in injury of the stomach, 60

post-operative, 282

Hæmoptysis, 23

Hahn's operation, 201

Hair in stomach, 35

Hegar's dilators in pylorodiosis, 54
Heineke's operation, 349

Hemmeter's bag in diagnosis, 90

Hernia after incision, 29, 30

Herniæ, internal, 257

Hey's coin-catcher, 40

Hodgkin's disease, 3

Hæmin test, 22

Hoffmann's symptom in gastric tetany, 410

Hour-glass stomach, I I 5, 21 5, 338 acquired, 340

cancerous, 375

cases, 354

congenital, 338

differential diagnosis, 346

divulsion, 353

recorded cases, 354

symptoms, 343

treatment, $34^{8}$

Hunterian lectures on surgery of stomach for 1900,184

Huschke, ligament of, I 3

Hydrochloric acid, free, Gunsberg's test for, 24

Hyperchlorhydria, 18, 202, 223

Incision for exposure of stomach, 29

Fenger's, in gastrostomy, 142

Inflammation of the submucosa, 463

Injury of the stomach, 59

Internal herniæ, 257

Intestinal anastomoses, 23I

Jejunostomy, 73, 105, I 29, I 3 I

Maydl's method for, I3I

Jejunum, peptic ulcer of, I30

Jonnesco, pyloric canal of, 8, I2

Kader's method for gastrostomy, I 43 Kidney, 1o, 11

Knee-elbow position in diagnosis, 17

Kocher's method of pylorectomy, i ro

Krönlein's cases tabulated, 97

Lactic acid, 9I

Latent ulcers of stomach, 175

Leube's method of estimating motor activity of stomach, 26

Ligament of Huschke, I 13

Ligamenta pylori, I2

Linitis plastica et suppurativa, 463

Lipoma, 75, 77

Lipo-myoma, 75, 77

Loreta's operation, 54, 201, 222, 382 statistics of, $3^{84}$

Lymphadenoma, 75, 77

Lymphatics of stomach, 4

Lymphoid tissue, 3

Lympho-sarcoma of stomach, 154

Maydl's method for jejuncstomy, I3I 
Mayo Robson's decalcified bone bobbin, 232

modification of Frank's operation, 137

McBurney's point, 337

Mesocolon, transverse, II

Mett's method for pepsin, 25

Mortality. See Statistics

Motor activity of stomach, 26

Ewald's method, 26, 90

Leube's method, 26

Müller's method for preventing adhesions, 438

Mural cancer, 105

Murphy button, 58 , I 10, 246, 247, 255. 257

method of gastro-enterostomy, 233

Muscular spasm, 422

Myoma, 75, 77

Myosarcoma, 78,158

Nerves of stomach, 4,5

Non-union in gastro-enterostomy, 255

Esophageal bougies, 20

Esophagotomy, 39, 40

Omentum, 5, ro

Oppler-Boas bacillus, 92, 159

Oral sepsis in gastric ulcer, 165

Otis's urethrotome as dilator, 42

Pancreas, Io, II

Pancreatic cyst, 217

Pancreatitis, 225

Paradoxical dilatation, 344

Partial gastrectomy, 96. I07, 114, 348

Pepsin, Mett's method for, 25

Peptic ulcer of the jejunum, r3o

Perforation of duodenal ulcer; 30r, 335,336

of gastric ulcer, 169,301 and appendicitis, 308

acute, 305

diaphragm phenomenon, 318

differential diagnosis, 307

drainage after, 312

frequency of, 302

operation, 3 Io

prognosis, $3{ }^{1} 3$

results, 314,324

sex in, 303

site of, 303

symptoms, 305

treatment, 309

Perigastric abscess, 59, 3 I 5

Perigastritis, 256, 426

Peripyloritis, 265

Peristalsis, I4, I6

Peritonitis, general septic, 312

Phenolphthalein, 25
Phlegmonous gastritis, 463

pathology, 467

recorded cases, $47 \mathrm{I}$

symptoms, 464

treatment, 468

Phlegmon ventriculi, 463

Pleurisy after stomach operations, 254

Pneumonia after stomach operations, 254

Polypus, gastrotomy for, 42

Post-operative dilatation, 401

hæmatemesis, 282

Prepyloric ulcero-cancer, 147

Pressure point in gastric ulcer, 182

Pus in vomited matters, 23

Pylorectomy, 107, 109, I1 2, 190, 387

Billroth's method, 109

- Doyen's hysterectomy clamps in, I08

method of operating, I I 2

Pylori ligamenta, I2

Pylorodiosis, 52, 54, 201, 222, 382

Hegar's dilators in, 54

Pyloroplasty, 54, 55, 151, 196, 222, 263,384

operation, 263

results, 266

statistics, 269

submucous, 267

Pylorus congenital atresia of the, $5^{8}$ calibre of, 2

Pyopneumothorax subphrenicus, 316

Rectal injections, 28I

Regurgitant vomiting, 235

statistics of, 240

Reichmann's disease, 209, 222

Resection of the stomach, 102

Retroperitoneal abscess, 320

Röntgen rays in diagnosis, 21 for foreign bodies, 34

Round-celled sarcoma, I 55

Roux's gastro-enterostomy, 23 I

Rupture of empyema, $23^{\circ}$ spontaneous, of stomach, 68

Salol in diagnosis, 26

Sarcoma of the stomach, 155

Saundby's treatment of gastric ulcer, 178

Schlatter's complete gastrectomy, I 23

Scirrhus of the pylorus, 44

Secretion, defective, 90

Senn's method for gastrostomy, 140 plates, 247

Sepsis, oral, in gastric ulcer, 165

Septic origin of gastric ulcer, 165

Sequelæ of gastric ulcer, 176

Shock on manipulation, 4,5

Shoulder-blade pain, 5 
Spasm, congenital gastric, 49

of pharyn $x, 21$

of cesophagus, 2 I

Spheroidal carcinomata, 80

Spindle-celled sarcoma, ${ }_{56}$

Spleen, I I

Spontaneous rupture of stomach, 68

Ssbanajew-Frank's method for gastrostomy, 137

Stab wounds, 28

Statistics, of adhesions, 427

carcinoma duodenum, 152

complete gastrectomy, I25

exploratory incision, 102

gastrectomy, 97-102

gastric tetany, 4I7

ulcer, $166-169,183$

gastro-enterostomy, 102, 207, 249, 261

gastrolysis, 431

gastroplication, 405

gastroptosis, 449

gastrorrhagia, 284-287

gastrostomy, 145

for foreign bodies, 34,36

gunshot wounds, 66

hour-glass stomach, 377

jejunostomy, 132

Loreta's operation, $3^{84}$

perforating ulcer, 302, 31.3, 314

phlegmonous gastritis, 463

pylorectomy, 12r

pyloroplasty, 269, 27 I, 384

regurgitant vomiting, 240

resection, 102

subphrenic abscess, 322

syphilis, $46 \mathrm{r}$

Stenosis of pylorus, 44,50

Stille's clamp, 124

Stomach, adeno-carcinoma, 8o

adenoma, 43,75

adhesions around, 427

after abdominal operations, 433

analysis of contents, 24

anatomical considerations, I

angiosarcoma, $15^{8}$

atonic dilatation, $16,226,403$, 404

' bed,' 10

bilocular, $33^{8}$

blood-supply of, 3

calibre of pylorus, 2

capacity of, 2

' chamber,' ro

chest complications after operations, 254

collapse after injury of, 60

congenital hour-glass, 338

contents, analysis of, 24

diagnosis, 14
Stomach, dilatation, 14,378 acute, 389

distended, 7

double, $33^{8}$

epithelial lining, 3

examination of contents, 89

Hunterian Lectures, 184

latent ulcers of, 175

lymphatics of, 4

lympho-sarcoma, I55

motility of, 17

motor activity of, 26

natural form of, 6

pain, 19

pleurisy after operations, 254

pneumonia after operations, 254

position of, $\mathbf{I}$

pyloric sphincter, 2, 6

rupture of, 68

sarcoma, 155

shape of, 6

spontaneous rupture of, 68

surfaces of, to

' uncovered area' of, 13

walls of, 2

tube, 23

Stricture, bridle, 339

œsophageal, r9-2 r

Subacute perforation of gastric ulcer, 306

Subdiaphragmatic abscess, 23

Submucous suppurative phlegmon, 463

Subphrenic abscess, 17, 19, 20, $3^{\text {I } 6}$ mortality, 322

treatment, 322

Succussion splash, I 7

Suprarenal extract, after gastro-enterostomy, 259

in gastrorrhagia, 28 $\mathrm{I}$

Syphilis of the stomach, $45^{8}$

Temperature in carcinoma, 88

Test breakfast, 23, 9 I

Tetany of gastric origin, 224 gastric, 408-425

Thoracic pain in gastric ulcer, 175

Tight-lacing, results of, 9, 450

Toepfer's reagent, 25

Traumatic ulcer of stomach, $6 r$

Treatment of gastric ulcer, 177,178

Trichobezoars, 35

Trifid stomach, 34 I

Tripier's rectal injections, 281, 283

Trousseau's symptom in gastric tetany, 410

Tuberculosis of the stomach, 455

Tumours of the stomach, 75

Uffelmann's reagent, 24,9 I

Ulcer, duodenal, 218 
Ulceration of stomach, I6, 20, 29 caustic, $7 \mathrm{I}$

Ulcero-cancer, prepyloric, 147

Ulcus carcinomatosum, 90, I46, 343 pepticum jejuni, 248

Vomited matters, 22

blood in, 22

pus in, 23

Von Hacker's operation, $7^{0}, 76,209$
Weiss's rectal dilator, 42

Westphalen's treatment of gastric ulcer, I79

Witzel's operation for gastrostomy, I3I, I42 : method for jejunostomy, I3I

Wölfler's compressorium, I24 operation for gastro-enterostomy, 209

Wounds of stomach, 62,69

THE END 

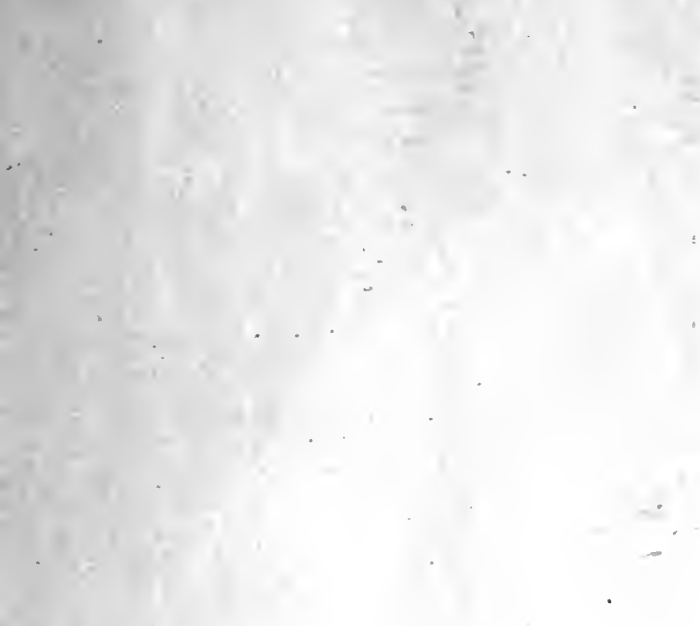

. 



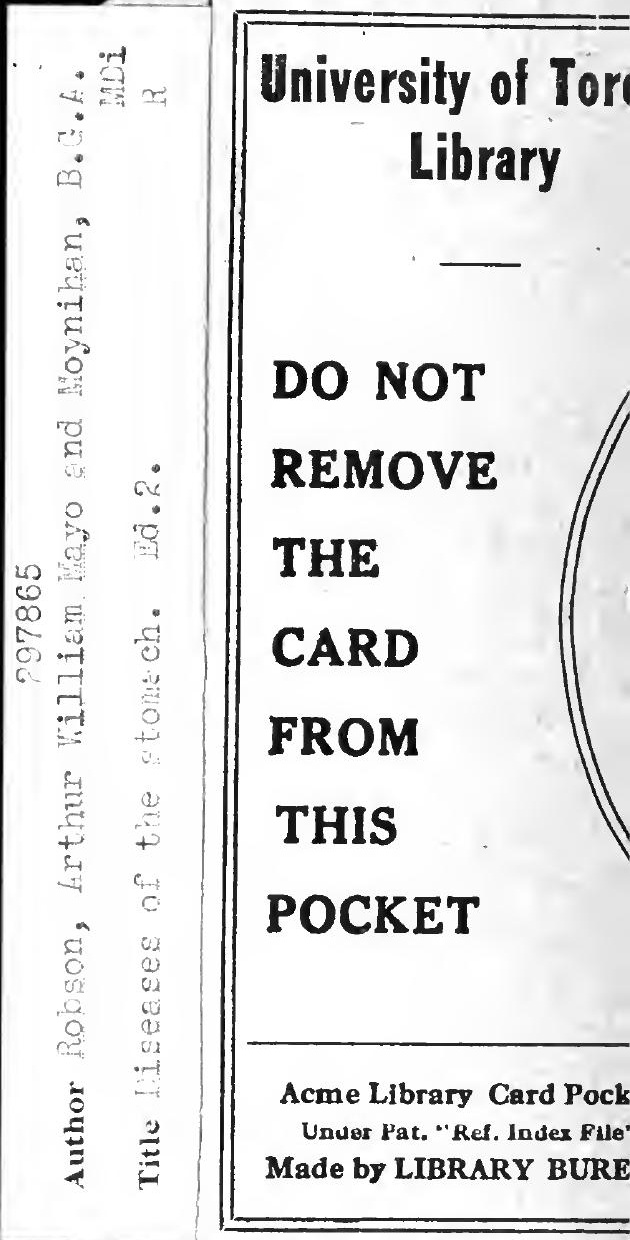


